\title{
Magnitude and Frequency of Floods in Alabama, 2015
}

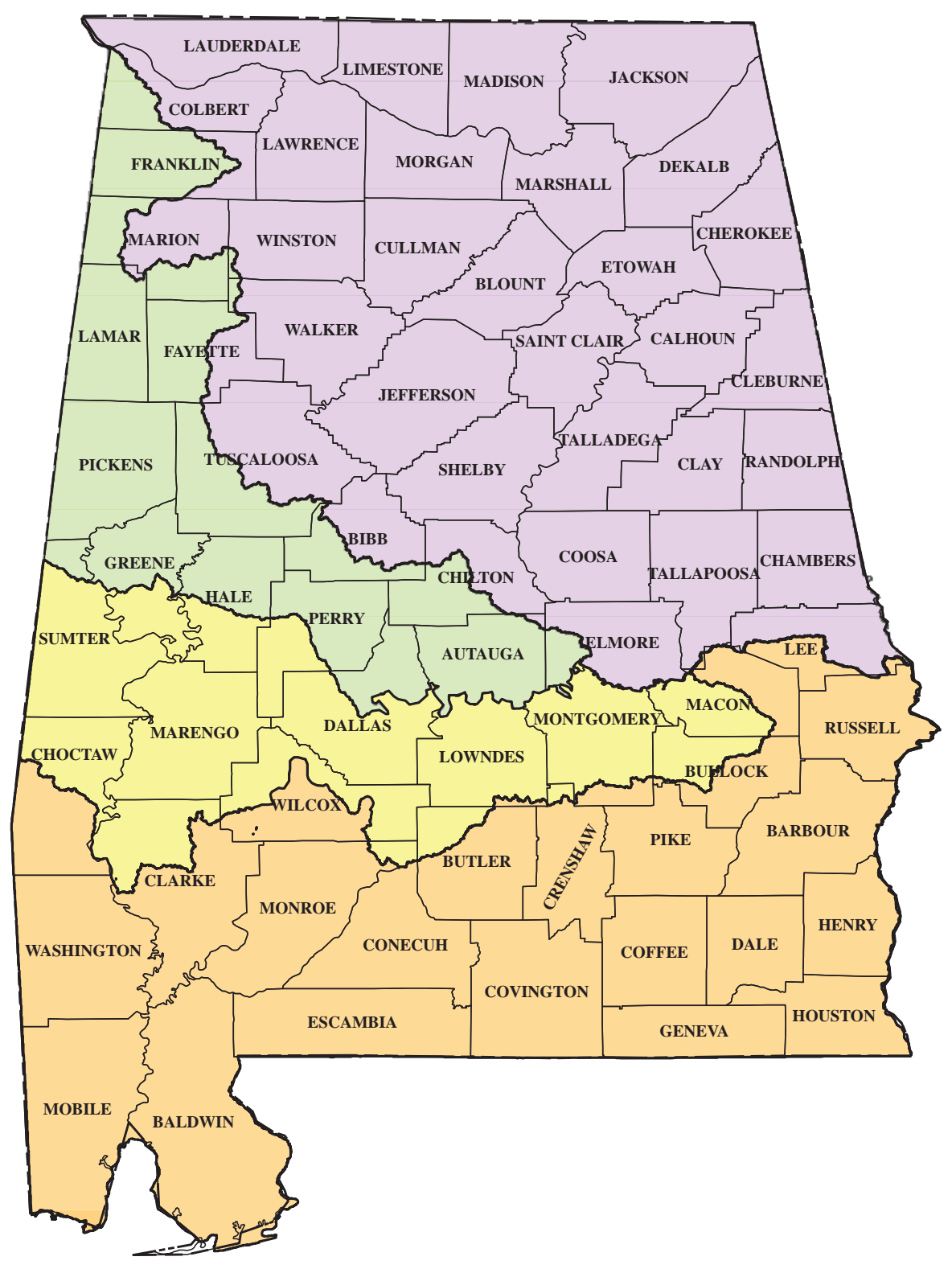

Scientific Investigations Report 2020-5032 



\title{
Magnitude and Frequency of Floods in Alabama, 2015
}

\author{
By Brandon T. Anderson
}

Prepared in cooperation with the Alabama Department of Transportation

Scientific Investigations Report 2020-5032 


\title{
U.S. Department of the Interior \\ DAVID BERNHARDT, Secretary
}

\author{
U.S. Geological Survey \\ James F. Reilly II, Director
}

U.S. Geological Survey, Reston, Virginia: 2020

For more information on the USGS - the Federal source for science about the Earth, its natural and living resources, natural hazards, and the environment-visit https://www.usgs.gov or call 1-888-ASK-USGS.

For an overview of USGS information products, including maps, imagery, and publications,

visit https://store.usgs.gov.

Any use of trade, firm, or product names is for descriptive purposes only and does not imply endorsement by the U.S. Government.

Although this information product, for the most part, is in the public domain, it also may contain copyrighted materials as noted in the text. Permission to reproduce copyrighted items must be secured from the copyright owner.

Suggested citation:

Anderson, B.T., 2020, Magnitude and frequency of floods in Alabama, 2015: U.S. Geological Survey Scientific Investigations Report 2020-5032, 148 p., https://doi.org/10.3133/sir20205032.

Associated data for this publication:

Anderson, B.T., 2020, Flood regions and annual exceedance probability flows for Alabama streams, data through 2015: U.S. Geological Survey data release, https://doi.org/10.5066/P9TYSZLL.

ISSN 2328-031X (print)

ISSN 2328-0328 (online)

ISBN 978-1-4113-4370-2 


\section{Acknowledgments}

The following U.S. Geological Survey personnel are acknowledged for their assistance in developing the computations for this project and helping to complete the report. T. Scott Hedgecock, hydrologist in the U.S. Geological Survey Lower Mississippi-Gulf Water Science Center (USGS LMGWSC) Montgomery, Alabama, office, provided mentorship during the project. Toby Feaster, hydrologist in the U.S. Geological Survey South Atlantic Water Science Center (USGS SAWSC) Columbia, South Carolina, office, provided analysis and writeup for large river sites. 



\section{Contents}

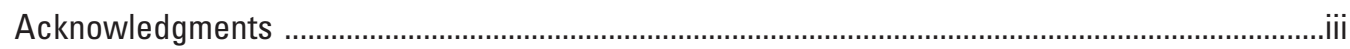

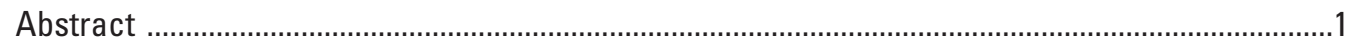

Introduction

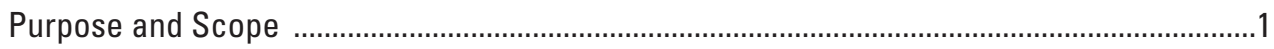

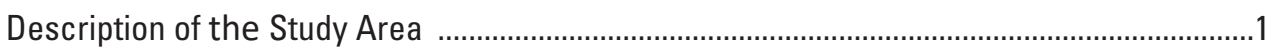

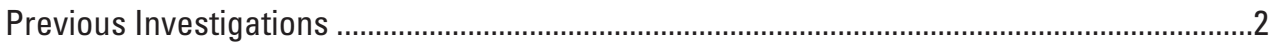

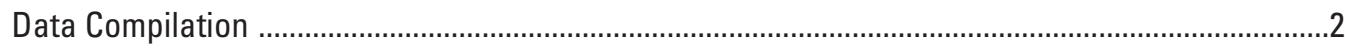

Peak-Flow Data and Basin Characteristics ......................................................................

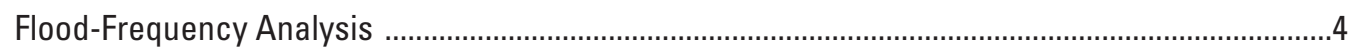

Analysis of Flow at Streamgages .................................................................................

Regional Regression Analysis ......................................................................................

Application and Limits of Methods ...............................................................................

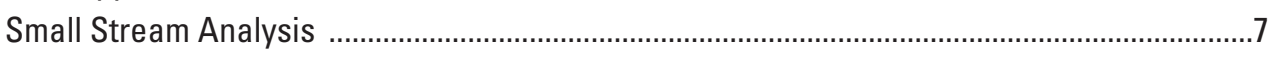

Large River Analysis .......................................................................................................

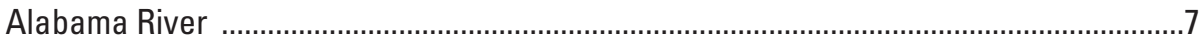

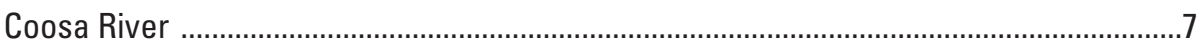

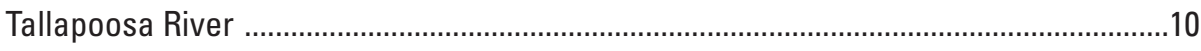

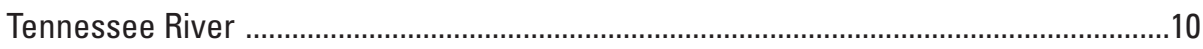

Tombigbee River ..........................................................................................................

Black Warrior River .........................................................................................................

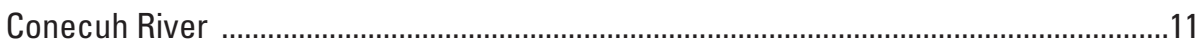

Flood-Frequency Estimates at Streamgages in Alabama ..........................................................12

Flood-Frequency Estimates at Ungaged Locations on Gaged Streams ........................................12

Flood-Frequency Estimates at Locations on Ungaged Streams .................................................13

Accuracy and Limitations of Regional Regression Equations ........................................................13

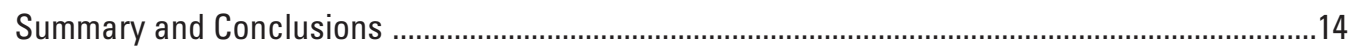

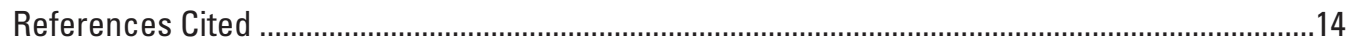

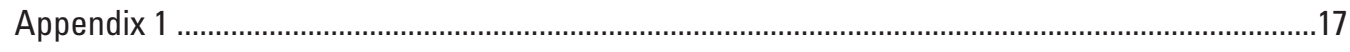

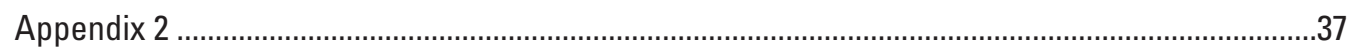

\section{Plate}

[Plate in pocket and available at https://doi.org/10.3133/sir20205032]

1. Locations of flood regions and streamgages in Alabama.

\section{Figures}

1. Map showing locations of streamgages used in regional regression analysis and selected large-river streamgages in Alabama .............................................................

2. Map showing locations of flood regions in Alabama .................................................

3. Map showing locations of flood regions and small streamgages in Alabama ................8 


\section{Tables}

1. Regional flood-frequency relations for urban streams in Alabama

2. $T$-year recurrence intervals with corresponding annual exceedance probability and $P$-percent chance exceedance for flood-frequency flow estimates

3. Final regional regression equations for estimating annual exceedance probability flows and generalized least squares model diagnostics for unregulated streams in Alabama

4. Small stream regional regression equations for estimating annual exceedance probability flows and generalized least squares model diagnostics for unregulated small streams in Alabama

5. Ranges of expanatory variable data used to develop regional regression equations

\section{Conversion Factors}

U.S. customary units to International System of Units

\begin{tabular}{lcl}
\hline & Multiply & \multicolumn{1}{c}{ To obtain } \\
\hline & Length & \\
\hline inch (in.) & 2.54 & centimeter $(\mathrm{cm})$ \\
inch (in.) & 25.4 & millimeter $(\mathrm{mm})$ \\
foot (ft) & 0.3048 & meter $(\mathrm{m})$ \\
mile (mi) & 1.609 & kilometer $(\mathrm{km})$ \\
\hline & Area & \\
\hline square mile $\left(\mathrm{mi}^{2}\right)$ & 259.0 & hectare $($ ha) \\
square mile $\left(\mathrm{mi}^{2}\right)$ & 2.590 & square kilometer $\left(\mathrm{km}^{2}\right)$ \\
\hline & Flow rate & \\
\hline cubic foot per second $\left(\mathrm{ft}^{3} / \mathrm{s}\right)$ & 0.02832 & cubic meter per second $\left(\mathrm{m}^{3} / \mathrm{s}\right)$ \\
\hline & Hydraulic gradient & \\
\hline foot per mile $(\mathrm{ft} / \mathrm{mi})$ & 0.1894 & meter per kilometer $\left(\mathrm{m} / \mathrm{km}^{2}\right)$ \\
\hline
\end{tabular}

Temperature in degrees Fahrenheit $\left({ }^{\circ} \mathrm{F}\right)$ may be converted to degrees Celsius $\left({ }^{\circ} \mathrm{C}\right)$ as follows:

$$
{ }^{\circ} \mathrm{C}=\left({ }^{\circ} \mathrm{F}-32\right) / 1.8 \text {. }
$$

\section{Datum}

Horizontal coordinate information is referenced to the North American Datum of 1983 (NAD 83).

Vertical coordinate information is referenced to the National Geodetic Vertical Datum of 1929 (NGVD 29).

Elevation, as used in this report, refers to distance above the vertical datum. 


\section{Abbreviations}

$\begin{array}{ll}\text { AEP } & \text { annual exceedance probability } \\ \text { AVP } & \text { average variance of prediction } \\ \text { EMA } & \text { Expected Moments Algorithm } \\ \text { GIS } & \text { geographic information system } \\ \text { GLS } & \text { generalized least squares } \\ \text { LP3 } & \text { log-Pearson type III } \\ \text { MGB } & \text { multiple Grubbs-Beck test } \\ \text { MSE } & \text { mean square error } \\ \text { MSEp } & \text { mean square error of prediction } \\ \text { NWIS } & \text { National Water Information System } \\ \text { OLS } & \text { ordinary least squares } \\ \text { PILFs } & \text { potentially influential low floods } \\ \text { RRE } & \text { regional regression equation } \\ \text { SEP } & \text { standard error of prediction } \\ \text { USGS } & \text { U.S. Geological Survey } \\ \text { WREG } & \text { weighted-multiple-linear regression program }\end{array}$





\title{
Magnitude and Frequency of Floods in Alabama, 2015
}

\author{
By Brandon T. Anderson
}

\section{Abstract}

To improve flood-frequency estimates at rural streams in Alabama, annual exceedance probability flows at gaged locations and regional regression equations used to estimate annual exceedance probability flows at ungaged locations were developed by using current geospatial data, new analytical methods, and annual peak-flow data through September 2015 at 242 streamgages in Alabama and surrounding States. The regional regression equations were derived from statistical analyses of annual peak-flow data and basin characteristics for a subset of 217 streamgages. Four flood regions were identified based on residuals from the regional regression analyses and contain sites with similar basin characteristics. A separate set of equations was derived for estimating flood frequency and magnitude for small rural streams using a subset of 40 small basin streamgages. A large river analysis was also completed for 14 selected large-river streamgages in Alabama. Annual exceedance probability flows presented in this report reflect additional streamflow data collected since the previous study of flood magnitude and frequency in Alabama, which included streamflow through September 2003.

\section{Introduction}

Improved flood-frequency information is important for the effective management of flood plains, including the safe and economic design of bridges, culverts, dams, levees, and other structures near streams. The last flood-frequency study for Alabama was published more than 11 years ago (Hedgecock and Feaster, 2007). Since that time, improvements in statistical techniques, specifically the Expected Moments Algorithm (EMA) and the multiple Grubbs-Beck (MGB) test for potentially influential low floods, have increased the accuracy of flood-frequency estimates (Cohn and others, 1997, 2013). The EMA allows for the incorporation of censored observations, historic flood data, low outliers, and uncertain data points in the flood-frequency analysis, while the MGB test increases the accuracy of peak-flow statistics by objectively and systematically detecting and removing low, highly variable peak flows and is recommended for use with the EMA.
In 2016, the U.S. Geological Survey (USGS), in cooperation with the Alabama Department of Transportation, began a study to update the regional flood-frequency equations (regional regression equations [RREs]) and annual exceedance probability (AEP) flows for rural streams in Alabama by using recent geospatial data, new analytical methods, and additional annual peak-flow data through the 2015 water year. ${ }^{1}$ Results of flood frequency studies, including AEP flows and RRE information, are published and also incorporated into the USGS StreamStats application, an online tool that provides flood-response planners and water managers in Alabama with basin characteristics and estimates of flow statistics at locations on both gaged and ungaged streams (http://water. usgs.gov/osw/streamstats/; USGS, 2017a).

\section{Purpose and Scope}

The purpose of this report is to describe the methods and results of a study to (1) update flood regions for all areas in Alabama; (2) update the AEP flows for rural streams at gaged locations; (3) update RREs for use at rural ungaged locations in Alabama; and (4) update the AEP flows for streamgages on large, regulated streams. All data used in support of the analysis and presented in this report, including geographic information system (GIS) data for the four flood regions in Alabama, are available from Anderson (2020). Urban stream flood frequency equations were not updated as part of this study and the results from Hedgecock and Lee (2010) are provided in table 1 for convenience. This report updates the flood-frequency analysis published by Hedgecock and Feaster (2007).

\section{Description of the Study Area}

The study area (fig. 1) includes the State of Alabama and selected locations in Georgia, Mississippi, Tennessee, and Florida. Rainfall in Alabama generally is associated with the movement of warm and cold fronts across the State from November through April and isolated thunderstorms from May through October. From June to September, tropical storms or

\footnotetext{
${ }^{1}$ The water year is the annual period from October 1 through September 30 and is designated by the year in which the period ends. For example, the 2013 water year is from October 1, 2012, through September 30, 2013.
} 
Table 1. Regional flood-frequency relations for urban streams in Alabama (Hedgecock and Lee, 2010).

$[Q$, flood flow, in cubic feet per second; $A$, contributing drainage area, in square miles; $P D$, percentage of basin developed]

\begin{tabular}{cl}
\hline $\begin{array}{c}\text { Exceedance probability } \\
\text { (percent) }\end{array}$ & Urban regression equations \\
\hline 50 & $Q=95 A^{0.648} P D^{0.407}$ \\
20 & $Q=226 A^{0.670} P D^{0.298}$ \\
10 & $Q=306 A^{0.675} P D^{0.276}$ \\
4 & $Q=417 A^{0.670} P D^{0.253}$ \\
2 & $Q=513 A^{0.663} P D^{0.237}$ \\
1 & $Q=618 A^{0.656} P D^{0.223}$ \\
0.5 & $Q=733 A^{0.650} P D^{0.210}$ \\
0.2 & $Q=897 A^{0.642} P D^{0.196}$ \\
\hline
\end{tabular}

hurricanes occasionally enter the State along the Gulf Coast and produce unusually large amounts of rainfall. The average annual precipitation for Alabama is 53.05 inches (U.S. Climate Data, 2018). The average annual high and low temperatures are 76.5 and 53.5 degrees Fahrenheit, respectively (U.S. Climate Data, 2018).

\section{Previous Investigations}

Magnitude and frequency of floods in Alabama have been described by Pierce (1954), Speer and Gamble (1964), Gamble (1965), Barnes and Golden (1966), Hains (1973), Olin (1984), Atkins (1996), and Hedgecock and Feaster (2007). Magnitude and frequency of floods for rural streams with small drainage areas have been described by Olin and Bingham (1977) and Hedgecock (2004), and for urban streams by Olin and Bingham (1982) and Hedgecock and Lee (2010).

\section{Data Compilation}

USGS streamgages in Alabama, Georgia, Mississippi, Florida, and Tennessee that had 15 or more years of annual peak-flow data were used in this analysis. A 50-mile (mi) buffer was used from the Alabama State boundary for rural streams, and a 90-mi buffer was used for small rural streams. The gages used in this study were either continuous-record or crest-stage gages. Continuous-record gages are equipped with instrumentation to record the height of the water surface above the gage datum, or stage, of the water body at fixed time intervals. The stage data are transmitted by satellite to USGS offices and are applied to a stage-streamflow rating to determine flow for the given stage value. Crest-stage gages record only the peak stage of a flood; the peak stage is then applied to a stage-streamflow rating to determine the associated flow. Hereinafter, these two types of gages collectively are referred to as streamgages. A rainfall event of high magnitude occurred in the latter part of December 2015 for many gaged locations in Alabama. This was the peak of the 2016 water year and was included in the analysis for those locations.

\section{Peak-Flow Data and Basin Characteristics}

The RRE analysis was conducted using data from 217 streamgages (152 in Alabama and 65 from adjacent States) that had 15 or more years of annual peak-flow data collected through the end of the 2015 water year (Anderson, 2020). Annual peak-flow data for the streamgages were downloaded from the USGS National Water Information System (NWIS) database (U.S. Geological Survey, 2017b). The drainage areas of the 217 streamgages range from 0.13 to 1,766 square miles $\left(\mathrm{mi}^{2}\right)$. In addition, there were 14 large river streamgages, included in a separate analysis, whose drainage areas range from 1,675 to $30,810 \mathrm{mi}^{2}$.

Basin characteristics for each streamgage included in this study were obtained by using the USGS map-based web application StreamStats (U.S. Geological Survey, 2017a). The following basin characteristics were tested for significance in the generalized least-squares (GLS) regression analysis:

- Contributing drainage area $(A)$, in square miles, upstream from the streamgage;

- Main channel slope $(S)$, in feet per mile, between points 10 and 85 percent of the distance from the streamgage to the basin divide;

- Main channel length $(L)$, in miles, between the streamgage and the basin divide;

- Lag-time factor (T), defined by the ratio $L / S^{0.5}$ with $L$ and $S$ defined above;

- Forest cover $(F)$, in percent, percentage of the total contributing drainage area covered by forests;

- Storage $(S t)$, in percent, percentage of the total contributing drainage area covered by lakes, ponds, and swamps; and

- Width-to-length ratio $(W / L)$, the average basin width to basin length. The average basin width $(W)$ is the drainage area $(A)$ divided by the main channel length $(L)$. This ratio is essentially a basin shape factor. 


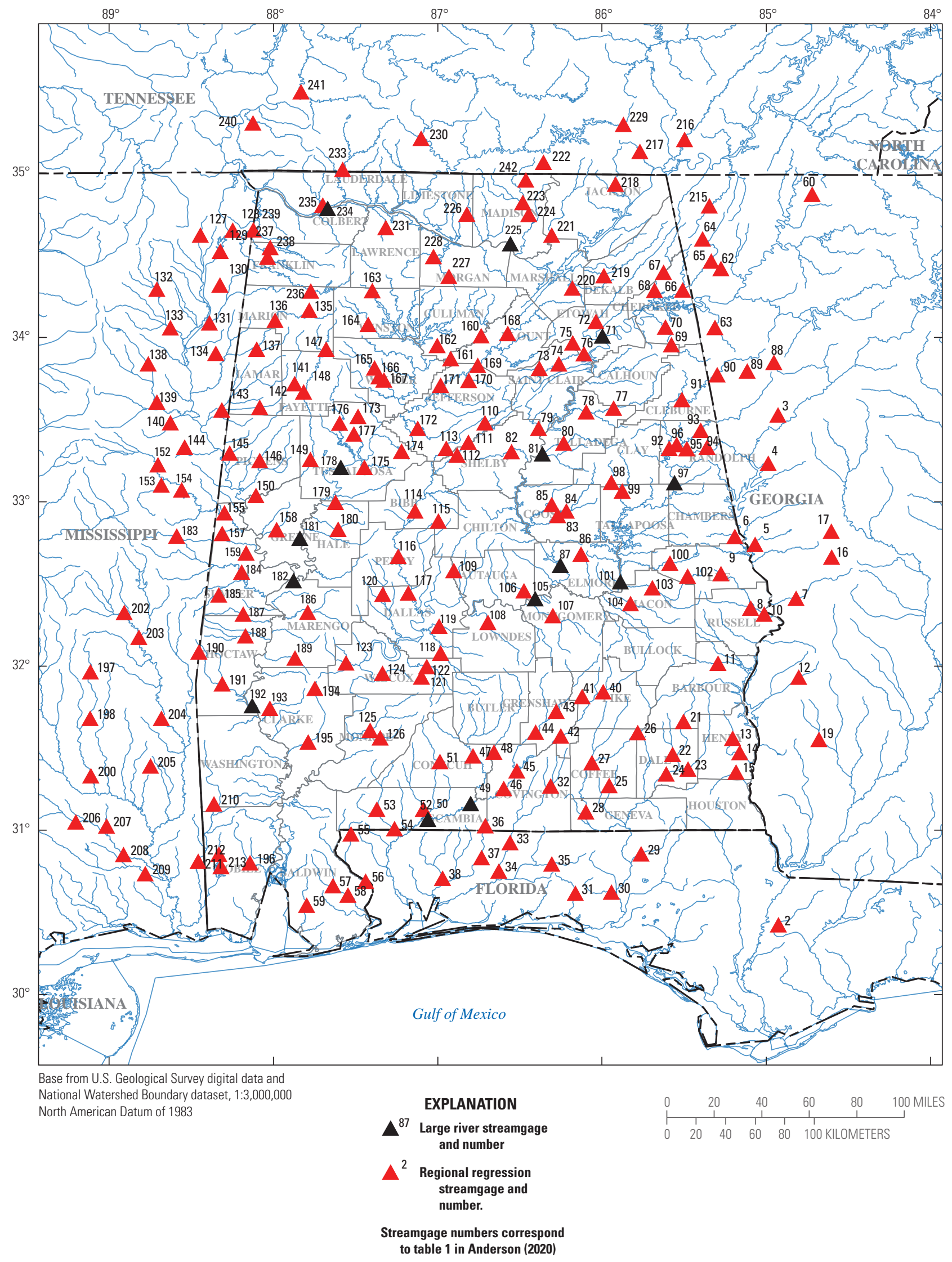

Figure 1. Locations of streamgages used in regional regression analysis and selected large-river streamgages in Alabama. 
Percentage of developed (urban) land and average percentage of impervious area taken from the 2011 National Land Cover Database and available in StreamStats, were computed for each basin because soil types (Hedgecock and Feaster, 2007) and land use can influence runoff. Initial GLS regression analyses were performed for all the streamgages included in the study, incorporating multiple combinations of the above-mentioned explanatory variables. These regressions were used in the development of statewide equations for estimating AEPs. Statewide regression equations that included drainage area as the only explanatory variable had standard errors of prediction that were within 1 percent of the standard errors, produced from equations that included main channel slope, forest cover, and storage. Therefore, equations that included drainage area as the only explanatory variable were used for regionalization purposes.

\section{Flood-Frequency Analysis}

Annual peak flood flow data for the streamgages in this study were analyzed to produce AEP flows for each streamgage. Results are available from Anderson (2020) and can be found in Appendix 1. A regional analysis was conducted based on the residuals of the streamgages in order to group basins with similar hydrologic characteristics. As a result, four flood regions were determined from the regional analysis, and regional flood frequency equations for ungaged streams were developed from the annual peak flow data at streamgages that fell within these regions. Separate studies were also conducted to derive equations for small stream basins and update the previously published large river basins analysis.

\section{Analysis of Flow at Streamgages}

For each streamgage, a log-Pearson type III (LP3) mathematical probability distribution was fit to annual peak flow data and then used to estimate streamflow values for the range of recurrence-intervals at each streamgage as described in Bulletin 17C (England and others, 2019). The LP3 distribution is a three-parameter distribution that requires estimates of the mean, standard deviation, and skew coefficient of the population of base 10 logarithms of annual peak flows at each streamgage (Parrett and others, 2011). The EMA method improves upon the standard LP3 method by allowing for the analysis of historic peak datasets containing censored observations, historic data, low outliers, and uncertain data points and accommodates the interval data by using perception thresholds and flow intervals (Cohn and others, 1997). In the EMA analysis the perception thresholds are used to describe the flood knowledge in each year within the flood record and represents the observable range in floods (England and others, 2019). If no historic, censored, or interval data are incorporated, the EMA method produces estimates of the three LP3 statistics that are identical to those produced by the standard LP3 method described in Bulletin 17B (Interagency Advisory Committee on Water Data, 1982). Bulletin 17C did not publish regional skew values therefore general-skew and corresponding mean square error (MSE) values from Bulletin 17B were used to weight the AEP flow values.

The basic equation for fitting the LP3 distribution to a measured series of annual peak flows is

$$
\log Q_{p}=\overline{\mathrm{X}}+K_{p} S,
$$

where

$$
\begin{aligned}
& Q_{p} \quad \text { is the } P \text {-percent AEP flow, in cubic feet per } \\
& \text { second; } \\
& \overline{\mathrm{X}} \quad \text { is the mean of the logarithms of the annual } \\
& \text { peak flows; } \\
& K_{p} \quad \text { is a factor based on the skew coefficient and } \\
& \text { the given percentage of annual exceedance } \\
& \text { probability, which can be obtained from } \\
& \text { appendix 3 of Bulletin 17B (Interagency } \\
& \text { Advisory Committee on Water Data, } \\
& \text { 1982); and } \\
& \text { is the standard deviation of the logarithms of } \\
& \text { the annual peak flows. }
\end{aligned}
$$

In previous USGS reports about floods in Alabama, the term "recurrence interval, in years" was used to characterize flood frequency (50-year flood and so forth). The USGS and other Federal agencies now refer to the $P$-percent chance of occurrence as an AEP. For example, the $0.02 \mathrm{AEP}\left(\mathrm{Q}_{2 \%}\right)$ has a 2-percent chance of occurring in any given year and corresponds to a recurrence interval of 50 years (reciprocal of the AEP, table 2) (Griffis and Stedinger, 2007). An increase in the number of years of annual peak-flow record at a streamgage increases the level of confidence in AEP flow estimates. For example, 30 years of annual peak-flow record likely will have a lower variance than 10 years of record, thus increasing the confidence of the estimated AEP flows.

Table 2. T-year recurrence intervals with corresponding annual exceedance probability and $P$-percent chance exceedance for flood-frequency flow estimates.

\begin{tabular}{ccc}
\hline $\begin{array}{c}\text { Corresponding } \\
\text { recurrence } \\
\text { interval }\end{array}$ & $\begin{array}{c}\text { Annual } \\
\text { exceedance } \\
\text { probability }\end{array}$ & $\begin{array}{c}\text { P-percent annual } \\
\text { exceedance } \\
\text { probability }\end{array}$ \\
\hline 2 & 0.5 & 50 \\
5 & 0.2 & 20 \\
10 & 0.1 & 10 \\
25 & 0.04 & 4 \\
50 & 0.02 & 2 \\
100 & 0.01 & 1 \\
200 & 0.005 & 0.5 \\
500 & 0.002 & 0.2 \\
\hline
\end{tabular}


The MGB test, a generalization of the Grubbs-Beck method, provides a standard procedure for identifying lowflow outliers and multiple potentially influential low floods (PILFs) (Cohn and others, 2013). PILFs are annual peaks that meet three criteria: (1) their magnitude is much smaller than the flood quantile of interest; (2) they occur below a statistically significant break in the flood-frequency plot; and (3) they can have excessive influence on the estimated frequency of large floods. The USGS PeakFQ software, version 7.1, available at https://water.usgs.gov/software/ PeakFQ/ (U.S. Geological Survey, 2014), was used to conduct the flood-frequency analyses following the EMA methodology, including the MGB test. The estimates of the AEP flows at gaged sites were computed by using the EMA and MGB to test for PILFs. These PILFs were excluded from the AEP flow computations at the streamgage. The final AEP flows at streamgages should be determined by weighting the station skew coefficient with the generalized skew coefficient from Bulletin 17B (Interagency Advisory Committee on Water Data, 1982).

\section{Regional Regression Analysis}

Initially, 242 streamgages were considered for inclusion in the regional regression analysis. Streamgage information and additional data related to the regional regression analysis are provided in Anderson (2020). Annual peak-flow records of the streamgages were evaluated for backwater, regulation, diversion, channelization, and urbanization by inspecting NWIS peak-flow data qualification codes, and sites having any of these attributes were removed. Annual peak-flow data for the sites are available from the NWIS database (U.S. Geological Survey, 2017b) and can also be found in Appendix 2. Streamgages in basins that have more than 10 percent impervious area were also removed, leaving 217 streamgages suitable for use in the regional regression analysis (fig. 1).

To determine the flood regions, a statewide ordinary least squares (OLS) regression equation was developed by using drainage area as the only explanatory variable for the 1-percent AEP $\left(Q_{1 \%}\right)$. The residuals for each streamgage, which represent the difference between observed values and the predicted values of streamflow, were evaluated to detect any geographic biases or clusters. Four flood regions were delineated in Alabama (fig. 2), based upon review of residual plots, previous reports, eight-digit hydrologic unit code maps, geologic maps, and physiographic maps. The four flood regions in this report differ slightly from the previous report by Hedgecock and Feaster (2007). These differences include a streamgage originally located in region 1 (USGS station no. 02445245, plate 1; Hedgecock and Feaster, 2007) that is now located in region 2 . One streamgage originally located in region 4 (USGS station no. 02419000, plate 1; Hedgecock and Feaster, 2007) is now located in region 3.

AEP estimates obtained from flood-frequency analysis of the 217 streamgages were related to basin characteristics by using OLS multiple linear regression analysis to evaluate the statistical significance of each basin characteristic (Wagner and others, 2016). The USGS weighted-multiple-linear regression program (WREG) version 1.05 (https://water. usgs.gov/software/WREG/) was then used to complete the final GLS regression analysis (Eng and others, 2009; U.S. Geological Survey, 2013). In GLS regression, streamgages are weighted according to differences in streamflow record length, the variance of streamflow measurements in the record, and spatial cross correlations of concurrent flows among streamgages.

Regression diagnostics were reviewed to identify streamgages that have high leverage and (or) high influence metrics. The leverage metric was used to compare the values of independent variables at one streamgage to the values of the same variables at all other streamgages. The influence metric was used to determine if a streamgage had a high influence on the estimated regression values (Eng and others, 2009). A streamgage may have a high leverage metric, indicating that its independent variables are significantly different from those at all other streamgages, but the same streamgage may not have a high influence on the regression metrics. A streamgage with a high influence may not have a high leverage metric. Sometimes high leverage or influence metrics are indicative of incorrect values for a given independent variable. These data were reviewed resulting in one site being removed from the dataset, leaving a total of 217 streamgages used in the analysis. Standard errors of prediction of the generalized least-squares models ranged from 9 to 58 percent. Pseudo coefficients of determination of the models ranged from 75 to 100 percent.

\section{Application and Limits of Methods}

When applying the RREs, users are advised not to interpret the empirical results as exact. Regression equations are statistical models that must be interpreted and applied within the limits of the data and with the understanding that the results are best-fit estimates with an associated variance. Methods for estimating AEP flows in Alabama differ between gaged locations, ungaged locations on gaged streams, and locations on ungaged streams. 


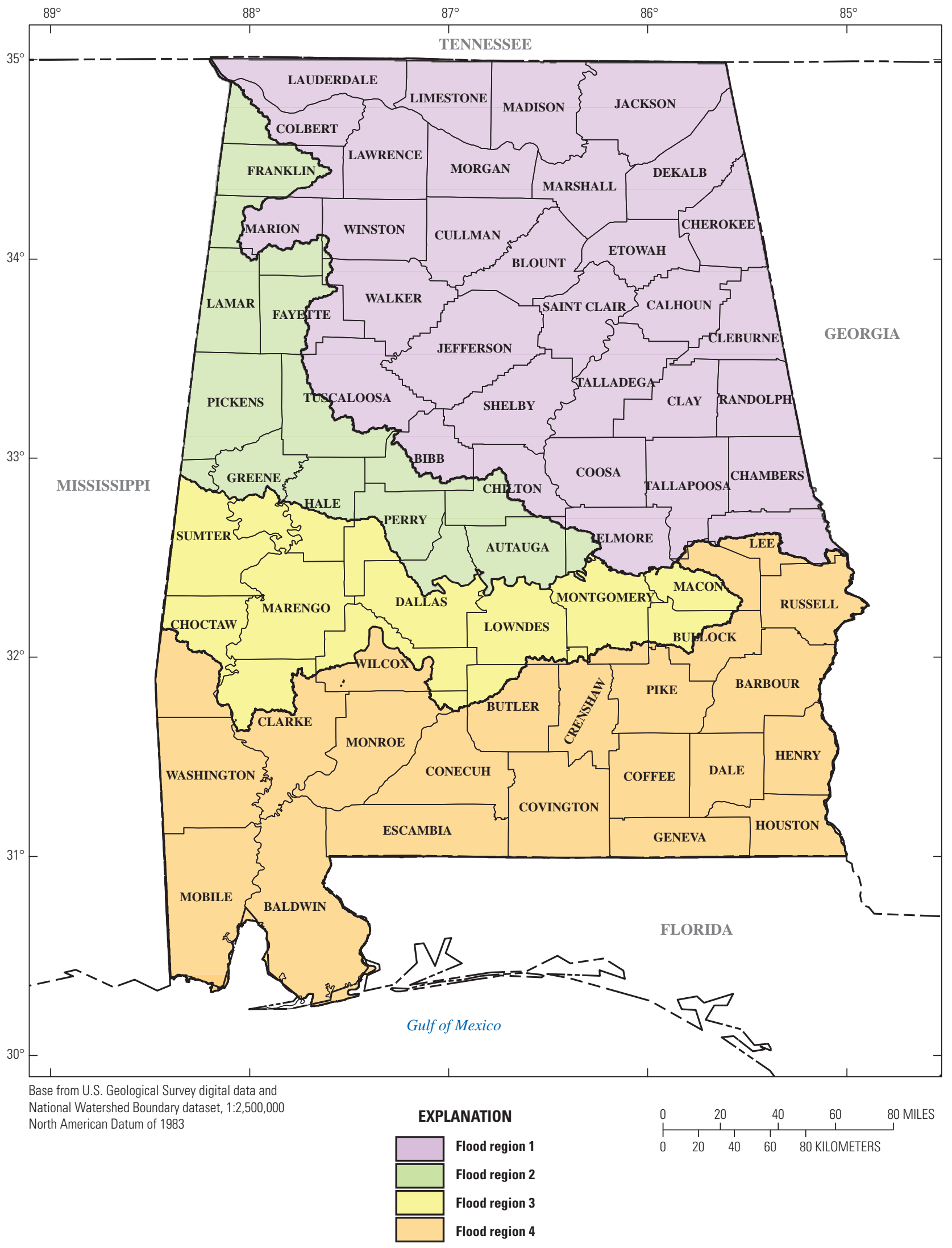

Figure 2. Locations of flood regions in Alabama. 


\section{Small Stream Analysis}

Data from 40 streamgages were used to conduct a separate GLS regression analysis on the rural small streams in Alabama with drainage areas ranging from 0.13 to $14 \mathrm{mi}^{2}$. The distance outside of the Alabama State line for streamgage consideration was increased from $50 \mathrm{mi}$ used in the streamgage selection for the regional equations to $90 \mathrm{mi}$. This change in distance was needed to increase the number of streamgages in the analysis (fig. 3). If a rural ungaged small stream is within 0.13 to $14 \mathrm{mi}^{2}$, AEPs could be computed with either regional or small stream equations (tables 3 and 4, respectively). It is recommended that if the drainage area at the ungaged location is within the range of 0.13 to $5 \mathrm{mi}^{2}$, then the small stream equations should be used because the AEP accuracy is improved.

\section{Large River Analysis}

Flood-frequency analysis for streamgages along the main stems of the Alabama, Coosa, Conecuh, Tallapoosa, Tennessee, Tombigbee, and Black Warrior Rivers were not included in the regional flood-frequency analysis because the drainage areas of these rivers are substantially larger than those of the streamgages used in the regional analyses and, in some cases, encompass more than one flood region. In addition, most of these large rivers are subject to varying degrees of regulation. Where the data analysis indicated it was appropriate, analysis of flood magnitudes to drainage area were determined for selected AEPs. The flood-frequency analyses are summarized in the following sections for each river listed and AEP flows are available from Anderson (2020) and in Appendix 1. The estimated AEP flows were based on LP3 analysis of peak-flow data at the gaged sites on the streams, as described in Bulletin 17C (England and others, 2019). Graphical inspection of the data from each analysis indicated that an LP3 distribution yielded a reasonable fit of the frequency curves for the selected streamgages. Because most of these large rivers have some degree of regulation, trend analyses were performed on the annual peak flows for the selected streamgages to determine if regulated flow patterns have changed with time. Trends in regulation were assessed by using the Mann-Kendall test and cumulative plots of daily mean flows (single mass curves, Helsel and Hirsch, 1995). The single mass curve is a basic analytical tool showing a plot of cumulative values against time. The slope of the mass curve represents the constant of proportionality between the two quantities (Searcy and Hardison, 1960). A change in the slope of the curve indicates a change in the proportionality constant. In the case of regulated streams, the single mass curve can be used to assess whether patterns of regulation have remained relatively consistent over time. For the large rivers, a flood-frequency analysis was performed if the annual peak-flow record at a streamgage showed no trend in either the entire dataset or a part of the dataset. AEP estimates at multiple gages on a stream will vary as a result of several factors, including length of the annual peak-flow records, concurrent periods of record, intervening tributaries, and areal coverage of storm systems causing the floods. For example, if a storm system affected only the lower half of a large drainage basin, streamgages in the upper half of the basin would not record runoff from the storm. The flood of record at a streamgage in the lower half of the basin may not necessarily be the flood of record at a streamgage in the upper half of the basin. As a result of these factors, interpolation of AEP estimates between two streamgages on the same stream may not be linear. However, linear interpolation between two streamgages with relatively similar lengths of record should provide a reasonable estimate of flood magnitude for locations between the two streamgages.

\section{Alabama River}

Flow in the Alabama River is regulated by upstream reservoirs on both the Coosa and Tallapoosa Rivers. For the Alabama River near Montgomery (USGS station no. 02420000 , plate 1), two different datasets were analyzed (1886-2015 and 1928-2015) that represent different periods of record with varying degrees of regulation. The 1928-2015 dataset represents the period of regulation and was used for this analysis.

No new data were available for the following streamgages on the Alabama River: Alabama River at Selma (USGS station no. 02423000), Alabama River near Millers Ferry (USGS station no. 02427500), and Alabama River at Claiborne (USGS station no. 02429500). Because no new analyses were conducted, the values found in the previous report (Hedgecock and Feaster, 2007) should be used.

\section{Coosa River}

Flow in the Coosa River is regulated by reservoirs at Carters Pond, Allatoona, Weiss, H. Neely Henry, Logan Martin, Lay, Mitchell, and Jordan Dams. For the Coosa River at Gadsden (USGS station no. 02400500, plate 1), no additional data had been collected since the last floodfrequency report (Hedgecock and Feaster, 2007); therefore, no new analysis was done. 


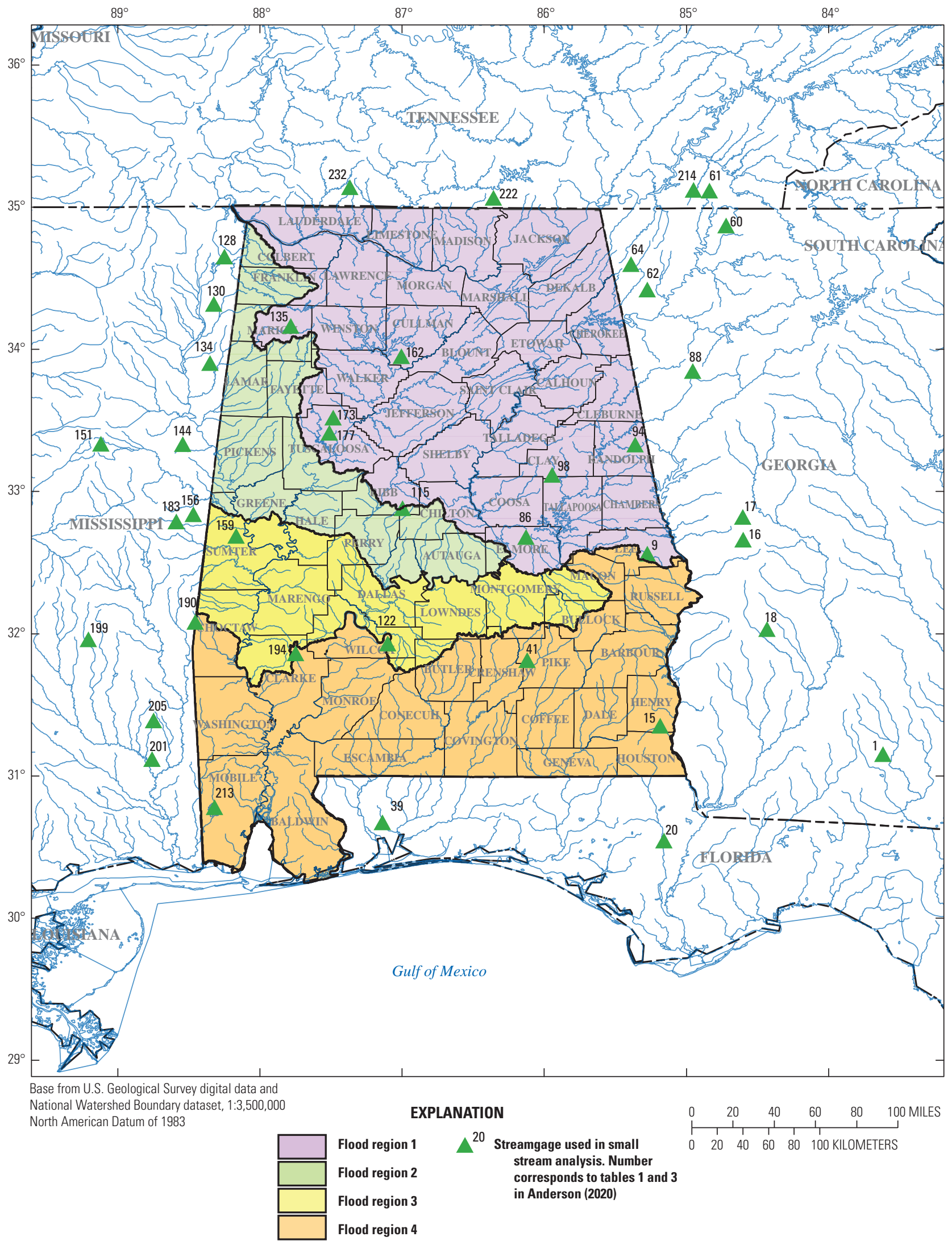

Figure 3. Locations of flood regions and small streamgages in Alabama. 
Table 3. Final regional regression equations for estimating annual exceedance probability flows and generalized least squares model diagnostics for unregulated streams in Alabama.

[MSE, mean square error in $\log 10$ cubic feet per second; $\mathrm{ft}^{3} / \mathrm{s}$, cubic foot per second; AVP, average variance of prediction in $\log 10$ cubic feet per second; SEP, standard error of prediction; pseudo- $R^{2}$, pseudo coefficient of determination; $A$, contributing drainage area in square miles]

\begin{tabular}{|c|c|c|c|c|}
\hline $\begin{array}{c}\text { Annual exceedance } \\
\text { probability flow equation }\end{array}$ & $\begin{array}{c}\text { MSE } \\
\left(\log \mathrm{ft}^{3} / \mathrm{s}\right)\end{array}$ & $\begin{array}{c}\text { AVP } \\
\left(\log \mathrm{ft}^{3} / \mathrm{s}\right)^{2}\end{array}$ & $\begin{array}{c}\text { SEP } \\
\text { (percent) }\end{array}$ & $\begin{array}{l}\text { Pseudo- } R^{2} \\
\text { (percent) }\end{array}$ \\
\hline \multicolumn{5}{|c|}{ Flood region 1 (90 streamgages) } \\
\hline$Q_{50 \%}=235(A)^{0.668}$ & 0.018 & 0.016 & 29 & 93 \\
\hline$Q_{20 \%}=424(A)^{0.646}$ & 0.017 & 0.014 & 27 & 93 \\
\hline$Q_{10 \%}=568(A)^{0.638}$ & 0.017 & 0.013 & 27 & 93 \\
\hline$Q_{4 \%}=762(A)^{0.632}$ & 0.019 & 0.014 & 28 & 93 \\
\hline$Q_{2 \%}=916(A)^{0.630}$ & 0.021 & 0.015 & 28 & 92 \\
\hline$Q_{1 \%}=1,076(A)^{0.628}$ & 0.023 & 0.015 & 29 & 92 \\
\hline$Q_{0.5 \%}=1,239(A)^{0.627}$ & 0.025 & 0.017 & 30 & 91 \\
\hline$Q_{0.2 \%}=1,462(A)^{0.627}$ & 0.029 & 0.018 & 32 & 90 \\
\hline \multicolumn{5}{|c|}{ Flood region 2 (33 streamgages) } \\
\hline$Q_{50 \%}=215(A)^{0.637}$ & 0.044 & 0.044 & 51 & 91 \\
\hline$Q_{20 \%}=340(A)^{0.646}$ & 0.029 & 0.026 & 38 & 95 \\
\hline$Q_{10 \%}=437(A)^{0.650}$ & 0.022 & 0.019 & 32 & 96 \\
\hline$Q_{4 \%}=570(A)^{0.653}$ & 0.018 & 0.014 & 28 & 97 \\
\hline$Q_{2 \%}=668(A)^{0.657}$ & 0.017 & 0.013 & 27 & 98 \\
\hline$Q_{1 \%}=794(A)^{0.655}$ & 0.017 & 0.012 & 25 & 98 \\
\hline$Q_{0.5 \%}=920(A)^{0.655}$ & 0.019 & 0.013 & 27 & 98 \\
\hline$Q_{0.2 \%}=1,109(A)^{0.654}$ & 0.024 & 0.017 & 31 & 97 \\
\hline \multicolumn{5}{|c|}{ Flood region 3 (22 streamgages) } \\
\hline$Q_{50 \%}=461(A)^{0.492}$ & 0.026 & 0.022 & 35 & 85 \\
\hline$Q_{20 \%}=805(A)^{0.503}$ & 0.014 & 0.008 & 21 & 95 \\
\hline$Q_{10 \%}=1,094(A)^{0.510}$ & 0.011 & 0.004 & 15 & 98 \\
\hline$Q_{4 \%}=1,439(A)^{0.525}$ & 0.009 & 0.003 & 12 & 99 \\
\hline$Q_{2 \%}=1,687(A)^{0.538}$ & 0.008 & 0.002 & 11 & 100 \\
\hline$Q_{1 \%}=1,901(A)^{0.553}$ & 0.009 & 0.003 & 12 & 100 \\
\hline$Q_{0.5 \%}=2,109(A)^{0.568}$ & 0.012 & 0.003 & 13 & 100 \\
\hline$Q_{0.2 \%}=2,388(A)^{0.586}$ & 0.017 & 0.004 & 15 & 100 \\
\hline \multicolumn{5}{|c|}{ Flood region 4 (72 streamgages) } \\
\hline$Q_{50 \%}=242(A)^{0.568}$ & 0.011 & 0.006 & 19 & 96 \\
\hline$Q_{20 \%}=455(A)^{0.565}$ & 0.007 & 0.003 & 12 & 98 \\
\hline$Q_{10 \%}=641(A)^{0.562}$ & 0.007 & 0.002 & 9 & 99 \\
\hline$Q_{4 \%}=908(A)^{0.560}$ & 0.009 & 0.002 & 11 & 99 \\
\hline$Q_{2 \%}=1,135(A)^{0.560}$ & 0.011 & 0.003 & 12 & 98 \\
\hline$Q_{1 \%}=1,380(A)^{0.561}$ & 0.015 & 0.004 & 14 & 98 \\
\hline$Q_{0.5 \%}=1,648(A)^{0.563}$ & 0.020 & 0.005 & 17 & 97 \\
\hline$Q_{0.2 \%}=2,032(A)^{0.567}$ & 0.027 & 0.008 & 21 & 96 \\
\hline
\end{tabular}


Table 4. Small stream regional regression equations for estimating annual exceedance probability flows and generalized least squares model diagnostics for unregulated small streams in Alabama.

[MSE, mean square error in $\log 10$ cubic feet per second; $\mathrm{ft}^{3} / \mathrm{s}$, cubic feet per second; AVP, average variance of prediction in $\log 10$ cubic feet per second; SEP, standard error of prediction; pseudo- $R^{2}$, pseudo coefficient of determination; $A$, contributing drainage area in square miles]

\begin{tabular}{|c|c|c|c|c|}
\hline $\begin{array}{l}\text { Annual exceedance } \\
\text { probability flow equation }\end{array}$ & $\begin{array}{c}\text { MSE } \\
\left(\log \mathrm{ft}^{3} / \mathbf{s}\right)\end{array}$ & $\begin{array}{c}\text { AVP } \\
\left(\log \mathrm{ft}^{3} / \mathbf{s}\right)^{2}\end{array}$ & $\begin{array}{c}\text { SEP } \\
\text { (percent) }\end{array}$ & $\begin{array}{l}\text { Pseudo- } R^{2} \\
\text { (percent) }\end{array}$ \\
\hline \multicolumn{5}{|c|}{ Small streams (40 streamgages) } \\
\hline$Q_{50 \%}=208(A)^{0.695}$ & 0.058 & 0.056 & 58 & 75 \\
\hline$Q_{20 \%}=360(A)^{0.688}$ & 0.028 & 0.023 & 36 & 88 \\
\hline$Q_{10 \%}=482(A)^{0.690}$ & 0.018 & 0.012 & 26 & 93 \\
\hline$Q_{4 \%}=659(A)^{0.692}$ & 0.014 & 0.007 & 20 & 96 \\
\hline$Q_{2 \%}=807(A)^{0.693}$ & 0.014 & 0.006 & 17 & 97 \\
\hline$Q_{1 \%}=962(A)^{0.696}$ & 0.017 & 0.007 & 19 & 97 \\
\hline$Q_{0.5 \%}=1,125(A)^{0.700}$ & 0.022 & 0.009 & 23 & 95 \\
\hline$Q_{0.2 \%}=1,361(A)^{0.705}$ & 0.032 & 0.016 & 30 & 92 \\
\hline
\end{tabular}

For the Coosa River at Childersburg, Ala. (USGS station no. 02407000, plate 1), a trend analysis of the entire period of record (1916-2016) indicated a change in high-flow patterns in the early to mid-1960s. This trend coincides with the construction of Weiss, H. Neely Henry, and Logan Martin Reservoirs (1961, 1966, and 1964, respectively). No trends were detected for the period prior to 1961, indicating that from 1916 to 1960 , flow patterns were relatively consistent. Flow at Childersburg was only regulated by the dam at Allatoona prior to 1961, and the effects of regulation were considered minimal. An LP3 analysis was conducted on the 1967-2016 period of record, which is considered indicative of current, post-regulation flood-frequency conditions. A comparison of flood-frequency estimates for the pre-regulation period (1916-60) by Hedgecock and Feaster (2007) with those for the post-regulation period (1967-2016) indicates that the estimates of the magnitudes of larger floods (lower AEP) are not substantially different.

For the Coosa River at Jordan Dam near Wetumpka, Ala. (USGS station no. 02411000, plate 1), two periods were considered for analysis (1913-1984 and 1985-2013). The period 1985-2013 was considered to better represent current regulated conditions.

\section{Tallapoosa River}

Annual peak flows in the Tallapoosa River is regulated by reservoirs at R. L. Harris, Martin, Yates, and Thurlow Dams. For the Tallapoosa River at Wadley, Ala. (USGS station no. 02414500 , plate 1 ), a trend analysis of the entire period of record (1924-2016), which included a mixture of unregulated and regulated flows, indicated no significant change in highflow patterns. Using the entire period of record was considered acceptable because there was no trend apparent in the dataset, and the graphical fit of an LP3 distribution was reasonable.

For the Tallapoosa River below Tallassee, Ala. (USGS station no. 02418500 , plate 1), flood-frequency analyses were run on four different periods of record: (1) 1920-2013, which includes annual peaks from the various regulation periods and the 1920 historic peak from the unregulated period; (2) 19292013, which excludes the 1920 historic peak; (3) 1929-1983, which is the period prior to completion of the R.L. Harris Dam; and (4) 1984-2013, which is the period after completion of the R.L. Harris Dam. Results of the Mann-Kendall test indicated no statistically significant trend in any of the four periods. The LP3 curve fit the data reasonably well for all four periods. Given the similarities in the frequency curves, the uncertainty of how storage from Martin and R.L. Harris Dams would have affected the 1920 historic peak, and the fact that the frequency curve fits the data reasonably well, it seems appropriate to publish flood-frequency estimates computed by using the data from the period 1929-2013 (app. 1).

\section{Tennessee River}

A flood-frequency analysis was performed for the Tennessee River at Whitesburg, Ala. (USGS station no. 03575500, plate 1). For comparison, four LP3 analyses were made: (1) the complete period of record (1925-2011), (2) 1944-2011, (3) 1944-2011 with a historical period of 1867-1943, and (4) 1968-2011, which is the period after all major upstream dams were in place. The period of record selected for flood-frequency analysis was 1944-2011, during which all the dams on the Tennessee River were in place. The results of the Mann-Kendall test and the results of singlemass curves plotted for this streamgage indicate that the 
high-flow patterns have been relatively consistent since 1944 . Inspection of the flow record for the streamgage upstream of Whitesburg, the Tennessee River at Chattanooga, Tenn. (USGS station no. 03568000, plate 1), indicates that floods in $1867,1875,1886$, and 1917 were the four largest that occurred at this location between 1867 and 2003. These flood events occurred primarily before regulation of the Tennessee River and before the streamgage at Whitesburg became active (1925). One of these flood peaks could have been the largest at the Whitesburg streamgage, but would not be reflected in this flood-frequency analysis.

For the Tennessee River at Florence, Ala. (USGS station no. 03589500 , plate 1), three LP3 analyses were made: (1) the complete period of record from 1895 to 2013, including the 1867 historic peak and associated historical period 18671894; (2) 1944-2013; and (3) 1944-2013 with a historical period 1867-1943. The period of record from 1944 to 2013, with a historical period 1867-1943, was used for the final flood-frequency analysis. This dataset represents a period during which all dams on the Tennessee River were in place. The results of the Mann-Kendall test and the single-mass curves plotted for this streamgage indicate that high-flow patterns have been relatively consistent since 1944 .

\section{Tombigbee River}

Annual peak flows in the Tombigbee River are regulated by locks and dams at Bevill, Heflin, Demopolis, and Coffeeville, and by numerous locks and dams on the Tennessee-Tombigbee Waterway in Mississippi. For the Tombigbee River at Gainesville, Ala. (USGS station no. 02449000, plate 1), no data were collected at this streamgage since the previous report (Hedgecock and Feaster, 2007); therefore, no update was performed.

For the Tombigbee River near Coatopa, Ala. (USGS station no. 02467000, plate 1), a trend analysis for the entire period of record (1893-2015) indicated a change in highflow patterns in the early to mid-1970s. This trend most likely coincided with the beginning of construction of the Tennessee-Tombigbee Waterway in 1973. Trend analyses of the period prior to 1974 indicate that relatively consistent flow patterns were present. Therefore, an LP3 analysis was performed on the period of record 1974-2015, which was considered indicative of regulated flood-frequency conditions. Annual peak flows resulting from floods occurring after 1973 may be somewhat attenuated by the effects of the TennesseeTombigbee Waterway.

For the Tombigbee River near Coffeeville, Ala. (USGS station no. 02469761, plate 1), the period of record available is 1961-2015. Two LP3 analyses were made, one of the complete periods of record (1961-2015) and another of the period 1974-2015. Floods occurring after 1973 may be of somewhat lesser magnitude because of the effects of the Tennessee-Tombigbee Waterway, although the 1979 peak was the peak of record and was flagged as being the highest since 1874. Therefore, the period of record 1974-2015 was used and is considered indicative of regulated flood-frequency conditions.

\section{Black Warrior River}

For the Black Warrior River at Northport, Ala. (USGS station no. 02465000, plate 1), the complete period of record (1929-2015) was used for the LP3 analysis. Two reservoirs are located upstream from the streamgage at Northport. The Mann-Kendall test and single-mass curves for the period 1929-2015 indicated consistent peak-flow patterns.

For the Black Warrior River near Eutaw, Ala. (USGS station no. 02466030, plate 1), the complete period of record from 1977 to 2012 was used for the LP3 analysis. The MannKendall test indicated no trend for this period, and the LP3 distribution fits the dataset reasonably well. This period of record is indicative of current, regulated flood-frequency conditions. Other streamgages on the Black Warrior River were not analyzed because they either did not have enough record with a consistent flow pattern or the record was maximum daily average flow.

\section{Conecuh River}

For the Conecuh River near Brooklyn, Ala. (USGS station no. 02374000, plate 1 ), the systematic period of record is 1936-1957 with historic peaks in 1929 and 1975; the 1929 annual peak is considered to have been the highest flow since 1865. The historical period of record used for this analysis was 1865-1975.

For the Conecuh River at State Highway 41 near Brewton, Ala. (USGS station no. 02374250, plate 1), the systematic period of record is 1999-2016 with historic peaks in 1929 and 1975; the 1929 annual peak is considered to have been the highest flow since 1865 . The historical period of record used for this analysis was 1865-2016. A comparison of the LP3 analysis for USGS station no. 02374250 with that for USGS station no. 02374000 showed that the flood-frequency estimates are not substantially different, which was expected because there is only a 7-percent difference in the sizes of the drainage areas. For the 10- to 0.2-percent AEP floods, the results from USGS station no. 02374000 are within the same confidence limits as those from USGS station no. 02374250. 


\section{Flood-Frequency Estimates at Streamgages in Alabama}

The estimates of the AEP flows at streamgages were computed by using the EMA and MGB to test for PILFs. AEP flows at streamgages should be determined by weighting the station skew coefficient with the generalized skew coefficient from Bulletin 17B (Interagency Advisory Committee on Water Data, 1982). The accuracy of AEP flows at streamgages can be further improved by weighting those flows with RREpredicted AEP flows. The variance of prediction is a function of the regression equations and the independent variables used to develop the flow estimate. If the estimated AEP flows at streamgages and RRE-predicted AEP flows are assumed to be independent and are weighted in inverse proportion to the associated variances, the variance of the weighted estimate will be less than the variance of either of the independent estimates. Once the variances have been computed, the two independent flow estimates can be weighted by using the following equation:

$$
\log _{10} Q_{P(g) w}=\frac{V_{p, P(g) r} * \log _{10} Q_{P(g) s}+V_{p, P(g) s} * \log _{10} Q_{P(g) r}}{V_{p, P(g) s}+V_{p, P(g) r}},
$$

where

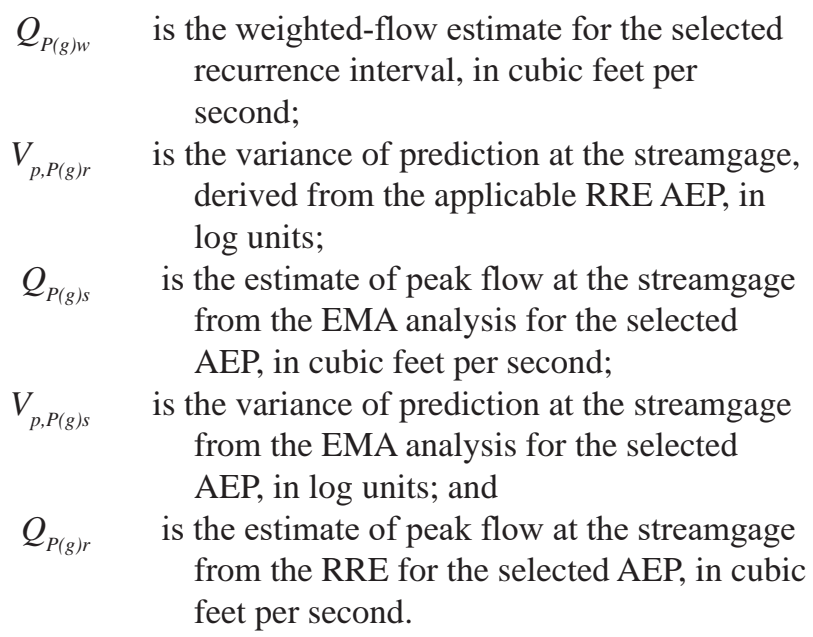

The estimates of streamflow at streamgages were weighted with the regional estimates of streamflow to compute a final set of AEP flows. The weighted-flow estimates for the streamgages in Alabama are provided in the data release associated with this report (Anderson, 2020).

\section{Flood-Frequency Estimates at Ungaged Locations on Gaged Streams}

The AEP flows for a streamgage can be transferred to an ungaged location on the same stream by using the areaweighting method. Equation 3 can be used if the drainage area at an ungaged location on the stream is within 50 percent of the drainage area at the streamgage (drainage area ratio is more than 0.5 or less than 1.5) (Ries and Dillow, 2006). The drainage area ratio estimate for an ungaged location can be calculated by using the following equation:

$$
Q_{P(u)}=\left(\frac{A_{(u)}}{A_{(g)}}\right)^{b} Q_{P(g) w},
$$

where

$Q_{P(u)} \quad$ is the drainage area ratio estimate of flood flow for the selected $P$-percent AEP for the ungaged location, $u$, in cubic feet per second;

$A_{(u)} \quad$ is the drainage area of the ungaged location, in square miles;

$A_{(g)} \quad$ is the drainage area of the upstream or downstream streamgage, in square miles;

$Q_{P(g) w} \quad$ is the weighted estimate of flood flow for the selected $P$-percent AEP for the upstream or downstream streamgage, in cubic feet per second; and

$b$ is the exponent of drainage area from the appropriate RRE (tables 3 and 4).

This method, however, does not weight the area-weighted flows with the RRE flows for the ungaged location. The AEP flows from the streamgage can be transferred and weighted with RRE flows for the ungaged location by using the following equation:

$$
Q_{P(u) w}=\left(\frac{2|\Delta A|}{A_{g}}\right) Q_{P(u) r}+\left(1-\frac{2|\Delta A|}{A_{g}}\right) Q_{P(u)},
$$

where

$$
\begin{aligned}
& Q_{P(u) w} \quad \text { is the weighted-flow estimate at the ungaged } \\
& \text { location, in cubic feet per second; } \\
& Q_{P(u) r} \quad \text { is the RRE flood estimate at the ungaged } \\
& \text { location for the selected AEP, in cubic feet } \\
& \text { per second; } \\
& A_{g} \quad \text { is the drainage area of the streamgage, in } \\
& \text { square miles; } \\
& |\Delta A| \quad \text { is the absolute difference in drainage areas } \\
& \text { between the ungaged location and the } \\
& \text { streamgage, in square miles; and } \\
& Q_{P(u)} \quad \text { is the estimate of flood flow for the selected } \\
& P \text {-percent AEP for the ungaged location, } \\
& u \text {, in cubic feet per second computed from } \\
& \text { equation } 3 .
\end{aligned}
$$

If the drainage area at an ungaged location differs by more than 50 percent from that of the streamgage, the RRE estimates should be used. If an ungaged location is between two streamgages on the same stream, the site with the closest drainage area ratio and longest period of record should be used (Sauer, 1974). 


\section{Flood-Frequency Estimates at Locations on Ungaged Streams}

For locations on ungaged streams, the flood region should be determined by using figure 2 or StreamStats (http:// water.usgs.gov/osw/streamstats/; USGS, 2017a). The RREs for the appropriate flood region and size of drainage area should then be used to compute flows for the desired range of AEPs (table 3 or 4). The standard error of prediction (SEP) is a measure of the accuracy of AEP flow estimates for ungaged basins in each region.

\section{Accuracy and Limitations of Regional Regression Equations}

The RREs and small-stream equations only apply to rural streams that can be described by using the basin characteristics and having drainage areas within the range of streamgages in each region that were used to develop the equations (table 5). These methods should not be used for sites in stream basins that are substantially affected by regulation from impoundments, channelization, levees, or other man-made structures. The RREs and small-stream equations also should not be applied to locations on streams in urban areas where more than 10 percent of the basin is covered by impervious area. The methods do not apply where flooding is influenced by extreme ocean storm surge or tidal events. Reliability of the RREs and small-stream equations for a location in a flood
Table 5. Ranges of expanatory variable data used to develop regional regression equations.

[RREs, regional regression equations; $\mathrm{mi}^{2}$, square mile]

\begin{tabular}{ccc}
\hline \multicolumn{2}{c}{ Ranges of data used to develop RREs } \\
\hline Region & $\begin{array}{c}\text { Number } \\
\text { of gages }\end{array}$ & $\begin{array}{c}\text { Range of } \\
\text { drainage area }\end{array}$ \\
\hline 1 & 90 & 0.94 to $1,027 \mathrm{mi}^{2}$ \\
2 & 33 & 0.13 to $1,766 \mathrm{mi}^{2}$ \\
3 & 22 & 0.34 to $1,097 \mathrm{mi}^{2}$ \\
4 & 72 & 0.69 to $1,650 \mathrm{mi}^{2}$ \\
Small streams & 40 & 0.13 to $14 \mathrm{mi}^{2}$ \\
\hline
\end{tabular}

region that has basin characteristics outside the limits of the streamgages confined in that flood region is unknown.

The accuracy of a flood-frequency estimate traditionally has been expressed in two ways - as the mean standard error of the model or as the mean SEP. The mean standard error of the model is a measure of how well the regression model fits the input data and represents the standard deviation of the differences between streamgage data and the corresponding prediction from the regression equation. The SEP is a measure of how well the regression model estimates flood magnitudes for ungaged basins. The SEP is the square root of the mean square error of prediction (MSEp). The MSEp is the sum of two components- the MSE resulting from the model and the sampling MSE resulting from estimating the model parameters from samples of the population. 


\section{Summary and Conclusions}

Flood-frequency estimates for rural Alabama streams were last updated in 2007; since that time, estimation techniques have improved, and additional streamflow data are available to improve the accuracy of such estimates. Thus, the U.S. Geological Survey, in cooperation with the Alabama Department of Transportation, performed flood-frequency analyses to estimate annual exceedance probability (AEP) flows at streamgages, developed regional regression equations (RREs) to estimate AEPs at ungaged locations in rural Alabama, and performed flood-frequency analyses for large river basins. Many basin characteristics were analyzed, but drainage area was the only characteristic that was statistically significant and was used as an explanatory variable in the RREs. The boundaries of four flood regions in the State of Alabama that were defined in the 2007 study were confirmed and adjusted through use of the RREs.

Additionally, equations were developed for small streams in Alabama having a drainage area of 14 square miles or less. These RREs are recommended in order to improve the accuracy of the AEP flow estimates for sites with drainage areas less than 5 square miles.

Extreme flow events have the potential for devastating impacts to the economy, infrastructure, and the landscape. Keeping flood-frequency analyses and RREs updated can provide water-resource managers the information needed during flood-response planning. By broadening the regional approach for the development of flood-frequency RREs across State lines, flood-frequency estimates have the potential to be more accurate than previously calculated estimates and applicable to a larger study area. Finally, providing the analysis results on a publicly accessible web interface, StreamStats (https://water.usgs.gov/osw/streamstats/), allows a user to select a site of interest and obtain the AEP flows for that site at any time.

\section{References Cited}

Anderson, B.T., 2020, Flood regions and annual exceedance probability flows for Alabama streams, data through 2015: U.S. Geological Survey data release, https://doi. org/10.5066/P9TYSZLL.

Atkins, J.B., 1996, Magnitude and frequency of floods in Alabama: U.S. Geological Survey Water-Resources Investigations Report 95-4199, 234 p.

Barnes, H.H., Jr., and Golden, H.G., 1966, Magnitude and frequency of floods in the United States, Part 2-B, South Atlantic and eastern Gulf of Mexico basins, Ogeechee River to Pearl River: U.S. Geological Survey Water-Supply Paper 1674, 409 p.
Cohn, T.A., England, J.F., Berenbrock, C.E., Mason, R.R., Stedinger, J.R., and Lamontagne, J.R., 2013, A generalized Grubbs-Beck test statistic for detecting multiple potentially influential low outliers in flood series: Water Resources Research, v. 49, no. 8, p. 5047-5058, accessed June 12, 2018, at http://dx.doi.org/10.1002/wrcr.20392.

Cohn, T.A., Lane, W.L., and Baier, W.G., 1997, An algorithm for computing moments-based flood quantile estimates when historical flood information is available: Water Resources Research, v. 33, no. 9, p. 2089-2096, June 12, 2018, at https://doi.org/10.1029/97WR01640.

Eng, K., Chen, Y.Y., and Kiang, J.E., 2009, User's guide to the weighted-multiple-linear regression program (WREG version 1.0): U.S. Geological Survey Techniques and Methods, book 4, chap. A8, 21 p. [Also available at http:// pubs.usgs.gov/tm/tm4a8/.]

England, J.F., Jr., Cohn, T.A., Faber, B.A., Stedinger, J.R., Thomas, W.O., Jr., Veilleux, A.G., Kiang, J.E., and Mason, R.R., Jr., 2019, Guidelines for determining flood flow frequency_Bulletin 17C (ver. 1.1, May 2019): U.S. Geological Survey Techniques and Methods, book 4, chap. B5, 148 p., June 13, 2018, at https://doi.org/10.3133/ tm4B5.

Gamble, C.R., 1965, Magnitude and frequency of floods in Alabama: Alabama Highway Department HPR Report No. 5, 42 p.

Griffis, V.W., and Stedinger, J.R., 2007, Log-Pearson type 3 distribution and its application in flood frequency analysis. II. Parameter estimation methods: Journal of Hydrologic Engineering, v. 12, no. 5, p. 492-500, accessed June 12, 2018, at https://doi.org/10.1061/(ASCE)10840699(2007)12:5(492).

Hains, C.F., 1973, Floods in Alabama, magnitude and frequency: Alabama Highway Department, 174 p.

Hedgecock, T.S., 2004, Magnitude and frequency of floods on small rural streams in Alabama: U.S. Geological Survey Scientific Investigations Report 2004-5135, 10 p.

Hedgecock, T.S., and Feaster, T.D., 2007, Magnitude and frequency of floods in Alabama, 2003: U.S. Geological Survey Scientific Investigations Report 2007-5204, 28 p., + app. [Also available at https://pubs.water.usgs.gov/ sir2007-5204.]

Hedgecock, T.S., and Lee, K.G., 2010, Magnitude and frequency of floods for urban streams in Alabama, 2007: U.S. Geological Survey Scientific Investigations Report 2010-5012, 17 p. [Also available at https://doi.org/10.3133/ sir20105012.]

Helsel, R.M., and Hirsch, D.R., 1995, Studies in environmental science 49-Statistical methods in water resources: Amsterdam, Elsevier Science, 529 p. 
Interagency Advisory Committee on Water Data, 1982, Guidelines for determining flood-flow frequency: Bulletin 17B, 183 p.

Olin, D.A., 1984, Magnitude and frequency of floods in Alabama: U.S. Geological Survey Water-Resources Investigations Report 84-4191, 105 p.

Olin, D.A., and Bingham, R.H., 1977, Flood frequency of small streams in Alabama: Alabama Highway Department, HPR Report No. 83, 44 p.

Olin, D.A., and Bingham, R.H., 1982, Synthesized flood frequency of urban streams in Alabama: U.S. Geological Survey Water-Resources Investigations Report 82-683, $35 \mathrm{p}$.

Parrett, C., Veilleux, A., Stedinger, J.R., Barth, N.A., Knifong, D.L., and Ferris, J.C., 2011, Regional skew for California, and flood frequency for selected sites in the SacramentoSan Joaquin River Basin, based on data through water year 2006: U.S. Geological Survey Scientific Investigations Report 2010-5260, 94 p.

Pierce, L.B., 1954, Floods in Alabama, magnitude and frequency: U.S. Geological Survey Circular 342, 105 p.

Ries, K.G., III, and Dillow, J.J.A., 2006, Magnitude and frequency of floods on nontidal streams in Delaware: U.S. Geological Survey Scientific Investigations Report 20065146, 59 p.

Sauer, V.B., 1974, Flood characteristics of Oklahoma streams, techniques for calculating magnitude and frequency of floods in Oklahoma, with compilations of flood data through 1971: U.S. Geological Survey Water-Resources Investigations Report 73-52, 307 p.
Searcy, J.K., and Hardison, C.H., 1960, Double-mass curves: U.S. Geological Survey Water-Supply Paper 1541-B, p. 31-66.

Speer, P.R., and Gamble, C.R., 1964, Magnitude and frequency of floods in the United States, Part 3-B, Cumberland and Tennessee River Basins: U.S. Geological Survey Water-Supply Paper 1676, 340 p.

U.S. Climate Data, 2018, Climate data for Montgomery, Alabama, accessed February 7, 2017, at https://www. usclimatedata.com/climate/montgomery/alabama/unitedstates/usal0375.

U.S. Geological Survey, 2013, WREG, weighted-multiplelinear regression program, accessed June 1, 2014, at https:// water.usgs.gov/software/WREG/.

U.S. Geological Survey, 2014, PeakFQ, accessed July 3, 2013, at https://water.usgs.gov/software/PeakFQ/.

U.S. Geological Survey, 2017a, Welcome to StreamStats, accessed July 3, 2015, at https://water.usgs.gov/osw/ streamstats/.

U.S. Geological Survey, 2017b, USGS water data for the Nation: U.S. Geological Survey National Water Information System database, accessed October 25, 2018, at http:// dx.doi.org/10.5066/F7P55KJN. [Peak-flow data directly accessible at https://nwis.waterdata.usgs.gov/usa/nwis/ peak.]

Wagner, D.M., Krieger, J.D., and Veilleux, A.G., 2016, Methods for estimating annual exceedance probability discharges for streams in Arkansas, based on data through water year 2013: U.S. Geological Survey Scientific Investigations Report 2016-5081, 136 p., accessed July 30, 2014, at https://doi.org/10.3133/sir20165081. 

Appendix 1 

Appendix 1. Annual exceedance probability flows for U.S. Geological Survey streamgages used in this analysis.

[USGS, U.S. Geological Survey; $\mathrm{ft}^{3} / \mathrm{s}$, cubic foot per second; Ala., Alabama; EMA, expected moments algorithm; RRE, regional regression equations; *, large or regulated streamgage that required separate analysis]

\begin{tabular}{|c|c|c|c|c|c|c|c|c|c|c|c|c|}
\hline \multirow{2}{*}{$\begin{array}{l}\text { USGS station } \\
\text { number } \\
\text { (pl. 1) }\end{array}$} & \multirow{2}{*}{$\begin{array}{c}\text { Site } \\
\text { number } \\
\text { (fig. 1) }\end{array}$} & \multirow[b]{2}{*}{ USGS streamgage name } & \multirow{2}{*}{$\begin{array}{l}\text { Flood } \\
\text { region } \\
\text { (fig. 2) }\end{array}$} & \multirow[b]{2}{*}{ Method } & \multicolumn{8}{|c|}{ Annual exceedance probability flow (ft $\mathrm{ft}^{3} \mathrm{~s}$ ) } \\
\hline & & & & & $\begin{array}{c}50 \\
\text { percent }\end{array}$ & $\begin{array}{c}20 \\
\text { percent }\end{array}$ & $\begin{array}{c}10 \\
\text { percent }\end{array}$ & $\begin{array}{c}4 \\
\text { percent }\end{array}$ & $\begin{array}{c}2 \\
\text { percent }\end{array}$ & $\begin{array}{c}1 \\
\text { percent }\end{array}$ & $\begin{array}{c}0.5 \\
\text { percent }\end{array}$ & $\begin{array}{c}0.2 \\
\text { percent }\end{array}$ \\
\hline \multirow[t]{3}{*}{02340750} & \multirow[t]{3}{*}{6} & \multirow[t]{3}{*}{ Osanippa Creek near Fairfax, Ala. } & \multirow[t]{3}{*}{1} & EMA & 3,880 & 6,300 & 8,240 & 11,100 & 13,600 & 16,300 & 19,400 & 24,100 \\
\hline & & & & RRE & 5,080 & 8,290 & 10,700 & 14,000 & 16,600 & 19,400 & 22,200 & 26,200 \\
\hline & & & & Weighted & 3,950 & 6,440 & 8,440 & 11,400 & 14,000 & 16,800 & 19,900 & 24,600 \\
\hline \multirow[t]{3}{*}{02342150} & \multirow[t]{3}{*}{8} & \multirow[t]{3}{*}{ Uchee Creek near Seale, Ala. } & \multirow[t]{3}{*}{4} & EMA & 4,342 & 8,573 & 12,050 & 17,140 & 21,390 & 26,000 & 30,980 & 38,160 \\
\hline & & & & RRE & 3,910 & 7,240 & 10,100 & 14,100 & 17,600 & 21,500 & 26,000 & 32,700 \\
\hline & & & & Weighted & 4,100 & 7,590 & 10,400 & 14,400 & 18,100 & 22,100 & 26,600 & 33,500 \\
\hline \multirow[t]{3}{*}{02342200} & \multirow[t]{3}{*}{9} & \multirow[t]{3}{*}{ Phelps Creek near Opelika, Ala. } & \multirow[t]{3}{*}{1} & EMA & 812 & 1,490 & 2,020 & 2,760 & 3,350 & 3,980 & 4,650 & 5,580 \\
\hline & & & & RRE & 835 & 1,440 & 1,910 & 2,530 & 3,030 & 3,540 & 4,070 & 4,800 \\
\hline & & & & Weighted & 813 & 1,490 & 2,010 & 2,730 & 3,300 & 3,880 & 4,500 & 5,330 \\
\hline \multirow[t]{3}{*}{02342200} & \multirow[t]{3}{*}{9} & \multirow[t]{3}{*}{ Phelps Creek near Opelika, Ala. } & \multirow{3}{*}{$\begin{array}{c}\text { Small } \\
\text { stream }\end{array}$} & EMA & 812 & 1,492 & 2,020 & 2,759 & 3,354 & 3,983 & 4,646 & 5,576 \\
\hline & & & & RRE & 1,170 & 2,090 & 2,880 & 3,900 & 4,680 & 5,430 & 6,200 & 7,260 \\
\hline & & & & Weighted & 846 & 1,620 & 2,370 & 3,480 & 4,310 & 5,070 & 5,810 & 6,740 \\
\hline \multirow[t]{3}{*}{02342500} & \multirow[t]{3}{*}{10} & \multirow[t]{3}{*}{ Uchee Creek near Fort Mitchell, Ala. } & \multirow[t]{3}{*}{4} & EMA & 8,380 & 14,790 & 19,870 & 27,210 & 33,310 & 39,940 & 47,140 & 57,610 \\
\hline & & & & $\mathrm{RRE}$ & 6,430 & 11,900 & 16,500 & 23,000 & 28,800 & 35,200 & 42,500 & 53,700 \\
\hline & & & & Weighted & 7,970 & 13,600 & 18,000 & 24,300 & 30,300 & 36,700 & 43,900 & 55,000 \\
\hline \multirow[t]{3}{*}{02342933} & 11 & South Fork Cowikee Creek near & 4 & EMA & 5,536 & 8,824 & 11,450 & 15,330 & 18,650 & 22,350 & 26,490 & 32,730 \\
\hline & & Batesville, Ala. & & RRE & 3,530 & 6,540 & 9,090 & 12,800 & 15,900 & 19,500 & 23,500 & 29,500 \\
\hline & & & & Weighted & 5,140 & 7,890 & 10,000 & 13,500 & 16,700 & 20,300 & 24,300 & 30,500 \\
\hline 02343275 & 13 & Abbie Creek near Abbeville, Ala. & 4 & EMA & 1,607 & 3,591 & 5,618 & 9,248 & 12,910 & 17,570 & 23,450 & 33,570 \\
\hline & & & & RRE & 2,180 & 4,040 & 5,680 & 8,020 & 10,000 & 12,200 & 14,700 & 18,400 \\
\hline & & & & Weighted & 1,890 & 3,910 & 5,670 & 8,170 & 10,400 & 12,800 & 15,600 & 20,100 \\
\hline 02343300 & 14 & Abbie Creek near Haleburg, Ala. & 4 & EMA & 2,817 & 5,285 & 7,627 & 11,620 & 15,500 & 20,320 & 26,290 & 36,350 \\
\hline & & & & RRE & 4,070 & 7,520 & 10,500 & 14,800 & 18,600 & 22,600 & 27,300 & 34,300 \\
\hline & & & & Weighted & 3,180 & 6,540 & 9,790 & 14,300 & 18,100 & 22,300 & 27,200 & 34,600 \\
\hline 02343700 & 15 & Stevenson Creek near Headland, Ala. & 4 & EMA & 1,064 & 1,832 & 2,453 & 3,369 & 4,149 & 5,014 & 5,975 & 7,407 \\
\hline & & & & RRE & 1,080 & 2,020 & 2,820 & 3,980 & 4,980 & 6,070 & 7,280 & 9,070 \\
\hline & & & & Weighted & 1,070 & 1,960 & 2,750 & 3,900 & 4,860 & 5,920 & 7,090 & 8,790 \\
\hline 02343700 & 15 & Stevenson Creek near Headland, Ala. & Small & EMA & 1,064 & 1,832 & 2,453 & 3,369 & 4,149 & 5,014 & 5,975 & 7,407 \\
\hline & & & stream & RRE & 1,690 & 3,040 & 4,200 & 5,750 & 6,980 & 8,180 & 9,440 & 11,200 \\
\hline & & & & Weighted & 1,110 & 2,070 & 3,120 & 4,840 & 6,180 & 7,370 & 8,560 & 10,000 \\
\hline
\end{tabular}


Appendix 1. Annual exceedance probability flows for U.S. Geological Survey streamgages used in this analysis.-Continued

[USGS, U.S. Geological Survey; $\mathrm{ft}^{3} / \mathrm{s}$, cubic foot per second; Ala., Alabama; EMA, expected moments algorithm; RRE, regional regression equations; *, large or regulated streamgage that required separate analysis]

\begin{tabular}{|c|c|c|c|c|c|c|c|c|c|c|c|c|}
\hline \multirow{2}{*}{$\begin{array}{l}\text { USGS station } \\
\text { number } \\
\text { (pl. 1) }\end{array}$} & \multirow{2}{*}{$\begin{array}{c}\text { Site } \\
\text { number } \\
\text { (fig. 1) }\end{array}$} & \multirow[b]{2}{*}{ USGS streamgage name } & \multirow{2}{*}{$\begin{array}{l}\text { Flood } \\
\text { region } \\
\text { (fig. 2) } \\
\end{array}$} & \multirow[b]{2}{*}{ Method } & \multicolumn{8}{|c|}{ Annual exceedance probability flow (fts/s) } \\
\hline & & & & & $\begin{array}{c}50 \\
\text { percent }\end{array}$ & $\begin{array}{c}20 \\
\text { percent }\end{array}$ & $\begin{array}{c}10 \\
\text { percent }\end{array}$ & $\begin{array}{c}4 \\
\text { percent }\end{array}$ & $\begin{array}{c}2 \\
\text { percent }\end{array}$ & $\begin{array}{c}1 \\
\text { percent }\end{array}$ & $\begin{array}{c}0.5 \\
\text { percent }\end{array}$ & $\begin{array}{c}0.2 \\
\text { percent }\end{array}$ \\
\hline \multirow[t]{3}{*}{02360000} & \multirow[t]{3}{*}{21} & \multirow{3}{*}{$\begin{array}{l}\text { West Fork Choctawhatchee R at Blue } \\
\text { Springs, Ala. }\end{array}$} & \multirow[t]{3}{*}{4} & EMA & 2,007 & 4,029 & 5,948 & 9,187 & 12,300 & 16,110 & 20,750 & 28,420 \\
\hline & & & & RRE & 3,050 & 5,670 & 7,880 & 11,100 & 13,800 & 16,900 & 20,300 & 25,500 \\
\hline & & & & Weighted & 2,390 & 5,000 & 7,390 & 10,800 & 13,500 & 16,800 & 20,400 & 26,000 \\
\hline \multirow[t]{3}{*}{02360275} & \multirow[t]{3}{*}{22} & \multirow[t]{3}{*}{ Judy Creek near Ozark, Ala. } & \multirow[t]{3}{*}{4} & EMA & 3,118 & 6,450 & 9,749 & 15,540 & 21,300 & 28,570 & 37,680 & 53,260 \\
\hline & & & & RRE & 3,320 & 6,140 & 8,610 & 12,100 & 15,200 & 18,500 & 22,300 & 27,900 \\
\hline & & & & Weighted & 3,210 & 6,240 & 8,820 & 12,500 & 15,900 & 19,600 & 23,800 & 30,400 \\
\hline \multirow{3}{*}{02360500} & \multirow[t]{3}{*}{23} & \multirow{3}{*}{$\begin{array}{l}\text { East Fork Choctawhatchee R near } \\
\text { Midland City, Ala. }\end{array}$} & \multirow[t]{3}{*}{4} & EMA & 4,947 & 9,748 & 14,170 & 21,450 & 28,280 & 36,460 & 46,220 & 62,010 \\
\hline & & & & RRE & 6,020 & 11,100 & 15,500 & 21,800 & 27,300 & 33,300 & 40,200 & 50,600 \\
\hline & & & & Weighted & 5,500 & 10,700 & 15,300 & 21,800 & 27,400 & 33,700 & 41,000 & 52,200 \\
\hline \multirow[t]{3}{*}{02361000} & \multirow[t]{3}{*}{24} & \multirow{3}{*}{$\begin{array}{l}\text { Choctawhatchee River near Newton, } \\
\text { Ala. }\end{array}$} & \multirow[t]{3}{*}{4} & EMA & 8,746 & 16,540 & 24,120 & 37,310 & 50,420 & 66,990 & 87,840 & 123,800 \\
\hline & & & & RRE & 9,800 & 18,000 & 25,100 & 35,300 & 44,200 & 53,800 & 65,200 & 82,400 \\
\hline & & & & Weighted & 8,930 & 17,100 & 24,700 & 35,800 & 45,600 & 56,500 & 69,300 & 90,100 \\
\hline \multirow[t]{3}{*}{02362240} & \multirow[t]{3}{*}{25} & \multirow{3}{*}{$\begin{array}{l}\text { Little Double Bridges Creek nr } \\
\text { Enterprise, Ala. }\end{array}$} & \multirow[t]{3}{*}{4} & EMA & 1,176 & 2,664 & 4,197 & 6,956 & 9,751 & 13,320 & 17,830 & 25,600 \\
\hline & & & & $\mathrm{RRE}$ & 1,370 & 2,540 & 3,580 & 5,060 & 6,340 & 7,700 & 9,260 & 11,500 \\
\hline & & & & Weighted & 1,270 & 2,580 & 3,700 & 5,310 & 6,810 & 8,410 & 10,200 & 13,100 \\
\hline \multirow[t]{3}{*}{02363000} & 26 & Pea River near Ariton, Ala. & 4 & EMA & 6,821 & 13,240 & 18,950 & 28,030 & 36,280 & 45,910 & 57,120 & 74,690 \\
\hline & & & & $\mathrm{RRE}$ & 8,170 & 15,000 & 21,000 & 29,500 & 36,900 & 45,000 & 54,500 & 68,700 \\
\hline & & & & Weighted & 7,090 & 13,900 & 20,200 & 29,100 & 36,700 & 45,300 & 55,200 & 70,400 \\
\hline 02364000 & 27 & Pea River at Elba, Ala. & 4 & EMA & 12,570 & 21,340 & 28,520 & 39,270 & 48,560 & 59,010 & 70,750 & 88,530 \\
\hline & & & & RRE & 11,900 & 21,800 & 30,300 & 42,500 & 53,300 & 65,000 & 78,700 & 99,600 \\
\hline & & & & Weighted & 12,500 & 21,500 & 29,400 & 41,300 & 51,500 & 62,800 & 75,900 & 95,300 \\
\hline 02364500 & 28 & Pea River near Samson, Ala. & 4 & EMA & 12,620 & 20,690 & 27,270 & 37,120 & 45,660 & 55,280 & 66,150 & 82,690 \\
\hline & & & & RRE & 13,500 & 24,800 & 34,200 & 47,700 & 59,700 & 73,000 & 88,500 & 112,000 \\
\hline & & & & Weighted & 12,800 & 21,900 & 30,500 & 43,200 & 53,600 & 65,500 & 79,500 & 99,400 \\
\hline 02367500 & 32 & Lightwood Knot Creek at Babbie, & 4 & EMA & 3,454 & 6,983 & 10,430 & 16,440 & 22,380 & 29,820 & 39,120 & 54,940 \\
\hline & & Ala. & & RRE & 3,540 & 6,540 & 9,170 & 12,900 & 16,200 & 19,700 & 23,700 & 29,800 \\
\hline & & & & Weighted & 3,490 & 6,710 & 9,470 & 13,500 & 17,200 & 21,200 & 25,700 & 33,100 \\
\hline 02369800 & 36 & Blackwater River near Bradley, Ala. & 4 & EMA & 2,317 & 4,983 & 7,538 & 11,840 & 15,950 & 20,930 & 26,930 & 36,700 \\
\hline & & & & RRE & 3,050 & 5,640 & 7,910 & 11,100 & 14,000 & 17,000 & 20,500 & 25,700 \\
\hline & & & & Weighted & 2,560 & 5,370 & 7,820 & 11,200 & 14,300 & 17,600 & 21,400 & 27,300 \\
\hline
\end{tabular}


Appendix 1. Annual exceedance probability flows for U.S. Geological Survey streamgages used in this analysis.-Continued

[USGS, U.S. Geological Survey; ft³/s, cubic foot per second; Ala., Alabama; EMA, expected moments algorithm; RRE, regional regression equations; *, large or regulated streamgage that required separate analysis]

\begin{tabular}{|c|c|c|c|c|c|c|c|c|c|c|c|c|}
\hline \multirow{2}{*}{$\begin{array}{c}\text { USGS station } \\
\text { number } \\
\text { (pl. 1) }\end{array}$} & \multirow{2}{*}{$\begin{array}{c}\text { Site } \\
\text { number } \\
\text { (fig. 1) }\end{array}$} & \multirow[b]{2}{*}{ USGS streamgage name } & \multirow{2}{*}{$\begin{array}{l}\text { Flood } \\
\text { region } \\
\text { (fig. 2) } \\
\end{array}$} & \multirow[b]{2}{*}{ Method } & \multicolumn{8}{|c|}{ Annual exceedance probability flow (ft $\mathrm{ft}^{3}$ ) } \\
\hline & & & & & $\begin{array}{c}50 \\
\text { percent }\end{array}$ & $\begin{array}{c}20 \\
\text { percent }\end{array}$ & $\begin{array}{c}10 \\
\text { percent }\end{array}$ & $\begin{array}{c}4 \\
\text { percent }\end{array}$ & $\begin{array}{c}2 \\
\text { percent }\end{array}$ & $\begin{array}{c}1 \\
\text { percent }\end{array}$ & $\begin{array}{c}0.5 \\
\text { percent }\end{array}$ & $\begin{array}{c}0.2 \\
\text { percent }\end{array}$ \\
\hline \multirow[t]{3}{*}{02371000} & 40 & Conecuh River near Troy, Ala. & 4 & EMA & 5,370 & 10,930 & 15,770 & 23,230 & 29,770 & 37,170 & 45,480 & 58,010 \\
\hline & & & & RRE & 5,610 & 10,300 & 14,500 & 20,400 & 25,500 & 31,000 & 37,500 & 47,200 \\
\hline & & & & Weighted & 5,480 & 10,500 & 14,800 & 20,900 & 26,300 & 32,100 & 38,900 & 49,300 \\
\hline \multirow[t]{3}{*}{02371200} & 41 & Indian Creek near Troy, Ala. & 4 & EMA & 544 & 1,058 & 1,530 & 2,303 & 3,026 & 3,891 & 4,924 & 6,593 \\
\hline & & & & RRE & 829 & 1,540 & 2,180 & 3,090 & 3,870 & 4,700 & 5,640 & 7,000 \\
\hline & & & & Weighted & 642 & 1,320 & 1,980 & 2,920 & 3,680 & 4,530 & 5,500 & 6,920 \\
\hline \multirow[t]{3}{*}{02371200} & 41 & Indian Creek near Troy, Ala. & Small & EMA & 544 & 1,058 & 1,530 & 2,303 & 3,026 & 3,891 & 4,942 & 6,593 \\
\hline & & & stream & RRE & 1,350 & 2,410 & 3,330 & 4,530 & 5,460 & 6,360 & 7,290 & 8,580 \\
\hline & & & & Weighted & 577 & 1,210 & 1,950 & 3,320 & 4,470 & 5,480 & 6,510 & 7,850 \\
\hline \multirow[t]{3}{*}{02371500} & 42 & Conecuh River at Brantley, Ala. & 4 & EMA & 6,540 & 11,800 & 15,970 & 21,980 & 26,960 & 32,360 & 38,180 & 46,600 \\
\hline & & & & RRE & 8,190 & 15,100 & 21,000 & 29,500 & 37,000 & 45,100 & 54,600 & 68,800 \\
\hline & & & & Weighted & 6,820 & 12,900 & 18,400 & 26,500 & 32,800 & 39,800 & 48,100 & 59,200 \\
\hline \multirow[t]{3}{*}{02372000} & 43 & Patsaliga Creek at Luverne, Ala. & 4 & EMA & 6,270 & 11,820 & 16,520 & 23,640 & 29,830 & 36,800 & 44,630 & 56,420 \\
\hline & & & & RRE & 5,570 & 10,300 & 14,400 & 20,200 & 25,300 & 30,800 & 37,300 & 46,900 \\
\hline & & & & Weighted & 5,980 & 10,900 & 15,000 & 20,900 & 26,300 & 32,100 & 38,800 & 49,100 \\
\hline \multirow[t]{3}{*}{02372250} & 44 & Patsaliga Creek near Brantley, Ala. & 4 & EMA & 6,126 & 12,190 & 17,580 & 26,110 & 33,800 & 42,710 & 52,990 & 68,960 \\
\hline & & & & RRE & 7,640 & 14,100 & 19,600 & 27,600 & 34,500 & 42,100 & 50,900 & 64,200 \\
\hline & & & & Weighted & 6,630 & 13,300 & 19,100 & 27,300 & 34,400 & 42,200 & 51,300 & 65,100 \\
\hline \multirow[t]{3}{*}{02372422} & 45 & Conecuh River Bel Pt A Dam nr & 4 & EMA & 10,320 & 19,350 & 26,740 & 37,620 & 46,810 & 56,890 & 67,950 & 84,130 \\
\hline & & River Falls, Ala. & & RRE & 13,900 & 25,600 & 35,600 & 49,900 & 62,500 & 76,200 & 92,400 & 117,000 \\
\hline & & & & Weighted & 12,100 & 23,700 & 34,000 & 48,300 & 60,300 & 73,400 & 88,900 & 111,000 \\
\hline \multirow[t]{3}{*}{02372500} & 46 & Conecuh River near Andalusia, Ala. & 4 & EMA & 13,890 & 23,580 & 31,410 & 42,980 & 52,860 & 63,870 & 76,130 & 94,480 \\
\hline & & & & RRE & 14,400 & 26,300 & 36,700 & 51,400 & 64,400 & 78,500 & 95,200 & 121,000 \\
\hline & & & & Weighted & 14,000 & 24,500 & 34,100 & 48,300 & 60,000 & 73,100 & 88,400 & 111,000 \\
\hline \multirow[t]{3}{*}{02373000} & 47 & Sepulga River near Mckenzie, Ala. & 4 & EMA & 8,737 & 15,850 & 21,770 & 30,680 & 38,390 & 47,040 & 56,740 & 71,340 \\
\hline & & & & RRE & 7,970 & 14,700 & 20,400 & 28,500 & 35,600 & 43,500 & 52,600 & 66,500 \\
\hline & & & & Weighted & 8,590 & 15,400 & 21,000 & 29,200 & 36,600 & 44,700 & 53,900 & 68,200 \\
\hline \multirow[t]{3}{*}{02373500} & 48 & Pigeon Creek near Thad, Ala. & 4 & EMA & 5,393 & 10,900 & 15,790 & 23,480 & 30,380 & 38,320 & 47,420 & 61,440 \\
\hline & & & & RRE & 6,210 & 11,400 & 16,000 & 22,500 & 28,200 & 34,300 & 41,500 & 52,200 \\
\hline & & & & Weighted & 5,710 & 11,200 & 15,900 & 22,700 & 28,700 & 35,100 & 42,600 & 54,100 \\
\hline
\end{tabular}


Appendix 1. Annual exceedance probability flows for U.S. Geological Survey streamgages used in this analysis.-Continued

[USGS, U.S. Geological Survey; $\mathrm{ft}^{3} / \mathrm{s}$, cubic foot per second; Ala., Alabama; EMA, expected moments algorithm; RRE, regional regression equations; *, large or regulated streamgage that required separate analysis]

\begin{tabular}{|c|c|c|c|c|c|c|c|c|c|c|c|c|}
\hline \multirow{2}{*}{$\begin{array}{l}\text { USGS station } \\
\text { number } \\
\text { (pl. 1) }\end{array}$} & \multirow{2}{*}{$\begin{array}{c}\text { Site } \\
\text { number } \\
\text { (fig. 1) }\end{array}$} & \multirow[b]{2}{*}{ USGS streamgage name } & \multirow{2}{*}{$\begin{array}{l}\text { Flood } \\
\text { region } \\
\text { (fig. 2) }\end{array}$} & \multirow[b]{2}{*}{ Method } & \multicolumn{8}{|c|}{ Annual exceedance probability flow (fts/s) } \\
\hline & & & & & $\begin{array}{c}50 \\
\text { percent }\end{array}$ & $\begin{array}{c}20 \\
\text { percent }\end{array}$ & $\begin{array}{c}10 \\
\text { percent }\end{array}$ & $\begin{array}{c}4 \\
\text { percent }\end{array}$ & $\begin{array}{c}2 \\
\text { percent }\end{array}$ & $\begin{array}{c}1 \\
\text { percent }\end{array}$ & $\begin{array}{c}0.5 \\
\text { percent }\end{array}$ & $\begin{array}{c}0.2 \\
\text { percent }\end{array}$ \\
\hline 02374000 & 49 & Conecuh River near Brooklyn, Ala. & $*$ & EMA & 29,200 & 48,800 & 63,800 & 85,000 & 102,000 & 121,000 & 141,000 & 170,000 \\
\hline 02374250 & 50 & $\begin{array}{l}\text { Conecuh River at State Hwy } 41 \text { near } \\
\text { Brewton, Ala. }\end{array}$ & * & EMA & 19,200 & 36,300 & 51,300 & 74,900 & 96,100 & 121,000 & 149,000 & 194,000 \\
\hline \multirow[t]{3}{*}{02374500} & \multirow[t]{3}{*}{51} & \multirow[t]{3}{*}{ Murder Creek near Evergreen, Ala. } & \multirow[t]{3}{*}{4} & EMA & 3,328 & 7,030 & 10,890 & 18,010 & 25,430 & 35,190 & 47,930 & 70,750 \\
\hline & & & & RRE & 4,530 & 8,350 & 11,700 & 16,500 & 20,600 & 25,100 & 30,300 & 38,100 \\
\hline & & & & Weighted & 3,580 & 7,660 & 11,500 & 16,800 & 21,400 & 26,600 & 32,600 & 42,300 \\
\hline \multirow[t]{3}{*}{02374745} & \multirow[t]{3}{*}{52} & \multirow{3}{*}{$\begin{array}{l}\text { Burnt Corn Creek at State Hwy } 41 \\
\text { near Brewton, Ala. }\end{array}$} & \multirow[t]{3}{*}{4} & EMA & 3,011 & 6,482 & 10,090 & 16,710 & 23,580 & 32,530 & 44,130 & 64,730 \\
\hline & & & & RRE & 4,610 & 8,510 & 11,900 & 16,800 & 21,000 & 25,600 & 30,900 & 38,800 \\
\hline & & & & Weighted & 3,870 & 8,110 & 11,700 & 16,800 & 21,200 & 26,000 & 31,600 & 40,200 \\
\hline \multirow[t]{3}{*}{02374950} & \multirow[t]{3}{*}{53} & \multirow{3}{*}{$\begin{array}{l}\text { Big Escambia Cr at Sardine } \mathrm{Br} \mathrm{nr} \\
\text { Stanley Crossroad }\end{array}$} & \multirow[t]{3}{*}{4} & EMA & 3,660 & 6,549 & 8,996 & 12,750 & 16,060 & 19,830 & 24,140 & 30,760 \\
\hline & & & & $\mathrm{RRE}$ & 4,770 & 8,800 & 12,300 & 17,300 & 21,700 & 26,400 & 31,900 & 40,100 \\
\hline & & & & Weighted & 4,180 & 8,150 & 11,800 & 16,800 & 21,000 & 25,600 & 30,900 & 38,800 \\
\hline \multirow[t]{3}{*}{02375000} & \multirow[t]{3}{*}{54} & \multirow{3}{*}{$\begin{array}{l}\text { Big Escambia Creek at Flomaton, } \\
\text { Ala. }\end{array}$} & \multirow[t]{3}{*}{4} & EMA & 6,959 & 12,540 & 17,500 & 25,450 & 32,780 & 41,450 & 51,710 & 68,140 \\
\hline & & & & RRE & 6,470 & 11,900 & 16,700 & 23,400 & 29,300 & 35,700 & 43,200 & 54,400 \\
\hline & & & & Weighted & 6,820 & 12,200 & 16,900 & 23,800 & 30,100 & 36,900 & 44,900 & 57,400 \\
\hline \multirow[t]{3}{*}{02377500} & \multirow[t]{3}{*}{57} & \multirow[t]{3}{*}{ Styx River Near Loxley, Ala. } & \multirow[t]{3}{*}{4} & EMA & 3,261 & 6,766 & 10,220 & 16,250 & 22,220 & 29,720 & 39,070 & 54,970 \\
\hline & & & & RRE & 3,160 & 5,860 & 8,150 & 11,400 & 14,300 & 17,500 & 21,000 & 26,400 \\
\hline & & & & Weighted & 3,210 & 6,140 & 8,540 & 12,000 & 15,300 & 19,000 & 23,000 & 29,700 \\
\hline \multirow[t]{3}{*}{02377570} & 58 & Styx River Near Elsanor, Ala. & 4 & EMA & 5,459 & 11,110 & 16,190 & 24,270 & 31,590 & 40,100 & 49,940 & 65,250 \\
\hline & & & & RRE & 4,790 & 8,870 & 12,300 & 17,200 & 21,600 & 26,400 & 31,800 & 40,000 \\
\hline & & & & Weighted & 5,170 & 9,740 & 13,300 & 18,500 & 23,600 & 29,000 & 34,900 & 44,700 \\
\hline 02378500 & 59 & Fish River near Silver Hill, Ala. & 4 & EMA & 1,989 & 4,709 & 7,486 & 12,400 & 17,270 & 23,360 & 30,890 & 43,510 \\
\hline & & & & RRE & 2,340 & 4,340 & 6,100 & 8,610 & 10,800 & 13,100 & 15,800 & 19,800 \\
\hline & & & & Weighted & 2,130 & 4,470 & 6,390 & 9,120 & 11,700 & 14,400 & 17,500 & 22,600 \\
\hline 02398300 & 66 & Chattooga River above Gaylesville, & 1 & EMA & 8,380 & 12,700 & 16,000 & 20,400 & 24,000 & 27,900 & 32,000 & 37,900 \\
\hline & & กí. & & RRE & 12,100 & 19,200 & 24,500 & 31,800 & 37,700 & 43,800 & 50,200 & 59,200 \\
\hline & & & & Weighted & 9,160 & 14,600 & 19,300 & 25,700 & 31,200 & 37,400 & 43,400 & 52,400 \\
\hline 02399000 & 67 & Little River near Jamestown, Ala. & 1 & EMA & 9,860 & 14,900 & 18,400 & 23,100 & 26,600 & 30,300 & 34,100 & 39,200 \\
\hline & & & & RRE & 5,910 & 9,590 & 12,400 & 16,100 & 19,200 & 22,300 & 25,600 & 30,200 \\
\hline & & & & Weighted & 9,490 & 14,200 & 17,500 & 21,600 & 24,600 & 27,600 & 30,900 & 35,200 \\
\hline
\end{tabular}


Appendix 1. Annual exceedance probability flows for U.S. Geological Survey streamgages used in this analysis. - Continued

[USGS, U.S. Geological Survey; ft³/s, cubic foot per second; Ala., Alabama; EMA, expected moments algorithm; RRE, regional regression equations; *, large or regulated streamgage that required separate analysis]

\begin{tabular}{|c|c|c|c|c|c|c|c|c|c|c|c|c|}
\hline \multirow{2}{*}{$\begin{array}{l}\text { USGS station } \\
\text { number } \\
\text { (pl. 1) }\end{array}$} & \multirow{2}{*}{$\begin{array}{c}\text { Site } \\
\text { number } \\
\text { (fig. 1) }\end{array}$} & \multirow[b]{2}{*}{ USGS streamgage name } & \multirow{2}{*}{$\begin{array}{l}\text { Flood } \\
\text { region } \\
\text { (fig. 2) }\end{array}$} & \multirow[b]{2}{*}{ Method } & \multicolumn{8}{|c|}{ Annual exceedance probability flow (ft/s) } \\
\hline & & & & & $\begin{array}{c}50 \\
\text { percent }\end{array}$ & $\begin{array}{c}20 \\
\text { percent }\end{array}$ & $\begin{array}{c}10 \\
\text { percent }\end{array}$ & $\begin{array}{c}4 \\
\text { percent }\end{array}$ & $\begin{array}{c}2 \\
\text { percent }\end{array}$ & $\begin{array}{c}1 \\
\text { percent }\end{array}$ & $\begin{array}{c}0.5 \\
\text { percent }\end{array}$ & $\begin{array}{c}0.2 \\
\text { percent }\end{array}$ \\
\hline \multirow[t]{3}{*}{02399200} & 68 & Little River near Blue Pond, Ala. & 1 & EMA & 14,300 & 23,000 & 29,200 & 37,400 & 43,700 & 50,200 & 56,800 & 65,900 \\
\hline & & & & RRE & 8,070 & 13,000 & 16,600 & 21,600 & 25,700 & 29,900 & 34,200 & 40,400 \\
\hline & & & & Weighted & 13,700 & 21,700 & 27,000 & 33,800 & 38,600 & 42,900 & 47,700 & 53,800 \\
\hline \multirow[t]{3}{*}{02400000} & 69 & Terrapin Creek near Piedmont, Ala. & 1 & EMA & 10,100 & 13,500 & 15,800 & 18,800 & 21,000 & 23,300 & 25,600 & 28,800 \\
\hline & & & & RRE & 5,630 & 9,140 & 11,800 & 15,400 & 18,300 & 21,300 & 24,400 & 28,800 \\
\hline & & & & Weighted & 9,890 & 13,200 & 15,400 & 18,300 & 20,500 & 22,900 & 25,300 & 28,800 \\
\hline \multirow[t]{3}{*}{02400100} & 70 & Terrapin Creek at Ellisville, Ala. & 1 & EMA & 6,720 & 10,800 & 13,800 & 17,800 & 20,900 & 24,200 & 27,600 & 32,400 \\
\hline & & & & RRE & 9,450 & 15,100 & 19,300 & 25,100 & 29,800 & 34,700 & 39,700 & 46,800 \\
\hline & & & & Weighted & 6,830 & 11,100 & 14,400 & 18,800 & 22,500 & 26,600 & 30,700 & 36,800 \\
\hline 02400500 & 71 & Coosa River at Gadsden, Ala. & $*$ & EMA & 47,200 & 60,200 & 68,800 & 80,000 & 88,500 & 97,100 & 106,000 & 118,000 \\
\hline \multirow[t]{3}{*}{02401000} & 72 & Big Wills Creek near Reece City & 1 & EMA & 5,200 & 8,520 & 10,900 & 13,900 & 16,200 & 18,500 & 20,900 & 24,000 \\
\hline & & & & RRE & 7,600 & 12,200 & 15,700 & 20,400 & 24,300 & 28,300 & 32,400 & 38,200 \\
\hline & & & & Weighted & 5,330 & 8,840 & 11,500 & 15,100 & 18,100 & 21,400 & 24,700 & 29,700 \\
\hline \multirow[t]{3}{*}{02401370} & 73 & Big Canoe Creek near Springville, & 1 & EMA & 2,530 & 3,620 & 4,370 & 5,350 & 6,090 & 6,860 & 7,640 & 8,720 \\
\hline & & & & RRE & 2,990 & 4,960 & 6,440 & 8,450 & 10,100 & 11,700 & 13,500 & 15,900 \\
\hline & & & & Weighted & 2,550 & 3,680 & 4,500 & 5,620 & 6,530 & 7,550 & 8,590 & 10,200 \\
\hline \multirow[t]{3}{*}{02401390} & 74 & Big Canoe Creek at Ashville, Ala. & 1 & EMA & 5,620 & 7,790 & 9,330 & 11,400 & 13,000 & 14,700 & 16,500 & 19,000 \\
\hline & & & & RRE & 6,410 & 10,400 & 13,400 & 17,400 & 20,700 & 24,100 & 27,600 & 32,500 \\
\hline & & & & Weighted & 5,680 & 7,960 & 9,640 & 11,900 & 13,900 & 16,100 & 18,300 & 21,800 \\
\hline \multirow[t]{3}{*}{02401470} & 75 & Little Canoe Creek near Steele, Ala. & 1 & EMA & 1,620 & 2,420 & 3,000 & 3,780 & 4,400 & 5,060 & 5,750 & 6,740 \\
\hline & & & & RRE & 1,870 & 3,150 & 4,120 & 5,420 & 6,480 & 7,560 & 8,680 & 10,200 \\
\hline & & & & Weighted & 1,630 & 2,450 & 3,060 & 3,880 & 4,560 & 5,320 & 6,090 & 7,270 \\
\hline \multirow[t]{3}{*}{02401500} & 76 & Big Canoe Creek near Gadsden, Ala. & 1 & EMA & 7,650 & 12,200 & 15,900 & 21,200 & 25,800 & 30,900 & 36,600 & 45,100 \\
\hline & & & & RRE & 9,470 & 15,100 & 19,400 & 25,200 & 29,900 & 34,800 & 39,800 & 47,000 \\
\hline & & & & Weighted & 7,730 & 12,400 & 16,300 & 21,800 & 26,500 & 31,800 & 37,400 & 45,700 \\
\hline \multirow[t]{3}{*}{02404000} & 77 & Choccolocco Creek near Jenifer, Ala. & 1 & EMA & 7,070 & 13,200 & 18,000 & 24,600 & 30,000 & 35,600 & 41,400 & 49,600 \\
\hline & & & & RRE & 10,100 & 16,000 & 20,500 & 26,600 & 31,700 & 36,800 & 42,100 & 49,700 \\
\hline & & & & Weighted & 7,150 & 13,300 & 18,100 & 24,800 & 30,200 & 35,800 & 41,500 & 49,600 \\
\hline
\end{tabular}


Appendix 1. Annual exceedance probability flows for U.S. Geological Survey streamgages used in this analysis.-Continued

[USGS, U.S. Geological Survey; $\mathrm{ft}^{3} / \mathrm{s}$, cubic foot per second; Ala., Alabama; EMA, expected moments algorithm; RRE, regional regression equations; *, large or regulated streamgage that required separate analysis]

\begin{tabular}{|c|c|c|c|c|c|c|c|c|c|c|c|c|}
\hline \multirow{2}{*}{$\begin{array}{c}\text { USGS station } \\
\text { number } \\
\text { (pl. 1) }\end{array}$} & \multirow{2}{*}{$\begin{array}{c}\text { Site } \\
\text { number } \\
\text { (fig. 1) }\end{array}$} & \multirow[b]{2}{*}{ USGS streamgage name } & \multirow{2}{*}{$\begin{array}{l}\text { Flood } \\
\text { region } \\
\text { (fig. 2) } \\
\end{array}$} & \multirow[b]{2}{*}{ Method } & \multicolumn{8}{|c|}{ Annual exceedance probability flow $\left(\mathrm{ft}^{3} / \mathrm{s}\right)$} \\
\hline & & & & & $\begin{array}{c}50 \\
\text { percent }\end{array}$ & $\begin{array}{c}20 \\
\text { percent }\end{array}$ & $\begin{array}{c}10 \\
\text { percent }\end{array}$ & $\begin{array}{c}4 \\
\text { percent }\end{array}$ & $\begin{array}{c}2 \\
\text { percent }\end{array}$ & $\begin{array}{c}1 \\
\text { percent }\end{array}$ & $\begin{array}{c}0.5 \\
\text { percent }\end{array}$ & $\begin{array}{c}0.2 \\
\text { percent }\end{array}$ \\
\hline \multirow[t]{3}{*}{02404400} & \multirow[t]{3}{*}{78} & \multirow{3}{*}{$\begin{array}{l}\text { Choccolocco Creek at Jackson Shoal } \\
\text { nr Linc }\end{array}$} & \multirow[t]{3}{*}{1} & EMA & 10,500 & 18,400 & 24,800 & 34,300 & 42,400 & 51,500 & 61,500 & 76,500 \\
\hline & & & & RRE & 14,500 & 22,900 & 29,200 & 37,800 & 44,800 & 52,000 & 59,500 & 70,300 \\
\hline & & & & Weighted & 10,600 & 18,500 & 25,000 & 34,500 & 42,500 & 51,500 & 61,300 & 75,700 \\
\hline \multirow[t]{3}{*}{02405500} & \multirow[t]{3}{*}{79} & \multirow[t]{3}{*}{ Kelly Creek near Vincent, Ala. } & \multirow[t]{3}{*}{1} & EMA & 6,800 & 11,000 & 14,500 & 19,600 & 24,100 & 29,200 & 35,000 & 43,800 \\
\hline & & & & RRE & 7,900 & 12,700 & 16,300 & 21,200 & 25,200 & 29,300 & 33,600 & 39,600 \\
\hline & & & & Weighted & 6,850 & 11,100 & 14,600 & 19,800 & 24,200 & 29,200 & 34,700 & 42,800 \\
\hline \multirow[t]{3}{*}{02406500} & \multirow[t]{3}{*}{80} & \multirow[t]{3}{*}{ Talladega Creek at Alpine, Ala. } & \multirow[t]{3}{*}{1} & EMA & 5,050 & 9,480 & 13,200 & 18,900 & 23,700 & 29,200 & 35,400 & 44,600 \\
\hline & & & & RRE & 6,680 & 10,800 & 13,900 & 18,100 & 21,500 & 25,000 & 28,700 & 33,800 \\
\hline & & & & Weighted & 5,440 & 9,880 & 13,500 & 18,500 & 22,500 & 26,600 & 31,100 & 37,000 \\
\hline 02407000 & 81 & Coosa River at Childersburg, Ala. & $*$ & EMA & 64,600 & 83,300 & 95,900 & 112,000 & 124,000 & 137,000 & 149,000 & 167,000 \\
\hline \multirow[t]{3}{*}{02407500} & \multirow[t]{3}{*}{82} & \multirow{3}{*}{$\begin{array}{l}\text { Yellowleaf Creek near Wilsonville, } \\
\text { Ala. }\end{array}$} & \multirow[t]{3}{*}{1} & EMA & 2,900 & 6,120 & 9,250 & 14,600 & 19,700 & 26,100 & 33,800 & 46,700 \\
\hline & & & & RRE & 4,970 & 8,120 & 10,500 & 13,700 & 16,300 & 19,000 & 21,700 & 25,700 \\
\hline & & & & Weighted & 3,040 & 6,300 & 9,390 & 14,500 & 19,000 & 24,200 & 30,100 & 38,400 \\
\hline \multirow[t]{3}{*}{02408500} & \multirow[t]{3}{*}{83} & \multirow[t]{3}{*}{ Hatchet Creek near Rockford, Ala. } & \multirow[t]{3}{*}{1} & EMA & 8,840 & 15,500 & 21,200 & 30,100 & 38,100 & 47,300 & 58,100 & 75,000 \\
\hline & & & & RRE & 8,960 & 14,300 & 18,400 & 23,900 & 28,400 & 33,000 & 37,800 & 44,600 \\
\hline & & & & Weighted & 8,850 & 15,400 & 20,900 & 29,300 & 36,600 & 44,500 & 53,500 & 66,000 \\
\hline \multirow[t]{3}{*}{02408540} & \multirow[t]{3}{*}{84} & Hatchet Creek below Rockford, Ala. & 1 & EMA & 9,970 & 18,000 & 24,600 & 34,600 & 43,100 & 52,600 & 63,200 & 79,000 \\
\hline & & & & RRE & 9,720 & 15,500 & 19,900 & 25,800 & 30,700 & 35,600 & 40,800 & 48,100 \\
\hline & & & & Weighted & 9,960 & 17,800 & 24,200 & 33,500 & 41,200 & 49,200 & 58,000 & 70,000 \\
\hline 02409000 & 85 & Weogufka Creek near Weogufka, & 1 & EMA & 3,150 & 6,350 & 9,300 & 14,100 & 18,600 & 23,900 & 30,300 & 40,400 \\
\hline & & & & RRE & 4,140 & 6,800 & 8,800 & 11,500 & 13,700 & 16,000 & 18,300 & 21,600 \\
\hline & & & & Weighted & 3,360 & 6,460 & 9,160 & 13,300 & 17,000 & 20,900 & 25,400 & 31,500 \\
\hline 02410000 & 86 & Paterson Creek near Central, Ala. & 1 & EMA & 627 & 1,080 & 1,460 & 2,050 & 2,580 & 3,180 & 3,870 & 4,940 \\
\hline & & & & RRE & 680 & 1,190 & 1,570 & 2,080 & 2,500 & 2,920 & 3,360 & 3,970 \\
\hline & & & & Weighted & 630 & 1,090 & 1,470 & 2,050 & 2,570 & 3,130 & 3,750 & 4,660 \\
\hline 02410000 & 86 & Paterson Creek near Central, Ala. & Small & EMA & 627 & 1,080 & 1,463 & 2,053 & 2,576 & 3,177 & 3,867 & 4,938 \\
\hline & & & & RRE & 1,010 & 1,790 & 2,460 & 3,320 & 3,970 & 4,580 & 5,210 & 6,070 \\
\hline & & & & Weighted & 638 & 1,150 & 1,690 & 2,640 & 3,410 & 4,080 & 4,760 & 5,640 \\
\hline
\end{tabular}


Appendix 1. Annual exceedance probability flows for U.S. Geological Survey streamgages used in this analysis. - Continued

[USGS, U.S. Geological Survey; ft³/s, cubic foot per second; Ala., Alabama; EMA, expected moments algorithm; RRE, regional regression equations; *, large or regulated streamgage that required separate analysis]

\begin{tabular}{|c|c|c|c|c|c|c|c|c|c|c|c|c|}
\hline \multirow{2}{*}{$\begin{array}{c}\text { USGS station } \\
\text { number } \\
\text { (pl. 1) }\end{array}$} & \multirow{2}{*}{$\begin{array}{c}\text { Site } \\
\text { number } \\
\text { (fig. 1) }\end{array}$} & \multirow[b]{2}{*}{ USGS streamgage name } & \multirow{2}{*}{$\begin{array}{l}\text { Flood } \\
\text { region } \\
\text { (fig. 2) }\end{array}$} & \multirow[b]{2}{*}{ Method } & \multicolumn{8}{|c|}{ Annual exceedance probability flow (ft³/s) } \\
\hline & & & & & $\begin{array}{c}50 \\
\text { percent }\end{array}$ & $\begin{array}{c}20 \\
\text { percent }\end{array}$ & $\begin{array}{c}10 \\
\text { percent }\end{array}$ & $\begin{array}{c}4 \\
\text { percent }\end{array}$ & $\begin{array}{c}2 \\
\text { percent }\end{array}$ & $\begin{array}{c}1 \\
\text { percent }\end{array}$ & $\begin{array}{c}0.5 \\
\text { percent }\end{array}$ & $\begin{array}{c}0.2 \\
\text { percent }\end{array}$ \\
\hline 02411000 & 87 & $\begin{array}{l}\text { Coosa River at Jordan Dam near } \\
\text { Wetumpka, Ala. }\end{array}$ & $*$ & EMA & 87,900 & 123,000 & 144,000 & 170,000 & 188,000 & 205,000 & 222,000 & 242,000 \\
\hline \multirow[t]{3}{*}{02412000} & \multirow[t]{3}{*}{91} & \multirow[t]{3}{*}{ Tallapoosa River near Heflin, Ala. } & \multirow[t]{3}{*}{1} & EMA & 7,470 & 11,400 & 14,500 & 18,900 & 22,600 & 26,600 & 31,000 & 37,600 \\
\hline & & & & RRE & 13,900 & 21,900 & 27,900 & 36,100 & 42,900 & 49,800 & 56,900 & 67,200 \\
\hline & & & & Weighted & 7,690 & 11,900 & 15,500 & 20,800 & 25,400 & 31,000 & 36,700 & 45,900 \\
\hline \multirow[t]{3}{*}{02412500} & \multirow[t]{3}{*}{92} & \multirow[t]{3}{*}{ Tallapoosa River near Ofelia, Ala. } & \multirow[t]{3}{*}{1} & EMA & 14,700 & 22,600 & 28,200 & 35,600 & 41,400 & 47,300 & 53,500 & 61,900 \\
\hline & & & & RRE & 20,300 & 31,600 & 40,200 & 51,800 & 61,400 & 71,200 & 81,400 & 96,000 \\
\hline & & & & Weighted & 14,800 & 22,900 & 28,800 & 36,800 & 43,500 & 50,600 & 58,000 & 69,100 \\
\hline \multirow[t]{3}{*}{02413300} & \multirow[t]{3}{*}{93} & \multirow{3}{*}{$\begin{array}{l}\text { Little Tallapoosa River near Newell, } \\
\text { Ala. }\end{array}$} & \multirow[t]{3}{*}{1} & EMA & 6,890 & 11,000 & 14,000 & 18,400 & 22,000 & 25,800 & 29,900 & 35,900 \\
\hline & & & & RRE & 13,000 & 20,500 & 26,200 & 33,900 & 40,300 & 46,800 & 53,500 & 63,200 \\
\hline & & & & Weighted & 7,570 & 12,600 & 16,900 & 23,400 & 29,000 & 35,300 & 41,500 & 51,200 \\
\hline \multirow[t]{3}{*}{02413400} & \multirow[t]{3}{*}{94} & \multirow{3}{*}{$\begin{array}{l}\text { Wedowee Creek above Wedowee, } \\
\text { Ala. }\end{array}$} & \multirow[t]{3}{*}{1} & EMA & 956 & 1,310 & 1,560 & 1,870 & 2,110 & 2,350 & 2,590 & 2,930 \\
\hline & & & & RRE & 851 & 1,470 & 1,940 & 2,580 & 3,080 & 3,610 & 4,150 & 4,890 \\
\hline & & & & Weighted & 923 & 1,360 & 1,710 & 2,200 & 2,600 & 3,060 & 3,510 & 4,190 \\
\hline \multirow[t]{3}{*}{02413400} & \multirow[t]{3}{*}{94} & \multirow{3}{*}{$\begin{array}{l}\text { Wedowee Creek above Wedowee, } \\
\text { Ala. }\end{array}$} & \multirow{3}{*}{$\begin{array}{l}\text { Small } \\
\text { stream }\end{array}$} & EMA & 956 & 1,314 & 1,557 & 1,869 & 2,106 & 2,347 & 2,593 & 2,930 \\
\hline & & & & RRE & 1,190 & 2,120 & 2,920 & 3,960 & 4,760 & 5,520 & 6,300 & 7,390 \\
\hline & & & & Weighted & 963 & 1,380 & 1,790 & 2,560 & 3,240 & 3,770 & 4,230 & 4,560 \\
\hline \multirow[t]{3}{*}{02413475} & 95 & Wedowee Creek near Wedowee, Ala. & 1 & EMA & 2,910 & 4,000 & 4,620 & 5,290 & 5,740 & 6,140 & 6,500 & 6,920 \\
\hline & & & & RRE & 3,060 & 5,070 & 6,590 & 8,640 & 10,300 & 12,000 & 13,800 & 16,300 \\
\hline & & & & Weighted & 2,920 & 4,110 & 4,880 & 5,850 & 6,640 & 7,550 & 8,420 & 9,810 \\
\hline 02413500 & 96 & Little Tallapoosa River near & 1 & EMA & 12,500 & 18,700 & 22,600 & 27,400 & 30,800 & 34,100 & 37,200 & 41,200 \\
\hline & & Wedowee, Ala. & & RRE & 16,700 & 26,200 & 33,300 & 43,000 & 51,000 & 59,200 & 67,700 & 79,900 \\
\hline & & & & Weighted & 13,100 & 19,200 & 23,500 & 29,500 & 34,500 & 40,100 & 45,500 & 53,700 \\
\hline 02414500 & 97 & Tallapoosa River at Wadley, Ala. & * & EMA & 27,200 & 40,700 & 51,200 & 66,000 & 78,300 & 91,800 & 106,000 & 128,000 \\
\hline 02414800 & 98 & Harbuck Creek near Hackneyville, & 1 & EMA & 1,150 & 2,020 & 2,760 & 3,940 & 4,990 & 6,220 & 7,640 & 9,880 \\
\hline & & Ala. & & RRE & 940 & 1,620 & 2,140 & 2,830 & 3,390 & 3,960 & 4,550 & 5,370 \\
\hline & & & & Weighted & 1,120 & 1,950 & 2,610 & 3,580 & 4,390 & 5,200 & 6,130 & 7,350 \\
\hline 02414800 & 98 & Harbuck Creek near Hackneyville, & Small & EMA & 1,147 & 2,015 & 2,763 & 3,935 & 4,990 & 6,217 & 7,642 & 9,880 \\
\hline & & 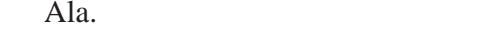 & stream & RRE & 1,280 & 2,290 & 3,150 & 4,280 & 5,150 & 5,990 & 6,860 & 8,060 \\
\hline & & & & Weighted & 1,150 & 2,060 & 2,890 & 4,140 & 5,100 & 6,050 & 7,040 & 8,550 \\
\hline
\end{tabular}


Appendix 1. Annual exceedance probability flows for U.S. Geological Survey streamgages used in this analysis.-Continued

[USGS, U.S. Geological Survey; $\mathrm{ft}^{3} / \mathrm{s}$, cubic foot per second; Ala., Alabama; EMA, expected moments algorithm; RRE, regional regression equations; *, large or regulated streamgage that required separate analysis]

\begin{tabular}{|c|c|c|c|c|c|c|c|c|c|c|c|c|}
\hline \multirow{2}{*}{$\begin{array}{c}\text { USGS station } \\
\text { number } \\
\text { (pl. 1) }\end{array}$} & \multirow{2}{*}{$\begin{array}{c}\text { Site } \\
\text { number } \\
\text { (fig. 1) }\end{array}$} & \multirow[b]{2}{*}{ USGS streamgage name } & \multirow{2}{*}{$\begin{array}{l}\text { Flood } \\
\text { region } \\
\text { (fig. 2) } \\
\end{array}$} & \multirow[b]{2}{*}{ Method } & \multicolumn{8}{|c|}{ Annual exceedance probability flow (ft3/s) } \\
\hline & & & & & $\begin{array}{c}50 \\
\text { percent }\end{array}$ & $\begin{array}{c}20 \\
\text { percent }\end{array}$ & $\begin{array}{c}10 \\
\text { percent }\end{array}$ & $\begin{array}{c}4 \\
\text { percent }\end{array}$ & $\begin{array}{c}2 \\
\text { percent }\end{array}$ & $\begin{array}{c}1 \\
\text { percent }\end{array}$ & $\begin{array}{c}0.5 \\
\text { percent }\end{array}$ & $\begin{array}{c}0.2 \\
\text { percent }\end{array}$ \\
\hline \multirow[t]{3}{*}{02415000} & \multirow[t]{3}{*}{99} & \multirow{3}{*}{$\begin{array}{l}\text { Hillabee Creek near Hackneyville, } \\
\text { Ala. }\end{array}$} & \multirow[t]{3}{*}{1} & EMA & 7,720 & 11,500 & 14,100 & 17,500 & 20,100 & 22,800 & 25,500 & 29,300 \\
\hline & & & & RRE & 7,820 & 12,600 & 16,200 & 21,000 & 25,000 & 29,000 & 33,300 & 39,200 \\
\hline & & & & Weighted & 7,740 & 11,700 & 14,600 & 18,500 & 21,600 & 25,100 & 28,600 & 33,800 \\
\hline \multirow[t]{3}{*}{02418230} & \multirow[t]{3}{*}{100} & \multirow{3}{*}{$\begin{array}{l}\text { Sougahatchee Creek at Co Rd } 188 \mathrm{nr} \\
\text { Loachap }\end{array}$} & \multirow[t]{3}{*}{1} & EMA & 3,260 & 5,760 & 7,860 & 11,100 & 13,900 & 17,100 & 20,700 & 26,300 \\
\hline & & & & RRE & 4,060 & 6,680 & 8,640 & 11,300 & 13,500 & 15,700 & 18,000 & 21,200 \\
\hline & & & & Weighted & 3,340 & 5,900 & 8,010 & 11,200 & 13,800 & 16,600 & 19,500 & 23,700 \\
\hline 02418500 & 101 & $\begin{array}{l}\text { Tallapoosa River below Tallassee, } \\
\text { Ala. }\end{array}$ & $*$ & EMA & 26,700 & 54,100 & 75,200 & 104,100 & 126,000 & 149,000 & 172,000 & 203,000 \\
\hline \multirow[t]{3}{*}{02418760} & \multirow[t]{3}{*}{102} & \multirow{3}{*}{$\begin{array}{l}\text { Chewacla Creek at Chewacla State } \\
\text { Park nr Auburn }\end{array}$} & \multirow[t]{3}{*}{4} & EMA & 2,419 & 4,625 & 6,463 & 9,207 & 11,550 & 14,150 & 17,030 & 21,280 \\
\hline & & & & RRE & 2,110 & 3,900 & 5,490 & 7,750 & 9,700 & 11,800 & 14,200 & 17,800 \\
\hline & & & & Weighted & 2,240 & 4,050 & 5,600 & 7,870 & 9,870 & 12,000 & 14,500 & 18,200 \\
\hline \multirow[t]{3}{*}{02419000} & \multirow[t]{3}{*}{103} & \multirow[t]{3}{*}{ Uphapee Creek near Tuskegee, Ala. } & \multirow[t]{3}{*}{4} & EMA & 8,435 & 15,420 & 20,900 & 28,670 & 35,000 & 41,750 & 48,940 & 59,140 \\
\hline & & & & RRE & 6,550 & 12,100 & 16,800 & 23,500 & 29,300 & 35,900 & 43,400 & 54,700 \\
\hline & & & & Weighted & 8,020 & 14,200 & 18,700 & 25,400 & 31,500 & 38,100 & 45,400 & 56,500 \\
\hline \multirow[t]{3}{*}{02419625} & \multirow[t]{3}{*}{104} & \multirow[t]{3}{*}{ Calebee Creek near Tuskegee, Ala. } & \multirow[t]{3}{*}{3} & EMA & 4,034 & 8,800 & 13,200 & 20,290 & 26,760 & 34,300 & 43,020 & 56,580 \\
\hline & & & & RRE & 4,940 & 9,090 & 12,800 & 18,100 & 22,600 & 27,300 & 32,600 & 40,300 \\
\hline & & & & Weighted & 4,280 & 8,960 & 12,900 & 18,400 & 22,900 & 27,800 & 33,200 & 41,100 \\
\hline 02420000 & 105 & $\begin{array}{l}\text { Alabama River near Montgomery, } \\
\text { Ala. }\end{array}$ & $*$ & EMA & 115,000 & 157,000 & 186,000 & 225,000 & 254,000 & 285,000 & 316,000 & 360,000 \\
\hline \multirow[t]{3}{*}{02420500} & \multirow[t]{3}{*}{106} & \multirow[t]{3}{*}{ Autauga Creek at Prattville, Ala. } & 2 & EMA & 1,950 & 3,860 & 5,720 & 8,960 & 12,200 & 16,200 & 21,300 & 30,000 \\
\hline & & & & RRE & 4,440 & 7,330 & 9,600 & 12,700 & 15,200 & 17,900 & 20,700 & 24,800 \\
\hline & & & & Weighted & 2,070 & 4,250 & 6,520 & 10,400 & 13,800 & 17,300 & 20,900 & 26,100 \\
\hline 02421000 & 107 & Catoma Creek near Montgomery, & 3 & EMA & 9,330 & 16,870 & 23,360 & 33,460 & 42,480 & 52,900 & 64,900 & 83,560 \\
\hline & & & & RRE & 7,500 & 13,900 & 19,700 & 28,200 & 35,600 & 43,700 & 52,800 & 66,200 \\
\hline & & & & Weighted & 9,200 & 16,300 & 21,900 & 30,300 & 37,200 & 45,900 & 55,100 & 69,200 \\
\hline 02422000 & 108 & Big Swamp Creek near & 3 & EMA & 6,992 & 13,690 & 19,580 & 28,800 & 37,050 & 46,540 & 57,440 & 74,260 \\
\hline & & Lowndesboro, Ala. & & RRE & 6,890 & 12,800 & 18,100 & 25,800 & 32,500 & 39,700 & 47,900 & 59,800 \\
\hline & & & & Weighted & 6,980 & 13,400 & 18,800 & 26,800 & 33,300 & 41,000 & 49,300 & 61,800 \\
\hline
\end{tabular}


Appendix 1. Annual exceedance probability flows for U.S. Geological Survey streamgages used in this analysis. - Continued

[USGS, U.S. Geological Survey; ft³/s, cubic foot per second; Ala., Alabama; EMA, expected moments algorithm; RRE, regional regression equations; *, large or regulated streamgage that required separate analysis]

\begin{tabular}{|c|c|c|c|c|c|c|c|c|c|c|c|c|}
\hline \multirow{2}{*}{$\begin{array}{l}\text { USGS station } \\
\text { number } \\
\text { (pl. 1) }\end{array}$} & \multirow{2}{*}{$\begin{array}{c}\text { Site } \\
\text { number } \\
\text { (fig. 1) }\end{array}$} & \multirow[b]{2}{*}{ USGS streamgage name } & \multirow{2}{*}{$\begin{array}{l}\text { Flood } \\
\text { region } \\
\text { (fig. 2) }\end{array}$} & \multirow[b]{2}{*}{ Method } & \multicolumn{8}{|c|}{ Annual exceedance probability flow (ft/s) } \\
\hline & & & & & $\begin{array}{c}50 \\
\text { percent }\end{array}$ & $\begin{array}{c}20 \\
\text { percent }\end{array}$ & $\begin{array}{c}10 \\
\text { percent }\end{array}$ & $\begin{array}{c}4 \\
\text { percent }\end{array}$ & $\begin{array}{c}2 \\
\text { percent }\end{array}$ & $\begin{array}{c}1 \\
\text { percent }\end{array}$ & $\begin{array}{c}0.5 \\
\text { percent }\end{array}$ & $\begin{array}{c}0.2 \\
\text { percent }\end{array}$ \\
\hline \multirow[t]{3}{*}{02422500} & \multirow[t]{3}{*}{109} & \multirow[t]{3}{*}{ Mulberry Creek at Jones, Ala. } & \multirow[t]{3}{*}{2} & EMA & 5,680 & 9,730 & 13,300 & 19,000 & 24,200 & 30,300 & 37,700 & 49,400 \\
\hline & & & & RRE & 6,340 & 10,500 & 13,800 & 18,300 & 21,900 & 25,800 & 29,900 & 35,800 \\
\hline & & & & Weighted & 5,690 & 9,770 & 13,400 & 18,800 & 23,400 & 28,200 & 33,500 & 41,500 \\
\hline \multirow[t]{3}{*}{02423380} & \multirow[t]{3}{*}{110} & \multirow{3}{*}{$\begin{array}{l}\text { Cahaba River near Mountain Brook, } \\
\text { Ala. }\end{array}$} & \multirow[t]{3}{*}{1} & EMA & 7,030 & 11,400 & 14,500 & 18,800 & 22,300 & 25,800 & 29,600 & 34,900 \\
\hline & & & & RRE & 6,380 & 10,300 & 13,300 & 17,300 & 20,600 & 24,000 & 27,500 & 32,400 \\
\hline & & & & Weighted & 6,940 & 11,200 & 14,300 & 18,500 & 21,900 & 25,300 & 28,900 & 33,900 \\
\hline \multirow[t]{3}{*}{02423500} & \multirow[t]{3}{*}{111} & \multirow[t]{3}{*}{ Cahaba River near Acton, Ala. } & \multirow[t]{3}{*}{1} & EMA & 8,550 & 13,800 & 17,900 & 23,800 & 28,700 & 34,100 & 40,100 & 48,900 \\
\hline & & & & RRE & 8,890 & 14,200 & 18,200 & 23,700 & 28,200 & 32,700 & 37,500 & 44,200 \\
\hline & & & & Weighted & 8,660 & 13,900 & 18,000 & 23,800 & 28,400 & 33,300 & 38,500 & 45,600 \\
\hline \multirow[t]{3}{*}{02423555} & \multirow[t]{3}{*}{112} & \multirow[t]{3}{*}{ Cahaba River near Helena, Ala. } & \multirow[t]{3}{*}{1} & EMA & 10,600 & 16,100 & 20,300 & 26,100 & 31,000 & 36,200 & 41,800 & 50,000 \\
\hline & & & & RRE & 11,400 & 18,100 & 23,200 & 30,000 & 35,700 & 41,500 & 47,500 & 56,000 \\
\hline & & & & Weighted & 10,800 & 16,700 & 21,300 & 27,700 & 33,100 & 39,000 & 45,000 & 53,700 \\
\hline \multirow[t]{3}{*}{02423630} & \multirow[t]{3}{*}{113} & \multirow[t]{3}{*}{ Shades Creek near Greenwood, Ala. } & \multirow[t]{3}{*}{1} & EMA & 3,900 & 5,130 & 5,970 & 7,060 & 7,900 & 8,760 & 9,650 & 10,900 \\
\hline & & & & RRE & 4,100 & 6,740 & 8,720 & 11,400 & 13,600 & 15,800 & 18,100 & 21,400 \\
\hline & & & & Weighted & 3,920 & 5,390 & 6,590 & 8,370 & 9,940 & 11,800 & 13,600 & 16,500 \\
\hline \multirow[t]{3}{*}{02424000} & \multirow[t]{3}{*}{114} & Cahaba River at Centreville, Ala. & 1 & EMA & 28,900 & 47,700 & 61,300 & 79,600 & 93,800 & 108,000 & 123,000 & 144,000 \\
\hline & & & & RRE & 24,100 & 37,400 & 47,400 & 61,000 & 72,300 & 83,800 & 95,800 & 113,000 \\
\hline & & & & Weighted & 28,400 & 46,600 & 59,200 & 76,200 & 89,300 & 102,000 & 116,000 & 135,000 \\
\hline 02424010 & 115 & Sandy Creek near Centreville, Ala. & 2 & EMA & 194 & 321 & 424 & 578 & 710 & 859 & 1,030 & 1,280 \\
\hline & & & & RRE & 154 & 242 & 310 & 404 & 472 & 562 & 651 & 785 \\
\hline & & & & Weighted & 190 & 304 & 385 & 486 & 560 & 648 & 746 & 905 \\
\hline 02424010 & 115 & Sandy Creek near Centreville, Ala. & Small & EMA & 187 & 300 & 389 & 519 & 629 & 751 & 886 & 1,087 \\
\hline & & & stream & RRE & 356 & 617 & 836 & 1,090 & 1,270 & 1,420 & 1,560 & 1,750 \\
\hline & & & & Weighted & 194 & 335 & 493 & 774 & 994 & 1,160 & 1,310 & 1,470 \\
\hline 02424500 & 116 & Cahaba River at Sprott, Ala. & 2 & EMA & 24,800 & 43,800 & 60,000 & 85,300 & 108,000 & 134,000 & 164,000 & 211,000 \\
\hline & & & & RRE & 21,400 & 36,100 & 47,800 & 63,700 & 76,800 & 90,000 & 104,000 & 125,000 \\
\hline & & & & Weighted & 24,600 & 42,700 & 56,900 & 75,900 & 90,900 & 105,000 & 122,000 & 149,000 \\
\hline
\end{tabular}


Appendix 1. Annual exceedance probability flows for U.S. Geological Survey streamgages used in this analysis.-Continued

[USGS, U.S. Geological Survey; $\mathrm{ft}^{3} / \mathrm{s}$, cubic foot per second; Ala., Alabama; EMA, expected moments algorithm; RRE, regional regression equations; *, large or regulated streamgage that required separate analysis]

\begin{tabular}{|c|c|c|c|c|c|c|c|c|c|c|c|c|}
\hline \multirow{2}{*}{$\begin{array}{l}\text { USGS station } \\
\text { number } \\
\text { (pl. 1) }\end{array}$} & \multirow{2}{*}{$\begin{array}{c}\text { Site } \\
\text { number } \\
\text { (fig. 1) }\end{array}$} & \multirow[b]{2}{*}{ USGS streamgage name } & \multirow{2}{*}{$\begin{array}{l}\text { Flood } \\
\text { region } \\
\text { (fig. 2) }\end{array}$} & \multirow[b]{2}{*}{ Method } & \multicolumn{8}{|c|}{ Annual exceedance probability flow (fts/s) } \\
\hline & & & & & $\begin{array}{c}50 \\
\text { percent }\end{array}$ & $\begin{array}{c}20 \\
\text { percent }\end{array}$ & $\begin{array}{c}10 \\
\text { percent }\end{array}$ & $\begin{array}{c}4 \\
\text { percent }\end{array}$ & $\begin{array}{c}2 \\
\text { percent }\end{array}$ & $\begin{array}{c}1 \\
\text { percent }\end{array}$ & $\begin{array}{c}0.5 \\
\text { percent }\end{array}$ & $\begin{array}{c}0.2 \\
\text { percent }\end{array}$ \\
\hline \multirow[t]{3}{*}{02425000} & \multirow[t]{3}{*}{117} & \multirow{3}{*}{$\begin{array}{l}\text { Cahaba River near Marion Junction, } \\
\text { Ala. }\end{array}$} & \multirow[t]{3}{*}{2} & EMA & 25,400 & 41,500 & 54,500 & 73,600 & 90,000 & 108,000 & 129,000 & 159,000 \\
\hline & & & & $\mathrm{RRE}$ & 25,200 & 42,600 & 56,400 & 75,200 & 90,800 & 106,000 & 123,000 & 147,000 \\
\hline & & & & Weighted & 25,400 & 41,600 & 54,700 & 73,900 & 90,200 & 107,000 & 126,000 & 154,000 \\
\hline \multirow[t]{3}{*}{02425500} & \multirow[t]{3}{*}{118} & \multirow[t]{3}{*}{ Cedar Creek at Minter, Ala. } & \multirow[t]{3}{*}{3} & EMA & 7,932 & 13,540 & 18,280 & 25,560 & 32,030 & 39,450 & 47,990 & 61,230 \\
\hline & & & & RRE & 6,420 & 11,900 & 16,800 & 23,900 & 30,000 & 36,700 & 44,100 & 55,000 \\
\hline & & & & Weighted & 7,760 & 13,000 & 17,500 & 24,400 & 30,400 & 37,300 & 44,700 & 55,900 \\
\hline \multirow[t]{3}{*}{02425655} & \multirow[t]{3}{*}{119} & \multirow[t]{3}{*}{ Mush Creek near Selma, Ala. } & \multirow[t]{3}{*}{3} & EMA & 4,269 & 7,480 & 10,180 & 14,310 & 17,940 & 22,090 & 26,820 & 34,080 \\
\hline & & & & RRE & 2,980 & 5,430 & 7,570 & 10,500 & 13,000 & 15,500 & 18,200 & 22,000 \\
\hline & & & & Weighted & 4,100 & 6,930 & 8,970 & 11,900 & 14,000 & 16,900 & 19,500 & 23,700 \\
\hline \multirow[t]{3}{*}{02426000} & \multirow[t]{3}{*}{120} & \multirow[t]{3}{*}{ Boguechitto Creek near Browns, Ala. } & \multirow[t]{3}{*}{3} & EMA & 4,292 & 7,590 & 10,250 & 14,150 & 17,440 & 21,070 & 25,070 & 30,960 \\
\hline & & & & RRE & 4,340 & 7,970 & 11,200 & 15,800 & 19,600 & 23,600 & 28,100 & 34,500 \\
\hline & & & & Weighted & 4,300 & 7,710 & 10,800 & 15,300 & 19,200 & 23,100 & 27,700 & 34,000 \\
\hline \multirow[t]{3}{*}{02427250} & \multirow[t]{3}{*}{121} & \multirow{3}{*}{$\begin{array}{l}\text { Pine Barren Creek near Snow Hill, } \\
\text { Ala. }\end{array}$} & \multirow[t]{3}{*}{3} & EMA & 7,349 & 13,080 & 18,170 & 26,320 & 33,840 & 42,750 & 53,290 & 70,210 \\
\hline & & & & RRE & 7,120 & 13,200 & 18,700 & 26,700 & 33,700 & 41,200 & 49,700 & 62,300 \\
\hline & & & & Weighted & 7,320 & 13,100 & 18,500 & 26,600 & 33,700 & 41,400 & 50,000 & 62,900 \\
\hline \multirow[t]{3}{*}{02427300} & 122 & Prairie Creek near Oak Hill, Ala. & 4 & EMA & 1,029 & 1,394 & 1,650 & 1,993 & 2,261 & 2,541 & 2,835 & 3,248 \\
\hline & & & & RRE & 910 & 1,700 & 2,380 & 3,350 & 4,190 & 5,110 & 6,130 & 7,620 \\
\hline & & & & Weighted & 1,000 & 1,520 & 2,080 & 2,900 & 3,490 & 4,140 & 4,890 & 5,750 \\
\hline 02427300 & 122 & Prairie Creek near Oak Hill, Ala. & Small & EMA & 1,029 & 1,394 & 1,650 & 1,993 & 2,261 & 2,541 & 2,835 & 3,248 \\
\hline & & & stream & RRE & 1,450 & 2,600 & 3,590 & 4,900 & 5,920 & 6,900 & 7,930 & 9,370 \\
\hline & & & & Weighted & 1,040 & 1,480 & 1,960 & 2,920 & 3,810 & 4,490 & 5,100 & 5,530 \\
\hline 02427700 & 123 & Turkey Creek at Kimbrough, Ala. & 3 & EMA & 4,349 & 7,874 & 11,050 & 16,230 & 21,060 & 26,840 & 33,770 & 45,000 \\
\hline & & & & RRE & 4,390 & 8,060 & 11,300 & 15,900 & 19,800 & 23,900 & 28,400 & 35,000 \\
\hline & & & & Weighted & 4,350 & 7,930 & 11,200 & 16,000 & 20,000 & 24,400 & 29,100 & 36,100 \\
\hline 02427875 & 124 & Pursley Creek near Camden, Ala. & 4 & EMA & 3,396 & 5,250 & 6,757 & 9,014 & 10,970 & 13,200 & 15,720 & 19,580 \\
\hline & & & & RRE & 2,580 & 4,780 & 6,650 & 9,350 & 11,700 & 14,300 & 17,200 & 21,500 \\
\hline & & & & Weighted & 3,140 & 4,980 & 6,680 & 9,290 & 11,600 & 14,100 & 16,900 & 21,100 \\
\hline 02428500 & 125 & Big Flat Creek near Fountain, Ala. & 4 & EMA & 4,774 & 9,435 & 13,920 & 21,620 & 29,150 & 38,510 & 50,090 & 69,620 \\
\hline & & & & RRE & 5,490 & 10,100 & 14,200 & 19,900 & 24,900 & 30,400 & 36,700 & 46,100 \\
\hline & & & & Weighted & 5,070 & 9,900 & 14,200 & 20,100 & 25,300 & 31,200 & 37,900 & 48,300 \\
\hline
\end{tabular}


Appendix 1. Annual exceedance probability flows for U.S. Geological Survey streamgages used in this analysis.-Continued

[USGS, U.S. Geological Survey; ft³/s, cubic foot per second; Ala., Alabama; EMA, expected moments algorithm; RRE, regional regression equations; *, large or regulated streamgage that required separate analysis]

\begin{tabular}{|c|c|c|c|c|c|c|c|c|c|c|c|c|}
\hline \multirow{2}{*}{$\begin{array}{l}\text { USGS station } \\
\text { number } \\
\text { (pl. 1) }\end{array}$} & \multirow{2}{*}{$\begin{array}{c}\text { Site } \\
\text { number } \\
\text { (fig. 1) }\end{array}$} & \multirow[b]{2}{*}{ USGS streamgage name } & \multirow{2}{*}{$\begin{array}{l}\text { Flood } \\
\text { region } \\
\text { (fig. 2) } \\
\end{array}$} & \multirow[b]{2}{*}{ Method } & \multicolumn{8}{|c|}{ Annual exceedance probability flow (ft/s) } \\
\hline & & & & & $\begin{array}{c}50 \\
\text { percent }\end{array}$ & $\begin{array}{c}20 \\
\text { percent }\end{array}$ & $\begin{array}{c}10 \\
\text { percent }\end{array}$ & $\begin{array}{c}4 \\
\text { percent }\end{array}$ & $\begin{array}{c}2 \\
\text { percent }\end{array}$ & $\begin{array}{c}1 \\
\text { percent }\end{array}$ & $\begin{array}{c}0.5 \\
\text { percent }\end{array}$ & $\begin{array}{c}0.2 \\
\text { percent }\end{array}$ \\
\hline \multirow[t]{3}{*}{02429000} & \multirow[t]{3}{*}{126} & \multirow{3}{*}{$\begin{array}{l}\text { Limestone Creek near Monroeville, } \\
\text { Ala. }\end{array}$} & \multirow[t]{3}{*}{4} & EMA & 3,835 & 7,342 & 10,540 & 15,770 & 20,660 & 26,510 & 33,480 & 44,740 \\
\hline & & & & RRE & 3,660 & 6,760 & 9,480 & 13,300 & 16,700 & 20,300 & 24,600 & 30,800 \\
\hline & & & & Weighted & 3,760 & 6,950 & 9,690 & 13,600 & 17,300 & 21,200 & 25,800 & 32,800 \\
\hline \multirow[t]{3}{*}{02437800} & \multirow[t]{3}{*}{135} & \multirow[t]{3}{*}{ Barn Creek near Hackleburg, Ala. } & \multirow[t]{3}{*}{1} & EMA & 1,310 & 2,390 & 3,340 & 4,850 & 6,230 & 7,840 & 9,720 & 12,700 \\
\hline & & & & RRE & 1,310 & 2,230 & 2,930 & 3,870 & 4,630 & 5,410 & 6,220 & 7,340 \\
\hline & & & & Weighted & 1,310 & 2,380 & 3,310 & 4,760 & 6,050 & 7,490 & 9,140 & 11,600 \\
\hline \multirow[t]{3}{*}{02437800} & \multirow[t]{3}{*}{135} & \multirow[t]{3}{*}{ Barn Creek near Hackleburg, Ala. } & \multirow{3}{*}{$\begin{array}{l}\text { Small } \\
\text { stream }\end{array}$} & EMA & 1,305 & 2,389 & 3,340 & 4,852 & 6,226 & 7,836 & 9,717 & 12,690 \\
\hline & & & & RRE & 1,630 & 2,940 & 4,060 & 5,550 & 6,730 & 7,890 & 9,090 & 10,800 \\
\hline & & & & Weighted & 1,340 & 2,540 & 3,700 & 5,360 & 6,640 & 7,880 & 9,190 & 11,200 \\
\hline \multirow[t]{3}{*}{02438000} & \multirow[t]{3}{*}{136} & \multirow{3}{*}{$\begin{array}{l}\text { Buttahatchee River below Hamilton, } \\
\text { Ala. }\end{array}$} & \multirow[t]{3}{*}{1} & EMA & 14,900 & 21,300 & 25,500 & 30,700 & 34,500 & 38,200 & 41,900 & 46,800 \\
\hline & & & & RRE & 10,100 & 16,000 & 20,500 & 26,600 & 31,700 & 36,800 & 42,100 & 49,700 \\
\hline & & & & Weighted & 14,500 & 20,900 & 25,000 & 30,200 & 34,100 & 38,000 & 41,900 & 47,400 \\
\hline \multirow[t]{3}{*}{02439000} & \multirow[t]{3}{*}{137} & \multirow{3}{*}{$\begin{array}{l}\text { Buttahatchee River near Sulligent, } \\
\text { Ala. }\end{array}$} & \multirow[t]{3}{*}{2} & EMA & 15,600 & 25,500 & 32,600 & 42,000 & 49,300 & 56,600 & 64,200 & 74,400 \\
\hline & & & & RRE & 10,900 & 18,100 & 23,900 & 31,800 & 38,200 & 44,800 & 51,900 & 62,200 \\
\hline & & & & Weighted & 15,300 & 24,800 & 31,200 & 39,300 & 45,500 & 51,500 & 58,400 & 68,500 \\
\hline \multirow[t]{3}{*}{02442000} & 141 & Luxapallila Creek near Fayette, Ala. & 2 & EMA & 5,790 & 8,210 & 9,890 & 12,100 & 13,800 & 15,600 & 17,400 & 20,000 \\
\hline & & & & RRE & 4,780 & 7,890 & 10,300 & 13,700 & 16,400 & 19,300 & 22,300 & 26,800 \\
\hline & & & & Weighted & 5,770 & 8,200 & 9,920 & 12,300 & 14,300 & 16,600 & 18,900 & 22,100 \\
\hline 02442500 & 142 & Luxapallila Creek at Millport, Ala. & 2 & EMA & 6,910 & 10,000 & 12,200 & 15,100 & 17,400 & 19,700 & 22,200 & 25,600 \\
\hline & & & & RRE & 7,190 & 11,900 & 15,700 & 20,800 & 24,900 & 29,300 & 34,000 & 40,700 \\
\hline & & & & Weighted & 6,920 & 10,100 & 12,400 & 15,800 & 18,600 & 21,900 & 25,300 & 29,800 \\
\hline 02444000 & 145 & Coal Fire Creek nr Pickensville, Ala. & 2 & EMA & 2,490 & 4,860 & 7,030 & 10,600 & 13,800 & 17,800 & 22,400 & 29,800 \\
\hline & & & & RRE & 4,680 & 7,730 & 10,100 & 13,400 & 16,000 & 18,900 & 21,900 & 26,200 \\
\hline & & & & Weighted & 2,650 & 5,340 & 7,930 & 12,000 & 15,100 & 18,600 & 22,000 & 27,000 \\
\hline 02445000 & 146 & Lubbub Creek nr Carrollton, Ala. & 2 & EMA & 2,630 & 5,190 & 7,590 & 11,600 & 15,400 & 20,100 & 25,700 & 35,000 \\
\hline & & & & RRE & 4,340 & 7,170 & 9,390 & 12,400 & 14,800 & 17,500 & 20,200 & 24,300 \\
\hline & & & & Weighted & 2,870 & 5,750 & 8,360 & 12,100 & 15,000 & 18,100 & 21,200 & 26,100 \\
\hline 02445245 & 147 & New River near Winfield, Ala. & 2 & EMA & 3,780 & 5,700 & 7,020 & 8,730 & 10,000 & 11,400 & 12,700 & 14,500 \\
\hline & & & & RRE & 2,900 & 4,750 & 6,210 & 8,200 & 9,770 & 11,500 & 13,300 & 16,000 \\
\hline & & & & Weighted & 3,730 & 5,600 & 6,880 & 8,580 & 9,920 & 11,400 & 13,000 & 15,200 \\
\hline
\end{tabular}


Appendix 1. Annual exceedance probability flows for U.S. Geological Survey streamgages used in this analysis.-Continued

[USGS, U.S. Geological Survey; $\mathrm{ft}^{3} / \mathrm{s}$, cubic foot per second; Ala., Alabama; EMA, expected moments algorithm; RRE, regional regression equations; *, large or regulated streamgage that required separate analysis]

\begin{tabular}{|c|c|c|c|c|c|c|c|c|c|c|c|c|}
\hline \multirow{2}{*}{$\begin{array}{l}\text { USGS station } \\
\text { number } \\
\text { (pl. 1) }\end{array}$} & \multirow{2}{*}{$\begin{array}{c}\text { Site } \\
\text { number } \\
\text { (fig. 1) }\end{array}$} & \multirow[b]{2}{*}{ USGS streamgage name } & \multirow{2}{*}{$\begin{array}{l}\text { Flood } \\
\text { region } \\
\text { (fig. 2) }\end{array}$} & \multirow[b]{2}{*}{ Method } & \multicolumn{8}{|c|}{ Annual exceedance probability flow (fts/s) } \\
\hline & & & & & $\begin{array}{c}50 \\
\text { percent }\end{array}$ & $\begin{array}{c}20 \\
\text { percent }\end{array}$ & $\begin{array}{c}10 \\
\text { percent }\end{array}$ & $\begin{array}{c}4 \\
\text { percent }\end{array}$ & $\begin{array}{c}2 \\
\text { percent }\end{array}$ & $\begin{array}{c}1 \\
\text { percent }\end{array}$ & $\begin{array}{c}0.5 \\
\text { percent }\end{array}$ & $\begin{array}{c}0.2 \\
\text { percent }\end{array}$ \\
\hline \multirow[t]{3}{*}{02445500} & \multirow[t]{3}{*}{148} & \multirow[t]{3}{*}{ Sipsey River at Fayette, Ala. } & \multirow[t]{3}{*}{2} & EMA & 7,680 & 12,300 & 15,800 & 20,700 & 24,800 & 29,100 & 33,800 & 40,500 \\
\hline & & & & RRE & 7,820 & 13,000 & 17,100 & 22,700 & 27,200 & 32,000 & 37,000 & 44,400 \\
\hline & & & & Weighted & 7,690 & 12,300 & 16,000 & 21,200 & 25,500 & 30,300 & 35,300 & 42,400 \\
\hline \multirow[t]{3}{*}{02446500} & \multirow[t]{3}{*}{149} & \multirow[t]{3}{*}{ Sipsey River nr Elrod, Ala. } & \multirow[t]{3}{*}{2} & EMA & 8,840 & 14,300 & 18,200 & 23,400 & 27,200 & 31,200 & 35,200 & 40,600 \\
\hline & & & & RRE & 11,700 & 19,500 & 25,700 & 34,200 & 41,100 & 48,200 & 55,900 & 66,900 \\
\hline & & & & Weighted & 8,900 & 14,500 & 18,600 & 24,600 & 29,500 & 35,000 & 40,600 & 47,800 \\
\hline \multirow[t]{3}{*}{02447000} & \multirow[t]{3}{*}{150} & \multirow{3}{*}{$\begin{array}{l}\text { Sipsey River near Pleasant Ridge, } \\
\text { Ala. }\end{array}$} & \multirow[t]{3}{*}{2} & EMA & 8,780 & 14,800 & 19,600 & 26,800 & 32,900 & 39,700 & 47,300 & 58,800 \\
\hline & & & & RRE & 14,800 & 24,900 & 32,800 & 43,700 & 52,600 & 61,700 & 71,500 & 85,600 \\
\hline & & & & Weighted & 9,170 & 16,200 & 22,500 & 33,200 & 42,200 & 52,200 & 62,100 & 75,600 \\
\hline \multirow[t]{3}{*}{02448500} & \multirow[t]{3}{*}{155} & \multirow[t]{3}{*}{ Noxubee River nr Geiger, Ala. } & \multirow[t]{3}{*}{3} & EMA & 13,370 & 22,100 & 29,970 & 42,850 & 54,980 & 69,680 & 87,480 & 116,800 \\
\hline & & & & RRE & 14,400 & 27,200 & 38,900 & 56,800 & 72,900 & 91,200 & 112,000 & 144,000 \\
\hline & & & & Weighted & 13,400 & 22,900 & 33,200 & 51,200 & 68,900 & 86,300 & 108,000 & 140,000 \\
\hline \multirow[t]{3}{*}{02448900} & \multirow[t]{3}{*}{157} & \multirow[t]{3}{*}{ Bodka Creek near Geiger, Ala. } & \multirow[t]{3}{*}{3} & EMA & 8,417 & 12,760 & 15,760 & 19,640 & 22,590 & 25,560 & 28,580 & 32,670 \\
\hline & & & & $\mathrm{RRE}$ & 5,560 & 10,300 & 14,500 & 20,500 & 25,700 & 31,200 & 37,400 & 46,400 \\
\hline & & & & Weighted & 8,120 & 12,100 & 15,200 & 20,200 & 24,900 & 29,600 & 35,300 & 43,000 \\
\hline \multirow[t]{3}{*}{02449245} & 158 & Brush Creek near Eutaw, Ala. & 2 & EMA & 2,410 & 3,740 & 4,770 & 6,260 & 7,510 & 8,880 & 10,400 & 12,600 \\
\hline & & & & $\mathrm{RRE}$ & 2,370 & 3,870 & 5,050 & 6,670 & 7,930 & 9,360 & 10,800 & 13,000 \\
\hline & & & & Weighted & 2,410 & 3,750 & 4,820 & 6,390 & 7,690 & 9,130 & 10,600 & 12,800 \\
\hline 02449400 & 159 & Jones Creek near Epes, Ala. & 3 & EMA & 2,198 & 2,970 & 3,515 & 4,243 & 4,815 & 5,411 & 6,038 & 6,920 \\
\hline & & & & RRE & 1,550 & 2,790 & 3,850 & 5,260 & 6,360 & 7,440 & 8,570 & 10,100 \\
\hline & & & & Weighted & 2,150 & 2,930 & 3,660 & 4,850 & 5,970 & 6,870 & 7,970 & 9,350 \\
\hline 02449400 & 159 & Jones Creek near Epes, Ala. & Small & EMA & 2,198 & 2,970 & 3,515 & 4,243 & 4,815 & 5,411 & 6,038 & 6,920 \\
\hline & & & stream & RRE & 1,550 & 2,790 & 3,850 & 5,260 & 6,360 & 7,440 & 8,570 & 10,100 \\
\hline & & & & Weighted & 2,180 & 2,950 & 3,580 & 4,640 & 5,580 & 6,470 & 7,350 & 8,350 \\
\hline 02450000 & 160 & Mulberry Fork near Garden City, & 1 & EMA & 25,400 & 39,100 & 48,400 & 60,100 & 68,700 & 77,300 & 85,800 & 97,000 \\
\hline & & & & RRE & 12,100 & 19,200 & 24,500 & 31,700 & 37,700 & 43,700 & 50,100 & 59,100 \\
\hline & & & & Weighted & 21,800 & 32,300 & 38,700 & 46,600 & 52,400 & 57,400 & 63,600 & 71,300 \\
\hline 02450180 & 161 & Mulberry Fork near Arkadelphia, & 1 & EMA & 17,700 & 28,400 & 35,900 & 46,000 & 53,700 & 61,500 & 69,600 & 80,600 \\
\hline & & Ala. & & RRE & 14,700 & 23,100 & 29,400 & 38,100 & 45,200 & 52,400 & 60,000 & 70,800 \\
\hline & & & & Weighted & 16,800 & 26,100 & 32,500 & 41,100 & 48,000 & 54,900 & 62,400 & 72,900 \\
\hline
\end{tabular}


Appendix 1. Annual exceedance probability flows for U.S. Geological Survey streamgages used in this analysis. - Continued

[USGS, U.S. Geological Survey; ft³/s, cubic foot per second; Ala., Alabama; EMA, expected moments algorithm; RRE, regional regression equations; *, large or regulated streamgage that required separate analysis]

\begin{tabular}{|c|c|c|c|c|c|c|c|c|c|c|c|c|}
\hline \multirow{2}{*}{$\begin{array}{c}\text { USGS station } \\
\text { number } \\
\text { (pl. 1) }\end{array}$} & \multirow{2}{*}{$\begin{array}{c}\text { Site } \\
\text { number } \\
\text { (fig. 1) }\end{array}$} & \multirow[b]{2}{*}{ USGS streamgage name } & \multirow{2}{*}{$\begin{array}{l}\text { Flood } \\
\text { region } \\
\text { (fig. 2) } \\
\end{array}$} & \multirow[b]{2}{*}{ Method } & \multicolumn{8}{|c|}{ Annual exceedance probability flow (ft $\mathrm{ft}^{3}$ ) } \\
\hline & & & & & $\begin{array}{c}50 \\
\text { percent }\end{array}$ & $\begin{array}{c}20 \\
\text { percent }\end{array}$ & $\begin{array}{c}10 \\
\text { percent }\end{array}$ & $\begin{array}{c}4 \\
\text { percent }\end{array}$ & $\begin{array}{c}2 \\
\text { percent }\end{array}$ & $\begin{array}{c}1 \\
\text { percent }\end{array}$ & $\begin{array}{c}0.5 \\
\text { percent }\end{array}$ & $\begin{array}{c}0.2 \\
\text { percent }\end{array}$ \\
\hline \multirow[t]{3}{*}{02450200} & 162 & Dorsey Creek near Arkadelphia, Ala. & 1 & EMA & 1,560 & 2,240 & 2,700 & 3,290 & 3,730 & 4,180 & 4,650 & 5,270 \\
\hline & & & & RRE & 1,300 & 2,220 & 2,920 & 3,850 & 4,610 & 5,390 & 6,190 & 7,300 \\
\hline & & & & Weighted & 1,530 & 2,240 & 2,730 & 3,390 & 3,910 & 4,470 & 5,050 & 5,900 \\
\hline \multirow[t]{3}{*}{02450200} & 162 & Dorsey Creek near Arkadelphia, Ala. & Small & EMA & 1,564 & 2,237 & 2,695 & 3,285 & 3,732 & 4,185 & 4,646 & 5,272 \\
\hline & & & & RRE & 1,630 & 2,920 & 4,050 & 5,530 & 6,710 & 7,850 & 9,050 & 10,700 \\
\hline & & & & Weighted & 1,570 & 2,310 & 2,980 & 4,180 & 5,220 & 6,090 & 6,910 & 7,660 \\
\hline \multirow[t]{3}{*}{02450250} & 163 & Sipsey Fork near Grayson, Ala. & 1 & EMA & 6,940 & 9,960 & 12,000 & 14,500 & 16,400 & 18,200 & 20,100 & 22,600 \\
\hline & & & & RRE & 4,820 & 7,880 & 10,200 & 13,300 & 15,800 & 18,400 & 21,100 & 24,900 \\
\hline & & & & Weighted & 6,620 & 9,590 & 11,600 & 14,200 & 16,200 & 18,300 & 20,500 & 23,700 \\
\hline \multirow[t]{3}{*}{02450825} & 164 & Clear C at New Hope Church nr & 1 & EMA & 4,770 & 6,950 & 8,420 & 10,300 & 11,700 & 13,100 & 14,500 & 16,400 \\
\hline & & Poplar Sprin & & RRE & 5,130 & 8,360 & 10,800 & 14,100 & 16,800 & 19,500 & 22,400 & 26,400 \\
\hline & & & & Weighted & 4,780 & 7,010 & 8,540 & 10,500 & 12,100 & 13,800 & 15,400 & 18,000 \\
\hline \multirow[t]{3}{*}{02453950} & 165 & Lost Creek near Jasper, Ala. & 1 & EMA & 6,200 & 9,030 & 10,900 & 13,400 & 15,200 & 17,100 & 18,900 & 21,400 \\
\hline & & & & RRE & 5,590 & 9,090 & 11,700 & 15,300 & 18,200 & 21,200 & 24,300 & 28,600 \\
\hline & & & & Weighted & 6,120 & 9,040 & 11,100 & 13,900 & 16,200 & 18,700 & 21,100 & 24,700 \\
\hline \multirow[t]{3}{*}{02454000} & 166 & Lost Creek near Oakman, Ala. & 1 & EMA & 4,970 & 7,340 & 9,110 & 11,600 & 13,600 & 15,700 & 18,100 & 21,400 \\
\hline & & & & RRE & 6,190 & 10,000 & 12,900 & 16,800 & 20,000 & 23,300 & 26,700 & 31,500 \\
\hline & & & & Weighted & 5,090 & 7,620 & 9,560 & 12,400 & 14,700 & 17,300 & 20,100 & 24,300 \\
\hline \multirow[t]{3}{*}{02454055} & 167 & Lost Creek above Parrish, Ala. & 1 & EMA & 5,590 & 7,920 & 9,640 & 12,000 & 13,900 & 16,000 & 18,200 & 21,300 \\
\hline & & & & RRE & 6,470 & 10,500 & 13,500 & 17,500 & 20,900 & 24,300 & 27,800 & 32,800 \\
\hline & & & & Weighted & 5,660 & 8,110 & 9,940 & 12,500 & 14,600 & 17,100 & 19,600 & 23,400 \\
\hline \multirow[t]{3}{*}{02455000} & 168 & Locust Fork near Cleveland, Ala. & 1 & EMA & 10,600 & 15,200 & 18,500 & 22,800 & 26,200 & 29,700 & 33,300 & 38,400 \\
\hline & & & & RRE & 10,700 & 17,000 & 21,800 & 28,200 & 33,500 & 38,900 & 44,600 & 52,600 \\
\hline & & & & Weighted & 10,600 & 15,400 & 18,900 & 23,600 & 27,600 & 31,800 & 36,200 & 42,900 \\
\hline \multirow[t]{3}{*}{02455500} & 169 & Locust Fork at Trafford, Ala. & 1 & EMA & 23,800 & 34,100 & 41,400 & 51,200 & 58,900 & 67,000 & 75,400 & 87,300 \\
\hline & & & & RRE & 17,300 & 27,100 & 34,500 & 44,500 & 52,800 & 61,300 & 70,100 & 82,700 \\
\hline & & & & Weighted & 22,900 & 32,700 & 39,600 & 49,000 & 56,600 & 64,500 & 72,900 & 84,900 \\
\hline \multirow[t]{3}{*}{02456000} & 170 & Turkey Creek at Morris, Ala. & 1 & EMA & 6,220 & 9,820 & 12,200 & 15,300 & 17,600 & 19,800 & 22,000 & 24,800 \\
\hline & & & & RRE & 4,420 & 7,240 & 9,370 & 12,200 & 14,600 & 17,000 & 19,500 & 23,000 \\
\hline & & & & Weighted & 6,140 & 9,680 & 12,000 & 15,000 & 17,300 & 19,400 & 21,600 & 24,400 \\
\hline
\end{tabular}


Appendix 1. Annual exceedance probability flows for U.S. Geological Survey streamgages used in this analysis.-Continued

[USGS, U.S. Geological Survey; $\mathrm{ft}^{3} / \mathrm{s}$, cubic foot per second; Ala., Alabama; EMA, expected moments algorithm; RRE, regional regression equations; *, large or regulated streamgage that required separate analysis]

\begin{tabular}{|c|c|c|c|c|c|c|c|c|c|c|c|c|}
\hline \multirow{2}{*}{$\begin{array}{c}\text { USGS station } \\
\text { number } \\
\text { (pl. 1) }\end{array}$} & \multirow{2}{*}{$\begin{array}{c}\text { Site } \\
\text { number } \\
\text { (fig. 1) }\end{array}$} & \multirow[b]{2}{*}{ USGS streamgage name } & \multirow{2}{*}{$\begin{array}{l}\text { Flood } \\
\text { region } \\
\text { (fig. 2) } \\
\end{array}$} & \multirow[b]{2}{*}{ Method } & \multicolumn{8}{|c|}{ Annual exceedance probability flow (ft3/s) } \\
\hline & & & & & $\begin{array}{c}50 \\
\text { percent }\end{array}$ & $\begin{array}{c}20 \\
\text { percent }\end{array}$ & $\begin{array}{c}10 \\
\text { percent }\end{array}$ & $\begin{array}{c}4 \\
\text { percent }\end{array}$ & $\begin{array}{c}2 \\
\text { percent }\end{array}$ & $\begin{array}{c}1 \\
\text { percent }\end{array}$ & $\begin{array}{c}0.5 \\
\text { percent }\end{array}$ & $\begin{array}{c}0.2 \\
\text { percent }\end{array}$ \\
\hline \multirow[t]{3}{*}{02456500} & \multirow[t]{3}{*}{171} & \multirow[t]{3}{*}{ Locust Fork at Sayre, Ala. } & \multirow[t]{3}{*}{1} & EMA & 23,200 & 33,500 & 40,300 & 48,700 & 54,900 & 61,000 & 67,000 & 74,900 \\
\hline & & & & RRE & 21,900 & 34,000 & 43,100 & 55,500 & 65,800 & 76,300 & 87,300 & 103,000 \\
\hline & & & & Weighted & 23,100 & 33,600 & 40,700 & 49,800 & 57,100 & 64,800 & 72,700 & 84,200 \\
\hline \multirow[t]{3}{*}{02462000} & \multirow[t]{3}{*}{164} & \multirow[t]{3}{*}{ Valley Creek near Oak Grove, Ala. } & \multirow[t]{3}{*}{1} & EMA & 7,910 & 12,100 & 15,400 & 20,200 & 24,200 & 28,600 & 33,400 & 40,700 \\
\hline & & & & RRE & 6,620 & 10,700 & 13,800 & 17,900 & 21,300 & 24,800 & 28,400 & 33,600 \\
\hline & & & & Weighted & 7,580 & 11,700 & 14,800 & 19,200 & 22,700 & 26,400 & 30,400 & 36,000 \\
\hline \multirow[t]{3}{*}{02462600} & \multirow[t]{3}{*}{173} & \multirow[t]{3}{*}{ Blue Creek near Oakman, Ala. } & \multirow[t]{3}{*}{1} & EMA & 1,400 & 2,410 & 3,150 & 4,170 & 4,960 & 5,790 & 6,640 & 7,810 \\
\hline & & & & RRE & 718 & 1,250 & 1,650 & 2,190 & 2,630 & 3,070 & 3,530 & 4,170 \\
\hline & & & & Weighted & 1,300 & 2,210 & 2,840 & 3,700 & 4,340 & 4,940 & 5,590 & 6,370 \\
\hline \multirow[t]{3}{*}{02462600} & \multirow[t]{3}{*}{173} & \multirow[t]{3}{*}{ Blue Creek near Oakman, Ala. } & \multirow{3}{*}{$\begin{array}{r}\text { Small } \\
\text { stream }\end{array}$} & EMA & 1,398 & 2,407 & 3,154 & 4,167 & 4,962 & 5,785 & 6,638 & 7,813 \\
\hline & & & & RRE & 1,050 & 1,870 & 2,570 & 3,460 & 4,150 & 4,790 & 5,450 & 6,360 \\
\hline & & & & Weighted & 1,370 & 2,340 & 3,020 & 3,900 & 4,570 & 5,270 & 6,020 & 7,140 \\
\hline \multirow[t]{3}{*}{02462800} & \multirow[t]{3}{*}{174} & \multirow[t]{3}{*}{ Davis Creek below Abernant, Ala. } & \multirow[t]{3}{*}{1} & EMA & 2,220 & 3,220 & 3,960 & 4,960 & 5,770 & 6,630 & 7,540 & 8,850 \\
\hline & & & & RRE & 3,000 & 4,980 & 6,470 & 8,480 & 10,100 & 11,800 & 13,500 & 16,000 \\
\hline & & & & Weighted & 2,340 & 3,610 & 4,700 & 6,270 & 7,610 & 9,180 & 10,700 & 13,000 \\
\hline \multirow[t]{3}{*}{02463500} & \multirow[t]{3}{*}{175} & Hurricane Creek near Holt, Ala. & 1 & EMA & 5,260 & 8,450 & 11,000 & 14,600 & 17,600 & 21,000 & 24,600 & 30,100 \\
\hline & & & & RRE & 5,360 & 8,730 & 11,300 & 14,700 & 17,500 & 20,400 & 23,300 & 27,500 \\
\hline & & & & Weighted & 5,270 & 8,480 & 11,000 & 14,600 & 17,600 & 20,800 & 24,100 & 29,000 \\
\hline 02464000 & 176 & North River near Samantha, Ala. & 1 & EMA & 8,300 & 11,900 & 14,500 & 18,100 & 20,900 & 24,000 & 27,200 & 31,700 \\
\hline & & & & RRE & 8,700 & 13,900 & 17,900 & 23,200 & 27,600 & 32,100 & 36,800 & 43,400 \\
\hline & & & & Weighted & 8,340 & 12,100 & 14,900 & 18,900 & 22,000 & 25,600 & 29,300 & 34,800 \\
\hline 02464146 & 177 & Turkey Creek near Tuscaloosa & 1 & EMA & 701 & 1,310 & 1,870 & 2,790 & 3,660 & 4,710 & 5,970 & 8,040 \\
\hline & & & & RRE & 792 & 1,370 & 1,810 & 2,400 & 2,880 & 3,370 & 3,870 & 4,570 \\
\hline & & & & Weighted & 714 & 1,320 & 1,850 & 2,640 & 3,300 & 3,970 & 4,710 & 5,690 \\
\hline 02464146 & 177 & Turkey Creek near Tuscaloosa & Small & EMA & 701 & 1,310 & 1,869 & 2,789 & 3,658 & 4,707 & 5,971 & 8,037 \\
\hline & & & stream & RRE & 1,130 & 2,010 & 2,770 & 3,740 & 4,490 & 5,200 & 5,920 & 6,930 \\
\hline & & & & Weighted & 718 & 1,410 & 2,150 & 3,340 & 4,250 & 5,080 & 5,930 & 7,210 \\
\hline 02465000 & 178 & $\begin{array}{l}\text { Black Warrior River at Northport, } \\
\text { Ala. }\end{array}$ & * & EMA & 114,000 & 160,000 & 188,000 & 221,000 & 244,000 & 265,000 & 285,000 & 311,000 \\
\hline
\end{tabular}


Appendix 1. Annual exceedance probability flows for U.S. Geological Survey streamgages used in this analysis. - Continued

[USGS, U.S. Geological Survey; ft³/s, cubic foot per second; Ala., Alabama; EMA, expected moments algorithm; RRE, regional regression equations; *, large or regulated streamgage that required separate analysis]

\begin{tabular}{|c|c|c|c|c|c|c|c|c|c|c|c|c|}
\hline \multirow{2}{*}{$\begin{array}{c}\text { USGS station } \\
\text { number } \\
\text { (pl. 1) }\end{array}$} & \multirow{2}{*}{$\begin{array}{c}\text { Site } \\
\text { number } \\
\text { (fig. 1) }\end{array}$} & \multirow[b]{2}{*}{ USGS streamgage name } & \multirow{2}{*}{$\begin{array}{l}\text { Flood } \\
\text { region } \\
\text { (fig. 2) }\end{array}$} & \multirow[b]{2}{*}{ Method } & \multicolumn{8}{|c|}{ Annual exceedance probability flow (ft $3 / \mathrm{s})$} \\
\hline & & & & & $\begin{array}{c}50 \\
\text { percent }\end{array}$ & $\begin{array}{c}20 \\
\text { percent }\end{array}$ & $\begin{array}{c}10 \\
\text { percent }\end{array}$ & $\begin{array}{c}4 \\
\text { percent }\end{array}$ & $\begin{array}{c}2 \\
\text { percent }\end{array}$ & $\begin{array}{c}1 \\
\text { percent }\end{array}$ & $\begin{array}{c}0.5 \\
\text { percent }\end{array}$ & $\begin{array}{c}0.2 \\
\text { percent }\end{array}$ \\
\hline \multirow[t]{3}{*}{02465493} & \multirow[t]{3}{*}{179} & \multirow[t]{3}{*}{ Elliotts Creek at Moundville, Ala. } & \multirow[t]{3}{*}{2} & EMA & 568 & 1,260 & 2,000 & 3,360 & 4,790 & 6,660 & 9,110 & 13,500 \\
\hline & & & & RRE & 1,970 & 3,210 & 4,180 & 5,510 & 6,550 & 7,730 & 8,960 & 10,800 \\
\hline & & & & Weighted & 635 & 1,530 & 2,590 & 4,430 & 5,900 & 7,450 & 8,990 & 11,300 \\
\hline \multirow[t]{3}{*}{02465500} & \multirow[t]{3}{*}{180} & \multirow{3}{*}{$\begin{array}{l}\text { Fivemile Creek near Greensboro, } \\
\text { Ala. }\end{array}$} & \multirow[t]{3}{*}{2} & EMA & 1,710 & 3,310 & 4,640 & 6,610 & 8,290 & 10,100 & 12,200 & 15,200 \\
\hline & & & & RRE & 3,320 & 5,460 & 7,140 & 9,440 & 11,300 & 13,300 & 15,400 & 18,400 \\
\hline & & & & Weighted & 1,840 & 3,640 & 5,270 & 7,790 & 9,850 & 12,100 & 14,300 & 17,400 \\
\hline 02466030 & 181 & $\begin{array}{l}\text { Black Warrior River at Selden L \& D } \\
\text { near Eutaw, Ala. }\end{array}$ & $*$ & EMA & 76,100 & 112,000 & 136,000 & 166,000 & 187,000 & 208,000 & 229,000 & 256,000 \\
\hline 02467000 & 182 & $\begin{array}{l}\text { Tombigbee R At Demopolis L\&D } \\
\text { near Coatopa, Ala. }\end{array}$ & $*$ & EMA & 153,000 & 195,000 & 221,000 & 250,000 & 271,000 & 291,000 & 310,000 & 334,000 \\
\hline \multirow[t]{3}{*}{02467500} & \multirow[t]{3}{*}{184} & \multirow{3}{*}{$\begin{array}{l}\text { Sucarnoochee River at Livingston, } \\
\text { Ala. }\end{array}$} & \multirow[t]{3}{*}{3} & EMA & 7,628 & 13,860 & 19,380 & 28,180 & 36,240 & 45,750 & 56,930 & 74,750 \\
\hline & & & & RRE & 10,800 & 20,200 & 28,700 & 41,600 & 53,000 & 65,800 & 80,300 & 102,000 \\
\hline & & & & Weighted & 7,780 & 14,900 & 22,800 & 35,900 & 48,900 & 60,700 & 75,700 & 97,000 \\
\hline \multirow[t]{3}{*}{02468000} & \multirow[t]{3}{*}{185} & \multirow[t]{3}{*}{ Alamuchee Creek near Cuba, Ala. } & \multirow[t]{3}{*}{3} & EMA & 1,555 & 3,621 & 5,754 & 9,582 & 13,440 & 18,340 & 24,490 & 35,010 \\
\hline & & & & RRE & 3,520 & 6,430 & 9,000 & 12,600 & 15,600 & 18,700 & 22,000 & 26,900 \\
\hline & & & & Weighted & 2,050 & 5,070 & 8,080 & 12,100 & 15,400 & 18,700 & 22,200 & 27,400 \\
\hline \multirow[t]{3}{*}{02468500} & \multirow[t]{3}{*}{186} & \multirow[t]{3}{*}{ Chickasaw Bogue near Linden, Ala. } & \multirow[t]{3}{*}{3} & EMA & 13,850 & 19,990 & 24,520 & 30,780 & 35,840 & 41,240 & 47,040 & 55,390 \\
\hline & & & & RRE & 7,070 & 13,100 & 18,500 & 26,500 & 33,400 & 40,900 & 49,300 & 61,700 \\
\hline & & & & Weighted & 13,400 & 18,800 & 22,400 & 28,600 & 34,200 & 41,000 & 48,700 & 60,000 \\
\hline \multirow[t]{3}{*}{02469000} & \multirow[t]{3}{*}{187} & \multirow[t]{3}{*}{ Kinterbish Creek near York, Ala. } & 3 & EMA & 1,868 & 4,193 & 6,675 & 11,330 & 16,240 & 22,730 & 31,250 & 46,590 \\
\hline & & & & RRE & 4,240 & 7,780 & 10,900 & 15,400 & 19,100 & 23,000 & 27,300 & 33,600 \\
\hline & & & & Weighted & 2,430 & 6,360 & 10,200 & 15,100 & 19,000 & 23,000 & 27,400 & 33,900 \\
\hline 02469500 & 188 & Tuckabum Creek near Butler, Ala. & 3 & EMA & 3,713 & 8,090 & 12,470 & 20,170 & 27,830 & 37,440 & 49,430 & 69,770 \\
\hline & & & & RRE & 4,760 & 8,760 & 12,300 & 17,400 & 21,700 & 26,200 & 31,200 & 38,500 \\
\hline & & & & Weighted & 3,930 & 8,450 & 12,300 & 17,700 & 22,000 & 26,800 & 32,000 & 39,700 \\
\hline 02469550 & 189 & Horse Creek near Sweetwater, Ala. & 3 & EMA & 3,689 & 7,291 & 10,470 & 15,460 & 19,940 & 25,100 & 31,030 & 40,180 \\
\hline & & & & RRE & 3,470 & 6,330 & 8,860 & 12,400 & 15,300 & 18,400 & 21,700 & 26,400 \\
\hline & & & & Weighted & 3,650 & 6,940 & 9,590 & 13,400 & 16,200 & 19,800 & 23,200 & 28,500 \\
\hline
\end{tabular}


Appendix 1. Annual exceedance probability flows for U.S. Geological Survey streamgages used in this analysis.-Continued

[USGS, U.S. Geological Survey; $\mathrm{ft}^{3} / \mathrm{s}$, cubic foot per second; Ala., Alabama; EMA, expected moments algorithm; RRE, regional regression equations; *, large or regulated streamgage that required separate analysis]

\begin{tabular}{|c|c|c|c|c|c|c|c|c|c|c|c|c|}
\hline \multirow{2}{*}{$\begin{array}{c}\text { USGS station } \\
\text { number } \\
\text { (pl. 1) }\end{array}$} & \multirow{2}{*}{$\begin{array}{c}\text { Site } \\
\text { number } \\
\text { (fig. 1) }\end{array}$} & \multirow[b]{2}{*}{ USGS streamgage name } & \multirow{2}{*}{$\begin{array}{l}\text { Flood } \\
\text { region } \\
\text { (fig. 2) } \\
\end{array}$} & \multirow[b]{2}{*}{ Method } & \multicolumn{8}{|c|}{ Annual exceedance probability flow (ft/s) } \\
\hline & & & & & $\begin{array}{c}50 \\
\text { percent }\end{array}$ & $\begin{array}{c}20 \\
\text { percent }\end{array}$ & $\begin{array}{c}10 \\
\text { percent }\end{array}$ & $\begin{array}{c}4 \\
\text { percent }\end{array}$ & $\begin{array}{c}2 \\
\text { percent }\end{array}$ & $\begin{array}{c}1 \\
\text { percent }\end{array}$ & $\begin{array}{c}0.5 \\
\text { percent }\end{array}$ & $\begin{array}{c}0.2 \\
\text { percent }\end{array}$ \\
\hline \multirow[t]{3}{*}{02469700} & \multirow[t]{3}{*}{191} & \multirow[t]{3}{*}{ Okatuppa Creek at Gilbertown, Ala. } & \multirow[t]{3}{*}{4} & EMA & 4,568 & 7,083 & 9,042 & 11,870 & 14,240 & 16,840 & 19,710 & 23,970 \\
\hline & & & & RRE & 4,100 & 7,580 & 10,600 & 14,900 & 18,700 & 22,800 & 27,500 & 34,500 \\
\hline & & & & Weighted & 4,390 & 7,390 & 10,200 & 14,400 & 17,900 & 21,600 & 26,000 & 32,200 \\
\hline 02469761 & 192 & $\begin{array}{l}\text { Tombigbee } \mathrm{R} \text { at Coffeeville L\&D nr } \\
\text { Coffeeville, Ala. }\end{array}$ & * & EMA & 146,000 & 190,000 & 214,000 & 240,000 & 257,000 & 272,000 & 286,000 & 302,000 \\
\hline \multirow[t]{3}{*}{02469800} & \multirow[t]{3}{*}{193} & \multirow[t]{3}{*}{ Satilpa Creek near Coffeeville, Ala. } & \multirow[t]{3}{*}{3} & EMA & 4,557 & 8,575 & 12,460 & 19,190 & 25,850 & 34,230 & 44,750 & 62,800 \\
\hline & & & & RRE & 5,670 & 10,500 & 14,700 & 20,900 & 26,200 & 31,900 & 38,200 & 47,400 \\
\hline & & & & Weighted & 4,630 & 9,050 & 13,600 & 20,400 & 26,200 & 32,200 & 38,800 & 48,700 \\
\hline \multirow[t]{3}{*}{02470072} & \multirow[t]{3}{*}{194} & \multirow{3}{*}{$\begin{array}{l}\text { Bassett Creek at US Highway } 43 \mathrm{nr} \\
\text { Thomasville, Ala. }\end{array}$} & \multirow[t]{3}{*}{4} & EMA & 1,191 & 1,601 & 1,886 & 2,262 & 2,553 & 2,853 & 3,166 & 3,602 \\
\hline & & & & RRE & 913 & 1,700 & 2,400 & 3,400 & 4,250 & 5,160 & 6,200 & 7,700 \\
\hline & & & & Weighted & 1,100 & 1,630 & 2,150 & 3,010 & 3,640 & 4,330 & 5,130 & 6,060 \\
\hline \multirow[t]{3}{*}{02470072} & \multirow[t]{3}{*}{194} & \multirow{3}{*}{$\begin{array}{l}\text { Bassett Creek at US Highway } 43 \mathrm{nr} \\
\text { Thomasville, Ala. }\end{array}$} & \multirow{3}{*}{$\begin{array}{l}\text { Small } \\
\text { stream }\end{array}$} & EMA & 1,191 & 1,601 & 1,886 & 2,262 & 2,553 & 2,853 & 3,166 & 3,602 \\
\hline & & & & RRE & 1,470 & 2,630 & 3,630 & 4,950 & 5,980 & 6,980 & 8,020 & 9,470 \\
\hline & & & & Weighted & 1,200 & 1,650 & 2,100 & 3,090 & 4,030 & 4,790 & 5,450 & 5,960 \\
\hline \multirow[t]{3}{*}{02470100} & \multirow[t]{3}{*}{195} & \multirow[t]{3}{*}{ Bassett Creek at Walker Springs, Ala. } & \multirow[t]{3}{*}{4} & EMA & 5,602 & 9,478 & 12,380 & 16,370 & 19,540 & 22,870 & 26,370 & 31,260 \\
\hline & & & & RRE & 4,800 & 8,850 & 12,400 & 17,400 & 21,800 & 26,600 & 32,100 & 40,400 \\
\hline & & & & Weighted & 5,280 & 9,100 & 12,400 & 17,200 & 21,300 & 25,800 & 30,800 & 38,000 \\
\hline \multirow[t]{3}{*}{02471001} & \multirow[t]{3}{*}{196} & Chickasaw Creek near Kushla, Ala. & 4 & EMA & 4,650 & 8,738 & 12,240 & 17,650 & 22,430 & 27,880 & 34,080 & 43,580 \\
\hline & & & & RRE & 3,730 & 6,890 & 9,650 & 13,600 & 17,000 & 20,700 & 25,000 & 31,400 \\
\hline & & & & Weighted & 4,420 & 7,880 & 10,600 & 14,600 & 18,400 & 22,400 & 27,100 & 34,400 \\
\hline 02479431 & 210 & Pond Creek near Deer Park, Ala. & 4 & EMA & 1,540 & 2,449 & 3,134 & 4,088 & 4,861 & 5,688 & 6,572 & 7,840 \\
\hline & & & & RRE & 1,330 & 2,470 & 3,480 & 4,930 & 6,170 & 7,490 & 9,010 & 11,200 \\
\hline & & & & Weighted & 1,470 & 2,460 & 3,370 & 4,730 & 5,830 & 7,020 & 8,380 & 10,200 \\
\hline 02479560 & 211 & Escatawpa River near Agricola, Miss. & 4 & EMA & 9,622 & 15,120 & 19,370 & 25,440 & 30,490 & 36,000 & 42,020 & 50,870 \\
\hline & & & & RRE & 8,750 & 16,100 & 22,500 & 31,500 & 39,500 & 48,100 & 58,300 & 73,600 \\
\hline & & & & Weighted & 9,520 & 15,400 & 20,700 & 28,800 & 35,400 & 42,700 & 51,200 & 63,000 \\
\hline 02479945 & 212 & Big Creek at County Rd 63 near & 4 & EMA & 1,113 & 2,469 & 3,750 & 5,863 & 7,830 & 10,160 & 12,910 & 17,250 \\
\hline & & Wilmer, Ala. & & RRE & 1,700 & 3,160 & 4,450 & 6,280 & 7,870 & 9,560 & 11,500 & 14,400 \\
\hline & & & & Weighted & 1,400 & 2,980 & 4,350 & 6,240 & 7,870 & 9,620 & 11,600 & 14,700 \\
\hline
\end{tabular}


Appendix 1. Annual exceedance probability flows for U.S. Geological Survey streamgages used in this analysis. - Continued

[USGS, U.S. Geological Survey; ft³/s, cubic foot per second; Ala., Alabama; EMA, expected moments algorithm; RRE, regional regression equations; *, large or regulated streamgage that required separate analysis]

\begin{tabular}{|c|c|c|c|c|c|c|c|c|c|c|c|c|}
\hline \multirow{2}{*}{$\begin{array}{l}\text { USGS station } \\
\text { number } \\
\text { (pl. 1) }\end{array}$} & \multirow{2}{*}{$\begin{array}{c}\text { Site } \\
\text { number } \\
\text { (fig. 1) }\end{array}$} & \multirow[b]{2}{*}{ USGS streamgage name } & \multirow{2}{*}{$\begin{array}{l}\text { Flood } \\
\text { region } \\
\text { (fig. 2) }\end{array}$} & \multirow[b]{2}{*}{ Method } & \multicolumn{8}{|c|}{ Annual exceedance probability flow (ft 3 s) } \\
\hline & & & & & $\begin{array}{c}50 \\
\text { percent }\end{array}$ & $\begin{array}{c}20 \\
\text { percent }\end{array}$ & $\begin{array}{c}10 \\
\text { percent }\end{array}$ & $\begin{array}{c}4 \\
\text { percent }\end{array}$ & $\begin{array}{c}2 \\
\text { percent }\end{array}$ & $\begin{array}{c}1 \\
\text { percent }\end{array}$ & $\begin{array}{c}0.5 \\
\text { percent }\end{array}$ & $\begin{array}{c}0.2 \\
\text { percent }\end{array}$ \\
\hline \multirow[t]{3}{*}{02479980} & \multirow[t]{3}{*}{213} & \multirow[t]{3}{*}{ Crooked Creek near Fairview, Ala. } & \multirow[t]{3}{*}{4} & EMA & 693 & 1,101 & 1,407 & 1,830 & 2,172 & 2,535 & 2,923 & 3,475 \\
\hline & & & & RRE & 786 & 1,470 & 2,070 & 2,930 & 3,670 & 4,460 & 5,350 & 6,640 \\
\hline & & & & Weighted & 718 & 1,280 & 1,830 & 2,630 & 3,220 & 3,880 & 4,630 & 5,590 \\
\hline \multirow[t]{3}{*}{02479980} & \multirow[t]{3}{*}{213} & \multirow[t]{3}{*}{ Crooked Creek near Fairview, Ala. } & \multirow{3}{*}{$\begin{array}{c}\text { Small } \\
\text { stream }\end{array}$} & EMA & 693 & 1,101 & 1,407 & 1,830 & 2,172 & 2,535 & 2,923 & 3,475 \\
\hline & & & & RRE & 1,290 & 2,300 & 3,180 & 4,310 & 5,190 & 6,040 & 6,910 & 8,120 \\
\hline & & & & Weighted & 711 & 1,200 & 1,740 & 2,770 & 3,670 & 4,400 & 5,060 & 5,680 \\
\hline \multirow[t]{3}{*}{03572110} & \multirow[t]{3}{*}{218} & \multirow[t]{3}{*}{ Crow Creek at Bass } & \multirow[t]{3}{*}{1} & EMA & 9,050 & 12,500 & 14,900 & 18,000 & 20,400 & 22,800 & 25,200 & 28,600 \\
\hline & & & & RRE & 6,100 & 9,890 & 12,700 & 16,600 & 19,800 & 23,000 & 26,300 & 31,100 \\
\hline & & & & Weighted & 8,580 & 12,200 & 14,600 & 17,800 & 20,300 & 22,800 & 25,400 & 29,200 \\
\hline \multirow[t]{3}{*}{03572900} & \multirow[t]{3}{*}{219} & \multirow[t]{3}{*}{ Town Creek near Geraldine, Ala. } & \multirow[t]{3}{*}{1} & EMA & 8,600 & 12,700 & 15,500 & 19,100 & 21,700 & 24,400 & 27,200 & 30,800 \\
\hline & & & & RRE & 6,410 & 10,400 & 13,400 & 17,400 & 20,700 & 24,100 & 27,600 & 32,500 \\
\hline & & & & Weighted & 8,040 & 12,100 & 14,900 & 18,600 & 21,400 & 24,300 & 27,300 & 31,500 \\
\hline \multirow[t]{3}{*}{03573000} & \multirow[t]{3}{*}{220} & \multirow[t]{3}{*}{ Short Creek near Albertville, Ala. } & \multirow[t]{3}{*}{1} & EMA & 6,420 & 10,300 & 13,400 & 17,900 & 21,800 & 26,100 & 30,900 & 38,100 \\
\hline & & & & RRE & 4,800 & 7,850 & 10,100 & 13,200 & 15,800 & 18,400 & 21,000 & 24,800 \\
\hline & & & & Weighted & 6,180 & 9,930 & 12,800 & 17,000 & 20,600 & 24,200 & 28,300 & 33,900 \\
\hline \multirow[t]{3}{*}{03574500} & \multirow[t]{3}{*}{221} & Paint Rock River near Woodville, & 1 & EMA & 16,700 & 26,200 & 32,900 & 41,700 & 48,500 & 55,400 & 62,500 & 72,100 \\
\hline & & & & RRE & 11,100 & 17,600 & 22,500 & 29,200 & 34,700 & 40,300 & 46,100 & 54,400 \\
\hline & & & & Weighted & 15,900 & 24,900 & 31,200 & 39,600 & 46,000 & 52,200 & 58,700 & 67,300 \\
\hline 03575000 & 223 & Flint River near Chase, Ala. & 1 & EMA & 16,200 & 30,600 & 42,800 & 61,100 & 77,000 & 94,800 & 115,000 & 145,000 \\
\hline & & & & $\mathrm{RRE}$ & 11,600 & 18,400 & 23,500 & 30,400 & 36,200 & 42,000 & 48,100 & 56,700 \\
\hline & & & & Weighted & 15,900 & 29,300 & 39,900 & 54,800 & 66,500 & 77,600 & 90,100 & 105,000 \\
\hline 03575100 & 224 & Flint River at Brownsboro, Ala. & 1 & EMA & 17,000 & 30,200 & 40,400 & 54,900 & 66,600 & 79,100 & 92,500 & 111,000 \\
\hline & & & & $\mathrm{RRE}$ & 12,300 & 19,500 & 24,900 & 32,300 & 38,300 & 44,500 & 50,900 & 60,100 \\
\hline & & & & Weighted & 16,700 & 29,500 & 39,000 & 52,000 & 61,900 & 71,500 & 81,800 & 94,900 \\
\hline 03575500 & 225 & Tennessee River at Whitesburg, Ala. & $*$ & EMA & 203,000 & 254,000 & 276,000 & 297,000 & 308,000 & 316,000 & 323,000 & 329,000 \\
\hline 03576250 & 226 & Limestone Creek near Athens, Ala. & 1 & EMA & 7,280 & 12,800 & 17,200 & 23,300 & 28,300 & 33,600 & 39,400 & 47,600 \\
\hline & & & & RRE & 5,720 & 9,290 & 12,000 & 15,600 & 18,600 & 21,600 & 24,800 & 29,300 \\
\hline & & & & Weighted & 7,070 & 12,200 & 16,000 & 21,000 & 24,900 & 28,500 & 32,600 & 37,800 \\
\hline
\end{tabular}


Appendix 1. Annual exceedance probability flows for U.S. Geological Survey streamgages used in this analysis.-Continued

[USGS, U.S. Geological Survey; $\mathrm{ft}^{3} / \mathrm{s}$, cubic foot per second; Ala., Alabama; EMA, expected moments algorithm; RRE, regional regression equations; *, large or regulated streamgage that required separate analysis]

\begin{tabular}{|c|c|c|c|c|c|c|c|c|c|c|c|c|}
\hline \multirow{2}{*}{$\begin{array}{c}\text { USGS station } \\
\text { number } \\
\text { (pl. 1) }\end{array}$} & \multirow{2}{*}{$\begin{array}{c}\text { Site } \\
\text { number } \\
\text { (fig. 1) }\end{array}$} & \multirow[b]{2}{*}{ USGS streamgage name } & \multirow{2}{*}{$\begin{array}{l}\text { Flood } \\
\text { region } \\
\text { (fig. 2) }\end{array}$} & \multirow[b]{2}{*}{ Method } & \multicolumn{8}{|c|}{ Annual exceedance probability flow (ft $3 / \mathrm{s})$} \\
\hline & & & & & $\begin{array}{c}50 \\
\text { percent }\end{array}$ & $\begin{array}{c}20 \\
\text { percent }\end{array}$ & $\begin{array}{c}10 \\
\text { percent }\end{array}$ & $\begin{array}{c}4 \\
\text { percent }\end{array}$ & $\begin{array}{c}2 \\
\text { percent }\end{array}$ & $\begin{array}{c}1 \\
\text { percent }\end{array}$ & $\begin{array}{c}0.5 \\
\text { percent }\end{array}$ & $\begin{array}{c}0.2 \\
\text { percent }\end{array}$ \\
\hline \multirow[t]{3}{*}{03576500} & 227 & Flint Creek near Falkville, Ala. & 1 & EMA & 5,240 & 7,730 & 9,570 & 12,100 & 14,200 & 16,400 & 18,700 & 22,100 \\
\hline & & & & RRE & 4,620 & 7,550 & 9,760 & 12,800 & 15,200 & 17,700 & 20,300 & 23,900 \\
\hline & & & & Weighted & 5,140 & 7,700 & 9,600 & 12,200 & 14,500 & 16,800 & 19,300 & 22,900 \\
\hline \multirow[t]{3}{*}{03577110} & 228 & West Flint Creek near Hartselle, Ala. & 1 & EMA & 4,800 & 8,080 & 10,600 & 14,000 & 16,700 & 19,700 & 22,700 & 27,100 \\
\hline & & & & RRE & 6,910 & 11,200 & 14,400 & 18,700 & 22,200 & 25,900 & 29,600 & 35,000 \\
\hline & & & & Weighted & 5,020 & 8,570 & 11,500 & 15,400 & 18,600 & 22,300 & 25,800 & 31,200 \\
\hline \multirow[t]{3}{*}{03586500} & 231 & Big Nance Creek at Courtland, Ala. & 1 & EMA & 6,120 & 9,420 & 11,700 & 14,800 & 17,200 & 19,600 & 22,100 & 25,500 \\
\hline & & & & RRE & 7,150 & 11,500 & 14,800 & 19,300 & 22,900 & 26,700 & 30,600 & 36,100 \\
\hline & & & & Weighted & 6,210 & 9,580 & 12,000 & 15,300 & 17,900 & 20,700 & 23,600 & 27,900 \\
\hline 03589500 & 234 & Tennessee River at Florence, Ala. & $*$ & EMA & 262,000 & 328,000 & 364,000 & 402,000 & 426,000 & 447,000 & 465,000 & 487,000 \\
\hline \multirow[t]{3}{*}{03590000} & 235 & Cypress Creek near Florence, Ala. & 1 & EMA & 9,260 & 16,300 & 21,800 & 29,600 & 35,900 & 42,600 & 49,800 & 60,100 \\
\hline & & & & RRE & 8,340 & 13,400 & 17,200 & 22,300 & 26,500 & 30,800 & 35,300 & 41,700 \\
\hline & & & & Weighted & 9,140 & 15,800 & 20,800 & 27,600 & 32,800 & 37,900 & 43,600 & 51,000 \\
\hline \multirow[t]{3}{*}{03591800} & 236 & Bear Creek near Hackleburg, Ala. & 1 & EMA & 7,420 & 11,500 & 14,400 & 18,200 & 21,000 & 23,900 & 26,800 & 30,800 \\
\hline & & & & RRE & 6,470 & 10,500 & 13,500 & 17,500 & 20,900 & 24,300 & 27,800 & 32,800 \\
\hline & & & & Weighted & 7,300 & 11,300 & 14,200 & 18,000 & 21,000 & 24,100 & 27,300 & 32,000 \\
\hline \multirow[t]{3}{*}{03592200} & 237 & Cedar Creek near Pleasant Site, Ala. & 2 & EMA & 8,420 & 12,800 & 15,900 & 20,000 & 23,300 & 26,600 & 30,100 & 35,000 \\
\hline & & & & RRE & 6,060 & 10,000 & 13,200 & 17,500 & 20,900 & 24,600 & 28,500 & 34,200 \\
\hline & & & & Weighted & 8,290 & 12,500 & 15,500 & 19,300 & 22,500 & 25,700 & 29,300 & 34,600 \\
\hline \multirow[t]{3}{*}{03592300} & 238 & Little Bear Creek near Halltown, Ala. & 2 & EMA & 3,710 & 6,070 & 8,010 & 10,900 & 13,500 & 16,400 & 19,700 & 24,800 \\
\hline & & & & RRE & 3,450 & 5,680 & 7,430 & 9,820 & 11,700 & 13,800 & 16,000 & 19,200 \\
\hline & & & & Weighted & 3,690 & 6,020 & 7,870 & 10,500 & 12,600 & 14,800 & 17,200 & 20,900 \\
\hline \multirow[t]{3}{*}{03592500} & 239 & Bear Creek at Bishop, Ala. & 2 & EMA & 15,800 & 24,400 & 30,700 & 39,200 & 46,000 & 53,100 & 60,600 & 71,100 \\
\hline & & & & RRE & 13,500 & 22,700 & 29,900 & 39,800 & 47,900 & 56,200 & 65,100 & 78,000 \\
\hline & & & & Weighted & 15,700 & 24,300 & 30,600 & 39,300 & 46,500 & 54,200 & 62,400 & 74,000 \\
\hline \multirow[t]{3}{*}{0357479650} & 242 & Hester Creek at Buddy Williamson & 1 & EMA & 2,330 & 3,790 & 4,980 & 6,750 & 8,280 & 10,000 & 11,900 & 14,900 \\
\hline & & Rd nr Plevna, Ala. & & RRE & 2,430 & 4,060 & 5,290 & 6,940 & 8,290 & 9,670 & 11,100 & 13,100 \\
\hline & & & & Weighted & 2,340 & 3,820 & 5,040 & 6,800 & 8,280 & 9,870 & 11,600 & 14,000 \\
\hline
\end{tabular}




\section{Appendix 2}

The following tables contain gage height and discharge (flow) data for streamgages in Alabama. The tables contain a brief description of the gage location, type of gage, gage datum (if known), drainage area in square miles, historical data, and explanatory remarks. Elevations are referenced to the National Geodetic Vertical Datum of 1929 (NGVD 29). The tables of peak stages and discharges show only the annual maximums. The qualification codes in the tables correspond to the peak data codes as used in the U.S. Geological Survey National Water Information System (NWIS).

The following qualification codes apply to the discharge data:

1 discharge is a maximum daily average;

2 discharge is an estimate;

4 discharge is less than indicated value, which is the minimum recordable discharge at this site;

5 discharge affected to unknown degree by regulation or diversion;

6 discharge affected by regulation or diversion;

7 discharge is a historic peak;

A year of occurrence is unknown or not exact;

B month or day of occurrence is unknown or not exact;

E only maximum peak available for this year.

The following qualification codes apply to the gage height data:

1 gage height affected by backwater;

2 gage height not the maximum for the year;

3 gage height at different datum or at different site and datum;

5 gage height is an estimate. 

Appendix 2. Discharge and gage height data for streamgages in Alabama.

[--, date or data not available; $\mathrm{ft}$, foot; $\mathrm{ft}^{3} / \mathrm{s}$, cubic foot per second; mi, mile; $\mathrm{mi}^{2}$, square mile; qualification codes apply to the discharge data: 1 , discharge is a maximum daily average; 2 , discharge is an estimate; 4 , discharge is less than indicated value, which is the minimum recordable discharge at this site; 5 , discharge affected to unknown degree by regulation or diversion; 6 , discharge affected by regulation or diversion; 7, discharge is a historic peak; A, year of occurrence is unknown or not exact; $\mathrm{B}$, month or day of occurrence is unknown or not exact; E, only maximum peak available for this year. qualification codes apply to the gage height data: 1, gage height affected by backwater; 2, gage height not the maximum for the year; 3, gage height at different datum or at different site and datum; 5 , gage height is an estimate]

02340750 Osanippa Creek near Fairfax

Location-Lat $32^{\circ} 47^{\prime} 20^{\prime \prime}$, long $85^{\circ} 11^{\prime} 30^{\prime \prime}$, in NW $1 / 4$ sec.

25, T. 21 N., R. 28 E., Chambers County, Hydrologic Unit

03130002, at bridge on U.S. Highway 29, 1 mi southwest of

Fairfax.

Drainage area $-99.7 \mathrm{mi}^{2}$.

Gage Crest-stage gage. Datum not available.

\begin{tabular}{|c|c|c|c|c|c|}
\hline $\begin{array}{l}\text { Water } \\
\text { year }\end{array}$ & Date & Discharge & $\begin{array}{l}\text { Discharge } \\
\text { code }\end{array}$ & $\begin{array}{c}\text { Gage } \\
\text { height } \\
\text { (feet) }\end{array}$ & $\begin{array}{c}\text { Gage } \\
\text { height } \\
\text { code }\end{array}$ \\
\hline 1953 & May 1 & 3,500 & & 7.80 & \\
\hline 1954 & Dec. 5 & 3,100 & & 7.30 & \\
\hline 1955 & Apr. 14 & 1,320 & & 5.20 & \\
\hline 1956 & Sept. 26 & 8,000 & & 11.70 & \\
\hline 1957 & Dec. 24 & 5,300 & & 9.70 & \\
\hline 1958 & Feb. 6 & 1,800 & & 5.80 & \\
\hline 1959 & Mar. 22 & 4,100 & & 8.40 & \\
\hline 1960 & Apr. 3 & 5,800 & & 10.20 & \\
\hline 1961 & Feb. 25 & 19,500 & & 16.08 & \\
\hline 1962 & Apr. 12 & 4,290 & & 8.66 & \\
\hline 1963 & Nov. 22 & 4,250 & & 8.61 & \\
\hline 1964 & Apr. 8 & 3,860 & & 8.18 & \\
\hline 1965 & Oct. 5 & 4,490 & & 8.88 & \\
\hline 1966 & Feb. 13 & 4,020 & & 8.36 & \\
\hline 1967 & Feb. 6 & 1,670 & & 5.65 & \\
\hline 1968 & Mar. 11 & 3,100 & & 7.33 & \\
\hline 1969 & Apr. 19 & 3,700 & & 8.00 & \\
\hline 1970 & Mar. 19 & 5,250 & & 9.65 & \\
\hline 1971 & Mar. 3 & 6,800 & & 10.86 & \\
\hline 1972 & Jan. 11 & 3,380 & & 7.65 & \\
\hline 1973 & Feb. 2 & 3,930 & & 8.26 & \\
\hline 1974 & May 24 & 2,990 & & 7.21 & \\
\hline
\end{tabular}

02342200 Phelps Creek near Opelika

L ocation-Lat $32^{\circ} 33^{\prime} 49^{\prime \prime}$, long $85^{\circ} 16^{\prime} 36^{\prime \prime}$, in SW 1/4 sec. 7, T. 18 N., R. 28 E., Lee County, Hydrologic Unit 03130003, on county road, 1 mi upstream from mouth, and 9 mi southeast of Opelika. Drainage area-6.67 $\mathrm{mi}^{2}$.

G age -Water-stage recorder, 1959-68. Crest-stage gage 1969-74. Datum of gage is $530 \mathrm{ft}$ NGVD 29 (from topographic map).

\begin{tabular}{|c|c|c|c|c|c|}
\hline $\begin{array}{l}\text { Water } \\
\text { year }\end{array}$ & Date & Discharge & $\begin{array}{l}\text { Discharge } \\
\text { code }\end{array}$ & $\begin{array}{c}\text { Gage } \\
\text { height } \\
\text { (feet) }\end{array}$ & $\begin{array}{c}\text { Gage } \\
\text { height } \\
\text { code }\end{array}$ \\
\hline 1959 & Feb. 4 & 247 & & 6.50 & \\
\hline 1960 & Apr. 3 & 668 & & 8.13 & \\
\hline 1961 & Feb. 24 & 1,210 & & 8.81 & \\
\hline 1962 & Mar. 10 & 766 & & 8.28 & \\
\hline 1963 & Mar. 6 & 585 & & 7.99 & \\
\hline 1964 & Apr. 8 & 3,030 & & 9.85 & \\
\hline 1965 & Oct. 5 & 822 & & 8.36 & \\
\hline 1966 & Sept. 13 & 1,560 & & 9.14 & \\
\hline 1967 & Sept. 3 & 515 & & 7.85 & \\
\hline 1968 & Mar. 11 & 1,060 & & 8.66 & \\
\hline 1969 & Mar. 24 & 1,000 & & 8.60 & \\
\hline 1970 & Mar. 20 & 920 & & 8.50 & \\
\hline 1971 & Mar. 2 & 1,500 & & 9.10 & \\
\hline 1972 & Jan. 10 & 157 & & 5.01 & \\
\hline 1973 & Dec. 21 & 1,560 & & 9.14 & \\
\hline 1974 & Apr. 2 & 362 & & 7.36 & \\
\hline
\end{tabular}


Appendix 2. Discharge and gage height data for streamgages in Alabama.-Continued

[--, date or data not available; ft, foot; $\mathrm{ft}^{3} / \mathrm{s}$, cubic foot per second; mi, mile; $\mathrm{mi}^{2}$, square mile; qualification codes apply to the discharge data: 1 , discharge is a maximum daily average; 2 , discharge is an estimate; 4 , discharge is less than indicated value, which is the minimum recordable discharge at this site; 5 , discharge affected to unknown degree by regulation or diversion; 6 , discharge affected by regulation or diversion; 7, discharge is a historic peak; A, year of occurrence is unknown or not exact; B, month or day of occurrence is unknown or not exact; E, only maximum peak available for this year. qualification codes apply to the gage height data: 1 , gage height affected by backwater; 2, gage height not the maximum for the year; 3, gage height at different data or at different site and datum; 5 , gage height is an estimate]

\section{Chattooga River above Gaylesville}

L ocation-Lat $34^{\circ} 17^{\prime} 25^{\prime \prime}$, long $85^{\circ} 30^{\prime} 33^{\prime \prime}$, in NW 1/4 sec.

5, T. 9 S., R. 11 E., Cherokee County, Hydrologic Unit

03150105, on county road, $600 \mathrm{ft}$ downstream from Mills Creek,

3.5 mi northeast of Gaylesville, and 20.1 mi upstream from mouth.

Drainage area-366 $\mathrm{mi}^{2}$.

Gage -Water-stage recorder. Datum of gage is $562.11 \mathrm{ft}$ NGVD 29.

$\begin{array}{ccccc}\begin{array}{c}\text { Water } \\ \text { year }\end{array} \text { Date } & \text { Discharge } & \begin{array}{c}\text { Discharge } \\ \text { code }\end{array} & \begin{array}{c}\text { Gage } \\ \text { height } \\ \text { (feet) }\end{array} & \begin{array}{c}\text { Gage } \\ \text { height }\end{array}\end{array}$

$\begin{array}{llrlcc} & & & & \text { (feet) } & \text { code } \\ 1938 & \text { Apr. } 9 & 11,800 & 2 & -- & \end{array}$

194

1941

1942

1943

1944

1945

1946

1947

1948

Mar. 14

July 16

Feb. 17

Dec. 30

Mar. 29

Feb. 15

4,090

Feb. 11

Jan. 21

Feb. 14

Nov. 29

Mar. 14

Mar. 30

Mar. 12

Feb. 22

Jan. 17

Dec. 6

Apr. 17

Feb. 2

Nov. 20

Apr. 20

Mar. 30

Feb. 23

Jan. 28

Apr. 30

Mar. 26

Mar. 27

Mar. 5

Feb. 21

Apr. 27

Feb. 5

May 15

Mar. 18

Apr. 5

Sept. 24

Jan. 27

Apr. 5

1977

$$
5,990
$$

7,420

13,200

10,300

6,670

17,700

13,900

11,800

31,200

12,400

32,900

14,300

8,390

9,470

6,620

6,790

10,900

11,200

4,220

4,050

12,000

11,500

13,600

13,300

10,100

16,500

4,460

5,620

5,620

9,820

12,500

7,200

9,100

4,680

12,800

--

$-$

--

--

--

--

$-$

$-$

--

$-$

$-$

--

23.48

$--$

--

$-$

--

--

$-$

--

$--$

13.93

20.56

20.34

20.95

20.85

18.94

22.03

14.60

16.35

16.36

18.96

20.25

17.56

18.60

14.97

20.42
02398300 Chattooga River above Gaylesville-Continued L ocation-Lat $34^{\circ} 17^{\prime} 25^{\prime \prime}$, long 85 $30^{\prime} 33^{\prime \prime}$, in NW 1/4 sec. 5, T. 9 S., R. 11 E., Cherokee County, Hydrologic Unit 03150105 , on county road, $600 \mathrm{ft}$ downstream from Mills Creek, $3.5 \mathrm{mi}$ northeast of Gaylesville, and $20.1 \mathrm{mi}$ upstream from mouth.

Drainage area-366 $\mathrm{mi}^{2}$.

Gage -Water-stage recorder. Datum of gage is $562.11 \mathrm{ft}$ NGVD 29.

\begin{tabular}{llcccc}
\hline $\begin{array}{c}\text { Water } \\
\text { year }\end{array}$ & Date & Discharge & $\begin{array}{c}\text { Discharge } \\
\text { code }\end{array}$ & $\begin{array}{c}\text { Gage } \\
\text { height } \\
\text { (feet) }\end{array}$ & $\begin{array}{c}\text { Gage } \\
\text { height } \\
\text { code }\end{array}$ \\
\hline 1978 & Nov. 7 & 8,720 & & 18.43 & \\
1979 & Apr. 13 & 16,800 & & 22.17 & \\
1980 & Mar. 22 & 10,600 & & 19.38 & \\
1981 & Feb. 11 & 4,450 & & 13.79 &
\end{tabular}

1983 Dec. $2 \quad 11,400 \quad 19.77$

1984 Apr. $4 \quad 8,120 \quad 18.12$

1985 Feb. $2 \quad 7,840 \quad 17.94$

$1986 \quad$ Feb. $19 \quad 3,180 \quad 10.88$

1987 Mar. $1 \quad 7,330 \quad 17.76$

$1988 \quad$ Jan. $21 \quad 6,660 \quad 17.15$

1989 Feb. $28 \quad 6,230 \quad 16.65$

$1990 \quad$ Feb. $17 \quad 23,300 \quad 24.25$

$1991 \quad$ Feb. $21 \quad 7,860 \quad 18.04$

$1992 \quad$ Feb. $26 \quad 6,710 \quad 16.95$

$1993 \quad$ Jan. $13 \quad 5,320 \quad 14.71$

1994 Mar. $29 \quad 12,500 \quad 20.55$

1995 Feb. $18 \quad 9,110 \quad 18.83$

1996 Jan. $27 \quad 11,600 \quad 20.14$

1997 Mar. $1 \quad 6,870 \quad 17.12$

$1998 \quad$ Apr. $17 \quad 6,830 \quad 17.08$

$1999 \quad$ Jan. $23 \quad 4,980 \quad 14.17$

2000 Apr. $5 \quad 7,700 \quad 17.91$

2001 Mar. $21 \quad 6,630 \quad 16.84$

$2002 \quad$ Jan. $25 \quad 6,130 \quad 16.17$

2003 May $7 \quad 10,600 \quad 19.65$

$2004 \quad$ Jan. $26 \quad 7,110 \quad 16.64$

2005 Nov. $25 \quad 9,020 \quad 18.44$

$2006 \quad$ Jan. $18 \quad 1,910 \quad 8.21$

2007 Nov. $16 \quad 4,020 \quad 12.04$

2008 Mar. $5 \quad 4,070 \quad 12.14$

2009 Sept. $22 \quad 13,000 \quad 20.78$

2010 Dec. $10 \quad 9,650 \quad 18.96$

2011 Mar. $11 \quad 8,980 \quad 18.41$

$2012 \quad$ Nov. $30 \quad 5,480 \quad 14.55$

$2013 \quad$ Jan. $16 \quad 8,790 \quad 18.25$

2014 Apr. $7 \quad 7,160 \quad 16.70$

2015 Jan. $4 \quad 4,170 \quad 12.35$ 
Appendix 2. Discharge and gage height data for streamgages in Alabama.-Continued

[--, date or data not available; $\mathrm{ft}$, foot; $\mathrm{ft}^{3} / \mathrm{s}$, cubic foot per second; mi, mile; $\mathrm{mi}^{2}$, square mile; qualification codes apply to the discharge data: 1 , discharge is a maximum daily average; 2 , discharge is an estimate; 4 , discharge is less than indicated value, which is the minimum recordable discharge at this site; 5 , discharge affected to unknown degree by regulation or diversion; 6 , discharge affected by regulation or diversion; 7, discharge is a historic peak; A, year of occurrence is unknown or not exact; B, month or day of occurrence is unknown or not exact; E, only maximum peak available for this year. qualification codes apply to the gage height data: 1, gage height affected by backwater; 2, gage height not the maximum for the year; 3, gage height at different datum or at different site and datum; 5 , gage height is an estimate]

\section{Little River near Jamestown}

L ocation-Lat $34^{\circ} 23^{\prime} 51^{\prime \prime}$, long $85^{\circ} 37^{\prime} 36^{\prime \prime}$, in SW 1/4 sec. 30, T. 7 S., R. 10 E., Cherokee County, Hydrologic Unit 03150105, at site of former highway bridge, 0.2 mi upstream from Yellow Creek, $0.3 \mathrm{mi}$ upstream from present highway bridge, and $2.5 \mathrm{mi}$ west of Jamestown. Drainage area-125 $\mathrm{mi}^{2}$.

Gage -Water-stage recorder 1923-32, 1936-49. Crest-stage gage 1951-67. Datum of gage is 1,177.4 ft NGVD 29.

\begin{tabular}{|c|c|c|c|c|c|}
\hline $\begin{array}{l}\text { Water } \\
\text { year }\end{array}$ & Date & Discharge & $\begin{array}{l}\text { Discharge } \\
\text { code }\end{array}$ & $\begin{array}{c}\text { Gage } \\
\text { height } \\
\text { (feet) }\end{array}$ & $\begin{array}{c}\text { Gage } \\
\text { height } \\
\text { code }\end{array}$ \\
\hline 1929 & Mar. 14 & 14,600 & & 10.40 & \\
\hline 1930 & Nov. 14 & 11,900 & & 9.38 & \\
\hline 1931 & Nov. 16 & 5,210 & & 6.42 & \\
\hline 1932 & Jan. 30 & 9,830 & & 8.62 & \\
\hline 1936 & Feb. 4 & 18,800 & & 11.90 & \\
\hline 1937 & Jan. 2 & 11,100 & & 9.09 & \\
\hline 1938 & Apr. 8 & 10,900 & & 9.00 & \\
\hline 1939 & Feb. 28 & 9,830 & & 8.57 & \\
\hline 1940 & Feb. 18 & 4,990 & & 6.27 & \\
\hline 1941 & July 17 & 2,850 & & 4.91 & \\
\hline 1942 & Feb. 17 & 5,380 & & 6.52 & \\
\hline 1943 & Dec. 28 & 19,700 & & 12.20 & \\
\hline 1944 & Feb. 27 & 8,350 & & 7.97 & \\
\hline 1945 & Feb. 13 & 7,650 & & 7.73 & \\
\hline 1946 & Jan. 8 & 17,900 & & 11.56 & \\
\hline 1947 & Jan. 20 & 10,900 & & 9.02 & \\
\hline 1948 & Feb. 12 & 7,650 & & 7.70 & \\
\hline 1949 & Nov. 28 & 21,800 & & 12.90 & \\
\hline 1951 & Mar. 29 & 19,700 & & 12.20 & \\
\hline 1952 & Mar. 11 & 13,800 & & 10.10 & \\
\hline 1953 & Feb. 21 & 5,210 & & 6.40 & \\
\hline 1954 & Jan. 17 & 12,200 & & 9.50 & \\
\hline 1955 & Apr. 14 & 13,500 & & 10.00 & \\
\hline 1956 & Apr. 17 & 4,890 & & 6.20 & \\
\hline 1957 & Feb. 1 & 8,830 & & 8.20 & \\
\hline 1958 & July 9 & 7,010 & & 7.40 & \\
\hline 1959 & Jan. 21 & 9,330 & & 8.40 & \\
\hline 1960 & Mar. 30 & 2,050 & & 4.20 & \\
\hline 1961 & Feb. 22 & 7,470 & & 7.55 & \\
\hline 1962 & Dec. 18 & 13,000 & & 9.92 & \\
\hline 1964 & Mar. 25 & 10,800 & & 9.06 & \\
\hline 1965 & Mar. 26 & 9,110 & & 8.32 & \\
\hline 1966 & Mar. 3 & 25,000 & & 13.83 & \\
\hline 1967 & Feb. 20 & 10,100 & & 8.76 & \\
\hline
\end{tabular}

\section{Little River near Blue Pond}

L ocation-Lat $34^{\circ} 17^{\prime} 20^{\prime \prime}$, long $85^{\circ} 40^{\prime} 50^{\prime \prime}$, in NE $1 / 4$ sec. 3 , T. 9 S., R. 9 E., Cherokee County, Hydrologic Unit 03150105, at Canyon Mouth Park, 0.9 mi upstream from State Highway 176, 2.5 mi upstream from Wolf Creek, 4.2 mi northeast of Blue Pond, and 7.5 mi upstream from mouth.

Drainage area-199 $\mathrm{mi}^{2}$.

G age -Water-stage recorder. Datum of gage is $589.34 \mathrm{ft}$ NGVD 29. Prior to Aug. 1, 1959, nonrecording gage on highway bridge 0.9 mi downstream at datum $15.45 \mathrm{ft}$ lower. Prior to May 27, 1981, recording gage $0.7 \mathrm{mi}$ downstream at datum $7.96 \mathrm{ft}$ lower.

\begin{tabular}{|c|c|c|c|c|c|}
\hline $\begin{array}{c}\text { Water } \\
\text { year }\end{array}$ & Date & Discharge & $\begin{array}{l}\text { Discharge } \\
\text { code }\end{array}$ & $\begin{array}{c}\text { Gage } \\
\text { height } \\
\text { (feet) }\end{array}$ & $\begin{array}{c}\text { Gage } \\
\text { height } \\
\text { code }\end{array}$ \\
\hline 1929 & Mar. 15 & 21,500 & 2,7 & -- & \\
\hline 1948 & Feb. 12 & 7,650 & 7 & 7.70 & 3 \\
\hline 1958 & July 9 & 7,400 & 2 & 8.97 & 3 \\
\hline 1959 & Jan. 21 & 9,650 & & 10.00 & 3 \\
\hline 1960 & Nov. 24 & 3,810 & & 7.42 & 3 \\
\hline 1961 & Feb. 23 & 11,600 & & 10.08 & 3 \\
\hline 1962 & Dec. 18 & 19,200 & & 11.80 & 3 \\
\hline 1963 & Apr. 29 & 22,000 & & 13.55 & 3 \\
\hline 1964 & Mar. 26 & 17,800 & & 12.36 & 3 \\
\hline 1965 & Mar. 26 & 12,300 & & 10.71 & 3 \\
\hline 1966 & Mar. 4 & 32,000 & & 14.45 & 3 \\
\hline 1967 & Feb. 20 & 8,290 & & 9.28 & 3 \\
\hline 1968 & Dec. 18 & 11,400 & & 10.24 & 3 \\
\hline 1969 & May 18 & 17,100 & & 11.77 & 3 \\
\hline 1970 & Dec. 31 & 9,250 & & 9.59 & 3 \\
\hline 1971 & Feb. 5 & 10,100 & & 9.85 & 3 \\
\hline 1972 & Jan. 4 & 11,000 & & 10.12 & 3 \\
\hline 1973 & May 28 & 21,000 & & 12.59 & 3 \\
\hline 1974 & Nov. 28 & 13,000 & & 10.70 & 3 \\
\hline 1975 & Sept. 23 & 12,900 & & 10.68 & 3 \\
\hline 1976 & July 5 & 14,600 & & 11.15 & 3 \\
\hline 1977 & Mar. 30 & 15,400 & & 11.34 & 3 \\
\hline 1978 & Nov. 5 & 18,200 & & 12.02 & 3 \\
\hline 1979 & Apr. 13 & 31,100 & & 14.30 & 3 \\
\hline 1980 & Mar. 21 & 21,800 & & 12.74 & 3 \\
\hline 1981 & Mar. 31 & 10,000 & & 9.90 & 3,6 \\
\hline 1982 & Jan. 4 & 22,000 & & 12.08 & \\
\hline 1983 & Dec. 1 & 23,600 & & 12.42 & \\
\hline 1984 & Nov. 28 & 16,400 & & 10.85 & \\
\hline 1985 & July 24 & 53,800 & & 16.98 & \\
\hline 1986 & Feb. 18 & 5,320 & & 7.05 & \\
\hline
\end{tabular}


Appendix 2. Discharge and gage height data for streamgages in Alabama.-Continued

[--, date or data not available; $\mathrm{ft}$, foot; $\mathrm{ft}^{3} / \mathrm{s}$, cubic foot per second; $\mathrm{mi}$, mile; $\mathrm{mi}^{2}$, square mile; qualification codes apply to the discharge data: 1 , discharge is a maximum daily average; 2 , discharge is an estimate; 4 , discharge is less than indicated value, which is the minimum recordable discharge at this site; 5 , discharge affected to unknown degree by regulation or diversion; 6 , discharge affected by regulation or diversion; 7, discharge is a historic peak; A, year of occurrence is unknown or not exact; B, month or day of occurrence is unknown or not exact; E, only maximum peak available for this year. qualification codes apply to the gage height data: 1, gage height affected by backwater; 2 , gage height not the maximum for the year; 3 , gage height at different data or at different site and datum;

5 , gage height is an estimate]

\section{Little River near Blue Pond-Continued}

L ocation-Lat $34^{\circ} 17^{\prime} 20^{\prime \prime}$, long $85^{\circ} 40^{\prime} 50^{\prime \prime}$, in NE $1 / 4$ sec. 3, T. 9 S., R. 9 E., Cherokee County, Hydrologic Unit 03150105, at Canyon Mouth Park, 0.9 mi upstream from State Highway 176, $2.5 \mathrm{mi}$ upstream from Wolf Creek, $4.2 \mathrm{mi}$ northeast of Blue Pond, and 7.5 mi upstream from mouth.

Drainage area-199 $\mathrm{mi}^{2}$.

G age -Water-stage recorder. Datum of gage is $589.34 \mathrm{ft}$ NGVD 29. Prior to Aug. 1, 1959, nonrecording gage on highway bridge 0.9 mi downstream at datum $15.45 \mathrm{ft}$ lower. Prior to May 27, 1981, recording gage $0.7 \mathrm{mi}$ downstream at datum $7.96 \mathrm{ft}$ lower.

\begin{tabular}{|c|c|c|c|c|c|}
\hline $\begin{array}{l}\text { Water } \\
\text { year }\end{array}$ & Date & Discharge & $\begin{array}{l}\text { Discharge } \\
\text { code }\end{array}$ & $\begin{array}{c}\text { Gage } \\
\text { height } \\
\text { (feet) }\end{array}$ & $\begin{array}{c}\text { Gage } \\
\text { height } \\
\text { code }\end{array}$ \\
\hline 1987 & Mar. 1 & 16,700 & & 10.86 & \\
\hline 1988 & Jan. 20 & 20,800 & & 11.81 & \\
\hline 1989 & June 15 & 10,700 & & 9.16 & \\
\hline 1990 & Feb. 16 & 40,700 & & 15.22 & \\
\hline 1991 & Feb. 19 & 12,800 & & 9.79 & \\
\hline 1992 & Feb. 26 & 7,140 & & 7.86 & \\
\hline 1993 & Nov. 4 & 7,510 & & 8.01 & \\
\hline 1994 & Mar. 27 & 17,900 & & 11.15 & \\
\hline 1995 & Feb. 16 & 14,700 & & 10.34 & \\
\hline 1996 & Oct. 5 & 40,700 & & 15.26 & \\
\hline 1997 & May 3 & 24,000 & & 12.48 & \\
\hline 1998 & Jan. 7 & 18,200 & & 11.22 & \\
\hline 1999 & Jan. 23 & 12,500 & & 9.76 & \\
\hline 2000 & Apr. 4 & 13,400 & & 10.03 & \\
\hline 2001 & Mar. 20 & 8,020 & & 8.39 & \\
\hline 2002 & Jan. 25 & 13,000 & & 9.91 & \\
\hline 2003 & May 6 & 24,700 & & 12.62 & \\
\hline 2004 & Sept. 17 & 39,600 & & 15.11 & \\
\hline 2005 & Nov. 24 & 21,500 & & 11.96 & \\
\hline 2006 & Jan. 18 & 3,040 & & 6.94 & \\
\hline 2007 & Nov. 16 & 4,640 & & 7.45 & \\
\hline 2008 & Mar. 4 & 6,420 & & 8.03 & \\
\hline 2009 & Sept. 21 & 23,600 & & 12.35 & \\
\hline 2010 & Dec. 9 & 20,800 & & 11.78 & \\
\hline 2011 & Mar. 9 & 17,900 & & 11.14 & \\
\hline 2012 & Nov. 28 & 13,900 & & 10.17 & \\
\hline 2013 & Jan. 15 & 11,900 & & 9.65 & \\
\hline 2014 & Apr. 7 & 11,100 & & 9.42 & \\
\hline 2015 & Jan. 4 & 6,160 & & 7.91 & \\
\hline
\end{tabular}

02400000 Terrapin Creek near Piedmont

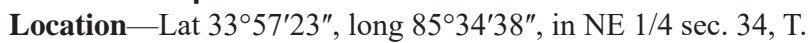
12 S., R. 10 E., Calhoun County, Hydrologic Unit 03150105, on U.S. Highway 278 and State Highway 74, 0.5 mi upstream from Ladiga Creek, and $3 \mathrm{mi}$ northeast of Piedmont.

Drainage area-116 $\mathrm{mi}^{2}$.

G age - Water-stage recorder. Datum of gage is $649.79 \mathrm{ft}$ NGVD 29.

\begin{tabular}{|c|c|c|c|c|c|}
\hline $\begin{array}{l}\text { Water } \\
\text { year }\end{array}$ & Date & Discharge & $\begin{array}{l}\text { Discharge } \\
\text { code }\end{array}$ & $\begin{array}{c}\text { Gage } \\
\text { height } \\
\text { (feet) }\end{array}$ & $\begin{array}{c}\text { Gage } \\
\text { height } \\
\text { code }\end{array}$ \\
\hline 1945 & May 13 & 3,950 & & 8.44 & \\
\hline 1946 & Mar. 28 & 14,600 & & 12.03 & \\
\hline 1947 & Jan. 20 & 11,000 & & 11.20 & \\
\hline 1948 & Feb. 7 & 3,830 & & 8.30 & \\
\hline 1949 & Nov. 28 & 21,000 & & 13.30 & \\
\hline 1950 & Nov. 28 & 5,900 & & 9.60 & \\
\hline 1951 & Mar. 29 & 17,800 & & 12.70 & \\
\hline 1952 & Mar. 23 & 10,000 & & 11.00 & \\
\hline 1953 & Jan. 9 & 5,690 & & 9.50 & \\
\hline 1954 & Jan. 16 & 12,000 & & 11.54 & \\
\hline 1955 & Feb. 7 & 6,380 & & 9.80 & \\
\hline 1956 & Mar. 17 & 4,790 & & 9.00 & \\
\hline 1957 & Apr. 5 & 10,600 & & 11.10 & \\
\hline 1958 & Sept. 21 & 5,160 & & 9.20 & \\
\hline 1959 & Jan. 21 & 3,500 & & 8.00 & \\
\hline 1960 & Jan. 31 & 2,310 & & 6.40 & \\
\hline 1961 & Feb. 23 & 14,000 & & 12.00 & \\
\hline 1962 & Dec. 12 & 10,900 & & 11.32 & \\
\hline 1963 & Mar. 12 & 13,800 & & 11.97 & \\
\hline 1979 & Mar. 3 & 17,600 & 2,7 & -- & \\
\hline
\end{tabular}


Appendix 2. Discharge and gage height data for streamgages in Alabama.-Continued

[--, date or data not available; $\mathrm{ft}$, foot; $\mathrm{ft}^{3} / \mathrm{s}$, cubic foot per second; mi, mile; $\mathrm{mi}^{2}$, square mile; qualification codes apply to the discharge data: 1 , discharge is a maximum daily average; 2 , discharge is an estimate; 4 , discharge is less than indicated value, which is the minimum recordable discharge at this site; 5 , discharge affected to unknown degree by regulation or diversion; 6 , discharge affected by regulation or diversion; 7, discharge is a historic peak; A, year of occurrence is unknown or not exact; $\mathrm{B}$, month or day of occurrence is unknown or not exact; E, only maximum peak available for this year. qualification codes apply to the gage height data: 1, gage height affected by backwater; 2, gage height not the maximum for the year; 3, gage height at different datum or at different site and datum; 5 , gage height is an estimate]

\section{Terrapin Creek at Ellisville}

L ocation-Lat $34^{\circ} 03^{\prime} 54^{\prime \prime}$, long $85^{\circ} 36^{\prime} 51^{\prime \prime}$, in SW 1/4 sec. 20, T.

11 S., R. 10 E., Cherokee County, Hydrologic Unit 03150105, on State Highway 9, $0.2 \mathrm{mi}$ southwest of Ellisville, and $6.7 \mathrm{mi}$ upstream from mouth.

Drainage area-252 $\mathrm{mi}^{2}$.

Gage -Water-stage recorder. Datum of gage is $539.07 \mathrm{ft}$ NGVD 29.

\begin{tabular}{cccccc}
\hline $\begin{array}{c}\text { Water } \\
\text { year }\end{array}$ & Date & Discharge & $\begin{array}{c}\text { Discharge } \\
\text { code }\end{array}$ & $\begin{array}{c}\text { Gage } \\
\text { height } \\
\text { (feet) }\end{array}$ & $\begin{array}{c}\text { Gage } \\
\text { height } \\
\text { code }\end{array}$ \\
\hline 1950 & Nov. 28 & 24,000 & 2,7 & -- &
\end{tabular}

196

1964

1965

1966

Mar. 13

13,200

17.90

17.35

Mar. $15 \quad$ 11,500

Feb. $12 \quad 3,500$

11.86

16.94

1967

Mar. $4 \quad$ 10,300

15.81

1968

Aug. 25

7,740

Jan. 10

8,910

16.36

12.91

1970

Jan. 20

4,300

18.00

1971

Mar. 20

13,500

18.10

17.55

1972

Apr. 24

13,800

13.61

19.07

1974

Jan. 8

4,900

17.30

16.43

16.12

14.29

19.82

16.30

17.10

16.10

13.35

1983

1984

Feb. 2

8,610

15.58

1985

July 31

7,420

12.22

7.17

1986 Mar. $19 \quad 917$

15.01

1988
Mar. 1 6,250

3,420
Jan. 20
02400100 Terrapin Creek at Ellisville-Continued

L ocation-Lat $34^{\circ} 03^{\prime} 54^{\prime \prime}$, long $85^{\circ} 36^{\prime} 51^{\prime \prime}$, in SW 1/4 sec. 20, T. 11 S., R. 10 E., Cherokee County, Hydrologic Unit 03150105, on State Highway 9, $0.2 \mathrm{mi}$ southwest of Ellisville, and $6.7 \mathrm{mi}$ upstream from mouth.

Drainage area-252 $\mathrm{mi}^{2}$

G age - Water-stage recorder. Datum of gage is $539.07 \mathrm{ft}$ NGVD 29.

\begin{tabular}{lccccc}
\hline $\begin{array}{c}\text { Water } \\
\text { year }\end{array}$ & Date & Discharge & $\begin{array}{c}\text { Discharge } \\
\text { code }\end{array}$ & $\begin{array}{c}\text { Gage } \\
\text { height } \\
\text { (feet) }\end{array}$ & $\begin{array}{c}\text { Gage } \\
\text { height } \\
\text { code }\end{array}$ \\
\hline 1989 & June 21 & 5,720 & & 14.59 & \\
1990 & Mar. 16 & 15,500 & & 18.60 & \\
1991 & Mar. 29 & 5,650 & & 14.42 & \\
1992 & Feb. 25 & 6,190 & & 14.77 & \\
1993 & Jan. 12 & 5,940 & & 14.61 & \\
1994 & Mar. 29 & 7,000 & 2 & 15.30 & 5
\end{tabular}

$1995 \quad$ Mar. $8 \quad 6,740 \quad 15.11$

1996 Oct. $5 \quad 16,900 \quad 19.10$

1997 May $3 \quad 5,210 \quad 14.15$

$1998 \quad$ Apr. $9 \quad 5,480 \quad 14.41$

1999 Jan. $23 \quad 3,220 \quad 12.02$

2000 Apr. $4 \quad 5,720 \quad 14.62$

2001 Mar. $20 \quad 6,620 \quad 15.42$

$2002 \quad$ Jan. $25 \quad 4,920 \quad 13.85$

2003 Mar. $6 \quad 6,430 \quad 15.26$

$2004 \quad$ Sept. $17 \quad 3,710 \quad 12.52$

2005 Mar. $31 \quad 4,980 \quad 13.48$

2006 Mar. $21 \quad 2,650 \quad 10.89$

2007 Nov. $15 \quad 2,490 \quad 10.77$

2008 Mar. $4 \quad 1,770 \quad 10.55$

$2009 \quad$ Jan. $6 \quad 8,180 \quad 15.61$

2010 Nov. $11 \quad 8,790 \quad 15.96$

2011 Mar. $9 \quad 8,560 \quad 15.83$

2012 Mar. $3 \quad 2,620 \quad 11.13$

$2013 \quad$ July $7 \quad 4,900 \quad 13.41$

2014 Apr. $7 \quad 7,020 \quad 14.91$

2015 Jan. $4 \quad 7,080 \quad 15.04$ 
Appendix 2. Discharge and gage height data for streamgages in Alabama.-Continued

[--, date or data not available; $\mathrm{ft}$, foot; $\mathrm{ft}^{3} / \mathrm{s}$, cubic foot per second; $\mathrm{mi}$, mile; $\mathrm{mi}^{2}$, square mile; qualification codes apply to the discharge data: 1 , discharge is a maximum daily average; 2 , discharge is an estimate; 4 , discharge is less than indicated value, which is the minimum recordable discharge at this site; 5 , discharge affected to unknown degree by regulation or diversion; 6 , discharge affected by regulation or diversion; 7, discharge is a historic peak; A, year of occurrence is unknown or not exact; $\mathrm{B}$, month or day of occurrence is unknown or not exact; E, only maximum peak available for this year. qualification codes apply to the gage height data: 1, gage height affected by backwater; 2 , gage height not the maximum for the year; 3 , gage height at different data or at different site and datum;

5 , gage height is an estimate]

\section{Big Wills Creek near Reece City}

Location-Lat $34^{\circ} 05^{\prime} 53^{\prime \prime}$, long $86^{\circ} 02^{\prime} 17^{\prime \prime}$, in SE $1 / 4$ sec. 6, T. 11 S., R. 6 E., Etowah County, Hydrologic Unit 03150106, on county road, 1 mi upstream from Fisher Creek, 1.8 mi northwest of Reece City, and at mile 25.0.

Drainage area-182 $\mathrm{mi}^{2}$.

Gage -Water-stage recorder. Datum of gage is $570 \mathrm{ft} \mathrm{NGVD} 29$ (by barometer).

\begin{tabular}{|c|c|c|c|c|c|}
\hline $\begin{array}{l}\text { Water } \\
\text { year }\end{array}$ & Date & Discharge & $\begin{array}{l}\text { Discharge } \\
\text { code }\end{array}$ & $\begin{array}{c}\text { Gage } \\
\text { height } \\
\text { (feet) }\end{array}$ & $\begin{array}{c}\text { Gage } \\
\text { height } \\
\text { code }\end{array}$ \\
\hline 1884 & -- & 20,500 & 7,B & 16.30 & \\
\hline 1943 & -- & 11,000 & B & 13.90 & 5 \\
\hline 1944 & Mar. 29 & 5,410 & & 10.76 & \\
\hline 1945 & Feb. 14 & 2,920 & & 9.52 & \\
\hline 1946 & Feb. 10 & 9,530 & & 13.04 & \\
\hline 1947 & Jan. 21 & 7,030 & & 11.80 & \\
\hline 1948 & Feb. 14 & 5,480 & & 11.00 & \\
\hline 1949 & Jan. 5 & 11,800 & & 14.20 & \\
\hline 1950 & Mar. 14 & 7,430 & & 12.00 & \\
\hline 1951 & Mar. 29 & 14,800 & & 14.50 & \\
\hline 1952 & Dec. 21 & 4,340 & & 10.60 & \\
\hline 1953 & Feb. 22 & 5,180 & & 11.10 & \\
\hline 1954 & Jan. 16 & 5,180 & & 11.10 & \\
\hline 1955 & Feb. 7 & 3,040 & & 9.64 & \\
\hline 1956 & Apr. 17 & 3,760 & & 10.20 & \\
\hline 1957 & Feb. 2 & 5,360 & & 11.20 & \\
\hline 1958 & Nov. 19 & 6,250 & & 12.00 & \\
\hline 1959 & Feb. 14 & 1,400 & 1 & -- & \\
\hline 1960 & Mar. 3 & 1,710 & & 7.88 & \\
\hline 1961 & Feb. 23 & 6,310 & & 11.56 & \\
\hline 1962 & Jan. 28 & 9,180 & & 12.76 & \\
\hline 1963 & Apr. 30 & 11,800 & & 14.37 & \\
\hline 1964 & Mar. 26 & 10,600 & & 13.76 & \\
\hline 1965 & Mar. 27 & 6,510 & & 11.72 & \\
\hline 1966 & Mar. 4 & 10,400 & & 13.66 & \\
\hline 1967 & Feb. 21 & 3,420 & & 9.80 & \\
\hline 1968 & Jan. 10 & 7,000 & 2 & -- & \\
\hline 1969 & May 19 & 4,800 & & 10.81 & \\
\hline 1970 & Apr. 27 & 4,150 & & 10.39 & \\
\hline 1971 & Feb. 27 & 3,820 & & 10.15 & \\
\hline 1972 & Jan. 5 & 3,150 & & 9.55 & \\
\hline 1973 & May 28 & 6,850 & & 11.90 & \\
\hline 1974 & Dec. 27 & 2,900 & & 9.30 & \\
\hline
\end{tabular}

02401000 Big Wills Creek near Reece City-Continued

L ocation-Lat $34^{\circ} 05^{\prime} 53^{\prime \prime}$, long $86^{\circ} 02^{\prime} 17^{\prime \prime}$, in SE $1 / 4 \mathrm{sec} .6$, T. $11 \mathrm{~S}$., R. 6 E., Etowah County, Hydrologic Unit 03150106 , on county road, 1 mi upstream from Fisher Creek, 1.8 mi northwest of Reece City, and at mile 25.0.

Drainage area $-182 \mathrm{mi}^{2}$.

Gage -Water-stage recorder. Datum of gage is $570 \mathrm{ft} \mathrm{NGVD} 29$ (by barometer).

\begin{tabular}{lccccc}
\hline $\begin{array}{c}\text { Water } \\
\text { year }\end{array}$ & Date & Discharge & $\begin{array}{c}\text { Discharge } \\
\text { code }\end{array}$ & $\begin{array}{c}\text { Gage } \\
\text { height } \\
\text { (feet) }\end{array}$ & $\begin{array}{c}\text { Gage } \\
\text { height } \\
\text { code }\end{array}$ \\
\hline 1975 & Mar. 15 & 4,490 & & 10.62 & \\
1976 & Jan. 27 & 3,760 & & 10.10 & \\
1977 & Apr. 5 & 7,780 & & 12.37 &
\end{tabular}

1977 Apr. $5 \quad 7,780 \quad 12.37$

$1978 \quad$ Nov. $6 \quad 4,860 \quad 10.81$

1979 Apr. $13 \quad 10,500 \quad 13.48$

1989 Mar. $1 \quad 3,780 \quad 10.14$

1990 Feb. $17 \quad 15,800 \quad 15.07$

1991 Feb. $20 \quad 4,050 \quad 10.33$

$1992 \quad$ Feb. $27 \quad 2,340 \quad 8.56$

$1993 \quad$ Jan. $13 \quad 2,440 \quad 8.73$

$1994 \quad$ Mar. $29 \quad 4,760 \quad 10.78$

$1995 \quad$ Feb. $17 \quad 6,770 \quad 11.89$

1996 Oct. $6 \quad 7,760 \quad 12.37$

1997 May $4 \quad 9,480 \quad 13.11$

$1998 \quad$ Jan. $8 \quad 4,280 \quad 10.48$

1999 Jan. $24 \quad 3,580 \quad 10.00$

2000 Apr. $4 \quad 4,060 \quad 10.34$

$2001 \quad$ Mar. $21 \quad 3,480 \quad 9.91$

$2002 \quad$ Jan. $25 \quad 4,530 \quad 10.64$

$2003 \quad$ May $7 \quad 8,840 \quad 12.85$

2004 Sept. $18 \quad 7,280 \quad 12.14$

$2005 \quad$ Nov. $25 \quad 8,980 \quad 12.91$

$2006 \quad$ Jan. $18 \quad 997$

2007 Jan. $8 \quad 1,010 \quad 5.42$

2008 Jan. $12 \quad 2,020 \quad 7.99$

$2009 \quad$ Jan. $7 \quad 8,400 \quad 12.66$

$2010 \quad$ Dec. $10 \quad 4,950 \quad 10.89$

2011 Sept. $7 \quad 6,040 \quad 11.51$

$2012 \quad$ Nov. $29 \quad 2,910 \quad 9.34$

$2013 \quad$ Jan. $16 \quad 5,940 \quad 11.46$

2014 Apr. $8 \quad 4,730 \quad 10.76$

2015 Jan. $5 \quad 2,000 \quad 7.95$ 
Appendix 2. Discharge and gage height data for streamgages in Alabama.-Continued

[--, date or data not available; $\mathrm{ft}$, foot; $\mathrm{ft}^{3} / \mathrm{s}$, cubic foot per second; mi, mile; $\mathrm{mi}^{2}$, square mile; qualification codes apply to the discharge data: 1 , discharge is a maximum daily average; 2 , discharge is an estimate; 4 , discharge is less than indicated value, which is the minimum recordable discharge at this site; 5 , discharge affected to unknown degree by regulation or diversion; 6 , discharge affected by regulation or diversion; 7, discharge is a historic peak; A, year of occurrence is unknown or not exact; $\mathrm{B}$, month or day of occurrence is unknown or not exact; E, only maximum peak available for this year. qualification codes apply to the gage height data: 1, gage height affected by backwater; 2, gage height not the maximum for the year; 3 , gage height at different datum or at different site and datum; 5 , gage height is an estimate]

\section{Big Canoe Creek near Springville}

Location-Lat $33^{\circ} 48^{\prime} 49^{\prime \prime}$, long $86^{\circ} 22^{\prime} 54^{\prime \prime}$, in SE $1 / 4 \mathrm{sec} .13$, T. 14 S., R. 2 E., St. Clair County, Hydrologic Unit 03150106, on U.S. Highway 11, $1 \mathrm{mi}$ west of Caldwell, 4 mi northwest of Springville, and $37.0 \mathrm{mi}$ upstream from mouth.

Drainage area $-45.0 \mathrm{mi}^{2}$.

Gage -Water-stage recorder. Datum of gage is $587.42 \mathrm{ft}$ NGVD 29.

\begin{tabular}{lllccc}
\hline $\begin{array}{c}\text { Water } \\
\text { year }\end{array}$ & Date & Discharge & $\begin{array}{c}\text { Discharge } \\
\text { code }\end{array}$ & $\begin{array}{c}\text { Gage } \\
\text { height } \\
\text { (feet) }\end{array}$ & $\begin{array}{c}\text { Gage } \\
\text { height } \\
\text { code }\end{array}$ \\
\hline 1979 & Apr. 13 & 4,690 & & 12.76 & \\
1980 & Mar. 17 & 4,130 & & 12.33 & \\
1981 & Mar. 30 & 3,030 & & 11.33 & \\
1982 & Apr. 17 & 1,610 & & 9.26 & \\
1983 & Dec. 1 & 4,870 & & 12.79 \\
1984 & Nov. 28 & 3,670 & & 11.77 & 10.12 \\
1985 & Feb. 1 & 2,190 & $\mathrm{E}$ & 5.79 & \\
1986 & Mar. 19 & 540 & & -- & \\
1987 & Jan. 19 & 1,400 & 1 & 9.88 \\
1988 & Jan. 20 & 2,010 & $\mathrm{E}$ & 10.32 \\
1989 & Feb. 28 & 2,340 & & 12.19 \\
1990 & Feb. 16 & 4,130 & & 9.90 \\
1991 & Feb. 20 & 2,020 & & 10.53 \\
1992 & Sept. 5 & 2,500 & & 10.94 \\
1993 & Jan. 12 & 2,860 & & 10.37 \\
1994 & Mar. 27 & 2,370 & & 9.71 \\
1995 & Mar. 8 & 1,890 & &
\end{tabular}

02401390 Big Canoe Creek at Ashville

L ocation-Lat $33^{\circ} 50^{\prime} 23^{\prime \prime}$, long $86^{\circ} 15^{\prime} 46^{\prime \prime}$, in SE $1 / 4$ sec. 6, T. 14 S., R. 4 E., St. Clair County, Hydrologic Unit 03150106, on U.S. Highway 231, $0.5 \mathrm{mi}$ west-northwest of Ashville, $1.7 \mathrm{mi}$ down-stream from Muckleroy Creek, and $22.3 \mathrm{mi}$ upstream from mouth.

Drainage area-141 $\mathrm{mi}^{2}$.

G age - Water-stage recorder. Datum of gage is $529.56 \mathrm{ft}$ NGVD 29.

\begin{tabular}{llcccc}
\hline $\begin{array}{c}\text { Water } \\
\text { year }\end{array}$ & Date & Discharge & $\begin{array}{c}\text { Discharge } \\
\text { code }\end{array}$ & $\begin{array}{c}\text { Gage } \\
\text { height } \\
\text { (feet) }\end{array}$ & $\begin{array}{c}\text { Gage } \\
\text { height } \\
\text { code }\end{array}$ \\
\hline 1966 & Mar. 4 & 4,040 & & 14.95 & \\
1967 & Sept. 9 & 4,410 & & 15.22 & \\
1968 & Jan. 10 & 4,250 & 15.11 & \\
1969 & May 19 & 3,810 & 14.74 & \\
1970 & Mar. 20 & 9,560 & & 17.34 &
\end{tabular}

$1971 \quad$ July $16 \quad 7,260 \quad 16.14$

$1972 \quad$ Jan. $11 \quad 6,870 \quad 15.93$

1973 June $13 \quad 4,790 \quad 14.66$

$1974 \quad$ Dec. $27 \quad 5,500 \quad 15.13$

1975 Jan. $25 \quad 7,350 \quad 16.19$

$1976 \quad$ Mar. $31 \quad 5,600 \quad 15.19$

1977 Apr. $6 \quad 9,140 \quad 17.06$

1978 Oct. $9 \quad 4,980 \quad 14.79$

1979 Apr. $13 \quad 13,600 \quad 18.75$

$1980 \quad$ Mar. $18 \quad 7,500 \quad 16.26$

1981 Mar. $30 \quad 5,260 \quad 14.97$

$1982 \quad$ Jan. $4 \quad 3,710 \quad 13.85$

$1983 \quad$ Dec. $1 \quad 8,270 \quad 16.74$

1984 Dec. $4 \quad 6,000 \quad 15.47$

$1985 \quad$ Feb. $1 \quad 4,600 \quad 14.56$

1986 Dec. $13 \quad 1,100 \quad 9.75$

1987 Jan. $19 \quad 4,190 \quad 14.26$

$1988 \quad$ Sept. $17 \quad 4,990 \quad 14.83$

1989 Mar. $5 \quad 4,550 \quad 14.53$

$1990 \quad$ Feb. $16 \quad 8,380 \quad 16.71$

$1991 \quad$ Feb. $20 \quad 4,790 \quad 14.65$

$1992 \quad$ Sept. $5 \quad 4,000 \quad 13.99$

$1993 \quad$ Jan. $13 \quad 5,300 \quad 15.03$

1994 Mar. $28 \quad 5,560 \quad 15.20$

1995 Dec. $5 \quad 4,640 \quad 14.53$

1996 Oct. $5 \quad 9,440 \quad 17.18$

1997 June $18 \quad 6,500 \quad 15.76$

$1998 \quad$ Jan. $8 \quad 8,380 \quad 16.71$ 
Appendix 2. Discharge and gage height data for streamgages in Alabama.-Continued

[--, date or data not available; $\mathrm{ft}$, foot; $\mathrm{ft}^{3} / \mathrm{s}$, cubic foot per second; $\mathrm{mi}$, mile; $\mathrm{mi}^{2}$, square mile; qualification codes apply to the discharge data: 1 , discharge is a maximum daily average; 2 , discharge is an estimate; 4 , discharge is less than indicated value, which is the minimum recordable discharge at this site; 5 , discharge affected to unknown degree by regulation or diversion; 6 , discharge affected by regulation or diversion; 7, discharge is a historic peak; A, year of occurrence is unknown or not exact; B, month or day of occurrence is unknown or not exact; E, only maximum peak available for this year. qualification codes apply to the gage height data: 1, gage height affected by backwater; 2 , gage height not the maximum for the year; 3 , gage height at different data or at different site and datum; 5 , gage height is an estimate]

\section{Big Canoe Creek at Ashville-Continued}

L ocation-Lat $33^{\circ} 50^{\prime} 23^{\prime \prime}$, long $86^{\circ} 15^{\prime} 46^{\prime \prime}$, in SE $1 / 4$ sec. 6, T. 14 S., R. 4 E., St. Clair County, Hydrologic Unit 03150106, on U.S. Highway 231, $0.5 \mathrm{mi}$ west-northwest of Ashville, $1.7 \mathrm{mi}$ down- stream from Muckleroy Creek, and 22.3 mi upstream from mouth.

Drainage area-141 $\mathrm{mi}^{2}$.

Gage -Water-stage recorder. Datum of gage is $529.56 \mathrm{ft}$ NGVD 29.

\begin{tabular}{clcccc}
\hline $\begin{array}{c}\text { Water } \\
\text { year }\end{array}$ & \multicolumn{1}{c}{ Date } & Discharge & $\begin{array}{c}\text { Discharge } \\
\text { code }\end{array}$ & $\begin{array}{c}\text { Gage } \\
\text { height } \\
\text { (feet) }\end{array}$ & $\begin{array}{c}\text { Gage } \\
\text { height } \\
\text { code }\end{array}$ \\
\hline 1999 & Jan. 23 & 4,120 & 14.10 & \\
2000 & Apr. 4 & 7,900 & 16.87 & \\
2001 & Sept. 4 & 8,110 & 16.96 & \\
2002 & Jan. 25 & 4,060 & 14.70 & \\
2003 & May 8 & 9,170 & 17.32 & \\
2004 & Sept. 17 & 9,360 & 17.38 & \\
2005 & Nov. 24 & 14,800 & 19.05 & \\
2006 & Mar. 21 & 3,140 & 13.61 & \\
2007 & Jan. 1 & 1,240 & 9.46 & \\
2008 & Aug. 26 & 4,380 & 14.81 & \\
2009 & Jan. 7 & 7,830 & 16.84 & \\
2010 & Nov. 11 & 5,600 & 15.69 & \\
2011 & Mar. 10 & 6,250 & 16.08 & \\
2012 & Jan. 27 & 4,030 & 14.50 & \\
2013 & May 19 & 5,400 & 15.56 \\
2014 & Apr. 8 & 6,720 & 16.32 \\
2015 & Jan. 5 & 3,980 & 14.46 & \\
& & & &
\end{tabular}

02401470 Little Canoe Creek near Steele

L ocation-Lat $33^{\circ} 58^{\prime} 09^{\prime \prime}$, long $86^{\circ} 10^{\prime} 40^{\prime \prime}$, in SW 1/4 sec. 24, T. 12 S., R. 4 E., St. Clair County, Hydrologic Unit 03150106, on U.S. Highway 11, 2.3 mi north of Steele, and 7.2 mi upstream from mouth.

Drainage area-22.3 $\mathrm{mi}^{2}$.

G age - Water-stage recorder. Datum of gage is $554.62 \mathrm{ft}$ NGVD 29.

\begin{tabular}{|c|c|c|c|c|c|}
\hline $\begin{array}{l}\text { Water } \\
\text { year }\end{array}$ & Date & Discharge & $\begin{array}{l}\text { Discharge } \\
\text { code }\end{array}$ & $\begin{array}{c}\text { Gage } \\
\text { height } \\
\text { (feet) }\end{array}$ & $\begin{array}{c}\text { Gage } \\
\text { height } \\
\text { code }\end{array}$ \\
\hline 1970 & Mar. 19 & 3,350 & 7 & 8.23 & \\
\hline 1979 & Apr. 13 & 3,600 & 7 & 8.36 & \\
\hline 1983 & May 18 & 3,250 & & 8.19 & \\
\hline 1984 & Dec. 28 & 1,700 & & 7.27 & \\
\hline 1985 & Feb. 1 & 1,400 & $\mathrm{E}$ & 7.06 & \\
\hline 1986 & Mar. 19 & 250 & & 3.77 & \\
\hline 1987 & July 4 & 1,700 & & 7.28 & \\
\hline 1988 & Sept. 17 & 2,200 & & 7.64 & \\
\hline 1989 & Feb. 28 & 1,190 & & 6.85 & \\
\hline 1990 & Feb. 16 & 3,310 & & 8.20 & \\
\hline 1991 & Feb. 19 & 1,370 & & 7.01 & \\
\hline 1992 & Feb. 25 & 442 & & 5.28 & \\
\hline 1993 & Jan. 12 & 1,050 & & 6.61 & \\
\hline 1994 & Mar. 27 & 1,820 & 2 & 7.40 & \\
\hline 1995 & Oct. 3 & 1,370 & & 7.01 & \\
\hline
\end{tabular}


Appendix 2. Discharge and gage height data for streamgages in Alabama.-Continued

[--, date or data not available; $\mathrm{ft}$, foot; $\mathrm{ft}^{3} / \mathrm{s}$, cubic foot per second; mi, mile; $\mathrm{mi}^{2}$, square mile; qualification codes apply to the discharge data: 1 , discharge is a maximum daily average; 2 , discharge is an estimate; 4 , discharge is less than indicated value, which is the minimum recordable discharge at this site; 5 , discharge affected to unknown degree by regulation or diversion; 6 , discharge affected by regulation or diversion; 7, discharge is a historic peak; A, year of occurrence is unknown or not exact; $\mathrm{B}$, month or day of occurrence is unknown or not exact; E, only maximum peak available for this year. qualification codes apply to the gage height data: 1, gage height affected by backwater; 2, gage height not the maximum for the year; 3, gage height at different datum or at different site and datum; 5 , gage height is an estimate]

\section{Big Canoe Creek near Gadsden}

Location-Lat $33^{\circ} 54^{\prime} 11^{\prime \prime}$, long $86^{\circ} 06^{\prime} 37^{\prime \prime}$, in NW $1 / 4$ sec. 15, T. 13 S., R. 5 E., Etowah County, Hydrologic Unit 03150106, on U.S. Highway 411, $400 \mathrm{ft}$ downstream from Rock Creek, 5 mi upstream from mouth, and 10 mi southwest of Gadsden.

Drainage area $-253 \mathrm{mi}^{2}$.

Gage -Water-stage recorder. Datum of gage is $490.56 \mathrm{ft}$ NGVD 29. Remarks - Since 1966, site affected by H. Neely Henry Reservoir.

\begin{tabular}{|c|c|c|c|c|c|}
\hline $\begin{array}{l}\text { Water } \\
\text { year }\end{array}$ & Date & Discharge & $\begin{array}{c}\text { Discharge } \\
\text { code }\end{array}$ & $\begin{array}{c}\text { Gage } \\
\text { height } \\
\text { (feet) }\end{array}$ & $\begin{array}{c}\text { Gage } \\
\text { height } \\
\text { code }\end{array}$ \\
\hline 1938 & Apr. 8 & 19,500 & & 23.00 & \\
\hline 1939 & Feb. 28 & 4,640 & & 13.80 & \\
\hline 1940 & Feb. 18 & 5,240 & & 14.70 & \\
\hline 1941 & July 7 & 3,370 & & 11.70 & \\
\hline 1942 & Feb. 17 & 8,300 & & 17.30 & \\
\hline 1943 & Dec. 29 & 36,200 & & 29.10 & \\
\hline 1944 & Mar. 29 & 7,800 & & 16.95 & \\
\hline 1945 & Feb. 13 & 5,100 & & 14.53 & \\
\hline 1946 & Feb. 10 & 13,600 & & 20.20 & \\
\hline 1947 & Jan. 20 & 8,800 & & 17.60 & \\
\hline 1948 & Feb. 9 & 5,840 & & 15.20 & \\
\hline 1949 & Jan. 6 & 18,500 & & 22.60 & \\
\hline 1950 & Mar. 14 & 11,300 & & 19.20 & \\
\hline 1951 & Mar. 30 & 15,800 & & 21.40 & \\
\hline 1952 & Dec. 21 & 6,470 & & 15.70 & \\
\hline 1953 & Jan. 10 & 7,520 & & 16.60 & \\
\hline 1954 & Jan. 16 & 8,130 & & 17.10 & \\
\hline 1955 & Feb. 6 & 6,690 & & 15.94 & \\
\hline 1956 & Apr. 6 & 5,480 & & 14.80 & \\
\hline 1957 & Apr. 6 & 5,920 & & 15.20 & \\
\hline 1958 & Sept. 21 & 6,690 & & 15.90 & \\
\hline 1959 & Feb. 15 & 4,490 & & 13.70 & \\
\hline 1960 & Mar. 3 & 6,690 & & 15.90 & \\
\hline 1961 & Feb. 23 & 20,300 & & 23.58 & \\
\hline 1962 & Dec. 13 & 9,700 & & 18.27 & \\
\hline 1963 & Jan. 20 & 4,900 & & 14.22 & \\
\hline 1964 & Apr. 8 & 6,470 & & 15.70 & \\
\hline 1965 & Feb. 12 & 4,250 & & 13.34 & \\
\hline
\end{tabular}

02404000 Choccolocco Creek near Jenifer

L ocation-Lat $33^{\circ} 34^{\prime} 14^{\prime \prime}$, long $85^{\circ} 55^{\prime} 50^{\prime \prime}$, in NW $1 / 4$ sec. 8 , T. 17 S., R. 7 E., Talladega County, Hydrologic Unit 03150106, $0.8 \mathrm{mi}$ upstream from Salt Creek, and 1.5 mi north of Jenifer.

Drainage area-277 $\mathrm{mi}^{2}$.

G age -Water-stage recorder. Datum of gage is $554.15 \mathrm{ft}$ NGVD 29.

\begin{tabular}{|c|c|c|c|c|c|}
\hline $\begin{array}{c}\text { Water } \\
\text { year }\end{array}$ & Date & Discharge & $\begin{array}{l}\text { Discharge } \\
\text { code }\end{array}$ & $\begin{array}{c}\text { Gage } \\
\text { height } \\
\text { (feet) }\end{array}$ & $\begin{array}{c}\text { Gage } \\
\text { height } \\
\text { code }\end{array}$ \\
\hline 1904 & Aug. 12 & 1,100 & & 4.00 & \\
\hline 1905 & Feb. 9 & 4,400 & & 8.00 & \\
\hline 1906 & Mar. 20 & 14,600 & & 14.20 & \\
\hline 1907 & Feb. 1 & 5,740 & & 9.10 & \\
\hline 1930 & Mar. 7 & 14,900 & & 14.30 & \\
\hline 1931 & Nov. 18 & 1,930 & & 5.75 & \\
\hline 1932 & Jan. 31 & 4,450 & & 8.35 & \\
\hline 1936 & Feb. 4 & 21,900 & & 17.20 & \\
\hline 1937 & Apr. 30 & 8,550 & & 11.50 & \\
\hline 1938 & Apr. 8 & 18,800 & & 16.00 & \\
\hline 1939 & Mar. 2 & 3,700 & & 7.52 & \\
\hline 1940 & July 13 & 6,200 & & 9.60 & \\
\hline 1941 & Mar. 22 & 1,550 & & 5.01 & \\
\hline 1942 & Mar. 21 & 6,460 & & 9.74 & \\
\hline 1943 & Mar. 21 & 7,030 & & 10.17 & \\
\hline 1944 & Mar. 30 & 5,200 & & 8.82 & \\
\hline 1945 & May 15 & 3,200 & & 7.03 & \\
\hline 1946 & Jan. 7 & 12,800 & & 13.56 & \\
\hline 1947 & Jan. 20 & 11,800 & & 13.00 & \\
\hline 1948 & Feb. 9 & 6,100 & & 9.50 & \\
\hline 1949 & Nov. 29 & 16,600 & & 15.10 & \\
\hline 1950 & Mar. 15 & 2,020 & & 5.70 & \\
\hline 1951 & Mar. 29 & 20,400 & & 16.60 & \\
\hline 1952 & Mar. 23 & 9,770 & & 11.70 & \\
\hline 1953 & Jan. 10 & 8,440 & & 10.90 & \\
\hline 1954 & Jan. 17 & 7,950 & & 10.60 & \\
\hline 1955 & Apr. 14 & 6,070 & & 9.30 & \\
\hline 1956 & Mar. 16 & 6,630 & & 9.73 & \\
\hline 1957 & Apr. 5 & 11,800 & & 12.90 & \\
\hline 1958 & Apr. 16 & 4,600 & & 8.50 & \\
\hline
\end{tabular}


Appendix 2. Discharge and gage height data for streamgages in Alabama.-Continued

[--, date or data not available; $\mathrm{ft}$, foot; $\mathrm{ft}^{3} / \mathrm{s}$, cubic foot per second; mi, mile; $\mathrm{mi}^{2}$, square mile; qualification codes apply to the discharge data: 1 , discharge is a maximum daily average; 2 , discharge is an estimate; 4 , discharge is less than indicated value, which is the minimum recordable discharge at this site; 5 , discharge affected to unknown degree by regulation or diversion; 6 , discharge affected by regulation or diversion; 7, discharge is a historic peak; A, year of occurrence is unknown or not exact; B, month or day of occurrence is unknown or not exact; E, only maximum peak available for this year. qualification codes apply to the gage height data: 1, gage height affected by backwater; 2 , gage height not the maximum for the year; 3 , gage height at different data or at different site and datum;

5 , gage height is an estimate]

02404000 Choccolocco Creek near Jenifer-Continued

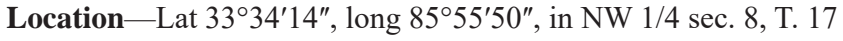
S., R. 7 E., Talladega County, Hydrologic Unit 03150106, 0.8 mi upstream from Salt Creek, and 1.5 mi north of Jenifer.

Drainage area $-277 \mathrm{mi}^{2}$.

Gage-Water-stage recorder. Datum of gage is $554.15 \mathrm{ft}$ NGVD 29.

\begin{tabular}{|c|c|c|c|c|c|}
\hline $\begin{array}{c}\text { Water } \\
\text { year }\end{array}$ & Date & Discharge & $\begin{array}{c}\text { Discharge } \\
\text { code }\end{array}$ & $\begin{array}{c}\text { Gage } \\
\text { height } \\
\text { (feet) }\end{array}$ & $\begin{array}{c}\text { Gage } \\
\text { height } \\
\text { code }\end{array}$ \\
\hline 1959 & June 1 & 3,300 & & 7.30 & \\
\hline 1960 & Feb. 1 & 2,220 & & 6.10 & \\
\hline 1961 & Feb. 22 & 15,800 & & 15.12 & \\
\hline 1962 & Feb. 23 & 13,800 & & 13.99 & \\
\hline 1963 & Apr. 30 & 22,500 & & 17.68 & \\
\hline 1964 & Mar. 16 & 9,480 & & 11.60 & \\
\hline 1965 & Mar. 24 & 3,070 & & 6.96 & \\
\hline 1966 & Feb. 17 & 6,730 & & 9.95 & \\
\hline 1967 & Aug. 25 & 21,600 & & 17.47 & \\
\hline 1968 & May 16 & 20,000 & & 16.82 & \\
\hline 1969 & May 20 & 2,200 & & 5.80 & \\
\hline 1970 & Mar. 20 & 14,400 & & 14.48 & \\
\hline 1979 & Apr. 13 & 23,000 & 7 & 17.85 & \\
\hline
\end{tabular}

02404400 Choccolocco Creek at Jackson Shoals near Lincoln

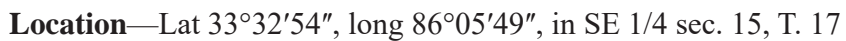
S., R. 5 E., Talladega County, Hydrologic Unit 03150106, at foot of Jackson Shoals, 1.8 mi downstream from Eastaboga Creek, and 4.5 mi southeast of Lincoln.

Drainage area $481 \mathrm{mi}^{2}$.

G age -Water-stage recorder. Datum of gage is $448.50 \mathrm{ft}$ NGVD 29.

\begin{tabular}{llcccc}
\hline $\begin{array}{c}\text { Water } \\
\text { year }\end{array}$ & Date & Discharge & $\begin{array}{c}\text { Discharge } \\
\text { code }\end{array}$ & $\begin{array}{c}\text { Gage } \\
\text { height } \\
\text { (feet) }\end{array}$ & $\begin{array}{c}\text { Gage } \\
\text { height } \\
\text { code }\end{array}$ \\
\hline 1886 & -- & 70,000 & $2,7, B$ & -- & \\
1951 & Mar & 45,000 & $7, B$ & 42.40 & \\
1961 & Feb. 22 & 25,900 & & 36.78 & \\
1962 & & & & 31.00 &
\end{tabular}

1963 Apr. $30 \quad 36,900 \quad 39.98$

1964 Mar. $15 \quad$ 15,200 31.80

1965 Feb. $12 \quad 4,720 \quad 24.01$

$1966 \quad$ Feb. $17 \quad 10,700 \quad 28.66$

1967 Aug. $26 \quad 21,600 \quad 34.65$

1968 May $16 \quad 16,800 \quad 32.28$

1969 May $9 \quad 3,670 \quad 23.00$

1970 Mar. $20 \quad 23,500 \quad 35.97$

1971 Mar. $2 \quad 7,230 \quad 26.10$

1975 July $7 \quad 9,950 \quad 28.11$

1976 Mar. $16 \quad 31,600 \quad 38.25$

1977 Mar. $30 \quad 35,800 \quad 39.54$

$1978 \quad$ Jan. $26 \quad 6,790 \quad 25.76$

1979 Apr. $13 \quad 30,100 \quad 37.76$

1980 Apr. $13 \quad 10,400 \quad 28.45$

1981 Feb. $10 \quad 6,000 \quad 25.12$

1982 Feb. $3 \quad 12,000 \quad 29.59$

1983 Feb. $2 \quad 8,860 \quad 27.33$

1984 Dec. $6 \quad 14,200 \quad 30.91$

1985 Feb. $5 \quad 4,560 \quad 23.58$

1986 Aug. $28 \quad 1,530 \quad 20.28$

$1987 \quad$ Feb. $28 \quad 8,070 \quad 26.66$

$1988 \quad$ Jan. $20 \quad 5,410 \quad 24.55$

$1989 \quad$ Mar. $6 \quad 7,700 \quad 26.38$

1990 Mar. $17 \quad 27,400 \quad 36.67$

1991 Feb. $20 \quad 7,170 \quad 25.98$ 
Appendix 2. Discharge and gage height data for streamgages in Alabama.-Continued

[--, date or data not available; $\mathrm{ft}$, foot; $\mathrm{ft}^{3} / \mathrm{s}$, cubic foot per second; mi, mile; $\mathrm{mi}^{2}$, square mile; qualification codes apply to the discharge data: 1 , discharge is a maximum daily average; 2 , discharge is an estimate; 4 , discharge is less than indicated value, which is the minimum recordable discharge at this site; 5 , discharge affected to unknown degree by regulation or diversion; 6 , discharge affected by regulation or diversion; 7, discharge is a historic peak; A, year of occurrence is unknown or not exact; $\mathrm{B}$, month or day of occurrence is unknown or not exact; E, only maximum peak available for this year. qualification codes apply to the gage height data: 1, gage height affected by backwater; 2, gage height not the maximum for the year; 3 , gage height at different datum or at different site and datum; 5 , gage height is an estimate]

\section{Choccolocco Creek at Jackson Shoals near Lincoln- Continued}

Location-Lat $33^{\circ} 32^{\prime} 54^{\prime \prime}$, long $86^{\circ} 05^{\prime} 49^{\prime \prime}$, in SE $1 / 4$ sec. 15 , T. 17 S., R. 5 E., Talladega County, Hydrologic Unit 03150106, at foot of Jackson Shoals, $1.8 \mathrm{mi}$ downstream from Eastaboga Creek, and 4.5 mi southeast of Lincoln.

Drainage area $481 \mathrm{mi}^{2}$.

G age - Water-stage recorder. Datum of gage is $448.50 \mathrm{ft}$ NGVD 29.

\begin{tabular}{|c|c|c|c|c|c|}
\hline $\begin{array}{c}\text { Water } \\
\text { year }\end{array}$ & Date & Discharge & $\begin{array}{l}\text { Discharge } \\
\text { code }\end{array}$ & $\begin{array}{c}\text { Gage } \\
\text { height } \\
\text { (feet) }\end{array}$ & $\begin{array}{c}\text { Gage } \\
\text { height } \\
\text { code }\end{array}$ \\
\hline
\end{tabular}

\begin{tabular}{llrl}
\hline 1992 & Feb. 26 & 5,410 & 24.55 \\
1993 & Jan. 12 & 10,100 & 28.05 \\
1994 & Apr. 16 & 8,890 & 27.24
\end{tabular}

1994

1995

1996

1997

1998

1999

2000

2001

2002

2003

2004

2005

2006

2007

2008

2009

2010

2011

2012

2013

2014

2015
Apr. 16

Mar. 8

9,790

Oct. 5

18,100

June 14

16,000

Mar. $8 \quad 10,000$

Jan. 23

3,670

Apr. 4

8,220

Apr. 4

16,000

Jan. 25

6,170

May 8

27,700

Sept. 17

8,010

Nov. 23

8,930

Feb. 7

9,410

Nov. 15

3,100

5,310

Aug. 26

6,290

Mar. 11

16,200

14,200

8,870

Mar. 3

May 18

27,000

14,000

Jan. 4

16,200
Apr. 7
27.24

27.86

32.48

31.39

28.02

22.92

26.76

31.38

25.19

36.79

26.61

27.27

27.60

22.31

24.47

25.29

31.49

30.43

27.23

36.53

30.32

31.49
02405500 Kelly Creek near Vincent

L ocation-Lat $33^{\circ} 26^{\prime} 51^{\prime \prime}$, long $86^{\circ} 23^{\prime} 13^{\prime \prime}$, in SW 1/4 sec. 24, T. 18 S., R. 2 E., Shelby County, Hydrologic Unit 03150106, on U.S. Highway 231, 1.5 mi downstream from Little Creek, 4.2 mi north of Vincent, and 5 mi upstream from mouth.

Drainage area-193 $\mathrm{mi}^{2}$.

G age - Water-stage recorder. Datum of gage is $404.09 \mathrm{ft}$ NGVD 29.

\begin{tabular}{|c|c|c|c|c|c|}
\hline $\begin{array}{c}\text { Water } \\
\text { year }\end{array}$ & Date & Discharge & $\begin{array}{c}\text { Discharge } \\
\text { code }\end{array}$ & $\begin{array}{c}\text { Gage } \\
\text { height } \\
\text { (feet) }\end{array}$ & $\begin{array}{r}\text { Gage } \\
\text { height } \\
\text { code }\end{array}$ \\
\hline 1952 & Dec. 21 & 8,770 & & 19.51 & \\
\hline 1953 & Jan. 10 & 9,970 & & 20.50 & \\
\hline 1954 & Jan. 17 & 7,190 & & 17.88 & \\
\hline
\end{tabular}

$1955 \quad$ Feb. $7 \quad 10,500 \quad 20.86$

1956 Mar. $16 \quad 6,000 \quad 16.53$

1957 Apr. $5 \quad 9,840 \quad 20.40$

$1958 \quad$ Feb. $7 \quad 5,040 \quad 15.24$

$1959 \quad$ Jan. $22 \quad 3,730 \quad 12.90$

1960 Mar. $3 \quad 3,880 \quad 13.20$

$1961 \quad$ Feb. $22 \quad 30,900 \quad 27.08$

1962 Dec. $13 \quad 13,700 \quad 22.80$

1963 Apr. $30 \quad 8,850 \quad 19.90$

1964 Apr. $7 \quad 6,940 \quad 18.65$

1965 Feb. $13 \quad 3,000 \quad 11.40$

1966 Feb. $14 \quad 7,100 \quad 17.80$

1967 May $6 \quad 2,950 \quad 11.53$

1968 Mar. $13 \quad 8,270 \quad 19.03$

1969 Dec. $23 \quad 4,580 \quad 14.47$

1970 Mar. $20 \quad 16,100 \quad 23.69$

$1979 \quad$ Apr. $13 \quad 33,400 \quad 7 \quad 27.39$

1987 Jan. $19 \quad 7,020 \quad 17.72$

$1988 \quad$ Jan. $20 \quad 3,300 \quad 11.48$

$1989 \quad$ June $22 \quad 8,070 \quad 19.18$

$1990 \quad$ Feb. $16 \quad 18,700 \quad 25.31$

$1991 \quad$ Feb. $21 \quad 6,710 \quad 17.58$

$1992 \quad$ Feb. $26 \quad 4,210 \quad 13.50$

$1993 \quad$ Jan. $13 \quad 5,610 \quad 15.79$

1994 Sept. $24 \quad 4,450 \quad 13.90$

1995 Dec. $5 \quad 5,120 \quad 15.05$

1996 Mar. $8 \quad 11,000 \quad 21.98$ 
Appendix 2. Discharge and gage height data for streamgages in Alabama._- Continued

[--, date or data not available; ft, foot; $\mathrm{ft}^{3} / \mathrm{s}$, cubic foot per second; mi, mile; $\mathrm{mi}^{2}$, square mile; qualification codes apply to the discharge data: 1 , discharge is a maximum daily average; 2 , discharge is an estimate; 4 , discharge is less than indicated value, which is the minimum recordable discharge at this site; 5 , discharge affected to unknown degree by regulation or diversion; 6 , discharge affected by regulation or diversion; 7, discharge is a historic peak; A, year of occurrence is unknown or not exact; $\mathrm{B}$, month or day of occurrence is unknown or not exact; E, only maximum peak available for this year. qualification codes apply to the gage height data: 1 , gage height affected by backwater; 2, gage height not the maximum for the year; 3, gage height at different data or at different site and datum; 5 , gage height is an estimate]

\section{Kelly Creek near Vincent-Continued}

L ocation-Lat $33^{\circ} 26^{\prime} 51^{\prime \prime}$, long $86^{\circ} 23^{\prime} 13^{\prime \prime}$, in SW 1/4 sec. 24 , T. 18 S., R. 2 E., Shelby County, Hydrologic Unit 03150106, on U.S.

Highway 231, 1.5 mi downstream from Little Creek, 4.2 mi north of Vincent, and $5 \mathrm{mi}$ upstream from mouth.

Drainage area-193 $\mathrm{mi}^{2}$.

Gage - Water-stage recorder. Datum of gage is $404.09 \mathrm{ft}$ NGVD 29.

\begin{tabular}{|c|c|c|c|c|c|}
\hline $\begin{array}{c}\text { Water } \\
\text { year }\end{array}$ & Date & Discharge & $\begin{array}{l}\text { Discharge } \\
\text { code }\end{array}$ & $\begin{array}{c}\text { Gage } \\
\text { height } \\
\text { (feet) }\end{array}$ & $\begin{array}{c}\text { Gage } \\
\text { height } \\
\text { code }\end{array}$ \\
\hline 1997 & June 23 & 5,960 & & 16.42 & \\
\hline 1998 & Jan. 8 & 6,790 & & 17.69 & \\
\hline 1999 & June 29 & 5,830 & & 16.21 & \\
\hline 2000 & Apr. 4 & 10,700 & & 21.79 & \\
\hline 2001 & Apr. 4 & 8,540 & & 19.75 & \\
\hline 2002 & Jan. 25 & 5,140 & & 15.63 & \\
\hline 2003 & May 8 & 11,400 & & 22.71 & \\
\hline 2005 & Nov. 25 & 8,610 & & 19.82 & \\
\hline 2006 & Feb. 7 & 6,490 & & 17.24 & \\
\hline 2007 & Jan. 8 & 2,840 & & 10.76 & \\
\hline 2008 & Aug. 26 & 3,890 & & 12.87 & \\
\hline 2009 & Jan. 7 & 6,290 & & 16.93 & \\
\hline 2010 & Nov. 11 & 8,410 & & 19.62 & \\
\hline 2011 & Mar. 10 & 8,220 & & 19.43 & \\
\hline 2012 & Mar. 3 & 2,510 & & 10.03 & \\
\hline 2013 & May 19 & 7,430 & & 18.55 & \\
\hline 2014 & Apr. 8 & 10,400 & & 21.55 & \\
\hline 2015 & Dec. 29 & 6,620 & & 17.44 & \\
\hline
\end{tabular}

\section{Talladega Creek at Alpine}

L ocation-Lat $33^{\circ} 21^{\prime} 34^{\prime \prime}$, long $86^{\circ} 14^{\prime} 03^{\prime \prime}$, in SW 1/4 sec. 21, T. 19 S., R. 4 E., Talladega County, Hydrologic Unit 03150106, on county road 207, 1 mi north of Alpine, 9 mi southwest of Talladega, and 11.0 mi upstream from mouth.

Drainage area $-150 \mathrm{mi}^{2}$.

Gage - Water-stage recorder. Datum of gage is $431.24 \mathrm{ft}$ NGVD 29.

\begin{tabular}{lccccc}
\hline $\begin{array}{c}\text { Water } \\
\text { year }\end{array}$ & Date & Discharge & $\begin{array}{c}\text { Discharge } \\
\text { code }\end{array}$ & $\begin{array}{c}\text { Gage } \\
\text { height } \\
\text { (feet) }\end{array}$ & $\begin{array}{c}\text { Gage } \\
\text { height } \\
\text { code }\end{array}$ \\
\hline 1939 & Feb. 28 & 3,760 & & 12.84 & \\
1940 & July 13 & 5,500 & & 13.20 & \\
1941 & Mar. 21 & 2,140 & & 10.58 & \\
1942 & & & &
\end{tabular}

1942 Mar. $21 \quad 17,000$

1943 Apr. $19 \quad 6,500 \quad 13.42$

1944 Mar. $29 \quad 3,460 \quad 12.60$

1945 May $13 \quad 3,160 \quad 12.31$

$1946 \quad$ Jan. $7 \quad 15,000 \quad 14.99$

$1947 \quad$ Jan. $20 \quad 14,700 \quad 14.89$

1948 Mar. $23 \quad 7,200 \quad 13.64$

1949 Nov. $28 \quad 9,000 \quad 14.00$

$1950 \quad$ Mar. $13 \quad 2,200 \quad 10.74$

$1951 \quad$ Mar. $29 \quad 39,000 \quad 16.60$

1952 Dec. $21 \quad 6,000 \quad 13.36$

$1953 \quad$ Jan. $8 \quad 3,080 \quad 12.20$

1954 Jan. $23 \quad 980 \quad 7.38$

1955 Apr. $13 \quad 10,100 \quad 14.30$

1956 Mar. $16 \quad 12,100 \quad 14.60$

1957 Apr. $5 \quad 12,700 \quad 14.70$

$1958 \quad$ Feb. $6 \quad 5,900 \quad 13.30$

1959 Jan. $22 \quad 1,480 \quad 9.00$

$1960 \quad$ Jan. $31 \quad 1,410 \quad 8.80$

$1961 \quad$ Feb. $22 \quad 12,200 \quad 14.63$

1962 Dec. $18 \quad 8,400 \quad 13.85$

1963 Mar. $13 \quad 8,300 \quad 13.81$

1964 Apr. $15 \quad 8,350 \quad 13.82$

1965 Feb. $13 \quad 2,300 \quad 10.90$

$1966 \quad$ Feb. $16 \quad 5,100 \quad 13.00$

1967 May $23 \quad 2,750 \quad 11.74$

1968 Apr. $5 \quad 7,300 \quad 13.68$

1969 Mar. $24 \quad 1,610 \quad 9.36$ 
Appendix 2. Discharge and gage height data for streamgages in Alabama._-Continued

[--, date or data not available; $\mathrm{ft}$, foot; $\mathrm{ft}^{3} / \mathrm{s}$, cubic foot per second; mi, mile; $\mathrm{mi}^{2}$, square mile; qualification codes apply to the discharge data: 1 , discharge is a maximum daily average; 2 , discharge is an estimate; 4 , discharge is less than indicated value, which is the minimum recordable discharge at this site; 5 , discharge affected to unknown degree by regulation or diversion; 6 , discharge affected by regulation or diversion; 7, discharge is a historic peak; A, year of occurrence is unknown or not exact; B, month or day of occurrence is unknown or not exact; E, only maximum peak available for this year. qualification codes apply to the gage height data: 1, gage height affected by backwater; 2, gage height not the maximum for the year; 3, gage height at different datum or at different site and datum; 5 , gage height is an estimate]

\section{Talladega Creek at Alpine-Continued}

L ocation-Lat $33^{\circ} 21^{\prime} 34^{\prime \prime}$, long $86^{\circ} 14^{\prime} 03^{\prime \prime}$, in SW 1/4 sec. 21 , T. 19 S., R. 4 E., Talladega County, Hydrologic Unit 03150106, on county road 207, $1 \mathrm{mi}$ north of Alpine, 9 mi southwest of Talladega, and $11.0 \mathrm{mi}$ upstream from mouth.

Drainage area $-150 \mathrm{mi}^{2}$.

Gage - Water-stage recorder. Datum of gage is $431.24 \mathrm{ft}$ NGVD 29.

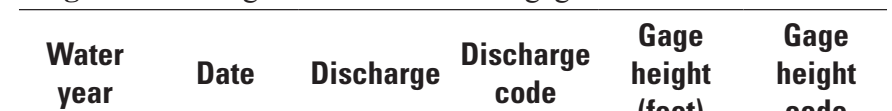

\begin{tabular}{lllccc} 
& & & code & (feet) & code \\
\hline 1970 & Mar. 19 & 14,800 & & 14.95 & \\
1979 & Apr. 13 & 14,400 & 7 & 14.81
\end{tabular}

1988

1989

1990

1991

1992

1993

1994

1995

1996

1997

1998

1999

2000

2001

2002

2003

2004

2005

2006

2007

2008

2009

2010

2011

2012

2013

2014

2015
Jan. 20

3,250

Apr. 5

3,410

Mar. 17

13,000

Feb. 20

4,110

Feb. 26

1,790

Nov. 5

4,040

Apr. 16

3,400

Feb. 11

9,950

Oct. 5

10,100

Mar. 1

6,560

Feb. 4

4,920

Jan. 23

1,470

Apr. 4

5,860

Apr. 4

9,950

Jan. 20

3,400

May 8

15,300

Sept. 17

5,320

Apr. 1

2,800

Mar. $21 \quad 2,810$

Nov. 16

2,290

Feb. 22

2,050

Mar. 28

3,030

Mar. 11

8,650

Mar. 10

6,540

Mar. 3

2,820

May 19

4,470

Apr. 7

9,840

Jan. 4

6,210
10.85

10.43

11.81

14.40

13.74

11.56

13.64

14.60

14.16
02407500 Yellowleaf Creek near Wilsonville

L ocation-Lat $33^{\circ} 18^{\prime} 23^{\prime \prime}$, long $86^{\circ} 33^{\prime} 04^{\prime \prime}$, in NW 1/4 sec. 9, T. 20 S., R. 1 E., Shelby County, Hydrologic Unit 03150107, on county road, $3.5 \mathrm{mi}$ south of U.S. Highway $280,4 \mathrm{mi}$ upstream from Muddy Prong, and $6 \mathrm{mi}$ northwest of Wilsonville.

Drainage area-96.5 $\mathrm{mi}^{2}$.

G age-Water-stage recorder. Datum of gage is $430.56 \mathrm{ft}$ NGVD 29.

\begin{tabular}{|c|c|c|c|c|c|}
\hline $\begin{array}{c}\text { Water } \\
\text { year }\end{array}$ & Date & Discharge & $\begin{array}{l}\text { Discharge } \\
\text { code }\end{array}$ & $\begin{array}{c}\text { Gage } \\
\text { height } \\
\text { (feet) }\end{array}$ & $\begin{array}{c}\text { Gage } \\
\text { height } \\
\text { code }\end{array}$ \\
\hline 1951 & Mar. 29 & 19,300 & & 23.85 & \\
\hline 1952 & Dec. 22 & 4,190 & & 19.60 & \\
\hline 1953 & Jan. 9 & 3,590 & & 19.10 & \\
\hline 1954 & Jan. 17 & 1,050 & & 11.30 & \\
\hline 1955 & Apr. 14 & 3,210 & & 18.60 & \\
\hline 1956 & Mar. 16 & 3,280 & & 18.68 & \\
\hline 1957 & Apr. 5 & 4,050 & & 19.50 & \\
\hline 1958 & Feb. 7 & 2,200 & & 16.40 & \\
\hline 1959 & Jan. 22 & 982 & & 11.20 & \\
\hline 1960 & Mar. 16 & 1,010 & & 11.38 & \\
\hline 1961 & Feb. 21 & 26,700 & & 25.20 & \\
\hline 1962 & Feb. 23 & 3,390 & & 18.86 & \\
\hline 1963 & June 23 & 5,300 & 1,3 & 28.31 & \\
\hline 1964 & Apr. 7 & 2,690 & & 17.64 & \\
\hline 1965 & Feb. 13 & 1,400 & & 13.41 & \\
\hline 1966 & Feb. 14 & 3,260 & & 18.67 & \\
\hline 1967 & Nov. 11 & 1,120 & & 11.93 & \\
\hline 1968 & Jan. 11 & 1,960 & & 15.60 & \\
\hline 1969 & Jan. 20 & 3,030 & & 18.30 & \\
\hline 1970 & Mar. 19 & 8,820 & & 21.47 & \\
\hline 1979 & Apr. 13 & 16,200 & 7 & 23.17 & \\
\hline
\end{tabular}


Appendix 2. Discharge and gage height data for streamgages in Alabama.-Continued

[--, date or data not available; $\mathrm{ft}$, foot; $\mathrm{ft}^{3} / \mathrm{s}$, cubic foot per second; $\mathrm{mi}$, mile; $\mathrm{mi}^{2}$, square mile; qualification codes apply to the discharge data: 1 , discharge is a maximum daily average; 2 , discharge is an estimate; 4 , discharge is less than indicated value, which is the minimum recordable discharge at this site; 5 , discharge affected to unknown degree by regulation or diversion; 6 , discharge affected by regulation or diversion; 7, discharge is a historic peak; A, year of occurrence is unknown or not exact; B, month or day of occurrence is unknown or not exact; E, only maximum peak available for this year. qualification codes apply to the gage height data: 1, gage height affected by backwater; 2 , gage height not the maximum for the year; 3 , gage height at different data or at different site and datum;

5 , gage height is an estimate]

\section{Hatchet Creek near Rockford}

L ocation-Lat $32^{\circ} 56^{\prime} 42^{\prime \prime}$, long $86^{\circ} 13^{\prime} 06^{\prime \prime}$, in NE $1 / 4 \mathrm{sec}$. 36, T. 23 N., R. 18 E., Coosa County, Hydrologic Unit 03150107, on county road, $1 \mathrm{mi}$ downstream from U.S. Highway 231, $1.5 \mathrm{mi}$ downstream from Socapatoy Creek, and 4 mi north of Rockford. Drainage area-233 $\mathrm{mi}^{2}$.

Gage -Water-stage recorder. Datum of gage is $449 \mathrm{ft}$ NGVD 29. Prior to Sept. 30, 1964, at same site and at datum $1.00 \mathrm{ft}$ higher.

\begin{tabular}{|c|c|c|c|c|c|c|c|c|c|c|c|}
\hline $\begin{array}{c}\text { Water } \\
\text { year }\end{array}$ & Date & Discharge & $\begin{array}{l}\text { Discharge } \\
\text { code }\end{array}$ & $\begin{array}{c}\text { Gage } \\
\text { height } \\
\text { (feet) }\end{array}$ & $\begin{array}{c}\text { Gage } \\
\text { height } \\
\text { code }\end{array}$ & $\begin{array}{c}\text { Water } \\
\text { year }\end{array}$ & Date & Discharge & $\begin{array}{l}\text { Discharge } \\
\text { code }\end{array}$ & $\begin{array}{c}\text { Gage } \\
\text { height } \\
\text { (feet) }\end{array}$ & $\begin{array}{c}\text { Gage } \\
\text { height } \\
\text { code }\end{array}$ \\
\hline 1945 & Apr. 25 & 13,300 & & 20.80 & & 1979 & Apr. 13 & 65,000 & 2,7 & -- & \\
\hline 1947 & Jan. 20 & 11,000 & & 19.60 & & 1982 & Feb. 3 & 23,500 & & 25.65 & \\
\hline 1948 & Mar. 23 & 7,500 & & 16.80 & & 1983 & Apr. 8 & 15,200 & & 19.69 & \\
\hline 1951 & Mar. 29 & 15,750 & & 21.70 & & 1986 & Dec. 13 & 2,020 & & 6.84 & \\
\hline 1952 & Dec. 21 & 9,200 & & 18.42 & & 1987 & Jan. 19 & 7,820 & & 14.31 & \\
\hline 1953 & Apr. 30 & 5,750 & & 15.20 & & 1988 & Sept. 17 & 21,200 & & 24.40 & \\
\hline 1954 & Apr. 16 & 2,450 & & 8.10 & & 1989 & June 19 & 12,800 & & 18.53 & \\
\hline 1955 & Apr. 13 & 9,700 & & 18.82 & & 1990 & Feb. 16 & 26,200 & & 27.10 & \\
\hline 1959 & Jan. 22 & 4,550 & & 12.60 & & 1994 & Apr. 16 & 10,900 & & 17.03 & \\
\hline 1960 & Mar. 30 & 4,650 & & 12.80 & & 1995 & Mar. 8 & 7,230 & & 13.68 & \\
\hline 1961 & Mar. 31 & 8,800 & & 18.15 & & 1996 & Oct. 5 & 27,800 & & 27.90 & \\
\hline 1962 & Dec. 18 & 11,300 & & 19.87 & & 1997 & Feb. 28 & 12,000 & & 17.94 & \\
\hline 1963 & Mar. 13 & 7,500 & & 16.92 & & 1998 & Mar. 8 & 15,300 & & 20.32 & \\
\hline 1964 & Apr. 6 & 18,200 & & 22.52 & & 1999 & Jan. 31 & 6,760 & & 13.11 & \\
\hline 1965 & Jan. 23 & 3,860 & & 11.30 & & 2000 & Apr. 4 & 4,090 & & 9.72 & \\
\hline 1966 & Feb. 13 & 7,880 & & 17.37 & & 2001 & Mar. 20 & 9,160 & & 15.55 & \\
\hline 1967 & Oct. 18 & 4,430 & & 12.44 & & 2002 & Jan. 25 & 3,250 & & 8.57 & \\
\hline 1968 & Apr. 5 & 9,210 & & 18.46 & & 2003 & May 8 & 10,700 & & 16.85 & \\
\hline 1976 & Jan. 26 & 20,500 & & 23.46 & & 2011 & Mar. 9 & 6,650 & & 12.98 & \\
\hline 1977 & Mar. 30 & 15,600 & & 21.72 & & 2012 & Jan. 23 & 7,280 & & 13.74 & \\
\hline 1978 & Jan. 25 & 5,580 & & 14.39 & & 2013 & Apr. 12 & 6,410 & & 12.06 & \\
\hline \multirow[t]{2}{*}{1979} & Apr. 13 & 60,000 & 2 & 31.83 & & 2014 & Apr. 7 & 14,900 & & 20.07 & \\
\hline & & & & & & 2015 & Jan. 4 & 9,000 & & 15.41 & \\
\hline
\end{tabular}

02408540 Hatchet Creek below Rockford

L ocation-Lat $32^{\circ} 55^{\prime} 00^{\prime \prime}$, long $86^{\circ} 16^{\prime} 13^{\prime \prime}$, in SE $1 / 4$ sec. $4, \mathrm{~T}$. 22 N., R. 18 E., Coosa County, Hydrologic Unit 03150107, on county road, $2.1 \mathrm{mi}$ downstream from Jack Creek, and $4 \mathrm{mi}$ northwest of Rockford.

Drainage area-263 $\mathrm{mi}^{2}$

G age - Water-stage recorder. Datum of gage is $377 \mathrm{ft} \mathrm{NGVD} 29$ (from topographic map).

(1)


Appendix 2. Discharge and gage height data for streamgages in Alabama.-Continued

[--, date or data not available; $\mathrm{ft}$, foot; $\mathrm{ft}^{3} / \mathrm{s}$, cubic foot per second; mi, mile; $\mathrm{mi}^{2}$, square mile; qualification codes apply to the discharge data: 1 , discharge is a maximum daily average; 2 , discharge is an estimate; 4 , discharge is less than indicated value, which is the minimum recordable discharge at this site; 5 , discharge affected to unknown degree by regulation or diversion; 6 , discharge affected by regulation or diversion; 7, discharge is a historic peak; A, year of occurrence is unknown or not exact; $\mathrm{B}$, month or day of occurrence is unknown or not exact; E, only maximum peak available for this year. qualification codes apply to the gage height data: 1, gage height affected by backwater; 2, gage height not the maximum for the year; 3 , gage height at different datum or at different site and datum; 5 , gage height is an estimate]

\section{Weogufka Creek near Weogufka}

L ocation-Lat $32^{\circ} 59^{\prime} 01^{\prime \prime}$, long $86^{\circ} 18^{\prime} 26^{\prime \prime}$, in NE $1 / 4$ sec. $18, \mathrm{~T}$. 23 N., R. 18 E., Coosa County, Hydrologic Unit 03150107, on county road, 2 mi south of Weogufka and 6 mi upstream from Phinikochika Creek.

Drainage area $-73.4 \mathrm{mi}^{2}$.

G age - Water-stage recorder 1951-58. Crest-stage gage 1959-70. Datum of gage is $593.08 \mathrm{ft}$ NGVD 29.

\begin{tabular}{|c|c|c|c|c|c|}
\hline $\begin{array}{l}\text { Water } \\
\text { year }\end{array}$ & Date & Discharge & $\begin{array}{l}\text { Discharge } \\
\text { code }\end{array}$ & $\begin{array}{c}\text { Gage } \\
\text { height } \\
\text { (feet) }\end{array}$ & $\begin{array}{c}\text { Gage } \\
\text { height } \\
\text { code }\end{array}$ \\
\hline 1951 & Mar. 29 & 24,200 & & 16.80 & \\
\hline 1952 & Dec. 21 & 3,220 & & 11.00 & \\
\hline 1953 & Apr. 30 & 2,700 & & 10.50 & \\
\hline 1954 & Mar. 27 & 838 & & 7.20 & \\
\hline 1955 & Apr. 14 & 5,100 & & 12.30 & \\
\hline 1956 & Mar. 16 & 4,450 & & 11.93 & \\
\hline 1957 & Apr. 5 & 6,600 & & 13.00 & \\
\hline 1958 & Feb. 7 & 1,800 & & 9.45 & \\
\hline 1959 & Jan. 21 & 1,680 & & 9.20 & \\
\hline 1960 & Mar. 30 & 1,370 & & 8.60 & \\
\hline 1961 & Feb. 25 & 3,130 & & 10.92 & \\
\hline 1962 & Dec. 18 & 5,420 & & 12.46 & \\
\hline 1963 & Apr. 30 & 2,310 & & 10.08 & \\
\hline 1964 & Apr. 6 & 8,260 & & 13.62 & \\
\hline 1965 & Jan. 23 & 1,600 & & 9.06 & \\
\hline 1966 & Feb. 13 & 3,250 & & 11.03 & \\
\hline 1967 & Aug. 24 & 4,420 & & 11.88 & \\
\hline 1968 & Apr. 5 & 13,700 & & 15.08 & \\
\hline 1969 & Jan. 20 & 1,480 & & 8.83 & \\
\hline 1970 & Mar. 19 & 1,100 & & 7.95 & \\
\hline 1979 & Apr. 13 & 15,200 & 7 & 15.41 & \\
\hline
\end{tabular}

02410000 Paterson Creek near Central

L ocation-Lat $32^{\circ} 40^{\prime} 54^{\prime \prime}$, long $86^{\circ} 07^{\prime} 40^{\prime \prime}$, in SE $1 / 4$ sec. 26 , T. 20 N., R. 19 E., Elmore County, Hydrologic Unit 03150107, on county road, 2 mi west of Central, and 11 mi northeast of Wetumpka.

Drainage area-4.91 $\mathrm{mi}^{2}$.

G age - Water-stage recorder. Datum of gage is $440 \mathrm{ft} \mathrm{NGVD} 29$ (by barometer).

\begin{tabular}{|c|c|c|c|c|c|}
\hline $\begin{array}{l}\text { Water } \\
\text { year }\end{array}$ & Date & Discharge & $\begin{array}{c}\text { Discharge } \\
\text { code }\end{array}$ & $\begin{array}{c}\text { Gage } \\
\text { height } \\
\text { (feet) }\end{array}$ & $\begin{array}{c}\text { Gage } \\
\text { height } \\
\text { code }\end{array}$ \\
\hline 1952 & Mar. 3 & 604 & & 5.40 & \\
\hline 1953 & Apr. 29 & 716 & & 6.20 & \\
\hline 1954 & June 2 & 258 & & 3.28 & \\
\hline 1955 & Feb. 6 & 410 & & 4.20 & \\
\hline 1956 & Apr. 5 & 640 & & 5.50 & \\
\hline 1957 & Apr. 4 & 828 & & 7.00 & \\
\hline 1958 & Feb. 6 & 856 & & 7.20 & \\
\hline 1959 & June 6 & 204 & & 2.85 & \\
\hline 1960 & Mar. 29 & 856 & & 7.16 & \\
\hline 1961 & Feb. 25 & 1,770 & & 9.10 & \\
\hline 1962 & Dec. 10 & 1,500 & & 8.84 & \\
\hline 1963 & June 23 & 393 & & 3.95 & \\
\hline 1964 & Apr. 6 & 1,010 & & 7.82 & \\
\hline 1965 & Jan. 23 & 863 & & 7.25 & \\
\hline 1966 & Mar. 3 & 729 & & 6.29 & \\
\hline 1967 & Oct. 9 & 618 & & 5.50 & \\
\hline 1968 & Apr. 5 & 477 & & 4.50 & \\
\hline 1969 & Aug. 2 & 4,310 & & 10.10 & \\
\hline 1970 & Mar. 19 & 396 & & 3.97 & \\
\hline 1971 & Mar. 3 & 715 & & 5.39 & \\
\hline 1972 & Jan. 11 & 472 & & 4.47 & \\
\hline 1973 & May 28 & 446 & & 4.29 & \\
\hline 1974 & Jan. 20 & 228 & & 2.92 & \\
\hline 1975 & Apr. 2 & 1,200 & & 8.40 & \\
\hline 1976 & Mar. 13 & 604 & & 5.56 & \\
\hline 1977 & Mar. 12 & 228 & & 2.76 & \\
\hline 1978 & Jan. 25 & 645 & & 5.67 & \\
\hline 1979 & Apr. 13 & 1,110 & & 8.12 & \\
\hline 1980 & Mar. 8 & 449 & & 4.32 & \\
\hline 1981 & Apr. 1 & 918 & & 7.47 & \\
\hline 1982 & Feb. 2 & 660 & & 5.80 & \\
\hline 1983 & Apr. 4 & 1,250 & & 7.73 & \\
\hline 1984 & Aug. 2 & 1,140 & & 7.19 & \\
\hline 1985 & Feb. 5 & 246 & $\mathrm{E}$ & 2.83 & \\
\hline 1986 & Mar. 13 & 633 & & 4.73 & \\
\hline 1987 & Feb. 15 & 548 & & 4.27 & \\
\hline 1988 & Sept. 16 & 662 & & 4.88 & \\
\hline
\end{tabular}


Appendix 2. Discharge and gage height data for streamgages in Alabama.-Continued

[--, date or data not available; $\mathrm{ft}$, foot; $\mathrm{ft}^{3} / \mathrm{s}$, cubic foot per second; mi, mile; $\mathrm{mi}^{2}$, square mile; qualification codes apply to the discharge data: 1 , discharge is a maximum daily average; 2 , discharge is an estimate; 4 , discharge is less than indicated value, which is the minimum recordable discharge at this site; 5 , discharge affected to unknown degree by regulation or diversion; 6 , discharge affected by regulation or diversion; 7, discharge is a historic peak; A, year of occurrence is unknown or not exact; $\mathrm{B}$, month or day of occurrence is unknown or not exact; E, only maximum peak available for this year. qualification codes apply to the gage height data: 1, gage height affected by backwater; 2 , gage height not the maximum for the year; 3 , gage height at different data or at different site and datum;

5 , gage height is an estimate]

\section{Tallapoosa River near Heflin}

L ocation-Lat $33^{\circ} 37^{\prime} 22^{\prime \prime}$, long $85^{\circ} 30^{\prime} 48^{\prime \prime}$, in NW 1/4 sec. 20 , T. 16 S., R. 11 E., Cleburne County, Hydrologic Unit 03150108, 2.2 mi upstream from Cane Creek, 4 mi southeast of Heflin, and at mile 186.8.

Drainage area $-448 \mathrm{mi}^{2}$.

Gage -Water-stage recorder. Datum of gage is $830 \mathrm{ft}$ NGVD 29 (by barometer).

\begin{tabular}{|c|c|c|c|c|c|}
\hline $\begin{array}{l}\text { Water } \\
\text { year }\end{array}$ & Date & Discharge & $\begin{array}{l}\text { Discharge } \\
\text { code }\end{array}$ & $\begin{array}{c}\text { Gage } \\
\text { height } \\
\text { (feet) }\end{array}$ & $\begin{array}{c}\text { Gage } \\
\text { height } \\
\text { code }\end{array}$ \\
\hline 1953 & Jan. 10 & 8,110 & & 20.40 & \\
\hline 1954 & Jan. 18 & 7,020 & & 19.00 & \\
\hline 1955 & Apr. 14 & 4,770 & & 14.67 & \\
\hline 1956 & Mar. 17 & 6,840 & & 18.70 & \\
\hline 1957 & Apr. 5 & 9,140 & & 21.40 & \\
\hline 1958 & Feb. 7 & 4,820 & & 14.80 & \\
\hline 1959 & June 1 & 6,840 & & 18.70 & \\
\hline 1960 & Feb. 1 & 4,950 & & 15.07 & \\
\hline 1961 & Feb. 22 & 19,300 & & 26.39 & \\
\hline 1962 & Feb. 24 & 9,100 & & 21.34 & \\
\hline 1963 & Apr. 30 & 11,400 & & 22.95 & \\
\hline 1964 & Mar. 16 & 8,690 & & 20.29 & \\
\hline 1965 & Apr. 6 & 4,320 & & 13.36 & \\
\hline 1966 & Feb. 17 & 6,710 & & 17.43 & \\
\hline 1967 & Aug. 26 & 10,000 & & 22.02 & \\
\hline 1968 & May 16 & 17,100 & & 25.65 & \\
\hline 1969 & Apr. 16 & 3,940 & & 12.85 & \\
\hline 1970 & Mar. 21 & 13,700 & & 24.21 & \\
\hline 1971 & Mar. 3 & 6,400 & & 17.96 & \\
\hline 1972 & Jan. 12 & 9,470 & & 21.08 & \\
\hline 1973 & Mar. 18 & 8,160 & & 19.65 & \\
\hline 1974 & Jan. 2 & 9,600 & & 21.20 & \\
\hline 1975 & Sept. 25 & 7,780 & & 19.12 & \\
\hline 1976 & Mar. 16 & 14,200 & & 24.33 & \\
\hline 1977 & Mar. 31 & 32,500 & & 31.34 & \\
\hline 1978 & Nov. 8 & 7,670 & & 18.96 & \\
\hline 1979 & Mar. 5 & 23,100 & & 28.05 & \\
\hline 1980 & Apr. 15 & 8,300 & & 19.82 & \\
\hline 1981 & Feb. 12 & 6,080 & & 16.64 & \\
\hline 1982 & Feb. 4 & 19,100 & & 26.34 & \\
\hline 1983 & Apr. 10 & 8,130 & & 19.59 & \\
\hline 1984 & Dec. 7 & 10,500 & & 21.96 & \\
\hline 1985 & Feb. 1 & 4,810 & & 13.51 & \\
\hline
\end{tabular}

02412000 Tallapoosa River near Heflin-Continued

L ocation-Lat $33^{\circ} 37^{\prime} 22^{\prime \prime}$, long $85^{\circ} 30^{\prime} 48^{\prime \prime}$, in NW 1/4 sec. 20, T. 16 S., R. 11 E., Cleburne County, Hydrologic Unit 03150108, $2.2 \mathrm{mi}$ upstream from Cane Creek, $4 \mathrm{mi}$ southeast of Heflin, and at mile 186.8.

Drainage area $448 \mathrm{mi}^{2}$.

Gage -Water-stage recorder. Datum of gage is $830 \mathrm{ft}$ NGVD 29 (by barometer).

\begin{tabular}{|c|c|c|c|c|c|}
\hline $\begin{array}{c}\text { Water } \\
\text { year }\end{array}$ & Date & Discharge & $\begin{array}{l}\text { Discharge } \\
\text { code }\end{array}$ & $\begin{array}{c}\text { Gage } \\
\text { height } \\
\text { (feet) }\end{array}$ & $\begin{array}{c}\text { Gage } \\
\text { height } \\
\text { code }\end{array}$ \\
\hline 1986 & Dec. 13 & 1,910 & & 7.71 & \\
\hline 1987 & Mar. 1 & 6,770 & & 17.20 & \\
\hline 1988 & Jan. 21 & 5,520 & & 14.91 & \\
\hline 1989 & June 23 & 6,680 & & 17.03 & \\
\hline 1990 & Mar. 18 & 24,000 & & 28.33 & \\
\hline 1991 & Feb. 21 & 6,750 & & 17.16 & \\
\hline 1992 & Feb. 27 & 6,670 & & 17.01 & \\
\hline 1993 & Jan. 13 & 6,630 & & 16.95 & \\
\hline 1994 & July 28 & 5,880 & & 15.60 & \\
\hline 1995 & Feb. 18 & 7,930 & & 19.20 & \\
\hline 1996 & Oct. 6 & 12,600 & & 23.37 & \\
\hline 1997 & Mar. 1 & 8,920 & & 20.51 & \\
\hline 1998 & Mar. 9 & 9,800 & & 21.38 & \\
\hline 1999 & July 1 & 4,400 & & 12.55 & \\
\hline 2000 & Apr. 4 & 5,740 & & 15.33 & \\
\hline 2001 & Mar. 21 & 7,220 & & 18.03 & \\
\hline 2002 & Mar. 31 & 5,360 & & 14.58 & \\
\hline 2003 & May 8 & 12,900 & & 23.55 & \\
\hline 2004 & Sept. 18 & 5,660 & & 15.18 & \\
\hline 2005 & July 12 & 6,200 & & 16.18 & \\
\hline 2006 & Feb. 7 & 6,070 & & 15.95 & \\
\hline 2007 & Nov. 16 & 4,990 & & 13.80 & \\
\hline 2008 & Feb. 23 & 3,340 & & 10.20 & \\
\hline 2009 & Mar. 1 & 5,790 & & 15.42 & \\
\hline 2010 & Mar. 12 & 8,150 & & 19.54 & \\
\hline 2011 & Mar. 11 & 7,340 & & 18.24 & \\
\hline 2012 & Jan. 24 & 4,190 & & 12.09 & \\
\hline 2013 & Jan. 31 & 5,670 & & 15.20 & \\
\hline 2014 & Dec. 24 & 8,440 & & 19.97 & \\
\hline 2015 & Jan. 5 & 8,330 & & 19.13 & \\
\hline
\end{tabular}


Appendix 2. Discharge and gage height data for streamgages in Alabama.-Continued

[--, date or data not available; $\mathrm{ft}$, foot; $\mathrm{ft}^{3} / \mathrm{s}$, cubic foot per second; mi, mile; $\mathrm{mi}^{2}$, square mile; qualification codes apply to the discharge data: 1 , discharge is a maximum daily average; 2 , discharge is an estimate; 4 , discharge is less than indicated value, which is the minimum recordable discharge at this site; 5 , discharge affected to unknown degree by regulation or diversion; 6 , discharge affected by regulation or diversion; 7, discharge is a historic peak; A, year of occurrence is unknown or not exact; $\mathrm{B}$, month or day of occurrence is unknown or not exact; E, only maximum peak available for this year. qualification codes apply to the gage height data: 1, gage height affected by backwater; 2, gage height not the maximum for the year; 3 , gage height at different datum or at different site and datum; 5 , gage height is an estimate]

\section{Tallapoosa River near Ofelia}

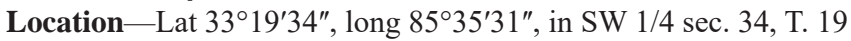
S., R. 10 E., Randolph County, Hydrologic Unit 03150108, 1 mi northeast of Ofelia, 1.5 mi upstream from Little Tallapoosa River, and 9 mi east of Lineville.

Drainage area $-792 \mathrm{mi}^{2}$.

Gage-Water-stage recorder 1939-51. Crest-stage gage 1952-70. Datum of gage is $665 \mathrm{ft} \mathrm{NGVD} 29$ (from topographic map).

Remarks - Since 1982, site affected by Harris Dam.

\begin{tabular}{|c|c|c|c|c|c|}
\hline $\begin{array}{c}\text { Water } \\
\text { year }\end{array}$ & Date & Discharge & $\begin{array}{c}\text { Discharge } \\
\text { code }\end{array}$ & $\begin{array}{c}\text { Gage } \\
\text { height } \\
\text { (feet) }\end{array}$ & $\begin{array}{c}\text { Gage } \\
\text { height } \\
\text { code }\end{array}$ \\
\hline 1920 & Dec & 41,000 & 2,7,B & 21.00 & \\
\hline 1939 & Mar. 2 & 8,690 & & 8.80 & \\
\hline 1940 & Mar. 14 & 8,460 & & 8.71 & \\
\hline 1941 & July 12 & 3,990 & & 4.94 & \\
\hline 1942 & Mar. 21 & 20,500 & & 14.62 & \\
\hline 1943 & Mar. 21 & 16,900 & & 12.92 & \\
\hline 1944 & Apr. 11 & 14,900 & & 11.87 & \\
\hline 1945 & Apr. 25 & 8,530 & & 8.40 & \\
\hline 1946 & Jan. 6 & 21,200 & & 14.92 & \\
\hline 1947 & Jan. 20 & 22,100 & & 15.34 & \\
\hline 1948 & Mar. 23 & 14,500 & & 11.90 & \\
\hline 1949 & Nov. 29 & 24,500 & & 16.20 & \\
\hline 1950 & Feb. 10 & 5,530 & & 6.40 & \\
\hline 1951 & Mar. 29 & 16,600 & & 13.00 & \\
\hline 1952 & Dec. 21 & 19,600 & & 14.30 & \\
\hline 1953 & Jan. 9 & 13,700 & & 11.50 & \\
\hline 1954 & Jan. 18 & 7,470 & & 7.80 & \\
\hline 1955 & Apr. 15 & 8,590 & & 8.50 & \\
\hline 1956 & Mar. 18 & 21,900 & & 15.20 & \\
\hline 1957 & Apr. 5 & 21,400 & & 15.00 & \\
\hline 1958 & Apr. 16 & 9,550 & & 9.10 & \\
\hline 1959 & Jan. 22 & 3,880 & & 4.90 & \\
\hline 1960 & Jan. 31 & 7,020 & & 7.50 & \\
\hline 1961 & Feb. 23 & 22,400 & & 15.42 & \\
\hline 1962 & Dec. 15 & 16,300 & & 12.84 & \\
\hline 1963 & Apr. 30 & 38,000 & & 20.40 & \\
\hline 1964 & Apr. 17 & 15,900 & & 12.64 & \\
\hline 1965 & Dec. 27 & 6,420 & & 7.08 & \\
\hline 1966 & Feb. 16 & 12,000 & & 10.58 & \\
\hline 1967 & Aug. 26 & 17,900 & & 13.60 & \\
\hline 1968 & May 16 & 26,000 & & 16.70 & \\
\hline 1969 & May 19 & 5,980 & & 6.75 & \\
\hline 1970 & Mar. 4 & 23,700 & & 15.90 & \\
\hline
\end{tabular}

02413300 Little Tallapoosa River near Newell

L ocation-Lat $33^{\circ} 26^{\prime} 14^{\prime \prime}$, long $85^{\circ} 23^{\prime} 57^{\prime \prime}$, in SW $1 / 4 \mathrm{sec}$. 21, T. 18 S., R. 12 E., Randolph County, Hydrologic Unit 03150108 , on county highway $82,1.0$ mi upstream from Cut Nose Creek, and 2.0 mi east of Newell.

Drainage area $-406 \mathrm{mi}^{2}$.

G age - Water-stage recorder. Datum of gage is $842.92 \mathrm{ft}$ NGVD 29.

\begin{tabular}{|c|c|c|c|c|c|}
\hline $\begin{array}{l}\text { Water } \\
\text { year }\end{array}$ & Date & Discharge & $\begin{array}{l}\text { Discharge } \\
\text { code }\end{array}$ & $\begin{array}{c}\text { Gage } \\
\text { height } \\
\text { (feet) }\end{array}$ & $\begin{array}{c}\text { Gage } \\
\text { height } \\
\text { code }\end{array}$ \\
\hline 1976 & Mar. 16 & 14,100 & & 18.18 & \\
\hline 1977 & Mar. 30 & 8,510 & & 15.82 & \\
\hline 1978 & Jan. 25 & 6,610 & & 13.89 & \\
\hline 1979 & Apr. 13 & 12,700 & & 17.80 & \\
\hline 1980 & Mar. 13 & 5,730 & & 12.76 & \\
\hline 1981 & May 26 & 5,580 & & 12.54 & \\
\hline 1982 & Feb. 3 & 11,600 & & 17.34 & \\
\hline 1983 & Apr. 8 & 9,100 & & 15.81 & \\
\hline 1984 & Dec. 6 & 6,610 & & 13.18 & \\
\hline 1985 & Feb. 5 & 4,150 & & 9.97 & \\
\hline
\end{tabular}

1986 Dec. $14 \quad 1,840 \quad 2 \quad 6.50$

$1987 \quad$ Jan. $19 \quad 6,610 \quad 13.06$

$1988 \quad$ Jan. $20 \quad 3,650 \quad 9.30$

$1989 \quad$ June $21 \quad 5,940 \quad 12.18$

1990 Mar. $17 \quad 13,000 \quad 17.85$

$1991 \quad$ June $26 \quad 5,300 \quad 11.23$

$1992 \quad$ Nov. $22 \quad 6,140 \quad 12.33$

1993 Dec. $17 \quad 7,290 \quad 13.75$

1994 Apr. $16 \quad 5,010 \quad 10.84$

1995 Feb. $11 \quad 11,200 \quad 17.11$

1996 Oct. $5 \quad 13,500 \quad 18.03$

$1997 \quad$ Feb. $28 \quad 9,220 \quad 15.60$

1998 Mar. $8 \quad 11,200 \quad 17.08$

$1999 \quad$ June $28 \quad 4,320 \quad 9.88$

2000 Apr. $4 \quad 3,690 \quad 8.94$

2001 Mar. $20 \quad 6,570 \quad 12.86$

2002 Sept. $22 \quad 4,740 \quad 10.45$

2003 May $8 \quad 13,200 \quad 17.91$

2004 Sept. $16 \quad 6,530 \quad 12.80$

2005 July $12 \quad 6,150 \quad 12.32$ 
Appendix 2. Discharge and gage height data for streamgages in Alabama.-Continued

[--, date or data not available; ft, foot; $\mathrm{ft}^{3} / \mathrm{s}$, cubic foot per second; mi, mile; $\mathrm{mi}^{2}$, square mile; qualification codes apply to the discharge data: 1 , discharge is a maximum daily average; 2 , discharge is an estimate; 4 , discharge is less than indicated value, which is the minimum recordable discharge at this site; 5 , discharge affected to unknown degree by regulation or diversion; 6 , discharge affected by regulation or diversion; 7, discharge is a historic peak; A, year of occurrence is unknown or not exact; $\mathrm{B}$, month or day of occurrence is unknown or not exact; E, only maximum peak available for this year. qualification codes apply to the gage height data: 1 , gage height affected by backwater; 2, gage height not the maximum for the year; 3, gage height at different data or at different site and datum;

5 , gage height is an estimate]

$\mathbf{0 2 4 1 3 3 0 0}$ Little Tallapoosa River near Newell-Continued L ocation-Lat $33^{\circ} 26^{\prime} 14^{\prime \prime}$, long 85 $23^{\prime} 57^{\prime \prime}$, in SW 1/4 sec. 21, T. 18 S., R. 12 E., Randolph County, Hydrologic Unit 03150108, on county highway 82, $1.0 \mathrm{mi}$ upstream from Cut

Nose Creek, and 2.0 mi east of Newell.

Drainage area-406 $\mathrm{mi}^{2}$.

Gage -Water-stage recorder. Datum of gage is $842.92 \mathrm{ft}$ NGVD 29.

\begin{tabular}{|c|c|c|c|c|c|}
\hline $\begin{array}{c}\text { Water } \\
\text { year }\end{array}$ & Date & Discharge & $\begin{array}{c}\text { Discharge } \\
\text { code }\end{array}$ & $\begin{array}{c}\text { Gage } \\
\text { height } \\
\text { (feet) }\end{array}$ & $\begin{array}{c}\text { Gage } \\
\text { height } \\
\text { code }\end{array}$ \\
\hline 2006 & Feb. 7 & 3,520 & & 8.68 & \\
\hline 2007 & Nov. 15 & 3,470 & & 8.61 & \\
\hline 2008 & Nov. 15 & 3,470 & & 8.61 & \\
\hline 2009 & Sept. 23 & 6,520 & & 12.79 & \\
\hline 2010 & Mar. 11 & 6,880 & & 13.24 & \\
\hline 2011 & Mar. 9 & 4,430 & & 10.03 & \\
\hline 2012 & Mar. 3 & 5,390 & & 11.36 & \\
\hline 2013 & Feb. 26 & 4,070 & & 9.52 & \\
\hline 2014 & Apr. 7 & 11,600 & & 17.53 & \\
\hline 2015 & Dec. 28 & 5,900 & & 12.02 & \\
\hline
\end{tabular}

\section{Wedowee Creek above Wedowee}

L ocation-Lat $33^{\circ} 19^{\prime} 20^{\prime \prime}$, long $85^{\circ} 20^{\prime} 35^{\prime \prime}$, in SE $1 / 4$ sec. 36, T. 19 S., R. 12 E., Randolph County, Hydrologic Unit 03150108, on County Highway 56, 8 mi east of Wedowee.

Drainage area $-6.87 \mathrm{mi}^{2}$.

Gage -Water-stage recorder 1960-66. Crest-stage gage 1967-72. Datum of gage is 1,050 ft NGVD 29 (from topographic map).

\begin{tabular}{llrccc}
\hline $\begin{array}{c}\text { Water } \\
\text { year }\end{array}$ & Date & Discharge & $\begin{array}{c}\text { Discharge } \\
\text { code }\end{array}$ & $\begin{array}{c}\text { Gage } \\
\text { height } \\
\text { (feet) }\end{array}$ & $\begin{array}{c}\text { Gage } \\
\text { height } \\
\text { code }\end{array}$ \\
\hline 1960 & Apr. 3 & 870 & & 5.58 & \\
1961 & Mar. 31 & 1,080 & & 6.30 & \\
1962 & Dec. 18 & 1,220 & 6.71 & \\
1963 & Mar. 5 & 858 & 5.53 & \\
1964 & Jan. 25 & 1,210 & 6.67 & \\
1965 & Dec. 25 & 638 & & 4.65 & \\
1966 & Oct. 1 & 1,490 & & 7.38 & \\
1967 & July 8 & 570 & & 4.38 & \\
1968 & May 15 & 888 & & 5.65 & \\
1969 & May 18 & 815 & & 5.36 & \\
1970 & June 4 & 512 & & 4.15 & \\
1971 & Mar. 3 & 898 & & 5.69 & \\
1972 & Jan. 10 & 845 & & 5.48 & \\
1979 & Apr. 13 & 1,900 & 7 & 8.22 &
\end{tabular}

02413475 Wedowee Creek near Wedowee

L ocation-Lat $33^{\circ} 19^{\prime} 30^{\prime \prime}$, long $85^{\circ} 29^{\prime} 02^{\prime \prime}$, in SE $1 / 4$ sec. 34 , T. 19

S., R. 11 E., Randolph County, Hydrologic Unit

03150108, at bridge on U.S. Highway 431, $1.5 \mathrm{mi}$ north of

Wedowee.

Drainage area $46.6 \mathrm{mi}^{2}$.

Gage - Crest-stage gage. Datum not available.

\begin{tabular}{|c|c|c|c|c|c|}
\hline $\begin{array}{l}\text { Water } \\
\text { year }\end{array}$ & Date & Discharge & $\begin{array}{l}\text { Discharge } \\
\text { code }\end{array}$ & $\begin{array}{c}\text { Gage } \\
\text { height } \\
\text { (feet) }\end{array}$ & $\begin{array}{c}\text { Gage } \\
\text { height } \\
\text { code }\end{array}$ \\
\hline 1951 & Mar. 29 & 2,100 & & 8.08 & \\
\hline 1952 & Mar. 3 & 3,850 & & 12.02 & \\
\hline 1953 & Apr. 30 & 3,600 & & 11.60 & \\
\hline 1954 & Mar. 28 & 1,220 & & 5.63 & \\
\hline 1955 & Feb. 7 & 2,330 & & 8.80 & \\
\hline 1956 & Mar. 16 & 4,100 & & 12.70 & \\
\hline 1957 & Apr. 4 & 3,920 & & 12.60 & \\
\hline 1958 & Feb. 6 & 3,180 & & 11.00 & \\
\hline 1959 & Mar. 10 & 1,260 & & 5.80 & \\
\hline 1960 & July 28 & 2,550 & & 9.40 & \\
\hline 1961 & Feb. 25 & 4,300 & & 13.01 & \\
\hline 1962 & Apr. 12 & 2,780 & & 9.94 & \\
\hline 1963 & Mar. 6 & 1,975 & & 7.93 & \\
\hline 1964 & Jan. 25 & 3,950 & & 12.37 & \\
\hline 1965 & Dec. 25 & 2,675 & & 9.60 & \\
\hline 1966 & Feb. 13 & 2,850 & & 10.05 & \\
\hline 1967 & Nov. 10 & 940 & & 4.67 & \\
\hline 1968 & Apr. 5 & 2,950 & & 10.38 & \\
\hline 1969 & Apr. 18 & 2,950 & & 10.38 & \\
\hline 1970 & June 4 & 1,730 & & 7.23 & \\
\hline 1971 & Mar. 2 & 3,950 & & 12.36 & \\
\hline 1972 & Jan. 11 & 3,950 & & 12.37 & \\
\hline 1973 & May 21 & 3,540 & & 11.87 & \\
\hline 1974 & Jan. 2 & 3,360 & & 11.40 & \\
\hline 1975 & Sept. 23 & 3,200 & & 10.86 & \\
\hline 1979 & Apr. 13 & 4,870 & 7 & 14.10 & \\
\hline
\end{tabular}


Appendix 2. Discharge and gage height data for streamgages in Alabama.-Continued

[--, date or data not available; $\mathrm{ft}$, foot; $\mathrm{ft}^{3} / \mathrm{s}$, cubic foot per second; mi, mile; $\mathrm{mi}^{2}$, square mile; qualification codes apply to the discharge data: 1 , discharge is a maximum daily average; 2 , discharge is an estimate; 4 , discharge is less than indicated value, which is the minimum recordable discharge at this site; 5 , discharge affected to unknown degree by regulation or diversion; 6 , discharge affected by regulation or diversion; 7, discharge is a historic peak; A, year of occurrence is unknown or not exact; $\mathrm{B}$, month or day of occurrence is unknown or not exact; E, only maximum peak available for this year. qualification codes apply to the gage height data: 1, gage height affected by backwater; 2, gage height not the maximum for the year; 3 , gage height at different datum or at different site and datum; 5 , gage height is an estimate]

\section{Little Tallapoosa River near Wedowee}

L ocation-Lat $33^{\circ} 20^{\prime} 57^{\prime \prime}$, long $85^{\circ} 32^{\prime} 43^{\prime \prime}$, in SE 1/4 sec. 24, T. 19 S., R. 10 E., Randolph County, Hydrologic Unit 03150108, 4.5 mi northwest of Wedowee and 5.5 mi upstream from mouth.

Drainage area-591 $\mathrm{mi}^{2}$.

Gage -Water-stage recorder 1940-52. Crest-stage gage 1953-70. Datum of gage is $680 \mathrm{ft} \mathrm{NGVD} 29$ (from topographic map).

Remarks - Since 1982, site affected by Harris Dam.

\begin{tabular}{|c|c|c|c|c|c|}
\hline $\begin{array}{l}\text { Water } \\
\text { year }\end{array}$ & Date & Discharge & $\begin{array}{l}\text { Discharge } \\
\text { code }\end{array}$ & $\begin{array}{c}\text { Gage } \\
\text { height } \\
\text { (feet) }\end{array}$ & $\begin{array}{c}\text { Gage } \\
\text { height } \\
\text { code }\end{array}$ \\
\hline 1919 & Dec. & 27,000 & $7, \mathrm{~B}$ & 23.00 & 5 \\
\hline 1938 & Apr. 8 & 18,900 & & 20.00 & \\
\hline 1940 & July 9 & 9,600 & & 14.92 & \\
\hline 1941 & July 12 & 4,600 & & 10.10 & \\
\hline 1942 & Mar. 21 & 19,800 & & 20.40 & \\
\hline 1943 & Mar. 21 & 14,000 & & 17.47 & \\
\hline 1944 & Apr. 11 & 14,200 & & 16.65 & \\
\hline 1945 & Apr. 25 & 12,000 & & 16.33 & \\
\hline 1946 & Jan. 6 & 16,300 & & 18.67 & \\
\hline 1947 & Jan. 20 & 15,400 & & 18.37 & \\
\hline 1948 & Mar. 23 & 16,400 & & 18.90 & \\
\hline 1949 & Nov. 28 & 20,800 & & 20.80 & \\
\hline 1950 & Mar. 13 & 4,810 & & 10.70 & \\
\hline 1951 & Mar. 29 & 6,920 & & 12.80 & \\
\hline 1952 & Dec. 21 & 16,000 & & 18.70 & \\
\hline 1953 & Apr. 30 & 9,790 & & 15.10 & \\
\hline 1954 & Jan. 18 & 3,300 & & 8.90 & \\
\hline 1955 & Apr. 15 & 7,480 & & 13.30 & \\
\hline 1956 & Mar. 18 & 20,600 & & 20.70 & \\
\hline 1957 & Apr. 5 & 19,600 & & 20.30 & \\
\hline 1958 & Nov. 23 & 12,500 & & 16.90 & \\
\hline 1959 & June 2 & 6,480 & & 12.40 & \\
\hline 1960 & Jan. 31 & 7,140 & & 13.00 & \\
\hline 1961 & Feb. 25 & 25,500 & & 22.58 & \\
\hline 1962 & Dec. 19 & 16,400 & & 18.90 & \\
\hline 1963 & Apr. 30 & 24,600 & & 22.34 & \\
\hline 1964 & Apr. 8 & 14,300 & & 17.26 & \\
\hline 1965 & Dec. 27 & 9,100 & & 14.60 & \\
\hline 1966 & Feb. 14 & 10,100 & & 15.29 & \\
\hline 1967 & Nov. 10 & 8,200 & & 13.90 & \\
\hline 1968 & May 16 & 16,700 & & 19.02 & \\
\hline 1969 & May 19 & 9,230 & & 14.70 & \\
\hline 1970 & Mar. 21 & 16,300 & & 18.83 & \\
\hline 1979 & Apr. 13 & 26,000 & 7 & 22.72 & \\
\hline
\end{tabular}

02414800 Harbuck Creek near Hackneyville

L ocation-Lat $33^{\circ} 07^{\prime} 08^{\prime \prime}$, long $85^{\circ} 56^{\prime} 45^{\prime \prime}$, in SW 1/4 sec. 8, T. 22 S., R. 7 E., Clay County, Hydrologic Unit 03150109, on county road, 0.5 mi upstream from mouth, 1 mi north of county line, and 4 mi north of Hackneyville.

Drainage area-7.97 $\mathrm{mi}^{2}$.

G age -Water-stage recorder. Datum of gage is $710 \mathrm{ft}$ NGVD 29 (from topographic map).

\begin{tabular}{|c|c|c|c|c|c|}
\hline $\begin{array}{c}\text { Water } \\
\text { year }\end{array}$ & Date & Discharge & $\begin{array}{l}\text { Discharge } \\
\text { code }\end{array}$ & $\begin{array}{c}\text { Gage } \\
\text { height } \\
\text { (feet) }\end{array}$ & $\begin{array}{c}\text { Gage } \\
\text { height } \\
\text { code }\end{array}$ \\
\hline 1951 & Mar. 29 & 2,460 & & 7.80 & \\
\hline 1952 & Dec. 29 & 1,160 & & 4.90 & \\
\hline 1953 & Apr. 12 & 1,460 & & 5.70 & \\
\hline 1954 & Feb. 24 & 560 & & 3.30 & \\
\hline 1955 & May 22 & 3,320 & & 8.90 & \\
\hline 1956 & Mar. 16 & 1,620 & & 6.10 & \\
\hline 1957 & Apr. 5 & 2,340 & & 7.60 & \\
\hline 1958 & Mar. 11 & 2,680 & & 8.10 & \\
\hline 1959 & Oct. 1 & 1,060 & & 4.60 & \\
\hline 1960 & Mar. 29 & 515 & & 3.15 & \\
\hline 1961 & Mar. 31 & 1,350 & & 5.41 & \\
\hline 1962 & Dec. 18 & 1,720 & & 5.34 & \\
\hline 1963 & June 23 & 905 & & 4.18 & \\
\hline 1964 & Apr. 6 & 2,560 & & 7.95 & \\
\hline 1965 & Sept. 30 & 1,020 & & 4.51 & \\
\hline 1966 & Apr. 27 & 820 & & 3.95 & \\
\hline 1967 & Oct. 18 & 652 & & 3.53 & \\
\hline 1968 & Apr. 5 & 720 & & 3.70 & \\
\hline 1969 & Sept. 24 & 466 & & 2.98 & \\
\hline 1970 & Mar. 19 & 966 & & 4.35 & \\
\hline 1979 & Apr. 14 & 6,260 & 7 & 11.87 & \\
\hline
\end{tabular}


Appendix 2. Discharge and gage height data for streamgages in Alabama._- Continued

[--, date or data not available; ft, foot; $\mathrm{ft}^{3} / \mathrm{s}$, cubic foot per second; mi, mile; $\mathrm{mi}^{2}$, square mile; qualification codes apply to the discharge data: 1 , discharge is a maximum daily average; 2 , discharge is an estimate; 4 , discharge is less than indicated value, which is the minimum recordable discharge at this site; 5 , discharge affected to unknown degree by regulation or diversion; 6 , discharge affected by regulation or diversion; 7, discharge is a historic peak; A, year of occurrence is unknown or not exact; $\mathrm{B}$, month or day of occurrence is unknown or not exact; E, only maximum peak available for this year. qualification codes apply to the gage height data: 1 , gage height affected by backwater; 2, gage height not the maximum for the year; 3, gage height at different data or at different site and datum; 5 , gage height is an estimate]

\section{Hillabee Creek near Hackneyville}

L ocation-Lat $33^{\circ} 04^{\prime} 00^{\prime \prime}$, long 85 $52^{\prime} 45^{\prime \prime}$, in SW 1/4 sec.

17, T. 24 N., R. 22 E., Tallapoosa County, Hydrologic Unit

03150109 , on county road, $1 \mathrm{mi}$ downstream from Enitachopco

Creek, 3 mi east of Hackneyville, and 4 mi upstream from

Hackney Creek.

Drainage area-190 $\mathrm{mi}^{2}$.

Gage -Water-stage recorder. Datum of gage is $557.92 \mathrm{ft}$ NGVD 29

$\begin{array}{ccccc}\begin{array}{c}\text { Water } \\ \text { year }\end{array} \text { Date } \quad \text { Discharge } & \begin{array}{c}\text { Discharge } \\ \text { code }\end{array} & \begin{array}{c}\text { Gage } \\ \text { height }\end{array} & \begin{array}{c}\text { Gage } \\ \text { height }\end{array}\end{array}$

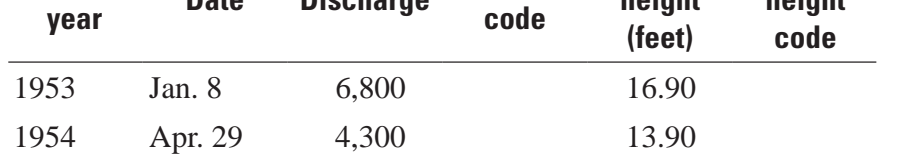

1955

1956

1957

1958

Apr. 14

8,600

Mar. 16

14,200

Apr. 5

17,800

Feb. 6

7,650

1959

Mar. 12

4,500

1960

Mar. 30

5,900

19.10

23.60

25.70

18.00

14.10

15.81

1961

Feb. 25

10,500

20.97

1962

Dec. 18

10,700

21.02

1963

Mar. 13

9,200

19.82

23.74

1964

Apr. 6

14,300

Mar. 17

4,900

14.41

18.06

1966

May 13

6,650

15.97

1967

Oct. 19

6,000

19.87

15.52

1969

Apr. 5

9,250

15.52

1970

Mar. 20

5,600

20.43

20.60

23.62

21.82

1973

1979

Jan. 11

10,000

14,000

May 8

11,500

Apr. 13

23,000

7

28.10

10.65

16.08

22.43

1988

Jan. 19

4,700

18.77
02415000 Hillabee Creek near Hackneyville-Continued L ocation-Lat $33^{\circ} 04^{\prime} 00^{\prime \prime}$, long 85 $52^{\prime} 45^{\prime \prime}$, in SW 1/4 sec. 17, T. 24 N., R. 22 E., Tallapoosa County, Hydrologic Unit 03150109, on county road, $1 \mathrm{mi}$ downstream from Enitachopco Creek, 3 mi east of Hackneyville, and 4 mi upstream from Hackney Creek.

Drainage area-190 $\mathrm{mi}^{2}$.

Gage - Water-stage recorder. Datum of gage is $557.92 \mathrm{ft}$ NGVD 29

\begin{tabular}{lccccc}
\hline $\begin{array}{c}\text { Water } \\
\text { year }\end{array}$ & Date & Discharge & $\begin{array}{c}\text { Discharge } \\
\text { code }\end{array}$ & $\begin{array}{c}\text { Gage } \\
\text { height } \\
\text { (feet) }\end{array}$ & $\begin{array}{c}\text { Gage } \\
\text { height } \\
\text { code }\end{array}$ \\
\hline 1990 & Mar. 16 & 17,700 & & 25.59 & \\
1991 & Mar. 29 & 3,200 & & 13.55 &
\end{tabular}

1992

1993

Feb. $25 \quad 4,600$

15.73

1993 Dec. $17 \quad 8,200 \quad 20.03$

1994 Apr. $16 \quad 8,250 \quad 20.14$

1995 Mar. $8 \quad 6,550 \quad 18.39$

1996 Oct. $5 \quad 12,200 \quad 23.02$

1997 Mar. $1 \quad 8,250 \quad 20.14$

1998 Mar. $8 \quad 10,000 \quad 21.54$

$1999 \quad$ Jan. $31 \quad 2,820 \quad 12.79$

2000 Apr. $4 \quad 2,010 \quad 11.04$

2001 Apr. $4 \quad 8,800 \quad 20.66$

$2002 \quad$ Jan. $25 \quad 2,250 \quad 11.55$

2003 May $8 \quad 17,700 \quad 25.59$

2004 Sept. $17 \quad 7,900 \quad 18.23$

$2005 \quad$ July $7 \quad 8,610 \quad 19.06$

2006 Mar. $21 \quad 3,000 \quad 11.86$

2007 Nov. $15 \quad 7,930 \quad 18.27$

2008 Nov. $15 \quad 7,930 \quad 18.27$

2009 Sept. $19 \quad 7,950 \quad 18.29$

2010 May $10 \quad 10,600 \quad 20.96$

$2011 \quad$ Mar. $9 \quad 5,980 \quad 15.91$

2012 Mar. $3 \quad 6,740 \quad 16.81$

$2013 \quad$ July $7 \quad 5,660 \quad 15.53$

2014 Apr. $7 \quad 10,900 \quad 21.24$

2015 Jan. $4 \quad 6,850 \quad 16.95$ 
Appendix 2. Discharge and gage height data for streamgages in Alabama.-Continued

[--, date or data not available; $\mathrm{ft}$, foot; $\mathrm{ft}^{3} / \mathrm{s}$, cubic foot per second; mi, mile; $\mathrm{mi}^{2}$, square mile; qualification codes apply to the discharge data: 1 , discharge is a maximum daily average; 2 , discharge is an estimate; 4 , discharge is less than indicated value, which is the minimum recordable discharge at this site; 5 , discharge affected to unknown degree by regulation or diversion; 6 , discharge affected by regulation or diversion; 7, discharge is a historic peak; A, year of occurrence is unknown or not exact; $\mathrm{B}$, month or day of occurrence is unknown or not exact; E, only maximum peak available for this year. qualification codes apply to the gage height data: 1, gage height affected by backwater; 2, gage height not the maximum for the year; 3, gage height at different datum or at different site and datum; 5 , gage height is an estimate]

\section{Sougahatchee Creek at Co Rd 188 nr Loachap}

Location-Lat $32^{\circ} 37^{\prime} 36^{\prime \prime}$, long $85^{\circ} 35^{\prime} 17^{\prime \prime}$ referenced to North American Datum of 1927, Lee County, AL, Hydrologic Unit 03150110, on downstream side of bridge on County Road 188, 1 mi upstream of Loblocklee Creek, and 2 mi north of Loachapoka.

Drainage area $-71 \mathrm{mi}^{2}$.

G age -Water-stage recorder. Elevation of gage is $520 \mathrm{ft}$ above NGVD of 1929 (from topographic map).

\begin{tabular}{|c|c|c|c|c|c|}
\hline $\begin{array}{l}\text { Water } \\
\text { year }\end{array}$ & Date & Discharge & $\begin{array}{l}\text { Discharge } \\
\text { code }\end{array}$ & $\begin{array}{c}\text { Gage } \\
\text { height } \\
\text { (feet) }\end{array}$ & $\begin{array}{c}\text { Gage } \\
\text { height } \\
\text { code }\end{array}$ \\
\hline 2000 & Jan. 10 & 1,590 & & 4.63 & \\
\hline 2001 & Mar. 4 & 6,000 & & 7.88 & \\
\hline 2002 & Mar. 21 & 1,280 & & 4.26 & \\
\hline 2003 & July 2 & 7,570 & & 8.64 & \\
\hline 2004 & Sept. 17 & 2,980 & & 5.91 & \\
\hline 2005 & Mar. 28 & 6,350 & & 8.06 & \\
\hline 2006 & Mar. 21 & 6,490 & & 8.13 & \\
\hline 2007 & Nov. 16 & 2,440 & & 5.45 & \\
\hline 2008 & Nov. 16 & 2,440 & & 5.45 & \\
\hline 2009 & Feb. 28 & 2,870 & & 5.82 & \\
\hline 2010 & Nov. 11 & 4,410 & & 6.95 & \\
\hline 2011 & July 20 & 1,620 & & 4.66 & \\
\hline 2012 & Feb. 19 & 1,410 & & 4.42 & \\
\hline 2013 & Feb. 23 & 2,520 & & 5.52 & \\
\hline 2014 & Apr. 7 & 4,460 & & 6.98 & \\
\hline 2015 & May 29 & 2,800 & & 5.76 & \\
\hline 2016 & Dec. 25 & 12,800 & & 10.38 & \\
\hline
\end{tabular}

02423380 Cahaba River Near Mountain Brook Ala.

L ocation - Lat $33^{\circ} 28^{\prime} 54^{\prime \prime}$, long 86 $46^{\circ} 46^{\prime \prime}$ referenced to North American Datum of 1927, Jefferson County, Ala., Hydrologic Unit 03150202 , on downstream side bridge on county road, 0.1 mi upstream from Fuller Creek, 3.5 mi east of Mountain Brook, 5.4 mi upstream from Little Cahaba River, and at mi 153.6.

Drainage area-140 $\mathrm{mi}^{2}$.

G age -Water-stage recorder. Datum of gage is $443.85 \mathrm{ft}$ above NGVD of 1929 (levels by Jefferson County Engineering Department).

\begin{tabular}{|c|c|c|c|c|c|}
\hline $\begin{array}{c}\text { Water } \\
\text { year }\end{array}$ & Date & Discharge & $\begin{array}{l}\text { Discharge } \\
\text { code }\end{array}$ & $\begin{array}{c}\text { Gage } \\
\text { height } \\
\text { (feet) }\end{array}$ & $\begin{array}{c}\text { Gage } \\
\text { height } \\
\text { code }\end{array}$ \\
\hline 1979 & Apr. 13 & 19,000 & 7 & 20.60 & \\
\hline 1981 & Mar. 30 & 6,750 & & 10.84 & \\
\hline 1985 & Feb. 1 & 5,340 & & 9.18 & \\
\hline 1986 & July 21 & 1,420 & & 3.75 & \\
\hline 1987 & Jan. 19 & 4,110 & & 8.80 & \\
\hline 1988 & Jan. 20 & 3,140 & & 7.29 & \\
\hline 1989 & Mar. 5 & 6,410 & & 12.10 & \\
\hline 1990 & Mar. 16 & 6,880 & & 11.99 & \\
\hline 1991 & Feb. 20 & 5,240 & & 9.84 & \\
\hline 1992 & Sept. 4 & 3,370 & & 7.15 & \\
\hline 1993 & Jan. 13 & 4,320 & & 8.55 & \\
\hline 1994 & July 27 & 5,190 & & 9.87 & \\
\hline 1995 & Mar. 8 & 4,310 & & 8.75 & \\
\hline 1996 & Jan. 27 & 10,500 & & 15.60 & \\
\hline 1997 & June 14 & 8,050 & & 13.15 & \\
\hline 1998 & Jan. 8 & 12,500 & & 16.19 & \\
\hline 1999 & June 28 & 11,000 & & 15.11 & \\
\hline 2000 & Apr. 4 & 10,300 & & 14.58 & \\
\hline 2001 & Apr. 4 & 13,200 & & 16.70 & \\
\hline 2002 & Sept. 22 & 6,780 & & 11.65 & \\
\hline 2003 & May 8 & 23,500 & & 25.08 & \\
\hline 2004 & Sept. 16 & 12,700 & & 16.94 & \\
\hline 2005 & Nov. 23 & 8,760 & & 13.40 & \\
\hline 2006 & Feb. 6 & 2,980 & & 6.29 & \\
\hline 2007 & Jan. 8 & 1,250 & & 3.90 & \\
\hline 2008 & Aug. 26 & 5,420 & & 9.52 & \\
\hline 2009 & Sept. 18 & 11,200 & & 15.42 & \\
\hline 2010 & Nov. 11 & 8,010 & & 12.33 & \\
\hline 2011 & Mar. 9 & 8,540 & & 12.86 & \\
\hline 2012 & Jan. 27 & 5,740 & & 9.89 & \\
\hline 2013 & May 18 & 12,100 & & 16.15 & \\
\hline 2014 & Apr. 7 & 13,300 & & 16.25 & \\
\hline 2015 & Jan. 4 & 7,980 & & 10.52 & \\
\hline
\end{tabular}


Appendix 2. Discharge and gage height data for streamgages in Alabama.-Continued

[--, date or data not available; $\mathrm{ft}$, foot; $\mathrm{ft}^{3} / \mathrm{s}$, cubic foot per second; $\mathrm{mi}$, mile; $\mathrm{mi}^{2}$, square mile; qualification codes apply to the discharge data: 1 , discharge is a maximum daily average; 2 , discharge is an estimate; 4 , discharge is less than indicated value, which is the minimum recordable discharge at this site; 5 , discharge affected to unknown degree by regulation or diversion; 6 , discharge affected by regulation or diversion; 7, discharge is a historic peak; A, year of occurrence is unknown or not exact; B, month or day of occurrence is unknown or not exact; E, only maximum peak available for this year. qualification codes apply to the gage height data: 1, gage height affected by backwater; 2 , gage height not the maximum for the year; 3 , gage height at different data or at different site and datum;

5 , gage height is an estimate]

\section{Cahaba River near Acton}

L ocation-Lat $33^{\circ} 21^{\prime} 48^{\prime \prime}$, long $86^{\circ} 48^{\prime} 47$, in SE $1 / 4$ sec. 23 , T. 19 S., R. 3 W., Jefferson County, Hydrologic Unit 03150202 , on right bank at downstream side of highway bridge (Bains Bridge), 0.5 mi upstream from Patton Creek, 1 mi downstream from U.S. Highway 31, 1 mi northwest of Acton, 16 mi south of Birmingham, and at mile 136.8 .

Drainage area-230 $\mathrm{mi}^{2}$.

Gage -Water-stage recorder. Datum of gage is $375.00 \mathrm{ft}$ NGVD 29.

\begin{tabular}{|c|c|c|c|c|c|}
\hline $\begin{array}{l}\text { Water } \\
\text { year }\end{array}$ & Date & Discharge & $\begin{array}{l}\text { Discharge } \\
\text { code }\end{array}$ & $\begin{array}{c}\text { Gage } \\
\text { height } \\
\text { (feet) }\end{array}$ & $\begin{array}{c}\text { Gage } \\
\text { height } \\
\text { code }\end{array}$ \\
\hline 1939 & Feb. 28 & 5,570 & & 19.03 & \\
\hline 1940 & Feb. 6 & 6,010 & & 20.03 & \\
\hline 1941 & Aug. 3 & 4,800 & & 17.10 & \\
\hline 1942 & Mar. 21 & 6,200 & & 20.45 & \\
\hline 1943 & Dec. 28 & 34,000 & & 43.70 & \\
\hline 1944 & Mar. 29 & 9,000 & & 26.43 & \\
\hline 1945 & Feb. 13 & 6,900 & & 22.00 & \\
\hline 1946 & Feb. 10 & 13,700 & & 33.10 & \\
\hline 1947 & Jan. 20 & 10,800 & & 29.30 & 2 \\
\hline 1948 & Feb. 9 & 6,180 & & 20.30 & 2 \\
\hline 1949 & Nov. 29 & 23,000 & & 39.80 & 2 \\
\hline 1950 & Mar. 14 & 6,100 & & 20.20 & \\
\hline 1951 & Mar. 29 & 17,800 & & 36.80 & 2 \\
\hline 1952 & Dec. 21 & 7,720 & & 24.30 & 2 \\
\hline 1953 & Jan. 9 & 8,700 & & 26.00 & \\
\hline 1954 & Jan. 16 & 7,300 & & 23.60 & \\
\hline 1955 & Feb. 7 & 9,340 & & 26.80 & \\
\hline 1956 & Apr. 6 & 6,590 & & 21.20 & \\
\hline 1957 & Apr. 5 & 11,000 & & 29.40 & \\
\hline 1961 & Feb. 22 & 30,000 & 7 & 42.66 & \\
\hline 1979 & Apr. 13 & 31,000 & 7 & 42.84 & \\
\hline 1984 & Dec. 3 & 15,000 & & 34.62 & \\
\hline 1985 & Feb. 1 & 5,380 & & 18.30 & \\
\hline 1986 & July 21 & 1,820 & & 8.05 & \\
\hline 1987 & Nov. 26 & 4,270 & & 16.02 & \\
\hline 1988 & Jan. 20 & 3,230 & & 12.82 & \\
\hline 1989 & Mar. 5 & 9,220 & & 25.81 & \\
\hline
\end{tabular}

02423500 Cahaba River near Acton-Continued

L ocation-Lat $33^{\circ} 21^{\prime} 48^{\prime \prime}$, long $86^{\circ} 48^{\prime} 47$, in SE 1/4 sec. $23, \mathrm{~T}$. 19 S., R. 3 W., Jefferson County, Hydrologic Unit 03150202, on right bank at downstream side of highway bridge (Bains Bridge), 0.5 mi upstream from Patton Creek, 1 mi downstream from U.S. Highway 31, 1 mi northwest of Acton, 16 mi south of Birmingham, and at mile 136.8.

Drainage area $-230 \mathrm{mi}^{2}$.

G age - Water-stage recorder. Datum of gage is $375.00 \mathrm{ft}$ NGVD 29.

\begin{tabular}{lccccc}
\hline $\begin{array}{c}\text { Water } \\
\text { year }\end{array}$ & Date & Discharge & $\begin{array}{c}\text { Discharge } \\
\text { code }\end{array}$ & $\begin{array}{c}\text { Gage } \\
\text { height } \\
\text { (feet) }\end{array}$ & $\begin{array}{c}\text { Gage } \\
\text { height } \\
\text { code }\end{array}$ \\
\hline 1990 & Feb. 16 & 14,000 & & 33.11 & \\
1991 & Feb. 20 & 7,350 & & 22.14 & \\
1992 & Sept. 4 & 4,400 & & 16.00 &
\end{tabular}

$1993 \quad$ Jan. $13 \quad 8,100 \quad 24.51$

$1994 \quad$ July $27 \quad 4,400 \quad 16.06$

1995 Mar. $8 \quad 5,200 \quad 18.12$

1996 Mar. $7 \quad 14,500 \quad 33.35$

$1997 \quad$ June $15 \quad 6,480 \quad 20.99$

$1998 \quad$ Jan. $8 \quad 13,300 \quad 32.42$

$1999 \quad$ June $29 \quad 9,900 \quad 28.10$

2000 Apr. $4 \quad 15,200 \quad 34.40$

2001 Apr. $4 \quad 12,100 \quad 30.85$

2002 Sept. $22 \quad 8,630 \quad 25.63$

2003 May $8 \quad 19,100 \quad 37.74$

2004 Sept. $17 \quad 13,400 \quad 32.62$

2005 Nov. $25 \quad 11,500 \quad 30.05$

$2006 \quad$ Feb. $7 \quad 6,990 \quad 21.58$

$2007 \quad$ Jan. $8 \quad 1,800 \quad 8.95$

2008 Aug. $26 \quad 6,340 \quad 20.29$

$2009 \quad$ Jan. $7 \quad 9,150 \quad 26.06$

$2010 \quad$ Nov. $11 \quad 10,100 \quad 27.72$

2011 Mar. $10 \quad 9,830 \quad 27.32$

$2012 \quad$ Jan. $27 \quad 6,190 \quad 19.98$

2013 May $19 \quad 8,170 \quad 25.19$

2014 Apr. $7 \quad 14,500 \quad 34.98$

$2015 \quad$ Jan. $4 \quad 7,320 \quad 23.28$ 
Appendix 2. Discharge and gage height data for streamgages in Alabama.-Continued

[--, date or data not available; $\mathrm{ft}$, foot; $\mathrm{ft}^{3} / \mathrm{s}$, cubic foot per second; mi, mile; $\mathrm{mi}^{2}$, square mile; qualification codes apply to the discharge data: 1 , discharge is a maximum daily average; 2 , discharge is an estimate; 4 , discharge is less than indicated value, which is the minimum recordable discharge at this site; 5 , discharge affected to unknown degree by regulation or diversion; 6 , discharge affected by regulation or diversion; 7, discharge is a historic peak; A, year of occurrence is unknown or not exact; B, month or day of occurrence is unknown or not exact; E, only maximum peak available for this year. qualification codes apply to the gage height data: 1, gage height affected by backwater; 2, gage height not the maximum for the year; 3, gage height at different datum or at different site and datum; 5 , gage height is an estimate]

02423555 Cahaba River near Helena

Location-Lat $33^{\circ} 17^{\prime} 04^{\prime \prime}$, long $86^{\circ} 52^{\prime} 57^{\prime \prime}$, in NE $1 / 4$ sec. 19 , T. 20 S., R. 3 W., Shelby County, Hydrologic Unit 03150202, 2 mi southwest of Helena.

Drainage area-335 $\mathrm{mi}^{2}$.

Gage - Crest-stage gage. Datum not available.

\begin{tabular}{|c|c|c|c|c|c|}
\hline $\begin{array}{l}\text { Water } \\
\text { year }\end{array}$ & Date & Discharge & $\begin{array}{l}\text { Discharge } \\
\text { code }\end{array}$ & $\begin{array}{c}\text { Gage } \\
\text { height } \\
\text { (feet) }\end{array}$ & $\begin{array}{c}\text { Gage } \\
\text { height } \\
\text { code }\end{array}$ \\
\hline 1961 & Feb. 22 & 36,000 & 2,7 & -- & \\
\hline 1965 & Jan. 24 & 5,280 & & 13.07 & \\
\hline 1966 & Apr. 28 & 9,180 & & 20.30 & \\
\hline 1967 & Aug. 27 & 4,520 & & 11.29 & \\
\hline 1969 & Jan. 20 & 8,660 & & 19.43 & \\
\hline 1970 & Mar. 20 & 21,000 & & 33.35 & \\
\hline 1971 & Feb. 6 & 8,260 & & 18.76 & \\
\hline 1972 & Jan. 11 & 10,740 & & 22.45 & \\
\hline 1973 & Apr. 1 & 6,620 & & 15.85 & \\
\hline 1974 & Dec. 26 & 9,250 & & 20.42 & \\
\hline 1975 & Feb. 26 & 10,200 & & 21.97 & \\
\hline 1976 & Mar. 16 & 22,800 & & 34.85 & \\
\hline 1977 & Apr. 6 & 13,700 & & 26.70 & \\
\hline 1979 & Apr. 13 & 37,200 & 2,7 & -- & \\
\hline 1996 & Mar. 7 & 17,500 & & 30.37 & \\
\hline 1997 & Mar. 1 & 7,890 & & 18.16 & \\
\hline 1998 & Jan. 8 & 14,800 & & 27.80 & \\
\hline 1999 & June 29 & 9,770 & & 21.28 & \\
\hline 2000 & Apr. 4 & 18,200 & & 30.97 & \\
\hline 2001 & Apr. 5 & 12,400 & & 25.14 & \\
\hline 2002 & Sept. 22 & 10,000 & & 21.68 & \\
\hline 2003 & May 9 & 17,700 & & 30.54 & \\
\hline 2004 & Sept. 17 & 13,900 & & 26.90 & \\
\hline 2005 & Nov. 25 & 14,900 & & 27.93 & \\
\hline 2006 & Feb. 7 & 7,600 & & 17.63 & \\
\hline 2007 & Jan. 8 & 1,800 & 1 & -- & \\
\hline 2008 & Aug. 26 & 6,570 & & 15.75 & \\
\hline 2009 & Jan. 7 & 10,200 & & 22.06 & \\
\hline 2010 & Mar. 11 & 14,100 & & 27.08 & \\
\hline 2011 & Mar. 10 & 11,000 & & 23.22 & \\
\hline 2012 & July 31 & 7,720 & & 17.87 & \\
\hline 2013 & May 19 & 7,760 & & 17.94 & \\
\hline 2014 & Apr. 7 & 19,000 & & 31.69 & \\
\hline 2015 & Dec. 28 & 10,300 & & 22.23 & \\
\hline
\end{tabular}

02423630 Shades Creek near Greenwood Ala.

L ocation - Lat 3319'34", long 86 ${ }^{\circ} 56^{\prime} 59^{\prime \prime}$ referenced to North American Datum of 1927, Jefferson County, AL, Hydrologic Unit 03150202, near left bank on downstream side of bridge on county road, $1.4 \mathrm{mi}$ southwest of Greenwood, $5.5 \mathrm{mi}$ south of Bessemer, and at mile 20.8 .

Drainage area-72 $\mathrm{mi}^{2}$.

G age -Water-stage recorder. Datum of gage is $478.37 \mathrm{ft}$ above NGVD of 1929. Prior to October 1, 1997, gage was at datum $480.37 \mathrm{ft}$ above NGVD of 1929.

\begin{tabular}{|c|c|c|c|c|c|}
\hline $\begin{array}{c}\text { Water } \\
\text { year }\end{array}$ & Date & Discharge & $\begin{array}{l}\text { Discharge } \\
\text { code }\end{array}$ & $\begin{array}{c}\text { Gage } \\
\text { height } \\
\text { (feet) }\end{array}$ & $\begin{array}{c}\text { Gage } \\
\text { height } \\
\text { code }\end{array}$ \\
\hline 1965 & Feb. 12 & 1,800 & & 10.78 & 3 \\
\hline 1966 & Feb. 16 & 3,200 & & 11.86 & 3 \\
\hline 1967 & Aug. 27 & 3,080 & & 11.80 & 3 \\
\hline 1968 & Dec. 22 & 2,800 & & 11.66 & 3 \\
\hline 1969 & Jan. 20 & 4,020 & & 12.18 & 3 \\
\hline 1970 & Mar. 20 & 7,220 & & 13.04 & 3 \\
\hline 1971 & Feb. 5 & 4,570 & & 12.36 & 3 \\
\hline 1972 & Jan. 11 & 3,400 & & 11.94 & 3 \\
\hline 1973 & Mar. 31 & 2,980 & & 11.75 & 3 \\
\hline 1974 & Dec. 27 & 3,500 & & 12.04 & 3 \\
\hline 1975 & Jan. 25 & 4,000 & & 12.22 & 3 \\
\hline 1976 & Mar. 16 & 6,470 & & 12.87 & 3 \\
\hline 1977 & Apr. 5 & 4,870 & & 12.45 & 3 \\
\hline 1978 & Oct. 26 & 3,600 & & 12.06 & 3 \\
\hline 1979 & Apr. 13 & 7,800 & & 13.19 & 3 \\
\hline 1980 & Mar. 17 & 5,200 & & 12.59 & 3 \\
\hline 1981 & Mar. 30 & 4,070 & & 12.04 & 3 \\
\hline 1998 & Jan. 8 & 5,200 & & 14.56 & 6 \\
\hline 1999 & June 29 & 3,350 & & 13.83 & \\
\hline 2000 & Apr. 3 & 6,110 & & 14.84 & \\
\hline 2001 & Sept. 3 & 4,160 & & 14.24 & \\
\hline 2002 & Sept. 22 & 4,140 & & 14.23 & \\
\hline 2003 & May 18 & 4,800 & & 14.46 & \\
\hline 2004 & Sept. 17 & 7,000 & & 15.02 & \\
\hline 2005 & Nov. 24 & 3,990 & & 14.16 & \\
\hline 2006 & Feb. 7 & 3,310 & & 13.80 & \\
\hline 2007 & Jan. 8 & 1,200 & & 10.27 & \\
\hline 2008 & Aug. 26 & 1,870 & & 12.58 & \\
\hline 2009 & Feb. 28 & 3,000 & & 13.69 & \\
\hline 2010 & Nov. 11 & 4,400 & & 14.31 & \\
\hline 2011 & Sept. 6 & 4,130 & & 14.23 & \\
\hline 2012 & Jan. 27 & 3,340 & & 13.82 & \\
\hline 2013 & Jan. 14 & 3,520 & & 13.92 & \\
\hline 2014 & Apr. 7 & 4,450 & & 14.25 & \\
\hline 2015 & Dec. 28 & 2,690 & & 13.66 & \\
\hline
\end{tabular}


Appendix 2. Discharge and gage height data for streamgages in Alabama.-Continued

[--, date or data not available; ft, foot; $\mathrm{ft}^{3} / \mathrm{s}$, cubic foot per second; mi, mile; $\mathrm{mi}^{2}$, square mile; qualification codes apply to the discharge data: 1 , discharge is a maximum daily average; 2 , discharge is an estimate; 4 , discharge is less than indicated value, which is the minimum recordable discharge at this site; 5 , discharge affected to unknown degree by regulation or diversion; 6 , discharge affected by regulation or diversion; 7, discharge is a historic peak; A, year of occurrence is unknown or not exact; $\mathrm{B}$, month or day of occurrence is unknown or not exact; E, only maximum peak available for this year. qualification codes apply to the gage height data: 1 , gage height affected by backwater; 2, gage height not the maximum for the year; 3, gage height at different data or at different site and datum; 5 , gage height is an estimate]

\section{Cahaba River at Centreville}

L ocation-Lat $32^{\circ} 56^{\prime} 42^{\prime \prime}$, long $87^{\circ} 08^{\prime} 21^{\prime \prime}$, in SE $1 / 4$ sec. $26, \mathrm{~T}$. 23 N. R. 9 E., Bibb County, Hydrologic Unit 03150202, 60 $\mathrm{ft}$ downstream from U.S. Highway 82 bridge. $0.2 \mathrm{mi}$ west of Centreville, $2.5 \mathrm{mi}$ upstream from Sandy Creek, and at mile 81.2. Drainage area-1,027 $\mathrm{mi}^{2}$.

Gage - Water-stage recorder. Datum of gage is $180.74 \mathrm{ft}$ NGVD 29. Prior to Jan. 31, 1939, nonrecording gage at same site. Prior to May 1929, at datum $1.15 \mathrm{ft}$ lower.

Remarks - Flow affected by regulation from Lake Purdy and diversion by Birmingham Water Works.

\begin{tabular}{|c|c|c|c|c|c|}
\hline $\begin{array}{c}\text { Water } \\
\text { year }\end{array}$ & Date & Discharge & $\begin{array}{l}\text { Discharge } \\
\text { code }\end{array}$ & $\begin{array}{c}\text { Gage } \\
\text { height } \\
\text { (feet) }\end{array}$ & $\begin{array}{c}\text { Gage } \\
\text { height } \\
\text { code }\end{array}$ \\
\hline 1902 & Mar. 28 & 100,000 & & 36.70 & 3 \\
\hline 1903 & Feb. 8 & 51,000 & & 31.60 & 3 \\
\hline 1904 & Feb. 8 & 5,400 & & 11.50 & 3 \\
\hline 1905 & Feb. 9 & 25,000 & & 25.90 & 3 \\
\hline 1906 & Mar. 19 & 84,000 & & 35.50 & 3 \\
\hline 1907 & May 15 & 23,000 & & 25.20 & 3 \\
\hline 1916 & July 8 & 90,000 & & 36.20 & 3 \\
\hline 1917 & Mar. 4 & 28,600 & & 27.10 & 3 \\
\hline 1918 & Jan & 17,600 & B & 21.50 & 3 \\
\hline 1919 & Oct. 30 & 60,000 & & 33.00 & 3 \\
\hline 1920 & Dec. 9 & 59,000 & & 32.80 & 3 \\
\hline 1921 & Apr. 17 & 46,000 & & 31.00 & 3 \\
\hline 1922 & Mar. 11 & 22,500 & & 25.00 & 3 \\
\hline 1923 & Feb. 13 & 41,500 & & 30.00 & 3 \\
\hline 1924 & Feb. 24 & 17,600 & & 21.50 & 3 \\
\hline 1925 & Jan. 18 & 36,000 & & 29.10 & 3 \\
\hline 1926 & Jan & 20,500 & & 24.00 & 3 \\
\hline 1927 & Feb. 14 & 28,500 & & 27.00 & 3 \\
\hline 1928 & Apr. 22 & 38,200 & & 29.50 & 3 \\
\hline 1929 & Jan. 14 & 20,500 & & 24.00 & 3,6 \\
\hline 1930 & Nov. 15 & 67,000 & & 34.00 & \\
\hline 1931 & Nov. 16 & 16,200 & & 21.75 & \\
\hline 1932 & Mar. 31 & 41,000 & & 30.10 & \\
\hline 1933 & Mar. 30 & 32,000 & & 28.00 & \\
\hline 1934 & Mar. 3 & 52,000 & & 32.00 & \\
\hline 1935 & Mar. 7 & 32,000 & & 28.00 & \\
\hline 1936 & Feb. 4 & 87,000 & & 35.80 & \\
\hline 1937 & Mar. 20 & 35,000 & & 28.80 & \\
\hline
\end{tabular}

02424000 Cahaba River at Centreville-Continued

L ocation-Lat $32^{\circ} 56^{\prime} 42^{\prime \prime}$, long $87^{\circ} 08^{\prime} 21^{\prime \prime}$, in SE $1 / 4$ sec. 26 , T. 23 N. R. 9 E., Bibb County, Hydrologic Unit 03150202, 60 $\mathrm{ft}$ downstream from U.S. Highway 82 bridge. $0.2 \mathrm{mi}$ west of Centreville, 2.5 mi upstream from Sandy Creek, and at mile 81.2.

Drainage area- $1,027 \mathrm{mi}^{2}$.

Gage -Water-stage recorder. Datum of gage is $180.74 \mathrm{ft}$ NGVD 29. Prior to Jan. 31, 1939, nonrecording gage at same site. Prior to May 1929, at datum $1.15 \mathrm{ft}$ lower.

Remarks - Flow affected by regulation from Lake Purdy and diversion by Birmingham Water Works.

\begin{tabular}{|c|c|c|c|c|c|}
\hline $\begin{array}{c}\text { Water } \\
\text { year }\end{array}$ & Date & Discharge & $\begin{array}{l}\text { Discharge } \\
\text { code }\end{array}$ & $\begin{array}{c}\text { Gage } \\
\text { height } \\
\text { (feet) }\end{array}$ & $\begin{array}{c}\text { Gage } \\
\text { height } \\
\text { code }\end{array}$ \\
\hline 1938 & Apr. 8 & 97,000 & & 36.63 & \\
\hline 1939 & Aug. 16 & 50,000 & & 31.56 & \\
\hline 1940 & Feb. 6 & 28,500 & & 26.96 & \\
\hline 1941 & Mar. 7 & 18,000 & & 21.79 & \\
\hline 1942 & June 13 & 43,000 & & 30.48 & \\
\hline 1943 & Dec. 28 & 72,000 & & 34.38 & \\
\hline 1944 & Mar. 29 & 31,600 & & 28.02 & \\
\hline 1945 & May 13 & 30,100 & & 27.63 & \\
\hline 1946 & Feb. 10 & 37,200 & & 29.31 & \\
\hline 1947 & Jan. 20 & 43,900 & & 30.59 & \\
\hline 1948 & Feb. 9 & 24,800 & & 26.00 & \\
\hline 1949 & Nov. 28 & 57,400 & & 32.60 & \\
\hline 1950 & Mar. 14 & 13,900 & & 21.16 & \\
\hline 1951 & Mar. 29 & 83,600 & & 34.80 & \\
\hline 1952 & Dec. 21 & 26,300 & & 26.40 & \\
\hline 1953 & Jan. 10 & 20,600 & & 24.60 & \\
\hline 1954 & Jan. 17 & 13,800 & & 21.00 & \\
\hline 1955 & Apr. 14 & 27,900 & & 27.05 & \\
\hline 1956 & Mar. 16 & 31,600 & & 28.01 & \\
\hline 1957 & Apr. 5 & 35,400 & & 28.90 & \\
\hline 1958 & Feb. 7 & 17,800 & & 23.14 & \\
\hline 1959 & Jan. 21 & 10,200 & & 16.84 & \\
\hline 1960 & Mar. 4 & 9,930 & & 17.56 & \\
\hline 1961 & Feb. 23 & 82,200 & & 35.35 & \\
\hline 1962 & Dec. 18 & 37,800 & & 29.41 & \\
\hline 1963 & June 24 & 22,100 & & 25.13 & \\
\hline 1964 & Apr. 6 & 31,800 & & 28.06 & \\
\hline 1965 & Feb. 12 & 17,900 & & 23.35 & \\
\hline
\end{tabular}


Appendix 2. Discharge and gage height data for streamgages in Alabama._-Continued

[--, date or data not available; $\mathrm{ft}$, foot; $\mathrm{ft}^{3} / \mathrm{s}$, cubic foot per second; mi, mile; $\mathrm{mi}^{2}$, square mile; qualification codes apply to the discharge data: 1 , discharge is a maximum daily average; 2 , discharge is an estimate; 4 , discharge is less than indicated value, which is the minimum recordable discharge at this site; 5 , discharge affected to unknown degree by regulation or diversion; 6 , discharge affected by regulation or diversion; 7, discharge is a historic peak; A, year of occurrence is unknown or not exact; B, month or day of occurrence is unknown or not exact; E, only maximum peak available for this year. qualification codes apply to the gage height data: 1, gage height affected by backwater; 2, gage height not the maximum for the year; 3, gage height at different datum or at different site and datum; 5 , gage height is an estimate]

\section{Cahaba River at Centreville - Continued}

Location-Lat $32^{\circ} 56^{\prime} 42^{\prime \prime}$, long $87^{\circ} 08^{\prime} 21^{\prime \prime}$, in SE $1 / 4$ sec. 26 , T.

23 N. R. 9 E., Bibb County, Hydrologic Unit 03150202, 60

$\mathrm{ft}$ downstream from U.S. Highway 82 bridge. 0.2 mi west of

Centreville, 2.5 mi upstream from Sandy Creek, and at mile 81.2.

Drainage area $-1,027 \mathrm{mi}^{2}$.

Gage - Water-stage recorder. Datum of gage is $180.74 \mathrm{ft}$ NGVD 29. Prior to Jan. 31, 1939, nonrecording gage at same site. Prior to May 1929, at datum $1.15 \mathrm{ft}$ lower.

Remarks - Flow affected by regulation from Lake Purdy and diversion by Birmingham Water Works.

\begin{tabular}{|c|c|c|c|c|c|}
\hline $\begin{array}{l}\text { Water } \\
\text { year }\end{array}$ & Date & Discharge & $\begin{array}{l}\text { Discharge } \\
\text { code }\end{array}$ & $\begin{array}{c}\text { Gage } \\
\text { height } \\
\text { (feet) }\end{array}$ & $\begin{array}{c}\text { Gage } \\
\text { height } \\
\text { code }\end{array}$ \\
\hline 1966 & Feb. 16 & 29,300 & & 27.40 & \\
\hline 1967 & Aug. 27 & 9,460 & & 17.29 & \\
\hline 1968 & Apr. 5 & 19,900 & & 24.30 & \\
\hline 1969 & Jan. 20 & 28,500 & & 27.08 & \\
\hline 1970 & Mar. 20 & 45,100 & & 30.86 & \\
\hline 1971 & Feb. 22 & 24,300 & & 25.90 & \\
\hline 1972 & Jan. 11 & 36,400 & & 29.13 & \\
\hline 1973 & Mar. 31 & 20,600 & & 24.49 & \\
\hline 1974 & Jan. 1 & 31,800 & & 28.01 & \\
\hline 1975 & Jan. 25 & 22,200 & & 25.17 & \\
\hline 1976 & Mar. 16 & 64,300 & & 33.73 & \\
\hline 1977 & Mar. 30 & 46,700 & & 31.15 & \\
\hline 1978 & June 9 & 16,800 & & 22.89 & \\
\hline 1979 & Apr. 13 & 78,400 & & 35.03 & \\
\hline 1980 & Mar. 13 & 42,400 & & 30.33 & \\
\hline 1981 & Feb. 11 & 21,600 & & 24.98 & \\
\hline 1982 & Apr. 20 & 34,400 & & 28.65 & \\
\hline 1983 & Feb. 2 & 34,000 & & 28.55 & \\
\hline 1984 & Dec. 4 & 40,800 & & 29.99 & \\
\hline 1985 & Feb. 6 & 14,400 & $\mathrm{E}$ & 21.60 & \\
\hline 1986 & Dec. 13 & 4,470 & & 10.03 & \\
\hline 1987 & Jan. 19 & 39,500 & $\mathrm{E}$ & 29.74 & \\
\hline 1988 & Jan. 20 & 12,500 & $\mathrm{E}$ & 20.10 & \\
\hline 1989 & Mar. 6 & 21,800 & & 24.68 & \\
\hline 1990 & Feb. 16 & 65,900 & & 33.88 & \\
\hline
\end{tabular}

02424000 Cahaba River at Centreville-Continued

L ocation-Lat $32^{\circ} 56^{\prime} 42^{\prime \prime}$, long $87^{\circ} 08^{\prime} 21^{\prime \prime}$, in SE $1 / 4 \mathrm{sec}$. 26, T. 23 N. R. 9 E., Bibb County, Hydrologic Unit 03150202, 60 $\mathrm{ft}$ downstream from U.S. Highway 82 bridge. $0.2 \mathrm{mi}$ west of Centreville, $2.5 \mathrm{mi}$ upstream from Sandy Creek, and at mile 81.2.

Drainage area-1,027 $\mathrm{mi}^{2}$.

G age - Water-stage recorder. Datum of gage is $180.74 \mathrm{ft}$ NGVD 29. Prior to Jan. 31, 1939, nonrecording gage at same site. Prior to May 1929, at datum $1.15 \mathrm{ft}$ lower.

R emarks - Flow affected by regulation from Lake Purdy and diversion by Birmingham Water Works.

\begin{tabular}{|c|c|c|c|c|c|}
\hline $\begin{array}{l}\text { Water } \\
\text { year }\end{array}$ & Date & Discharge & $\begin{array}{l}\text { Discharge } \\
\text { code }\end{array}$ & $\begin{array}{c}\text { Gage } \\
\text { height } \\
\text { (feet) }\end{array}$ & $\begin{array}{c}\text { Gage } \\
\text { height } \\
\text { code }\end{array}$ \\
\hline 1991 & Feb. 20 & 26,300 & & 26.50 & \\
\hline 1992 & Sept. 4 & 11,600 & & 17.34 & \\
\hline 1993 & Jan. 21 & 18,800 & & 23.17 & \\
\hline 1994 & Apr. 16 & 13,900 & & 19.73 & \\
\hline 1995 & Feb. 11 & 18,200 & & 22.81 & \\
\hline 1996 & Mar. 7 & 33,400 & & 28.42 & \\
\hline 1997 & Mar. 1 & 21,200 & & 24.34 & \\
\hline 1998 & Jan. 8 & 41,800 & & 30.21 & \\
\hline 1999 & Jan. 31 & 24,400 & & 25.76 & \\
\hline 2000 & Apr. 4 & 36,300 & & 29.09 & \\
\hline 2001 & Apr. 5 & 18,900 & & 23.22 & \\
\hline 2002 & Jan. 25 & 18,300 & & 22.10 & \\
\hline 2003 & May 19 & 24,200 & & 25.47 & \\
\hline 2004 & Sept. 18 & 20,700 & & 23.73 & \\
\hline 2005 & Nov. 24 & 23,500 & & 25.12 & \\
\hline 2006 & Mar. 21 & 14,600 & & 18.98 & \\
\hline 2007 & Jan. 8 & 6,450 & & 10.97 & \\
\hline 2008 & Feb. 22 & 12,300 & & 16.93 & \\
\hline 2009 & Sept. 21 & 32,600 & & 28.22 & \\
\hline 2010 & Mar. 11 & 40,700 & & 29.98 & \\
\hline 2011 & Mar. 10 & 30,000 & & 27.56 & \\
\hline 2012 & Mar. 3 & 16,000 & & 20.20 & \\
\hline 2013 & Feb. 12 & 15,300 & & 19.62 & \\
\hline 2014 & Apr. 7 & 53,200 & & 32.23 & \\
\hline 2015 & Dec. 28 & 26,100 & & 26.29 & \\
\hline
\end{tabular}


Appendix 2. Discharge and gage height data for streamgages in Alabama.-Continued

[--, date or data not available; ft, foot; $\mathrm{ft}^{3} / \mathrm{s}$, cubic foot per second; mi, mile; $\mathrm{mi}^{2}$, square mile; qualification codes apply to the discharge data: 1 , discharge is a maximum daily average; 2 , discharge is an estimate; 4 , discharge is less than indicated value, which is the minimum recordable discharge at this site; 5 , discharge affected to unknown degree by regulation or diversion; 6 , discharge affected by regulation or diversion; 7, discharge is a historic peak; A, year of occurrence is unknown or not exact; $\mathrm{B}$, month or day of occurrence is unknown or not exact; E, only maximum peak available for this year. qualification codes apply to the gage height data: 1, gage height affected by backwater; 2, gage height not the maximum for the year; 3, gage height at different data or at different site and datum;

5 , gage height is an estimate]

\section{Barn Creek near Hackleburg}

L ocation-Lat $34^{\circ} 10^{\prime} 34^{\prime \prime}$, long $87^{\circ} 47^{\prime} 21^{\prime \prime}$, in NW 1/4 sec. 22 , T. 10 S., R. 12 W., Marion County, Hydrologic Unit 03160103, on county road, 4 mi upstream from mouth, and $8 \mathrm{mi}$ southeast of Hackleburg.

Drainage area-13.1 $\mathrm{mi}^{2}$.

Gage -Water-stage recorder 1959-70. Crest-stage gage 1971-73. Datum of gage is $575 \mathrm{ft} \mathrm{NGVD} 29$ (from topographic map).

\begin{tabular}{|c|c|c|c|c|c|}
\hline $\begin{array}{l}\text { Water } \\
\text { year }\end{array}$ & Date & Discharge & $\begin{array}{l}\text { Discharge } \\
\text { code }\end{array}$ & $\begin{array}{c}\text { Gage } \\
\text { height } \\
\text { (feet) }\end{array}$ & $\begin{array}{c}\text { Gage } \\
\text { height } \\
\text { code }\end{array}$ \\
\hline 1959 & Aug. 31 & 466 & 2 & -- & \\
\hline 1960 & Mar. 2 & 935 & & 6.84 & \\
\hline 1961 & Feb. 21 & 960 & & 6.33 & \\
\hline 1962 & Apr. 11 & 3,960 & & 13.39 & \\
\hline 1963 & May 26 & 2,480 & & 11.27 & \\
\hline 1964 & Apr. 7 & 1,010 & & 6.59 & \\
\hline 1965 & Mar. 25 & 972 & & 6.39 & \\
\hline 1966 & Apr. 21 & 606 & & 4.56 & \\
\hline 1967 & Apr. 26 & 1,220 & & 7.62 & \\
\hline 1968 & Dec. 18 & 3,110 & & 12.23 & \\
\hline 1969 & Feb. 2 & 926 & & 6.16 & \\
\hline 1970 & Apr. 25 & 2,000 & & 10.25 & \\
\hline 1971 & Feb. 26 & 1,030 & & 6.66 & \\
\hline 1972 & Jan. 4 & 982 & & 6.44 & \\
\hline 1973 & Mar. 16 & 5,160 & & 14.76 & \\
\hline
\end{tabular}

02438000 Buttahatchee River below Hamilton

L ocation-Lat $34^{\circ} 06^{\prime} 22^{\prime \prime}$, long $87^{\circ} 59^{\prime} 22^{\prime \prime}$, in NE $1 / 4$ sec. 15 , T. 11 S., R. 14 W., Marion County, Hydrologic Unit 03160103, on U.S. Highway 78, 0.5 mi downstream from Woods Creek, 2 mi south of Hamilton, and at mile 82.6.

Drainage area $-277 \mathrm{mi}^{2}$.

Gage - Water-stage recorder. Datum of gage is $360.50 \mathrm{ft}$ NGVD 29.

\begin{tabular}{llllll}
\hline $\begin{array}{c}\text { Water } \\
\text { year }\end{array}$ & Date & Discharge & $\begin{array}{c}\text { Discharge } \\
\text { code }\end{array}$ & $\begin{array}{c}\text { Gage } \\
\text { height } \\
\text { (feet) }\end{array}$ & $\begin{array}{c}\text { Gage } \\
\text { height } \\
\text { code }\end{array}$ \\
\hline 1951 & Mar. 29 & 24,200 & & 26.30 & \\
1952 & Dec. 8 & 18,900 & & 23.46 & \\
1953 & Apr. 30 & 15,000 & & 20.10 &
\end{tabular}

1954 Jan. $22 \quad 15,200 \quad 20.30$

1955 Mar. $21 \quad 17,200 \quad 22.10$

$1956 \quad$ Feb. $3 \quad 13,500 \quad 18.90$

1957 Jan. $31 \quad 11,000 \quad 16.60$

$1958 \quad$ Nov. $17 \quad 10,200 \quad 15.80$

$1959 \quad$ Feb. $13 \quad 4,770 \quad 9.50$

1960 Mar. $2 \quad 15,100 \quad 20.20$

1961 Feb. $21 \quad 15,400 \quad 20.58$

1962 Apr. $11 \quad 23,000 \quad 26.75$

1963 May $26 \quad 21,900 \quad 25.90$

1964 Apr. $13 \quad 10,600 \quad 16.18$

1965 Feb. $12 \quad 14,500 \quad 19.84$

1966 Apr. $21 \quad 7,250 \quad 12.65$

1967 Apr. $26 \quad 11,000 \quad 16.65$

1968 Dec. $18 \quad 27,900 \quad 28.33$

1969 Feb. $2 \quad 13,500 \quad 18.90$

1970 Dec. $30 \quad 23,300 \quad 26.13$

$1971 \quad$ Feb. $26 \quad 15,600 \quad 20.71$

1972 Jan. $4 \quad 15,400 \quad 20.61$

1973 Mar. $16 \quad 49,500 \quad 35.49$

1975 Mar. $13 \quad 24,000 \quad 26.95$

1976 Oct. $17 \quad 16,200 \quad 22.92$

1977 Mar. $4 \quad 27,700 \quad 28.23$

1978 May $8 \quad 14,500 \quad 21.39$

1979 Mar. 4 16,200 21.22

1980 Mar. $17 \quad 19,000 \quad 23.39$

$1981 \quad$ Mar. $30 \quad 8,000 \quad 13.98$

1982 Jan. $3 \quad 17,600 \quad 22.32$

1983 May $19 \quad 23,500 \quad$ E 26.56 
Appendix 2. Discharge and gage height data for streamgages in Alabama._-Continued

[--, date or data not available; $\mathrm{ft}$, foot; $\mathrm{ft}^{3} / \mathrm{s}$, cubic foot per second; mi, mile; $\mathrm{mi}^{2}$, square mile; qualification codes apply to the discharge data: 1 , discharge is a maximum daily average; 2 , discharge is an estimate; 4 , discharge is less than indicated value, which is the minimum recordable discharge at this site; 5 , discharge affected to unknown degree by regulation or diversion; 6 , discharge affected by regulation or diversion; 7, discharge is a historic peak; A, year of occurrence is unknown or not exact; B, month or day of occurrence is unknown or not exact; E, only maximum peak available for this year. qualification codes apply to the gage height data: 1, gage height affected by backwater; 2, gage height not the maximum for the year; 3, gage height at different datum or at different site and datum; 5 , gage height is an estimate]

02438000 Buttahatchee River below Hamilton-Continued

L ocation-Lat $34^{\circ} 06^{\prime} 22^{\prime \prime}$, long $87^{\circ} 59^{\prime} 22^{\prime \prime}$, in NE 1/4 sec. 15, T. 11 S., R. 14 W., Marion County, Hydrologic Unit 03160103, on U.S. Highway 78, 0.5 mi downstream from Woods Creek, 2 mi south of Hamilton, and at mile 82.6.

Drainage area $-277 \mathrm{mi}^{2}$.

G age - Water-stage recorder. Datum of gage is $360.50 \mathrm{ft}$ NGVD 29.

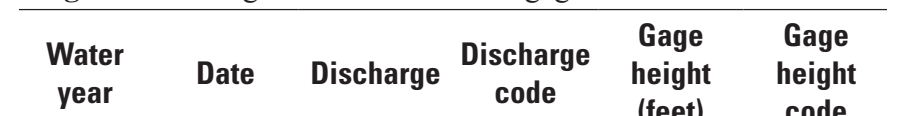

\begin{tabular}{llrrr}
\hline 1984 & Dec. 3 & 19,900 & E & 24.10 \\
1985 & May 2 & 21,600 & & 25.10 \\
1986 & May 28 & 5,550 & & 10.58
\end{tabular}

1986

1987

1988

1989

Nov. 26

5,550

10.58

19.71

13.32

21.99

Feb. $28 \quad$ 15,300

1990

Oct. 1

12,900

1991

Dec. 23

29,000

19.90

28.87

1992

1993

Dec. 22,700

Mar. 23

11,300

26.24

18.25

1994

Feb. 11

20,600

1995

Feb. 16

13,300

25.27

20.29

13.05

1997

Dec. 18

6,890

25.92

22.54

21.97

20.29

23.40

24.38

22.06

24.93

26.95

14.81

12.12

16.70

21.53

18.97

22.57

12.43

16.07

16.03

2014

Feb. 21

9,500

9,470

July 4

12,300

$2015 \quad$ July $4 \quad 12,300 \quad 19.26$

02450000 Mulberry Fork near Garden City

L ocation-Lat $33^{\circ} 59^{\prime} 42^{\prime \prime}$, long $86^{\circ} 44^{\prime} 56^{\prime \prime}$, in NE $1 / 4$ sec. 16 , T. 12 S., R. 2 W., Blount County, Hydrologic Unit 03160109, on U.S. Highway 31 (old), 1 mi southwest of Garden City, $5.5 \mathrm{mi}$ downstream from Mud Creek, and at mile 79.2.

Drainage area-365 $\mathrm{mi}^{2}$.

G age -Water-stage recorder. Datum of gage is $380.54 \mathrm{ft}$ NGVD 29.

\begin{tabular}{lccccc}
\hline $\begin{array}{c}\text { Water } \\
\text { year }\end{array}$ & Date & Discharge & $\begin{array}{c}\text { Discharge } \\
\text { code }\end{array}$ & $\begin{array}{c}\text { Gage } \\
\text { height } \\
\text { (feet) }\end{array}$ & $\begin{array}{c}\text { Gage } \\
\text { height } \\
\text { code }\end{array}$ \\
\hline 1929 & Mar. 14 & 26,000 & & 16.40 & \\
1930 & Nov. 14 & 31,900 & & 17.96 & \\
1931 & Nov. 16 & 5,700 & 8.59 & \\
1932 & Dec. 14 & 13,500 & 12.20 & \\
1933 & Oct. 16 & 25,500 & 16.30 &
\end{tabular}

1934 Mar. $3 \quad 13,500 \quad 12.18$

1935 Mar. $12 \quad 14,800 \quad 12.35$

1936 Feb. $4 \quad 60,000 \quad 24.00$

$1937 \quad$ Apr. $29 \quad 27,500 \quad 16.80$

1938 Apr. $8 \quad 24,600 \quad 16.00$

$1939 \quad$ Feb. $28 \quad 45,000 \quad 20.77$

$1940 \quad$ July $13 \quad 20,500 \quad 14.58$

$1941 \quad$ Aug. $1 \quad 41,000 \quad 20.13$

1942 Aug. $19 \quad 11,800 \quad 11.51$

1943 Dec. $28 \quad 57,500 \quad 23.62$

$1944 \quad$ Mar. $28 \quad 25,500 \quad 16.32$

$1945 \quad$ Feb. $13 \quad 19,500 \quad 14.40$

$1946 \quad$ Jan. $8 \quad 42,000 \quad 20.30$

$1947 \quad$ Jan. $20 \quad 26,000 \quad 16.34$

$1948 \quad$ Feb. $12 \quad 32,000 \quad 17.90$

$1949 \quad$ Jan. $5 \quad 43,000 \quad 20.60$

$1950 \quad$ Mar. $13 \quad 32,000 \quad 18.10$

1951 Mar. $29 \quad 43,500 \quad 20.62$

1952 Dec. $21 \quad 17,200 \quad 13.75$

1953 Apr. $30 \quad 20,000 \quad 14.50$

1954 Jan. $16 \quad 22,500 \quad 15.40$

$1955 \quad$ Mar. $22 \quad 22,500 \quad 15.40$

$1956 \quad$ Feb. $20 \quad 13,000 \quad 12.30$

1957 Jan. $31 \quad 17,500 \quad 13.70$

1958 Nov. $18 \quad 32,000 \quad 18.10$ 
Appendix 2. Discharge and gage height data for streamgages in Alabama._- Continued

[--, date or data not available; ft, foot; $\mathrm{ft}^{3} / \mathrm{s}$, cubic foot per second; mi, mile; $\mathrm{mi}^{2}$, square mile; qualification codes apply to the discharge data: 1 , discharge is a maximum daily average; 2 , discharge is an estimate; 4 , discharge is less than indicated value, which is the minimum recordable discharge at this site; 5 , discharge affected to unknown degree by regulation or diversion; 6 , discharge affected by regulation or diversion; 7, discharge is a historic peak; A, year of occurrence is unknown or not exact; $\mathrm{B}$, month or day of occurrence is unknown or not exact; E, only maximum peak available for this year. qualification codes apply to the gage height data: 1 , gage height affected by backwater; 2, gage height not the maximum for the year; 3, gage height at different data or at different site and datum;

5 , gage height is an estimate]

\section{$\mathbf{0 2 4 5 0 0 0 0}$ Mulberry Fork near Garden City-Continued}

L ocation-Lat $33^{\circ} 59^{\prime} 42^{\prime \prime}$, long $86^{\circ} 44^{\prime} 56^{\prime \prime}$, in NE $1 / 4$ sec. 16 , T. 12 S., R. 2 W., Blount County, Hydrologic Unit 03160109, on U.S. Highway 31 (old), $1 \mathrm{mi}$ southwest of Garden City, $5.5 \mathrm{mi}$ downstream from Mud Creek, and at mile 79.2.

Drainage area-365 $\mathrm{mi}^{2}$.

Gage - Water-stage recorder. Datum of gage is $380.54 \mathrm{ft}$ NGVD 29.

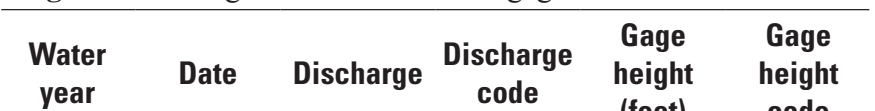

\begin{tabular}{lllll} 
& & & (feet) & code \\
\hline 1959 & Jan. 21 & 14,700 & 12.70 \\
1960 & Mar. 3 & 23,900 & 15.85 \\
1961 & Feb. 22 & 34,000 & 18.54 \\
1962 & Dec. 18 & 45,100 & 20.93 \\
1963 & Apr. 30 & 22,500 & 15.42
\end{tabular}

1964

1965

1966

1967

Apr. 13

45,000

Mar. 26

35,000

Mar. 4 33,000

Apr. 26

21,000

1969

May 18

26,250

1970

1971

Apr. 26

23,750

1972

July 16

46,000

Jan. 4

21,900

1973

Mar. 16

34,000

1974

Dec. 26

46,000

Mar. 13

32,500

1976

May 14

13,400

1977

Mar. 12

39,950

1978

Oct. 26

6,100

1979

Mar. 4

46,500

1980

Mar. 21

37,000

1981

1982

Mar. 30

7,240

Jan. 3

53,000

1983

May 19

16,800

1984

May 3

28,100

1985

July 27

21,000

Mar. 13

5,460

1987

$$
17,700
$$

20.81

18.82

18.27

14.92

16.47

15.78

20.97

16.00

18.40

20.95

17.96

12.18

19.62

8.94

20.99

19.27

9.43

22.45

13.55

17.00

14.96

8.41

13.90
02450000 Mulberry Fork near Garden City-Continued

L ocation-Lat $33^{\circ} 59^{\prime} 42^{\prime \prime}$, long 86 $44^{\prime} 56^{\prime \prime}$, in NE 1/4 sec. 16, T. 12 S., R. 2 W., Blount County, Hydrologic Unit 03160109, on U.S. Highway 31 (old), 1 mi southwest of Garden City, $5.5 \mathrm{mi}$ downstream from Mud Creek, and at mile 79.2.

Drainage area- $365 \mathrm{mi}^{2}$.

G age - Water-stage recorder. Datum of gage is $380.54 \mathrm{ft}$ NGVD 29.

\begin{tabular}{lllccc}
\hline $\begin{array}{c}\text { Water } \\
\text { year }\end{array}$ & Date & Discharge & $\begin{array}{c}\text { Discharge } \\
\text { code }\end{array}$ & $\begin{array}{c}\text { Gage } \\
\text { height } \\
\text { (feet) }\end{array}$ & $\begin{array}{c}\text { Gage } \\
\text { height } \\
\text { code }\end{array}$ \\
\hline 1988 & Jan. 20 & 13,600 & & 12.30 & \\
1989 & Feb. 28 & 21,500 & & 15.42 & \\
1990 & Feb. 16 & 67,000 & & 25.04 &
\end{tabular}

1991 Dec. $23 \quad 29,600 \quad 17.18$

$1992 \quad$ Feb. $15 \quad 8,200 \quad 9.93$

$1993 \quad$ Jan. $12 \quad 17,800 \quad 13.92$

1994 Feb. $11 \quad 49,600 \quad 21.97$

$1995 \quad$ Feb. $16 \quad 15,600 \quad 13.12$

1996 Mar. $7 \quad 19,400 \quad 14.48$

1997 June $22 \quad 33,700 \quad 18.42$

$1998 \quad$ Jan. $7 \quad 42,000 \quad 20.08$

1999 Jan. $23 \quad 29,000 \quad 17.12$

2001 Mar. $20 \quad 24,700 \quad 16.07$

2002 Jan. $25 \quad 28,200 \quad 17.02$

2003 Feb. $27 \quad 22,200 \quad 15.33$

2004 Feb. $6 \quad 36,500 \quad 19.08$

2005 Dec. $9 \quad 35,800 \quad 18.93$

$2006 \quad$ Jan. $17 \quad 7,760 \quad 9.78$

2007 Oct. $20 \quad 9,680 \quad 10.66$

$2008 \quad$ Feb. $6 \quad 8,480 \quad 10.13$

$2009 \quad$ Jan. $6 \quad 65,200 \quad 24.81$

2010 May $3 \quad 46,100 \quad 21.24$

2011 Mar. $9 \quad 47,100 \quad 21.46$

$2012 \quad$ Jan. $11 \quad 19,500 \quad 14.49$

2013 Jan. $15 \quad 25,200 \quad 21.04$

2014 Apr. $7 \quad 22,500 \quad 19.99$

2015 Jan. $4 \quad 16,000 \quad 17.13$

$2016 \quad--\quad 90,000$ 
Appendix 2. Discharge and gage height data for streamgages in Alabama.-Continued

[--, date or data not available; $\mathrm{ft}$, foot; $\mathrm{ft}^{3} / \mathrm{s}$, cubic foot per second; mi, mile; $\mathrm{mi}^{2}$, square mile; qualification codes apply to the discharge data: 1 , discharge is a maximum daily average; 2 , discharge is an estimate; 4 , discharge is less than indicated value, which is the minimum recordable discharge at this site; 5 , discharge affected to unknown degree by regulation or diversion; 6 , discharge affected by regulation or diversion; 7, discharge is a historic peak; A, year of occurrence is unknown or not exact; $\mathrm{B}$, month or day of occurrence is unknown or not exact; E, only maximum peak available for this year. qualification codes apply to the gage height data: 1, gage height affected by backwater; 2, gage height not the maximum for the year; 3, gage height at different datum or at different site and datum; 5 , gage height is an estimate]

\section{$\mathbf{0 2 4 5 0 1 8 0}$ Mulberry Fork near Arkadelphia}

L ocation-Lat $33^{\circ} 52^{\prime} 19^{\prime \prime}$, long $86^{\circ} 55^{\prime} 20^{\prime \prime}$, in NE 1/4 sec. 35 , T. 13 S., R. 4 W., Blount County, Hydrologic Unit 03160109, 200ft upstream from county road, $4 \mathrm{mi}$ south of Arkadelphia, and at mile 58.6.

Drainage area $-487 \mathrm{mi}^{2}$.

G age - Water-stage recorder. Datum of gage is $270.23 \mathrm{ft}$ NGVD 29.

\begin{tabular}{|c|c|c|c|c|c|}
\hline $\begin{array}{c}\text { Water } \\
\text { year }\end{array}$ & Date & Discharge & $\begin{array}{c}\text { Discharge } \\
\text { code }\end{array}$ & $\begin{array}{c}\text { Gage } \\
\text { height } \\
\text { (feet) }\end{array}$ & $\begin{array}{c}\text { Gage } \\
\text { height } \\
\text { code }\end{array}$ \\
\hline 1977 & Mar. 13 & 23,400 & & 34.28 & \\
\hline 1978 & Oct. 26 & 12,900 & & 26.28 & \\
\hline 1979 & Apr. 14 & 37,000 & & 39.11 & \\
\hline 1981 & Mar. 30 & 6,430 & & 18.36 & \\
\hline 1982 & Jan. 4 & 28,000 & & 36.32 & \\
\hline 1983 & May 20 & 13,600 & & 27.60 & \\
\hline 1984 & May 4 & 17,100 & & 30.06 & \\
\hline 1985 & July 28 & 13,300 & & 27.59 & \\
\hline 1986 & Mar. 13 & 5,050 & & 13.68 & \\
\hline 1989 & Jan. 13 & 18,400 & & 29.94 & \\
\hline 1990 & Feb. 16 & 51,700 & & 42.90 & \\
\hline 1991 & Feb. 20 & 20,000 & & 30.50 & \\
\hline 1992 & Feb. 25 & 7,800 & & 19.20 & \\
\hline 1993 & Jan. 12 & 13,300 & & 26.19 & \\
\hline 1994 & Feb. 11 & 32,300 & & 36.27 & \\
\hline 1995 & Feb. 17 & 12,700 & & 25.70 & \\
\hline 1996 & Jan. 27 & 16,500 & & 28.46 & \\
\hline 1997 & June 23 & 15,300 & & 27.61 & \\
\hline 1998 & Jan. 8 & 30,000 & 2 & 35.30 & 5 \\
\hline 1999 & Jan. 23 & 17,600 & & 29.90 & \\
\hline 2000 & Apr. 4 & 25,100 & & 33.16 & \\
\hline 2001 & Mar. 21 & 17,100 & & 28.86 & \\
\hline 2002 & Jan. 25 & 18,400 & & 29.67 & \\
\hline 2003 & Feb. 22 & 13,300 & & 26.18 & \\
\hline 2004 & Feb. 6 & 25,000 & & 33.15 & \\
\hline 2005 & Dec. 10 & 18,800 & & 29.92 & \\
\hline 2006 & May 11 & 6,910 & & 17.27 & \\
\hline 2007 & Jan. 1 & 6,540 & & 16.47 & \\
\hline 2008 & Feb. 6 & 6,120 & & 15.56 & \\
\hline 2009 & Jan. 7 & 44,500 & & 40.65 & \\
\hline 2010 & May 3 & 24,600 & & 32.95 & \\
\hline 2011 & Mar. 10 & 31,600 & & 36.00 & \\
\hline 2012 & Jan. 12 & 15,900 & & 28.17 & \\
\hline
\end{tabular}

02450180 Mulberry Fork near Arkadelphia-Continued

L ocation-Lat $33^{\circ} 52^{\prime} 19^{\prime \prime}$, long $86^{\circ} 55^{\prime} 20^{\prime \prime}$, in NE 1/4 sec. 35 , T. 13 S., R. 4 W., Blount County, Hydrologic Unit 03160109, $200 \mathrm{ft}$ upstream from county road, 4 mi south of Arkadelphia, and at mile 58.6.

Drainage area-487 $\mathrm{mi}^{2}$.

G age - Water-stage recorder. Datum of gage is $270.23 \mathrm{ft}$ NGVD 29.

\begin{tabular}{lllccc}
\hline $\begin{array}{c}\text { Water } \\
\text { year }\end{array}$ & Date & Discharge & $\begin{array}{c}\text { Discharge } \\
\text { code }\end{array}$ & $\begin{array}{c}\text { Gage } \\
\text { height } \\
\text { (feet) }\end{array}$ & $\begin{array}{c}\text { Gage } \\
\text { height } \\
\text { code }\end{array}$ \\
\hline 2013 & Jan. 15 & 26,000 & & 34.14 & \\
2014 & Apr. 7 & 19,400 & & 30.24 & \\
2015 & Jan. 4 & 14,500 & & 26.76 & \\
2016 & Dec. 26 & 14,000 & & 26.44 &
\end{tabular}

02450200 Dorsey Creek near Arkadelphia

L ocation-Lat $33^{\circ} 57^{\prime} 10^{\prime \prime}$, long $87^{\circ} 00^{\prime} 14^{\prime \prime}$, in SW 1/4 sec. 31 , T. 12 S., R. 4 W., Cullman County, Hydrologic Unit 03160109, on county road, 4 mi northwest of Arkadelphia, and 8 mi upstream from mouth.

Drainage area-13.0 $\mathrm{mi}^{2}$.

G age -Water-stage recorder 1959-67. Crest-stage gage 1968-74. Datum of gage is $430 \mathrm{ft}$ NGVD 29.

\begin{tabular}{|c|c|c|c|c|c|}
\hline $\begin{array}{l}\text { Water } \\
\text { year }\end{array}$ & Date & Discharge & $\begin{array}{l}\text { Discharge } \\
\text { code }\end{array}$ & $\begin{array}{c}\text { Gage } \\
\text { height } \\
\text { (feet) }\end{array}$ & $\begin{array}{c}\text { Gage } \\
\text { height } \\
\text { code }\end{array}$ \\
\hline 1959 & Jan. 21 & 894 & & 5.20 & \\
\hline 1960 & Mar. 2 & 1,370 & & 6.64 & \\
\hline 1961 & Feb. 20 & 2,550 & & 9.41 & \\
\hline 1962 & Apr. 11 & 2,630 & & 9.58 & \\
\hline 1963 & Mar. 12 & 1,040 & & 5.74 & \\
\hline 1964 & Apr. 13 & 2,850 & & 10.02 & \\
\hline 1965 & Mar. 26 & 1,740 & & 7.53 & \\
\hline 1966 & Mar. 3 & 2,140 & & 8.48 & \\
\hline 1967 & July 7 & 1,030 & & 5.71 & \\
\hline 1968 & Jan. 10 & 1,730 & & 7.50 & \\
\hline 1969 & Apr. 10 & 834 & & 5.13 & \\
\hline 1970 & Mar. 19 & 1,310 & & 6.45 & \\
\hline 1971 & Feb. 26 & 1,570 & & 7.11 & \\
\hline 1972 & Jan. 4 & 865 & & 5.22 & \\
\hline 1973 & Mar. 16 & 2,090 & & 8.37 & \\
\hline 1974 & Dec. 26 & 2,480 & & 9.25 & \\
\hline
\end{tabular}


Appendix 2. Discharge and gage height data for streamgages in Alabama.-Continued

[--, date or data not available; $\mathrm{ft}$, foot; $\mathrm{ft}^{3} / \mathrm{s}$, cubic foot per second; $\mathrm{mi}$, mile; $\mathrm{mi}^{2}$, square mile; qualification codes apply to the discharge data: 1 , discharge is a maximum daily average; 2 , discharge is an estimate; 4 , discharge is less than indicated value, which is the minimum recordable discharge at this site; 5 , discharge affected to unknown degree by regulation or diversion; 6 , discharge affected by regulation or diversion; 7, discharge is a historic peak; A, year of occurrence is unknown or not exact; B, month or day of occurrence is unknown or not exact; E, only maximum peak available for this year. qualification codes apply to the gage height data: 1, gage height affected by backwater; 2 , gage height not the maximum for the year; 3 , gage height at different data or at different site and datum; 5 , gage height is an estimate]

\section{Sipsey Fork near Grayson}

L ocation-Lat $34^{\circ} 17^{\prime} 07^{\prime \prime}$, long $87^{\circ} 23^{\prime} 56^{\prime \prime}$, in NW $1 / 4 \mathrm{sec} .8$, T. 9 S., R. 8 W., Winston County, Hydrologic Unit 03160110, Bankhead National Forest, on Cranal Road, 0.5 mi downstream from Borden Creek, $4.5 \mathrm{mi}$ west of Grayson, 14 mi northeast of Haleyville, and 64.1 mi upstream from mouth.

Drainage area $-92.1 \mathrm{mi}^{2}$.

Gage - Water-stage recorder. Datum of gage is $540 \mathrm{ft} \mathrm{NGVD} 29$ (from topographic map).

\begin{tabular}{|c|c|c|c|c|c|}
\hline $\begin{array}{c}\text { Water } \\
\text { year }\end{array}$ & Date & Discharge & $\begin{array}{l}\text { Discharge } \\
\text { code }\end{array}$ & $\begin{array}{c}\text { Gage } \\
\text { height } \\
\text { (feet) }\end{array}$ & $\begin{array}{c}\text { Gage } \\
\text { height } \\
\text { code }\end{array}$ \\
\hline 1967 & Dec. 9 & 6,600 & & 24.48 & \\
\hline 1968 & Dec. 18 & 14,200 & & 38.29 & \\
\hline 1969 & Feb. 2 & 6,700 & & 24.54 & \\
\hline 1970 & Dec. 30 & 11,000 & & 33.48 & \\
\hline 1971 & Feb. 26 & 7,000 & & 25.10 & \\
\hline 1972 & Jan. 4 & 6,030 & & 23.20 & \\
\hline 1973 & Mar. 16 & 20,300 & & 44.27 & \\
\hline 1974 & Feb. 2 & 4,260 & & 18.70 & \\
\hline 1975 & Jan. 10 & 2,850 & & 14.50 & \\
\hline 1976 & Oct. 17 & 13,000 & & 36.20 & \\
\hline 1977 & Mar. 4 & 11,000 & & 33.54 & \\
\hline 1978 & May 8 & 7,980 & & 26.98 & \\
\hline 1979 & Apr. 13 & 6,700 & & 24.69 & \\
\hline 1980 & Mar. 7 & 7,700 & & 26.93 & \\
\hline 1981 & Mar. 30 & 3,100 & & 15.25 & \\
\hline 1982 & Jan. 3 & 8,100 & & 27.87 & \\
\hline 1983 & May 19 & 11,100 & & 33.66 & \\
\hline 1984 & May 3 & 10,900 & & 33.37 & \\
\hline 1985 & May 1 & 6,900 & & 25.00 & \\
\hline 1986 & May 28 & 3,110 & & 15.24 & \\
\hline 1987 & Jan. 19 & 5,600 & & 21.93 & \\
\hline 1988 & Jan. 19 & 4,650 & & 19.68 & \\
\hline 1989 & Mar. 5 & 8,000 & & 27.55 & \\
\hline 1990 & Feb. 16 & 5,070 & & 20.52 & \\
\hline 1991 & Dec. 23 & 11,300 & & 33.66 & \\
\hline
\end{tabular}

02450250 Sipsey Fork near Grayson-Continued

L ocation-Lat $34^{\circ} 17^{\prime} 07^{\prime \prime}$, long $87^{\circ} 23^{\prime} 56^{\prime \prime}$, in NW 1/4 sec. 8, T. 9 S., R. 8 W., Winston County, Hydrologic Unit 03160110, Bankhead National Forest, on Cranal Road, 0.5 mi downstream from Borden Creek, 4.5 mi west of Grayson, 14 mi northeast of Haleyville, and 64.1 mi upstream from mouth.

Drainage area $-92.1 \mathrm{mi}^{2}$.

G age - Water-stage recorder. Datum of gage is $540 \mathrm{ft} \mathrm{NGVD} 29$ (from topographic map).

\begin{tabular}{llcccc}
\hline $\begin{array}{c}\text { Water } \\
\text { year }\end{array}$ & Date & Discharge & $\begin{array}{c}\text { Discharge } \\
\text { code }\end{array}$ & $\begin{array}{c}\text { Gage } \\
\text { height } \\
\text { (feet) }\end{array}$ & $\begin{array}{c}\text { Gage } \\
\text { height } \\
\text { code }\end{array}$ \\
\hline 1992 & Dec. 2 & 5,880 & & 22.48 & \\
1993 & Mar. 23 & 7,110 & & 25.37 & \\
1994 & Feb. 11 & 9,070 & & 29.66 & \\
1995 & Feb. 17 & 10,000 & 2 & 31.60 & 5
\end{tabular}

$1996 \quad$ Mar. $6 \quad 4,680 \quad 19.49$

1997 May $3 \quad 10,100 \quad 31.77$

$1998 \quad$ Jan. $7 \quad 9,130 \quad 29.79$

$1999 \quad$ Jan. $23 \quad 13,500 \quad 36.78$

2000 Apr. $3 \quad 6,970 \quad 25.04$

2001 Apr. $3 \quad 7,300 \quad 25.84$

2002 Dec. $14 \quad 8,500 \quad 28.68$

2003 Feb. $22 \quad 8,660 \quad 29.02$

2004 Feb. $6 \quad 10,800 \quad 33.13$

2005 Dec. $7 \quad 6,940 \quad 25.18$

$2006 \quad$ Jan. $17 \quad 4,560 \quad 19.33$

2007 Dec. $31 \quad 3,020 \quad 15.04$

2008 Mar. $4 \quad 2,940 \quad 14.80$

$2009 \quad$ Sept. $17 \quad 5,750 \quad 22.35$

2010 Dec. $9 \quad 7,850 \quad 27.22$

$2011 \quad$ Jan. $1 \quad 9,340 \quad 30.45$

$2012 \quad$ Jan. $11 \quad 3,810 \quad 17.30$

2013 July $6 \quad 6,200 \quad 23.43$

$2014 \quad$ Feb. $21 \quad 4,760 \quad 19.85$

2015 Dec. $24 \quad 5,880 \quad 22.65$ 
Appendix 2. Discharge and gage height data for streamgages in Alabama.-Continued

[--, date or data not available; $\mathrm{ft}$, foot; $\mathrm{ft}^{3} / \mathrm{s}$, cubic foot per second; mi, mile; $\mathrm{mi}^{2}$, square mile; qualification codes apply to the discharge data: 1 , discharge is a maximum daily average; 2 , discharge is an estimate; 4 , discharge is less than indicated value, which is the minimum recordable discharge at this site; 5 , discharge affected to unknown degree by regulation or diversion; 6 , discharge affected by regulation or diversion; 7, discharge is a historic peak; A, year of occurrence is unknown or not exact; $\mathrm{B}$, month or day of occurrence is unknown or not exact; E, only maximum peak available for this year. qualification codes apply to the gage height data: 1, gage height affected by backwater; 2, gage height not the maximum for the year; 3, gage height at different datum or at different site and datum;

5 , gage height is an estimate]

\section{Clear Creek at New Hope Church near Poplar Spring}

L ocation-Lat $34^{\circ} 04^{\prime} 52^{\prime \prime}$, long $87^{\circ} 16^{\prime} 22^{\prime \prime}$, in NE $1 / 4$ sec. 19 , T. 11 S., R. 8 W., Winston County Hydrologic Unit 03160110 , on left bank $150 \mathrm{ft}$ downstream of bridge on county road at New Hope Church, 4.5 mi northeast of Poplar Spring, and 6.1 mi southeast of Double Springs.

Drainage area-101 $\mathrm{mi}^{2}$.

G age - Water-stage recorder. Datum of gage is $565 \mathrm{ft}$ NGVD 29 (from topographic map).

\begin{tabular}{|c|c|c|c|c|c|}
\hline $\begin{array}{c}\text { Water } \\
\text { year }\end{array}$ & Date & Discharge & $\begin{array}{c}\text { Discharge } \\
\text { code }\end{array}$ & $\begin{array}{c}\text { Gage } \\
\text { height } \\
\text { (feet) }\end{array}$ & $\begin{array}{c}\text { Gage } \\
\text { height } \\
\text { code }\end{array}$ \\
\hline 1946 & 1946 & 10,000 & $2,7, \mathrm{~B}$ & -- & \\
\hline 1981 & Nov. 24 & 2,510 & & 6.49 & \\
\hline 1982 & Jan. 3 & 6,750 & 7 & 12.25 & \\
\hline 1983 & Mar. 5 & 9,680 & 7 & 15.74 & \\
\hline 1994 & Feb. 11 & 7,920 & & 13.67 & \\
\hline 1995 & Apr. 21 & 4,440 & & 9.25 & \\
\hline 1996 & Jan. 27 & 2,340 & & 6.14 & \\
\hline 1997 & May 3 & 9,000 & 2 & 15.00 & 5 \\
\hline 1998 & Jan. 7 & 9,190 & & 15.18 & \\
\hline 1999 & Jan. 23 & 11,500 & & 17.74 & \\
\hline 2000 & Apr. 3 & 6,580 & & 12.04 & \\
\hline 2001 & Apr. 4 & 9,740 & & 15.82 & \\
\hline 2002 & Dec. 14 & 6,480 & & 11.91 & \\
\hline 2003 & Feb. 22 & 7,160 & & 13.59 & \\
\hline 2004 & Feb. 6 & 4,040 & & 8.87 & \\
\hline 2005 & Dec. 7 & 5,690 & & 11.43 & \\
\hline 2006 & Jan. 17 & 3,080 & & 7.31 & \\
\hline 2007 & Nov. 7 & 2,920 & & 7.03 & \\
\hline 2008 & Mar. 4 & 1,950 & & 5.34 & \\
\hline 2009 & Jan. 7 & 5,160 & & 10.63 & \\
\hline 2010 & Dec. 9 & 7,070 & & 13.46 & \\
\hline 2011 & Jan. 1 & 7,150 & & 13.59 & \\
\hline 2012 & Jan. 11 & 4,090 & & 8.98 & \\
\hline 2013 & July 4 & 4,040 & & 8.90 & \\
\hline 2014 & Apr. 7 & 3,060 & & 7.31 & \\
\hline 2015 & Dec. 24 & 4,110 & & 9.01 & \\
\hline
\end{tabular}

02453950 Lost Creek near Jasper

L ocation-Lat $33^{\circ} 48^{\prime} 56^{\prime \prime}$, long $87^{\circ} 23^{\prime} 02^{\prime \prime}$, in NW 1/4 sec. 21, T. 14 S., R. 8 W., Walker County, Hydrologic Unit 03160109, at bridge on U.S. Highway 78, 6 mi west of Jasper.

Drainage area-115 $\mathrm{mi}^{2}$.

G age Crest-stage gage. Datum not available.

\begin{tabular}{|c|c|c|c|c|c|}
\hline $\begin{array}{c}\text { Water } \\
\text { year }\end{array}$ & Date & Discharge & $\begin{array}{c}\text { Discharge } \\
\text { code }\end{array}$ & $\begin{array}{c}\text { Gage } \\
\text { height } \\
\text { (feet) }\end{array}$ & $\begin{array}{c}\text { Gage } \\
\text { height } \\
\text { code }\end{array}$ \\
\hline 1951 & Mar. 29 & 11,600 & & 24.80 & \\
\hline 1952 & Mar. 11 & 4,900 & & 17.20 & \\
\hline 1954 & Jan. 16 & 7,100 & & 20.00 & \\
\hline 1955 & Feb. 6 & 8,100 & & 21.20 & \\
\hline 1956 & Mar. 15 & 5,100 & & 17.40 & \\
\hline 1957 & Apr. 5 & 5,300 & & 17.70 & \\
\hline 1958 & Nov. 19 & 10,000 & & 23.40 & \\
\hline 1959 & Jan. 21 & 2,990 & & 16.30 & \\
\hline 1960 & Mar. 3 & 6,800 & & 21.70 & \\
\hline 1961 & Feb. 23 & 11,900 & & 24.75 & \\
\hline 1962 & Dec. 18 & 8,800 & & 22.95 & \\
\hline 1963 & Mar. 13 & 3,600 & & 18.67 & \\
\hline 1964 & Apr. 14 & 10,100 & & 23.95 & \\
\hline 1965 & Feb. 12 & 6,530 & & 21.50 & \\
\hline 1966 & Apr. 28 & 4,050 & & 19.21 & \\
\hline 1967 & Feb. 20 & 3,070 & & 16.70 & \\
\hline 1968 & Jan. 10 & 9,060 & & 23.30 & \\
\hline 1969 & Feb. 2 & 3,090 & & 16.76 & \\
\hline 1970 & Mar. 20 & 6,940 & & 21.80 & \\
\hline
\end{tabular}


Appendix 2. Discharge and gage height data for streamgages in Alabama.-Continued

[--, date or data not available; ft, foot; $\mathrm{ft}^{3} / \mathrm{s}$, cubic foot per second; mi, mile; $\mathrm{mi}^{2}$, square mile; qualification codes apply to the discharge data: 1 , discharge is a maximum daily average; 2 , discharge is an estimate; 4 , discharge is less than indicated value, which is the minimum recordable discharge at this site; 5 , discharge affected to unknown degree by regulation or diversion; 6 , discharge affected by regulation or diversion; 7, discharge is a historic peak; A, year of occurrence is unknown or not exact; $\mathrm{B}$, month or day of occurrence is unknown or not exact; E, only maximum peak available for this year. qualification codes apply to the gage height data: 1 , gage height affected by backwater; 2, gage height not the maximum for the year; 3, gage height at different data or at different site and datum;

5 , gage height is an estimate]

\section{Lost Creek near Oakman}

L ocation-Lat $33^{\circ} 45^{\prime} 50^{\prime \prime}$, long $87^{\circ} 21^{\prime} 30^{\prime \prime}$, in SE $1 / 4$ sec. 3 , T. 15 S., R. 8 W., Walker County, Hydrologic Unit 03160109, on State Highway 69, 0.2 mi upstream from Wolf Branch, $0.8 \mathrm{mi}$ downstream from Pumpkin Creek, 4.0 mi northeast of Oakman, 6.5 mi southwest of Jasper, and at mile 24.8.

Drainage area-134 $\mathrm{mi}^{2}$.

Gage - Water-stage recorder. Datum of gage is $280 \mathrm{ft}$ NGVD 29 (from topographic map).

\begin{tabular}{|c|c|c|c|c|c|}
\hline $\begin{array}{l}\text { Water } \\
\text { year }\end{array}$ & Date & Discharge & $\begin{array}{l}\text { Discharge } \\
\text { code }\end{array}$ & $\begin{array}{c}\text { Gage } \\
\text { height } \\
\text { (feet) }\end{array}$ & $\begin{array}{c}\text { Gage } \\
\text { height } \\
\text { code }\end{array}$ \\
\hline 1951 & Mar. 29 & 13,300 & 2 & -- & \\
\hline 1952 & Dec. 21 & 3,550 & & 16.50 & \\
\hline 1953 & Feb. 22 & 4,000 & & 17.50 & \\
\hline 1954 & Jan. 16 & 4,100 & & 17.68 & \\
\hline 1955 & Feb. 7 & 4,500 & & 18.80 & \\
\hline 1956 & Feb. 4 & 3,200 & & 15.00 & \\
\hline 1957 & Feb. 1 & 3,290 & & 15.40 & \\
\hline 1958 & Nov. 19 & 7,700 & & 24.90 & \\
\hline 1959 & Jan. 21 & 3,260 & & 15.30 & \\
\hline 1960 & Mar. 3 & 5,180 & & 20.50 & \\
\hline 1961 & Feb. 23 & 19,400 & & 30.73 & \\
\hline 1962 & Dec. 18 & 7,600 & & 24.86 & \\
\hline 1963 & Mar. 19 & 3,550 & & 16.19 & \\
\hline 1964 & Apr. 13 & 10,100 & & 27.35 & \\
\hline 1965 & Feb. 12 & 4,960 & & 19.83 & \\
\hline 1966 & Apr. 28 & 4,210 & & 18.18 & \\
\hline 1967 & Feb. 20 & 3,370 & & 15.56 & \\
\hline 1968 & Jan. 11 & 7,600 & & 24.70 & \\
\hline 1969 & Feb. 2 & 3,500 & & 15.89 & \\
\hline 1970 & Mar. 20 & 6,700 & & 23.49 & \\
\hline 1979 & Apr. 13 & 10,800 & & 27.92 & \\
\hline 1980 & Mar. 21 & 5,350 & & 21.02 & \\
\hline 1981 & Mar. 31 & 3,600 & & 16.31 & \\
\hline
\end{tabular}

02454055 Lost Creek above Parrish, Ala.

L ocation-Lat $33^{\circ} 44^{\prime} 30^{\prime \prime}$, long $87^{\circ} 19^{\prime} 37^{\prime \prime}$ referenced to North American Datum of 1927, Walker County, AL, Hydrologic Unit 03160109, on downstream side of bridge on Browns Bridge Road, 1 mi north of County Road 20 (East), 3 mi northwest of Parrish, 3.4 mi west of Oakman, and $16.8 \mathrm{mi}$ above mouth.

Drainage area-143 $\mathrm{mi}^{2}$.

G age - Water-stage recorder. Elevation of gage is $280 \mathrm{ft}$ above NGVD of 1929, from topographic map.

\begin{tabular}{|c|c|c|c|c|c|}
\hline $\begin{array}{l}\text { Water } \\
\text { year }\end{array}$ & Date & Discharge & $\begin{array}{l}\text { Discharge } \\
\text { code }\end{array}$ & $\begin{array}{c}\text { Gage } \\
\text { height } \\
\text { (feet) }\end{array}$ & $\begin{array}{c}\text { Gage } \\
\text { height } \\
\text { code }\end{array}$ \\
\hline 1993 & Jan. 12 & 3,900 & & 18.36 & \\
\hline 1994 & Mar. 28 & 10,600 & & 29.48 & \\
\hline 1995 & Feb. 17 & 4,170 & & 18.63 & \\
\hline 1996 & Jan. 27 & 5,210 & & 20.72 & \\
\hline 1997 & May 4 & 6,210 & & 22.58 & \\
\hline 1998 & Jan. 8 & 7,290 & & 24.22 & \\
\hline 1999 & Jan. 24 & 5,240 & & 20.55 & \\
\hline 2000 & Apr. 4 & 6,650 & & 23.14 & \\
\hline 2001 & Apr. 5 & 5,990 & & 22.79 & \\
\hline 2002 & Dec. 15 & 6,520 & & 23.70 & \\
\hline 2003 & May 7 & 6,160 & & 23.08 & \\
\hline 2004 & Feb. 7 & 8,200 & & 26.07 & \\
\hline 2005 & Dec. 10 & 5,170 & & 21.30 & \\
\hline 2006 & Feb. 23 & 3,200 & & 16.74 & \\
\hline 2007 & Jan. 1 & 4,240 & & 18.22 & \\
\hline 2008 & Mar. 5 & 4,360 & & 18.47 & \\
\hline 2009 & Jan. 7 & 8,770 & & 29.58 & \\
\hline 2010 & Apr. 25 & 4,500 & & 20.62 & \\
\hline 2011 & Mar. 10 & 6,280 & & 24.64 & \\
\hline 2012 & Jan. 12 & 3,420 & & 17.88 & \\
\hline 2013 & Jan. 16 & 5,420 & & 22.76 & \\
\hline 2014 & Apr. 7 & 4,680 & & 21.04 & \\
\hline 2015 & Jan. 4 & 4,120 & & 19.69 & \\
\hline 2016 & Dec. 27 & 5,820 & & 25.72 & \\
\hline
\end{tabular}


Appendix 2. Discharge and gage height data for streamgages in Alabama.-Continued

[--, date or data not available; $\mathrm{ft}$, foot; $\mathrm{ft}^{3} / \mathrm{s}$, cubic foot per second; mi, mile; $\mathrm{mi}^{2}$, square mile; qualification codes apply to the discharge data: 1 , discharge is a maximum daily average; 2 , discharge is an estimate; 4 , discharge is less than indicated value, which is the minimum recordable discharge at this site; 5 , discharge affected to unknown degree by regulation or diversion; 6 , discharge affected by regulation or diversion; 7, discharge is a historic peak; A, year of occurrence is unknown or not exact; $\mathrm{B}$, month or day of occurrence is unknown or not exact; E, only maximum peak available for this year. qualification codes apply to the gage height data: 1, gage height affected by backwater; 2, gage height not the maximum for the year; 3 , gage height at different datum or at different site and datum; 5 , gage height is an estimate]

\section{Locust Fork near Cleveland}

L ocation-Lat $34^{\circ} 01^{\prime} 28^{\prime \prime}$, long $86^{\circ} 34^{\prime} 27^{\prime \prime}$, in NE $1 / 4$ sec. 6 , T.

12 S., R. 1 E., Blount County, Hydrologic Unit 03160111, 200

$\mathrm{ft}$ upstream from U.S. Highway 231, $2.5 \mathrm{mi}$ downstream from

Graves Creek, 3 mi north of Cleveland, and at mile 98.6.

Drainage area-303 $\mathrm{mi}^{2}$.

Gage -Water-stage recorder. Datum of gage is $536.94 \mathrm{ft}$ NGVD 29.

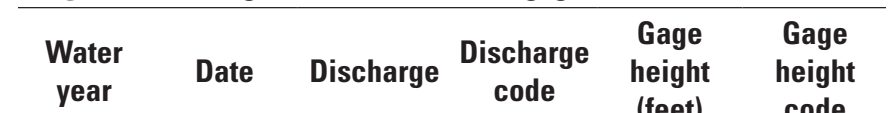

\begin{tabular}{llrrr} 
& & & (feet) & code \\
\hline 1941 & Aug. 1 & 17,000 & 14.00 & \\
1942 & Feb. 17 & 8,100 & 10.00
\end{tabular}

1943

1944

1945

1946

Dec. 28

34,000

Feb. 27

9,600

19.20

10.80

12.00

12.90

1947

Feb. 10

14,000

Jan. 20

11,500

1948

Feb. 14

10,000

Jan. 5

22,500

1950

Mar. 13

16,000

Mar. 29

18,000

Dec. 21

9,600

7,400

Jan. 10

11,700

1954

Jan. 16

7,800

Feb. 6

7,700

Feb. 3

5,600

1958

1959

1960

Nov. 19

5,600

Jan. 22

5,500

Mar. 3

8,100

Feb. 22

16,900

1962

1963

Dec. 18

13,500

Apr. 30

13,000

Apr. 14

15,000

1965

1966

Mar. 26

9,570

Mar. 4

9,400

1967

Apr. 26

9,000

Jan. 10

9,850

1969 May 18

6,100

1970
Mar. 20

7,650
11.76

11.00

15.90

13.70

14.54

10.90

9.60

11.80

9.80

9.70

8.60

8.60

8.40

10.00

13.91

12.62

12.51

--

10.70

10.61

10.49

10.94

8.88

9.66
02455000 Locust Fork near Cleveland-Continued

L ocation-Lat $34^{\circ} 01^{\prime} 28^{\prime \prime}$, long $86^{\circ} 34^{\prime} 27^{\prime \prime}$, in NE $1 / 4$ sec. 6 , T. 12 S., R. 1 E., Blount County, Hydrologic Unit 03160111, 200 ft upstream from U.S. Highway 231, 2.5 mi downstream from Graves Creek, 3 mi north of Cleveland, and at mile 98.6.

Drainage area-303 $\mathrm{mi}^{2}$.

G age - Water-stage recorder. Datum of gage is $536.94 \mathrm{ft}$ NGVD 29.

\begin{tabular}{cccccc}
\hline $\begin{array}{c}\text { Water } \\
\text { year }\end{array}$ & Date & Discharge & $\begin{array}{c}\text { Discharge } \\
\text { code }\end{array}$ & $\begin{array}{c}\text { Gage } \\
\text { height } \\
\text { (feet) }\end{array}$ & $\begin{array}{c}\text { Gage } \\
\text { height } \\
\text { code }\end{array}$ \\
\hline 1971 & July 16 & 13,900 & & 12.75 & \\
1972 & Jan 4 & 7,800 & & 9.80 &
\end{tabular}

1972 Jan. $4 \quad 7,800 \quad 9.80$

1973 May $28 \quad 8,600 \quad 10.29$

1974 Dec. $26 \quad 6,500 \quad 9.05$

1975 Mar. $14 \quad 8,700 \quad 10.36$

1976 Mar. $30 \quad 10,800 \quad 11.35$

1977 Mar. $13 \quad 17,500 \quad 14.13$

1978 May $9 \quad 8,100 \quad 9.99$

1979 Apr. $13 \quad 20,200 \quad 15.30$

1980 Mar. $17 \quad 12,800 \quad 12.35$

$1981 \quad$ Mar. $31 \quad 6,600 \quad 9.11$

1982 Jan. $4 \quad 26,400 \quad 17.05$

1983 Dec. $1 \quad 14,600 \quad 12.91$

1984 Dec. $28 \quad 12,300 \quad 11.88$

$\begin{array}{lllll}1985 & \text { Feb. } 1 & 7,550 & \mathrm{E} & 9.69\end{array}$

1986 May $28 \quad 6,400 \quad 9.00$

$1987 \quad$ Jan. $19 \quad 8,450 \quad 10.20$

1988 Sept. $18 \quad 5,400 \quad 8.34$

1989 Feb. $28 \quad 11,400 \quad 11.45$

$1990 \quad$ Feb. $16 \quad 24,400 \quad 16.46$

$1991 \quad$ Feb. $20 \quad 12,300 \quad 11.85$

$1992 \quad$ Feb. $26 \quad 7,200 \quad 9.45$

$1993 \quad$ Jan. $12 \quad 9,500 \quad 10.73$

1994 Mar. $28 \quad 9,000 \quad 10.44$

1995 Feb. $16 \quad 10,700 \quad 11.30$

1996 Oct. $5 \quad 16,800 \quad 13.76$

1997 May $3 \quad 13,300 \quad 13.96$

$1998 \quad$ Jan. $8 \quad 17,800 \quad 14.13$

1999 Jan. $23 \quad 8,500 \quad 10.22$

2000 Apr. $4 \quad 14,500 \quad 12.78$ 
Appendix 2. Discharge and gage height data for streamgages in Alabama.-Continued

[--, date or data not available; $\mathrm{ft}$, foot; $\mathrm{ft}^{3} / \mathrm{s}$, cubic foot per second; $\mathrm{mi}$, mile; $\mathrm{mi}^{2}$, square mile; qualification codes apply to the discharge data: 1 , discharge is a maximum daily average; 2 , discharge is an estimate; 4 , discharge is less than indicated value, which is the minimum recordable discharge at this site; 5 , discharge affected to unknown degree by regulation or diversion; 6 , discharge affected by regulation or diversion; 7, discharge is a historic peak; A, year of occurrence is unknown or not exact; $\mathrm{B}$, month or day of occurrence is unknown or not exact; E, only maximum peak available for this year. qualification codes apply to the gage height data: 1, gage height affected by backwater; 2 , gage height not the maximum for the year; 3 , gage height at different data or at different site and datum;

5 , gage height is an estimate]

\section{Locust Fork near Cleveland-Continued}

L ocation-Lat $34^{\circ} 01^{\prime} 28^{\prime \prime}$, long $86^{\circ} 34^{\prime} 27^{\prime \prime}$, in NE $1 / 4$ sec. 6 , T.

12 S., R. 1 E., Blount County, Hydrologic Unit 03160111, 200

$\mathrm{ft}$ upstream from U.S. Highway 231, $2.5 \mathrm{mi}$ downstream from

Graves Creek, 3 mi north of Cleveland, and at mile 98.6.

Drainage area-303 $\mathrm{mi}^{2}$.

Gage-Water-stage recorder. Datum of gage is $536.94 \mathrm{ft}$ NGVD 29.

\begin{tabular}{|c|c|c|c|c|c|}
\hline $\begin{array}{c}\text { Water } \\
\text { year }\end{array}$ & Date & Discharge & $\begin{array}{l}\text { Discharge } \\
\text { code }\end{array}$ & $\begin{array}{c}\text { Gage } \\
\text { height } \\
\text { (feet) }\end{array}$ & $\begin{array}{c}\text { Gage } \\
\text { height } \\
\text { code }\end{array}$ \\
\hline 2001 & Mar. 20 & 9,520 & & 10.76 & \\
\hline 2002 & Jan. 25 & 11,500 & & 11.47 & \\
\hline 2003 & Mar. 6 & 11,800 & & 11.57 & \\
\hline 2004 & Feb. 6 & 12,600 & & 12.21 & \\
\hline 2005 & Nov. 24 & 14,600 & & 13.06 & \\
\hline 2006 & May 11 & 4,480 & & 7.54 & \\
\hline 2007 & Jan. 8 & 3,860 & & 7.53 & \\
\hline 2008 & Aug. 26 & 5,370 & & 8.28 & \\
\hline 2009 & Jan. 7 & 21,500 & & 15.62 & \\
\hline 2010 & May 3 & 10,400 & & 11.20 & \\
\hline 2011 & Sept. 6 & 16,000 & & 13.64 & \\
\hline 2012 & Jan. 26 & 13,700 & & 12.69 & \\
\hline 2013 & Jan. 15 & 13,600 & & 12.65 & \\
\hline 2014 & Feb. 21 & 8,430 & & 10.18 & \\
\hline 2015 & Jan. 4 & 7,410 & & 9.61 & \\
\hline
\end{tabular}

02455500 Locust Fork at Trafford

L ocation-Lat $33^{\circ} 49^{\prime} 49^{\prime \prime}$, long 86 $45^{\prime} 21^{\prime \prime}$, in SW 1/4 sec. 9, T. 14 S., R. 2 W., Jefferson County, Hydrologic Unit 03160111, $0.8 \mathrm{mi}$ northwest of Trafford, $1.5 \mathrm{mi}$ east of Coaldale, $2.8 \mathrm{mi}$ upstream from Gurley Creek, and at mile 67.4.

Drainage area $-624 \mathrm{mi}^{2}$.

Gage - Water-stage recorder. Datum of gage is $309.12 \mathrm{ft}$ NGVD 29.

R emarks - Records include effects of diversion upstream on Blackburn Fork by Birmingham Water Works.

\begin{tabular}{cccccc}
\hline $\begin{array}{c}\text { Water } \\
\text { year }\end{array}$ & Date & Discharge & $\begin{array}{c}\text { Discharge } \\
\text { code }\end{array}$ & $\begin{array}{c}\text { Gage } \\
\text { height } \\
\text { (feet) }\end{array}$ & $\begin{array}{c}\text { Gage } \\
\text { height } \\
\text { code }\end{array}$ \\
\hline 1908 & -- & 62,400 & $2,7, B$ & 60.00 & \\
1929 & -- & 37,000 & $2, B$ & -- & \\
1930 & -- & 54,000 & $2, B$ & -- & \\
\end{tabular}

$\mathbf{0 2 4 5 5 5 0 0}$ Locust Fork at Trafford-Continued

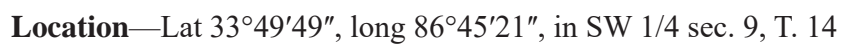
S., R. 2 W., Jefferson County, Hydrologic Unit 03160111, $0.8 \mathrm{mi}$ northwest of Trafford, $1.5 \mathrm{mi}$ east of Coaldale, $2.8 \mathrm{mi}$ upstream from Gurley Creek, and at mile 67.4.

Drainage area $-624 \mathrm{mi}^{2}$.

Gage - Water-stage recorder. Datum of gage is $309.12 \mathrm{ft}$ NGVD 29.

Remarks - Records include effects of diversion upstream on Blackburn Fork by Birmingham Water Works.

\begin{tabular}{|c|c|c|c|c|c|}
\hline $\begin{array}{l}\text { Water } \\
\text { year }\end{array}$ & Date & Discharge & $\begin{array}{l}\text { Discharge } \\
\text { code }\end{array}$ & $\begin{array}{c}\text { Gage } \\
\text { height } \\
\text { (feet) }\end{array}$ & $\begin{array}{c}\text { Gage } \\
\text { height } \\
\text { code }\end{array}$ \\
\hline 1931 & Nov. 17 & 20,000 & & 31.50 & \\
\hline 1932 & Jan. 30 & 22,200 & & 33.50 & \\
\hline 1933 & Dec. 12 & 19,700 & & 31.15 & \\
\hline 1934 & Mar. 4 & 22,000 & & 33.91 & \\
\hline 1935 & Oct. 11 & 28,100 & & 38.95 & \\
\hline 1936 & Feb. 4 & 45,500 & & 50.25 & \\
\hline 1937 & Jan. 3 & 30,300 & & 40.30 & 2 \\
\hline 1938 & Apr. 9 & 37,000 & & 44.80 & 2 \\
\hline 1939 & Feb. 28 & 17,600 & & 29.10 & 2 \\
\hline 1940 & Apr. 4 & 13,700 & & -- & \\
\hline 1941 & Aug. 1 & 28,000 & & 38.03 & \\
\hline 1942 & Feb. 17 & 24,300 & & 35.36 & 2 \\
\hline 1943 & Dec. 29 & 55,800 & & 56.38 & \\
\hline 1944 & Mar. 29 & 24,300 & & 35.60 & 2 \\
\hline 1945 & Feb. 13 & 23,500 & & 34.60 & 2 \\
\hline 1946 & Feb. 10 & 38,000 & & 45.60 & \\
\hline 1947 & Jan. 16 & 32,400 & & 41.50 & 2 \\
\hline 1948 & Feb. 14 & 22,600 & & 33.80 & 2 \\
\hline 1949 & Jan. 6 & 60,700 & & 59.10 & \\
\hline 1950 & Mar. 14 & 43,000 & & 48.50 & 2 \\
\hline 1951 & Mar. 29 & 51,100 & & 53.20 & 2 \\
\hline 1952 & Dec. 21 & 18,000 & 1 & -- & \\
\hline 1953 & Jan. 10 & 16,100 & & 28.20 & \\
\hline 1954 & Jan. 16 & 30,000 & & 40.40 & \\
\hline 1955 & Feb. 7 & 18,600 & & 30.70 & \\
\hline 1956 & Apr. 16 & 19,000 & & 31.10 & \\
\hline 1957 & Feb. 4 & 13,600 & & 25.50 & \\
\hline 1958 & Nov. 19 & 10,800 & & 22.20 & \\
\hline
\end{tabular}


Appendix 2. Discharge and gage height data for streamgages in Alabama.-Continued

[--, date or data not available; $\mathrm{ft}$, foot; $\mathrm{ft}^{3} / \mathrm{s}$, cubic foot per second; mi, mile; $\mathrm{mi}^{2}$, square mile; qualification codes apply to the discharge data: 1 , discharge is a maximum daily average; 2 , discharge is an estimate; 4 , discharge is less than indicated value, which is the minimum recordable discharge at this site; 5 , discharge affected to unknown degree by regulation or diversion; 6 , discharge affected by regulation or diversion; 7, discharge is a historic peak; A, year of occurrence is unknown or not exact; $\mathrm{B}$, month or day of occurrence is unknown or not exact; E, only maximum peak available for this year. qualification codes apply to the gage height data: 1, gage height affected by backwater; 2, gage height not the maximum for the year; 3 , gage height at different datum or at different site and datum; 5 , gage height is an estimate]

\section{$\mathbf{0 2 4 5 5 5 0 0}$ Locust Fork at Trafford-Continued}

L ocation-Lat $33^{\circ} 49^{\prime} 49^{\prime \prime}$, long $86^{\circ} 45^{\prime} 21^{\prime \prime}$, in SW 1/4 sec. 9, T. 14 S., R. 2 W., Jefferson County, Hydrologic Unit 03160111, $0.8 \mathrm{mi}$ northwest of Trafford, $1.5 \mathrm{mi}$ east of Coaldale, $2.8 \mathrm{mi}$ upstream from Gurley Creek, and at mile 67.4.

Drainage area $-624 \mathrm{mi}^{2}$.

G age - Water-stage recorder. Datum of gage is $309.12 \mathrm{ft}$ NGVD 29.

Remarks - Records include effects of diversion upstream on Blackburn Fork by Birmingham Water Works.

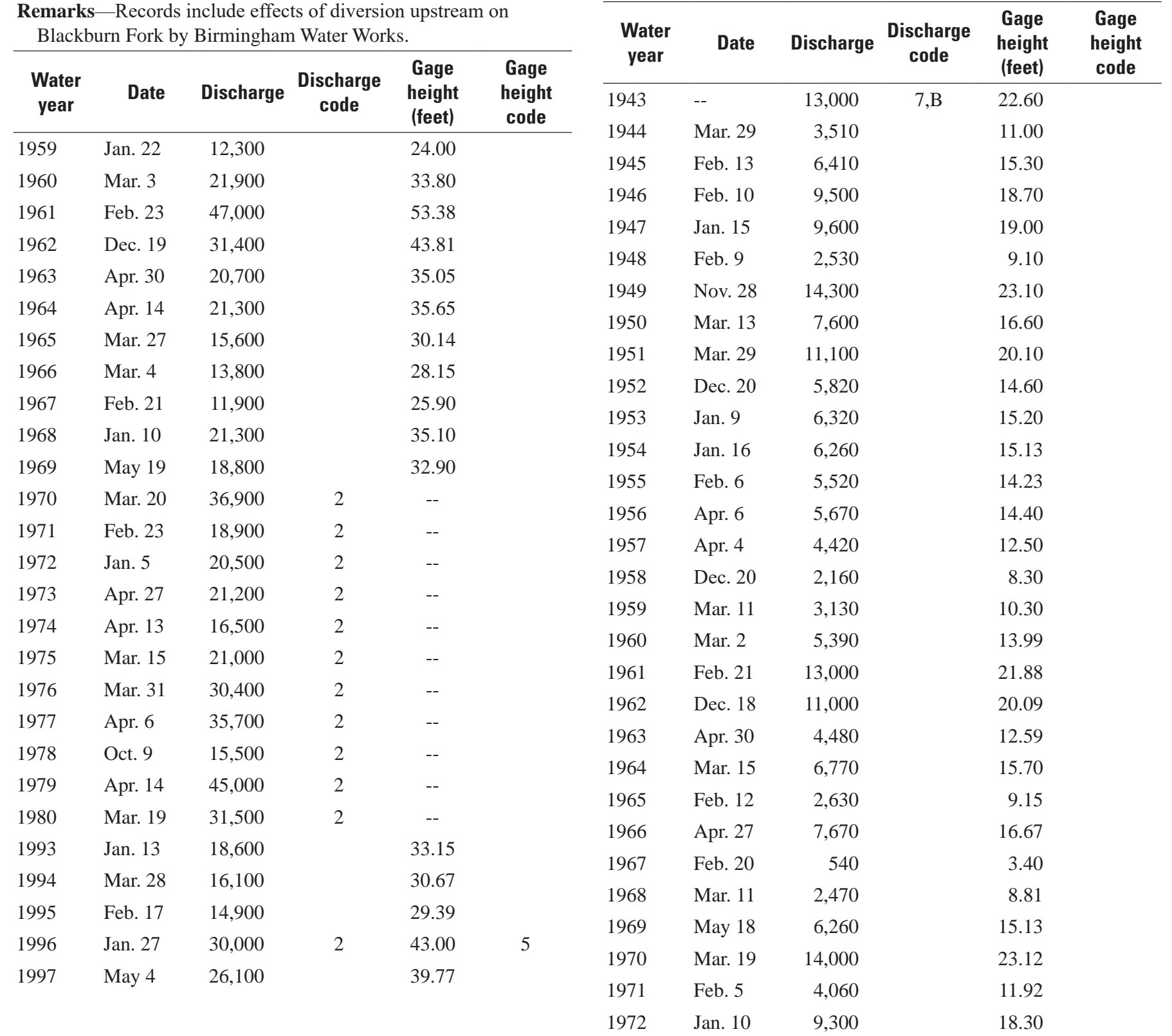

02456000 Turkey Creek at Morris

L ocation-Lat $33^{\circ} 44^{\prime} 25^{\prime \prime}$, long $86^{\circ} 48^{\prime} 45^{\prime \prime}$, in SW 1/4 sec. 12, T. 15 S., R. 3 W., Jefferson County, Hydrologic Unit 03160111, on (county road) former U.S. Highway 31 at Morris, $0.8 \mathrm{mi}$ downstream from Cunningham Creek, and at mile 4.0.

Drainage area-80.9 $\mathrm{mi}^{2}$.

G age - Water-stage recorder. Datum of gage is $345.18 \mathrm{ft}$ NGVD 29. 
Appendix 2. Discharge and gage height data for streamgages in Alabama.-Continued

[--, date or data not available; $\mathrm{ft}$, foot; $\mathrm{ft}^{3} / \mathrm{s}$, cubic foot per second; $\mathrm{mi}$, mile; $\mathrm{mi}^{2}$, square mile; qualification codes apply to the discharge data: 1 , discharge is a maximum daily average; 2 , discharge is an estimate; 4 , discharge is less than indicated value, which is the minimum recordable discharge at this site; 5 , discharge affected to unknown degree by regulation or diversion; 6 , discharge affected by regulation or diversion; 7, discharge is a historic peak; A, year of occurrence is unknown or not exact; $\mathrm{B}$, month or day of occurrence is unknown or not exact; E, only maximum peak available for this year. qualification codes apply to the gage height data: 1, gage height affected by backwater; 2 , gage height not the maximum for the year; 3 , gage height at different data or at different site and datum;

5 , gage height is an estimate]

\section{Turkey Creek at Morris-Continued}

L ocation-Lat $33^{\circ} 44^{\prime} 25^{\prime \prime}$, long $86^{\circ} 48^{\prime} 45^{\prime \prime}$, in SW 1/4 sec. 12, T. 15 S., R. 3 W., Jefferson County, Hydrologic Unit 03160111, on (county road) former U.S. Highway 31 at Morris, $0.8 \mathrm{mi}$ downstream from Cunningham Creek, and at mile 4.0.

Drainage area - $80.9 \mathrm{mi}^{2}$.

Gage-Water-stage recorder. Datum of gage is $345.18 \mathrm{ft}$ NGVD 29.

\begin{tabular}{|c|c|c|c|c|c|}
\hline $\begin{array}{c}\text { Water } \\
\text { year }\end{array}$ & Date & Discharge & $\begin{array}{l}\text { Discharge } \\
\text { code }\end{array}$ & $\begin{array}{c}\text { Gage } \\
\text { height } \\
\text { (feet) }\end{array}$ & $\begin{array}{c}\text { Gage } \\
\text { height } \\
\text { code }\end{array}$ \\
\hline 1973 & Mar. 31 & 5,260 & & 13.80 & \\
\hline 1974 & Apr. 13 & 6,860 & & 15.80 & \\
\hline 1975 & Jan. 25 & 5,990 & & 14.81 & \\
\hline 1976 & Mar. 31 & 6,380 & & 15.20 & \\
\hline 1977 & Apr. 4 & 11,700 & & 21.14 & \\
\hline 1978 & Oct. 9 & 6,780 & & 15.43 & \\
\hline 1979 & Apr. 13 & 10,700 & & 19.55 & \\
\hline 2003 & May 7 & 15,800 & & 24.95 & \\
\hline 2004 & Feb. 6 & 13,200 & & 22.54 & \\
\hline 2005 & Nov. 24 & 10,700 & & 20.10 & \\
\hline 2006 & Feb. 6 & 4,190 & & 11.91 & \\
\hline 2007 & Dec. 31 & 1,110 & & 5.00 & \\
\hline 2008 & May 11 & 3,740 & & 11.18 & \\
\hline 2009 & Jan. 7 & 5,470 & & 13.82 & \\
\hline 2010 & Nov. 10 & 4,580 & & 12.52 & \\
\hline 2011 & Sept. 6 & 10,200 & & 19.57 & \\
\hline
\end{tabular}

02456500 Locust Fork at Sayre

L ocation-Lat $33^{\circ} 42^{\prime} 35^{\prime \prime}$, long 86 $89^{\prime} 00^{\prime \prime}$, in NW 1/4 sec. 29 , T. 15 S., R. 4 W., Jefferson county, Hydrologic Unit 03160111, 150 $\mathrm{ft}$ upstream from county road at Sayre, $1.5 \mathrm{mi}$ downstream from Camp Creek, and at mile 33.9.

Drainage area $-885 \mathrm{mi}^{2}$.

G age - Water-stage recorder. Datum of gage is $258.64 \mathrm{ft}$ NGVD 29.

Remarks - Record include effects of diversion upstream on Blackburn Fork by Birmingham Water Works.

\begin{tabular}{|c|c|c|c|c|c|}
\hline $\begin{array}{c}\text { Water } \\
\text { year }\end{array}$ & Date & Discharge & $\begin{array}{l}\text { Discharge } \\
\text { code }\end{array}$ & $\begin{array}{c}\text { Gage } \\
\text { height } \\
\text { (feet) }\end{array}$ & $\begin{array}{c}\text { Gage } \\
\text { height } \\
\text { code }\end{array}$ \\
\hline 1908 & -- & 56,000 & $2,7, B$ & -- & \\
\hline 1942 & Aug. 21 & 6,500 & 2 & 13.20 & \\
\hline 1943 & Dec. 29 & 49,400 & & 45.00 & \\
\hline 1944 & Mar. 30 & 27,500 & & 32.01 & \\
\hline 1945 & Feb. 14 & 22,800 & & 29.50 & \\
\hline 1946 & Feb. 11 & 37,500 & & 38.90 & \\
\hline 1947 & Jan. 16 & 29,800 & & 34.50 & \\
\hline 1948 & Feb. 15 & 20,900 & & 27.90 & \\
\hline 1949 & Jan. 7 & 55,300 & & 47.90 & \\
\hline 1950 & Mar. 14 & 39,400 & & 40.00 & \\
\hline 1951 & Mar. 30 & 47,200 & & 43.90 & \\
\hline 1952 & Dec. 21 & 23,500 & & 29.96 & \\
\hline 1953 & Jan. 10 & 18,900 & & 26.20 & \\
\hline 1954 & Jan. 17 & 27,600 & & 33.00 & \\
\hline 1955 & Feb. 7 & 20,200 & & 27.32 & \\
\hline 1956 & Apr. 17 & 17,400 & & 24.80 & \\
\hline 1957 & Apr. 5 & 15,300 & & 22.80 & \\
\hline 1958 & Nov. 19 & 11,300 & & 18.50 & \\
\hline 1959 & Jan. 22 & 12,300 & & 19.70 & \\
\hline 1960 & Mar. 4 & 20,500 & & 27.60 & \\
\hline 1961 & Feb. 23 & 54,700 & & 48.60 & \\
\hline 1962 & Dec. 19 & 32,600 & & 35.73 & \\
\hline 1963 & May 1 & 20,100 & & 26.98 & \\
\hline 1964 & Mar. 16 & 23,600 & & 29.51 & \\
\hline 1965 & Mar. 27 & 16,000 & & 23.45 & \\
\hline 1966 & Mar. 5 & 16,500 & & 23.99 & \\
\hline 1967 & Feb. 21 & 12,700 & & 20.24 & \\
\hline 1968 & Jan. 11 & 23,100 & & 29.14 & \\
\hline 1969 & May 19 & 26,200 & & 31.38 & \\
\hline 1970 & Mar. 20 & 34,900 & & 37.16 & \\
\hline 1971 & Feb. 23 & 19,300 & & 26.38 & \\
\hline 1972 & Jan. 5 & 20,800 & & 27.47 & \\
\hline 1973 & Apr. 27 & 21,300 & & 27.89 & \\
\hline 1974 & Apr. 13 & 17,000 & & 24.41 & \\
\hline
\end{tabular}


Appendix 2. Discharge and gage height data for streamgages in Alabama.-Continued

[--, date or data not available; $\mathrm{ft}$, foot; $\mathrm{ft}^{3} / \mathrm{s}$, cubic foot per second; mi, mile; $\mathrm{mi}^{2}$, square mile; qualification codes apply to the discharge data: 1 , discharge is a maximum daily average; 2 , discharge is an estimate; 4 , discharge is less than indicated value, which is the minimum recordable discharge at this site; 5 , discharge affected to unknown degree by regulation or diversion; 6 , discharge affected by regulation or diversion; 7, discharge is a historic peak; A, year of occurrence is unknown or not exact; $\mathrm{B}$, month or day of occurrence is unknown or not exact; E, only maximum peak available for this year. qualification codes apply to the gage height data: 1, gage height affected by backwater; 2, gage height not the maximum for the year; 3, gage height at different datum or at different site and datum; 5 , gage height is an estimate]

$\mathbf{0 2 4 5 6 5 0 0}$ Locust Fork at Sayre-Continued

L ocation-Lat $33^{\circ} 42^{\prime} 35^{\prime \prime}$, long $86^{\circ} 59^{\prime} 00^{\prime \prime}$, in NW 1/4 sec. 29 , T. 15 S., R. 4 W., Jefferson county, Hydrologic Unit 03160111, 150 $\mathrm{ft}$ upstream from county road at Sayre, $1.5 \mathrm{mi}$ downstream from Camp Creek, and at mile 33.9.

Drainage area $-885 \mathrm{mi}^{2}$.

G age - Water-stage recorder. Datum of gage is $258.64 \mathrm{ft}$ NGVD 29.

Remarks - Record include effects of diversion upstream on Blackburn Fork by Birmingham Water Works.

\begin{tabular}{|c|c|c|c|c|c|}
\hline $\begin{array}{l}\text { Water } \\
\text { year }\end{array}$ & Date & Discharge & $\begin{array}{l}\text { Discharge } \\
\text { code }\end{array}$ & $\begin{array}{c}\text { Gage } \\
\text { height } \\
\text { (feet) }\end{array}$ & $\begin{array}{c}\text { Gage } \\
\text { height } \\
\text { code }\end{array}$ \\
\hline 1975 & Mar. 15 & 21,100 & & 26.69 & \\
\hline 1976 & Mar. 31 & 29,200 & & 33.41 & \\
\hline 1977 & Apr. 6 & 33,900 & & 36.54 & \\
\hline 1978 & Oct. 9 & 16,200 & & 23.64 & \\
\hline 1979 & Apr. 14 & 41,800 & & 41.40 & \\
\hline 1980 & Mar. 19 & 30,300 & & 34.17 & \\
\hline 1981 & Mar. 30 & 18,100 & & 25.04 & \\
\hline 1982 & Jan. 5 & 30,200 & & 34.17 & \\
\hline 1983 & May 21 & 34,200 & & 36.77 & \\
\hline 1984 & Dec. 4 & 24,200 & & 29.96 & \\
\hline 1985 & July 28 & 21,500 & & 27.88 & \\
\hline 1986 & May 29 & 5,900 & & 12.44 & \\
\hline 1987 & Mar. 1 & 20,400 & & 27.06 & \\
\hline 1988 & Jan. 20 & 8,940 & & 16.12 & \\
\hline 1989 & Mar. 1 & 21,600 & & 27.96 & \\
\hline 1990 & Feb. 17 & 42,400 & & 41.79 & \\
\hline 1991 & Feb. 21 & 18,800 & & 25.74 & \\
\hline 1992 & Feb. 26 & 14,700 & & 22.00 & \\
\hline 1993 & Jan. 13 & 18,000 & & 25.00 & \\
\hline 1994 & Mar. 29 & 21,300 & & 27.80 & \\
\hline 1995 & Mar. 9 & 17,200 & & 24.33 & \\
\hline 1996 & Jan. 28 & 32,200 & & 35.47 & \\
\hline 1997 & May 4 & 24,100 & & 29.92 & \\
\hline 1998 & Jan. 9 & 33,000 & & 36.02 & \\
\hline 1999 & June 29 & 17,800 & & 24.89 & \\
\hline 2000 & Apr. 4 & 32,500 & & 35.68 & \\
\hline 2001 & Apr. 4 & 28,700 & & 33.19 & \\
\hline 2002 & Jan. 26 & 20,400 & & 27.01 & \\
\hline 2003 & May 8 & 34,200 & & 36.75 & \\
\hline 2004 & Feb. 7 & 28,000 & & 32.71 & \\
\hline 2005 & Nov. 25 & 33,400 & & 36.28 & \\
\hline 2006 & Apr. 21 & 9,580 & & 16.84 & \\
\hline 2007 & Jan. 8 & 5,710 & & 12.20 & \\
\hline 2008 & May 11 & 7,950 & & 14.97 & \\
\hline
\end{tabular}

\section{Locust Fork at Sayre-Continued}

L ocation-Lat $33^{\circ} 42^{\prime} 35^{\prime \prime}$, long $86^{\circ} 59^{\prime} 00^{\prime \prime}$, in NW 1/4 sec. 29 , T. 15 S., R. 4 W., Jefferson county, Hydrologic Unit 03160111, 150 $\mathrm{ft}$ upstream from county road at Sayre, $1.5 \mathrm{mi}$ downstream from Camp Creek, and at mile 33.9.

Drainage area-885 $\mathrm{mi}^{2}$.

G age - Water-stage recorder. Datum of gage is $258.64 \mathrm{ft}$ NGVD 29.

Remarks - Record include effects of diversion upstream on Blackburn Fork by Birmingham Water Works.

\begin{tabular}{|c|c|c|c|c|c|}
\hline $\begin{array}{c}\text { Water } \\
\text { year }\end{array}$ & Date & Discharge & $\begin{array}{c}\text { Discharge } \\
\text { code }\end{array}$ & $\begin{array}{c}\text { Gage } \\
\text { height } \\
\text { (feet) }\end{array}$ & $\begin{array}{c}\text { Gage } \\
\text { height } \\
\text { code }\end{array}$ \\
\hline 2009 & Jan. 8 & 39,800 & & 39.10 & \\
\hline 2010 & Mar. 11 & 21,000 & & 27.52 & \\
\hline 2011 & Sept. 7 & 26,100 & & 31.30 & \\
\hline 2012 & Jan. 28 & 17,200 & & 24.40 & \\
\hline 2013 & Jan. 16 & 24,800 & & 30.41 & \\
\hline 2014 & Apr. 8 & 25,000 & & 30.55 & \\
\hline 2015 & Jan. 5 & 15,000 & & 22.26 & \\
\hline 2016 & Dec. 27 & 37,000 & & 37.39 & \\
\hline
\end{tabular}

02462000 Valley Creek near Oak Grove

Location-Lat $33^{\circ} 26^{\prime} 50^{\prime \prime}$, long $87^{\circ} 07^{\prime} 20^{\prime \prime}$, in NW $1 / 4 \mathrm{sec} .25$, T. 18 S., R. 6 W., Jefferson County, Hydrologic Unit 03160112, near center of span on downstream side of highway bridge, $1,000 \mathrm{ft}$ down- stream from Raccoon Branch, 1.5 mi east of Oak Grove, 10.5 mi west of Bessemer, and 18.2 mi upstream from mouth.

Drainage area-148 $\mathrm{mi}^{2}$.

G age - Water-stage recorder. Datum of gage is $438.64 \mathrm{ft} \mathrm{NGVD} 29$.

\begin{tabular}{|c|c|c|c|c|c|}
\hline $\begin{array}{c}\text { Water } \\
\text { year }\end{array}$ & Date & Discharge & $\begin{array}{l}\text { Discharge } \\
\text { code }\end{array}$ & $\begin{array}{c}\text { Gage } \\
\text { height } \\
\text { (feet) }\end{array}$ & $\begin{array}{c}\text { Gage } \\
\text { height } \\
\text { code }\end{array}$ \\
\hline 1916 & -- & 26,800 & $7, \mathrm{~B}$ & 29.60 & \\
\hline 1936 & -- & 20,000 & $7, \mathrm{~B}$ & 28.00 & \\
\hline 1954 & Jan. 16 & 8,570 & & 20.70 & \\
\hline 1955 & Feb. 6 & 8,520 & & 20.55 & \\
\hline 1956 & Apr. 6 & 8,520 & & 20.65 & \\
\hline 1957 & Apr. 4 & 8,170 & & 19.80 & \\
\hline 1958 & Feb. 6 & 6,390 & & 15.75 & \\
\hline 1959 & May 31 & 7,510 & & 18.30 & \\
\hline 1960 & Mar. 3 & 5,650 & & 14.20 & \\
\hline 1961 & Feb. 21 & 23,000 & & 28.86 & \\
\hline 1962 & Dec. 18 & 12,700 & & 25.12 & \\
\hline
\end{tabular}


Appendix 2. Discharge and gage height data for streamgages in Alabama._- Continued

[--, date or data not available; ft, foot; $\mathrm{ft}^{3} / \mathrm{s}$, cubic foot per second; mi, mile; $\mathrm{mi}^{2}$, square mile; qualification codes apply to the discharge data: 1 , discharge is a maximum daily average; 2 , discharge is an estimate; 4 , discharge is less than indicated value, which is the minimum recordable discharge at this site; 5 , discharge affected to unknown degree by regulation or diversion; 6 , discharge affected by regulation or diversion; 7, discharge is a historic peak; A, year of occurrence is unknown or not exact; B, month or day of occurrence is unknown or not exact; E, only maximum peak available for this year. qualification codes apply to the gage height data: 1, gage height affected by backwater; 2, gage height not the maximum for the year; 3, gage height at different data or at different site and datum;

5 , gage height is an estimate]

\section{Valley Creek near Oak Grove-Continued}

Location-Lat $33^{\circ} 26^{\prime} 50^{\prime \prime}$, long $87^{\circ} 07^{\prime} 20^{\prime \prime}$, in NW 1/4 sec. 25 , T. 18 S., R. 6 W., Jefferson County, Hydrologic Unit 03160112, near center of span on downstream side of highway bridge, $1,000 \mathrm{ft}$ down- stream from Raccoon Branch, 1.5 mi east of Oak Grove, 10.5 mi west of Bessemer, and 18.2 mi upstream from mouth.

Drainage area-148 $\mathrm{mi}^{2}$.

Gage -Water-stage recorder. Datum of gage is $438.64 \mathrm{ft}$ NGVD 29.

\begin{tabular}{ccccc}
$\begin{array}{c}\text { Water } \\
\text { year }\end{array}$ & Date $\quad$ Discharge $\begin{array}{c}\text { Discharge } \\
\text { code }\end{array}$ & $\begin{array}{c}\text { Gage } \\
\text { height } \\
\text { (feet) }\end{array}$ & $\begin{array}{c}\text { Gage } \\
\text { height } \\
\text { code }\end{array}$ \\
\hline
\end{tabular}

\begin{tabular}{llrl}
\hline 1963 & Jan. 19 & 7,680 & 19.09 \\
1964 & Apr. 6 & 10,200 & 23.29 \\
1965 & Feb. 11 & 7,690 & 19.12
\end{tabular}

196

1967

1968

1969

1970

Feb. 11

6,490

16.50

Apr. 27

July 30

3,840

16.50

Dec. 15

6,540

10.76

16.62

24.73

28.43

1971

May 19

11,800

18.36

1972

Feb. 5

7,340

19.73

1973

Jan. 11

7,970

19.95

1974 Apr. 13

8,070

21.09

1975

Jan. 25

8,650

21.61

25.39

1976

Mar. 27

13,300

Sept. 7

8,680

1978

Oct. 25

8,860

21.14

21.44

29.80

1979

Apr. 13

26,300

22.96

1981 Mar. 30

9,920

21.21

1982

Jan. 19

8,730

18.61

25.41

1984

May 19

14,000

33.98

1985 Feb. 5

32,000

13.90

1986

Nov. 28

3,360

9.41

1987

Jan. 19

6,420

16.06

1988 Jan. 20

5,000

12.92

1989

7,070

17.44
02462000 Valley Creek near Oak Grove-Continued

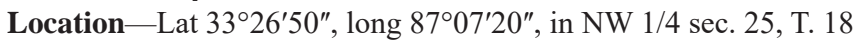
S., R. 6 W., Jefferson County, Hydrologic Unit 03160112, near center of span on downstream side of highway bridge, $1,000 \mathrm{ft}$ down- stream from Raccoon Branch, 1.5 mi east of Oak Grove, $10.5 \mathrm{mi}$ west of Bessemer, and $18.2 \mathrm{mi}$ upstream from mouth.

Drainage area-148 $\mathrm{mi}^{2}$.

G age -Water-stage recorder. Datum of gage is $438.64 \mathrm{ft}$ NGVD 29.

\begin{tabular}{|c|c|c|c|c|c|}
\hline $\begin{array}{c}\text { Water } \\
\text { year }\end{array}$ & Date & Discharge & $\begin{array}{l}\text { Discharge } \\
\text { code }\end{array}$ & $\begin{array}{c}\text { Gage } \\
\text { height } \\
\text { (feet) }\end{array}$ & $\begin{array}{c}\text { Gage } \\
\text { height } \\
\text { code }\end{array}$ \\
\hline 1990 & Feb. 16 & 10,100 & & 22.33 & \\
\hline 1991 & May 12 & 7,340 & & 17.98 & \\
\hline 1992 & Feb. 26 & 3,540 & & 9.79 & \\
\hline 1993 & Jan. 12 & 6,220 & & 15.61 & \\
\hline 1994 & Apr. 6 & 6,000 & & 15.12 & \\
\hline 1995 & Mar. 8 & 4,830 & & 12.55 & \\
\hline
\end{tabular}

$1996 \quad$ Jan. $27 \quad 16,400 \quad 26.71$

1997 Apr. $27 \quad 3,710 \quad 10.14$

$1998 \quad$ Jan. $7 \quad 10,700 \quad 22.87$

$1999 \quad$ Jan. $23 \quad 6,480 \quad 16.18$

2000 Mar. $11 \quad 9,230 \quad 21.23$

2001 Sept. $3 \quad 9,160 \quad 21.13$

2002 Dec. $14 \quad 6,780 \quad 16.86$

2003 Dec. $24 \quad 6,670 \quad 16.62$

2004 Feb. $6 \quad 11,200 \quad 23.38$

2005 July $11 \quad 6,480 \quad 16.18$

$2006 \quad$ Feb. $6 \quad 5,860 \quad 14.41$

2007 Oct. $19 \quad 2,460 \quad 7.40$

2008 May $15 \quad 4,500 \quad 11.83$

2009 Feb. $28 \quad 5,840 \quad 14.77$

2010 Nov. $11 \quad 8,060 \quad 19.43$

2011 Sept. $6 \quad 9,190 \quad 21.17$

$2012 \quad$ Jan. $26 \quad 6,200 \quad 15.57$

2013 Jan. $14 \quad 6,140 \quad 15.44$

2014 Apr. $7 \quad 11,100 \quad 23.28$

2015 Jan. $4 \quad 7,570 \quad 18.45$ 
Appendix 2. Discharge and gage height data for streamgages in Alabama._-Continued

[--, date or data not available; $\mathrm{ft}$, foot; $\mathrm{ft}^{3} / \mathrm{s}$, cubic foot per second; mi, mile; $\mathrm{mi}^{2}$, square mile; qualification codes apply to the discharge data: 1 , discharge is a maximum daily average; 2 , discharge is an estimate; 4 , discharge is less than indicated value, which is the minimum recordable discharge at this site; 5 , discharge affected to unknown degree by regulation or diversion; 6 , discharge affected by regulation or diversion; 7, discharge is a historic peak; A, year of occurrence is unknown or not exact; B, month or day of occurrence is unknown or not exact; E, only maximum peak available for this year. qualification codes apply to the gage height data: 1, gage height affected by backwater; 2, gage height not the maximum for the year; 3, gage height at different datum or at different site and datum; 5 , gage height is an estimate]

\section{Blue Creek near Oakman}

L ocation-Lat $33^{\circ} 31^{\prime} 17^{\prime \prime}$, long $87^{\circ} 29^{\prime} 07^{\prime \prime}$, in SW 1/4 sec. 33, T. 17 S., R. 9 W., Tuscaloosa County, Hydrologic Unit 03160112, on State Highway 69, 1.5 mi southwest of Wiley, 2 mi upstream from McDuff Spring Branch, 12.6 mi upstream from mouth, and 14 mi southwest of Oakman.

Drainage area $-5.32 \mathrm{mi}^{2}$.

G age - Water-stage recorder. Datum of gage is $420 \mathrm{ft}$ NGVD 29 (from topographic map).

\begin{tabular}{|c|c|c|c|c|c|}
\hline $\begin{array}{c}\text { Water } \\
\text { year }\end{array}$ & Date & Discharge & $\begin{array}{l}\text { Discharge } \\
\text { code }\end{array}$ & $\begin{array}{c}\text { Gage } \\
\text { height } \\
\text { (feet) }\end{array}$ & $\begin{array}{c}\text { Gage } \\
\text { height } \\
\text { code }\end{array}$ \\
\hline 1960 & Nov. 23 & 887 & & 4.50 & \\
\hline 1961 & Feb. 21 & 3,430 & & 7.16 & \\
\hline 1962 & Dec. 18 & 1,240 & & 5.33 & \\
\hline 1963 & Mar. 5 & 473 & & 3.56 & \\
\hline 1964 & Apr. 27 & 1,660 & & 5.90 & \\
\hline 1965 & Feb. 9 & 1,200 & & 5.26 & \\
\hline 1966 & Apr. 27 & 1,450 & & 5.64 & \\
\hline 1967 & Aug. 25 & 1,260 & & 5.35 & \\
\hline 1968 & July 8 & 3,700 & & 7.37 & \\
\hline 1969 & Apr. 13 & 308 & & 2.85 & \\
\hline 1970 & Mar. 19 & 4,250 & & 7.70 & \\
\hline 1971 & Feb. 22 & 665 & & 4.10 & \\
\hline 1972 & Jan. 4 & 2,000 & & 6.22 & \\
\hline 1973 & Mar. 30 & 1,940 & & 6.16 & \\
\hline 1977 & Apr. 4 & 3,000 & & 6.93 & \\
\hline 1978 & Oct. 25 & 1,720 & & 6.00 & \\
\hline 1979 & Apr. 12 & 3,000 & & 6.93 & \\
\hline 1980 & Apr. 12 & 2,110 & & 6.34 & \\
\hline 1981 & Mar. 30 & 700 & & 4.38 & \\
\hline 1982 & Apr. 20 & 566 & & 3.94 & \\
\hline 1983 & May 19 & 3,600 & & 7.30 & \\
\hline 1984 & Dec. 3 & 3,240 & & 7.04 & \\
\hline
\end{tabular}

02462800 Davis Creek below Abernant

L ocation-Lat $33^{\circ} 18^{\prime} 30^{\prime \prime}$, long $87^{\circ} 13^{\prime} 10^{\prime \prime}$, in SE $1 / 4$ sec. 12 , T. 20 S., R. 7 W., Tuscaloosa County, Hydrologic Unit 03160112, on county road, $0.2 \mathrm{mi}$ downstream from Lye Branch, $0.6 \mathrm{mi}$ downstream from Texas Creek, 2 mi northwest of Abernant, and 2.8 mi downstream from Rockcastle Creek.

Drainage area $-45.3 \mathrm{mi}^{2}$.

G age - Water-stage recorder. Datum of gage is $410 \mathrm{ft}$ NGVD 29 (from topographic map).

\begin{tabular}{|c|c|c|c|c|c|}
\hline $\begin{array}{l}\text { Water } \\
\text { year }\end{array}$ & Date & Discharge & $\begin{array}{l}\text { Discharge } \\
\text { code }\end{array}$ & $\begin{array}{c}\text { Gage } \\
\text { height } \\
\text { (feet) }\end{array}$ & $\begin{array}{c}\text { Gage } \\
\text { height } \\
\text { code }\end{array}$ \\
\hline 1957 & Apr. 4 & 2,630 & & 11.10 & \\
\hline 1958 & May 2 & 1,750 & & 8.30 & \\
\hline 1959 & Jan. 21 & 1,450 & & 7.45 & \\
\hline 1960 & Mar. 2 & 1,290 & & 6.92 & \\
\hline 1961 & Feb. 21 & 5,800 & & 18.30 & \\
\hline 1962 & Dec. 18 & 3,850 & & 14.00 & \\
\hline 1963 & Apr. 30 & 2,690 & & 11.10 & \\
\hline 1964 & Apr. 6 & 3,490 & & 13.10 & \\
\hline 1965 & Feb. 12 & 1,560 & & 7.88 & \\
\hline 1966 & Feb. 13 & 2,060 & & 9.33 & \\
\hline 1967 & June 30 & 580 & & 4.57 & \\
\hline 1968 & Dec. 22 & 1,650 & & 8.11 & \\
\hline 1969 & May 18 & 3,170 & & 12.29 & \\
\hline 1970 & Mar. 19 & 2,890 & & 11.60 & \\
\hline 1971 & Feb. 5 & 2,350 & & 10.15 & \\
\hline 1972 & Jan. 10 & 2,020 & & 9.21 & \\
\hline 1973 & Mar. 31 & 2,280 & & 9.95 & \\
\hline
\end{tabular}


Appendix 2. Discharge and gage height data for streamgages in Alabama._- Continued

[--, date or data not available; ft, foot; $\mathrm{ft}^{3} / \mathrm{s}$, cubic foot per second; mi, mile; $\mathrm{mi}^{2}$, square mile; qualification codes apply to the discharge data: 1 , discharge is a maximum daily average; 2 , discharge is an estimate; 4 , discharge is less than indicated value, which is the minimum recordable discharge at this site; 5 , discharge affected to unknown degree by regulation or diversion; 6 , discharge affected by regulation or diversion; 7, discharge is a historic peak; A, year of occurrence is unknown or not exact; $\mathrm{B}$, month or day of occurrence is unknown or not exact; E, only maximum peak available for this year. qualification codes apply to the gage height data: 1, gage height affected by backwater; 2, gage height not the maximum for the year; 3, gage height at different data or at different site and datum;

5 , gage height is an estimate]

\section{Hurricane Creek near Holt}

L ocation-Lat $33^{\circ} 12^{\prime} 45^{\prime \prime}$, long $87^{\circ} 26^{\prime} 55^{\prime \prime}$, in SE 1/4 sec. 14, T. 21

S., R. 9 W., Tuscaloosa County, Hydrologic Unit 03160112, on

State Highway 116, 0.5 mi downstream from Cottondale Creek,

$2.8 \mathrm{mi}$ southeast of Holt, and at mile 7.1.

Drainage area-108 $\mathrm{mi}^{2}$.

Gage-Water-stage recorder. Datum of gage is $173.70 \mathrm{ft}$ NGVD 29.

\begin{tabular}{|c|c|c|c|c|c|}
\hline $\begin{array}{c}\text { Water } \\
\text { year }\end{array}$ & Date & Discharge & $\begin{array}{l}\text { Discharge } \\
\text { code }\end{array}$ & $\begin{array}{c}\text { Gage } \\
\text { height } \\
\text { (feet) }\end{array}$ & $\begin{array}{c}\text { Gage } \\
\text { height } \\
\text { code }\end{array}$ \\
\hline 1953 & Jan. 9 & 4,530 & & 8.84 & \\
\hline 1954 & Jan. 16 & 6,200 & & 10.50 & \\
\hline 1955 & Feb. 6 & 7,420 & & 13.71 & \\
\hline 1956 & Apr. 6 & 8,380 & & 14.64 & \\
\hline 1957 & Apr. 4 & 5,500 & & 10.90 & \\
\hline 1958 & May 2 & 6,940 & & 12.84 & \\
\hline 1959 & Jan. 21 & 2,770 & & 7.00 & \\
\hline 1960 & Jan. 30 & 2,900 & & 7.83 & \\
\hline 1961 & Feb. 21 & 16,800 & & 22.33 & \\
\hline 1962 & Dec. 18 & 12,300 & & 18.97 & \\
\hline 1963 & Apr. 30 & 5,980 & & 11.63 & \\
\hline 1964 & Mar. 15 & 8,080 & & 14.55 & \\
\hline 1965 & Feb. 12 & 3,900 & & 8.86 & \\
\hline 1966 & Feb. 6 & 4,280 & & 9.43 & \\
\hline 1967 & July 1 & 920 & & 5.45 & \\
\hline 1968 & Dec. 20 & 2,870 & & 7.88 & \\
\hline 1969 & May 19 & 4,550 & & 9.76 & \\
\hline
\end{tabular}

02464000 North River near Samantha

L ocation-Lat $33^{\circ} 28^{\prime} 45^{\prime \prime}$, long $87^{\circ} 35^{\prime} 50^{\prime \prime}$, in SW 1/4 sec. 16 , T. 18 S., R. 10 W., Tuscaloosa County, Hydrologic Unit 03160112, 200 $\mathrm{ft}$ downstream from bridge on county road, $1.2 \mathrm{mi}$ upstream from Cripple Creek, 4 mi north of Samantha, and at mile 36.9.

Drainage area-223 $\mathrm{mi}^{2}$.

Gage-Water-stage recorder. Datum of gage is $232.39 \mathrm{ft}$ NGVD 29.

\begin{tabular}{llcccc}
\hline $\begin{array}{c}\text { Water } \\
\text { year }\end{array}$ & Date & Discharge & $\begin{array}{c}\text { Discharge } \\
\text { code }\end{array}$ & $\begin{array}{c}\text { Gage } \\
\text { height } \\
\text { (feet) }\end{array}$ & $\begin{array}{c}\text { Gage } \\
\text { height } \\
\text { code }\end{array}$ \\
\hline 1916 & -- & 20,500 & $2,7, B$ & 31.00 & \\
1936 & -- & 20,500 & $2,7, B$ & 31.00 & \\
1939 & Feb. 28 & 12,000 & & 22.96 &
\end{tabular}

$1940 \quad$ July $4 \quad 6,600 \quad 14.35$

$1941 \quad$ Mar. $7 \quad 3,900 \quad 9.83$

1942 Feb. $17 \quad 6,000 \quad 13.43$

1943 Dec. $28 \quad 7,430 \quad 2 \quad 15.95$

1944 Mar. $29 \quad 8,100 \quad 16.96$

1945 Mar. $4 \quad 6,040 \quad 13.68$

1946 Feb. $10 \quad 9,950 \quad 19.69$

1947 Jan. $20 \quad 6,590 \quad 14.63$

$1948 \quad$ Feb. $9 \quad 5,680 \quad 13.10$

1949 Nov. $28 \quad 10,400 \quad 20.50$

1950 Mar. $13 \quad 14,000 \quad 26.00$

1951 Mar. $29 \quad 18,000 \quad 30.70$

1952 Dec. $21 \quad 7,650 \quad 16.30$

1953 Feb. $21 \quad 6,380 \quad 14.20$

$1954 \quad$ Jan. $16 \quad 8,160 \quad 17.06$

1955 Feb. $6 \quad 9,140 \quad 18.60$

1956 Apr. $6 \quad 5,380 \quad 12.20$

1957 Dec. $23 \quad 6,590 \quad 14.60$

1958 Nov. $19 \quad 7,020 \quad 15.30$

$1959 \quad$ Jan. $22 \quad 6,710 \quad 14.80$

1960 Mar. $3 \quad 9,530 \quad 19.20$

1961 Feb. $22 \quad 17,600 \quad 30.32$

1962 Dec. $18 \quad 10,300 \quad 20.36$

1963 July $16 \quad 6,320 \quad 14.17$

1964 Apr. $27 \quad 10,200 \quad 20.16$

1965 Feb. $12 \quad 7,760 \quad 16.46$

1966 Apr. $28 \quad 5,250 \quad 11.94$ 
Appendix 2. Discharge and gage height data for streamgages in Alabama.-Continued

[--, date or data not available; $\mathrm{ft}$, foot; $\mathrm{ft}^{3} / \mathrm{s}$, cubic foot per second; mi, mile; $\mathrm{mi}^{2}$, square mile; qualification codes apply to the discharge data: 1 , discharge is a maximum daily average; 2 , discharge is an estimate; 4 , discharge is less than indicated value, which is the minimum recordable discharge at this site; 5 , discharge affected to unknown degree by regulation or diversion; 6 , discharge affected by regulation or diversion; 7, discharge is a historic peak; A, year of occurrence is unknown or not exact; $\mathrm{B}$, month or day of occurrence is unknown or not exact; E, only maximum peak available for this year. qualification codes apply to the gage height data: 1, gage height affected by backwater; 2, gage height not the maximum for the year; 3, gage height at different datum or at different site and datum; 5 , gage height is an estimate]

\section{North River near Samantha-Continued}

L ocation-Lat $33^{\circ} 28^{\prime} 45^{\prime \prime}$, long $87^{\circ} 35^{\prime} 50^{\prime \prime}$, in SW 1/4 sec. 16, T. 18

S., R. 10 W., Tuscaloosa County, Hydrologic Unit 03160112, 200

$\mathrm{ft}$ downstream from bridge on county road, $1.2 \mathrm{mi}$ upstream from

Cripple Creek, 4 mi north of Samantha, and at mile 36.9.

Drainage area-223 $\mathrm{mi}^{2}$.

Gage -Water-stage recorder. Datum of gage is $232.39 \mathrm{ft}$ NGVD 29.

$\begin{array}{ccccc}\begin{array}{c}\text { Water } \\ \text { year }\end{array} \text { Date Discharge } & \begin{array}{c}\text { Discharge } \\ \text { code }\end{array} & \begin{array}{c}\text { Gage } \\ \text { height } \\ \text { (feet) }\end{array} & \begin{array}{c}\text { Gage } \\ \text { height }\end{array}\end{array}$

\begin{tabular}{lllll}
\hline 1967 & Feb. 20 & 5,010 & (feet) & code \\
1968 & Dec. 11 & 8,940 & 11.46 & \\
1969 & Apr. 14 & 5,840 & 18.30 &
\end{tabular}

1969

1970

1971

Apr. 14

5,840

Mar. 20

25,500

Feb. 22

7,610

Jan. 10

8,440

1973

Mar. 31

8,780

\section{4}

Dec. 26

13,400

Jan. 11

8,980

6,760

1976

Oct. 8

11,800

Apr. 5

7,210

1978

Oct. 9

19,400

1980

Apr. 13

9,820

1981

Mar. 17

7,540

1982

Mar. 30

6,640

1983

Jan. 4

14,500

1984

Mar. 6

19,700

1985

Dec. 3

5,700

May 2

2,200

1987

1988

Mar. 13

2,200

Jan. 19

6,970

Feb. 3

2,500

Jan. 13

7,140

1990

Feb. 16

14,700

1991

Feb. 20

9,870
13.12

35.08

16.18

17.46

17.99

24.85

18.29

14.88

22.55

15.57

30.55

19.58

16.07

14.68

26.58

30.82

12.84

6.18

15.19

6.79

15.47

26.21

19.67
02464000 North River near Samantha-Continued

L ocation-Lat $33^{\circ} 28^{\prime} 45^{\prime \prime}$, long $87^{\circ} 35^{\prime} 50^{\prime \prime}$, in SW $1 / 4$ sec. 16 , T. 18 S., R. 10 W., Tuscaloosa County, Hydrologic Unit 03160112, 200 $\mathrm{ft}$ downstream from bridge on county road, $1.2 \mathrm{mi}$ upstream from Cripple Creek, 4 mi north of Samantha, and at mile 36.9.

Drainage area-223 $\mathrm{mi}^{2}$.

G age - Water-stage recorder. Datum of gage is $232.39 \mathrm{ft}$ NGVD 29.

\begin{tabular}{|c|c|c|c|c|c|}
\hline $\begin{array}{c}\text { Water } \\
\text { year }\end{array}$ & Date & Discharge & $\begin{array}{l}\text { Discharge } \\
\text { code }\end{array}$ & $\begin{array}{c}\text { Gage } \\
\text { height } \\
\text { (feet) }\end{array}$ & $\begin{array}{c}\text { Gage } \\
\text { height } \\
\text { code }\end{array}$ \\
\hline 1992 & Feb. 26 & 3,960 & & 9.66 & \\
\hline 1993 & Jan. 12 & 7,480 & & 15.99 & \\
\hline
\end{tabular}

$1993 \quad$ Jan. $12 \quad 7,480$

1994 Mar. $28 \quad 8,960 \quad 18.28$

$1995 \quad$ Feb. $17 \quad 4,310 \quad 10.25$

$1996 \quad$ Jan. $27 \quad 10,500 \quad 20.68$

$1997 \quad$ Feb. $21 \quad 6,130 \quad 13.70$

$1998 \quad$ Jan. $7 \quad 10,300 \quad 20.30$

$1999 \quad$ Jan. $31 \quad 8,450 \quad 17.48$

2000 Mar. $11 \quad 11,700 \quad 22.35$

2001 Feb. $13 \quad 5,950 \quad 13.13$

2002 Dec. $14 \quad 9,700 \quad 19.30$

2003 Feb. $22 \quad 8,670 \quad 16.51$

$2004 \quad$ Feb. $6 \quad 12,300 \quad 21.58$

2005 Apr. $7 \quad 9,360 \quad 17.50$

2006 Feb. $6 \quad 6,930 \quad 13.96$

2007 Dec. $31 \quad 6,240 \quad 12.91$

2008 Mar. $4 \quad 6,120 \quad 12.61$

2009 Jan. $7 \quad 15,600 \quad 24.55$

2010 May $3 \quad 10,100 \quad 17.93$

2011 Mar. $9 \quad 11,900 \quad 20.26$

2012 Dec. $27 \quad 5,550 \quad 11.78$

$2013 \quad$ Jan. $15 \quad 8,450 \quad 15.82$

2014 Apr. $7 \quad 13,000 \quad 22.24$

2015 Jan. $4 \quad 8,050 \quad 15.73$ 
Appendix 2. Discharge and gage height data for streamgages in Alabama.-Continued

[--, date or data not available; $\mathrm{ft}$, foot; $\mathrm{ft}^{3} / \mathrm{s}$, cubic foot per second; $\mathrm{mi}$, mile; $\mathrm{mi}^{2}$, square mile; qualification codes apply to the discharge data: 1 , discharge is a maximum daily average; 2 , discharge is an estimate; 4 , discharge is less than indicated value, which is the minimum recordable discharge at this site; 5 , discharge affected to unknown degree by regulation or diversion; 6 , discharge affected by regulation or diversion; 7, discharge is a historic peak; A, year of occurrence is unknown or not exact; B, month or day of occurrence is unknown or not exact; E, only maximum peak available for this year. qualification codes apply to the gage height data: 1, gage height affected by backwater; 2 , gage height not the maximum for the year; 3 , gage height at different data or at different site and datum;

5 , gage height is an estimate]

\section{Turkey Creek near Tuscaloosa}

L ocation-Lat $33^{\circ} 24^{\prime} 48^{\prime \prime}$, long $87^{\circ} 30^{\prime} 38^{\prime \prime}$ referenced to North American Datum of 1927, Tuscaloosa County, Ala., Hydrologic Unit 03160112, on left bank 1,400 ft downstream from State Highway 69, $1.1 \mathrm{mi}$ upstream from Long Creek, $4.7 \mathrm{mi}$ upstream from mouth, $5.5 \mathrm{mi}$ east of Samantha, and $14 \mathrm{mi}$ north of Tuscaloosa.

Drainage area $-6 \mathrm{mi}^{2}$.

G age -Water-stage recorder. Elevation of gage is $270 \mathrm{ft}$ above NGVD of 1929, from topographic map.

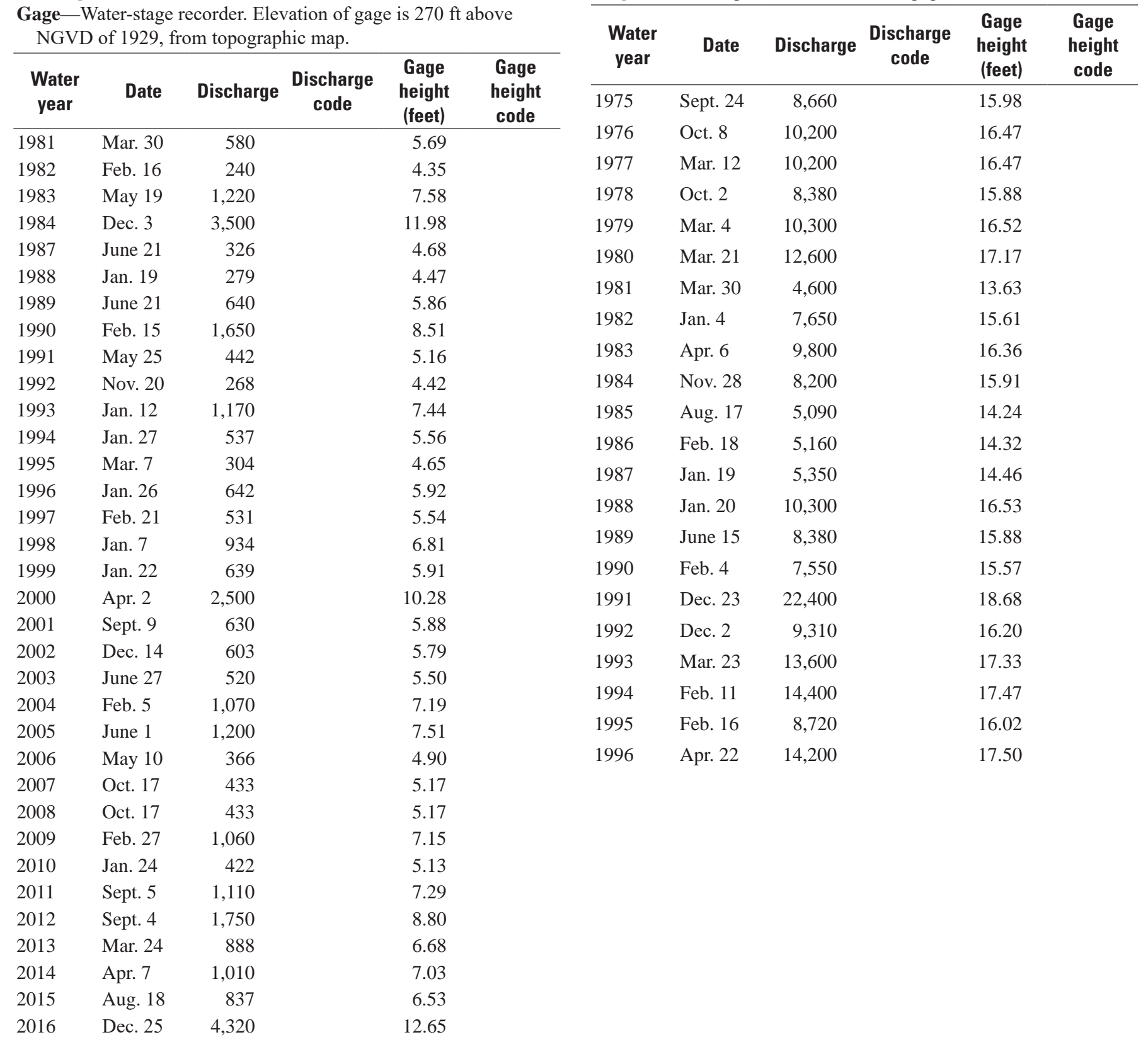

03572110 Crow Creek at Bass

L ocation-Lat $34^{\circ} 56^{\prime} 03^{\prime \prime}$, long $85^{\circ} 55^{\prime} 03^{\prime \prime}$, in SW 1/4 sec. 20, T. 1 S., R. 7 E., Jackson County, Hydrologic Unit 06030001, on State Highway 117, 0.3 mi northwest of Bass, $1 \mathrm{mi}$ upstream from Bennett Cove Creek, $3.7 \mathrm{mi}$ south of Alabama-Tennessee State line, and 15.8 mi upstream from mouth.

Drainage area-131 $\mathrm{mi}^{2}$.

G age - Water-stage recorder. Datum of gage is $598.76 \mathrm{ft}$ NGVD 29. 
Appendix 2. Discharge and gage height data for streamgages in Alabama.-Continued

[--, date or data not available; $\mathrm{ft}$, foot; $\mathrm{ft}^{3} / \mathrm{s}$, cubic foot per second; mi, mile; $\mathrm{mi}^{2}$, square mile; qualification codes apply to the discharge data: 1 , discharge is a maximum daily average; 2 , discharge is an estimate; 4 , discharge is less than indicated value, which is the minimum recordable discharge at this site; 5 , discharge affected to unknown degree by regulation or diversion; 6 , discharge affected by regulation or diversion; 7, discharge is a historic peak; A, year of occurrence is unknown or not exact; $\mathrm{B}$, month or day of occurrence is unknown or not exact; E, only maximum peak available for this year. qualification codes apply to the gage height data: 1, gage height affected by backwater; 2, gage height not the maximum for the year; 3, gage height at different datum or at different site and datum; 5 , gage height is an estimate]

\section{Town Creek near Geraldine}

L ocation-Lat $34^{\circ} 22^{\prime} 42^{\prime \prime}$, long $85^{\circ} 59^{\prime} 25^{\prime \prime}$, in SE $1 / 4$ sec. 34 , T. 7 S., R. 6 E., De Kalb County, Hydrologic Unit 06030001, on State Highway 75, 0.3 mi downstream from Reedy Creek, 2 mi northnortheast of Geraldine, and at mile 20.4.

Drainage area $-141 \mathrm{mi}^{2}$.

Gage -Water-stage recorder. Datum of gage is 1,000 ft NGVD 29 (from topographic map).

\begin{tabular}{ccccc}
$\begin{array}{c}\text { Water } \\
\text { year }\end{array} \quad$ Date & Discharge $\begin{array}{c}\text { Discharge } \\
\text { code }\end{array}$ & $\begin{array}{c}\text { Gage } \\
\text { height } \\
\text { (feet) }\end{array}$ & $\begin{array}{c}\text { Gage } \\
\text { height } \\
\text { code }\end{array}$ \\
\hline
\end{tabular}

\begin{tabular}{llrr}
\hline 1958 & Nov. 18 & 10,600 & 15.60 \\
1959 & Jan. 21 & 4,620 & 9.40 \\
1960 & Mar. 3 & 2,120 & 6.12 \\
1961 & Feb. 23 & 8,080 & 13.08
\end{tabular}

1962 Dec. $18 \quad 15,400 \quad 19.90$

1963 Apr. $29 \quad 17,700 \quad 21.70$

1964 Mar. $25 \quad$ 10,700

15.72

1965 Mar. $26 \quad 8,190$

13.19

1966 Mar. 4 9,850

$1967 \quad$ Feb. $21 \quad 3,420$

14.85

1968 Dec. $22 \quad 7,480$

1969 Feb. $2 \quad$ 10,400

1970

1971

1972

1973

1974

1975

1976

1977

1978

1979

1980

Apr. 26

4,440

Feb. 26

5,780

Jan. 4

6,530

Mar. 16

9,430

Feb. 16

5,770

Feb. 18

9,030

July 5

9,510

Mar. 30

15,300

Nov. 5

8,140

Mar. 4

15,000

Mar. 21

11,900

1990

23,500

7.90

12.48

15.35

9.17

10.76

11.53

14.43

10.74

14.03

14.55

20.45

13.73

20.20

17.43

27.27
03573000 Short Creek near Albertville

L ocation-Lat $34^{\circ} 18^{\prime} 05^{\prime \prime}$, long $86^{\circ} 10^{\prime} 53^{\prime \prime}$, in NE $1 / 4$ sec. 35 , T. 8 S., R. 4 E., Marshall County, Hydrologic Unit 06030001, $800 \mathrm{ft}$ downstream from Turkey Creek, 3 mi northeast of Albertville, and 4.4 mi upstream from Scarham Creek.

Drainage area-91.6 $\mathrm{mi}^{2}$.

G age -Water-stage recorder 1946-53. Crest-stage gage 1954-69. Datum of gage is $865.80 \mathrm{ft}$ NGVD 29.

\begin{tabular}{|c|c|c|c|c|c|}
\hline $\begin{array}{l}\text { Water } \\
\text { year }\end{array}$ & Date & Discharge & $\begin{array}{l}\text { Discharge } \\
\text { code }\end{array}$ & $\begin{array}{c}\text { Gage } \\
\text { height } \\
\text { (feet) }\end{array}$ & $\begin{array}{c}\text { Gage } \\
\text { height } \\
\text { code }\end{array}$ \\
\hline 1943 & Dec & 25,000 & $7, \mathrm{~B}$ & 21.20 & \\
\hline 1946 & Feb. 10 & 8,300 & & 12.77 & \\
\hline 1947 & Jan. 15 & 6,800 & & 11.70 & \\
\hline 1948 & Feb. 14 & 6,790 & & 11.65 & \\
\hline 1949 & Jan. 5 & 14,800 & & 16.37 & \\
\hline 1950 & Mar. 13 & 8,100 & & 12.64 & \\
\hline 1951 & Mar. 29 & 13,200 & & 15.55 & \\
\hline 1952 & Dec. 21 & 4,800 & & 10.14 & \\
\hline 1953 & June 16 & 4,890 & & 10.23 & \\
\hline 1954 & Jan. 16 & 2,700 & & 7.90 & \\
\hline 1955 & Feb. 6 & 3,660 & & 8.97 & \\
\hline 1956 & Apr. 16 & 4,760 & & 10.11 & \\
\hline 1957 & June 9 & 6,650 & & 11.65 & \\
\hline 1958 & Nov. 14 & 3,760 & & 9.10 & \\
\hline 1961 & Feb. 22 & 6,770 & & 11.86 & \\
\hline 1962 & Dec. 18 & 10,400 & & 14.10 & \\
\hline 1963 & Apr. 29 & 11,800 & & 14.79 & \\
\hline 1964 & Mar. 26 & 5,060 & & 10.40 & \\
\hline 1966 & Mar. 4 & 9,420 & & 13.54 & \\
\hline 1967 & July 14 & 3,760 & & 9.10 & \\
\hline 1968 & Jan. 10 & 4,820 & & 10.16 & \\
\hline 1969 & Feb. 2 & 3,950 & & 9.29 & \\
\hline
\end{tabular}


Appendix 2. Discharge and gage height data for streamgages in Alabama._- Continued

[--, date or data not available; ft, foot; $\mathrm{ft}^{3} / \mathrm{s}$, cubic foot per second; mi, mile; $\mathrm{mi}^{2}$, square mile; qualification codes apply to the discharge data: 1 , discharge is a maximum daily average; 2 , discharge is an estimate; 4 , discharge is less than indicated value, which is the minimum recordable discharge at this site; 5 , discharge affected to unknown degree by regulation or diversion; 6 , discharge affected by regulation or diversion; 7, discharge is a historic peak; A, year of occurrence is unknown or not exact; $\mathrm{B}$, month or day of occurrence is unknown or not exact; E, only maximum peak available for this year. qualification codes apply to the gage height data: 1, gage height affected by backwater; 2, gage height not the maximum for the year; 3, gage height at different data or at different site and datum;

5 , gage height is an estimate]

\section{Paint Rock River near Woodville}

L ocation-Lat $34^{\circ} 37^{\prime} 27^{\prime \prime}$, long $86^{\circ} 18^{\prime} 23^{\prime \prime}$, in NW 1/4 sec. 10, T. 5

S., R. 3 E., Jackson County, Hydrologic Unit 06030002, on U.S.

Highway 72, 2 mi west of Woodville, 4.1 mi upstream from Little

Paint Creek, and at mile 26.6.

Drainage area-320 $\mathrm{mi}^{2}$.

Gage-Water-stage recorder. Datum of gage is $570.95 \mathrm{ft}$ NGVD 29.

\begin{tabular}{|c|c|c|c|c|c|}
\hline $\begin{array}{l}\text { Water } \\
\text { year }\end{array}$ & Date & Discharge & $\begin{array}{l}\text { Discharge } \\
\text { code }\end{array}$ & $\begin{array}{c}\text { Gage } \\
\text { height } \\
\text { (feet) }\end{array}$ & $\begin{array}{r}\text { Gage } \\
\text { heigh } \\
\text { code }\end{array}$ \\
\hline
\end{tabular}

\begin{tabular}{llll}
\hline 1936 & Feb. 4 & 19,600 & 19.24 \\
1937 & Jan. 3 & 16,400 & 18.85 \\
1938 & Apr. 9 & 11,300 & 18.01
\end{tabular}

Apr. $9-11,300$

$1939 \quad$ Feb. $4 \quad 27,000$

$1940 \quad$ Feb. $19 \quad 7,920 \quad 16.89$

$1941 \quad$ Mar. $8 \quad 6,260 \quad 16.40$

1942 Mar. $22 \quad 4,570$

1943 Dec. 28 28,000

1944 Mar. $29 \quad 14,600$

1945 Feb. $18 \quad$ 13,200

$1946 \quad$ Jan. $9 \quad 16,400$

1947 Jan. $21 \quad 9,500$

1948 Feb. $13 \quad 23,600$

1949 Jan. $5 \quad 28,700$

1950 Jan. $7 \quad 19,400$

1951 Mar. $29 \quad$ 27,500

1952 Mar. $11 \quad$ 15,300

1953 Feb. $22 \quad 7,990$

1954 Jan. $16 \quad 22,800$

1955 Mar. $22 \quad 9,640$

1956 Feb. 4 23,600

1957 Feb. $1 \quad 25,900$

$1958 \quad$ Nov. $19 \quad 22,700$

1959 Jan. $22 \quad 13,900$

1960 Dec. $20 \quad 13,000$

1961 Feb. $23 \quad 21,700$

1962 Dec. $18 \quad 24,000$

1963 Mar. $12 \quad 46,700$

1964 Mar. $15 \quad 20,000$

1965 Mar. $27 \quad$ 16,000

1966 Feb. $14 \quad 11,000$

1967 July $13 \quad 8,400$

1968 Dec. $18 \quad 24,000$

\section{Paint Rock River near Woodville-Continued}

L ocation-Lat $34^{\circ} 37^{\prime} 27^{\prime \prime}$, long $86^{\circ} 18^{\prime} 23^{\prime \prime}$, in NW 1/4 sec. 10, T. 5 S., R. 3 E., Jackson County, Hydrologic Unit 06030002, on U.S. Highway 72, 2 mi west of Woodville, $4.1 \mathrm{mi}$ upstream from Little Paint Creek, and at mile 26.6.

Drainage area-320 $\mathrm{mi}^{2}$.

G age - Water-stage recorder. Datum of gage is $570.95 \mathrm{ft}$ NGVD 29.

\begin{tabular}{lllccc}
\hline $\begin{array}{c}\text { Water } \\
\text { year }\end{array}$ & Date & Discharge & $\begin{array}{c}\text { Discharge } \\
\text { code }\end{array}$ & $\begin{array}{c}\text { Gage } \\
\text { height } \\
\text { (feet) }\end{array}$ & $\begin{array}{c}\text { Gage } \\
\text { height } \\
\text { code }\end{array}$ \\
\hline 1969 & Feb. 2 & 20,000 & & 19.32 & \\
1970 & Dec. 31 & 27,000 & & 20.49 & 2 \\
1971 & Feb. 22 & 15,200 & 18.39 & \\
1972 & Dec. 7 & 18,000 & 19.01 & \\
1973 & Mar. 16 & 74,200 & & 24.40 &
\end{tabular}

$1973 \quad$ Mar. $16 \quad 74,200 \quad 24.40$

1974 Dec. $27 \quad 32,000 \quad 21.06$

1975 Mar. $14 \quad 27,000 \quad 20.40$

1976 Oct. $9 \quad 20,000 \quad 19.57$

1977 Apr. $5 \quad 14,000 \quad 18.60$

$1978 \quad$ Nov. $6 \quad 20,100 \quad 19.62$

1979 Mar. $4 \quad 19,700 \quad 19.49$

1980 Mar. $21 \quad 31,200 \quad 21.39$

$1981 \quad$ Mar. $31 \quad 9,000 \quad 16.93$

1982 Jan. $4 \quad 20,000 \quad 19.57$

1983 Apr. $6 \quad 22,000 \quad 19.96$

1984 May $4 \quad 16,000 \quad 18.94$

$1985 \quad$ Feb. $2 \quad 9,000 \quad 17.01$

1986 Mar. $14 \quad 11,000 \quad 17.75$

1987 Jan. $20 \quad 14,000 \quad 18.35$

$1988 \quad$ Jan. $20 \quad 19,000 \quad 19.36$

1989 Apr. $5 \quad 12,000 \quad 17.85$

$1990 \quad$ Feb. $16 \quad 23,000 \quad 19.89$

1991 Dec. $23 \quad 58,000 \quad 23.42$

1992 Dec. $2 \quad 34,000 \quad 21.36$

1993 Mar. $24 \quad 28,000 \quad 20.64$

$1994 \quad$ Feb. $11 \quad 32,000 \quad 21.08$

1995 Mar. $9 \quad 9,500 \quad 17.07$

1996 Mar. $8 \quad 15,000 \quad 18.55$

1997 Mar. $20 \quad 10,000 \quad 17.31$

1998 Jan. $8 \quad 29,000 \quad 20.87$

$1999 \quad$ Jan. $24 \quad 14,000 \quad 18.35$

2000 Apr. $4 \quad 21,200 \quad 19.73$

$2001 \quad$ Jan. $20 \quad 16,500 \quad 18.84$ 
Appendix 2. Discharge and gage height data for streamgages in Alabama._-Continued

[--, date or data not available; $\mathrm{ft}$, foot; $\mathrm{ft}^{3} / \mathrm{s}$, cubic foot per second; mi, mile; $\mathrm{mi}^{2}$, square mile; qualification codes apply to the discharge data: 1 , discharge is a maximum daily average; 2 , discharge is an estimate; 4 , discharge is less than indicated value, which is the minimum recordable discharge at this site; 5 , discharge affected to unknown degree by regulation or diversion; 6 , discharge affected by regulation or diversion; 7, discharge is a historic peak; A, year of occurrence is unknown or not exact; B, month or day of occurrence is unknown or not exact; E, only maximum peak available for this year. qualification codes apply to the gage height data: 1, gage height affected by backwater; 2, gage height not the maximum for the year; 3, gage height at different datum or at different site and datum; 5 , gage height is an estimate]

\section{Paint Rock River near Woodville-Continued}

L ocation-Lat $34^{\circ} 37^{\prime} 27^{\prime \prime}$, long $86^{\circ} 18^{\prime} 23^{\prime \prime}$, in NW 1/4 sec. 10, T. 5

S., R. 3 E., Jackson County, Hydrologic Unit 06030002, on U.S.

Highway 72, 2 mi west of Woodville, 4.1 mi upstream from Little

Paint Creek, and at mile 26.6.

Drainage area-320 $\mathrm{mi}^{2}$.

Gage -Water-stage recorder. Datum of gage is $570.95 \mathrm{ft}$ NGVD 29.

\begin{tabular}{|c|c|c|c|c|c|}
\hline $\begin{array}{c}\text { Water } \\
\text { year }\end{array}$ & Date & Discharge & $\begin{array}{c}\text { Discharge } \\
\text { code }\end{array}$ & $\begin{array}{c}\text { Gage } \\
\text { height } \\
\text { (feet) }\end{array}$ & $\begin{array}{c}\text { Gage } \\
\text { height } \\
\text { code }\end{array}$ \\
\hline 2002 & Dec. 15 & 17,000 & & 18.87 & \\
\hline 2003 & Feb. 16 & 24,500 & & 20.20 & \\
\hline 2004 & Feb. 7 & 19,800 & & 20.10 & \\
\hline 2005 & Dec. 7 & 29,000 & & 20.68 & \\
\hline 2006 & Jan. 19 & 7,410 & & 16.31 & \\
\hline 2007 & Nov. 17 & 4,830 & & 15.15 & \\
\hline 2008 & Mar. 5 & 4,290 & & 14.84 & \\
\hline 2009 & Dec. 11 & 13,100 & & 18.90 & \\
\hline 2010 & Dec. 10 & 17,300 & & 19.76 & \\
\hline 2011 & Mar. 10 & 12,200 & & 18.65 & \\
\hline 2012 & Jan. 24 & 12,900 & & 18.85 & \\
\hline 2013 & Jan. 16 & 12,900 & & 18.85 & \\
\hline 2014 & Apr. 8 & 6,560 & & 16.64 & \\
\hline 2015 & Jan. 5 & 6,760 & & 16.74 & \\
\hline
\end{tabular}

\section{Flint River near Chase}

L ocation-Lat $34^{\circ} 49^{\prime} 22^{\prime \prime}$, long 86 $28^{\prime} 59^{\prime \prime}$, in NE 1/4 sec. 35, T.

2 S., R. 1 E., Madison County, Hydrologic Unit 06030002, on

Winchester Road, $400 \mathrm{ft}$ downstream from Brier Fork, $4.3 \mathrm{mi}$

northeast of Chase, and at mile 36.2.

Drainage area $342 \mathrm{mi}^{2}$.

Gage -Water-stage recorder. Datum of gage is $640.37 \mathrm{ft}$ NGVD 29.

\begin{tabular}{|c|c|c|c|c|c|}
\hline $\begin{array}{c}\text { Water } \\
\text { year }\end{array}$ & Date & Discharge & $\begin{array}{l}\text { Discharge } \\
\text { code }\end{array}$ & $\begin{array}{c}\text { Gage } \\
\text { height } \\
\text { (feet) }\end{array}$ & $\begin{array}{c}\text { Gage } \\
\text { height } \\
\text { code }\end{array}$ \\
\hline 1929 & Sep & 57,000 & B & 25.00 & \\
\hline 1931 & Apr. 4 & 2,600 & & 6.20 & \\
\hline 1932 & Aug. 18 & 6,800 & & 10.10 & \\
\hline 1933 & May 10 & 14,200 & & 15.00 & \\
\hline 1934 & Mar. 2 & 20,300 & & 17.30 & \\
\hline 1935 & Mar. 12 & 14,500 & & 14.90 & \\
\hline 1936 & July 4 & 22,000 & & 18.13 & \\
\hline 1937 & Jan. 2 & 13,000 & & 14.48 & \\
\hline
\end{tabular}

03575000 Flint River near Chase-Continued

L ocation-Lat $34^{\circ} 49^{\prime} 22^{\prime \prime}$, long $86^{\circ} 28^{\prime} 59^{\prime \prime}$, in NE $1 / 4$ sec. 35 , T. 2 S., R. 1 E., Madison County, Hydrologic Unit 06030002, on Winchester Road, $400 \mathrm{ft}$ downstream from Brier Fork, $4.3 \mathrm{mi}$ northeast of Chase, and at mile 36.2.

Drainage area-342 $\mathrm{mi}^{2}$.

G age - Water-stage recorder. Datum of gage is $640.37 \mathrm{ft}$ NGVD 29.

\begin{tabular}{llcccc}
\hline $\begin{array}{c}\text { Water } \\
\text { year }\end{array}$ & Date & Discharge & $\begin{array}{c}\text { Discharge } \\
\text { code }\end{array}$ & $\begin{array}{c}\text { Gage } \\
\text { height } \\
\text { (feet) }\end{array}$ & $\begin{array}{c}\text { Gage } \\
\text { height } \\
\text { code }\end{array}$ \\
\hline 1938 & July 21 & 7,680 & & 10.52 & \\
1939 & Feb. 3 & 21,400 & 17.75 & \\
1940 & Mar. 14 & 3,870 & 7.44 & \\
1941 & Jan. 2 & 3,780 & 7.33 & \\
1942 & Feb. 17 & 4,150 & 7.72 & \\
1943 & Dec. 28 & 12,800 & & 14.18 &
\end{tabular}

1943 Dec. $28 \quad 12,800 \quad 14.18$

1944 Mar. $29 \quad 18,300 \quad 16.79$

$1945 \quad$ Feb. $17 \quad 14,500 \quad 15.14$

$1946 \quad$ Jan. $8 \quad 20,000 \quad 17.56$

1947 Jan. $20 \quad 7,950 \quad 11.43$

1948 Feb. $13 \quad 19,800 \quad 17.76$

$1949 \quad$ Jan. $5 \quad 44,600 \quad 23.61$

$1950 \quad$ Jan. $6 \quad 13,200 \quad 15.35$

1951 Feb. $1 \quad 32,300 \quad 21.98$

1952 Mar. $11 \quad$ 8,950 12.81

$1953 \quad$ Feb. $21 \quad 10,200 \quad 13.79$

$1954 \quad$ Jan. $21 \quad 58,500 \quad 25.00$

1955 Mar. $22 \quad 20,200 \quad 17.79$

1956 Feb. $3 \quad 14,900 \quad 16.00$

$1957 \quad$ Feb. $1 \quad 25,900 \quad 19.70$

1958 Nov. $19 \quad 17,200 \quad 16.80$

$1959 \quad$ July $18 \quad 5,620 \quad 9.60$

1960 Dec. $19 \quad 18,400 \quad 17.20$

1961 Mar. $8 \quad 16,300 \quad 16.37$

1962 Feb. $28 \quad 14,400 \quad 15.50$

1963 Mar. $12 \quad 84,000 \quad 27.55$

1964 Mar. $15 \quad 30,000 \quad 21.00$

1965 Mar. $30 \quad 11,200 \quad 13.67$

1966 Feb. $13 \quad 6,230 \quad 9.49$

1967 May $13 \quad 7,060 \quad 10.26$

1968 Dec. $18 \quad 29,900 \quad 20.97$ 
Appendix 2. Discharge and gage height data for streamgages in Alabama._- Continued

[--, date or data not available; ft, foot; $\mathrm{ft}^{3} / \mathrm{s}$, cubic foot per second; mi, mile; $\mathrm{mi}^{2}$, square mile; qualification codes apply to the discharge data: 1 , discharge is a maximum daily average; 2 , discharge is an estimate; 4 , discharge is less than indicated value, which is the minimum recordable discharge at this site; 5 , discharge affected to unknown degree by regulation or diversion; 6 , discharge affected by regulation or diversion; 7, discharge is a historic peak; A, year of occurrence is unknown or not exact; $\mathrm{B}$, month or day of occurrence is unknown or not exact; E, only maximum peak available for this year. qualification codes apply to the gage height data: 1, gage height affected by backwater; 2, gage height not the maximum for the year; 3, gage height at different data or at different site and datum; 5 , gage height is an estimate]

03575000 Flint River near Chase-Continued

L ocation-Lat $34^{\circ} 49^{\prime} 22^{\prime \prime}$, long $86^{\circ} 28^{\prime} 59^{\prime \prime}$, in NE $1 / 4$ sec. 35 , T. 2 S., R. 1 E., Madison County, Hydrologic Unit 06030002, on Winchester Road, $400 \mathrm{ft}$ downstream from Brier Fork, $4.3 \mathrm{mi}$ northeast of Chase, and at mile 36.2.

Drainage area-342 $\mathrm{mi}^{2}$.

Gage-Water-stage recorder. Datum of gage is $640.37 \mathrm{ft}$ NGVD 29.

\begin{tabular}{|c|c|c|c|c|c|}
\hline $\begin{array}{c}\text { Water } \\
\text { year }\end{array}$ & Date & Discharge & $\begin{array}{l}\text { Discharge } \\
\text { code }\end{array}$ & $\begin{array}{c}\text { Gage } \\
\text { height } \\
\text { (feet) }\end{array}$ & $\begin{array}{c}\text { Gage } \\
\text { height } \\
\text { code }\end{array}$ \\
\hline 1969 & May 18 & 15,200 & & 15.87 & \\
\hline 1970 & Dec. 30 & 23,700 & & 18.50 & \\
\hline 1971 & Feb. 22 & 14,400 & & 14.52 & \\
\hline 1972 & Jan. 10 & 6,960 & & 10.16 & \\
\hline 1973 & Mar. 16 & 104,000 & & 29.52 & \\
\hline 1974 & Dec. 26 & 24,800 & & 18.92 & \\
\hline 1975 & Mar. 14 & 29,800 & & 20.97 & \\
\hline 1976 & Oct. 17 & 24,000 & & 19.20 & \\
\hline 1977 & Apr. 5 & 33,100 & & 21.22 & \\
\hline 1978 & Oct. 2 & 27,500 & & 19.61 & \\
\hline 1979 & Mar. 4 & 18,100 & & 16.47 & \\
\hline 1980 & Mar. 21 & 18,000 & & 16.43 & \\
\hline 1981 & Mar. 30 & 4,460 & & 8.01 & \\
\hline 1983 & May 19 & 26,400 & & 19.35 & \\
\hline 1984 & May 3 & 15,500 & & 15.48 & \\
\hline 1985 & Nov. 28 & 8,470 & & 11.34 & \\
\hline 1986 & Feb. 18 & 9,090 & & 11.78 & \\
\hline 1987 & Nov. 24 & 11,900 & & 13.63 & \\
\hline 1988 & Jan. 20 & 11,600 & & 13.41 & \\
\hline 1989 & Mar. 6 & 26,000 & & 19.22 & \\
\hline 1990 & Oct. 2 & 17,700 & & 16.33 & \\
\hline 1991 & Dec. 23 & 120,000 & & 31.04 & \\
\hline 1992 & Dec. 2 & 30,900 & & 20.63 & \\
\hline 1993 & May 4 & 20,400 & & 17.36 & \\
\hline 1994 & Feb. 11 & 36,900 & & 22.07 & \\
\hline
\end{tabular}

03575100 Flint River at Brownsboro, Ala.

L ocation-Lat $34^{\circ} 44^{\prime} 57^{\prime \prime}$, long $86^{\circ} 26^{\prime} 48^{\prime \prime}$ referenced to North American Datum of 1927, Madison County, AL, Hydrologic Unit 06030002, on right bank on Brownsboro Road, 0.3 mi north of U.S. Highway 72, 10 mi east of Huntsville, at Brownsboro, and river mile 27.6.

Drainage area-375 $\mathrm{mi}^{2}$.

G age - Water-stage recorder. Datum of gage is $616.058 \mathrm{ft}$ above NAVD of 1988.

\begin{tabular}{llllll}
\hline $\begin{array}{c}\text { Water } \\
\text { year }\end{array}$ & Date & Discharge & $\begin{array}{c}\text { Discharge } \\
\text { code }\end{array}$ & $\begin{array}{c}\text { Gage } \\
\text { height } \\
\text { (feet) }\end{array}$ & $\begin{array}{c}\text { Gage } \\
\text { height } \\
\text { code }\end{array}$ \\
\hline 1999 & Jan. 23 & 26,300 & 20.61 & \\
2000 & Apr. 4 & 23,900 & 20.42 & \\
2001 & Dec. 17 & 11,900 & 18.57 & \\
2002 & Mar. 18 & 15,500 & 19.44 & \\
2003 & May 7 & 33,500 & 22.10 & \\
2004 & Feb. 6 & 36,400 & 23.03 & \\
2005 & Dec. 7 & 32,900 & 22.59 & \\
2006 & Apr. 8 & 6,540 & 16.16 & \\
2007 & Nov. 15 & 4,810 & 14.75 & \\
2008 & Apr. 5 & 5,440 & 15.33 & \\
2009 & Dec. 10 & 22,100 & 20.91 & \\
2010 & Dec. 9 & 21,600 & 20.83 & \\
2011 & Apr. 28 & 56,000 & 24.58 & \\
2012 & Jan. 12 & 16,800 & 19.63 \\
2013 & Jan. 16 & 15,100 & 19.28 \\
2014 & Dec. 22 & 9,160 & 17.62 \\
2015 & Mar. 11 & 12,800 & 18.75 &
\end{tabular}

\section{Limestone Creek near Athens}

L ocation-Lat $34^{\circ} 45^{\prime} 06^{\prime \prime}$, long $86^{\circ} 49^{\prime} 24^{\prime \prime}$, in SW 1/4 sec. 26 , T. 3 S., R. 3 W., Limestone County, Hydrologic Unit 06030002, on U.S. Highway 72, $10 \mathrm{mi}$ east of Athens, and at mile 17.0.

Drainage area-119 $\mathrm{mi}^{2}$.

G age - Water-stage recorder. Datum of gage is $626.34 \mathrm{ft}$ NGVD 29.

\begin{tabular}{llrcrc}
\hline $\begin{array}{c}\text { Water } \\
\text { year }\end{array}$ & Date & Discharge & $\begin{array}{c}\text { Discharge } \\
\text { code }\end{array}$ & $\begin{array}{c}\text { Gage } \\
\text { height } \\
\text { (feet) }\end{array}$ & $\begin{array}{c}\text { Gage } \\
\text { height } \\
\text { code }\end{array}$ \\
\hline 1940 & July 8 & 3,000 & & 8.00 & \\
1941 & Jan. 2 & 1,650 & 5.80 & \\
1942 & Feb. 17 & 2,400 & 7.20 & \\
1943 & Dec. 28 & 3,250 & 7.90 & \\
1944 & Mar. 29 & 10,000 & 11.50 &
\end{tabular}


Appendix 2. Discharge and gage height data for streamgages in Alabama.-Continued

[--, date or data not available; ft, foot; $\mathrm{ft}^{3} / \mathrm{s}$, cubic foot per second; mi, mile; $\mathrm{mi}^{2}$, square mile; qualification codes apply to the discharge data: 1 , discharge is a maximum daily average; 2 , discharge is an estimate; 4 , discharge is less than indicated value, which is the minimum recordable discharge at this site; 5 , discharge affected to unknown degree by regulation or diversion; 6 , discharge affected by regulation or diversion; 7, discharge is a historic peak; A, year of occurrence is unknown or not exact; B, month or day of occurrence is unknown or not exact; E, only maximum peak available for this year. qualification codes apply to the gage height data: 1 , gage height affected by backwater; 2, gage height not the maximum for the year; 3, gage height at different datum or at different site and datum; 5 , gage height is an estimate]

$\mathbf{0 3 5 7 6 2 5 0}$ Limestone Creek near Athens-Continued

L ocation-Lat $34^{\circ} 45^{\prime} 06^{\prime \prime}$, long $86^{\circ} 49^{\prime} 24^{\prime \prime}$, in SW 1/4 sec. 26, T. 3

S., R. 3 W., Limestone County, Hydrologic Unit 06030002, on

U.S. Highway 72, $10 \mathrm{mi}$ east of Athens, and at mile 17.0.

Drainage area-119 $\mathrm{mi}^{2}$.

Gage-Water-stage recorder. Datum of gage is $626.34 \mathrm{ft}$ NGVD 29.

\begin{tabular}{|c|c|c|c|c|c|}
\hline $\begin{array}{c}\text { Water } \\
\text { year }\end{array}$ & Date & Discharge & $\begin{array}{c}\text { Discharge } \\
\text { code }\end{array}$ & $\begin{array}{l}\text { Gage } \\
\text { height }\end{array}$ & $\begin{array}{l}\text { Gage } \\
\text { height }\end{array}$ \\
\hline
\end{tabular}

\begin{tabular}{llrrr} 
& & & (feet) & code \\
\hline 1945 & Feb. 17 & 6,600 & 10.10 & \\
1946 & Jan. 8 & 10,700 & 11.67 & \\
1947 & Jan. 20 & 3,250 & 7.90
\end{tabular}

1948

1949

1950

1951

1952

1953

1954

1955

1956

1957

1958

1959

1960

1961

1962

1963

1964

1965

1966

1967

1968

1969

1970

1971

1972

1973

1975

1976
Jan. 20

3,250

Feb. 13

9,950

Jan. 5

15,000

Jan. 6

8,950

Feb. $1 \quad$ 16,000

Dec. 8

6,650

Feb. 21

5,600

Jan. 21

21,000

Feb. 22

6,700

Feb. 3

4,800

Feb. 1

10,200

Nov. 18

8,600

July 18

4,300

Dec. 19

8,650

Mar. 8

9,000

Feb. 28

7,600

26,000

15,500

Mar. 15

Mar. 29

4,850

2,700

June 29

3,340

Dec. 18

12,500

Feb. 2

Dec. 30

6,100

10,800

6,750

2,800

38,000

Mar. 16

Mar. 13

12,500

Oct. 17

13,600
7.90

11.45

12.89

11.14

13.16

10.15

9.70

14.32

10.19

9.23

11.60

10.91

8.92

10.96

11.08

10.59

15.50

13.00

9.30

7.59

8.34

12.17

9.92

11.74

10.20

7.71

17.28

12.21

12.61
03576250 Limestone Creek near Athens-Continued

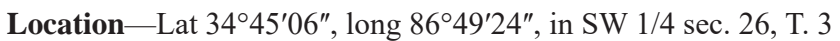
S., R. 3 W., Limestone County, Hydrologic Unit 06030002, on U.S. Highway 72, $10 \mathrm{mi}$ east of Athens, and at mile 17.0.

Drainage area-119 $\mathrm{mi}^{2}$.

G age -Water-stage recorder. Datum of gage is $626.34 \mathrm{ft}$ NGVD 29.

\begin{tabular}{|c|c|c|c|c|c|}
\hline $\begin{array}{c}\text { Water } \\
\text { year }\end{array}$ & Date & Discharge & $\begin{array}{l}\text { Discharge } \\
\text { code }\end{array}$ & $\begin{array}{c}\text { Gage } \\
\text { height } \\
\text { (feet) }\end{array}$ & $\begin{array}{c}\text { Gage } \\
\text { height } \\
\text { code }\end{array}$ \\
\hline 1977 & Apr. 5 & 13,000 & & 12.38 & \\
\hline 1978 & Nov. 5 & 7,930 & & 10.84 & \\
\hline 1979 & Mar. 4 & 5,700 & & 9.70 & \\
\hline
\end{tabular}

1979 Mar. $4 \quad 5,700-9.70$

1980 Mar. $20 \quad 12,200 \quad 12.12$

1981 June $4 \quad 1,860 \quad 6.26$

$1982 \quad$ Jan. $4 \quad 10,500 \quad$ E $\quad 11.81$

1984 May $3 \quad 8,500 \quad$ E $\quad 10.93$

$1985 \quad$ Aug. $17 \quad 3,650 \quad$ E $\quad 8.55$

1992 Dec. $23 \quad 25,000 \quad 7 \quad 15.20$

1995 Mar. $8 \quad 3,150 \quad 8.08$

$1996 \quad$ Mar. $7 \quad 5,700 \quad 9.72$

1997 Mar. $3 \quad 5,500 \quad 9.59$

$1998 \quad$ Jan. $7 \quad 10,300 \quad 11.76$

1999 Jan. $23 \quad 17,000 \quad 13.56$

2000 Apr. $3 \quad 8,700 \quad 11.01$

$2001 \quad$ July $5 \quad 5,050 \quad 9.36$

$2002 \quad$ Jan. $23 \quad 5,100 \quad 9.39$

2003 May $6 \quad 13,500 \quad 12.49$

2004 Feb. $6 \quad 15,400 \quad 12.99$

2005 Dec. $7 \quad 11,800 \quad 12.00$

$2006 \quad$ Jan. $23 \quad 2,940 \quad 7.46$

2007 Jan. $5 \quad 2,060 \quad 6.13$

2008 Apr. $5 \quad 2,360 \quad 6.56$

2009 Dec. $10 \quad 6,380 \quad 10.00$

2010 Dec. $9 \quad 12,800 \quad 12.82$

2011 Apr. $27 \quad 25,800 \quad 15.10$

$2012 \quad$ Jan. $23 \quad 6,170 \quad 10.44$

$2013 \quad$ July $5 \quad 12,200 \quad 12.67$

2014 Apr. $29 \quad 4,530 \quad 9.08$

2015 Mar. $11 \quad 4,900 \quad 9.44$

2016 Dec. $25 \quad 9,710 \quad 12.01$ 
Appendix 2. Discharge and gage height data for streamgages in Alabama._- Continued

[--, date or data not available; ft, foot; $\mathrm{ft}^{3} / \mathrm{s}$, cubic foot per second; mi, mile; $\mathrm{mi}^{2}$, square mile; qualification codes apply to the discharge data: 1 , discharge is a maximum daily average; 2 , discharge is an estimate; 4 , discharge is less than indicated value, which is the minimum recordable discharge at this site; 5 , discharge affected to unknown degree by regulation or diversion; 6 , discharge affected by regulation or diversion; 7, discharge is a historic peak; A, year of occurrence is unknown or not exact; $\mathrm{B}$, month or day of occurrence is unknown or not exact; E, only maximum peak available for this year. qualification codes apply to the gage height data: 1, gage height affected by backwater; 2, gage height not the maximum for the year; 3, gage height at different data or at different site and datum; 5 , gage height is an estimate]

\section{Flint Creek near Falkville}

L ocation-Lat $34^{\circ} 22^{\prime} 23^{\prime \prime}$, long $86^{\circ} 56^{\prime} 01^{\prime \prime}$, in SW 1/4 sec. 2 , T. 8 S., R. 4 W., Morgan County, Hydrologic Unit 06030002, $1.2 \mathrm{mi}$ downstream from Robinson Creek, $1.5 \mathrm{mi}$ west of Falkville, and 2.8 mi upstream from Cedar Creek.

Drainage area $-86.3 \mathrm{mi}^{2}$.

G age - Water-stage recorder. Datum of gage is $572.59 \mathrm{ft}$ NGVD 29.

\begin{tabular}{|c|c|c|c|c|c|}
\hline $\begin{array}{l}\text { Water } \\
\text { year }\end{array}$ & Date & Discharge & $\begin{array}{l}\text { Discharge } \\
\text { code }\end{array}$ & $\begin{array}{l}\text { Gage } \\
\text { height } \\
\text { (feet) }\end{array}$ & $\begin{array}{c}\text { Gage } \\
\text { height } \\
\text { code }\end{array}$ \\
\hline
\end{tabular}

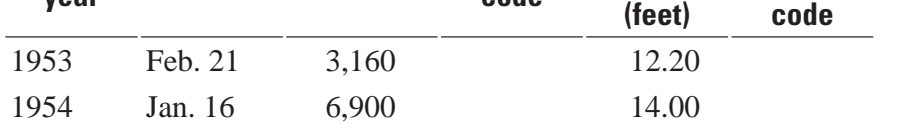

$1955 \quad$ Mar. $21 \quad 8,400 \quad 14.60$

$1956 \quad$ Feb. $4 \quad 2,410 \quad 11.80$

1957 Feb. $1 \quad 5,300 \quad 13.30$

1958 Nov. $19 \quad 7,400 \quad 14.20$

1959 Jan. $22 \quad 6,600 \quad 13.80$

$1960 \quad$ Mar. $3 \quad 5,920 \quad 13.54$

1961 Feb. $22 \quad 12,200 \quad 15.77$

1962 Apr. $11 \quad 10,100 \quad 15.15$

1963 Mar. $12 \quad 6,200 \quad 13.75$

1964 Mar. $26 \quad 9,420 \quad 14.94$

1965 Mar. $26 \quad 6,760 \quad 13.98$

1966 May $18 \quad 4,440 \quad 12.87$

$1967 \quad$ May $7 \quad 2,260 \quad 11.71$

$1968 \quad$ Jan. $10 \quad 6,600 \quad 13.84$

1969 Feb. $2 \quad 6,600 \quad 13.80$

1970 Dec. $31 \quad 7,630 \quad 14.33$

1971 Feb. $26 \quad 6,120 \quad 13.69$

1972 Dec. $7 \quad 4,050 \quad 12.67$

1973 Mar. $16 \quad 12,500 \quad 15.85$

$1992 \quad$ Dec. $23 \quad 30,000 \quad 2,7 \quad 19.28$

$1993 \quad$ Mar. $23 \quad 5,760 \quad 13.54$

$1994 \quad$ Feb. $11 \quad 8,310 \quad 14.57$

$1995 \quad$ Feb. $16 \quad 5,900 \quad 13.63$

$1996 \quad$ Mar. $7 \quad 2,900 \quad 12.16$

1997 May $3 \quad 5,700 \quad 13.51$

$1998 \quad$ Jan. $7 \quad 9,200 \quad 14.85$

$1999 \quad$ Jan. $23 \quad 3,160 \quad 12.20$

$2012 \quad$ Jan. $11 \quad 3,160 \quad 13.04$

$2013 \quad$ Jan. $15 \quad 3,330 \quad 13.13$

$2014 \quad$ Apr. $7 \quad 2,530 \quad 12.59$

2015 Jan. $4 \quad 3,220 \quad 12.53$
03577110 West Flint Creek near Hartselle

L ocation-Lat $34^{\circ} 29^{\prime} 38^{\prime \prime}$, long $87^{\circ} 01^{\prime} 34^{\prime \prime}$, in NW 1/4 NW 1/4 SE 1/4 sec. 26, T. 6 S., R. 5 W., Morgan County, Hydrologic Unit 06030002, on pier at right bank of Means Bridge on DecaturDanville Road, $7.5 \mathrm{mi}$ southwest of Decatur, and 6 mi northwest of Hartselle.

Drainage area-158 $\mathrm{mi}^{2}$.

Gage - Water-stage recorder. Datum of gage is $553.67 \mathrm{ft}$ NGVD 29.

Remarks - Station operated and records published by Tennessee Valley Authority.

\begin{tabular}{|c|c|c|c|c|c|}
\hline $\begin{array}{l}\text { Water } \\
\text { year }\end{array}$ & Date & Discharge & $\begin{array}{l}\text { Discharge } \\
\text { code }\end{array}$ & $\begin{array}{c}\text { Gage } \\
\text { height } \\
\text { (feet) }\end{array}$ & $\begin{array}{c}\text { Gage } \\
\text { height } \\
\text { code }\end{array}$ \\
\hline 1941 & Mar. 8 & 1,460 & & 11.85 & \\
\hline 1942 & Mar. 18 & 1,620 & & 13.01 & \\
\hline 1943 & Dec. 29 & 2,320 & & 14.21 & \\
\hline 1944 & Mar. 29 & 5,870 & 2 & 16.56 & \\
\hline 1945 & Feb. 13 & 2,970 & & 14.92 & \\
\hline 1946 & Jan. 8 & 8,660 & & 17.55 & \\
\hline 1947 & Jan. 20 & 2,760 & & 14.71 & \\
\hline 1948 & Feb. 13 & 7,060 & & 17.02 & \\
\hline 1949 & Jan. 5 & 10,300 & & 17.98 & \\
\hline 1950 & Jan. 7 & 10,300 & & 17.98 & \\
\hline 1951 & Mar. 29 & 9,550 & & 17.78 & \\
\hline 1952 & Mar. 11 & 3,080 & & 14.20 & \\
\hline 1953 & Feb. 21 & 3,300 & & 14.44 & \\
\hline 1954 & Jan. 22 & 4,950 & & 15.81 & \\
\hline 1955 & Mar. 22 & 4,830 & & 15.74 & \\
\hline 1956 & Feb. 4 & 4,530 & & 15.54 & \\
\hline 1957 & Feb. 1 & 6,190 & & 16.49 & \\
\hline 1958 & Nov. 18 & 5,910 & & 16.35 & \\
\hline 1992 & Dec. 24 & 11,400 & 2,7 & -- & \\
\hline
\end{tabular}


Appendix 2. Discharge and gage height data for streamgages in Alabama._-Continued

[--, date or data not available; $\mathrm{ft}$, foot; $\mathrm{ft}^{3} / \mathrm{s}$, cubic foot per second; mi, mile; $\mathrm{mi}^{2}$, square mile; qualification codes apply to the discharge data: 1 , discharge is a maximum daily average; 2 , discharge is an estimate; 4 , discharge is less than indicated value, which is the minimum recordable discharge at this site; 5 , discharge affected to unknown degree by regulation or diversion; 6 , discharge affected by regulation or diversion; 7, discharge is a historic peak; A, year of occurrence is unknown or not exact; B, month or day of occurrence is unknown or not exact; E, only maximum peak available for this year. qualification codes apply to the gage height data: 1, gage height affected by backwater; 2, gage height not the maximum for the year; 3, gage height at different datum or at different site and datum; 5 , gage height is an estimate]

\section{Big Nance Creek at Courtland}

L ocation-Lat $34^{\circ} 40^{\prime} 12^{\prime \prime}$, long $87^{\circ} 19^{\prime} 02^{\prime \prime}$, in SW 1/4 sec. 30, T. 4 S., R. 7 W., Lawrence County, Hydrologic Unit 06030005, on county road 25, at Courtland, and at mile 12.9.

Drainage area-166 $\mathrm{mi}^{2}$.

G age - Water-stage recorder. Datum of gage is $537.60 \mathrm{ft}$ NGVD 29.

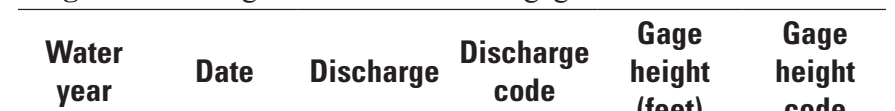

\begin{tabular}{lllll}
\hline 1936 & Apr. 3 & 6,980 & (feet) & code \\
1937 & Jan. 3 & 4,400 & 19.80 \\
1938 & Mar. 11 & 4,000 & 16.10 \\
1939 & Feb. 16 & 6,980 & 15.30 \\
\end{tabular}

1940

1946

1947

1948

1949

1950

1951

1952

1953

1954

1955

1956

1957

1958

1959

1960

1961

1962

1963

1964

1965

1966

1967

1968

1969

1970

1971

1972

1973

1974

1975

July 10

4,650

Feb. 10

8,180

Jan. 2

3,960

Feb. 13

9,000

Jan. 5

11,000

Jan. 7

12,500

Feb. 1

12,000

Dec. 9 8,140

Feb. $22 \quad 5,300$

Jan. $23 \quad 7,620$

Mar. 22

7,160

Apr. 7

Feb. 1

4,080

9,000

6,700

2,400

Apr. 19

Mar. 3

5,610

Feb. $23 \quad 3,760$

Feb. 24

7,600

Mar. 12

10,100

Mar. 16

6,800

Feb. 13

5,160

May 2

1,890

Feb. $21 \quad 4,300$

Dec. $19 \quad 8,560$

Feb. $3 \quad 7,400$

Dec. $31 \quad 8,800$

Feb. $23 \quad 4,590$

Jan. $5 \quad$ 4,330

Mar. 16

26,500

6,110

Dec. 27

10,200
17.00

21.00

15.70

21.50

22.25

22.60

22.45

20.92

17.99

20.49

20.27

16.50

21.50

19.52

12.02

18.94

15.77

20.54

21.75

19.89

18.43

10.72

16.14

21.09

20.42

21.22

17.54

17.04

24.97

19.48

21.94
03586500 Big Nance Creek at Courtland-Continued

L ocation-Lat $34^{\circ} 40^{\prime} 12^{\prime \prime}$, long $87^{\circ} 19^{\prime} 02^{\prime \prime}$, in SW 1/4 sec. 30, T. 4 S., R. 7 W., Lawrence County, Hydrologic Unit 06030005, on county road 25, at Courtland, and at mile 12.9 .

Drainage area-166 $\mathrm{mi}^{2}$.

G age-Water-stage recorder. Datum of gage is $537.60 \mathrm{ft}$ NGVD 29.

\begin{tabular}{|c|c|c|c|c|c|}
\hline $\begin{array}{c}\text { Water } \\
\text { year }\end{array}$ & Date & Discharge & $\begin{array}{c}\text { Discharge } \\
\text { code }\end{array}$ & $\begin{array}{c}\text { Gage } \\
\text { height } \\
\text { (feet) }\end{array}$ & $\begin{array}{c}\text { Gage } \\
\text { height } \\
\text { code }\end{array}$ \\
\hline 1976 & Oct. 18 & 10,700 & & 22.15 & \\
\hline 1977 & Apr. 5 & 6,000 & & 18.75 & \\
\hline 1978 & May 9 & 5,450 & & 18.13 & \\
\hline
\end{tabular}

1980 Mar. $21 \quad 7,270 \quad 20.34$

1981 Mar. $31 \quad 2,350 \quad 11.17$

1988 Apr. $13 \quad 1,980 \quad 10.09$

1989 Mar. $6 \quad 6,450 \quad 19.26$

1990 Oct. $2 \quad 7,500.36$

1991 Dec. $23 \quad 22,000 \quad 24.21$

1992 Dec. $3 \quad 8,750 \quad 21.13$

1993 Mar. $24 \quad 5,000 \quad 17.32$

1994 Mar. $28 \quad 7,400 \quad 20.32$

$1995 \quad$ Feb. $17 \quad 6,920 \quad 19.73$

1996 Mar. $8 \quad 5,230 \quad 17.70$

1997 June $23 \quad 5,660 \quad 18.33$

1998 Jan. $8 \quad 8,130 \quad 20.73$

1999 Jan. $24 \quad 11,200 \quad 21.98$

2000 Apr. $4 \quad 6,230 \quad 19.03$

2001 Jan. $20 \quad 5,190$

2002 May $5 \quad 6,200 \quad 19.00$

2003 Feb. $23 \quad 5,850 \quad 18.57$

$2004 \quad$ Feb. $7 \quad 10,600 \quad 21.76$

2005 Dec. $8 \quad 8,000 \quad 20.63$

2006 Jan. $18 \quad 3,760 \quad 14.55$

2007 Jan. $1 \quad 1,800 \quad 9.92$

2008 Mar. $5 \quad 1,980 \quad 10.44$

2009 Dec. $12 \quad 4,580 \quad 16.31$

2010 Dec. $10 \quad 5,030 \quad 17.15$

2011 Apr. $28 \quad 14,400 \quad 22.79$

2012 Jan. $24 \quad 3,920 \quad 15.03$

$2013 \quad$ July $7 \quad 6,770 \quad 19.58$

2014 Apr. $8 \quad 2,790 \quad 12.55$

2015 Dec. $25 \quad 3,990$ 
Appendix 2. Discharge and gage height data for streamgages in Alabama._- Continued

[--, date or data not available; ft, foot; $\mathrm{ft}^{3} / \mathrm{s}$, cubic foot per second; mi, mile; $\mathrm{mi}^{2}$, square mile; qualification codes apply to the discharge data: 1 , discharge is a maximum daily average; 2 , discharge is an estimate; 4 , discharge is less than indicated value, which is the minimum recordable discharge at this site; 5 , discharge affected to unknown degree by regulation or diversion; 6 , discharge affected by regulation or diversion; 7, discharge is a historic peak; A, year of occurrence is unknown or not exact; $\mathrm{B}$, month or day of occurrence is unknown or not exact; E, only maximum peak available for this year. qualification codes apply to the gage height data: 1, gage height affected by backwater; 2, gage height not the maximum for the year; 3, gage height at different data or at different site and datum; 5 , gage height is an estimate]

\section{Cypress Creek near Florence}

L ocation-Lat $34^{\circ} 48^{\prime} 27^{\prime \prime}$, long $87^{\circ} 42^{\prime} 02^{\prime \prime}$, in NE $1 / 4$ sec.9, T. 3

S., R. 11 W., Lauderdale County, Hydrologic Unit 06030005, on State Highway 2, 2 mi west of Florence, 4 mi downstream from

Cox Creek, and 4 mi upstream from mouth.

Drainage area-209 $\mathrm{mi}^{2}$.

Gage-Water-stage recorder. Datum of gage is $423.78 \mathrm{ft}$ NGVD 29.

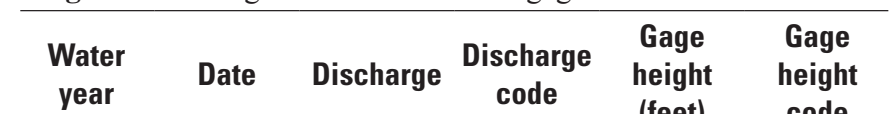

\begin{tabular}{|c|c|c|c|c|}
\hline & & & & (teet) \\
\hline 1935 & Mar. 12 & 8,000 & & 9.43 \\
\hline 1936 & Apr. 6 & 6,170 & & 7.95 \\
\hline 1937 & May 4 & 20,600 & & 16.60 \\
\hline 1938 & Aug. 29 & 4,760 & & 6.51 \\
\hline 1939 & Feb. 15 & 11,300 & & 11.73 \\
\hline 1940 & Apr. 19 & 13,000 & & 12.97 \\
\hline 1941 & July 4 & 2,400 & & 3.79 \\
\hline 1942 & Mar. 17 & 2,980 & & 4.53 \\
\hline 1943 & Dec. 28 & 6,230 & & 8.00 \\
\hline 1944 & Mar. 28 & 10,200 & & 11.06 \\
\hline 1945 & Feb. 22 & 13,000 & & 12.90 \\
\hline 1946 & Nov. 22 & 13,900 & & 13.45 \\
\hline 1947 & Apr. 16 & 4,400 & & 6.19 \\
\hline 1948 & Feb. 13 & 21,400 & & 17.40 \\
\hline 1949 & Mar. 27 & 13,400 & & 13.17 \\
\hline 1950 & Feb. 14 & 13,600 & & 13.36 \\
\hline 1951 & Mar. 28 & 25,100 & & 19.20 \\
\hline 1952 & Jan. 27 & 9,190 & & 10.35 \\
\hline 1953 & Feb. 12 & 11,900 & & 12.17 \\
\hline 1955 & Mar. 24 & 50,000 & 7 & 29.94 \\
\hline 2015 & Feb. 22 & 3,710 & & 5.65 \\
\hline
\end{tabular}

\section{Bear Creek near Hackleburg}

L ocation-Lat $34^{\circ} 17^{\prime} 01^{\prime \prime}$, long $87^{\circ} 46^{\prime} 26^{\prime \prime}$, in SW 1/4 sec. 11, T. 9 S., R. 12 W., Marion County, Hydrologic Unit 06030006, on State Highway 172, 2 mi upstream from Bluff Creek, 3.5 mi east of Hackleburg, and at mile 104.8.

Drainage area-143 $\mathrm{mi}^{2}$.

G age - Water-stage recorder. Datum of gage is $646.50 \mathrm{ft}$ NGVD 29.

\begin{tabular}{lccccc}
\hline $\begin{array}{c}\text { Water } \\
\text { year }\end{array}$ & Date & Discharge & $\begin{array}{c}\text { Discharge } \\
\text { code }\end{array}$ & $\begin{array}{c}\text { Gage } \\
\text { height } \\
\text { (feet) }\end{array}$ & $\begin{array}{c}\text { Gage } \\
\text { height } \\
\text { code }\end{array}$ \\
\hline 1957 & Jan. 31 & 7,300 & & 20.00 & \\
1958 & Nov. 18 & 6,600 & & 19.20 &
\end{tabular}

$1959 \quad$ Jan. $21 \quad 2,520 \quad 10.30$

1960 Mar. $2 \quad 7,000 \quad 19.50$

$1961 \quad$ Feb. $21 \quad 5,100 \quad 16.20$

1962 Apr. $11 \quad 14,300 \quad 28.88$

1963 May $26 \quad 13,800 \quad 27.36$

1964 Mar. $26 \quad 6,500 \quad 18.84$

1965 Feb. $12 \quad 5,880 \quad 17.77$

$1966 \quad$ Feb. $13 \quad 2,420 \quad 10.05$

1967 Dec. $9 \quad 6,350 \quad 18.58$

1968 Dec. $18 \quad 13,800 \quad 27.90$

1969 Feb. $2 \quad 6,560 \quad 18.93$

$1970 \quad$ Dec. $30 \quad 13,000 \quad 27.09$

1971 Feb. $26 \quad 7,310 \quad 20.16$

1972 Jan. $4 \quad 6,310 \quad 18.52$

1973 Mar. $16 \quad 24,000 \quad 39.00$

1974 Dec. $26 \quad 7,970 \quad 21.10$

1975 Mar. $13 \quad 13,400 \quad 27.83$

1976 Oct. $17 \quad 14,800 \quad 29.31$

1977 Mar. $4 \quad$ 15,400 30.03

$\begin{array}{lllll}1978 & \text { May } 8 & 5,930 & 5 & 17.87\end{array}$

1979 Apr. $13 \quad 6,660 \quad 5 \quad 19.02$

$\begin{array}{lllll}1981 & \text { Mar. } 30 & 2,790 & 5 & 10.78\end{array}$ 
Appendix 2. Discharge and gage height data for streamgages in Alabama.-Continued

[--, date or data not available; $\mathrm{ft}$, foot; $\mathrm{ft}^{3} / \mathrm{s}$, cubic foot per second; mi, mile; $\mathrm{mi}^{2}$, square mile; qualification codes apply to the discharge data: 1 , discharge is a maximum daily average; 2 , discharge is an estimate; 4 , discharge is less than indicated value, which is the minimum recordable discharge at this site; 5 , discharge affected to unknown degree by regulation or diversion; 6 , discharge affected by regulation or diversion; 7, discharge is a historic peak; A, year of occurrence is unknown or not exact; $\mathrm{B}$, month or day of occurrence is unknown or not exact; E, only maximum peak available for this year. qualification codes apply to the gage height data: 1, gage height affected by backwater; 2, gage height not the maximum for the year; 3, gage height at different datum or at different site and datum; 5 , gage height is an estimate]

$\mathbf{0 3 5 7 4 7 9 6 5 0}$ Hester Creek at Buddy Williamson Rd nr Ple

Location - Lat $34^{\circ} 57^{\prime} 39^{\prime \prime}$, long $86^{\circ} 27^{\prime} 49^{\prime \prime}$ referenced to North American Datum of 1927, Madison County, Ala., Hydrologic Unit 06030002, on right bank on Buddy Williamson Road, $2.7 \mathrm{mi}$ east of Plevna, 3.8 mi northwest of New Market, and 2 mi south of Alabama-Tennessee State line.

Drainage area $33 \mathrm{mi}^{2}$.

G age -Water-stage recorder. Datum of gage is $756.445 \mathrm{ft}$ above NAVD of 1988.

\begin{tabular}{|c|c|c|c|c|c|}
\hline $\begin{array}{l}\text { Water } \\
\text { year }\end{array}$ & Date & Discharge & $\begin{array}{c}\text { Discharge } \\
\text { code }\end{array}$ & $\begin{array}{c}\text { Gage } \\
\text { height } \\
\text { (feet) }\end{array}$ & $\begin{array}{c}\text { Gage } \\
\text { height } \\
\text { code }\end{array}$ \\
\hline 1999 & Jan. 23 & 2,300 & & 8.21 & \\
\hline 2000 & Apr. 3 & 3,970 & & 8.94 & \\
\hline 2001 & Dec. 16 & 2,160 & & 8.09 & \\
\hline 2002 & Mar. 18 & 2,040 & & 7.99 & \\
\hline 2003 & May 6 & 5,780 & & 9.55 & \\
\hline 2004 & Feb. 6 & 3,250 & & 8.68 & \\
\hline 2005 & Dec. 6 & 2,860 & & 8.52 & \\
\hline 2006 & Apr. 8 & 1,160 & & 6.82 & \\
\hline 2007 & Nov. 15 & 1,000 & & 6.50 & \\
\hline 2008 & Mar. 4 & 1,280 & & 6.96 & \\
\hline 2009 & Dec. 10 & 2,600 & & 8.37 & \\
\hline 2010 & Dec. 9 & 1,920 & & 7.87 & \\
\hline 2011 & Apr. 27 & 8,630 & & 10.32 & \\
\hline 2012 & Jan. 11 & 2,170 & & 7.99 & \\
\hline 2013 & Jan. 16 & 1,850 & & 7.70 & \\
\hline 2014 & Dec. 22 & 2,130 & & 7.97 & \\
\hline
\end{tabular}


Appendix 2. Discharge and gage height data for streamgages in Alabama.-Continued

[--, date or data not available; ft, foot; $\mathrm{ft}^{3} / \mathrm{s}$, cubic foot per second; mi, mile; $\mathrm{mi}^{2}$, square mile; qualification codes apply to the discharge data: 1 , discharge is a maximum daily average; 2 , discharge is an estimate; 4 , discharge is less than indicated value, which is the minimum recordable discharge at this site; 5 , discharge affected to unknown degree by regulation or diversion; 6 , discharge affected by regulation or diversion; 7, discharge is a historic peak; A, year of occurrence is unknown or not exact; $\mathrm{B}$, month or day of occurrence is unknown or not exact; E, only maximum peak available for this year. qualification codes apply to the gage height data: 1 , gage height affected by backwater; 2, gage height not the maximum for the year; 3, gage height at different data or at different site and datum;

5 , gage height is an estimate]

\section{Mulberry Creek at Jones}

L ocation-Lat $32^{\circ} 34^{\prime} 58^{\prime \prime}$, long $86^{\circ} 54^{\prime} 13^{\prime \prime}$, in SE $1 / 4$ sec. 31 , T. 19 N., R. 12 E., Dallas County, Hydrologic Unit 03150201, 75 $\mathrm{ft}$ downstream from highway bridge, $0.4 \mathrm{mi}$ west of Jones, $6 \mathrm{mi}$ upstream from Buck Creek, and 11 mi upstream from mouth.

Drainage area-203 $\mathrm{mi}^{2}$.

Gage-Water-stage recorder. Datum of gage is $165.23 \mathrm{ft}$ NGVD 29.

\begin{tabular}{|c|c|c|c|c|c|}
\hline $\begin{array}{c}\text { Water } \\
\text { year }\end{array}$ & Date & Discharge & $\begin{array}{c}\text { Discharge } \\
\text { code }\end{array}$ & $\begin{array}{c}\text { Gage } \\
\text { height } \\
\text { (feet) }\end{array}$ & $\begin{array}{c}\text { Gage } \\
\text { height } \\
\text { code }\end{array}$ \\
\hline 1938 & Apr. & 40,000 & & 33.60 & \\
\hline 1939 & Aug. 16 & 32,800 & & 30.38 & \\
\hline 1940 & July 5 & 5,000 & & 9.56 & \\
\hline 1941 & July 17 & 3,420 & & 8.00 & \\
\hline 1942 & Mar. 21 & 12,800 & & 20.03 & \\
\hline 1943 & Dec. 28 & 13,600 & & 18.43 & \\
\hline 1944 & Apr. 27 & 6,040 & & 11.80 & \\
\hline 1945 & Apr. 25 & 8,800 & & 14.20 & \\
\hline 1946 & Jan. 7 & 5,360 & & 9.98 & \\
\hline 1947 & Jan. 20 & 11,600 & & 18.18 & \\
\hline 1948 & Mar. 23 & 7,020 & & 12.40 & \\
\hline 1949 & Nov. 27 & 5,820 & & 10.80 & \\
\hline 1950 & July 15 & 3,400 & & 8.00 & \\
\hline 1951 & Mar. 29 & 18,200 & & 23.80 & \\
\hline 1952 & Mar. 4 & 5,250 & & 9.97 & \\
\hline 1953 & Feb. 25 & 3,500 & & 7.50 & \\
\hline 1954 & Apr. 16 & 2,850 & & 6.56 & \\
\hline 1955 & Apr. 13 & 9,740 & & 15.80 & \\
\hline 1956 & Mar. 16 & 8,460 & & 14.18 & \\
\hline 1957 & Apr. 5 & 7,800 & & -- & \\
\hline 1958 & July 13 & 3,450 & & 8.05 & \\
\hline 1959 & Mar. 12 & 2,440 & & 5.50 & \\
\hline 1960 & Mar. 30 & 3,380 & & 6.60 & \\
\hline 1961 & Feb. 25 & 5,340 & & 9.99 & \\
\hline 1962 & Dec. 12 & 8,410 & & 14.14 & \\
\hline 1963 & Mar. 13 & 5,570 & & 10.36 & \\
\hline 1964 & Apr. 6 & 11,600 & & 18.13 & \\
\hline 1965 & Jan. 23 & 3,510 & & 7.16 & \\
\hline 1966 & Feb. 17 & 5,670 & & 11.39 & \\
\hline 1967 & Feb. 7 & 2,670 & & 7.10 & \\
\hline 1968 & Apr. 6 & 4,760 & & 10.08 & \\
\hline 1969 & Jan. 19 & 4,550 & & 9.78 & \\
\hline 1970 & Apr. 27 & 2,810 & & 7.82 & \\
\hline 1971 & Mar. 3 & 10,200 & & 17.17 & \\
\hline 1972 & Jan. 11 & 10,600 & & 17.62 & \\
\hline 1973 & Mar. 16 & 5,490 & & 11.13 & \\
\hline 1974 & Feb. 15 & 3,610 & & 8.76 & \\
\hline 1975 & Apr. 3 & 9,550 & & 16.50 & \\
\hline 1976 & Mar. 31 & 7,060 & & 13.37 & \\
\hline
\end{tabular}

\section{Mulberry Creek at Jones-Continued}

L ocation-Lat $32^{\circ} 34^{\prime} 58^{\prime \prime}$, long $86^{\circ} 54^{\prime} 13^{\prime \prime}$, in SE $1 / 4 \mathrm{sec} .31$, T. 19 N., R. 12 E., Dallas County, Hydrologic Unit 03150201, 75 $\mathrm{ft}$ downstream from highway bridge, $0.4 \mathrm{mi}$ west of Jones, $6 \mathrm{mi}$ upstream from Buck Creek, and 11 mi upstream from mouth.

Drainage area-203 $\mathrm{mi}^{2}$.

G age - Water-stage recorder. Datum of gage is $165.23 \mathrm{ft}$ NGVD 29.

\begin{tabular}{llcccc}
\hline $\begin{array}{c}\text { Water } \\
\text { year }\end{array}$ & Date & Discharge & $\begin{array}{c}\text { Discharge } \\
\text { code }\end{array}$ & $\begin{array}{c}\text { Gage } \\
\text { height } \\
\text { (feet) }\end{array}$ & $\begin{array}{c}\text { Gage } \\
\text { height } \\
\text { code }\end{array}$ \\
\hline 1977 & Feb. 24 & 5,170 & & 10.67 & \\
1978 & May 8 & 3,420 & & 8.17 & \\
1979 & Apr. 14 & 21,800 & & 25.26 & \\
1980 & Mar. 21 & 5,610 & & 11.26 & \\
1981 & Apr. 1 & 10,700 & & 16.60 &
\end{tabular}

1981 Apr. $1 \quad 10,700 \quad 16.60$

1982 Feb. $3 \quad 10,700 \quad 16.57$

$1983 \quad$ Apr. $9 \quad 8,730 \quad 14.63$

1984 Dec. $28 \quad 4,010 \quad 9.02$

1986 May $29 \quad 1,200 \quad 4.72$

$1987 \quad$ Nov. $24 \quad 4,410 \quad 9.59$

1988 Jan. $20 \quad 3,220 \quad 7.89$

1989 Mar. $6 \quad 7,620 \quad 13.54$

1990 Mar. $16 \quad 19,600 \quad 23.26$

1991 May $13 \quad 7,180 \quad 12.62$

1992 Feb. $17 \quad 3,330 \quad 7.48$

1993 Jan. $21 \quad 7,260 \quad 12.72$

$1994 \quad$ Feb. $5 \quad 3,880 \quad 8.31$

1995 Mar. $8 \quad 3,960 \quad 8.43$

$1996 \quad$ Jan. $27 \quad 6,240 \quad 11.49$

$1997 \quad$ Feb. $28 \quad 5,450 \quad 10.48$

$1998 \quad$ Jan. $8 \quad 10,600 \quad 16.52$

$1999 \quad$ Jan. $31 \quad 6,370 \quad 11.65$

2000 Apr. $4 \quad 4,240 \quad 8.84$

2001 Mar. $12 \quad 5,150 \quad 10.09$

2002 Jan. $25 \quad 2,600 \quad 6.28$

2003 May $22 \quad 8,330 \quad 13.99$

$2004 \quad$ Sept. $16 \quad 3,750 \quad 8.12$

2005 Apr. $1 \quad 6,670 \quad 12.02$

2006 May $11 \quad 2,620 \quad 6.31$

2007 Nov. $15 \quad 3,240 \quad 7.35$

$2008 \quad$ Feb. $22 \quad 4,070 \quad 8.59$

2009 Mar. $27 \quad 6,980 \quad 12.39$

$2010 \quad$ Jan. $25 \quad 8,090 \quad 13.71$

2011 Mar. $9 \quad 7,790 \quad 13.35$

2012 Sept. $4 \quad 4,750 \quad 9.55$

2013 Feb. $11 \quad 4,310 \quad 8.63$

2014 Apr. $7 \quad 5,390 \quad 10.41$

2015 Jan. $4 \quad 5,180 \quad 10.13$ 
Appendix 2. Discharge and gage height data for streamgages in Alabama.-Continued

[--, date or data not available; $\mathrm{ft}$, foot; $\mathrm{ft}^{3} / \mathrm{s}$, cubic foot per second; mi, mile; $\mathrm{mi}^{2}$, square mile; qualification codes apply to the discharge data: 1 , discharge is a maximum daily average; 2 , discharge is an estimate; 4 , discharge is less than indicated value, which is the minimum recordable discharge at this site; 5 , discharge affected to unknown degree by regulation or diversion; 6 , discharge affected by regulation or diversion; 7, discharge is a historic peak; A, year of occurrence is unknown or not exact; $\mathrm{B}$, month or day of occurrence is unknown or not exact; E, only maximum peak available for this year. qualification codes apply to the gage height data: 1, gage height affected by backwater; 2, gage height not the maximum for the year; 3 , gage height at different datum or at different site and datum; 5 , gage height is an estimate]

02424010 Sandy Creek near Centreville

L ocation-Lat $32^{\circ} 54^{\prime \prime} 12^{\prime \prime}$, long $87^{\circ} 00^{\prime} 09^{\prime \prime}$, in NE $1 / 4$ sec. 7, T. 22 N., R. 11 E., Bibb County, Hydrologic Unit 03150202, at culvert on U.S. Highway $82,8 \mathrm{mi}$ southeast of Centreville, and about 10 mi upstream from mouth.

Drainage area $-0.59 \mathrm{mi}^{2}$.

Gage - Water-stage recorder 1970-74. Crest-stage gage 1975-80, 1990-91. Datum of gage is $417 \mathrm{ft}$ NGVD 29 (from topographic map).

\begin{tabular}{|c|c|c|c|c|c|}
\hline $\begin{array}{l}\text { Water } \\
\text { year }\end{array}$ & Date & Discharge & $\begin{array}{c}\text { Discharge } \\
\text { code }\end{array}$ & $\begin{array}{c}\text { Gage } \\
\text { height } \\
\text { (feet) }\end{array}$ & $\begin{array}{c}\text { Gage } \\
\text { height } \\
\text { code }\end{array}$ \\
\hline 1970 & Apr. 26 & 118 & & -- & \\
\hline 1971 & May 12 & 180 & & 4.45 & \\
\hline 1972 & Jan. 10 & 140 & & 3.87 & \\
\hline 1973 & June 19 & 313 & & 6.14 & \\
\hline 1974 & Feb. 14 & 188 & & 4.55 & \\
\hline 1975 & Apr. 15 & 146 & & 3.96 & \\
\hline 1976 & Apr. 16 & 104 & & 3.31 & \\
\hline 1978 & June 8 & 100 & & 3.25 & \\
\hline 1979 & Mar. 4 & 360 & & 6.72 & \\
\hline 1980 & Mar. 14 & 490 & & 8.14 & \\
\hline 1991 & June 20 & 524 & & 8.38 & \\
\hline 1992 & Sept. 4 & 426 & & 7.40 & \\
\hline 1993 & Aug. 7 & 97 & & 3.18 & \\
\hline 1994 & Mar. 24 & 150 & & 4.01 & \\
\hline 1995 & Apr. 21 & 151 & & 4.03 & \\
\hline 1996 & Mar. 7 & 262 & & 5.53 & \\
\hline
\end{tabular}


Appendix 2. Discharge and gage height data for streamgages in Alabama.-Continued

[--, date or data not available; ft, foot; $\mathrm{ft}^{3} / \mathrm{s}$, cubic foot per second; mi, mile; $\mathrm{mi}^{2}$, square mile; qualification codes apply to the discharge data: 1 , discharge is a maximum daily average; 2 , discharge is an estimate; 4 , discharge is less than indicated value, which is the minimum recordable discharge at this site; 5 , discharge affected to unknown degree by regulation or diversion; 6 , discharge affected by regulation or diversion; 7, discharge is a historic peak; A, year of occurrence is unknown or not exact; B, month or day of occurrence is unknown or not exact; E, only maximum peak available for this year. qualification codes apply to the gage height data: 1 , gage height affected by backwater; 2, gage height not the maximum for the year; 3, gage height at different data or at different site and datum;

5 , gage height is an estimate]

\section{Cahaba River Marion Junction}

L ocation-Lat $32^{\circ} 26^{\prime} 38^{\prime \prime}$, long $87^{\circ} 10^{\prime} 49^{\prime \prime}$, in SW 1/4 sec. 16, T. 17 N., R. 9 E., Dallas County, Hydrologic Unit 03150202, on U.S. Highway 80, 3.8 mi downstream from Oakmulgee Creek, $3.5 \mathrm{mi}$ east of Marion Junction, and 21.4 mi upstream from mouth.

Drainage area-1,766 $\mathrm{mi}^{2}$.

Gage -Water-stage recorder. Datum of gage is $86.72 \mathrm{ft}$ NGVD 29.

Remarks - Flow affected by regulation from Lake Purdy, and diversion by Birmingham Water Works.

\begin{tabular}{|c|c|c|c|c|c|}
\hline $\begin{array}{c}\text { Water } \\
\text { year }\end{array}$ & Date & Discharge & $\begin{array}{l}\text { Discharge } \\
\text { code }\end{array}$ & $\begin{array}{c}\text { Gage } \\
\text { height } \\
\text { (feet) }\end{array}$ & $\begin{array}{c}\text { Gage } \\
\text { height } \\
\text { code }\end{array}$ \\
\hline 1938 & Apr & 85,000 & $2,7, B$ & -- & \\
\hline 1939 & Aug. 16 & 83,400 & & 42.95 & \\
\hline 1940 & Feb. 9 & 22,000 & & 29.73 & \\
\hline 1941 & Mar. 10 & 14,800 & & 22.40 & \\
\hline 1942 & Mar. 24 & 33,000 & & 36.50 & \\
\hline 1943 & Dec. 30 & 77,600 & & 41.50 & \\
\hline 1944 & Apr. 1 & 35,000 & & 37.40 & \\
\hline 1945 & Feb. 24 & 23,000 & & 30.70 & \\
\hline 1946 & Feb. 14 & 31,000 & & 35.10 & \\
\hline 1947 & Jan. 22 & 54,000 & & 39.60 & \\
\hline 1948 & Feb. 13 & 25,000 & & 32.00 & \\
\hline 1949 & Dec. 1 & 52,000 & & 39.40 & \\
\hline 1950 & Mar. 17 & 12,500 & & 20.90 & \\
\hline 1951 & Mar. 31 & 80,400 & & 41.70 & \\
\hline 1952 & Dec. 24 & 17,500 & & 27.30 & \\
\hline 1953 & Jan. 13 & 16,100 & & 25.50 & \\
\hline 1954 & Jan. 19 & 10,600 & & 18.40 & \\
\hline 1961 & Feb. 24 & 92,000 & 2,7 & 43.80 & \\
\hline 1970 & Mar. 24 & 36,500 & & 37.44 & \\
\hline 1971 & Mar. 6 & 27,200 & & 33.50 & \\
\hline 1972 & Jan. 14 & 34,000 & & 36.60 & \\
\hline 1973 & Jan. 11 & 17,800 & 1 & 25.90 & \\
\hline 1974 & Jan. 4 & 23,100 & & 30.35 & \\
\hline 1975 & Feb. 8 & 21,000 & & 28.75 & 2 \\
\hline 1976 & Mar. 19 & 54,500 & & 39.53 & \\
\hline 1977 & Apr. 1 & 46,600 & & 38.86 & \\
\hline 1978 & Jan. 28 & 16,900 & & 25.13 & \\
\hline 1979 & Apr. 15 & 73,900 & & 41.13 & \\
\hline 1980 & Mar. 23 & 29,000 & & 34.39 & \\
\hline 1981 & Apr. 2 & 27,000 & & 33.37 & \\
\hline 1982 & Apr. 23 & 24,000 & & 31.41 & \\
\hline 1983 & Apr. 11 & 33,500 & & 36.37 & \\
\hline
\end{tabular}

02425000 Cahaba River Marion Junction-Continued

L ocation-Lat $32^{\circ} 26^{\prime} 38^{\prime \prime}$, long $87^{\circ} 10^{\prime} 49^{\prime \prime}$, in SW 1/4 sec. 16, T. 17 N., R. 9 E., Dallas County, Hydrologic Unit 03150202, on U.S. Highway 80, 3.8 mi downstream from Oakmulgee Creek, $3.5 \mathrm{mi}$ east of Marion Junction, and 21.4 mi upstream from mouth.

Drainage area- $1,766 \mathrm{mi}^{2}$.

Gage-Water-stage recorder. Datum of gage is $86.72 \mathrm{ft}$ NGVD 29.

Remarks - Flow affected by regulation from Lake Purdy, and diversion by Birmingham Water Works.

\begin{tabular}{|c|c|c|c|c|c|}
\hline $\begin{array}{c}\text { Water } \\
\text { year }\end{array}$ & Date & Discharge & $\begin{array}{l}\text { Discharge } \\
\text { code }\end{array}$ & $\begin{array}{c}\text { Gage } \\
\text { height } \\
\text { (feet) }\end{array}$ & $\begin{array}{c}\text { Gage } \\
\text { height } \\
\text { code }\end{array}$ \\
\hline 1984 & Dec. 7 & 32,600 & & 35.82 & \\
\hline 1985 & Feb. 8 & 16,900 & & 24.77 & \\
\hline 1986 & Dec. 14 & 6,400 & & 12.64 & \\
\hline 1987 & Jan. 22 & 32,500 & & 35.98 & \\
\hline 1988 & Jan. 22 & 14,800 & & 22.61 & \\
\hline 1989 & July 5 & 20,300 & & 28.02 & \\
\hline 1990 & Feb. 18 & 76,000 & & 41.21 & \\
\hline 1991 & Feb. 23 & 24,100 & & 31.40 & \\
\hline 1992 & Feb. 28 & 12,100 & & 19.63 & \\
\hline 1993 & Jan. 24 & 21,500 & & 29.12 & \\
\hline 1994 & Apr. 18 & 13,400 & & 21.11 & \\
\hline 1995 & Mar. 11 & 19,600 & & 27.29 & \\
\hline 1996 & Mar. 10 & 31,100 & & 35.28 & \\
\hline 1997 & Mar. 3 & 22,500 & & 29.95 & \\
\hline 1998 & Jan. 11 & 32,500 & & 36.01 & \\
\hline 1999 & Feb. 3 & 26,700 & & 33.12 & \\
\hline 2000 & Apr. 7 & 31,000 & & 35.25 & \\
\hline 2001 & Apr. 7 & 21,000 & & 28.69 & \\
\hline 2002 & Jan. 28 & 18,300 & & 26.06 & \\
\hline 2003 & May 22 & 24,300 & & 31.53 & \\
\hline 2004 & Sept. 20 & 18,800 & & 26.53 & \\
\hline 2005 & Apr. 4 & 29,100 & & 34.32 & \\
\hline 2006 & Mar. 23 & 15,600 & & 23.44 & \\
\hline 2007 & Jan. 9 & 8,600 & 1 & -- & \\
\hline 2008 & Feb. 24 & 13,800 & & 21.47 & \\
\hline 2009 & Sept. 24 & 29,800 & & 34.66 & \\
\hline 2010 & Mar. 14 & 33,800 & & 36.52 & \\
\hline 2011 & Mar. 12 & 29,800 & & 34.67 & \\
\hline 2012 & Mar. 5 & 16,600 & & 24.40 & \\
\hline 2013 & Feb. 15 & 19,000 & & 30.89 & \\
\hline 2014 & Apr. 11 & 29,600 & & 36.81 & \\
\hline 2015 & Jan. 7 & 18,100 & & 30.06 & \\
\hline
\end{tabular}


Appendix 2. Discharge and gage height data for streamgages in Alabama.-Continued

[--, date or data not available; $\mathrm{ft}$, foot; $\mathrm{ft}^{3} / \mathrm{s}$, cubic foot per second; mi, mile; $\mathrm{mi}^{2}$, square mile; qualification codes apply to the discharge data: 1 , discharge is a maximum daily average; 2 , discharge is an estimate; 4 , discharge is less than indicated value, which is the minimum recordable discharge at this site; 5 , discharge affected to unknown degree by regulation or diversion; 6 , discharge affected by regulation or diversion; 7, discharge is a historic peak; A, year of occurrence is unknown or not exact; $\mathrm{B}$, month or day of occurrence is unknown or not exact; E, only maximum peak available for this year. qualification codes apply to the gage height data: 1, gage height affected by backwater; 2, gage height not the maximum for the year; 3, gage height at different datum or at different site and datum; 5 , gage height is an estimate]

\section{Buttahatchee River near Sulligent}

L ocation-Lat $33^{\circ} 55^{\prime} 08^{\prime \prime}$, long $88^{\circ} 08^{\prime} 47^{\prime \prime}$, in NE $1 / 4$ sec. 19 , T. 13

S., R. 15 W., Lamar County, Hydrologic Unit 03160103, on State

Highway 17, 1 mi upstream from Bogue Creek, 1.5 mi northwest of Sulligent, and 2 mi downstream from Beaver Creek.

Drainage area $472 \mathrm{mi}^{2}$.

Gage -Water-stage recorder. Datum of gage is $287.58 \mathrm{ft}$ sea level. Prior to June 1, 1942, nonrecording gage at site $500 \mathrm{ft}$ upstream at datum $1.00 \mathrm{ft}$ higher. July 1, 1942 to Sept. 30, 1971, nonrecording gage at present site and datum. Nov. 3, 1948 to Sept. 30, 1971, supplemental nonrecording gage on side channel at datum $10.00 \mathrm{ft}$ lower. Since Aug. 12, 1971, supplemental water-stage recorder on side channel at datum $10.00 \mathrm{ft}$ lower.

\begin{tabular}{|c|c|c|c|c|c|}
\hline $\begin{array}{c}\text { Water } \\
\text { year }\end{array}$ & Date & Discharge & $\begin{array}{c}\text { Discharge } \\
\text { code }\end{array}$ & $\begin{array}{c}\text { Gage } \\
\text { height } \\
\text { (feet) }\end{array}$ & $\begin{array}{c}\text { Gage } \\
\text { height } \\
\text { code }\end{array}$ \\
\hline 1939 & May 23 & 3,470 & 2 & -- & 3 \\
\hline 1940 & Mar. 4 & 3,640 & 2 & -- & 3 \\
\hline 1941 & Mar. 8 & 3,390 & 2 & -- & 3 \\
\hline 1942 & Feb. 18 & 3,470 & 2 & -- & 3 \\
\hline 1943 & Dec. 29 & 6,920 & & 13.93 & \\
\hline 1944 & Mar. 29 & 26,000 & & 15.21 & \\
\hline 1945 & Feb. 23 & 11,200 & & 14.45 & \\
\hline 1946 & Jan. 8 & 33,000 & & 15.50 & \\
\hline 1947 & Apr. 12 & 11,800 & & 14.40 & \\
\hline 1948 & Feb. 13 & 25,400 & & 15.10 & \\
\hline 1949 & Jan. 5 & 27,400 & & 15.50 & \\
\hline 1950 & Jan. 7 & 32,800 & & 16.40 & \\
\hline 1951 & Mar. 29 & 29,700 & & 15.70 & \\
\hline 1952 & Dec. 9 & 16,000 & & 14.60 & \\
\hline 1953 & May 1 & 11,400 & & 14.20 & \\
\hline 1954 & Jan. 23 & 12,700 & & 14.50 & \\
\hline 1955 & Dec. 30 & 17,700 & & 14.95 & \\
\hline 1956 & Feb. 4 & 14,200 & & 14.70 & \\
\hline
\end{tabular}

02439000 Buttahatchee River near Sulligent-Continued

L ocation-Lat $33^{\circ} 55^{\prime} 08^{\prime \prime}$, long $88^{\circ} 08^{\prime} 47^{\prime \prime}$, in NE $1 / 4$ sec. 19, T. 13 S., R. 15 W., Lamar County, Hydrologic Unit 03160103, on State Highway 17, 1 mi upstream from Bogue Creek, 1.5 mi northwest of Sulligent, and 2 mi downstream from Beaver Creek.

Drainage area-472 $\mathrm{mi}^{2}$

G age -Water-stage recorder. Datum of gage is $287.58 \mathrm{ft}$ sea level. Prior to June 1, 1942, nonrecording gage at site $500 \mathrm{ft}$ upstream at datum $1.00 \mathrm{ft}$ higher. July 1, 1942 to Sept. 30, 1971, nonrecording gage at present site and datum. Nov. 3, 1948 to Sept. 30, 1971, supplemental nonrecording gage on side channel at datum $10.00 \mathrm{ft}$ lower. Since Aug. 12, 1971, supplemental water-stage recorder on side channel at datum $10.00 \mathrm{ft}$ lower.

\begin{tabular}{|c|c|c|c|c|c|}
\hline $\begin{array}{l}\text { Water } \\
\text { year }\end{array}$ & Date & Discharge & $\begin{array}{c}\text { Discharge } \\
\text { code }\end{array}$ & $\begin{array}{c}\text { Gage } \\
\text { height } \\
\text { (feet) }\end{array}$ & $\begin{array}{c}\text { Gage } \\
\text { height } \\
\text { code }\end{array}$ \\
\hline 1957 & Dec. 14 & 12,200 & & 14.80 & \\
\hline 1958 & Nov. 19 & 11,500 & & 14.70 & \\
\hline 1959 & Feb. 14 & 7,300 & & 14.90 & \\
\hline 1960 & Mar. 3 & 15,200 & & 14.80 & \\
\hline 1972 & Jan. 5 & 15,900 & & 15.26 & \\
\hline 1973 & Mar. 17 & 60,100 & & 17.31 & \\
\hline 1974 & Dec. 27 & 13,700 & & 15.37 & \\
\hline 1975 & Mar. 14 & 23,300 & & 15.96 & \\
\hline 1976 & Oct. 18 & 17,800 & & 15.56 & \\
\hline 1977 & Mar. 5 & 24,200 & & 16.17 & \\
\hline 1978 & May 9 & 16,000 & & 15.45 & \\
\hline 1979 & Apr. 13 & 15,900 & & 15.44 & \\
\hline 1980 & Mar. 18 & 17,700 & & 15.54 & \\
\hline 1981 & Mar. 31 & 7,260 & & 15.18 & \\
\hline 1982 & Jan. 4 & 22,800 & & 15.53 & \\
\hline 1983 & May 20 & 23,900 & & 15.82 & \\
\hline 1984 & Dec. 3 & 21,700 & & 15.81 & \\
\hline 1985 & May 2 & 24,400 & $\mathrm{E}$ & 16.09 & \\
\hline
\end{tabular}


Appendix 2. Discharge and gage height data for streamgages in Alabama.-Continued

[--, date or data not available; $\mathrm{ft}$, foot; $\mathrm{ft}^{3} / \mathrm{s}$, cubic foot per second; $\mathrm{mi}$, mile; $\mathrm{mi}^{2}$, square mile; qualification codes apply to the discharge data: 1 , discharge is a maximum daily average; 2 , discharge is an estimate; 4 , discharge is less than indicated value, which is the minimum recordable discharge at this site; 5 , discharge affected to unknown degree by regulation or diversion; 6 , discharge affected by regulation or diversion; 7, discharge is a historic peak; A, year of occurrence is unknown or not exact; $\mathrm{B}$, month or day of occurrence is unknown or not exact; E, only maximum peak available for this year. qualification codes apply to the gage height data: 1, gage height affected by backwater; 2 , gage height not the maximum for the year; 3 , gage height at different data or at different site and datum;

5 , gage height is an estimate]

\section{Luxapallila Creek near Fayette}

L ocation-Lat $33^{\circ} 43^{\prime} 10^{\prime \prime}$, long $87^{\circ} 52^{\prime} 14^{\prime \prime}$, in SW 1/4 sec. 26, T. 15 S., R. 13 W., Fayette County, Hydrologic Unit 03160105, on State Highway 18 and 2 mi northwest of Fayette.

Drainage area-130 $\mathrm{mi}^{2}$.

Gage -Water-stage recorder. Datum of gage is $322.33 \mathrm{ft} \mathrm{NGVD} 29$. Prior to Apr. 22, 1944, nonrecording gage at same site and datum.

\begin{tabular}{|c|c|c|c|c|c|}
\hline $\begin{array}{c}\text { Water } \\
\text { year }\end{array}$ & Date & Discharge & $\begin{array}{c}\text { Discharge } \\
\text { code }\end{array}$ & $\begin{array}{c}\text { Gage } \\
\text { height } \\
\text { (feet) }\end{array}$ & $\begin{array}{c}\text { Gage } \\
\text { height } \\
\text { code }\end{array}$ \\
\hline 1938 & -- & 9,500 & 2,B & 13.00 & \\
\hline 1940 & Apr. 4 & 6,260 & & 12.40 & \\
\hline 1941 & Aug. 1 & 4,940 & & 11.50 & \\
\hline 1942 & Mar. 17 & 4,710 & & 11.30 & \\
\hline 1943 & Mar. 12 & 5,600 & & 12.00 & \\
\hline 1944 & Mar. 28 & 5,750 & & 12.10 & \\
\hline 1945 & Feb. 22 & 4,710 & & 11.30 & \\
\hline 1946 & Jan. 8 & 12,500 & & 13.57 & \\
\hline 1947 & Apr. 11 & 4,600 & & 11.16 & \\
\hline 1948 & Feb. 12 & 5,060 & & 11.60 & \\
\hline 1949 & Jan. 5 & 14,000 & & 13.80 & \\
\hline 1950 & Jan. 6 & 9,700 & & 13.10 & \\
\hline 1951 & Mar. 29 & 10,100 & & 13.20 & \\
\hline 1952 & Mar. 11 & 4,710 & & 11.30 & \\
\hline 1953 & Feb. 21 & 5,060 & & 11.65 & \\
\hline 1954 & Jan. 22 & 3,860 & & 10.20 & \\
\hline 1955 & Mar. 22 & 4,280 & & 10.80 & \\
\hline 1956 & Feb. 20 & 4,060 & & 10.50 & \\
\hline 1957 & Apr. 4 & 4,960 & & 11.50 & \\
\hline 1958 & Nov. 19 & 5,330 & & 11.80 & \\
\hline 1959 & Feb. 13 & 2,970 & & 8.50 & \\
\hline 1960 & Mar. 3 & 5,330 & & 11.75 & \\
\hline 1961 & Feb. 21 & 9,150 & & 12.93 & \\
\hline 1962 & Dec. 18 & 8,000 & & 12.70 & \\
\hline 1963 & Mar. 12 & 2,380 & & 7.17 & \\
\hline 1964 & Apr. 27 & 6,340 & & 12.20 & \\
\hline 1965 & Feb. 12 & 5,000 & & 11.58 & \\
\hline 1966 & Apr. 27 & 6,200 & & 12.23 & \\
\hline 1967 & Feb. 20 & 3,150 & & 9.10 & \\
\hline 1968 & Dec. 18 & 12,000 & & 13.48 & \\
\hline 1969 & Apr. 10 & 8,250 & & 12.75 & \\
\hline 1970 & Mar. 20 & 7,560 & & 12.59 & \\
\hline 1979 & Apr. 13 & 9,500 & 7 & 13.00 & \\
\hline
\end{tabular}

02442500 Luxapallila Creek at Millport

L ocation-Lat $33^{\circ} 34^{\prime} 30^{\prime \prime}$, long $88^{\circ} 05^{\prime} 00^{\prime \prime}$, in SW 1/4 sec. 14 , T. 17 S., R. 15 W., Lamar County, Hydrologic Unit 03160105, on State Highway 17, 0.2 mi downstream from Driver Creek, 1.0 mi north of Millport, and at mile 31.6.

Drainage area $-247 \mathrm{mi}^{2}$.

Gage-Water-stage recorder. Datum of gage is $243.65 \mathrm{ft}$ NGVD 29.

\begin{tabular}{llcccc}
\hline $\begin{array}{c}\text { Water } \\
\text { year }\end{array}$ & \multicolumn{1}{c}{ Date } & Discharge & $\begin{array}{c}\text { Discharge } \\
\text { code }\end{array}$ & $\begin{array}{c}\text { Gage } \\
\text { height } \\
\text { (feet) }\end{array}$ & $\begin{array}{c}\text { Gage } \\
\text { height } \\
\text { code }\end{array}$ \\
\hline 1955 & Feb. 6 & 4,020 & & 10.20 & \\
1956 & Feb. 20 & 4,081 & & 10.30 & \\
1957 & Apr. 4 & 5,060 & & 11.80 & \\
1958 & Nov. 19 & 4,990 & & 11.70 & \\
1959 & Jan. 21 & 3,960 & & 10.10 & \\
1961 & Feb. 22 & 16,500 & 2,7 & 14.21 & \\
1979 & Apr. 13 & 14,000 & 7 & 13.93 &
\end{tabular}

1981 Mar. $31 \quad 3,650 \quad 10.20$

$1982 \quad$ Jan. $5 \quad 10,500 \quad 13.24$

1983 Mar. $6 \quad 10,800 \quad 13.30$

1984 Dec. $3 \quad 13,300 \quad 13.74$

1985 May $3 \quad 5,630 \quad 11.97$

1986 Mar. $13 \quad 4,490 \quad 11.04$

$1987 \quad$ Feb. $27 \quad 5,150 \quad 11.64$

1989 Jan. $13 \quad 6,740 \quad 12.46$

$1990 \quad$ Feb. $16 \quad 7,810 \quad 12.65$

1991 Dec. $24 \quad 15,500 \quad 14.07$

1992 Dec. $2 \quad 2,960 \quad 9.17$

$1993 \quad$ Mar. $31 \quad 5,290 \quad 11.74$

1994 Mar. $28 \quad 10,300 \quad 13.19$

1995 Apr. $24 \quad 6,000 \quad 12.70$

$1996 \quad$ Apr. $22 \quad 6,310 \quad 12.74$

1997 May $4 \quad 9,500 \quad 13.37$

1998 Jan. $8 \quad 12,500 \quad 13.72$

1999 Jan. $24 \quad 12,200 \quad 13.54$

2000 Apr. $3 \quad 8,550 \quad 13.22$

$2001 \quad$ June $8 \quad 5,400 \quad 12.48$

2002 Dec. $14 \quad 6,650 \quad 12.86$

$2003 \quad$ Feb. $23 \quad 9,150 \quad 13.35$

$2004 \quad$ Feb. $7 \quad 14,600 \quad 13.99$

2005 Dec. $9 \quad 6,500 \quad 12.88$

2006 Feb. $23 \quad 5,250 \quad 12.43$

2007 Nov. $7 \quad 5,380 \quad 12.49$

2008 Mar. $4 \quad 5,530 \quad 12.18$

$2009 \quad$ Jan. $7 \quad 11,700 \quad 14.32$

2010 Dec. $10 \quad 6,490 \quad 13.64$

2011 Mar. $9 \quad 7,220 \quad 13.87$ 
Appendix 2. Discharge and gage height data for streamgages in Alabama.-Continued

[--, date or data not available; $\mathrm{ft}$, foot; $\mathrm{ft}^{3} / \mathrm{s}$, cubic foot per second; mi, mile; $\mathrm{mi}^{2}$, square mile; qualification codes apply to the discharge data: 1 , discharge is a maximum daily average; 2 , discharge is an estimate; 4 , discharge is less than indicated value, which is the minimum recordable discharge at this site; 5 , discharge affected to unknown degree by regulation or diversion; 6 , discharge affected by regulation or diversion; 7, discharge is a historic peak; A, year of occurrence is unknown or not exact; $\mathrm{B}$, month or day of occurrence is unknown or not exact; E, only maximum peak available for this year. qualification codes apply to the gage height data: 1, gage height affected by backwater; 2, gage height not the maximum for the year; 3 , gage height at different datum or at different site and datum; 5 , gage height is an estimate]

\section{Coal Fire Creek near Pickensville}

L ocation-Lat $33^{\circ} 17^{\prime} 39^{\prime \prime}$, long $88^{\circ} 15^{\prime} 56^{\prime \prime}$, in NW 1/4 sec. 25 , T. 20 S., R. 17 W., Pickens County, Hydrologic Unit 03160106, on State Highway 14, 4.5 mi north of Pickensville, and at mile 4.5. Drainage area $-126 \mathrm{mi}^{2}$.

Gage-Water-stage recorder. Datum of gage is $148.50 \mathrm{ft}$ NGVD 29.

\begin{tabular}{|c|c|c|c|c|c|}
\hline $\begin{array}{l}\text { Water } \\
\text { year }\end{array}$ & Date & Discharge & $\begin{array}{l}\text { Discharge } \\
\text { code }\end{array}$ & $\begin{array}{c}\text { Gage } \\
\text { height } \\
\text { (feet) }\end{array}$ & $\begin{array}{c}\text { Gage } \\
\text { height } \\
\text { code }\end{array}$ \\
\hline
\end{tabular}

\begin{tabular}{llrr}
\hline 1955 & Feb. 24 & 860 & 6.53 \\
1956 & Apr. 8 & 1,130 & 7.12
\end{tabular}

1957

Dec. 24 1,101

1958 May $3 \quad 1,820$

1959 Jan. $24 \quad 1,000$

1960 Mar. 4 3,060

1961 Feb. $22 \quad 8,110$

1962 Dec. $19 \quad 2,890$

1963 July $16 \quad 2,960$

1964 Mar. $16 \quad 2,930$

1965 Feb. $12 \quad 2,210$

1966 Apr. $23 \quad 1,040$

1967 Aug. $12 \quad 590$

1968 Jan. $11 \quad 1,940$

1969 Apr. $15 \quad 1,920$

1970 Mar. $20 \quad 9,820$

1971 May $14 \quad 2,200$

1972 Jan. $11 \quad 2,700$

1973 Apr. $1 \quad 4,050$

1974 Apr. $14 \quad 3,200$

1975 Jan. $12 \quad 2,500$

1976 May $12 \quad 2,700$

1977 Apr. $4 \quad 6,760$

1978 May $11 \quad 3,770$

1979 Apr. $13 \quad$ 16,400

$1980 \quad$ Apr. $14 \quad 3,480$

\section{Lubbub Creek near Carrollton}

L ocation-Lat $33^{\circ} 14^{\prime} 47^{\prime \prime}$, long $88^{\circ} 04^{\prime} 53^{\prime \prime}$, in NE $1 / 4 \mathrm{sec} .10$, T. 21 S., R. 15 W., Pickens County, Hydrologic Unit 03160106, on county highway 12, 1 mi southeast of Carrollton, and $4 \mathrm{mi}$ upstream from Little Lubbub Creek.

Drainage area-112 $\mathrm{mi}^{2}$.

G age -Water-stage recorder 1955-64. Crest-stage gage 1965-69. Datum of gage is $174.24 \mathrm{ft}$ NGVD 29.

\begin{tabular}{llccrr}
\hline $\begin{array}{c}\text { Water } \\
\text { year }\end{array}$ & Date & Discharge & $\begin{array}{c}\text { Discharge } \\
\text { code }\end{array}$ & $\begin{array}{c}\text { Gage } \\
\text { height } \\
\text { (feet) }\end{array}$ & $\begin{array}{c}\text { Gage } \\
\text { height } \\
\text { code }\end{array}$ \\
\hline 1955 & Feb. 7 & 1,560 & & 8.70 & \\
1956 & Feb. 5 & 1,480 & & 8.65 & \\
1957 & Dec. 15 & 1,480 & 8.65 & \\
1958 & Nov. 20 & 1,410 & 8.58 & \\
1959 & May 31 & 1,410 & & 8.60 & \\
1960 & Mar. 3 & 2,600 & & 9.27 & \\
1961 & Feb. 22 & 8,210 & & 11.97 & \\
1962 & Dec. 18 & 3,600 & & 9.99 & \\
1963 & July 17 & 4,590 & & 10.31 & \\
1964 & Apr. 6 & 4,170 & & 10.10 & \\
1965 & Feb. 12 & 3,060 & & 9.53 & \\
1966 & Apr. 28 & 1,870 & & 8.89 & \\
1967 & Dec. 13 & 1,140 & & 8.39 & \\
1969 & Apr. 15 & 3,480 & 7 & 9.75 & \\
1979 & Apr. 13 & 21,000 & 7 & 16.00 &
\end{tabular}


Appendix 2. Discharge and gage height data for streamgages in Alabama._- Continued

[--, date or data not available; ft, foot; $\mathrm{ft}^{3} / \mathrm{s}$, cubic foot per second; mi, mile; $\mathrm{mi}^{2}$, square mile; qualification codes apply to the discharge data: 1 , discharge is a maximum daily average; 2 , discharge is an estimate; 4 , discharge is less than indicated value, which is the minimum recordable discharge at this site; 5 , discharge affected to unknown degree by regulation or diversion; 6 , discharge affected by regulation or diversion; 7, discharge is a historic peak; A, year of occurrence is unknown or not exact; $\mathrm{B}$, month or day of occurrence is unknown or not exact; E, only maximum peak available for this year. qualification codes apply to the gage height data: 1, gage height affected by backwater; 2, gage height not the maximum for the year; 3, gage height at different data or at different site and datum; 5 , gage height is an estimate]

\section{New River near Winfield}

Location-Lat $33^{\circ} 55^{\prime} 47^{\prime \prime}$, long $87^{\circ} 40^{\prime} 47^{\prime \prime}$, in SE 1/4 SE 1/4 SW 1/4 sec. 10, T. 13 S., R. 11 W., Marion County, Hydrologic Unit 03160107, at bridge on U.S. Highway 78, 8 mi east of Winfield.

Drainage area $-59.3 \mathrm{mi}^{2}$.

Gage Crest-stage gage. Datum of gage is $387.80 \mathrm{ft}$ NGVD 29.

\begin{tabular}{|c|c|c|c|c|c|}
\hline $\begin{array}{c}\text { Water } \\
\text { year }\end{array}$ & Date & Discharge & $\begin{array}{c}\text { Discharge } \\
\text { code }\end{array}$ & $\begin{array}{c}\text { Gage } \\
\text { height } \\
\text { (feet) }\end{array}$ & $\begin{array}{c}\text { Gage } \\
\text { height } \\
\text { code }\end{array}$ \\
\hline 1951 & Mar. 29 & 6,900 & & 23.20 & \\
\hline 1952 & Mar. 11 & 3,000 & & 16.50 & \\
\hline 1953 & Feb. 22 & 2,300 & & 14.80 & \\
\hline 1954 & Jan. 22 & 3,500 & & 17.50 & \\
\hline 1955 & Mar. 23 & 3,800 & & 18.20 & \\
\hline 1956 & Feb. 4 & 2,700 & & 16.00 & \\
\hline 1957 & Dec. 14 & 4,000 & & 18.50 & \\
\hline 1958 & Apr. 29 & 2,100 & & 14.50 & \\
\hline 1959 & Jan. 24 & 1,900 & & 13.80 & \\
\hline 1960 & Mar. 2 & 4,700 & & 19.80 & \\
\hline 1961 & Feb. 21 & 7,600 & & 23.88 & \\
\hline 1963 & Mar. 12 & 3,210 & & 17.02 & \\
\hline 1964 & Apr. 6 & 5,020 & & 20.36 & \\
\hline 1965 & Feb. 12 & 3,510 & & 17.62 & \\
\hline 1967 & Feb. 20 & 2,500 & & 15.50 & \\
\hline 1968 & Dec. 18 & 7,800 & & 24.11 & \\
\hline 1969 & Feb. 2 & 4,260 & & 19.04 & \\
\hline 1970 & Mar. 20 & 4,900 & & 20.16 & \\
\hline 1971 & Feb. 22 & 5,020 & & 20.37 & \\
\hline 1972 & Dec. 6 & 1,250 & & 11.66 & \\
\hline 1973 & Mar. 16 & 7,970 & & 24.30 & \\
\hline
\end{tabular}

\section{Sipsey River at Fayette}

L ocation-Lat $33^{\circ} 40^{\prime} 10^{\prime \prime}$, long $87^{\circ} 48^{\prime} 59^{\prime \prime}$, in SW $1 / 4$ sec. $8, \mathrm{~T}$. 16 S., R. 12 W., Fayette County, Hydrologic Unit 03160107, 1 mi southeast of Fayette, and 1.5 mi downstream from Southern Railway bridge.

Drainage area-282 $\mathrm{mi}^{2}$.

G age - Water-stage recorder. Datum of gage is $296.72 \mathrm{ft}$ NGVD 29.

\begin{tabular}{|c|c|c|c|c|c|}
\hline $\begin{array}{l}\text { Water } \\
\text { year }\end{array}$ & Date & Discharge & $\begin{array}{l}\text { Discharge } \\
\text { code }\end{array}$ & $\begin{array}{c}\text { Gage } \\
\text { height } \\
\text { (feet) }\end{array}$ & $\begin{array}{c}\text { Gage } \\
\text { height } \\
\text { code }\end{array}$ \\
\hline 1936 & -- & 20,000 & 2,B & 21.20 & \\
\hline 1939 & Feb. 16 & 11,500 & & 19.60 & \\
\hline 1940 & Apr. 5 & 8,200 & & 18.70 & \\
\hline 1941 & Mar. 6 & 2,950 & & 16.10 & \\
\hline 1942 & Mar. 19 & 3,420 & & 16.60 & \\
\hline 1943 & Dec. 29 & 8,800 & & 18.90 & \\
\hline 1944 & Mar. 29 & 14,400 & & 20.04 & \\
\hline 1945 & Mar. 5 & 10,500 & & 19.34 & \\
\hline 1946 & Jan. 8 & 24,500 & & 21.75 & \\
\hline 1947 & Jan. 21 & 7,200 & & 18.30 & \\
\hline 1948 & Feb. 14 & 9,600 & & 19.10 & \\
\hline 1949 & Jan. 6 & 20,000 & & 21.10 & \\
\hline 1950 & Jan. 7 & 20,500 & & 21.20 & \\
\hline 1951 & Mar. 29 & 20,500 & & 21.20 & \\
\hline 1952 & Dec. 22 & 5,601 & & 17.80 & \\
\hline 1953 & Feb. 22 & 10,100 & & 19.15 & \\
\hline 1954 & Jan. 23 & 7,200 & & 18.38 & \\
\hline 1955 & Mar. 23 & 8,100 & & 18.67 & \\
\hline 1956 & Feb. 5 & 5,600 & & 17.80 & \\
\hline 1957 & Dec. 15 & 6,601 & & 18.20 & \\
\hline 1958 & Nov. 19 & 11,500 & & 19.50 & \\
\hline 1959 & Jan. 24 & 3,500 & & 16.60 & \\
\hline 1979 & Apr. 16 & 12,000 & 7 & 19.59 & \\
\hline
\end{tabular}


Appendix 2. Discharge and gage height data for streamgages in Alabama.-Continued

[--, date or data not available; $\mathrm{ft}$, foot; $\mathrm{ft}^{3} / \mathrm{s}$, cubic foot per second; mi, mile; $\mathrm{mi}^{2}$, square mile; qualification codes apply to the discharge data: 1 , discharge is a maximum daily average; 2 , discharge is an estimate; 4 , discharge is less than indicated value, which is the minimum recordable discharge at this site; 5 , discharge affected to unknown degree by regulation or diversion; 6 , discharge affected by regulation or diversion; 7, discharge is a historic peak; A, year of occurrence is unknown or not exact; $\mathrm{B}$, month or day of occurrence is unknown or not exact; E, only maximum peak available for this year. qualification codes apply to the gage height data: 1, gage height affected by backwater; 2, gage height not the maximum for the year; 3 , gage height at different datum or at different site and datum; 5 , gage height is an estimate]

\section{Sipsey River near Elrod}

L ocation-Lat $33^{\circ} 15^{\prime} 25^{\prime \prime}$, long $87^{\circ} 46^{\prime} 35^{\prime \prime}$, in NE $1 / 4$ sec. 3 , T. 21 S., R. 12 W., Tuscaloosa County, Hydrologic Unit 03160107, on State Highway 140, $1.0 \mathrm{mi}$ east of Elrod, $2.0 \mathrm{mi}$ downstream from Box Creek, and at mile 50.7.

Drainage area $-528 \mathrm{mi}^{2}$.

G age - Water-stage recorder. Datum of gage is $197.81 \mathrm{ft}$ NGVD 29 Prior to Mar. 31, 1932, non- recording gage at railroad bridge 0.2 mi downstream from present site at datum $1.93 \mathrm{ft}$ higher. Nov. 1 to Dec. 11, 1939, nonrecording gage at present site and datum.

\begin{tabular}{|c|c|c|c|c|c|}
\hline $\begin{array}{c}\text { Water } \\
\text { year }\end{array}$ & Date & Discharge & $\begin{array}{c}\text { Discharge } \\
\text { code }\end{array}$ & $\begin{array}{c}\text { Gage } \\
\text { height } \\
\text { (feet) }\end{array}$ & $\begin{array}{c}\text { Gage } \\
\text { height } \\
\text { code }\end{array}$ \\
\hline 1930 & Nov. 17 & 15,500 & & 14.10 & 3 \\
\hline 1931 & Apr. 5 & 4,690 & & 11.70 & 3 \\
\hline 1932 & Feb. 22 & 7,090 & & 12.31 & 3 \\
\hline 1940 & July 13 & 8,260 & & 15.37 & \\
\hline 1941 & Aug. 1 & 6,560 & & 15.00 & \\
\hline 1942 & Mar. 23 & 3,630 & & 14.23 & \\
\hline 1943 & Mar. 17 & 7,760 & & 15.25 & \\
\hline 1944 & Mar. 31 & 12,200 & & 16.35 & \\
\hline 1945 & Feb. 17 & 7,401 & & 15.19 & \\
\hline 1946 & Jan. 11 & 18,600 & & 17.76 & \\
\hline 1947 & Jan. 23 & 6,500 & & 14.96 & \\
\hline 1948 & Feb. 16 & 9,200 & & 15.64 & \\
\hline 1949 & Jan. 7 & 17,100 & & 17.30 & \\
\hline 1950 & Jan. 9 & 21,000 & & 18.10 & \\
\hline 1951 & Mar. 31 & 21,000 & & 18.10 & \\
\hline 1952 & Dec. 26 & 5,400 & & 14.70 & \\
\hline 1953 & Feb. 25 & 9,400 & & 15.70 & \\
\hline 1954 & Jan. 26 & 6,400 & & 14.95 & \\
\hline 1955 & Mar. 27 & 4,230 & & 14.40 & \\
\hline 1956 & Feb. 9 & 5,190 & & 14.67 & \\
\hline 1957 & Feb. 4 & 6,190 & & 14.90 & \\
\hline 1958 & Nov. 21 & 8,990 & & 15.60 & \\
\hline 1959 & Feb. 19 & 3,020 & & 13.94 & \\
\hline 1960 & Mar. 7 & 7,190 & & 15.17 & \\
\hline 1961 & Feb. 23 & 27,800 & & 18.83 & \\
\hline 1962 & Dec. 20 & 15,700 & & 16.82 & \\
\hline 1963 & July 18 & 3,810 & & 14.27 & \\
\hline 1964 & Apr. 16 & 11,700 & & 16.15 & \\
\hline
\end{tabular}

02446500 Sipsey River near Elrod-Continued

L ocation-Lat $33^{\circ} 15^{\prime} 25^{\prime \prime}$, long $87^{\circ} 46^{\prime} 35^{\prime \prime}$, in NE $1 / 4$ sec. 3 , T. 21 S., R. 12 W., Tuscaloosa County, Hydrologic Unit 03160107, on State Highway 140, 1.0 mi east of Elrod, 2.0 mi downstream from Box Creek, and at mile 50.7.

Drainage area- $528 \mathrm{mi}^{2}$

G age -Water-stage recorder. Datum of gage is $197.81 \mathrm{ft}$ NGVD 29. Prior to Mar. 31, 1932, non- recording gage at railroad bridge 0.2 mi downstream from present site at datum $1.93 \mathrm{ft}$ higher. Nov. 1 to Dec. 11, 1939, nonrecording gage at present site and datum.

\begin{tabular}{|c|c|c|c|c|c|}
\hline $\begin{array}{c}\text { Water } \\
\text { year }\end{array}$ & Date & Discharge & $\begin{array}{l}\text { Discharge } \\
\text { code }\end{array}$ & $\begin{array}{c}\text { Gage } \\
\text { height } \\
\text { (feet) }\end{array}$ & $\begin{array}{c}\text { Gage } \\
\text { height } \\
\text { code }\end{array}$ \\
\hline 1965 & Feb. 14 & 9,840 & & 15.77 & \\
\hline 1966 & May 1 & 5,550 & & 14.74 & \\
\hline 1967 & Dec. 17 & 2,100 & & 13.32 & \\
\hline 1968 & Dec. 21 & 13,700 & & 16.48 & \\
\hline 1969 & Apr. 14 & 8,070 & & 15.37 & \\
\hline 1970 & Mar. 21 & 18,400 & & 17.27 & \\
\hline 1971 & Feb. 25 & 9,980 & & 15.80 & \\
\hline 1979 & Apr. 13 & 23,100 & & 17.85 & \\
\hline 1980 & Apr. 15 & 13,600 & & 16.25 & \\
\hline 1981 & Apr. 3 & 5,900 & & 14.72 & \\
\hline 1982 & Jan. 7 & 13,300 & & 16.20 & \\
\hline 1983 & Mar. 7 & 15,000 & & 16.50 & \\
\hline 1984 & Dec. 4 & 17,300 & & 16.88 & \\
\hline 1985 & May 5 & 7,800 & & 15.10 & \\
\hline 1986 & June 4 & 1,970 & & 13.15 & \\
\hline 1987 & Mar. 3 & 6,300 & & 14.80 & \\
\hline 1988 & Jan. 26 & 3,170 & & 13.86 & \\
\hline 1989 & Jan. 16 & 9,300 & & 15.40 & \\
\hline 1990 & Feb. 16 & 14,300 & & 16.38 & \\
\hline 1991 & Dec. 26 & 23,800 & & 17.97 & \\
\hline 1992 & Dec. 9 & 2,720 & & 13.63 & \\
\hline 1993 & Jan. 12 & 5,560 & & 14.64 & \\
\hline 1994 & Mar. 30 & 12,900 & & 16.12 & \\
\hline 1995 & Mar. 9 & 7,240 & & 15.09 & \\
\hline 1996 & Jan. 31 & 7,440 & & 15.14 & \\
\hline 1997 & May 7 & 9,520 & & 15.59 & \\
\hline 1998 & Jan. 10 & 12,800 & & 16.15 & \\
\hline 1999 & Jan. 26 & 14,000 & & 16.34 & \\
\hline
\end{tabular}


Appendix 2. Discharge and gage height data for streamgages in Alabama._- Continued

[--, date or data not available; ft, foot; $\mathrm{ft}^{3} / \mathrm{s}$, cubic foot per second; mi, mile; $\mathrm{mi}^{2}$, square mile; qualification codes apply to the discharge data: 1 , discharge is a maximum daily average; 2 , discharge is an estimate; 4 , discharge is less than indicated value, which is the minimum recordable discharge at this site; 5 , discharge affected to unknown degree by regulation or diversion; 6 , discharge affected by regulation or diversion; 7, discharge is a historic peak; A, year of occurrence is unknown or not exact; $\mathrm{B}$, month or day of occurrence is unknown or not exact; E, only maximum peak available for this year. qualification codes apply to the gage height data: 1, gage height affected by backwater; 2, gage height not the maximum for the year; 3, gage height at different data or at different site and datum; 5 , gage height is an estimate]

\section{Sipsey River near Elrod-Continued}

L ocation-Lat $33^{\circ} 15^{\prime} 25^{\prime \prime}$, long $87^{\circ} 46^{\prime} 35^{\prime \prime}$, in NE 1/4 sec. 3, T. 21 S., R. 12 W., Tuscaloosa County, Hydrologic Unit 03160107, on State Highway 140, $1.0 \mathrm{mi}$ east of Elrod, $2.0 \mathrm{mi}$ downstream from Box Creek, and at mile 50.7.

Drainage area-528 $\mathrm{mi}^{2}$.

G age - Water-stage recorder. Datum of gage is $197.81 \mathrm{ft}$ NGVD 29. Prior to Mar. 31, 1932, non- recording gage at railroad bridge 0.2 mi downstream from present site at datum $1.93 \mathrm{ft}$ higher. Nov. 1 to Dec. 11, 1939, nonrecording gage at present site and datum.

\begin{tabular}{|c|c|c|c|c|c|}
\hline $\begin{array}{c}\text { Water } \\
\text { year }\end{array}$ & Date & Discharge & $\begin{array}{l}\text { Discharge } \\
\text { code }\end{array}$ & $\begin{array}{c}\text { Gage } \\
\text { height } \\
\text { (feet) }\end{array}$ & $\begin{array}{c}\text { Gage } \\
\text { height } \\
\text { code }\end{array}$ \\
\hline 2000 & Apr. 6 & 11,300 & & 15.92 & \\
\hline 2001 & Apr. 8 & 6,960 & & 15.02 & \\
\hline 2002 & Dec. 17 & 10,800 & & 15.82 & \\
\hline 2003 & Feb. 25 & 12,500 & & 16.10 & \\
\hline 2004 & Feb. 9 & 15,200 & & 16.59 & \\
\hline 2005 & Dec. 11 & 13,800 & & 16.30 & \\
\hline 2006 & Jan. 24 & 5,830 & & 14.71 & \\
\hline 2007 & Jan. 5 & 4,050 & & 14.31 & \\
\hline 2008 & Mar. 10 & 3,760 & & 14.21 & \\
\hline 2009 & Jan. 9 & 18,300 & & 17.14 & \\
\hline 2010 & Dec. 13 & 12,100 & & 16.05 & \\
\hline 2011 & Mar. 13 & 12,000 & & 16.02 & \\
\hline 2012 & Jan. 1 & 3,550 & & 14.02 & \\
\hline 2013 & Jan. 18 & 10,500 & & 15.77 & \\
\hline 2014 & Apr. 8 & 7,000 & & 15.03 & \\
\hline 2015 & Dec. 29 & 7,200 & & 15.08 & \\
\hline
\end{tabular}

02447000 Sipsey River near Pleasant Ridge

L ocation-Lat $33^{\circ} 02^{\prime} 19^{\prime \prime}$, long $88^{\circ} 06^{\prime} 42^{\prime \prime}$, in NE $1 / 4$ sec. 29 , T. 24 N., R. 1 W., Greene County, Hydrologic Unit 03160107, on State Highway 40, $450 \mathrm{ft}$ downstream from Hughes Creek, 2.5 mi northwest of Pleasant Ridge, 6 mi upstream from mouth, and 6 mi south of Aliceville.

Drainage area-769 $\mathrm{mi}^{2}$.

Gage-Nonrecording. Datum of gage is $105.13 \mathrm{ft} \mathrm{NGVD} 29$.

\begin{tabular}{|c|c|c|c|c|c|}
\hline $\begin{array}{l}\text { Water } \\
\text { year }\end{array}$ & Date & Discharge & $\begin{array}{l}\text { Discharge } \\
\text { code }\end{array}$ & $\begin{array}{c}\text { Gage } \\
\text { height } \\
\text { (feet) }\end{array}$ & $\begin{array}{c}\text { Gage } \\
\text { height } \\
\text { code }\end{array}$ \\
\hline 1939 & Feb. 10 & 8,190 & & 19.05 & \\
\hline 1940 & July 16 & 7,020 & & 19.25 & \\
\hline 1941 & Aug. 5 & 4,825 & & 13.95 & \\
\hline 1942 & Mar. 28 & 3,350 & & 11.05 & \\
\hline 1943 & Mar. 21 & 12,800 & & 21.96 & \\
\hline 1944 & Apr. 3 & 10,800 & & 22.71 & \\
\hline 1945 & Feb. 22 & 9,600 & & 19.95 & \\
\hline 1946 & Jan. 13 & 15,000 & & 22.20 & \\
\hline 1947 & Jan. 21 & 7,700 & & 18.25 & \\
\hline 1948 & Feb. 15 & 10,000 & & 20.90 & \\
\hline 1949 & Jan. 10 & 19,500 & & 25.80 & \\
\hline 1950 & Jan. 11 & 17,500 & & 24.10 & \\
\hline 1951 & Apr. 2 & 19,250 & & 25.50 & \\
\hline 1952 & Dec. 30 & 5,200 & & 14.60 & \\
\hline 1953 & Feb. 27 & 7,900 & & 20.00 & \\
\hline 1954 & Jan. 29 & 5,630 & & 15.30 & \\
\hline 1955 & Apr. 15 & 4,520 & & 13.60 & \\
\hline 1957 & Feb. 7 & 7,800 & & 18.16 & \\
\hline 1958 & Nov. 25 & 11,200 & & 20.90 & \\
\hline 1959 & Feb. 22 & 3,550 & & 11.50 & \\
\hline 1961 & Feb. 25 & 35,000 & 7 & 26.60 & \\
\hline
\end{tabular}


Appendix 2. Discharge and gage height data for streamgages in Alabama.-Continued

[--, date or data not available; $\mathrm{ft}$, foot; $\mathrm{ft}^{3} / \mathrm{s}$, cubic foot per second; mi, mile; $\mathrm{mi}^{2}$, square mile; qualification codes apply to the discharge data: 1 , discharge is a maximum daily average; 2 , discharge is an estimate; 4 , discharge is less than indicated value, which is the minimum recordable discharge at this site; 5 , discharge affected to unknown degree by regulation or diversion; 6 , discharge affected by regulation or diversion; 7, discharge is a historic peak; A, year of occurrence is unknown or not exact; $\mathrm{B}$, month or day of occurrence is unknown or not exact; E, only maximum peak available for this year. qualification codes apply to the gage height data: 1, gage height affected by backwater; 2, gage height not the maximum for the year; 3, gage height at different datum or at different site and datum; 5 , gage height is an estimate]

02449245 Brush Creek near Eutaw

Location-Lat $32^{\circ} 49^{\prime} 51^{\prime \prime}$, long $87^{\circ} 58^{\prime} 56^{\prime \prime}$, in NE $1 / 4$ sec. $3, \mathrm{~T}$. 21 N., R. 1 E., Greene County, Hydrologic Unit 03160106, on county highway, 1.3 mi downstream from Pippan Creek, 2.2 mi upstream from Dry Creek, $5.5 \mathrm{mi}$ west of Eutaw, and $7.2 \mathrm{mi}$ upstream from mouth.

Drainage area $-43.2 \mathrm{mi}^{2}$.

G age -Water-stage recorder. Crest-stage gage 1971-75. Datum of gage is $105.92 \mathrm{ft}$ NGVD 29.

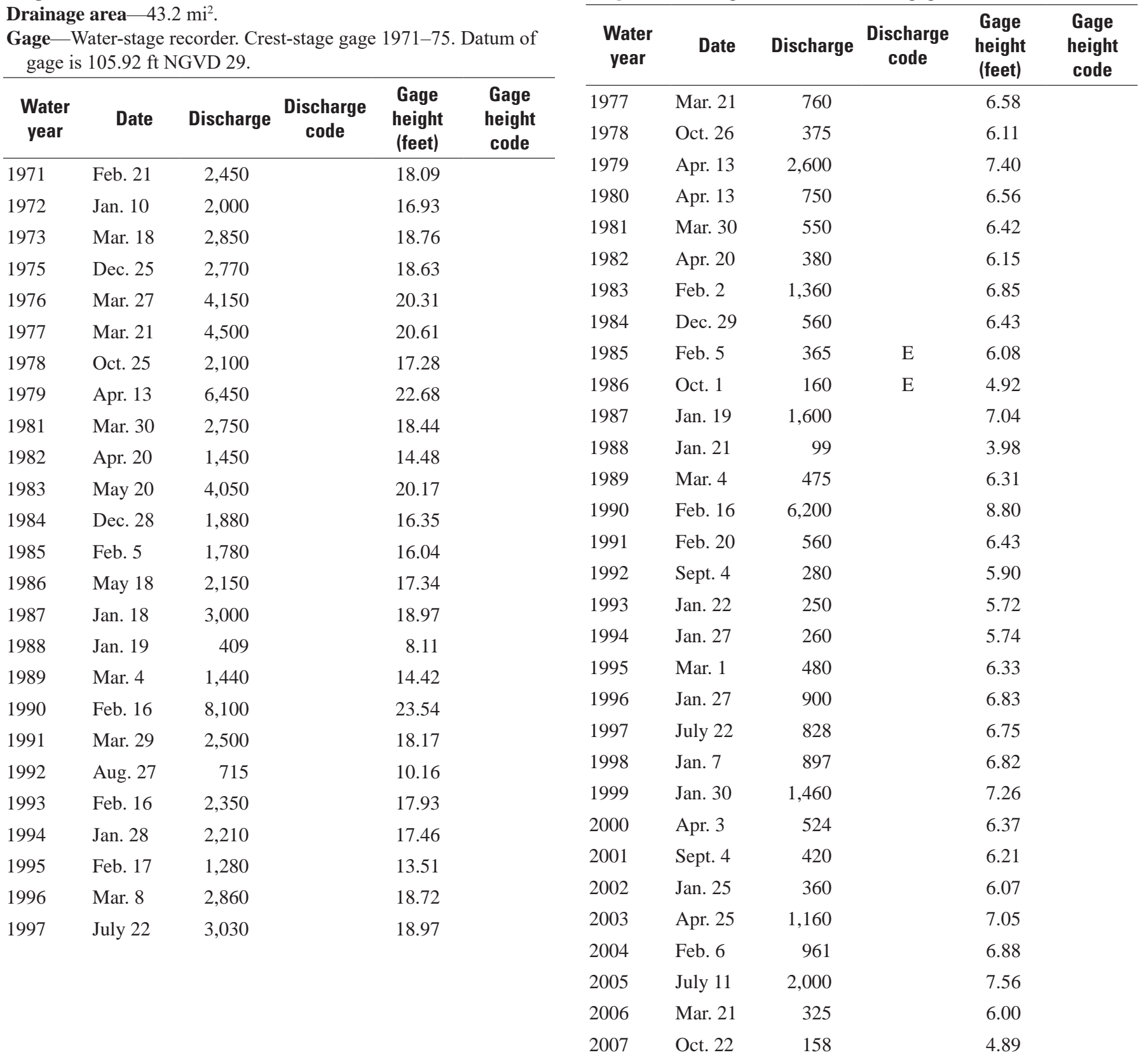

02465493 Elliots Creek at Moundville

L ocation-Lat $32^{\circ} 59^{\prime} 50^{\prime \prime}$, long $87^{\circ} 37^{\prime} 20^{\prime \prime}$, in SW 1/4 sec. 6, T. 23 N., R. 5 E., Hale County, Hydrologic Unit 03160113, on State Highway 69 at Moundville, and 6.6 mi upstream from mouth.

Drainage area-32.3 $\mathrm{mi}^{2}$.

G age - Water-stage recorder. Datum of gage is $147.81 \mathrm{ft}$ NGVD 29. 
Appendix 2. Discharge and gage height data for streamgages in Alabama.-Continued

[--, date or data not available; $\mathrm{ft}$, foot; $\mathrm{ft}^{3} / \mathrm{s}$, cubic foot per second; $\mathrm{mi}$, mile; $\mathrm{mi}^{2}$, square mile; qualification codes apply to the discharge data: 1 , discharge is a maximum daily average; 2 , discharge is an estimate; 4 , discharge is less than indicated value, which is the minimum recordable discharge at this site; 5 , discharge affected to unknown degree by regulation or diversion; 6 , discharge affected by regulation or diversion; 7, discharge is a historic peak; A, year of occurrence is unknown or not exact; B, month or day of occurrence is unknown or not exact; E, only maximum peak available for this year. qualification codes apply to the gage height data: 1, gage height affected by backwater; 2 , gage height not the maximum for the year; 3 , gage height at different data or at different site and datum;

5 , gage height is an estimate]

02465493 Elliots Creek at Moundville-Continued

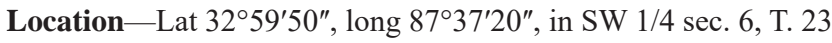

N., R. 5 E., Hale County, Hydrologic Unit 03160113, on State

Highway 69 at Moundville, and 6.6 mi upstream from mouth.

Drainage area-32.3 $\mathrm{mi}^{2}$.

Gage-Water-stage recorder. Datum of gage is $147.81 \mathrm{ft}$ NGVD 29.

\begin{tabular}{|c|c|c|c|c|c|}
\hline $\begin{array}{c}\text { Water } \\
\text { year }\end{array}$ & Date & Discharge & $\begin{array}{l}\text { Discharge } \\
\text { code }\end{array}$ & $\begin{array}{c}\text { Gage } \\
\text { height } \\
\text { (feet) }\end{array}$ & $\begin{array}{c}\text { Gage } \\
\text { height } \\
\text { code }\end{array}$ \\
\hline 2008 & Feb. 22 & 195 & & 5.27 & \\
\hline 2009 & Sept. 21 & 6,430 & & 9.76 & \\
\hline 2010 & Nov. 10 & 665 & & 6.87 & \\
\hline 2011 & Mar. 10 & 679 & & 6.89 & \\
\hline 2012 & Jan. 27 & 330 & & 5.91 & \\
\hline 2013 & Jan. 31 & 419 & & 6.31 & \\
\hline 2014 & Apr. 7 & 907 & & 7.16 & \\
\hline 2015 & Jan. 4 & 557 & & 6.69 & \\
\hline
\end{tabular}

\section{Fivemile Creek near Greensboro}

L ocation-Lat $32^{\circ} 49^{\prime} 46^{\prime \prime}$, long $87^{\circ} 36^{\prime} 15^{\prime \prime}$, in NW 1/4 sec. 5, T. 21

N., R. 5 E., Hale County, Hydrologic Unit 03160113, on State

Highway 69, 8.5 mi north of Greensboro, and 12 mi upstream

from mouth.

Drainage area $-73.6 \mathrm{mi}^{2}$.

Gage - Water-stage recorder. Datum of gage is $160 \mathrm{ft}$ NGVD 29 (by barometer).

\begin{tabular}{|c|c|c|c|c|c|}
\hline $\begin{array}{c}\text { Water } \\
\text { year }\end{array}$ & Date & Discharge & $\begin{array}{c}\text { Discharge } \\
\text { code }\end{array}$ & $\begin{array}{c}\text { Gage } \\
\text { height } \\
\text { (feet) }\end{array}$ & $\begin{array}{c}\text { Gage } \\
\text { height } \\
\text { code }\end{array}$ \\
\hline 1955 & Apr. 14 & 2,470 & & 7.90 & \\
\hline 1956 & Mar. 16 & 3,540 & & 8.37 & \\
\hline 1957 & Apr. 5 & 1,900 & & 7.65 & \\
\hline 1958 & Feb. 7 & 955 & & 6.86 & \\
\hline 1959 & Feb. 10 & 628 & & 6.40 & \\
\hline 1960 & Jan. 30 & 974 & & 6.88 & \\
\hline 1961 & Feb. 22 & 7,400 & & 9.84 & \\
\hline 1962 & Dec. 18 & 7,400 & & 9.84 & \\
\hline 1963 & Jan. 21 & 647 & & 6.36 & \\
\hline 1964 & Apr. 6 & 3,460 & & 8.49 & \\
\hline 1965 & Feb. 12 & 1,540 & & 7.43 & \\
\hline 1966 & Feb. 16 & 1,880 & & 7.67 & \\
\hline 1967 & Feb. 8 & 256 & & 5.16 & \\
\hline 1968 & Apr. 5 & 1,320 & & 7.25 & \\
\hline
\end{tabular}

02465500 Fivemile Creek near Greensboro-Continued

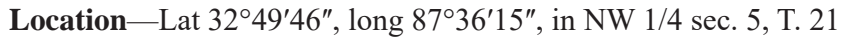

N., R. 5 E., Hale County, Hydrologic Unit 03160113, on State

Highway 69, 8.5 mi north of Greensboro, and 12 mi upstream from mouth.

Drainage area $-73.6 \mathrm{mi}^{2}$.

Gage - Water-stage recorder. Datum of gage is $160 \mathrm{ft} \mathrm{NGVD} 29$ (by barometer).

\begin{tabular}{lllccc}
\hline $\begin{array}{c}\text { Water } \\
\text { year }\end{array}$ & Date & Discharge & $\begin{array}{c}\text { Discharge } \\
\text { code }\end{array}$ & $\begin{array}{c}\text { Gage } \\
\text { height } \\
\text { (feet) }\end{array}$ & $\begin{array}{c}\text { Gage } \\
\text { height } \\
\text { code }\end{array}$ \\
\hline 1969 & Apr. 19 & 1,440 & & 7.35 & \\
1970 & June 4 & 2,970 & & 8.27 & \\
1971 & Mar. 3 & 1,840 & & 7.65 & \\
1972 & Jan. 11 & 2,930 & & 8.25 & \\
1973 & Apr. 8 & 1,120 & & 6.92 & \\
1974 & Dec. 26 & 3,280 & & 8.30 & \\
1979 & Apr. 13 & 2,700 & 7 & 8.14 &
\end{tabular}

03592200 Cedar Creek near Pleasant Site

L ocation-Lat $34^{\circ} 32^{\prime} 56^{\prime \prime}$, long $88^{\circ} 01^{\prime} 09^{\prime \prime}$, in SW 1/4 sec. 9, T. 6 S., R. 14 W., Franklin County, Hydrologic Unit 06030006, $2.6 \mathrm{mi}$ east of Pleasant Site, 4.3 mi upstream from Little Bear Creek, and at mile 19.1 .

Drainage area $-189 \mathrm{mi}^{2}$.

G age-Water-stage recorder. Datum of gage is $482.67 \mathrm{ft}$ NGVD 29.

\begin{tabular}{llcccc}
\hline $\begin{array}{c}\text { Water } \\
\text { year }\end{array}$ & Date & Discharge & $\begin{array}{c}\text { Discharge } \\
\text { code }\end{array}$ & $\begin{array}{c}\text { Gage } \\
\text { height } \\
\text { (feet) }\end{array}$ & $\begin{array}{c}\text { Gage } \\
\text { height } \\
\text { code }\end{array}$ \\
\hline 1948 & Feb. 13 & 15,000 & 7 & 22.90 & \\
1951 & Mar. 28 & 14,000 & 7 & 22.20 & \\
1955 & Mar. 21 & 17,800 & 7 & 24.40 & \\
1958 & Nov. 16 & 8,800 & & 18.60 & \\
1959 & Apr. 19 & 3,250 & & 12.00 & \\
1960 & Dec. 18 & 7,200 & & 18.10 & \\
1961 & Mar. 21 & 4,850 & & 15.20 & \\
1962 & Dec. 18 & 11,000 & & 20.73 & \\
1963 & Mar. 12 & 13,500 & & 22.00 & \\
1964 & Mar. 15 & 12,200 & & 20.78 & \\
1965 & Feb. 12 & 7,500 & & 17.81 & \\
1966 & Feb. 13 & 3,710 & & 12.92 & \\
1967 & Dec. 9 & 6,500 & & 17.13 & \\
1968 & Jan. 10 & 6,550 & & 17.16
\end{tabular}


Appendix 2. Discharge and gage height data for streamgages in Alabama.-Continued

[--, date or data not available; $\mathrm{ft}$, foot; $\mathrm{ft}^{3} / \mathrm{s}$, cubic foot per second; mi, mile; $\mathrm{mi}^{2}$, square mile; qualification codes apply to the discharge data: 1 , discharge is a maximum daily average; 2 , discharge is an estimate; 4 , discharge is less than indicated value, which is the minimum recordable discharge at this site; 5 , discharge affected to unknown degree by regulation or diversion; 6 , discharge affected by regulation or diversion; 7, discharge is a historic peak; A, year of occurrence is unknown or not exact; $\mathrm{B}$, month or day of occurrence is unknown or not exact; E, only maximum peak available for this year. qualification codes apply to the gage height data: 1, gage height affected by backwater; 2, gage height not the maximum for the year; 3, gage height at different datum or at different site and datum; 5 , gage height is an estimate]

\section{Cedar Creek near Pleasant Site-Continued}

L ocation-Lat $34^{\circ} 32^{\prime} 56^{\prime \prime}$, long $88^{\circ} 01^{\prime} 09^{\prime \prime}$, in SW 1/4 sec. 9, T. 6 S., R. 14 W., Franklin County, Hydrologic Unit 06030006, 2.6 mi east of Pleasant Site, 4.3 mi upstream from Little Bear Creek, and at mile 19.1 .

Drainage area-189 $\mathrm{mi}^{2}$.

Gage -Water-stage recorder. Datum of gage is $482.67 \mathrm{ft}$ NGVD 29.

\begin{tabular}{|c|c|c|c|c|c|}
\hline $\begin{array}{l}\text { Water } \\
\text { year }\end{array}$ & Date & Discharge & $\begin{array}{l}\text { Discharge } \\
\text { code }\end{array}$ & $\begin{array}{c}\text { Gage } \\
\text { height } \\
\text { (feet) }\end{array}$ & $\begin{array}{c}\text { Gage } \\
\text { height } \\
\text { code }\end{array}$ \\
\hline 1969 & Feb. 2 & 7,500 & & 17.80 & \\
\hline 1970 & Dec. 30 & 12,000 & & 20.79 & \\
\hline 1971 & Feb. 22 & 7,400 & & 17.23 & \\
\hline 1972 & May 7 & 4,150 & & 13.32 & \\
\hline 1973 & Mar. 16 & 27,000 & & 28.02 & \\
\hline 1974 & Jan. 11 & 7,500 & & 17.50 & \\
\hline 1975 & Mar. 14 & 12,900 & & 21.50 & \\
\hline 1976 & Oct. 18 & 8,250 & & 18.06 & \\
\hline 1977 & Mar. 24 & 10,300 & & 19.81 & \\
\hline
\end{tabular}

03592300 Little Bear Creek near Halltown

Location-Lat $34^{\circ} 29^{\prime} 19^{\prime \prime}$, long $88^{\circ} 02^{\prime} 07^{\prime \prime}$, in NW 1/4 sec. 5, T. 7

S. R. 14 W., Franklin County, Hydrologic Unit 06030006, $2.7 \mathrm{mi}$ northeast of Halltown, and at mile 4.3.

Drainage area $-78.2 \mathrm{mi}^{2}$.

Gage - Water-stage recorder. Datum of gage is $499.30 \mathrm{ft}$ NGVD 29.

\begin{tabular}{llcccc}
\hline $\begin{array}{c}\text { Water } \\
\text { year }\end{array}$ & Date & Discharge & $\begin{array}{c}\text { Discharge } \\
\text { code }\end{array}$ & $\begin{array}{c}\text { Gage } \\
\text { height } \\
\text { (feet) }\end{array}$ & $\begin{array}{c}\text { Gage } \\
\text { height } \\
\text { code }\end{array}$ \\
\hline 1951 & Mar. 28 & 3,900 & 7 & 11.70 & \\
1955 & Mar. 21 & 6,800 & 7 & 13.70 & \\
1958 & Nov. 18 & 4,300 & & 12.10 & \\
1959 & Apr. 19 & 1,750 & & 8.40 & \\
1960 & Mar. 3 & 3,900 & & 11.70 & \\
1961 & Mar. 21 & 2,500 & & 10.09 & \\
1962 & Dec. 17 & 5,990 & & 13.27 & \\
1963 & Mar. 12 & 5,810 & & 13.17 & \\
1964 & Mar. 14 & 4,100 & & 11.83 & \\
1965 & Feb. 11 & 3,900 & & 11.67 & \\
1966 & Feb. 13 & 1,600 & & 8.06 & \\
1967 & May 7 & 4,000 & & 11.78 &
\end{tabular}

\section{Little Bear Creek near Halltown-Continued}

L ocation-Lat $34^{\circ} 29^{\prime} 19^{\prime \prime}$, long $88^{\circ} 02^{\prime} 07^{\prime \prime}$, in NW 1/4 sec. 5, T. 7 S. R. 14 W., Franklin County, Hydrologic Unit 06030006, $2.7 \mathrm{mi}$ northeast of Halltown, and at mile 4.3.

Drainage area-78.2 $\mathrm{mi}^{2}$.

G age - Water-stage recorder. Datum of gage is $499.30 \mathrm{ft}$ NGVD 29.

\begin{tabular}{lllccc}
\hline $\begin{array}{c}\text { Water } \\
\text { year }\end{array}$ & Date & Discharge & $\begin{array}{c}\text { Discharge } \\
\text { code }\end{array}$ & $\begin{array}{c}\text { Gage } \\
\text { height } \\
\text { (feet) }\end{array}$ & $\begin{array}{c}\text { Gage } \\
\text { height } \\
\text { code }\end{array}$ \\
\hline 1968 & Dec. 18 & 4,340 & & 12.05 & \\
1969 & Apr. 10 & 3,130 & & 10.68 & \\
1970 & Dec. 30 & 7,070 & & 13.78 & \\
1971 & Feb. 22 & 3,420 & & 11.00 & \\
1972 & Jan. 4 & 2,920 & & 10.33 & \\
1973 & Mar. 16 & 20,400 & & 18.18 & \\
1974 & Nov. 28 & 3,540 & & 10.87 & \\
1975 & Mar. 13 & 8,200 & & 14.23 & \\
1976 & Oct. 17 & 2,000 & 6 & 8.46 & \\
1977 & Mar. 12 & 2,870 & 6 & 10.00
\end{tabular}

\section{Bear Creek at Bishop}

L ocation-Lat $34^{\circ} 39^{\prime} 21^{\prime \prime}$, long $88^{\circ} 07^{\prime} 21^{\prime \prime}$, in SE $1 / 4$ sec. 5 , T. 5 S., R. 15 W., Colbert County, Hydrologic Unit 06030006, 0.5 mi downstream from Cedar Creek, $0.8 \mathrm{mi}$ southwest of Bishop, and at mile 27.3.

Drainage area $-667 \mathrm{mi}^{2}$.

G age - Water-stage recorder. Datum of gage is $419.91 \mathrm{ft}$ NGVD 29. Nonrecording gage prior to June 23, 1928, and Feb. 10, 1929 to Mar. 31, 1932, at site $35 \mathrm{ft}$ downstream, and June 7, 1933 to May 28, 1934, at bridge $20 \mathrm{ft}$ downstream at datum $5.00 \mathrm{ft}$ lower.

Remarks - Flow regulated since March 1969 by Bear Creek Reservoir.

\begin{tabular}{|c|c|c|c|c|c|}
\hline $\begin{array}{c}\text { Water } \\
\text { year }\end{array}$ & Date & Discharge & $\begin{array}{c}\text { Discharge } \\
\text { code }\end{array}$ & $\begin{array}{c}\text { Gage } \\
\text { height } \\
\text { (feet) }\end{array}$ & $\begin{array}{c}\text { Gage } \\
\text { height } \\
\text { code }\end{array}$ \\
\hline 1927 & Dec. 26 & 40,000 & & 22.00 & \\
\hline 1928 & Apr. 24 & 15,800 & & 17.20 & \\
\hline 1929 & Mar. 24 & 36,500 & & 21.60 & \\
\hline 1930 & Mar. 7 & 11,000 & & 15.50 & \\
\hline 1931 & Apr. 1 & 6,500 & & 12.40 & \\
\hline 1932 & Dec. 14 & 16,400 & & 17.40 & \\
\hline 1934 & June 7 & 17,000 & & 17.63 & \\
\hline 1935 & Mar. 7 & 9,600 & & 15.23 & \\
\hline
\end{tabular}


Appendix 2. Discharge and gage height data for streamgages in Alabama.-Continued

[--, date or data not available; $\mathrm{ft}$, foot; $\mathrm{ft}^{3} / \mathrm{s}$, cubic foot per second; $\mathrm{mi}$, mile; $\mathrm{mi}^{2}$, square mile; qualification codes apply to the discharge data: 1 , discharge is a maximum daily average; 2 , discharge is an estimate; 4 , discharge is less than indicated value, which is the minimum recordable discharge at this site; 5 , discharge affected to unknown degree by regulation or diversion; 6 , discharge affected by regulation or diversion; 7, discharge is a historic peak; A, year of occurrence is unknown or not exact; B, month or day of occurrence is unknown or not exact; E, only maximum peak available for this year. qualification codes apply to the gage height data: 1, gage height affected by backwater; 2 , gage height not the maximum for the year; 3 , gage height at different data or at different site and datum; 5 , gage height is an estimate]

\section{Bear Creek at Bishop-Continued}

L ocation-Lat $34^{\circ} 39^{\prime} 21^{\prime \prime}$, long $88^{\circ} 07^{\prime} 21^{\prime \prime}$, in SE $1 / 4$ sec. 5, T. 5 S., R. 15 W., Colbert County, Hydrologic Unit 06030006, $0.5 \mathrm{mi}$ downstream from Cedar Creek, 0.8 mi southwest of Bishop, and at mile 27.3.

Drainage area $-667 \mathrm{mi}^{2}$.

Gage - Water-stage recorder. Datum of gage is $419.91 \mathrm{ft}$ NGVD 29. Nonrecording gage prior to June 23, 1928, and Feb. 10, 1929 to Mar. 31, 1932, at site $35 \mathrm{ft}$ downstream, and June 7, 1933 to May 28, 1934, at bridge $20 \mathrm{ft}$ downstream at datum $5.00 \mathrm{ft}$ lower.

Remarks -Flow regulated since March 1969 by Bear Creek Reservoir.

\begin{tabular}{|c|c|c|c|c|c|}
\hline $\begin{array}{c}\text { Water } \\
\text { year }\end{array}$ & Date & Discharge & $\begin{array}{l}\text { Discharge } \\
\text { code }\end{array}$ & $\begin{array}{c}\text { Gage } \\
\text { height } \\
\text { (feet) }\end{array}$ & $\begin{array}{c}\text { Gage } \\
\text { height } \\
\text { code }\end{array}$ \\
\hline 1936 & Apr. 6 & 14,000 & & 16.86 & \\
\hline 1937 & Jan. 2 & 10,800 & & 15.69 & \\
\hline 1938 & Mar. 12 & 8,800 & & 14.70 & \\
\hline 1939 & Feb. 15 & 17,700 & & 17.72 & \\
\hline 1940 & Apr. 19 & 8,600 & & 14.59 & \\
\hline 1941 & July 5 & 7,070 & & 13.34 & \\
\hline 1942 & Mar. 17 & 12,300 & & 16.30 & \\
\hline 1943 & Dec. 29 & 7,950 & & 14.08 & \\
\hline 1944 & Mar. 29 & 29,000 & & 20.18 & \\
\hline 1945 & Feb. 22 & 19,700 & & 18.27 & \\
\hline 1946 & Jan. 8 & 25,000 & & 19.38 & \\
\hline 1947 & Jan. 2 & 15,200 & & 17.30 & \\
\hline 1948 & Feb. 14 & 35,000 & & 21.44 & \\
\hline 1949 & Jan. 6 & 30,500 & & 20.48 & \\
\hline 1950 & Jan. 8 & 28,800 & & 20.06 & \\
\hline 1951 & Mar. 29 & 27,000 & & 19.73 & \\
\hline 1952 & Dec. 21 & 12,000 & & 16.07 & \\
\hline 1953 & Feb. 21 & 14,100 & & 16.83 & \\
\hline 1954 & Jan. 23 & 15,500 & & 17.23 & \\
\hline 1955 & Mar. 22 & 40,000 & & 21.98 & \\
\hline 1956 & Feb. 5 & 11,100 & & 15.81 & \\
\hline 1957 & Feb. 2 & 28,900 & & 20.22 & \\
\hline 1958 & Nov. 19 & 19,100 & & 18.08 & \\
\hline
\end{tabular}

03592500 Bear Creek at Bishop-Continued

L ocation-Lat $34^{\circ} 39^{\prime} 21^{\prime \prime}$, long $88^{\circ} 07^{\prime} 21^{\prime \prime}$, in SE $1 / 4$ sec. 5, T. 5 S., R. 15 W., Colbert County, Hydrologic Unit 06030006, 0.5 mi downstream from Cedar Creek, $0.8 \mathrm{mi}$ southwest of Bishop, and at mile 27.3.

Drainage area $-667 \mathrm{mi}^{2}$.

G age - Water-stage recorder. Datum of gage is $419.91 \mathrm{ft}$ NGVD 29. Nonrecording gage prior to June 23, 1928, and Feb. 10, 1929 to Mar. 31, 1932, at site $35 \mathrm{ft}$ downstream, and June 7, 1933 to May 28, 1934, at bridge $20 \mathrm{ft}$ downstream at datum $5.00 \mathrm{ft}$ lower.

Remarks-Flow regulated since March 1969 by Bear Creek Reservoir.

\begin{tabular}{|c|c|c|c|c|c|}
\hline $\begin{array}{c}\text { Water } \\
\text { year }\end{array}$ & Date & Discharge & $\begin{array}{c}\text { Discharge } \\
\text { code }\end{array}$ & $\begin{array}{c}\text { Gage } \\
\text { height } \\
\text { (feet) }\end{array}$ & $\begin{array}{c}\text { Gage } \\
\text { height } \\
\text { code }\end{array}$ \\
\hline 1959 & Feb. 14 & 6,600 & & 12.78 & \\
\hline 1960 & Dec. 19 & 13,000 & & 16.47 & \\
\hline 1961 & Mar. 8 & 9,480 & & 15.09 & \\
\hline 1962 & Dec. 18 & 24,900 & & 19.34 & \\
\hline 1963 & Mar. 12 & 24,400 & & 19.24 & \\
\hline 1964 & Mar. 15 & 22,800 & & 18.89 & \\
\hline 1965 & Feb. 12 & 14,600 & & 16.98 & \\
\hline 1966 & Feb. 13 & 7,400 & & 13.58 & \\
\hline 1967 & May 8 & 14,400 & & 16.91 & \\
\hline 1968 & Jan. 11 & 13,900 & & 16.76 & \\
\hline 1969 & Feb. 2 & 13,800 & & 16.75 & \\
\hline 1970 & Dec. 30 & 27,200 & 6 & 19.84 & \\
\hline 1971 & Feb. 22 & 16,200 & 6 & 17.40 & \\
\hline 1972 & Jan. 5 & 10,500 & 6 & 15.55 & \\
\hline 1973 & Mar. 17 & 60,800 & 6 & 24.12 & \\
\hline 1974 & Jan. 11 & 18,100 & 6 & 17.95 & \\
\hline 1975 & Mar. 14 & 31,200 & 6 & 20.62 & \\
\hline 1976 & Oct. 18 & 13,000 & 6 & 16.45 & \\
\hline 1977 & Mar. 4 & 17,700 & 6 & 17.78 & \\
\hline 1978 & May 8 & 18,100 & 6 & 17.86 & \\
\hline 1979 & Jan. 1 & 10,100 & 6 & 15.39 & \\
\hline 2014 & July 19 & 5,740 & 6 & 11.23 & \\
\hline 2015 & Oct. 14 & 10,100 & 6 & 15.63 & \\
\hline
\end{tabular}


Appendix 2. Discharge and gage height data for streamgages in Alabama.-Continued

[--, date or data not available; $\mathrm{ft}$, foot; $\mathrm{ft}^{3} / \mathrm{s}$, cubic foot per second; mi, mile; $\mathrm{mi}^{2}$, square mile; qualification codes apply to the discharge data: 1 , discharge is a maximum daily average; 2 , discharge is an estimate; 4 , discharge is less than indicated value, which is the minimum recordable discharge at this site; 5 , discharge affected to unknown degree by regulation or diversion; 6 , discharge affected by regulation or diversion; 7, discharge is a historic peak; A, year of occurrence is unknown or not exact; $\mathrm{B}$, month or day of occurrence is unknown or not exact; E, only maximum peak available for this year. qualification codes apply to the gage height data: 1, gage height affected by backwater; 2, gage height not the maximum for the year; 3, gage height at different datum or at different site and datum; 5 , gage height is an estimate]

02419625 Calebee Creek near Tuskegee Ala.

L ocation-Lat $32^{\circ} 22^{\prime} 48^{\prime \prime}$, long 85 49'36", Macon County, Hydrologic Unit 03150110.

Drainage area-124 $\mathrm{mi}^{2}$.

G age -Water-stage recorder. Datum of gage is 222.05 feet above NGVD 29.

\begin{tabular}{|c|c|c|c|c|c|}
\hline $\begin{array}{c}\text { Water } \\
\text { year }\end{array}$ & Date & Discharge & $\begin{array}{l}\text { Discharge } \\
\text { code }\end{array}$ & $\begin{array}{c}\text { Gage } \\
\text { height } \\
\text { (feet) }\end{array}$ & $\begin{array}{c}\text { Gage } \\
\text { height } \\
\text { code }\end{array}$ \\
\hline 1952 & Mar. 25 & 7,200 & & 15.50 & \\
\hline 1953 & May 5 & 7,800 & & 15.60 & \\
\hline 1954 & Dec. 5 & 12,500 & & 16.40 & \\
\hline 1955 & Apr. 14 & 2,100 & & 13.50 & \\
\hline 1956 & Mar. 17 & 2,100 & & 13.50 & \\
\hline 1957 & Apr. 6 & 5,200 & & 14.90 & \\
\hline 1958 & Mar. 7 & 19,000 & & 17.40 & \\
\hline 1959 & Feb. 6 & 960 & & 11.30 & \\
\hline 1960 & Apr. 3 & 2,600 & & 13.90 & \\
\hline 1961 & Feb. 25 & 13,800 & & 16.54 & \\
\hline 1962 & Apr. 1 & 3,460 & & 14.18 & \\
\hline 1963 & Jan. 21 & 2,150 & & 13.38 & \\
\hline 1964 & Apr. 9 & 16,200 & & 16.97 & \\
\hline 1965 & Jan. 23 & 2,940 & & 13.91 & \\
\hline 1966 & Feb. 14 & 6,240 & & 15.16 & \\
\hline 1967 & Jan. 3 & 338 & & 9.48 & \\
\hline 1968 & Mar. 12 & 5,330 & & 14.91 & \\
\hline 1970 & Mar. 21 & 1,900 & & 13.16 & \\
\hline
\end{tabular}

02421000 Catoma Creek near Montgomery

L ocation-Lat $32^{\circ} 18^{\prime} 26^{\prime \prime}$, long $86^{\circ} 17^{\prime} 58^{\prime \prime}$, in NW 1/4 sec. 6, T. 15 N., R. 18 E., Montgomery County,

Hydrologic Unit 03150201, on old U.S. Highway 331, 5 mi south of Montgomery.

Drainage area-290 $\mathrm{mi}^{2}$.

G age -Water-stage recorder. Datum of gage is $151.02 \mathrm{ft}$ NGVD 29.

\begin{tabular}{|c|c|c|c|c|c|}
\hline $\begin{array}{c}\text { Water } \\
\text { year }\end{array}$ & Date & Discharge & $\begin{array}{c}\text { Discharge } \\
\text { code }\end{array}$ & $\begin{array}{c}\text { Gage } \\
\text { height } \\
\text { (feet) }\end{array}$ & $\begin{array}{c}\text { Gage } \\
\text { height } \\
\text { code }\end{array}$ \\
\hline 1949 & Nov. 28 & 38,300 & 7 & 27.50 & \\
\hline 1953 & Feb. 27 & 6,420 & & 20.50 & \\
\hline 1954 & Dec. 5 & 10,700 & & 23.03 & \\
\hline 1955 & Apr. 15 & 5,560 & & 19.73 & \\
\hline 1956 & Sept. 26 & 6,500 & & 20.68 & \\
\hline 1957 & Apr. 6 & 15,000 & & 23.80 & \\
\hline 1958 & Mar. 8 & 25,600 & & 25.70 & \\
\hline 1959 & Mar. 7 & 3,660 & & 17.00 & \\
\hline 1960 & Apr. 4 & 13,500 & & 23.33 & \\
\hline 1961 & Feb. 25 & 48,600 & & 28.60 & 5 \\
\hline 1962 & Apr. 13 & 10,400 & & 22.35 & \\
\hline 1963 & Jan. 21 & 9,100 & & 21.85 & 2 \\
\hline 1964 & Apr. 28 & 21,700 & & 25.20 & \\
\hline 1965 & Jan. 24 & 12,800 & & 23.15 & \\
\hline 1966 & Feb. 13 & 11,500 & & 22.71 & \\
\hline 1967 & Jan. 3 & 4,790 & & 18.86 & \\
\hline 1968 & Mar. 12 & 10,800 & & 22.47 & \\
\hline 1969 & Mar. 25 & 5,400 & & 19.55 & \\
\hline 1970 & June 5 & 14,900 & & 23.80 & \\
\hline 1971 & Mar. 4 & 12,200 & & 22.94 & \\
\hline 1972 & Mar. 3 & 10,100 & & 22.23 & \\
\hline 1973 & Apr. 26 & 20,900 & & 25.07 & \\
\hline 1974 & Sept. 28 & 6,810 & & 20.64 & \\
\hline 1975 & Feb. 17 & 43,900 & & 28.13 & \\
\hline 1976 & Apr. 1 & 12,800 & & 23.12 & \\
\hline 1977 & Mar. 30 & 8,580 & & 21.59 & \\
\hline 1978 & Jan. 26 & 17,000 & & 24.76 & \\
\hline 1979 & Apr. 5 & 15,500 & & 24.44 & \\
\hline 1980 & Mar. 30 & 9,460 & & 22.38 & \\
\hline 1981 & Feb. 12 & 5,480 & & 18.97 & \\
\hline 1982 & Feb. 3 & 15,500 & & 24.46 & \\
\hline 1983 & Apr. 9 & 9,760 & & 22.53 & \\
\hline 1984 & Mar. 27 & 5,900 & & 19.57 & \\
\hline
\end{tabular}


Appendix 2. Discharge and gage height data for streamgages in Alabama._- Continued

[--, date or data not available; ft, foot; $\mathrm{ft}^{3} / \mathrm{s}$, cubic foot per second; mi, mile; $\mathrm{mi}^{2}$, square mile; qualification codes apply to the discharge data: 1 , discharge is a maximum daily average; 2 , discharge is an estimate; 4 , discharge is less than indicated value, which is the minimum recordable discharge at this site; 5 , discharge affected to unknown degree by regulation or diversion; 6 , discharge affected by regulation or diversion; 7, discharge is a historic peak; A, year of occurrence is unknown or not exact; $\mathrm{B}$, month or day of occurrence is unknown or not exact; E, only maximum peak available for this year. qualification codes apply to the gage height data: 1, gage height affected by backwater; 2, gage height not the maximum for the year; 3, gage height at different data or at different site and datum; 5 , gage height is an estimate]

\section{Catoma Creek near Montgomery-Continued}

L ocation-Lat $32^{\circ} 18^{\prime} 26^{\prime \prime}$, long $86^{\circ} 17^{\prime} 58^{\prime \prime}$, in NW 1/4 sec. 6, T. 15

N., R. 18 E., Montgomery County, Hydrologic Unit 03150201, on old U.S. Highway 331, 5 mi south of Montgomery.

Drainage area-290 $\mathrm{mi}^{2}$.

G age - Water-stage recorder. Datum of gage is $151.02 \mathrm{ft}$ NGVD 29.

\begin{tabular}{|c|c|c|c|c|c|}
\hline $\begin{array}{c}\text { Water } \\
\text { year }\end{array}$ & Date & Discharge & $\begin{array}{l}\text { Discharge } \\
\text { code }\end{array}$ & $\begin{array}{c}\text { Gage } \\
\text { height } \\
\text { (feet) }\end{array}$ & $\begin{array}{c}\text { Gage } \\
\text { height } \\
\text { code }\end{array}$ \\
\hline 1985 & Feb. 7 & 4,650 & $E$ & 17.36 & \\
\hline 1986 & Mar. 15 & 11,900 & & 22.77 & \\
\hline 1987 & Mar. 1 & 5,420 & & 18.29 & \\
\hline 1988 & Feb. 3 & 3,970 & & 15.59 & \\
\hline 1989 & June 21 & 18,400 & & 24.87 & \\
\hline 1990 & Mar. 17 & 50,000 & & 29.78 & \\
\hline 1991 & Mar. 30 & 7,210 & & 20.66 & \\
\hline 1992 & Jan. 15 & 5,430 & & 18.77 & \\
\hline 1993 & Nov. 27 & 7,930 & & 21.25 & \\
\hline 1994 & July 8 & 22,800 & & 26.07 & \\
\hline 1995 & Feb. 19 & 5,610 & & 19.01 & \\
\hline 1996 & Mar. 7 & 12,800 & & 23.55 & \\
\hline 1997 & Feb. 23 & 5,190 & & 18.43 & \\
\hline 1998 & Mar. 9 & 18,900 & & 24.99 & \\
\hline 1999 & Feb. 1 & 4,400 & & 17.17 & \\
\hline 2000 & Mar. 21 & 2,780 & & 13.25 & \\
\hline 2001 & Mar. 4 & 28,600 & & 27.20 & \\
\hline 2002 & Mar. 22 & 3,180 & & 14.39 & \\
\hline 2003 & Apr. 27 & 7,430 & & 20.85 & \\
\hline 2004 & Sept. 18 & 9,740 & & 22.39 & \\
\hline 2005 & Mar. 28 & 28,300 & & 27.14 & \\
\hline 2006 & Feb. 27 & 5,530 & & 18.91 & \\
\hline 2007 & Mar. 3 & 6,150 & & 19.62 & \\
\hline 2008 & Aug. 26 & 9,780 & & 22.41 & \\
\hline 2009 & Mar. 29 & 11,100 & & 23.03 & \\
\hline 2010 & Nov. 12 & 10,200 & & 22.64 & \\
\hline 2011 & Mar. 11 & 3,170 & & 14.36 & \\
\hline 2012 & Feb. 20 & 3,650 & & 15.58 & \\
\hline 2013 & Feb. 13 & 14,300 & & 23.96 & \\
\hline 2014 & Apr. 8 & 19,500 & & 25.15 & \\
\hline 2015 & May 29 & 2,720 & & 13.07 & \\
\hline
\end{tabular}

02422000 Big Swamp Creek near Lowndesboro

L ocation-Lat $32^{\circ} 15^{\prime} 58^{\prime \prime}$, long $86^{\circ} 41^{\prime} 40^{\prime \prime}$, in NE $1 / 4$ sec. 19 , T. 15 N., R. 14 E., Lowndes County, Hydrologic Unit 03150201, on U.S. Highway 80, $1 \mathrm{mi}$ downstream from Panther Creek, $5 \mathrm{mi}$ west of Lowndesboro, and 12 mi upstream from mouth.

Drainage area $-244 \mathrm{mi}^{2}$.

Gage-Water-stage recorder. Datum of gage is $127.95 \mathrm{ft}$ NGVD 29.

\begin{tabular}{|c|c|c|c|c|c|}
\hline $\begin{array}{c}\text { Water } \\
\text { year }\end{array}$ & Date & Discharge & $\begin{array}{c}\text { Discharge } \\
\text { code }\end{array}$ & $\begin{array}{c}\text { Gage } \\
\text { height } \\
\text { (feet) }\end{array}$ & $\begin{array}{c}\text { Gage } \\
\text { height } \\
\text { code }\end{array}$ \\
\hline 1938 & Apr. 1 & 29,500 & 7 & 20.00 & \\
\hline 1941 & Mar. 8 & 4,400 & & 16.60 & \\
\hline 1942 & Dec. 24 & 13,500 & & 18.20 & \\
\hline 1943 & Mar. 21 & 25,000 & & 19.50 & \\
\hline 1944 & Mar. 23 & 20,800 & & 19.00 & \\
\hline 1945 & Apr. 25 & 13,500 & & 18.20 & \\
\hline 1946 & June 2 & 14,000 & & 18.28 & \\
\hline 1947 & Apr. 3 & 3,400 & & 16.40 & \\
\hline 1948 & Mar. 7 & 6,000 & & 17.00 & \\
\hline 1949 & Nov. 27 & 48,000 & & 21.30 & \\
\hline 1950 & July 13 & 3,340 & & 15.63 & \\
\hline 1951 & Apr. 22 & 1,600 & & 14.98 & \\
\hline 1952 & Mar. 25 & 2,150 & & 15.84 & \\
\hline 1953 & May 4 & 6,600 & & 17.18 & \\
\hline 1954 & Dec. 6 & 2,850 & & 16.11 & \\
\hline 1955 & Apr. 14 & 11,000 & & 17.94 & \\
\hline 1956 & Mar. 16 & 5,400 & & 16.93 & \\
\hline 1957 & Apr. 5 & 14,500 & & 18.30 & \\
\hline 1958 & Mar. 7 & 21,000 & & 19.11 & \\
\hline 1959 & Apr. 12 & 3,000 & & 16.30 & \\
\hline 1960 & Jan. 18 & 4,400 & & 16.68 & \\
\hline 1961 & Feb. 25 & 30,300 & & 20.10 & \\
\hline 1962 & Apr. 12 & 5,400 & & 16.95 & \\
\hline 1963 & Jan. 21 & 4,700 & & 16.78 & \\
\hline 1964 & Apr. 8 & 11,000 & & 17.93 & \\
\hline 1965 & Jan. 24 & 14,700 & & 18.33 & \\
\hline 1966 & Feb. 13 & 4,500 & & 16.73 & \\
\hline 1967 & Feb. 7 & 2,750 & & 16.07 & \\
\hline 1968 & Mar. 12 & 5,400 & & 16.97 & \\
\hline 1969 & Mar. 26 & 2,300 & 1 & -- & \\
\hline 1970 & Mar. 22 & 11,000 & & 17.97 & \\
\hline 1971 & Mar. 3 & 14,400 & & 18.28 & \\
\hline 1972 & Mar. 3 & 14,400 & & 18.28 & \\
\hline 1973 & Apr. 26 & 13,300 & & 18.13 & \\
\hline 1990 & Mar. 17 & 20,300 & 7 & 19.07 & \\
\hline
\end{tabular}


Appendix 2. Discharge and gage height data for streamgages in Alabama.-Continued

[--, date or data not available; $\mathrm{ft}$, foot; $\mathrm{ft}^{3} / \mathrm{s}$, cubic foot per second; mi, mile; $\mathrm{mi}^{2}$, square mile; qualification codes apply to the discharge data: 1 , discharge is a maximum daily average; 2 , discharge is an estimate; 4 , discharge is less than indicated value, which is the minimum recordable discharge at this site; 5 , discharge affected to unknown degree by regulation or diversion; 6 , discharge affected by regulation or diversion; 7, discharge is a historic peak; A, year of occurrence is unknown or not exact; $\mathrm{B}$, month or day of occurrence is unknown or not exact; E, only maximum peak available for this year. qualification codes apply to the gage height data: 1, gage height affected by backwater; 2, gage height not the maximum for the year; 3, gage height at different datum or at different site and datum; 5 , gage height is an estimate]

\section{Cedar Creek at Minter}

Location-Lat $32^{\circ} 04^{\prime} 45^{\prime \prime}$, long $86^{\circ} 59^{\prime} 02^{\prime \prime}$, in SE $1 / 4$ sec. 20 , T.

13 N., R. 11 E., Dallas County, Hydrologic Unit 03150203, on county road, 0.2 mi downstream from Snake Creek, 0.5 mi east of Minter, and 4 mi upstream from Dry Cedar Creek.

Drainage area-211 $\mathrm{mi}^{2}$.

Gage -Water-stage recorder. Datum of gage is $123.50 \mathrm{ft}$ NGVD 29.

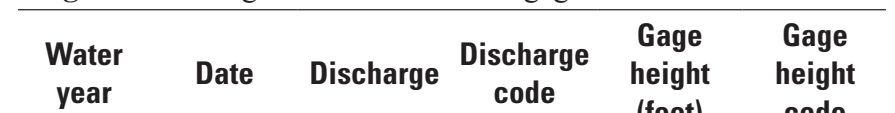

\begin{tabular}{|c|c|c|c|}
\hline yeal & & & (feet) \\
\hline 1953 & May 3 & 7,300 & 19.40 \\
\hline 1954 & Dec. 5 & 5,500 & 16.26 \\
\hline
\end{tabular}

1955

1956

1957

Apr. 10

7,200

19.15

Apr. 6

5,700

16.70

21.50

20.50

1958

Dec. 24

14,100

Mar. 7

8,500

17.10

19.49

1960

Mar. 28

6,000

24.58

1961

Feb. 25

9,630

18.34

16.48

17.71

20.10

17.11

16.59

14.75

15.64

17.85

19.15

1976

1977

1978

1979

1980

1981

1982
22.46

19.99

14.72

26.53

20.13

Mar. $2 \quad 14,500$

Apr. $26 \quad 7,770$

Jan. 21

4,900

Feb. 17 99,800

8,000

17.82

20.30

20.40

19.86

17.87

20.45

\section{Mush Creek near Selma}

L ocation-Lat $32^{\circ} 14^{\prime} 40^{\prime \prime}$, long $86^{\circ} 59^{\prime} 35^{\prime \prime}$, in SW 1/4 sec. 29 , T. 15 N., R. 11 E., Dallas County, Hydrologic Unit 03150203, at bridge on State Highway 41, 3 mi south of Sardis, and 12 mi south of Selma.

Drainage area- $44.4 \mathrm{mi}^{2}$.

G age Crest-stage gage. Datum not available.

\begin{tabular}{|c|c|c|c|c|c|}
\hline $\begin{array}{c}\text { Water } \\
\text { year }\end{array}$ & Date & Discharge & $\begin{array}{l}\text { Discharge } \\
\text { code }\end{array}$ & $\begin{array}{c}\text { Gage } \\
\text { height } \\
\text { (feet) }\end{array}$ & $\begin{array}{c}\text { Gage } \\
\text { height } \\
\text { code }\end{array}$ \\
\hline 1951 & Apr. 22 & 3,900 & & 11.50 & \\
\hline 1952 & Mar. 25 & 2,000 & & 9.50 & \\
\hline 1953 & May 4 & 12,500 & & 16.50 & \\
\hline 1954 & Mar. 14 & 1,400 & & 8.70 & \\
\hline 1955 & Apr. 11 & 22,100 & & 20.10 & \\
\hline 1956 & Mar. 16 & 5,600 & & 12.80 & \\
\hline 1957 & Apr. 17 & 4,700 & & 12.10 & \\
\hline 1958 & Mar. 7 & 11,000 & & 15.90 & \\
\hline 1959 & Mar. 12 & 2,100 & & 9.70 & \\
\hline 1960 & Mar. 30 & 6,500 & & 13.40 & \\
\hline 1961 & Mar. 31 & 13,000 & & 16.89 & \\
\hline 1962 & Dec. 13 & 19,100 & & 19.11 & \\
\hline 1963 & June 23 & 3,240 & & 10.85 & \\
\hline 1964 & Mar. 15 & 13,100 & & 16.95 & \\
\hline 1965 & Jan. 23 & 6,240 & & 13.23 & \\
\hline 1966 & May 20 & 8,260 & & 14.45 & \\
\hline 1967 & Feb. 7 & 3,540 & & 11.13 & \\
\hline 1968 & Apr. 6 & 5,540 & & 12.74 & \\
\hline 1969 & Mar. 24 & 4,620 & & 12.05 & \\
\hline 1970 & Mar. 21 & 2,220 & & 9.76 & \\
\hline 1971 & Mar. 2 & 5,580 & & 12.77 & \\
\hline 1990 & Mar. 16 & 15,000 & 7 & 17.76 & \\
\hline
\end{tabular}


Appendix 2. Discharge and gage height data for streamgages in Alabama.-Continued

[--, date or data not available; ft, foot; $\mathrm{ft}^{3} / \mathrm{s}$, cubic foot per second; mi, mile; $\mathrm{mi}^{2}$, square mile; qualification codes apply to the discharge data: 1 , discharge is a maximum daily average; 2 , discharge is an estimate; 4 , discharge is less than indicated value, which is the minimum recordable discharge at this site; 5 , discharge affected to unknown degree by regulation or diversion; 6 , discharge affected by regulation or diversion; 7, discharge is a historic peak; A, year of occurrence is unknown or not exact; B, month or day of occurrence is unknown or not exact; E, only maximum peak available for this year. qualification codes apply to the gage height data: 1, gage height affected by backwater; 2, gage height not the maximum for the year; 3, gage height at different data or at different site and datum;

5 , gage height is an estimate]

\section{Boguechitto Creek near Browns}

L ocation-Lat $32^{\circ} 26^{\prime} 21^{\prime \prime}$, long $87^{\circ} 20^{\prime} 06^{\prime \prime}$, in NW 1/4 sec. 24 , T. 17

N., R. 7 E., Dallas County, Hydrologic Unit 03150203, on U.S.

Highway 80, 0.3 mi upstream from Southern Railway bridge,

2 mi east of Browns, and $2.5 \mathrm{mi}$ downstream from Washington Creek.

Drainage area $-95.4 \mathrm{mi}^{2}$.

G age - Water-stage gage. Datum of gage is $129.39 \mathrm{ft}$ NGVD 29

\begin{tabular}{|c|c|c|c|c|c|}
\hline $\begin{array}{c}\text { Water } \\
\text { year }\end{array}$ & Date & Discharge & $\begin{array}{c}\text { Discharge } \\
\text { code }\end{array}$ & $\begin{array}{c}\text { Gage } \\
\text { height } \\
\text { (feet) }\end{array}$ & $\begin{array}{c}\text { Gage } \\
\text { height } \\
\text { code }\end{array}$ \\
\hline
\end{tabular}

\begin{tabular}{rrrccc} 
year & & & code & (feet) & code \\
\hline 1943 & Dec. 28 & 21,000 & 2 & 20.70 & \\
1944 & Mar. 23 & 7,000 & & 16.40
\end{tabular}

1945 Mar. $26 \quad 9,440$

$1946 \quad$ Mar. $28 \quad 2,800$

1947 Jan. $20 \quad 7,800$

1948 Mar. $23 \quad 3,500 \quad 14.80$

1949 Feb. $16 \quad 3,300 \quad 14.60$

$1950 \quad$ May $21 \quad 1,175$

1951 Mar. $29 \quad 14,200 \quad 19.00$

1952 Mar. $4 \quad 5,670 \quad 15.80$

$1953 \quad$ Feb. $21 \quad 2,850 \quad 14.30$

$1954 \quad$ Apr. $16 \quad 2,700$

1955 Apr. $13 \quad 4,930$

1956 Mar. $14 \quad 5,170$

1957 Apr. $4 \quad 820$

$1958 \quad$ Mar. $7 \quad 3,310$

$1960 \quad$ Oct. $15 \quad 2,050$

1961 Feb. $22 \quad 5,540$

1962 Dec. 18 4,200

1963 Mar. $6 \quad$ 3,490

1964 Apr. $8 \quad 10,200$

1965 Jan. $23 \quad 2,700$

1966 Feb. $13 \quad 3,440$

$1967 \quad$ Feb. $7 \quad 2,400$

1968 Apr. $5 \quad 4,500$

1969 Apr. $18 \quad 5,200$

$1970 \quad$ Mar. $20 \quad 2,640$

1971 Mar. $2 \quad 5,880$

$1972 \quad$ Jan. $5 \quad 5,980$

1973

1979
02427250 Pine Barren Creek near Snow Hill

L ocation-Lat $31^{\circ} 59^{\prime} 46^{\prime \prime}$, long $87^{\circ} 04^{\prime} 06^{\prime \prime}$, in SE $1 / 4 \mathrm{sec} .21$, T. 12 N., R. 10 E., Wilcox County, Hydrologic Unit 03150203, at bridge on State Highway 21, 4 mi west of Snow Hill.

Drainage area-261 $\mathrm{mi}^{2}$.

Gage -Water-stage recorder. Datum of gage is $126.60 \mathrm{ft}$ NGVD 29 (levels by Highway Department).

\begin{tabular}{lrrrrr}
\hline $\begin{array}{c}\text { Water } \\
\text { year }\end{array}$ & Date & Discharge & $\begin{array}{c}\text { Discharge } \\
\text { code }\end{array}$ & $\begin{array}{c}\text { Gage } \\
\text { height } \\
\text { (feet) }\end{array}$ & $\begin{array}{c}\text { Gage } \\
\text { height } \\
\text { code }\end{array}$ \\
\hline 1990 & Mar. 17 & 23,900 & & 24.30 & \\
1991 & May 11 & 5,160 & & 17.93 & \\
1992 & Feb. 18 & 4,870 & & 17.44 & \\
1993 & Dec. 18 & 10,100 & 22.19 &
\end{tabular}

1995 Mar. $8 \quad 4,950 \quad 17.58$

$1996 \quad$ Jan. $28 \quad 8,570 \quad 21.48$

1997 Dec. $2 \quad 5,870 \quad 19.07$

1998 Sept. $30 \quad 28,600 \quad 24.73$

1999 Mar. $15 \quad 7,190 \quad 20.48$

$2000 \quad$ Mar. $20 \quad 2,740 \quad 13.65$

$2001 \quad$ Mar. $4 \quad 30,400 \quad 25.70$

$2002 \quad$ Mar. $22 \quad 3,610 \quad 16.07$

2003 Dec. $25 \quad 10,800 \quad 21.28$

2004 Sept. $17 \quad 11,000 \quad 21.37$

2005 Apr. $2 \quad 10,200 \quad 21.07$

2006 May $11 \quad 4,650 \quad 18.00$

2007 Nov. $17 \quad 6,550 \quad 19.43$

$2008 \quad$ Aug. $26 \quad 3,460 \quad 15.68$

2009 Mar. $29 \quad 9,200 \quad 20.68$

$2010 \quad$ Dec. $14 \quad 9,590 \quad 20.84$

2011 Mar. $11 \quad 4,290 \quad 17.45$

2012 Sept. $5 \quad 8,300 \quad 21.68$

2013 Feb. $11 \quad 10,400 \quad 22.27$

2014 Apr. $8 \quad 17,900 \quad 24.10$

2015 Apr. $17 \quad 3,700 \quad 16.32$ 
Appendix 2. Discharge and gage height data for streamgages in Alabama.-Continued

[--, date or data not available; $\mathrm{ft}$, foot; $\mathrm{ft}^{3} / \mathrm{s}$, cubic foot per second; mi, mile; $\mathrm{mi}^{2}$, square mile; qualification codes apply to the discharge data: 1 , discharge is a maximum daily average; 2 , discharge is an estimate; 4 , discharge is less than indicated value, which is the minimum recordable discharge at this site; 5 , discharge affected to unknown degree by regulation or diversion; 6 , discharge affected by regulation or diversion; 7, discharge is a historic peak; A, year of occurrence is unknown or not exact; $\mathrm{B}$, month or day of occurrence is unknown or not exact; E, only maximum peak available for this year. qualification codes apply to the gage height data: 1, gage height affected by backwater; 2, gage height not the maximum for the year; 3, gage height at different datum or at different site and datum; 5 , gage height is an estimate]

\section{Turkey Creek at Kimbrough}

L ocation-Lat $32^{\circ} 01^{\prime} 15^{\prime \prime}$, long $87^{\circ} 33^{\prime} 30^{\prime \prime}$, in SE $1 / 4$ sec. 10 , T. 12 N., R. 5 E., Wilcox County, Hydrologic Unit 03150203, on county road, $0.6 \mathrm{mi}$ downstream from State Highway 5, $1 \mathrm{mi}$ south of Kimbrough, 2 mi upstream from mouth, and $6 \mathrm{mi}$ upstream from Alabama River.

Drainage area $-97.5 \mathrm{mi}^{2}$.

Gage Water-stage recorder. Datum of gage is $58.78 \mathrm{ft}$ NGVD 29.

\begin{tabular}{ccccc}
\hline $\begin{array}{c}\text { Water } \\
\text { year }\end{array}$ Date $\quad$ Discharge & $\begin{array}{c}\text { Discharge } \\
\text { code }\end{array}$ & $\begin{array}{c}\text { Gage } \\
\text { height } \\
\text { (feet) }\end{array}$ & $\begin{array}{c}\text { Gage } \\
\text { height } \\
\text { code }\end{array}$ \\
\hline
\end{tabular}

\begin{tabular}{lllrr}
\hline 1958 & Mar. 8 & 6,000 & 2 & 18.03 \\
1959 & Apr. 1 & 1,900 & & 6.51
\end{tabular}

1960

1961

1962

1963

June 2

7,310

19.90

21.39

Mar. $31 \quad$ 10,300

Dec. 10

39,600

Mar. 6

2,090

1964

Apr. 8

6,210

1965

Jan. 24

8,370

1966

Feb. 13

2,730

Feb. 7

2,250

1968

Oct. 31

8,220

1969

Mar. 24

2,460

1970

Aug. 10

3,840

1971

Mar. 3

6,290

1972

Dec. 7

4,940

1973

Mar. 7

8,800

1974

Sept. 9

6,150

1975

Feb. 17

6,870

1976

Mar. 31

9,340

1977

Mar. 13

5,620
25.02

7.46

18.33

20.40

11.35

8.31

20.27

9.75

14.30

18.44

16.33

20.64

18.24

19.24

20.92

17.47
02427700 Turkey Creek at Kimbrough —Continued

L ocation-Lat $32^{\circ} 01^{\prime} 15^{\prime \prime}$, long $87^{\circ} 33^{\prime} 30^{\prime \prime}$, in SE $1 / 4$ sec. 10 , T. 12 N., R. 5 E., Wilcox County, Hydrologic Unit 03150203, on county road, $0.6 \mathrm{mi}$ downstream from State Highway 5, $1 \mathrm{mi}$ south of Kimbrough, 2 mi upstream from mouth, and $6 \mathrm{mi}$ upstream from Alabama River.

Drainage area—97.5 $\mathrm{mi}^{2}$.

G age - Water-stage recorder. Datum of gage is $58.78 \mathrm{ft}$ NGVD 29.

\begin{tabular}{|c|c|c|c|c|c|}
\hline $\begin{array}{c}\text { Water } \\
\text { year }\end{array}$ & Date & Discharge & $\begin{array}{l}\text { Discharge } \\
\text { code }\end{array}$ & $\begin{array}{c}\text { Gage } \\
\text { height } \\
\text { (feet) }\end{array}$ & $\begin{array}{c}\text { Gage } \\
\text { height } \\
\text { code }\end{array}$ \\
\hline 1978 & May 9 & 2,690 & & 11.18 & \\
\hline 1979 & Mar. 4 & 20,000 & & 23.37 & \\
\hline 1980 & Mar. 28 & 5,500 & & 19.12 & \\
\hline 1981 & Feb. 11 & 3,210 & & 13.03 & \\
\hline 1982 & Feb. 3 & 3,320 & & 13.30 & \\
\hline 1983 & Feb. 2 & 8,520 & & 20.49 & \\
\hline 1984 & Dec. 28 & 2,850 & & 11.82 & \\
\hline 1985 & Feb. 26 & 2,070 & & 7.64 & \\
\hline 1986 & June 10 & 1,550 & & 5.41 & \\
\hline 1987 & Mar. 1 & 2,990 & & 12.35 & \\
\hline 1988 & Sept. 4 & 1,780 & & 6.25 & \\
\hline 1989 & Apr. 5 & 2,750 & & 11.37 & \\
\hline 1990 & Mar. 16 & 14,600 & & 22.61 & \\
\hline 1991 & Mar. 30 & 3,090 & & 12.66 & \\
\hline 1992 & Feb. 17 & 2,680 & & 11.06 & \\
\hline 1993 & Jan. 21 & 6,040 & & 18.06 & \\
\hline 1994 & Feb. 6 & 4,990 & & 16.41 & \\
\hline 1995 & Mar. 8 & 2,580 & & 10.45 & \\
\hline 1996 & Dec. 19 & 5,150 & & 16.66 & \\
\hline
\end{tabular}


Appendix 2. Discharge and gage height data for streamgages in Alabama.-Continued

[--, date or data not available; ft, foot; $\mathrm{ft}^{3} / \mathrm{s}$, cubic foot per second; mi, mile; $\mathrm{mi}^{2}$, square mile; qualification codes apply to the discharge data: 1 , discharge is a maximum daily average; 2 , discharge is an estimate; 4 , discharge is less than indicated value, which is the minimum recordable discharge at this site; 5 , discharge affected to unknown degree by regulation or diversion; 6 , discharge affected by regulation or diversion; 7, discharge is a historic peak; A, year of occurrence is unknown or not exact; B, month or day of occurrence is unknown or not exact; E, only maximum peak available for this year. qualification codes apply to the gage height data: 1, gage height affected by backwater; 2, gage height not the maximum for the year; 3, gage height at different data or at different site and datum;

5 , gage height is an estimate]

\section{Noxubee River near Geiger}

Location-Lat $32^{\circ} 55^{\prime} 57^{\prime \prime}$, long $88^{\circ} 17^{\prime} 52^{\prime \prime}$, in NE 1/4 sec. 33, T. 23

N., R. 3 W., Sumter County, Hydrologic Unit 03160108, on State Highway 17, $0.1 \mathrm{mi}$ upstream from Woodward Creek, 5 mi north of Geiger, and at mile 16.9.

Drainage area-1,097 $\mathrm{mi}^{2}$.

Gage-Water-stage recorder. Datum of gage is $86.08 \mathrm{ft}$ NGVD 29.

\begin{tabular}{|c|c|c|c|c|c|}
\hline $\begin{array}{c}\text { Water } \\
\text { year }\end{array}$ & Date & Discharge & $\begin{array}{c}\text { Discharge } \\
\text { code }\end{array}$ & $\begin{array}{c}\text { Gage } \\
\text { height } \\
\text { (feet) }\end{array}$ & $\begin{array}{c}\text { Gage } \\
\text { height } \\
\text { code }\end{array}$ \\
\hline
\end{tabular}

\begin{tabular}{lllcc}
\hline 1940 & July 10 & 33,000 & 41.30 & 3 \\
1945 & Feb. 24 & 19,700 & 38.70 & 3
\end{tabular}

$1946 \quad$ Feb. $14 \quad 20,300$

1947 Jan. $21 \quad 12,000$

$1948 \quad$ Feb. $16 \quad 14,000$

$1949 \quad$ Jan. $8 \quad 35,000$

$1950 \quad$ Jan. $11 \quad 22,400$

$1951 \quad$ Mar. $31 \quad 37,600$

1952 Mar. $12 \quad 6,600$

1953 May $6 \quad 11,900$

1954 Apr. $17 \quad 8,550$

1955 Apr. $14 \quad 8,270$

1956 Apr. $7 \quad 12,700$

1957 Apr. $5 \quad 7,690$

1958 May $7 \quad$ 13,500

$1959 \quad$ Feb. $10 \quad 6,240$

1960 Mar. 4 10,200

1961 Feb. $24 \quad 26,000$

1962 Dec. 18 44,000

1963 Mar. $13 \quad 6,500$

1964 Mar. $19 \quad$ 13,000

1965 Feb. $15 \quad 17,500$

1966 Apr. $28 \quad$ 11,600

1967 Aug. $26 \quad 6,330$

1968 Dec. $24 \quad 18,400$

1969 Apr. $15 \quad$ 23,600

1970 Mar. $24 \quad 9,900$

1971 Mar. $4 \quad 11,500$

1972 Jan. $12 \quad 20,000$

1973 Mar. $21 \quad 20,500$

1974 Apr. $16 \quad$ 18,600

1975 Mar. $18 \quad$ 23,100

1976 Mar. $18 \quad$ 18,200

1977 Apr. $7 \quad 28,000$

1978 May $9 \quad 9,260$

1979 Apr. $14 \quad$ 156,000
02448500 Noxubee River near Geiger-Continued

L ocation-Lat $32^{\circ} 55^{\prime} 57^{\prime \prime}$, long $88^{\circ} 17^{\prime} 52^{\prime \prime}$, in NE $1 / 4$ sec. 33 , T. 23 N., R. 3 W., Sumter County, Hydrologic Unit 03160108, on State Highway 17, 0.1 mi upstream from Woodward Creek, 5 mi north of Geiger, and at mile 16.9.

Drainage area-1,097 $\mathrm{mi}^{2}$.

Gage-Water-stage recorder. Datum of gage is $86.08 \mathrm{ft}$ NGVD 29.

\begin{tabular}{llrccc}
\hline $\begin{array}{c}\text { Water } \\
\text { year }\end{array}$ & Date & Discharge & $\begin{array}{c}\text { Discharge } \\
\text { code }\end{array}$ & $\begin{array}{c}\text { Gage } \\
\text { height } \\
\text { (feet) }\end{array}$ & $\begin{array}{c}\text { Gage } \\
\text { height } \\
\text { code }\end{array}$ \\
\hline 1980 & Apr. 17 & 23,400 & & 39.09 & 3 \\
1981 & Apr. 1 & 9,130 & & 30.76 & 3 \\
1982 & Apr. 26 & 11,100 & 33.02 & 3 \\
1983 & May 22 & 34,400 & 41.38 & 3 \\
1984 & Dec. 12 & 12,600 & 34.30 & 3 \\
1985 & Feb. 6 & 9,880 & 32.23 & 6 \\
1986 & Mar. 13 & 6,770 & & 26.94 & \\
1987 & Mar. 1 & 13,500 & & 35.61 & \\
1988 & Apr. 3 & 6,070 & & 25.24 & \\
1989 & Jan. 19 & 11,600 & 34.46 & \\
1990 & Feb. 18 & 23,300 & & 39.08 & \\
1991 & Feb. 24 & 28,500 & & 40.61 & \\
1992 & Aug. 28 & 7,940 & & 29.24 & \\
1993 & Jan. 15 & 16,000 & & 37.20 & \\
1994 & Jan. 29 & 10,900 & 33.64 & \\
1995 & Apr. 24 & 10,700 & 2 & 33.18 & 5
\end{tabular}

$1996 \quad$ Jan. $27 \quad 13,100 \quad 36.0$

1997 Apr. $28 \quad 9,300 \quad 31.23$

$1998 \quad$ Jan. $8 \quad 19,800 \quad 38.89$

1999 Feb. $3 \quad 19,200 \quad 38.69$

2000 Apr. $4 \quad 13,200 \quad 35.75$

2001 Mar. $16 \quad 9,310 \quad 31.24$

2002 Dec. $15 \quad 10,400 \quad 32.82$

2003 Feb. $25 \quad 21,700 \quad 39.49$

$2004 \quad$ Feb. $9 \quad 17,200 \quad 37.98$

2005 Dec. $10 \quad 11,300 \quad 33.89$

2006 Mar. $2 \quad 9,870 \quad 32.16$

2007 Jan. $1 \quad 7,840 \quad 27.83$

2008 Feb. $23 \quad 7,540 \quad 27.00$

$2009 \quad$ Jan. $11 \quad 14,800 \quad 36.11$

2010 Mar. $12 \quad 14,200 \quad 35.29$

2011 Mar. $10 \quad$ 13,400 34.11

2012 Mar. $23 \quad 11,400 \quad 30.79$

$2013 \quad$ Jan. $20 \quad 13,800 \quad 34.76$

2014 Apr. $9 \quad 15,500 \quad 37.10$

2015 Jan. $5 \quad 10,800 \quad 33.37$ 
Appendix 2. Discharge and gage height data for streamgages in Alabama.-Continued

[--, date or data not available; $\mathrm{ft}$, foot; $\mathrm{ft}^{3} / \mathrm{s}$, cubic foot per second; mi, mile; $\mathrm{mi}^{2}$, square mile; qualification codes apply to the discharge data: 1 , discharge is a maximum daily average; 2 , discharge is an estimate; 4 , discharge is less than indicated value, which is the minimum recordable discharge at this site; 5 , discharge affected to unknown degree by regulation or diversion; 6 , discharge affected by regulation or diversion; 7, discharge is a historic peak; A, year of occurrence is unknown or not exact; $\mathrm{B}$, month or day of occurrence is unknown or not exact; E, only maximum peak available for this year. qualification codes apply to the gage height data: 1, gage height affected by backwater; 2, gage height not the maximum for the year; 3, gage height at different datum or at different site and datum; 5 , gage height is an estimate]

\section{Bodka Creek near Geiger}

Location-Lat $32^{\circ} 48^{\prime} 25^{\prime \prime}$, long $88^{\circ} 18^{\prime} 43^{\prime \prime}$, in SE $1 / 4$ sec. 8 , T.

21 N., R. 3 E., Sumter County, Hydrologic Unit 03160108, at right bank on downstream side of State Highway 17, $1.6 \mathrm{mi}$ downstream from Tifallili Creek, 4.2 mi north of Geiger, and 9.2 mi upstream from mouth.

Drainage area $-158 \mathrm{mi}^{2}$.

Gage-Water-stage recorder. Datum of gage is $104.78 \mathrm{ft}$ NGVD 29.

\begin{tabular}{|c|c|c|c|c|c|}
\hline $\begin{array}{c}\text { Water } \\
\text { year }\end{array}$ & Date & Discharge & $\begin{array}{c}\text { Discharge } \\
\text { code }\end{array}$ & $\begin{array}{c}\text { Gage } \\
\text { height } \\
\text { (feet) }\end{array}$ & $\begin{array}{c}\text { Gage } \\
\text { height } \\
\text { code }\end{array}$ \\
\hline 1991 & Feb. 20 & 10,500 & & 21.89 & \\
\hline 1992 & Aug. 28 & 2,520 & & 16.44 & \\
\hline 1993 & Jan. 12 & 9,230 & & 21.44 & \\
\hline 1994 & Jan. 28 & 8,620 & & 21.23 & \\
\hline 1995 & Mar. 8 & 4,920 & & 18.88 & \\
\hline 1996 & Mar. 7 & 8,740 & & 21.28 & \\
\hline 1997 & Apr. 28 & 8,380 & & 21.13 & \\
\hline 1998 & Jan. 8 & 21,900 & & 24.20 & \\
\hline 1999 & Jan. 31 & 14,800 & & 22.87 & \\
\hline 2000 & Apr. 4 & 6,750 & & 20.36 & \\
\hline 2001 & Apr. 5 & 4,720 & & 18.67 & \\
\hline 2002 & Dec. 14 & 6,170 & & 20.01 & \\
\hline 2003 & Apr. 25 & 14,000 & & 22.71 & \\
\hline 2004 & Feb. 6 & 17,200 & & 23.41 & \\
\hline 2005 & June 12 & 7,090 & & 20.35 & \\
\hline 2006 & Mar. 21 & 7,440 & & 20.52 & \\
\hline 2007 & Jan. 1 & 3,290 & & 16.25 & \\
\hline 2008 & Feb. 22 & 7,830 & & 20.72 & \\
\hline 2009 & Mar. 28 & 5,710 & & 19.57 & \\
\hline 2010 & Mar. 11 & 13,600 & & 22.63 & \\
\hline 2011 & Mar. 10 & 9,520 & & 21.32 & \\
\hline 2012 & Mar. 23 & 11,200 & & 22.05 & \\
\hline 2013 & Feb. 13 & 6,830 & & 20.22 & \\
\hline 2014 & Apr. 7 & 15,400 & & 23.03 & \\
\hline 2015 & Jan. 4 & 7,270 & & 20.79 & \\
\hline
\end{tabular}

\section{Jones Creek near Epes}

L ocation-Lat $32^{\circ} 41^{\prime} 27^{\prime \prime}$, long $88^{\circ} 10^{\prime} 02^{\prime \prime}$, in SW 1/4 sec. 23 , T. 20 N., R. 2 W., Sumter County, Hydrologic Unit 03160106, on State Highway 39, $2.5 \mathrm{mi}$ west of Epes, and 6 mi upstream from mouth.

Drainage area-11.8 $\mathrm{mi}^{2}$.

G age -Water-stage recorder. Datum of gage is $125 \mathrm{ft} \mathrm{NGVD} 29$.

\begin{tabular}{|c|c|c|c|c|c|}
\hline $\begin{array}{c}\text { Water } \\
\text { year }\end{array}$ & Date & Discharge & $\begin{array}{l}\text { Discharge } \\
\text { code }\end{array}$ & $\begin{array}{c}\text { Gage } \\
\text { height } \\
\text { (feet) }\end{array}$ & $\begin{array}{c}\text { Gage } \\
\text { height } \\
\text { code }\end{array}$ \\
\hline 1959 & June 11 & 1,530 & & 13.11 & \\
\hline 1960 & Apr. 3 & 1,480 & & 12.75 & \\
\hline 1961 & Feb. 21 & 5,160 & & 21.46 & \\
\hline 1962 & Apr. 12 & 2,980 & & 18.55 & \\
\hline 1963 & Mar. 5 & 2,260 & & 16.43 & \\
\hline 1964 & Mar. 2 & 2,490 & & 17.22 & \\
\hline 1965 & Dec. 4 & 2,760 & & 18.20 & \\
\hline 1966 & Feb. 12 & 2,220 & & 16.27 & \\
\hline 1967 & July 6 & 574 & & 6.40 & \\
\hline 1968 & May 4 & 1,500 & & 12.91 & \\
\hline 1969 & Dec. 22 & 2,130 & & 15.93 & \\
\hline 1970 & Aug. 9 & 2,040 & & 15.57 & \\
\hline 1971 & Oct. 18 & 2,580 & & 17.52 & \\
\hline 1972 & July 31 & 2,010 & & 15.45 & \\
\hline 1973 & Jan. 21 & 3,100 & & 18.81 & \\
\hline 1974 & Dec. 26 & 2,470 & & 17.17 & \\
\hline
\end{tabular}


Appendix 2. Discharge and gage height data for streamgages in Alabama.-Continued

[--, date or data not available; ft, foot; $\mathrm{ft}^{3} / \mathrm{s}$, cubic foot per second; mi, mile; $\mathrm{mi}^{2}$, square mile; qualification codes apply to the discharge data: 1 , discharge is a maximum daily average; 2 , discharge is an estimate; 4 , discharge is less than indicated value, which is the minimum recordable discharge at this site; 5 , discharge affected to unknown degree by regulation or diversion; 6 , discharge affected by regulation or diversion; 7, discharge is a historic peak; A, year of occurrence is unknown or not exact; B, month or day of occurrence is unknown or not exact; E, only maximum peak available for this year. qualification codes apply to the gage height data: 1, gage height affected by backwater; 2, gage height not the maximum for the year; 3, gage height at different data or at different site and datum;

5 , gage height is an estimate]

\section{Sucarnoochee River at Livingston}

L ocation-Lat $32^{\circ} 34^{\prime} 25^{\prime \prime}$, long $88^{\circ} 11^{\prime} 36^{\prime \prime}$, in SW 1/4 sec. 33, T. 19 N., R. 2 W., Sumter County, Hydrologic Unit 03160202, $10 \mathrm{ft}$ downstream from bridge on U.S. Highway 11, 0.8 mi southwest of Livingston, and 9 mi upstream from Alamuchee Creek.

Drainage area $-607 \mathrm{mi}^{2}$.

Gage-Water-stage recorder. Datum of gage is $90.04 \mathrm{ft}$ NGVD 29.

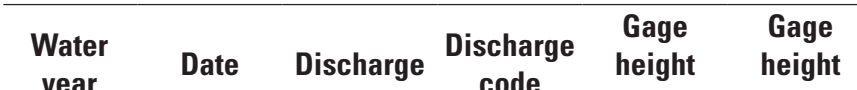

\begin{tabular}{|c|c|c|c|c|c|}
\hline year & Date & Discharge & code & $\begin{array}{l}\text { height } \\
\text { (feet) }\end{array}$ & $\begin{array}{c}\text { height } \\
\text { code }\end{array}$ \\
\hline 1939 & Mar. 30 & 6,800 & & 22.84 & \\
\hline 1940 & July 11 & 11,500 & & 24.60 & \\
\hline
\end{tabular}

1941

1942

1943

1944

1945

1946

1947

1949

1950

1951

1952

1953

1954

1955

1956

1957

1958

1959

1960

1961

July 11

11,500

Mar. 23

3,620

Mar. 23

10,300

10,500

Mar. 31

7,280

Feb. 16

5,510

Feb. 12

9,190

Jan. 20

12,200

Nov. 30

18,500

Jan. 9

16,600

Mar. 30

21,500

Dec. 21

3,100

7,090

Mar. 30

Apr. 14

3,600

Mar. 18

4,240

14,700

Apr. 8

4,040

May 3

6,630

Feb. 14

2,920

Mar. 4

4,170

Feb. 22

31,500

Dec. 19

19,600

1963

Mar. 5

3,520

Apr. $8 \quad$ 11,300

Feb. $15 \quad 5,920$

Feb. $16 \quad$ 10,900

Feb. $8 \quad 1,980$

Dec. 18

Apr. 18

Mar. 22

Feb. 25

Jan. 13

Apr. 27

Apr. 15

Jan. 13

Mar. 31

4,210

7,650

3,530

6,570

7,890

6,350

28,400

11,200

1978 May $10 \quad 4,290 \quad 19.11$

11,700

1978 May $10 \quad 4,290 \quad 19.11$
19.97

24.35

24.22

23.02

22.14

23.88

24.70

26.90

26.20

27.60

17.00

22.93

20.10

25.60

19.60

22.60

16.03

19.48

29.35

26.82

17.83

24.44

22.09

24.29

12.62

19.58

23.13

17.84

22.56

23.24

22.02

28.81

24.39

24.56
1978 May $10 \quad 4,290 \quad 19.11$
02467500 Sucarnoochee River at Livingston-Continued

L ocation-Lat $32^{\circ} 34^{\prime} 25^{\prime \prime}$, long $88^{\circ} 11^{\prime} 36^{\prime \prime}$, in SW 1/4 sec. 33, T. 19 N., R. 2 W., Sumter County, Hydrologic Unit 03160202, $10 \mathrm{ft}$ downstream from bridge on U.S. Highway 11, 0.8 mi southwest of Livingston, and $9 \mathrm{mi}$ upstream from Alamuchee Creek.

Drainage area $-607 \mathrm{mi}^{2}$.

Gage-Water-stage recorder. Datum of gage is $90.04 \mathrm{ft}$ NGVD 29.

\begin{tabular}{llrlll}
\hline $\begin{array}{c}\text { Water } \\
\text { year }\end{array}$ & Date & Discharge & $\begin{array}{c}\text { Discharge } \\
\text { code }\end{array}$ & $\begin{array}{c}\text { Gage } \\
\text { height } \\
\text { (feet) }\end{array}$ & $\begin{array}{c}\text { Gage } \\
\text { height } \\
\text { code }\end{array}$ \\
\hline 1979 & Apr. 14 & 62,200 & & 33.47 & \\
1980 & Apr. 14 & 20,800 & & 26.99 & \\
1981 & Apr. 2 & 10,600 & & 23.72 & \\
1982 & Feb. 4 & 4,110 & 1 & 17.28 & \\
1983 & May 22 & 14,900 & & 25.17 & \\
1984 & Dec. 28 & 4,240 & & 17.59 &
\end{tabular}

$1985 \quad$ Feb. $27 \quad 4,540 \quad$ E $\quad 18.35$

1986 Oct. $31 \quad 2,810 \quad 14.02$

1987 Jan. $21 \quad 11,200 \quad 23.70$

1988 Apr. $23 \quad 2,810 \quad 14.16$

1989 Mar. $8 \quad 4,660 \quad 18.64$

$1990 \quad$ Feb. $17 \quad 23,000 \quad 27.53$

1991 May $1 \quad 12,200 \quad 24.30$

1992 Apr. $24 \quad 3,630 \quad 15.92$

1993 Jan. $15 \quad 9,230 \quad 23.06$

$1994 \quad$ Jan. $31 \quad 9,980 \quad 23.40$

1995 Mar. $9 \quad 7,110 \quad 21.62$

1996 Jan. $30 \quad 7,660 \quad 22.19$

1997 Mar. $7 \quad 12,600 \quad 24.63$

$1998 \quad$ Jan. $9 \quad 19,100 \quad 26.64$

1999 Feb. $2 \quad 14,200 \quad 25.18$

$2000 \quad$ Apr. $7 \quad 4,250 \quad 16.55$

2001 Mar. $4 \quad 6,350 \quad 20.45$

2002 Dec. $18 \quad 6,690 \quad 20.91$

$2003 \quad$ Apr. $9 \quad 28,000 \quad 28.79$

2004 Feb. $8 \quad 15,800 \quad 25.90$

2005 June $15 \quad 7,070 \quad 21.39$

2006 Mar. $25 \quad 4,960 \quad 18.13$

2007 Jan. $1 \quad 2,520 \quad 11.92$

2008 Feb. $22 \quad 5,330 \quad 18.83$

2009 Mar. $29 \quad 8,070 \quad 21.89$

2010 Mar. $14 \quad 8,830 \quad 22.65$

2011 Mar. $10 \quad 6,710 \quad 20.15$

$2012 \quad$ Jan. $26 \quad 5,230 \quad 17.24$

2013 Feb. $13 \quad 7,830 \quad 21.63$

2014 Apr. $10 \quad 14,700 \quad 25.55$

2015 Jan. $7 \quad 8,060 \quad 21.88$ 
Appendix 2. Discharge and gage height data for streamgages in Alabama.-Continued

[--, date or data not available; $\mathrm{ft}$, foot; $\mathrm{ft}^{3} / \mathrm{s}$, cubic foot per second; mi, mile; $\mathrm{mi}^{2}$, square mile; qualification codes apply to the discharge data: 1 , discharge is a maximum daily average; 2 , discharge is an estimate; 4 , discharge is less than indicated value, which is the minimum recordable discharge at this site; 5 , discharge affected to unknown degree by regulation or diversion; 6 , discharge affected by regulation or diversion; 7, discharge is a historic peak; A, year of occurrence is unknown or not exact; $\mathrm{B}$, month or day of occurrence is unknown or not exact; E, only maximum peak available for this year. qualification codes apply to the gage height data: 1, gage height affected by backwater; 2, gage height not the maximum for the year; 3, gage height at different datum or at different site and datum; 5 , gage height is an estimate]

\section{Alamuchee Creek near Cuba}

Location-Lat $32^{\circ} 26^{\prime} 20^{\prime \prime}$, long $88^{\circ} 20^{\prime} 17^{\prime \prime}$, in NE $1 / 4$ sec. 24 , T. 17 N., R. 4 W., Sumter County, Hydrologic Unit 03160202, on U.S. Highway 80, 2.5 mi northeast of Cuba, and 4 mi upstream from Toomsuba Creek.

Drainage area $-62.3 \mathrm{mi}^{2}$.

Gage -Water-stage recorder 1955-67. Crest-stage gage 1968-70. Datum of gage is $161.50 \mathrm{ft}$ NGVD 29.

\begin{tabular}{|c|c|c|c|c|c|}
\hline $\begin{array}{l}\text { Water } \\
\text { year }\end{array}$ & Date & Discharge & $\begin{array}{l}\text { Discharge } \\
\text { code }\end{array}$ & $\begin{array}{c}\text { Gage } \\
\text { height } \\
\text { (feet) }\end{array}$ & $\begin{array}{c}\text { Gage } \\
\text { height } \\
\text { code }\end{array}$ \\
\hline 1955 & June 26 & 783 & & 10.06 & \\
\hline 1956 & Mar. 16 & 4,220 & & 15.98 & \\
\hline 1957 & Apr. 5 & 734 & & 9.73 & \\
\hline 1958 & Mar. 8 & 1,910 & & 14.60 & \\
\hline 1959 & June 9 & 460 & & 7.17 & \\
\hline 1960 & Feb. 5 & 861 & & 10.66 & \\
\hline 1961 & Feb. 22 & 12,000 & & 18.03 & \\
\hline 1962 & Dec. 18 & 4,220 & & 16.00 & \\
\hline 1963 & Mar. 7 & 838 & & 10.52 & \\
\hline 1964 & Apr. 6 & 12,700 & & 18.35 & \\
\hline 1965 & Dec. 12 & 1,470 & & 13.90 & \\
\hline 1966 & Feb. 13 & 3,380 & & 15.61 & \\
\hline 1967 & May 5 & 530 & & 7.03 & \\
\hline 1968 & Apr. 6 & 1,170 & & 11.29 & \\
\hline 1969 & Apr. 15 & 1,490 & & 13.98 & \\
\hline 1970 & Mar. 21 & 741 & & 9.51 & \\
\hline 1979 & Apr. 14 & 14,700 & 7 & 18.87 & \\
\hline
\end{tabular}

\section{Chickasaw Bogue near Linden}

L ocation-Lat $32^{\circ} 19^{\prime} 45^{\prime \prime}$, long $87^{\circ} 47^{\prime} 27^{\prime \prime}$, in SW 1/4 sec. 28 , T. 16 N., R. 3 E., Marengo County, Hydrologic Unit 03160201, on U.S. Highway 43, 1.5 mi north of Linden, 2 mi downstream from Atkin Creek, and 11 mi upstream from mouth.

Drainage area-257 $\mathrm{mi}^{2}$.

G age -Water-stage recorder. Datum of gage is $63.45 \mathrm{ft}$ NGVD 29. January 1944 to September 1946, nonrecording gage at site $75 \mathrm{ft}$ upstream at different datum.

\begin{tabular}{|c|c|c|c|c|c|}
\hline $\begin{array}{c}\text { Water } \\
\text { year }\end{array}$ & Date & Discharge & $\begin{array}{l}\text { Discharge } \\
\text { code }\end{array}$ & $\begin{array}{c}\text { Gage } \\
\text { height } \\
\text { (feet) }\end{array}$ & $\begin{array}{c}\text { Gage } \\
\text { height } \\
\text { code }\end{array}$ \\
\hline 1944 & Apr. 27 & 33,000 & & 30.02 & \\
\hline 1945 & Mar. 26 & 35,000 & & 30.33 & \\
\hline 1946 & May 21 & 7,230 & & 23.95 & \\
\hline 1966 & Feb. 13 & 11,200 & & 25.43 & \\
\hline 1967 & Feb. 7 & 6,710 & & 20.73 & \\
\hline 1968 & Apr. 5 & 11,500 & & 25.76 & \\
\hline 1969 & Apr. 18 & 11,600 & & 25.82 & \\
\hline 1970 & Mar. 4 & 8,150 & & 22.50 & \\
\hline 1971 & Mar. 2 & 14,500 & & 28.14 & \\
\hline 1972 & Dec. 7 & 12,500 & & 26.62 & \\
\hline 1973 & Mar. 31 & 10,700 & & 25.03 & \\
\hline 1974 & Sept. 8 & 16,700 & & 28.79 & \\
\hline 1975 & Dec. 24 & 12,900 & & 25.54 & \\
\hline 1976 & Mar. 31 & 25,000 & & 27.92 & \\
\hline 1977 & Mar. 13 & 20,400 & & 26.51 & \\
\hline 1978 & June 9 & 11,800 & & 22.50 & \\
\hline 1979 & Mar. 4 & 34,000 & & 30.18 & \\
\hline 1980 & Mar. 29 & 14,600 & & 24.03 & \\
\hline 1981 & Apr. 1 & 14,800 & & 24.14 & \\
\hline 1982 & Feb. 3 & 15,300 & & 24.40 & \\
\hline 1983 & Feb. 2 & 28,800 & & 28.23 & \\
\hline 1984 & Dec. 28 & 12,800 & & 22.98 & \\
\hline 1985 & Feb. 26 & 11,500 & & 22.11 & \\
\hline 1986 & Mar. 13 & 8,700 & & 20.39 & \\
\hline 1987 & Nov. 25 & 14,600 & & 24.02 & \\
\hline 1988 & Feb. 4 & 9,890 & & 21.06 & \\
\hline 1989 & July 3 & 13,900 & & 23.61 & \\
\hline 1990 & Mar. 16 & 23,900 & & 27.28 & \\
\hline 1991 & Feb. 20 & 16,300 & & 24.89 & \\
\hline
\end{tabular}


Appendix 2. Discharge and gage height data for streamgages in Alabama.-Continued

[--, date or data not available; $\mathrm{ft}$, foot; $\mathrm{ft}^{3} / \mathrm{s}$, cubic foot per second; $\mathrm{mi}$, mile; $\mathrm{mi}^{2}$, square mile; qualification codes apply to the discharge data: 1 , discharge is a maximum daily average; 2 , discharge is an estimate; 4 , discharge is less than indicated value, which is the minimum recordable discharge at this site; 5 , discharge affected to unknown degree by regulation or diversion; 6 , discharge affected by regulation or diversion; 7, discharge is a historic peak; A, year of occurrence is unknown or not exact; B, month or day of occurrence is unknown or not exact; E, only maximum peak available for this year. qualification codes apply to the gage height data: 1, gage height affected by backwater; 2 , gage height not the maximum for the year; 3 , gage height at different data or at different site and datum;

5 , gage height is an estimate]

\section{Kinterbish Creek near York}

L ocation-Lat $32^{\circ} 19^{\prime} 17^{\prime \prime}$, long $88^{\circ} 10^{\prime} 50^{\prime \prime}$, in NE $1 / 4$ sec. 33 , T. 16 N., R. 2 W., Sumter County, Hydrologic Unit 03160201, on State Highway 17, 0.8 mi north of Choctaw-Sumter Countyline, $5.5 \mathrm{mi}$ downstream from Little Kinterbish Creek, and 14 mi southeast of York.

Drainage area $-90.9 \mathrm{mi}^{2}$.

Gage - Water-stage recorder 1955-67. Crest-stage gage 1968-70. Datum of gage is $120 \mathrm{ft}$ NGVD 29 (by barometer).

\begin{tabular}{|c|c|c|c|c|c|}
\hline $\begin{array}{c}\text { Water } \\
\text { year }\end{array}$ & Date & Discharge & $\begin{array}{c}\text { Discharge } \\
\text { code }\end{array}$ & $\begin{array}{c}\text { Gage } \\
\text { height } \\
\text { (feet) }\end{array}$ & $\begin{array}{c}\text { Gage } \\
\text { height } \\
\text { code }\end{array}$ \\
\hline 1955 & Feb. 6 & 1,190 & & 11.40 & \\
\hline 1956 & Mar. 16 & 2,070 & & 16.00 & \\
\hline 1957 & Apr. 4 & 976 & & 10.10 & \\
\hline 1958 & Mar. 7 & 2,330 & & 17.30 & \\
\hline 1959 & June 12 & 1,000 & & 10.29 & \\
\hline 1960 & Oct. 21 & 2,330 & & 17.30 & \\
\hline 1961 & Feb. 22 & 11,500 & & 22.23 & \\
\hline 1962 & Dec. 18 & 2,580 & & 17.87 & \\
\hline 1963 & Jan. 20 & 510 & & 7.03 & \\
\hline 1964 & Apr. 6 & 15,500 & & 23.00 & \\
\hline 1965 & Oct. 5 & 2,170 & & 16.51 & \\
\hline 1966 & Feb. 17 & 2,650 & & 18.06 & \\
\hline 1967 & Dec. 31 & 640 & & 8.07 & \\
\hline 1968 & Apr. 5 & 1,910 & & 15.20 & \\
\hline 1969 & Apr. 18 & 2,950 & & 18.70 & \\
\hline 1970 & Mar. 20 & 1,090 & & 10.92 & \\
\hline
\end{tabular}


Appendix 2. Discharge and gage height data for streamgages in Alabama.-Continued

[--, date or data not available; $\mathrm{ft}$, foot; $\mathrm{ft}^{3} / \mathrm{s}$, cubic foot per second; mi, mile; $\mathrm{mi}^{2}$, square mile; qualification codes apply to the discharge data: 1 , discharge is a maximum daily average; 2 , discharge is an estimate; 4 , discharge is less than indicated value, which is the minimum recordable discharge at this site; 5 , discharge affected to unknown degree by regulation or diversion; 6 , discharge affected by regulation or diversion; 7, discharge is a historic peak; A, year of occurrence is unknown or not exact; $\mathrm{B}$, month or day of occurrence is unknown or not exact; E, only maximum peak available for this year. qualification codes apply to the gage height data: 1, gage height affected by backwater; 2, gage height not the maximum for the year; 3, gage height at different datum or at different site and datum; 5 , gage height is an estimate]

\section{Horse Creek near Sweetwater}

L ocation-Lat $32^{\circ} 02^{\prime} 53^{\prime \prime}$, long $87^{\circ} 52^{\prime} 32^{\prime \prime}$, in SW 1/4 sec. 34, T. 13 N., R. 2 E., Marengo County, Hydrologic Unit 03160201, on county road 25, $0.5 \mathrm{mi}$ downstream from Mill Creek, $0.8 \mathrm{mi}$ south of Exmoor, $1.2 \mathrm{mi}$ north of Hoboken, and $3.5 \mathrm{mi}$ south of Sweetwater.

Drainage area $-60.4 \mathrm{mi}^{2}$.

Gage -Water-stage recorder 1959-70. Crest-stage gage 1971-79. Datum of gage is $129 \mathrm{ft}$ NGVD 29 (from topographic map). Prior to Oct. 1,1965 , at datum $1.00 \mathrm{ft}$ higher.

\begin{tabular}{|c|c|c|c|c|c|}
\hline $\begin{array}{l}\text { Water } \\
\text { year }\end{array}$ & Date & Discharge & $\begin{array}{l}\text { Discharge } \\
\text { code }\end{array}$ & $\begin{array}{c}\text { Gage } \\
\text { height } \\
\text { (feet) }\end{array}$ & $\begin{array}{c}\text { Gage } \\
\text { height } \\
\text { code }\end{array}$ \\
\hline 1958 & Mar. 8 & 2,700 & 2 & 14.78 & \\
\hline 1959 & June 10 & 1,850 & 2 & 14.21 & \\
\hline 1960 & Mar. 30 & 2,500 & & 14.65 & \\
\hline 1961 & Mar. 31 & 9,700 & & 16.80 & \\
\hline 1962 & Dec. 10 & 14,500 & & 17.50 & \\
\hline 1963 & Mar. 6 & 800 & & 11.78 & \\
\hline 1964 & Mar. 15 & 2,400 & & 14.54 & \\
\hline 1965 & Jan. 23 & 6,200 & & 16.07 & \\
\hline 1966 & Feb. 16 & 2,750 & & 14.84 & \\
\hline 1967 & Feb. 7 & 1,200 & & 13.42 & \\
\hline 1968 & Oct. 31 & 3,700 & & 15.30 & \\
\hline 1969 & Mar. 24 & 1,400 & & 13.73 & \\
\hline 1970 & Mar. 4 & 4,500 & & 15.63 & \\
\hline 1971 & Mar. 2 & 8,500 & & 16.60 & \\
\hline 1972 & Dec. 7 & 9,900 & & 16.85 & \\
\hline 1973 & Apr. 26 & 17,000 & & 17.79 & \\
\hline 1974 & Apr. 14 & 5,060 & & 15.79 & \\
\hline 1975 & Feb. 17 & 16,400 & & 17.73 & \\
\hline 1976 & Mar. 31 & 14,500 & & 17.49 & \\
\hline 1977 & Apr. 1 & 6,400 & & 16.12 & \\
\hline 1978 & May 9 & 1,850 & & 14.19 & \\
\hline 1979 & Mar. 8 & 28,100 & & 18.66 & \\
\hline
\end{tabular}

\section{Satilpa Creek near Coffeeville}

L ocation-Lat $31^{\circ} 44^{\prime} 39^{\prime \prime}$, long $88^{\circ} 01^{\prime} 21^{\prime \prime}$, in SW 1/4 sec. 18, T. 9 N., R. 1 E., Clarke County, Hydrologic Unit 03160203, on U.S. Highway 84, 3 mi downstream from Harris Creek, and $3.8 \mathrm{mi}$ east of Coffeeville.

Drainage area-164 $\mathrm{mi}^{2}$.

G age-Water-stage recorder. Datum of gage is $39.80 \mathrm{ft}$ NGVD 29.

\begin{tabular}{|c|c|c|c|c|c|}
\hline $\begin{array}{l}\text { Water } \\
\text { year }\end{array}$ & Date & Discharge & $\begin{array}{l}\text { Discharge } \\
\text { code }\end{array}$ & $\begin{array}{c}\text { Gage } \\
\text { height } \\
\text { (feet) }\end{array}$ & $\begin{array}{c}\text { Gage } \\
\text { height } \\
\text { code }\end{array}$ \\
\hline 1956 & July 8 & 38,000 & & 18.37 & \\
\hline 1957 & Apr. 5 & 4,100 & & 13.93 & \\
\hline 1958 & Mar. 8 & 4,200 & & 14.00 & \\
\hline 1959 & June 10 & 3,310 & & 13.52 & \\
\hline 1960 & Mar. 30 & 4,000 & & 13.94 & \\
\hline 1961 & Feb. 21 & 18,000 & & 16.85 & \\
\hline 1962 & Dec. 10 & 14,850 & & 16.53 & \\
\hline 1963 & Mar. 6 & 1,500 & & 12.12 & \\
\hline 1964 & Mar. 15 & 3,400 & & 13.60 & \\
\hline 1965 & Jan. 24 & 6,000 & & 14.71 & \\
\hline 1966 & Feb. 17 & 4,000 & & 13.91 & \\
\hline 1967 & Feb. 7 & 1,800 & & 12.62 & \\
\hline 1968 & Nov. 1 & 3,100 & & 13.37 & \\
\hline 1969 & May 19 & 1,780 & & 12.56 & \\
\hline 1970 & Mar. 5 & 4,250 & & 14.04 & \\
\hline 1971 & Mar. 3 & 4,200 & & 14.00 & \\
\hline 1972 & Dec. 7 & 5,300 & & 14.52 & \\
\hline 1973 & Mar. 8 & 4,500 & & 14.13 & \\
\hline 1974 & Apr. 13 & 3,800 & & 13.86 & \\
\hline 1975 & Feb. 17 & 4,800 & & 14.33 & \\
\hline 1976 & Mar. 31 & 13,000 & & 16.06 & \\
\hline 1977 & Mar. 13 & 6,200 & & 14.80 & \\
\hline 1978 & June 9 & 3,150 & & 13.44 & \\
\hline 1979 & Mar. 4 & 23,400 & & 17.33 & \\
\hline 1980 & May 17 & 8,000 & & 15.22 & \\
\hline 1981 & Feb. 11 & 4,520 & & 14.14 & \\
\hline 1982 & Apr. 26 & 3,890 & & 13.73 & \\
\hline 1983 & Feb. 2 & 10,500 & & 15.77 & \\
\hline 1984 & Dec. 29 & 3,500 & $\mathrm{E}$ & 13.65 & \\
\hline 1985 & Feb. 6 & 2,000 & $\mathrm{E}$ & 12.77 & \\
\hline 1986 & Feb. 6 & 1,850 & & 12.69 & \\
\hline
\end{tabular}


Appendix 2. Discharge and gage height data for streamgages in Alabama._- Continued

[--, date or data not available; ft, foot; $\mathrm{ft}^{3} / \mathrm{s}$, cubic foot per second; mi, mile; $\mathrm{mi}^{2}$, square mile; qualification codes apply to the discharge data: 1 , discharge is a maximum daily average; 2 , discharge is an estimate; 4 , discharge is less than indicated value, which is the minimum recordable discharge at this site; 5 , discharge affected to unknown degree by regulation or diversion; 6 , discharge affected by regulation or diversion; 7, discharge is a historic peak; A, year of occurrence is unknown or not exact; B, month or day of occurrence is unknown or not exact; E, only maximum peak available for this year. qualification codes apply to the gage height data: 1 , gage height affected by backwater; 2, gage height not the maximum for the year; 3, gage height at different data or at different site and datum;

5 , gage height is an estimate]

\section{Satilpa Creek near Coffeeville-Continued}

L ocation-Lat $31^{\circ} 44^{\prime} 39^{\prime \prime}$, long $88^{\circ} 01^{\prime} 21^{\prime \prime}$, in SW 1/4 sec. 18 , T. 9 N., R. 1 E., Clarke County, Hydrologic Unit 03160203, on U.S. Highway 84, 3 mi downstream from Harris Creek, and $3.8 \mathrm{mi}$ east of Coffeeville.

Drainage area-164 $\mathrm{mi}^{2}$.

Gage -Water-stage recorder. Datum of gage is $39.80 \mathrm{ft}$ NGVD 29.

\begin{tabular}{|c|c|c|c|c|c|}
\hline $\begin{array}{l}\text { Water } \\
\text { year }\end{array}$ & Date & Discharge & $\begin{array}{l}\text { Discharge } \\
\text { code }\end{array}$ & $\begin{array}{c}\text { Gage } \\
\text { height } \\
\text { (feet) }\end{array}$ & $\begin{array}{c}\text { Gage } \\
\text { height } \\
\text { code }\end{array}$ \\
\hline
\end{tabular}

\begin{tabular}{llll}
\hline 1987 & Feb. 28 & 3,340 & 13.53 \\
1988 & Jan. 20 & 2,700 & 13.21
\end{tabular}

1989

1990

1991

1992

Apr. 5

4,500

Mar. 16

15,500

Mar. 2

4,200

Feb. 18

3,310

1993

Dec. 17

6,880

14.13

16.53

14.01

13.51

15.02

1994

July 15

2,400

1995

Mar. 8

3,430

13.03

13.59

1996

Dec. 19

6,070

14.77

1997

June 22

4,300

14.09

1998

Jan. 8

6,400

1999

Jan. 31

15,500

2000

Mar. 20

1,980

2001

2002

Mar. 4

9,990

Dec. 15

1,700

June 20

5,660

2004

2005

Sept. 17

3,810

July 11

8,190

Feb. 26

2,920

2007

Jan. 1

3,030

2008

2009

Aug. 26

2,280

Mar. 27

8,050

2010

Dec. 19

5,890

2011

2012

Mar. 10

5,580

Mar. 23

3,960

2013

Feb. 11

24,500

Feb. 21

3,460

2015

Apr. 17

7,470
14.92

16.54

12.73

15.67

12.50

14.63

13.83

15.32

13.32

13.36

12.95

15.29

14.71

14.60

13.92

17.45

13.61

15.16
02343275 Abbie Creek near Abbeville

L ocation-Lat $31^{\circ} 33^{\prime} 42^{\prime \prime}$, long $85^{\circ} 12^{\prime} 18^{\prime \prime}$, in SW 1/4 sec. 23, T. 7 N., R. 28 E., Henry County, Hydrologic Unit 03130004, at bridge on State Highway 10, 2.5 mi east of Abbeville.

Drainage area $-48.7 \mathrm{mi}^{2}$.

Gage Crest-stage gage. Datum not available.

\begin{tabular}{|c|c|c|c|c|c|}
\hline $\begin{array}{c}\text { Water } \\
\text { year }\end{array}$ & Date & Discharge & $\begin{array}{l}\text { Discharge } \\
\text { code }\end{array}$ & $\begin{array}{c}\text { Gage } \\
\text { height } \\
\text { (feet) }\end{array}$ & $\begin{array}{c}\text { Gage } \\
\text { height } \\
\text { code }\end{array}$ \\
\hline 1951 & -- & 7,020 & $2, \mathrm{~B}$ & 9.10 & \\
\hline 1952 & Feb. 16 & 860 & & 6.00 & \\
\hline 1953 & May 4 & 13,000 & 2 & 10.31 & \\
\hline 1954 & Dec. 6 & 1,300 & & 6.50 & \\
\hline 1955 & Apr. 13 & 330 & & 5.10 & \\
\hline 1956 & Sept. 25 & 3,200 & & 7.40 & \\
\hline 1957 & Apr. 6 & 1,970 & & 6.90 & \\
\hline 1958 & Apr. 10 & 500 & & 5.50 & \\
\hline 1959 & Feb. 5 & 1,100 & & 6.30 & \\
\hline 1960 & Apr. 5 & 1,100 & & 6.30 & \\
\hline 1961 & Mar. 31 & 1,020 & & 6.13 & \\
\hline 1962 & Apr. 1 & 2,290 & & 7.06 & \\
\hline 1963 & Feb. 12 & 1,970 & & 6.90 & \\
\hline 1964 & Mar. 3 & 2,080 & & 6.96 & \\
\hline 1965 & Feb. 18 & 674 & & 5.69 & \\
\hline 1966 & Mar. 4 & 1,400 & & 6.50 & \\
\hline 1967 & Jan. 3 & 1,300 & & 6.41 & \\
\hline 1968 & Nov. 1 & 420 & & 5.22 & \\
\hline 1969 & May 15 & 1,420 & & 6.52 & \\
\hline 1970 & Mar. 31 & 1,450 & & 6.54 & \\
\hline 1971 & Feb. 22 & 2,460 & & 7.14 & \\
\hline 1972 & Dec. 21 & 1,970 & & 6.90 & \\
\hline 1973 & Dec. 8 & 2,440 & & 7.39 & \\
\hline 1974 & Dec. 26 & 1,850 & & 6.99 & \\
\hline 1990 & Mar. 17 & 7,100 & 7 & 9.10 & \\
\hline 1994 & July 6 & 28,000 & 7 & 13.00 & \\
\hline
\end{tabular}


Appendix 2. Discharge and gage height data for streamgages in Alabama.-Continued

[--, date or data not available; $\mathrm{ft}$, foot; $\mathrm{ft}^{3} / \mathrm{s}$, cubic foot per second; mi, mile; $\mathrm{mi}^{2}$, square mile; qualification codes apply to the discharge data: 1 , discharge is a maximum daily average; 2 , discharge is an estimate; 4 , discharge is less than indicated value, which is the minimum recordable discharge at this site; 5 , discharge affected to unknown degree by regulation or diversion; 6 , discharge affected by regulation or diversion; 7, discharge is a historic peak; A, year of occurrence is unknown or not exact; $\mathrm{B}$, month or day of occurrence is unknown or not exact; E, only maximum peak available for this year. qualification codes apply to the gage height data: 1, gage height affected by backwater; 2, gage height not the maximum for the year; 3 , gage height at different datum or at different site and datum; 5 , gage height is an estimate]

\section{Abbie Creek near Haleburg}

L ocation-Lat $31^{\circ} 28^{\prime} 24^{\prime \prime}$, long $85^{\circ} 09^{\prime} 45^{\prime \prime}$, in SE $1 / 4 \mathrm{sec} .19$, T. 6 N., R. 29 E., Henry County, Hydrologic Unit 03130004, on State Highway 95, 1.2 mi upstream from Peterman Creek, $4.5 \mathrm{mi}$ northwest of Haleburg, $7.8 \mathrm{mi}$ upstream from mouth, and $9 \mathrm{mi}$ southeast of Abbeville.

Drainage area-146 $\mathrm{mi}^{2}$.

G age - Water-stage recorder. Datum of gage is $145.74 \mathrm{ft}$ NGVD 29.

\begin{tabular}{cccccc}
\hline $\begin{array}{c}\text { Water } \\
\text { year }\end{array}$ & Date & Discharge & $\begin{array}{c}\text { Discharge } \\
\text { code }\end{array}$ & $\begin{array}{c}\text { Gage } \\
\text { height } \\
\text { (feet) }\end{array}$ & $\begin{array}{c}\text { Gage } \\
\text { height } \\
\text { code }\end{array}$ \\
\hline 1958 & Apr. 10 & 1,000 & 2 & 6.57 & \\
1959 & Feb. 5 & 1,460 & & 8.13 &
\end{tabular}

1960

1961

1962

1963

1964

1965

1966

1967

1968

1969

1970

1971

1972

1973

1974

1975

1976

1977

1978

1979

1980

1981

1982

1983

1984

1985

1986

1987

1988

1989

1990

1991

1992

1993

1994

Feb. 5

Apr. 5

Mar. 31

Jan. 6

Feb. 12

Mar. 3

Dec. 26

Mar. 5

Jan. 3

Dec. 11

May 15

Mar. 31

Jan. 5

Dec. 21

--

--

Apr. 10

Jan. 26

Nov. 29

Jan. 26

Feb. 24

Mar. 13

Feb. 11

Feb. 3

Mar. 7

May 4

Feb. 7

Dec. 13

Jan. 22

Mar. 4

June 9

Mar. 18

Jan. 31

Jan. 13

Mar. 27

July 6

1,460

2,880

3,000

3,700

2,690

3,340

3,070

2,210

1,940

1,330

3,660

7,590

2,340

2,060

5,300

3,600

4,920

4,950

5,300

7,080

3,970

3,970

6,650

2,740

2,840

3,350

4,180

1,080

930

1,400

1,050

5,730

2,460

1,900

1,730

35,000

8.13
11.98
12.30

12.30

14.42

11.42

13.19

11.67

9.45

8.69

6.98

14.40

23.84

10.38

9.45

B $\quad 18.60$

2,B

17.63

17.70

18.38

23.07

15.01

15.00

22.00

11.21

11.54

13.13

15.59

6.80

6.29

7.56

6.69

19.72

10.73

9.17

8.68

$2,7 \quad 37.00$

02470072 Bassett Creek at US Highway 43 nr Thomasville, Ala.

L ocation-Lat $31^{\circ} 51^{\prime} 50^{\prime \prime}$, long $87^{\circ} 44^{\prime} 50^{\prime \prime}$ referenced to North American Datum of 1927, Clarke County, AL, Hydrologic Unit 03160203, on downstream side of bridge on U.S. Highway 43, 3 mi south of Thomasville.

Drainage area-10 $\mathrm{mi}^{2}$.

G age -Water-stage recorder. Elevation of gage is $292 \mathrm{ft}$ above NGVD of 1929, from topographic map. 4.00 feet added to stage on October 1, 2017 in order to prevent frequent negative gage heights.

\begin{tabular}{|c|c|c|c|c|c|}
\hline $\begin{array}{c}\text { Water } \\
\text { year }\end{array}$ & Date & Discharge & $\begin{array}{l}\text { Discharge } \\
\text { code }\end{array}$ & $\begin{array}{c}\text { Gage } \\
\text { height } \\
\text { (feet) }\end{array}$ & $\begin{array}{c}\text { Gage } \\
\text { height } \\
\text { code }\end{array}$ \\
\hline 1996 & Aug. 30 & 1,630 & & 10.33 & \\
\hline 1997 & June 21 & 633 & & 8.51 & \\
\hline 1998 & Mar. 8 & 1,330 & & 10.00 & \\
\hline 1999 & Jan. 30 & 1,150 & & 9.73 & \\
\hline 2000 & Apr. 4 & 194 & & 5.14 & \\
\hline 2001 & Mar. 12 & 786 & & 8.95 & \\
\hline 2002 & Dec. 14 & 475 & & 7.53 & \\
\hline 2003 & Dec. 24 & 1,310 & & 9.97 & \\
\hline 2004 & Sept. 16 & 1,780 & & 10.48 & \\
\hline 2005 & July 11 & 2,460 & & 11.03 & \\
\hline 2006 & May 11 & 695 & & 8.29 & \\
\hline 2007 & Dec. 31 & 590 & & 7.77 & \\
\hline 2008 & Dec. 31 & 590 & & 7.77 & \\
\hline 2009 & Mar. 27 & 1,850 & & 10.55 & \\
\hline 2010 & Feb. 5 & 1,290 & & 9.95 & \\
\hline 2011 & Mar. 9 & 1,190 & & 9.79 & \\
\hline 2012 & Feb. 19 & 578 & & 7.83 & \\
\hline 2013 & Feb. 11 & 1,690 & & 10.30 & \\
\hline 2014 & Apr. 6 & 797 & & 8.80 & \\
\hline 2015 & Apr. 17 & 1,630 & & 10.20 & 6 \\
\hline
\end{tabular}


Appendix 2. Discharge and gage height data for streamgages in Alabama.-Continued

[--, date or data not available; $\mathrm{ft}$, foot; $\mathrm{ft}^{3} / \mathrm{s}$, cubic foot per second; $\mathrm{mi}$, mile; $\mathrm{mi}^{2}$, square mile; qualification codes apply to the discharge data: 1 , discharge is a maximum daily average; 2 , discharge is an estimate; 4 , discharge is less than indicated value, which is the minimum recordable discharge at this site; 5 , discharge affected to unknown degree by regulation or diversion; 6 , discharge affected by regulation or diversion; 7, discharge is a historic peak; A, year of occurrence is unknown or not exact; B, month or day of occurrence is unknown or not exact; E, only maximum peak available for this year. qualification codes apply to the gage height data: 1, gage height affected by backwater; 2 , gage height not the maximum for the year; 3 , gage height at different data or at different site and datum; 5 , gage height is an estimate]

\section{Bassett Creek at Walker Springs}

Location-Lat $31^{\circ} 32^{\prime} 15^{\prime \prime}$, long $87^{\circ} 47^{\prime} 24^{\prime \prime}$, in NE $1 / 4$ sec. 32 , T. 7 N., R. 3 E., Clarke County, Hydrologic Unit 03160203, on county road, 1,000 ft southeast of Walker Springs, and 2.8 mi upstream from Rabbit Creek.

Drainage area-195 $\mathrm{mi}^{2}$.

Gage -Water-stage recorder 1956-70. Crest-stage gage 1971-78. Datum of gage is $60.02 \mathrm{ft} \mathrm{NGVD} 29$.

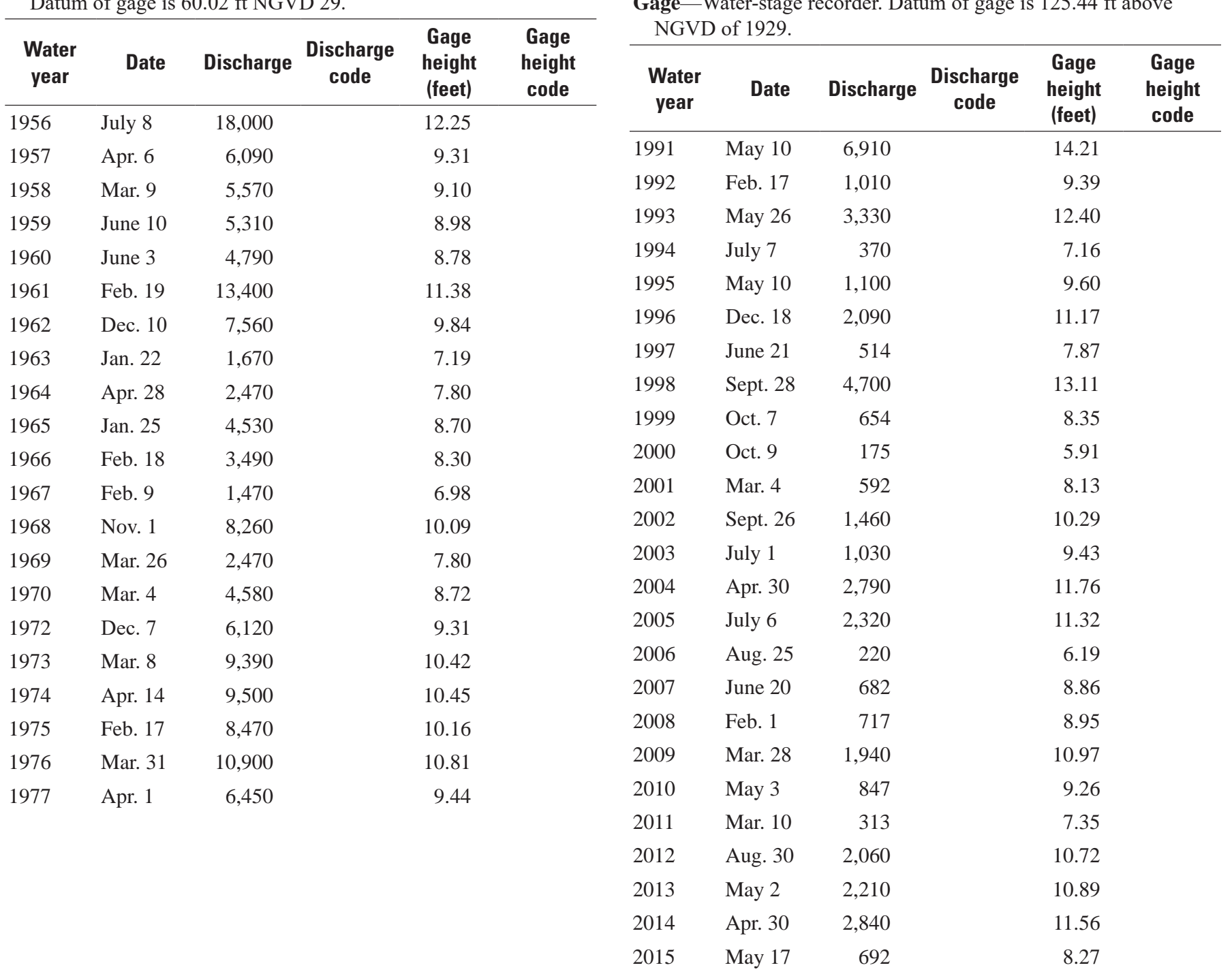

02479945 Big Creek at County Rd 63 near Wilmer, Ala.

L ocation-Lat $30^{\circ} 51^{\prime} 21^{\prime \prime}$, long $88^{\circ} 20^{\prime} 02^{\prime \prime}$ referenced to North American Datum of 1927, Mobile County, AL, Hydrologic Unit 03170008 , on downstream side of bridge at County Road 63, about 2.9 mi northeast of Wilmer, and 3.2 mi north of U.S. Highway 98.

Drainage area-31 $\mathrm{mi}^{2}$. 
Appendix 2. Discharge and gage height data for streamgages in Alabama.-Continued

[--, date or data not available; $\mathrm{ft}$, foot; $\mathrm{ft}^{3} / \mathrm{s}$, cubic foot per second; mi, mile; $\mathrm{mi}^{2}$, square mile; qualification codes apply to the discharge data: 1 , discharge is a maximum daily average; 2 , discharge is an estimate; 4 , discharge is less than indicated value, which is the minimum recordable discharge at this site; 5 , discharge affected to unknown degree by regulation or diversion; 6 , discharge affected by regulation or diversion; 7, discharge is a historic peak; A, year of occurrence is unknown or not exact; $\mathrm{B}$, month or day of occurrence is unknown or not exact; E, only maximum peak available for this year. qualification codes apply to the gage height data: 1, gage height affected by backwater; 2, gage height not the maximum for the year; 3, gage height at different datum or at different site and datum; 5 , gage height is an estimate]

\section{Big Escambia CR at Sardine Br nr Stanley Crossroad \\ L ocation-Lat $31^{\circ} 07^{\prime} 46^{\prime \prime}$, long $87^{\circ} 22^{\prime} 14^{\prime \prime}$ referenced to North American Datum of 1927, Escambia County, AL, Hydrologic Unit 03140305, on upstream side of Sardine Bridge on County Road 27, 2 mi west of Stanley Crossroads, 13 mi northwest of Flomaton.}

Drainage area-193 $\mathrm{mi}^{2}$.

G age -Water-stage recorder. Elevation of gage is $110 \mathrm{ft}$ above NGVD of 1929, from topographic map.

\begin{tabular}{|c|c|c|c|c|c|}
\hline $\begin{array}{l}\text { Water } \\
\text { year }\end{array}$ & Date & Discharge & $\begin{array}{l}\text { Discharge } \\
\text { code }\end{array}$ & $\begin{array}{c}\text { Gage } \\
\text { height } \\
\text { (feet) }\end{array}$ & $\begin{array}{c}\text { Gage } \\
\text { height } \\
\text { code }\end{array}$ \\
\hline 2001 & Mar. 5 & 11,400 & & 16.63 & \\
\hline 2002 & Sept. 26 & 4,910 & & 11.27 & \\
\hline 2003 & July 1 & 3,740 & & 9.99 & \\
\hline 2004 & Sept. 18 & 8,270 & & 14.30 & \\
\hline 2005 & July 12 & 3,620 & & 9.83 & \\
\hline 2006 & May 11 & 1,200 & & 5.22 & \\
\hline 2007 & Apr. 15 & 2,200 & & 7.69 & \\
\hline 2008 & Aug. 27 & 2,790 & & 8.70 & \\
\hline 2009 & Apr. 14 & 3,130 & & 9.18 & \\
\hline 2010 & Dec. 15 & 10,500 & & 18.02 & \\
\hline 2011 & Mar. 9 & 2,400 & & 7.51 & \\
\hline 2012 & Sept. 6 & 2,700 & & 8.05 & \\
\hline 2013 & Feb. 27 & 3,610 & & 8.47 & \\
\hline 2014 & May 15 & 7,550 & & 14.50 & \\
\hline 2015 & Apr. 19 & 1,490 & & 4.85 & \\
\hline
\end{tabular}

02375000 Big Escambia Creek at Flomaton

L ocation-Lat $31^{\circ} 00^{\prime} 38^{\prime \prime}$, long $87^{\circ} 15^{\prime} 46^{\prime \prime}$, in NE $1 / 4$ sec. 33 , T. 1

N., R. 8 E., Escambia County, Hydrologic Unit 03140305, on

U.S. Highway 31 at north edge of Flomaton, 1.5 mi upstream from Alabama-Florida State line, and 4 mi upstream from mouth.

Drainage area-330 $\mathrm{mi}^{2}$.

G age -Water-stage gage 1939-51. Crest-stage gage 1952-75. Datum of gage is $52.40 \mathrm{ft}$ NGVD 29.

\begin{tabular}{llcccc}
\hline $\begin{array}{c}\text { Water } \\
\text { year }\end{array}$ & Date & Discharge & $\begin{array}{c}\text { Discharge } \\
\text { code }\end{array}$ & $\begin{array}{c}\text { Gage } \\
\text { height } \\
\text { (feet) }\end{array}$ & $\begin{array}{c}\text { Gage } \\
\text { height } \\
\text { code }\end{array}$ \\
\hline 1939 & Sept. 27 & 41,400 & & 19.26 & \\
1940 & May 1 & 6,250 & & 10.36 & \\
1941 & Jan. 17 & 2,790 & 6.38 & \\
1942 & Jan. 2 & 9,100 & 11.90 & \\
1943 & Mar. 21 & 6,500 & 10.45 & \\
1944 & Mar. 23 & 9,400 & 12.25 &
\end{tabular}

02375000 Big Escambia Creek at Flomaton-Continued

L ocation-Lat $31^{\circ} 00^{\prime} 38^{\prime \prime}$, long $87^{\circ} 15^{\prime} 46^{\prime \prime}$, in NE $1 / 4 \mathrm{sec} .33$, T. 1 N., R. 8 E., Escambia County, Hydrologic Unit 03140305, on U.S. Highway 31 at north edge of Flomaton, 1.5 mi upstream from Alabama-Florida State line, and $4 \mathrm{mi}$ upstream from mouth. Drainage area-330 $\mathrm{mi}^{2}$.

G age -Water-stage gage 1939-51. Crest-stage gage 1952-75. Datum of gage is $52.40 \mathrm{ft}$ NGVD 29.

\begin{tabular}{|c|c|c|c|c|c|}
\hline $\begin{array}{l}\text { Water } \\
\text { year }\end{array}$ & Date & Discharge & $\begin{array}{l}\text { Discharge } \\
\text { code }\end{array}$ & $\begin{array}{c}\text { Gage } \\
\text { height } \\
\text { (feet) }\end{array}$ & $\begin{array}{c}\text { Gage } \\
\text { height } \\
\text { code }\end{array}$ \\
\hline 1945 & Apr. 29 & 5,400 & & 9.64 & \\
\hline 1946 & Mar. 29 & 6,300 & & 10.56 & \\
\hline 1947 & Mar. 8 & 7,400 & & 11.06 & \\
\hline 1948 & Mar. 7 & 6,500 & & 10.40 & \\
\hline 1949 & Nov. 28 & 15,400 & & 14.50 & \\
\hline 1950 & Apr. 5 & 4,570 & & 8.87 & \\
\hline 1951 & Mar. 19 & 6,330 & & 10.30 & \\
\hline 1952 & Mar. 24 & 3,000 & & 9.00 & \\
\hline 1953 & Apr. 26 & 3,590 & & 7.80 & \\
\hline 1954 & Dec. 6 & 8,870 & & 11.80 & \\
\hline 1955 & Apr. 14 & 45,600 & & 19.40 & \\
\hline 1956 & Sept. 25 & 2,930 & & 6.90 & \\
\hline 1957 & Dec. 25 & 4,270 & & 8.60 & \\
\hline 1958 & Sept. 22 & 3,500 & & 7.70 & \\
\hline 1959 & Sept. 14 & 5,100 & & 9.60 & \\
\hline 1960 & Apr. 3 & 8,540 & & 11.60 & \\
\hline 1961 & Apr. 12 & 10,900 & & 12.83 & \\
\hline 1962 & Apr. 1 & 14,300 & & 14.13 & \\
\hline 1963 & Jan. 21 & 5,100 & & 9.65 & \\
\hline 1964 & Apr. 27 & 6,710 & & 10.54 & \\
\hline 1965 & Jan. 23 & 16,200 & & 14.75 & \\
\hline 1966 & Oct. 2 & 10,600 & & 12.69 & \\
\hline 1967 & Jan. 3 & 9,200 & & 11.98 & \\
\hline 1968 & Oct. 31 & 3,140 & & 7.20 & \\
\hline 1969 & May 19 & 6,540 & & 10.43 & \\
\hline 1970 & Feb. 17 & 4,060 & & 8.37 & \\
\hline 1971 & Dec. 20 & 9,810 & & 12.30 & \\
\hline 1972 & May 9 & 8,760 & & 11.74 & \\
\hline 1973 & Mar. 31 & 8,190 & & 11.42 & \\
\hline 1974 & Sept. 9 & 17,500 & & 17.46 & \\
\hline 1975 & Feb & 22,200 & B & 17.92 & \\
\hline 2015 & Apr. 15 & 2,190 & & 11.38 & \\
\hline
\end{tabular}


Appendix 2. Discharge and gage height data for streamgages in Alabama.-Continued

[--, date or data not available; $\mathrm{ft}$, foot; $\mathrm{ft}^{3} / \mathrm{s}$, cubic foot per second; $\mathrm{mi}$, mile; $\mathrm{mi}^{2}$, square mile; qualification codes apply to the discharge data: 1 , discharge is a maximum daily average; 2 , discharge is an estimate; 4 , discharge is less than indicated value, which is the minimum recordable discharge at this site; 5 , discharge affected to unknown degree by regulation or diversion; 6 , discharge affected by regulation or diversion; 7, discharge is a historic peak; A, year of occurrence is unknown or not exact; B, month or day of occurrence is unknown or not exact; E, only maximum peak available for this year. qualification codes apply to the gage height data: 1, gage height affected by backwater; 2 , gage height not the maximum for the year; 3 , gage height at different data or at different site and datum;

5 , gage height is an estimate]

\section{Big Flat C reek near Fountain}

L ocation-Lat $31^{\circ} 36^{\prime} 30^{\prime \prime}$, Long $87^{\circ} 24^{\prime} 53^{\prime \prime}$, in NE 1/4 SEC. 1, T. 7 N., R. 6 E., Monroe County, Hydrologic Unit 03150204, on State Highway 41, 1 mi (1 mile) northwest of Foutain, 2 mi upstream from Bradley Mill Creek, $8 \mathrm{mi}$ upstream from mouth, and $8 \mathrm{mi}$ northwest of Monroeville.

Drainage area-247 $\mathrm{mi}^{2}$.

Gage -Water-stage recorder. Datum of gage is 45.43 FT NGVD 29.

\begin{tabular}{|c|c|c|c|c|c|}
\hline $\begin{array}{c}\text { Water } \\
\text { year }\end{array}$ & Date & Discharge & $\begin{array}{l}\text { Discharge } \\
\text { code }\end{array}$ & $\begin{array}{c}\text { Gage } \\
\text { height } \\
\text { (feet) }\end{array}$ & $\begin{array}{c}\text { Gage } \\
\text { height } \\
\text { code }\end{array}$ \\
\hline 1930 & -- & -- & & 16.70 & 5 \\
\hline 1931 & -- & -- & & 16.80 & 5 \\
\hline 1932 & -- & -- & & 14.50 & 5 \\
\hline 1933 & -- & -- & & 20.00 & 5 \\
\hline 1934 & -- & -- & & 17.20 & 5 \\
\hline 1935 & -- & -- & & 18.80 & 5 \\
\hline 1936 & -- & -- & & 20.20 & 5 \\
\hline 1937 & -- & -- & & 21.70 & 5 \\
\hline 1938 & -- & -- & & 22.80 & 5 \\
\hline 1939 & -- & -- & & 22.60 & 5 \\
\hline 1940 & -- & -- & & 17.20 & 5 \\
\hline 1941 & -- & -- & & 10.70 & 5 \\
\hline 1942 & -- & -- & & 15.10 & 5 \\
\hline 1943 & -- & -- & & 22.70 & 5 \\
\hline 1944 & Apr. 27 & 17,300 & & 21.19 & \\
\hline 1945 & Apr. 29 & 3,250 & & 12.68 & \\
\hline 1946 & May 21 & 9,700 & & 18.90 & \\
\hline 1947 & Apr. 3 & 7,950 & & 18.17 & \\
\hline 1948 & Dec. 11 & 8,900 & & 18.70 & \\
\hline 1949 & Nov. 27 & 29,800 & & 23.20 & \\
\hline 1950 & July 30 & 2,860 & & 11.60 & \\
\hline 1951 & Apr. 21 & 4,490 & & 14.70 & \\
\hline 1952 & Mar. 24 & 1,820 & & 8.40 & \\
\hline 1953 & Apr. 9 & 2,790 & & 11.40 & \\
\hline 1954 & Dec. 6 & 2,210 & & 9.70 & \\
\hline 1955 & Apr. 15 & 6,220 & & 16.80 & \\
\hline 1956 & July 8 & 3,960 & & 13.93 & \\
\hline 1957 & Apr. 6 & 15,100 & & 20.70 & \\
\hline 1958 & Mar. 10 & 3,160 & & 12.40 & \\
\hline 1959 & June 11 & 6,040 & & 16.60 & \\
\hline
\end{tabular}

02428500 Big Flat Creek near Fountain-Continued

L ocation-Lat $31^{\circ} 36^{\prime} 30^{\prime \prime}$, Long $87^{\circ} 24^{\prime} 53^{\prime \prime}$, in NE 1/4 SEC. 1, T. 7 N., R. 6 E., Monroe County, Hydrologic Unit 03150204, on State Highway 41, $1 \mathrm{mi}$ (1 mile) northwest of Foutain, 2 mi upstream from Bradley Mill Creek, $8 \mathrm{mi}$ upstream from mouth, and $8 \mathrm{mi}$ northwest of Monroeville.

Drainage area-247 $\mathrm{mi}^{2}$.

G age -Water-stage recorder. Datum of gage is 45.43 FT NGVD 29.

\begin{tabular}{|c|c|c|c|c|c|}
\hline $\begin{array}{l}\text { Water } \\
\text { year }\end{array}$ & Date & Discharge & $\begin{array}{l}\text { Discharge } \\
\text { code }\end{array}$ & $\begin{array}{c}\text { Gage } \\
\text { height } \\
\text { (feet) }\end{array}$ & $\begin{array}{c}\text { Gage } \\
\text { height } \\
\text { code }\end{array}$ \\
\hline 1960 & Mar. 31 & 6,040 & & 16.65 & \\
\hline 1961 & Feb. 25 & 21,300 & & 22.00 & \\
\hline 1962 & Dec. 14 & 5,900 & & 16.45 & \\
\hline 1963 & Jan. 20 & 2,710 & & 11.18 & \\
\hline 1964 & Apr. 16 & 5,890 & & 16.43 & \\
\hline 1965 & Jan. 25 & 4,280 & & 14.40 & \\
\hline 1966 & Oct. 1 & 6,210 & & 16.79 & \\
\hline 1967 & Feb. 8 & 1,970 & & 8.90 & \\
\hline 1968 & Dec. 12 & 1,510 & & 7.41 & \\
\hline 1969 & Mar. 25 & 2,550 & & 10.72 & \\
\hline 1970 & Mar. 24 & 3,380 & & 12.87 & \\
\hline
\end{tabular}

02369800 Blackwater River near Bradley

L ocation-Lat $31^{\circ} 01^{\prime} 39^{\prime \prime}$, long 86 $42^{\prime} 36^{\prime \prime}$, in SW 1/4 sec. 24, T. 1 N., R. 13 E., Escambia County, Hydrologic Unit 03140104, in Conecuh National Forest, on county road, and 1 mi east of Bradley.

Drainage area $-87.7 \mathrm{mi}^{2}$.

G age - Water-stage recorder. Datum of gage is $121.87 \mathrm{ft}$ NGVD 29.

\begin{tabular}{|c|c|c|c|c|c|}
\hline $\begin{array}{c}\text { Water } \\
\text { year }\end{array}$ & Date & Discharge & $\begin{array}{l}\text { Discharge } \\
\text { code }\end{array}$ & $\begin{array}{c}\text { Gage } \\
\text { height } \\
\text { (feet) }\end{array}$ & $\begin{array}{c}\text { Gage } \\
\text { height } \\
\text { code }\end{array}$ \\
\hline 1968 & Dec. 11 & 358 & & 4.84 & \\
\hline 1969 & Sept. 21 & 2,500 & & 14.10 & \\
\hline 1970 & June 4 & 15,000 & & 24.20 & \\
\hline 1971 & Aug. 1 & 786 & & 7.78 & \\
\hline 1972 & May 9 & 1,230 & & 9.84 & \\
\hline 1973 & Dec. 21 & 4,760 & & 17.10 & \\
\hline 1974 & Sept. 9 & 1,980 & & 12.56 & \\
\hline 1975 & July 31 & 8,700 & & 20.37 & \\
\hline 1976 & Nov. 13 & 2,280 & & 13.45 & \\
\hline
\end{tabular}


Appendix 2. Discharge and gage height data for streamgages in Alabama.-Continued

[--, date or data not available; $\mathrm{ft}$, foot; $\mathrm{ft}^{3} / \mathrm{s}$, cubic foot per second; mi, mile; $\mathrm{mi}^{2}$, square mile; qualification codes apply to the discharge data: 1 , discharge is a maximum daily average; 2 , discharge is an estimate; 4 , discharge is less than indicated value, which is the minimum recordable discharge at this site; 5 , discharge affected to unknown degree by regulation or diversion; 6 , discharge affected by regulation or diversion; 7, discharge is a historic peak; A, year of occurrence is unknown or not exact; $\mathrm{B}$, month or day of occurrence is unknown or not exact; E, only maximum peak available for this year. qualification codes apply to the gage height data: 1, gage height affected by backwater; 2, gage height not the maximum for the year; 3 , gage height at different datum or at different site and datum; 5 , gage height is an estimate]

\section{Blackwater River near Bradley-Continued}

L ocation-Lat $31^{\circ} 01^{\prime} 39^{\prime \prime}$, long $86^{\circ} 42^{\prime} 36^{\prime \prime}$, in SW 1/4 sec. 24, T. 1 N., R. 13 E., Escambia County, Hydrologic Unit 03140104, in Conecuh National Forest, on county road, and 1 mi east of Bradley.

Drainage area $-87.7 \mathrm{mi}^{2}$.

G age - Water-stage recorder. Datum of gage is $121.87 \mathrm{ft}$ NGVD 29.

\begin{tabular}{|c|c|c|c|c|c|}
\hline $\begin{array}{c}\text { Water } \\
\text { year }\end{array}$ & Date & Discharge & $\begin{array}{l}\text { Discharge } \\
\text { code }\end{array}$ & $\begin{array}{l}\text { Gage } \\
\text { height } \\
\text { (feet) }\end{array}$ & $\begin{array}{l}\text { Gage } \\
\text { height }\end{array}$ \\
\hline
\end{tabular}

\begin{tabular}{|c|c|c|c|}
\hline & & & (reet) \\
\hline 1977 & Mar. 31 & 1,760 & 11.86 \\
\hline 1978 & Jan. 25 & 5,200 & 17.33 \\
\hline 1979 & Mar. 4 & 2,380 & 13.70 \\
\hline 1980 & Mar. 30 & 2,760 & 14.52 \\
\hline 1981 & Feb. 11 & 2,220 & 13.31 \\
\hline 1982 & Feb. 3 & 2,040 & 12.79 \\
\hline 1983 & Feb. 2 & 1,720 & 11.72 \\
\hline 1984 & Mar. 6 & 2,010 & 12.69 \\
\hline 1985 & Feb. 6 & 2,890 & 14.78 \\
\hline 1986 & Mar. 14 & 2,310 & 13.54 \\
\hline 1987 & Aug. 14 & 1,850 & 12.44 \\
\hline 1988 & Sept. 17 & 4,670 & 16.88 \\
\hline 1989 & June 16 & 4,190 & 16.43 \\
\hline 1990 & Mar. 17 & 17,700 & 25.35 \\
\hline 1991 & Jan. 31 & 2,120 & 13.23 \\
\hline 1992 & Jan. 14 & 1,440 & 10.98 \\
\hline 1993 & Nov. 25 & 2,490 & 14.10 \\
\hline 1994 & July 7 & 5,850 & 17.83 \\
\hline 1995 & Feb. 11 & 4,200 & 16.44 \\
\hline 1996 & Oct. 5 & 9,560 & 19.98 \\
\hline 1997 & Feb. 15 & 989 & 8.80 \\
\hline 1998 & Mar. 8 & 17,500 & 25.30 \\
\hline 1999 & July 13 & 601 & 5.90 \\
\hline 2000 & Jan. 10 & 280 & 3.35 \\
\hline 2001 & Mar. 4 & 1,530 & 11.27 \\
\hline 2002 & Sept. 26 & 1,010 & 8.61 \\
\hline 2003 & Aug. 7 & 2,460 & 13.91 \\
\hline 2004 & Sept. 17 & 3,140 & 15.05 \\
\hline 2005 & Apr. 1 & 3,730 & 15.84 \\
\hline 2006 & Jan. 2 & 993 & 8.52 \\
\hline 2007 & Apr. 3 & 2,920 & 14.71 \\
\hline 2008 & Feb. 1 & 2,700 & 14.35 \\
\hline 2009 & Mar. 28 & 6,470 & 18.64 \\
\hline
\end{tabular}

\section{Blackwater River near Bradley-Continued}

L ocation-Lat $31^{\circ} 01^{\prime} 39^{\prime \prime}$, long 86 $42^{\prime} 36^{\prime \prime}$, in SW 1/4 sec. 24, T. 1 N., R. 13 E., Escambia County, Hydrologic Unit 03140104, in Conecuh National Forest, on county road, and 1 mi east of Bradley.

Drainage area-87.7 $\mathrm{mi}^{2}$.

G age - Water-stage recorder. Datum of gage is $121.87 \mathrm{ft}$ NGVD 29.

\begin{tabular}{llrrrr}
\hline $\begin{array}{c}\text { Water } \\
\text { year }\end{array}$ & Date & Discharge & $\begin{array}{c}\text { Discharge } \\
\text { code }\end{array}$ & $\begin{array}{c}\text { Gage } \\
\text { height } \\
\text { (feet) }\end{array}$ & $\begin{array}{c}\text { Gage } \\
\text { height } \\
\text { code }\end{array}$ \\
\hline 2010 & Jan. 21 & 3,060 & & 14.94 & \\
2011 & Jan. 6 & 824 & 7.43 & \\
2012 & June 11 & 1,110 & 9.26 & \\
2013 & Feb. 26 & 1,760 & 12.65 & \\
2014 & Apr. 19 & 2,320 & 13.82 & \\
2015 & Dec. 25 & 800 & 7.52 &
\end{tabular}

02374745 Burnt Corn Creek at State Hwy 41 near Brewton, Ala.

L ocation-Lat $31^{\circ} 07^{\prime} 47^{\prime \prime}$, long $87^{\circ} 05^{\prime} 14^{\prime \prime}$ referenced to North American Datum of 1927, Escambia County, AL, Hydrologic Unit 03140304, near right bank at bridge on State Highway 41, 1.8 mi northwest of Brewton, 3.2 mi upstream of Murder Creek, and at river mile 3.2.

Drainage area-182 $\mathrm{mi}^{2}$.

G age -Water-stage recorder. Elevation of gage is $75 \mathrm{ft}$ above NGVD of 1929, from topographic map.

\begin{tabular}{llrcrr}
\hline $\begin{array}{c}\text { Water } \\
\text { year }\end{array}$ & Date & Discharge & $\begin{array}{c}\text { Discharge } \\
\text { code }\end{array}$ & $\begin{array}{c}\text { Gage } \\
\text { height } \\
\text { (feet) }\end{array}$ & $\begin{array}{c}\text { Gage } \\
\text { height } \\
\text { code }\end{array}$ \\
\hline 1998 & Sept. 30 & 18,400 & 7 & 22.15 & \\
1999 & Mar. 14 & 3,500 & & 12.22 & \\
2000 & Oct. 10 & 946 & 7.71 & \\
2001 & Mar. 4 & 8,300 & 17.56 & \\
2002 & Sept. 26 & 2,250 & 10.54 & \\
2003 & July 2 & 5,810 & 15.68 & \\
2004 & Apr. 30 & 3,650 & 13.09 & \\
2005 & Nov. 3 & 3,180 & 12.35 & \\
2006 & May 11 & 1,040 & 7.53 & \\
2007 & Apr. 2 & 2,840 & 12.18 & \\
2008 & Dec. 30 & 1,760 & 9.78 & \\
2009 & May 6 & 2,760 & 12.03 & \\
2010 & Dec. 15 & 21,000 & 23.03 & \\
2011 & Mar. 10 & 1,500 & 8.98 & \\
2012 & Feb. 18 & 1,590 & 9.28 & \\
2013 & Feb. 12 & 3,090 & 11.53 \\
2014 & May 15 & 4,510 & 13.94 \\
2015 & Apr. 16 & 1,930 & 9.24 \\
& & & &
\end{tabular}


Appendix 2. Discharge and gage height data for streamgages in Alabama._- Continued

[--, date or data not available; ft, foot; $\mathrm{ft}^{3} / \mathrm{s}$, cubic foot per second; mi, mile; $\mathrm{mi}^{2}$, square mile; qualification codes apply to the discharge data: 1 , discharge is a maximum daily average; 2 , discharge is an estimate; 4 , discharge is less than indicated value, which is the minimum recordable discharge at this site; 5 , discharge affected to unknown degree by regulation or diversion; 6 , discharge affected by regulation or diversion; 7, discharge is a historic peak; A, year of occurrence is unknown or not exact; $\mathrm{B}$, month or day of occurrence is unknown or not exact; E, only maximum peak available for this year. qualification codes apply to the gage height data: 1 , gage height affected by backwater; 2, gage height not the maximum for the year; 3, gage height at different data or at different site and datum;

5 , gage height is an estimate]

\section{Chewacla Creek at Chewacla State Park nr Auburn}

L ocation-Lat $32^{\circ} 32^{\prime} 53^{\prime \prime}$, long $85^{\circ} 28^{\prime} 50^{\prime \prime}$ referenced to North American Datum of 1927, Lee County, AL, Hydrologic Unit 03150110, at abandoned bridge in Chewacla State Park, $0.2 \mathrm{mi}$ downstream of Moores Mill Creek, downstream of Lake Ogletree, and $4 \mathrm{mi}$ south of Auburn, Ala.

Drainage area $-45 \mathrm{mi}^{2}$.

Gage -Water-stage recorder. Elevation of gage is $390 \mathrm{ft}$ above NGVD of 1929, from topographic map.

\begin{tabular}{|c|c|c|c|c|c|}
\hline $\begin{array}{c}\text { Water } \\
\text { year }\end{array}$ & Date & Discharge & $\begin{array}{l}\text { Discharge } \\
\text { code }\end{array}$ & $\begin{array}{c}\text { Gage } \\
\text { height } \\
\text { (feet) }\end{array}$ & $\begin{array}{c}\text { Gage } \\
\text { height } \\
\text { code }\end{array}$ \\
\hline 2003 & July 1 & 5,100 & & 9.19 & \\
\hline 2004 & Sept. 16 & 1,890 & & 5.93 & \\
\hline 2005 & Mar. 27 & 4,500 & & 8.61 & \\
\hline 2006 & Mar. 21 & 1,300 & & 5.19 & \\
\hline 2007 & Mar. 2 & 1,670 & & 5.69 & \\
\hline 2008 & Feb. 22 & 652 & & 4.06 & \\
\hline 2009 & Feb. 28 & 3,800 & & 7.95 & \\
\hline 2010 & Nov. 10 & 3,010 & & 7.34 & \\
\hline 2011 & July 20 & 1,980 & & 6.19 & \\
\hline 2012 & Feb. 19 & 886 & & 4.55 & \\
\hline 2013 & Aug. 15 & 9,210 & & 13.87 & \\
\hline 2014 & Apr. 7 & 4,560 & & 9.17 & \\
\hline 2015 & May 28 & 2,770 & & 7.24 & \\
\hline 2016 & Dec. 24 & 4,690 & & 9.30 & \\
\hline
\end{tabular}

\section{Chickasaw Creek near Kushla}

L ocation-Lat $30^{\circ} 48^{\prime} 10^{\prime \prime}$, long $88^{\circ} 08^{\prime} 36^{\prime \prime}$, in NE $1 / 4$ sec. 11 , T. 3 S., R. 2 W., Mobile County, Hydrologic Unit 03160204, 0.7 mi upstream from Seabury Creek, $1.4 \mathrm{mi}$ southeast of Kushla, $7 \mathrm{mi}$ northwest of Mobile, and at mile 12.2.

Drainage area-125 $\mathrm{mi}^{2}$.

Gage -Water-stage recorder. Datum of gage is $3.85 \mathrm{ft}$ NGVD 29. Prior to Aug. 2, 1964, water- stage recorder, Aug. 3, 1964 to Dec. 9, 1965, nonrecording gage, and Dec. 10, 1965 to September 1968, water-stage recorder at site $1.4 \mathrm{mi}$ upstream at different datum.

Remarks - Record for October 1951 to September 1968 published as 02471000, Chickasaw Creek near Whistler.

\begin{tabular}{|c|c|c|c|c|c|}
\hline $\begin{array}{c}\text { Water } \\
\text { year }\end{array}$ & Date & Discharge & $\begin{array}{l}\text { Discharge } \\
\text { code }\end{array}$ & $\begin{array}{c}\text { Gage } \\
\text { height } \\
\text { (feet) }\end{array}$ & $\begin{array}{c}\text { Gage } \\
\text { height } \\
\text { code }\end{array}$ \\
\hline 1952 & May 20 & 1,540 & & 10.10 & 3 \\
\hline 1953 & Apr. 26 & 1,920 & & 11.96 & 3 \\
\hline 1954 & Dec. 13 & 1,030 & & 7.38 & 3 \\
\hline 1955 & Apr. 13 & 35,000 & & 25.40 & 3 \\
\hline 1956 & Mar. 12 & 4,270 & & 14.40 & 3 \\
\hline 1957 & Dec. 24 & 7,090 & & 15.90 & 3 \\
\hline 1958 & Nov. 15 & 2,730 & & 12.70 & 3 \\
\hline 1959 & June 2 & 17,000 & & 20.63 & 3 \\
\hline 1960 & May 7 & 5,590 & & 15.36 & 3 \\
\hline 1961 & June 20 & 6,340 & & 15.90 & 3 \\
\hline 1962 & Nov. 15 & 14,700 & & 19.97 & 3 \\
\hline 1963 & Jan. 20 & 1,430 & & 9.30 & 3 \\
\hline 1964 & Apr. 27 & 4,610 & & 14.74 & 3 \\
\hline 1965 & Jan. 23 & 12,000 & & 18.80 & 3 \\
\hline 1966 & Oct. 1 & 2,500 & & 12.38 & 3 \\
\hline 1967 & Jan. 2 & 4,120 & & 14.35 & 3 \\
\hline 1968 & Oct. 31 & 4,330 & & 14.70 & 3 \\
\hline 1969 & Aug. 18 & 6,830 & & 17.58 & \\
\hline 1970 & June 3 & 3,870 & & 15.91 & \\
\hline 1971 & Dec. 17 & 3,960 & & 15.98 & \\
\hline 1972 & May 9 & 4,060 & & 16.06 & \\
\hline 1973 & Sept. 14 & 3,060 & & 15.05 & \\
\hline 1974 & Sept. 8 & 4,590 & & 16.42 & \\
\hline 1975 & Aug. 1 & 7,270 & & 17.79 & \\
\hline 1976 & Oct. 17 & 4,800 & & 16.55 & \\
\hline 1977 & Apr. 1 & 3,020 & & 14.96 & \\
\hline 1978 & Jan. 26 & 3,930 & & 15.96 & \\
\hline 1979 & Mar. 4 & 7,000 & & 17.66 & \\
\hline 1980 & Apr. 13 & 12,500 & & 19.70 & \\
\hline 1981 & Feb. 11 & 5,620 & & 16.99 & \\
\hline 1982 & Feb. 4 & 2,160 & & 13.31 & \\
\hline
\end{tabular}


Appendix 2. Discharge and gage height data for streamgages in Alabama.-Continued

[--, date or data not available; $\mathrm{ft}$, foot; $\mathrm{ft}^{3} / \mathrm{s}$, cubic foot per second; mi, mile; $\mathrm{mi}^{2}$, square mile; qualification codes apply to the discharge data: 1 , discharge is a maximum daily average; 2 , discharge is an estimate; 4 , discharge is less than indicated value, which is the minimum recordable discharge at this site; 5 , discharge affected to unknown degree by regulation or diversion; 6 , discharge affected by regulation or diversion; 7, discharge is a historic peak; A, year of occurrence is unknown or not exact; B, month or day of occurrence is unknown or not exact; E, only maximum peak available for this year. qualification codes apply to the gage height data: 1, gage height affected by backwater; 2, gage height not the maximum for the year; 3, gage height at different datum or at different site and datum; 5 , gage height is an estimate]

\section{Chickasaw Creek near Kushla-Continued}

L ocation-Lat $30^{\circ} 48^{\prime} 10^{\prime \prime}$, long $88^{\circ} 08^{\prime} 36^{\prime \prime}$, in NE $1 / 4$ sec. 11 , T. 3 S., R. 2 W., Mobile County, Hydrologic Unit 03160204, $0.7 \mathrm{mi}$ upstream from Seabury Creek, $1.4 \mathrm{mi}$ southeast of Kushla, $7 \mathrm{mi}$ northwest of Mobile, and at mile 12.2.

Drainage area-125 $\mathrm{mi}^{2}$.

Gage-Water-stage recorder. Datum of gage is $3.85 \mathrm{ft} \mathrm{NGVD} 29$. Prior to Aug. 2, 1964, water- stage recorder, Aug. 3, 1964 to Dec. 9, 1965, nonrecording gage, and Dec. 10, 1965 to September 1968, water-stage recorder at site 1.4 mi upstream at different datum.

Remarks - Record for October 1951 to September 1968 published as 02471000, Chickasaw Creek near Whistler.

\begin{tabular}{|c|c|c|c|c|c|}
\hline $\begin{array}{c}\text { Water } \\
\text { year }\end{array}$ & Date & Discharge & $\begin{array}{c}\text { Discharge } \\
\text { code }\end{array}$ & $\begin{array}{c}\text { Gage } \\
\text { height } \\
\text { (feet) }\end{array}$ & $\begin{array}{c}\text { Gage } \\
\text { height } \\
\text { code }\end{array}$ \\
\hline 1983 & Apr. 7 & 15,800 & & 20.38 & \\
\hline 1984 & May 23 & 4,220 & & 16.18 & \\
\hline 1985 & Sept. 24 & 4,180 & & 16.15 & \\
\hline 1986 & Feb. 6 & 3,280 & & 15.27 & \\
\hline 1987 & Mar. 19 & 2,080 & $\mathrm{E}$ & 13.29 & \\
\hline 1988 & Sept. 17 & 3,410 & & 15.42 & \\
\hline 1989 & June 9 & 2,220 & & 13.36 & \\
\hline 1990 & Mar. 16 & 15,300 & & 20.29 & \\
\hline 1991 & May 11 & 9,810 & & 18.88 & \\
\hline 1992 & Feb. 18 & 3,510 & & 15.53 & \\
\hline 1993 & May 26 & 6,680 & & 17.55 & \\
\hline 1994 & June 25 & 1,400 & & 10.92 & \\
\hline 1995 & Aug. 5 & 5,820 & & 17.10 & \\
\hline 1996 & Dec. 19 & 10,800 & & 19.20 & \\
\hline 1997 & July 20 & 4,140 & & 16.11 & \\
\hline 1998 & Sept. 29 & 19,900 & & 22.36 & \\
\hline 2000 & Oct. 8 & 2,920 & & 14.43 & \\
\hline 2001 & Mar. 4 & 3,620 & & 15.38 & \\
\hline 2002 & Sept. 26 & 7,520 & & 18.16 & \\
\hline 2003 & July 1 & 4,050 & & 15.85 & \\
\hline 2004 & May 1 & 5,820 & & 17.15 & \\
\hline 2005 & Apr. 1 & 6,520 & & 17.60 & \\
\hline 2006 & Feb. 26 & 753 & & 8.40 & \\
\hline 2007 & Jan. 1 & 1,940 & & 12.59 & \\
\hline 2008 & Sept. 2 & 8,920 & & 18.80 & \\
\hline 2009 & Mar. 28 & 6,110 & & 17.34 & \\
\hline 2010 & May 4 & 5,070 & & 16.65 & \\
\hline 2011 & July 17 & 1,530 & & 11.48 & \\
\hline 2012 & Aug. 31 & 4,100 & & 15.90 & \\
\hline 2013 & Feb. 26 & 5,150 & & 16.70 & \\
\hline 2014 & Apr. 30 & 6,840 & & 17.88 & \\
\hline 2015 & May 17 & 9,610 & & 19.09 & \\
\hline
\end{tabular}

02361000 Choctawhatchee River near Newton

L ocation-Lat $31^{\circ} 20^{\prime} 30^{\prime \prime}$, long $85^{\circ} 36^{\prime} 43^{\prime \prime}$, in SE $1 / 4$ sec. 2, T. 4 N., R. 24 E., Dale County, Hydrologic Unit 03140201, on State Highway 123, 0.8 mi north of Newton, 1 mi downstream from Atlantic Coast Line Railroad bridge, and at mile 133.0.

Drainage area - $686 \mathrm{mi}^{2}$.

G age -Water-stage recorder. Datum of gage is $138.56 \mathrm{ft}$ NGVD 29. See Water-Supply Papers 1304 or 1724 for history of changes prior to Sept. 9, 1938.

\begin{tabular}{|c|c|c|c|c|c|}
\hline $\begin{array}{c}\text { Water } \\
\text { year }\end{array}$ & Date & Discharge & $\begin{array}{l}\text { Discharge } \\
\text { code }\end{array}$ & $\begin{array}{c}\text { Gage } \\
\text { height } \\
\text { (feet) }\end{array}$ & $\begin{array}{c}\text { Gage } \\
\text { height } \\
\text { code }\end{array}$ \\
\hline 1922 & June 2 & 11,400 & & 16.62 & \\
\hline 1923 & Mar. 20 & 13,200 & & 18.53 & \\
\hline 1924 & Jan. 25 & 7,340 & & 10.60 & \\
\hline 1925 & Jan. 17 & 28,000 & & 28.00 & \\
\hline 1926 & Sept. 22 & 14,700 & & 23.22 & \\
\hline 1927 & Feb. 18 & 3,760 & & 6.40 & \\
\hline 1929 & Mar. 15 & 110,000 & 7 & 42.00 & \\
\hline 1935 & July 14 & 5,550 & & 12.42 & \\
\hline 1936 & Jan. 20 & 25,800 & & 29.50 & \\
\hline 1937 & Sept. 2 & 16,200 & & 26.40 & \\
\hline 1938 & Nov. 14 & 9,650 & & 20.10 & \\
\hline 1939 & Mar. 1 & 9,430 & & 19.66 & \\
\hline 1940 & Feb. 19 & 9,600 & & 20.03 & \\
\hline 1941 & July 16 & 2,580 & & 6.98 & \\
\hline 1942 & Dec. 26 & 4,700 & & 10.76 & \\
\hline 1943 & Jan. 20 & 19,300 & & 27.40 & \\
\hline 1944 & Apr. 16 & 12,500 & & 23.40 & \\
\hline 1945 & Apr. 25 & 3,300 & & 9.61 & \\
\hline 1946 & Mar. 29 & 14,600 & & 25.00 & \\
\hline 1947 & Mar. 9 & 12,800 & & 23.66 & \\
\hline 1948 & Mar. 8 & 10,700 & & 21.80 & \\
\hline 1949 & Nov. 29 & 8,840 & & 18.90 & \\
\hline 1950 & Apr. 5 & 3,900 & & 10.70 & \\
\hline 1951 & Mar. 29 & 5,400 & & 12.60 & \\
\hline 1952 & Feb. 16 & 5,740 & & 12.60 & \\
\hline 1953 & May 4 & 23,900 & & 29.60 & \\
\hline 1954 & Dec. 7 & 12,000 & & 23.00 & \\
\hline 1955 & Apr. 15 & 6,780 & & 14.69 & \\
\hline 1956 & Sept. 27 & 13,000 & & 24.54 & \\
\hline 1957 & Apr. 6 & 16,800 & & 26.60 & \\
\hline 1958 & Mar. 9 & 5,000 & & 11.20 & \\
\hline 1959 & Feb. 5 & 6,980 & & 15.30 & \\
\hline
\end{tabular}


Appendix 2. Discharge and gage height data for streamgages in Alabama.-Continued

[--, date or data not available; $\mathrm{ft}$, foot; $\mathrm{ft}^{3} / \mathrm{s}$, cubic foot per second; $\mathrm{mi}$, mile; $\mathrm{mi}^{2}$, square mile; qualification codes apply to the discharge data: 1 , discharge is a maximum daily average; 2 , discharge is an estimate; 4 , discharge is less than indicated value, which is the minimum recordable discharge at this site; 5 , discharge affected to unknown degree by regulation or diversion; 6 , discharge affected by regulation or diversion; 7, discharge is a historic peak; A, year of occurrence is unknown or not exact; B, month or day of occurrence is unknown or not exact; E, only maximum peak available for this year. qualification codes apply to the gage height data: 1, gage height affected by backwater; 2 , gage height not the maximum for the year; 3 , gage height at different data or at different site and datum;

5 , gage height is an estimate]

\section{Choctawhatchee River near Newton-Continued}

L ocation-Lat $31^{\circ} 20^{\prime} 30^{\prime \prime}$, long $85^{\circ} 36^{\prime} 43^{\prime \prime}$, in SE 1/4 sec. 2, T. 4 N., R. 24 E., Dale County, Hydrologic Unit 03140201, on State Highway 123, 0.8 mi north of Newton, 1 mi downstream from Atlantic Coast Line Railroad bridge, and at mile 133.0.

Drainage area $-686 \mathrm{mi}^{2}$.

G age - Water-stage recorder. Datum of gage is $138.56 \mathrm{ft}$ NGVD 29. See Water-Supply Papers 1304 or 1724 for history of changes prior to Sept. 9, 1938.

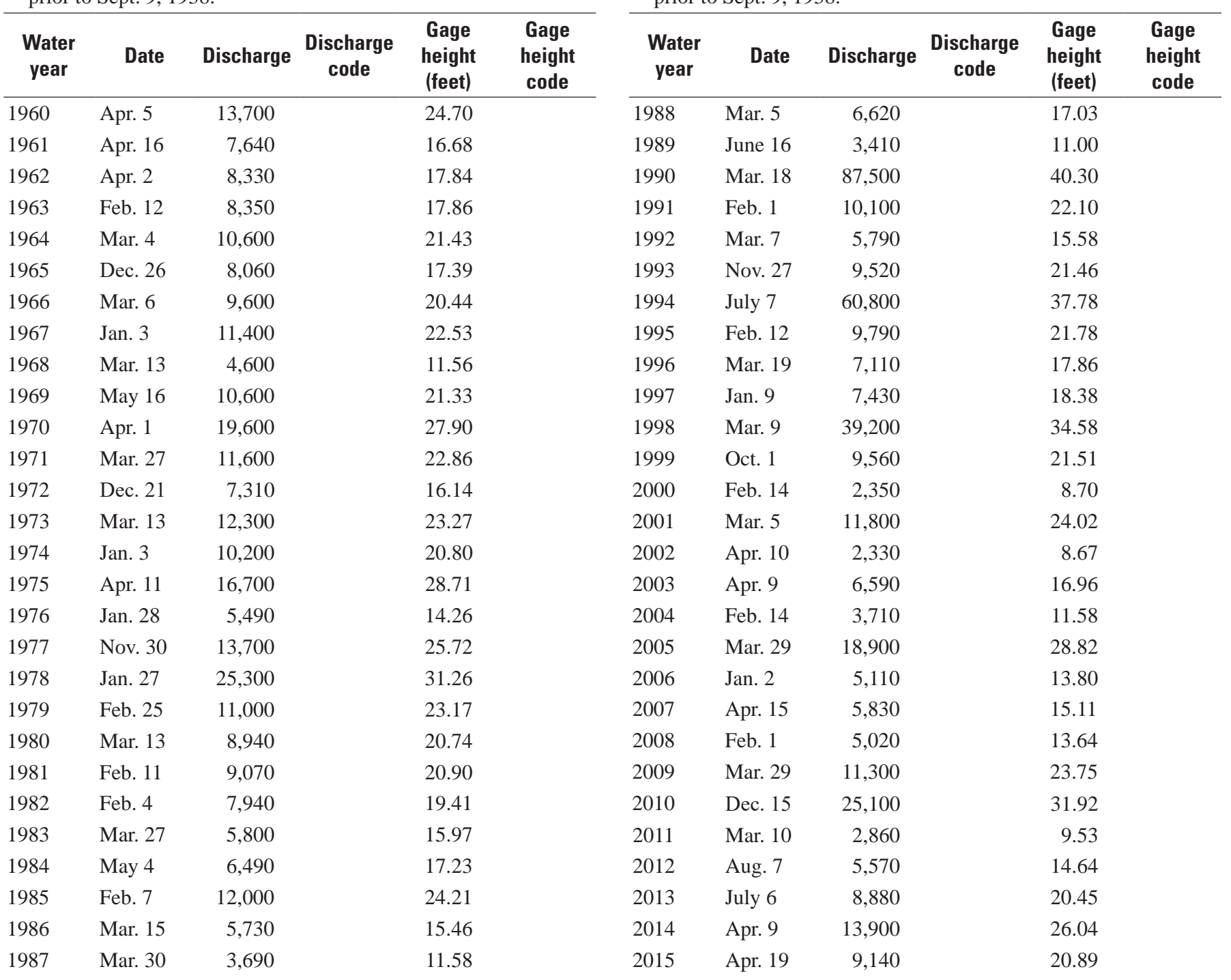

02361000 Choctawhatchee River near Newton-Continued

L ocation-Lat $31^{\circ} 20^{\prime} 30^{\prime \prime}$, long $85^{\circ} 36^{\prime} 43^{\prime \prime}$, in SE 1/4 sec. 2, T. 4 N., R. 24 E., Dale County, Hydrologic Unit 03140201, on State Highway 123, 0.8 mi north of Newton, 1 mi downstream from Atlantic Coast Line Railroad bridge, and at mile 133.0.

Drainage area $686 \mathrm{mi}^{2}$.

G age - Water-stage recorder. Datum of gage is $138.56 \mathrm{ft}$ NGVD 29. See Water-Supply Papers 1304 or 1724 for history of changes prior to Sept. 9, 1938. 
Appendix 2. Discharge and gage height data for streamgages in Alabama.-Continued

[--, date or data not available; $\mathrm{ft}$, foot; $\mathrm{ft}^{3} / \mathrm{s}$, cubic foot per second; mi, mile; $\mathrm{mi}^{2}$, square mile; qualification codes apply to the discharge data: 1 , discharge is a maximum daily average; 2 , discharge is an estimate; 4 , discharge is less than indicated value, which is the minimum recordable discharge at this site; 5 , discharge affected to unknown degree by regulation or diversion; 6 , discharge affected by regulation or diversion; 7, discharge is a historic peak; A, year of occurrence is unknown or not exact; $\mathrm{B}$, month or day of occurrence is unknown or not exact; E, only maximum peak available for this year. qualification codes apply to the gage height data: 1, gage height affected by backwater; 2, gage height not the maximum for the year; 3, gage height at different datum or at different site and datum; 5 , gage height is an estimate]

\section{Conecuh River near Andalusia}

L ocation-Lat $31^{\circ} 15^{\prime} 19^{\prime \prime}$, long $86^{\circ} 36^{\prime} 01^{\prime \prime}$, in NE $1 / 4$ sec. 1, T. 3 N., R. 14 E., Covington County, Hydrologic Unit 03140301, on county road, 0.5 mi upstream from Simmons Mill Creek, and $7.5 \mathrm{mi}$ southwest of Andalusia.

Drainage area-1,344 $\mathrm{mi}^{2}$.

Gage -Water-stage recorder 1905-19, 1930-52, 1966-70. Creststage gage 1953-65. Datum of gage is $106.77 \mathrm{ft}$ NGVD 29.

Remarks - Figures represent total period of record and reflect effects of regulation by Gantt and Point A Reservoirs and by hydroelectric plants.

\begin{tabular}{|c|c|c|c|c|c|}
\hline $\begin{array}{l}\text { Water } \\
\text { year }\end{array}$ & Date & Discharge & $\begin{array}{l}\text { Discharge } \\
\text { code }\end{array}$ & $\begin{array}{c}\text { Gage } \\
\text { height } \\
\text { (feet) }\end{array}$ & $\begin{array}{c}\text { Gage } \\
\text { height } \\
\text { code }\end{array}$ \\
\hline 1905 & Feb. 18 & 13,100 & & 24.10 & \\
\hline 1906 & Sept. 29 & 8,050 & & 16.60 & \\
\hline 1907 & Oct. 4 & 8,830 & & 17.90 & \\
\hline 1908 & Mar. 28 & 20,200 & & 32.10 & \\
\hline 1909 & June 6 & 14,100 & & 25.50 & \\
\hline 1910 & Apr. 21 & 7,940 & & 16.50 & \\
\hline 1911 & Apr. 2 & 6,750 & & 14.40 & \\
\hline 1912 & Apr. 22 & 19,600 & & 31.60 & \\
\hline 1913 & Mar. 18 & 50,000 & & 41.40 & \\
\hline 1914 & Feb. 10 & 2,970 & & 7.70 & \\
\hline 1915 & Jan. 25 & 7,380 & & 15.50 & \\
\hline 1916 & July 8 & 18,700 & & 30.70 & \\
\hline 1917 & Mar. 9 & 12,200 & & 22.80 & \\
\hline 1918 & Oct. 3 & 17,600 & & 29.50 & \\
\hline 1919 & Dec. 26 & 25,300 & & 34.60 & \\
\hline 1929 & Mar. 15 & 92,000 & & 47.60 & \\
\hline 1930 & Nov. 20 & 10,500 & & 23.92 & \\
\hline 1931 & Nov. 20 & 13,600 & & 26.90 & \\
\hline 1932 & Jan. 6 & 6,640 & & 17.42 & \\
\hline 1933 & Mar. 24 & 18,400 & & 30.43 & \\
\hline 1934 & Mar. 9 & 11,800 & & 24.68 & \\
\hline 1935 & Mar. 11 & 14,500 & & 27.77 & \\
\hline 1936 & Jan. 20 & 26,400 & & 34.65 & \\
\hline 1937 & Apr. 9 & 29,600 & & 36.17 & \\
\hline 1938 & Mar. 17 & 33,300 & & 37.30 & \\
\hline 1939 & Aug. 20 & 36,200 & & 38.50 & \\
\hline 1940 & Feb. 19 & 12,500 & & 26.40 & \\
\hline 1941 & Mar. 10 & 5,340 & & 14.34 & \\
\hline 1942 & Dec. 30 & 9,710 & & 22.46 & \\
\hline
\end{tabular}

02372500 Conecuh River near Andalusia-Continued

L ocation-Lat $31^{\circ} 15^{\prime} 19^{\prime \prime}$, long $86^{\circ} 36^{\prime} 01^{\prime \prime}$, in NE 1/4 sec. 1 , T. 3 N., R. 14 E., Covington County, Hydrologic Unit 03140301, on county road, $0.5 \mathrm{mi}$ upstream from Simmons Mill Creek, and 7.5 mi southwest of Andalusia.

Drainage area-1,344 $\mathrm{mi}^{2}$.

G age - Water-stage recorder 1905-19, 1930-52, 1966-70. Creststage gage 1953-65. Datum of gage is $106.77 \mathrm{ft}$ NGVD 29.

Remarks - Figures represent total period of record and reflect effects of regulation by Gantt and Point A Reservoirs and by hydroelectric plants.

\begin{tabular}{|c|c|c|c|c|c|}
\hline $\begin{array}{c}\text { Water } \\
\text { year }\end{array}$ & Date & Discharge & $\begin{array}{l}\text { Discharge } \\
\text { code }\end{array}$ & $\begin{array}{c}\text { Gage } \\
\text { height } \\
\text { (feet) }\end{array}$ & $\begin{array}{c}\text { Gage } \\
\text { height } \\
\text { code }\end{array}$ \\
\hline 1943 & Mar. 24 & 32,300 & & 37.40 & \\
\hline 1944 & Mar. 24 & 31,200 & & 36.80 & \\
\hline 1945 & Apr. 29 & 9,060 & & 21.40 & \\
\hline 1946 & May 22 & 18,100 & & 31.20 & \\
\hline 1947 & Apr. 6 & 16,800 & & 30.50 & \\
\hline 1948 & Mar. 8 & 22,100 & & 33.40 & \\
\hline 1949 & Nov. 30 & 35,400 & & 37.50 & \\
\hline 1950 & Apr. 9 & 10,300 & & 23.60 & \\
\hline 1951 & Apr. 21 & 7,300 & & 17.80 & \\
\hline 1952 & Mar. 29 & 16,900 & & 30.70 & \\
\hline 1953 & May 9 & 18,900 & & 31.80 & \\
\hline 1954 & Dec. 8 & 19,000 & & 31.90 & \\
\hline 1955 & Apr. 18 & 18,000 & & 31.30 & \\
\hline 1956 & Mar. 18 & 8,700 & & 20.40 & \\
\hline 1957 & Apr. 7 & 14,400 & & 29.00 & \\
\hline 1958 & Mar. 8 & 7,500 & & 18.20 & \\
\hline 1959 & Mar. 30 & 12,600 & & 26.70 & \\
\hline 1960 & Apr. 5 & 23,000 & & 33.80 & \\
\hline 1961 & Feb. 23 & 20,100 & & 32.45 & \\
\hline 1962 & Apr. 4 & 10,800 & & 24.51 & \\
\hline 1963 & Jan. 22 & 9,270 & & 21.54 & \\
\hline 1964 & May 6 & 11,100 & & 25.02 & \\
\hline 1965 & Feb. 22 & 10,300 & & 23.53 & \\
\hline 1966 & Mar. 6 & 19,800 & & 32.32 & \\
\hline 1967 & Jan. 2 & 10,400 & & 23.71 & \\
\hline 1968 & Dec. 13 & 4,580 & & 12.33 & \\
\hline 1969 & Sept. 23 & 5,510 & & 14.22 & \\
\hline 1970 & Apr. 11 & 26,500 & & 35.04 & \\
\hline 1975 & Apr. 11 & 32,000 & 7 & 36.70 & \\
\hline
\end{tabular}


Appendix 2. Discharge and gage height data for streamgages in Alabama.-Continued

[--, date or data not available; ft, foot; $\mathrm{ft}^{3} / \mathrm{s}$, cubic foot per second; mi, mile; $\mathrm{mi}^{2}$, square mile; qualification codes apply to the discharge data: 1 , discharge is a maximum daily average; 2 , discharge is an estimate; 4 , discharge is less than indicated value, which is the minimum recordable discharge at this site; 5 , discharge affected to unknown degree by regulation or diversion; 6 , discharge affected by regulation or diversion; 7, discharge is a historic peak; A, year of occurrence is unknown or not exact; $\mathrm{B}$, month or day of occurrence is unknown or not exact; E, only maximum peak available for this year. qualification codes apply to the gage height data: 1, gage height affected by backwater; 2, gage height not the maximum for the year; 3, gage height at different data or at different site and datum;

5 , gage height is an estimate]

\section{Conecuh River at Brantley}

L ocation-Lat $31^{\circ} 34^{\prime} 24^{\prime \prime}$, long $86^{\circ} 15^{\prime} 06^{\prime \prime}$, in SE 1/4 sec. 16, T. 7

N., R. 18 E., Crenshaw County, Hydrologic Unit 03140301, on

U.S. Highway 331 and State Highway 52, 0.8 mi southeast of

Brantley, and at mile 112.3.

Drainage area-500 $\mathrm{mi}^{2}$.

Gage-Water-stage recorder. Datum of gage is $226.2 \mathrm{ft}$ NGVD 29.

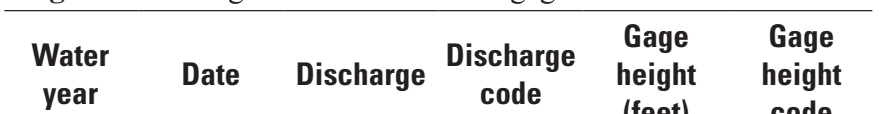

\begin{tabular}{lllllc}
\hline 1929 & -- & 32,000 & $2,7, B$ & 26.00 & 5 \\
1938 & Mar. 16 & 15,400 & & 22.75 & \\
1939 & Aug. 19 & 15,600 & & 22.87 &
\end{tabular}

194

1941

1942

July 2

6,500

17.49

Mar. 13

1,430

Dec. 27

5,700

1943

Mar. 23

15,600

1944

1945

Mar. 25

15,000

Apr. 30

1,970

May 22

9,920

1947

Apr. 4

10,800

Mar. 7

7,850

Nov. 29

15,800

1950

Apr. 8

3,330

1951

Apr. 20

1,810

1952

Mar. 26

11,300

May 7

15,000

1954

Dec. 7

9,250

1955

1956

Apr. 18

4,030

Mar. 19

3,940

1957

Apr. 8

8,610

1958

1959

Mar. 12

4,600

6,450

1960

Mar. 30

14,400

1961

1962

Apr. 4

Sept. 2

14,000

Apr. 3

5,460

Jan. 24

4,420

1964

May 5

5,280

1965

Dec. 29

6,630

Mar. 4

8,280

2,990
9.81

17.00

22.87

22.57

11.90

19.87

20.43

18.60

23.00

14.50

11.39

20.70

22.59

19.52

15.41

15.29

19.10

16.00

17.60

22.30

22.11

16.80

15.82

16.64

17.74

18.89

14.01
02371500 Conecuh River at Brantley-Continued

L ocation-Lat $31^{\circ} 34^{\prime} 24^{\prime \prime}$, long $86^{\circ} 15^{\prime} 06^{\prime \prime}$, in SE $1 / 4$ sec. 16, T. 7 N., R. 18 E., Crenshaw County, Hydrologic Unit 03140301, on U.S. Highway 331 and State Highway 52, $0.8 \mathrm{mi}$ southeast of Brantley, and at mile 112.3.

Drainage area $500 \mathrm{mi}^{2}$.

G age - Water-stage recorder. Datum of gage is $226.2 \mathrm{ft} \mathrm{NGVD} 29$.

\begin{tabular}{lccccc}
\hline $\begin{array}{c}\text { Water } \\
\text { year }\end{array}$ & Date & Discharge & $\begin{array}{c}\text { Discharge } \\
\text { code }\end{array}$ & $\begin{array}{c}\text { Gage } \\
\text { height } \\
\text { (feet) }\end{array}$ & $\begin{array}{c}\text { Gage } \\
\text { height } \\
\text { code }\end{array}$ \\
\hline 1968 & Dec. 11 & 1,560 & & 10.22 & \\
1969 & Mar. 29 & 2,250 & & 12.68 & \\
1970 & June 6 & 9,010 & & 19.35 &
\end{tabular}

$1971 \quad$ Jan. $7 \quad 11,300 \quad 20.69$

1972 Mar. $4 \quad 11,200 \quad 20.65$

1973 Apr. $2 \quad 15,500 \quad 22.84$

1974 Apr. $8 \quad 4,380 \quad 15.78$

1975 Feb. $19 \quad 21,000 \quad 24.51$

1976 May $18 \quad 5,880 \quad 17.15$

1977 Mar. $25 \quad 3,990 \quad 15.34$

1978 Jan. $27 \quad 15,200 \quad 22.72$

1979 Apr. $6 \quad 10,000 \quad 19.96$

1980 Apr. $16 \quad 8,270 \quad 18.87$

$1981 \quad$ Feb. $11 \quad 5,970 \quad 17.03$

$1982 \quad$ Feb. $6 \quad 7,550 \quad 18.35$

1983 Mar. $30 \quad 6,220 \quad 17.24$

1984 Mar. $7 \quad 2,820 \quad 13.25$

1985 Mar. $2 \quad 2,810 \quad$ E $\quad 13.23$

1986 Mar. $17 \quad$ 8,250 18.86

1987 Mar. $5 \quad 2,950 \quad$ E $\quad 13.53$

$1988 \quad$ Jan. $23 \quad 4,760 \quad$ E $\quad 15.76$

1989 June $19 \quad 7,090 \quad 18.49$

$1990 \quad$ Mar. $18 \quad 24,000 \quad 24.44$

1991 Mar. $6 \quad 4,900 \quad 16.22$

$1992 \quad$ Jan. $17 \quad 5,150 \quad 16.55$

$1993 \quad$ Nov. $28 \quad 11,400 \quad 20.58$

$1994 \quad$ July $9 \quad 17,000 \quad 22.37$

1995 Mar. $11 \quad 4,860 \quad 16.16$

1996 Oct. $8 \quad 6,630 \quad 18.11$

1997 May $1 \quad 4,430 \quad 15.56$

1998 Mar. $10 \quad 17,000 \quad 22.62$ 
Appendix 2. Discharge and gage height data for streamgages in Alabama.-Continued

[--, date or data not available; $\mathrm{ft}$, foot; $\mathrm{ft}^{3} / \mathrm{s}$, cubic foot per second; mi, mile; $\mathrm{mi}^{2}$, square mile; qualification codes apply to the discharge data: 1 , discharge is a maximum daily average; 2 , discharge is an estimate; 4 , discharge is less than indicated value, which is the minimum recordable discharge at this site; 5 , discharge affected to unknown degree by regulation or diversion; 6 , discharge affected by regulation or diversion; 7, discharge is a historic peak; A, year of occurrence is unknown or not exact; $\mathrm{B}$, month or day of occurrence is unknown or not exact; E, only maximum peak available for this year. qualification codes apply to the gage height data: 1, gage height affected by backwater; 2, gage height not the maximum for the year; 3, gage height at different datum or at different site and datum; 5 , gage height is an estimate]

\section{Conecuh River at Brantley-Continued}

L ocation-Lat $31^{\circ} 34^{\prime} 24^{\prime \prime}$, long $86^{\circ} 15^{\prime} 06^{\prime \prime}$, in SE $1 / 4$ sec. 16 , T. 7 N., R. 18 E., Crenshaw County, Hydrologic Unit 03140301, on U.S. Highway 331 and State Highway 52, $0.8 \mathrm{mi}$ southeast of Brantley, and at mile 112.3.

Drainage area $-500 \mathrm{mi}^{2}$.

Gage-Water-stage recorder. Datum of gage is $226.2 \mathrm{ft}$ NGVD 29.

\begin{tabular}{|c|c|c|c|c|c|}
\hline $\begin{array}{l}\text { Water } \\
\text { year }\end{array}$ & Date & Discharge & $\begin{array}{l}\text { Discharge } \\
\text { code }\end{array}$ & $\begin{array}{c}\text { Gage } \\
\text { height } \\
\text { (feet) }\end{array}$ & $\begin{array}{c}\text { Gage } \\
\text { height } \\
\text { code }\end{array}$ \\
\hline 1999 & July 1 & 6,980 & & 18.40 & \\
\hline 2000 & Mar. 25 & 1,660 & & 9.41 & \\
\hline 2001 & Mar. 6 & 16,900 & & 22.59 & \\
\hline 2002 & Mar. 26 & 2,100 & & 10.49 & \\
\hline 2003 & June 22 & 4,900 & & 16.21 & \\
\hline 2004 & Sept. 17 & 4,920 & & 16.25 & \\
\hline 2005 & Apr. 4 & 6,650 & & 18.10 & \\
\hline 2006 & May 15 & 2,880 & & 12.94 & \\
\hline 2007 & Apr. 20 & 2,250 & & 11.30 & \\
\hline 2008 & Aug. 28 & 6,460 & & 17.96 & \\
\hline 2009 & Mar. 31 & 8,510 & & 19.31 & \\
\hline 2010 & Dec. 17 & 11,900 & & 20.53 & \\
\hline 2011 & Mar. 13 & 3,550 & & 13.75 & \\
\hline 2012 & Feb. 24 & 2,100 & & 10.02 & \\
\hline 2013 & Feb. 15 & 7,230 & & 18.68 & \\
\hline 2014 & Apr. 10 & 8,580 & & 19.24 & \\
\hline 2015 & Apr. 22 & 2,830 & & 12.00 & \\
\hline
\end{tabular}

02372422 Conecuh River Bel Pt A Dam nr River Falls, Ala.

L ocation-Lat $31^{\circ} 21^{\prime} 40^{\prime \prime}$, long $86^{\circ} 31^{\prime} 11^{\prime \prime}$ referenced to North American Datum of 1927, Covington County, Ala., Hydrologic Unit 03140301, on right bank wingwall of Point A Dam, 1.65 miles upstream of U.S. Highway 84 bridge, 1.4 miles northeast of River Falls, 4 miles northwest of Andalusia, and at river mile 79.50 .

Drainage area-1,273 $\mathrm{mi}^{2}$.

G age -Water-stage recorder. Datum of gage is $120.80 \mathrm{ft}$ above NGVD of 1929.

\begin{tabular}{|c|c|c|c|c|c|}
\hline $\begin{array}{c}\text { Water } \\
\text { year }\end{array}$ & Date & Discharge & $\begin{array}{l}\text { Discharge } \\
\text { code }\end{array}$ & $\begin{array}{c}\text { Gage } \\
\text { height } \\
\text { (feet) }\end{array}$ & $\begin{array}{c}\text { Gage } \\
\text { height } \\
\text { code }\end{array}$ \\
\hline 1999 & July 2 & 13,100 & & 27.18 & \\
\hline 2000 & Mar. 21 & 3,020 & & 11.02 & \\
\hline 2001 & Mar. 7 & 32,100 & & 42.58 & \\
\hline 2002 & Mar. 26 & 4,920 & & 13.33 & \\
\hline 2003 & Dec. 24 & 11,700 & & 24.33 & \\
\hline 2004 & Sept. 21 & 12,900 & & 26.35 & \\
\hline 2005 & Apr. 1 & 17,800 & & 34.65 & \\
\hline 2006 & May 16 & 6,340 & & 15.24 & \\
\hline 2007 & Apr. 15 & 6,640 & & 15.72 & \\
\hline 2008 & Aug. 30 & 9,510 & & 20.55 & \\
\hline 2009 & Apr. 1 & 21,600 & & 37.63 & \\
\hline 2010 & Dec. 15 & 30,000 & & 42.34 & \\
\hline 2011 & Mar. 9 & 7,180 & & 16.58 & \\
\hline 2012 & Sept. 4 & 7,790 & & 17.76 & \\
\hline 2013 & Feb. 17 & 13,100 & & 27.23 & \\
\hline 2014 & Apr. 11 & 21,400 & & 35.11 & \\
\hline 2015 & Oct. 2 & 800 & 8 & 9.06 & \\
\hline
\end{tabular}


Appendix 2. Discharge and gage height data for streamgages in Alabama.-Continued

[--, date or data not available; ft, foot; $\mathrm{ft}^{3} / \mathrm{s}$, cubic foot per second; mi, mile; $\mathrm{mi}^{2}$, square mile; qualification codes apply to the discharge data: 1 , discharge is a maximum daily average; 2 , discharge is an estimate; 4 , discharge is less than indicated value, which is the minimum recordable discharge at this site; 5 , discharge affected to unknown degree by regulation or diversion; 6 , discharge affected by regulation or diversion; 7, discharge is a historic peak; A, year of occurrence is unknown or not exact; B, month or day of occurrence is unknown or not exact; E, only maximum peak available for this year. qualification codes apply to the gage height data: 1 , gage height affected by backwater; 2, gage height not the maximum for the year; 3, gage height at different data or at different site and datum;

5 , gage height is an estimate]

\section{Conecuh River near Troy}

L ocation-Lat $31^{\circ} 50^{\prime} 40^{\prime \prime}$, long $85^{\circ} 59^{\prime} 41^{\prime \prime}$, in NE $1 / 4$ sec. 13 , T. 10 N., R. 20 E., Pike County, Hydrologic Unit 03140301, on U.S. Highway 231, 1.5 mi downstream from Mannings Creek, and 3 mi north of Troy.

Drainage area-257 $\mathrm{mi}^{2}$.

Gage -Water-stage recorder 1943-53. Crest-stage gage 1954-68. Datum of gage is $313.3 \mathrm{ft} \mathrm{NGVD} 29$.

\begin{tabular}{|c|c|c|c|c|c|}
\hline $\begin{array}{l}\text { Water } \\
\text { year }\end{array}$ & Date & Discharge & $\begin{array}{l}\text { Discharge } \\
\text { code }\end{array}$ & $\begin{array}{c}\text { Gage } \\
\text { height } \\
\text { (feet) }\end{array}$ & $\begin{array}{c}\text { Gage } \\
\text { height } \\
\text { code }\end{array}$ \\
\hline 1944 & Mar. 23 & 16,500 & & 15.58 & \\
\hline 1945 & Feb. 25 & 1,260 & & 9.06 & \\
\hline 1946 & May 21 & 7,700 & & 13.05 & \\
\hline 1947 & Apr. 2 & 10,500 & & 14.00 & \\
\hline 1948 & Mar. 7 & 5,800 & & 12.20 & \\
\hline 1949 & Nov. 28 & 18,000 & & 16.10 & \\
\hline 1950 & Apr. 6 & 2,870 & & 10.50 & \\
\hline 1951 & Mar. 21 & 825 & & 8.30 & \\
\hline 1952 & Mar. 25 & 9,660 & & 14.20 & \\
\hline 1953 & May 6 & 18,000 & & 15.96 & \\
\hline 1954 & Dec. 7 & 7,820 & & 13.50 & \\
\hline 1955 & Apr. 13 & 4,750 & & 12.00 & \\
\hline 1956 & Mar. 18 & 3,430 & & 11.20 & \\
\hline 1957 & Apr. 5 & 8,070 & & 13.60 & \\
\hline 1958 & Nov. 24 & 2,760 & & 10.70 & \\
\hline 1959 & Mar. 29 & 5,120 & & 12.20 & \\
\hline 1960 & Apr. 4 & 8,580 & & 13.80 & \\
\hline 1961 & Aug. 31 & 10,800 & & 14.58 & \\
\hline 1962 & Apr. 3 & 4,710 & & 11.98 & \\
\hline 1963 & Jan. 23 & 3,430 & & 11.20 & \\
\hline 1964 & Apr. 27 & 4,480 & & 11.85 & \\
\hline 1965 & Dec. 28 & 4,200 & & 11.69 & \\
\hline 1966 & Mar. 4 & 4,370 & & 11.79 & \\
\hline 1967 & Aug. 27 & 1,860 & & 9.92 & \\
\hline 1968 & Dec. 11 & 1,290 & & 9.29 & \\
\hline 1990 & Mar. 17 & 36,000 & 7 & 19.41 & \\
\hline 1994 & July 8 & 15,000 & 7 & 15.58 & \\
\hline
\end{tabular}

\section{Crooked Creek near Fairview}

L ocation-Lat $30^{\circ} 46^{\prime} 48^{\prime \prime}$, long $88^{\circ} 19^{\prime} 08^{\prime \prime}$, in SW 1/4 sec. 18 , T. 3 S., R. 4 W., Mobile County, Hydrologic Unit 03170008, on right bank 1 mi southwest of Fairview, and 4 mi southeast of Wilmer. Drainage area. $-8.08 \mathrm{mi}^{2}$.

Gage.-Water Stage Recorder. Datum of gage is $115 \mathrm{ft}$ NGVD 29 (from topographic map).

\begin{tabular}{|c|c|c|c|c|c|}
\hline $\begin{array}{l}\text { Water } \\
\text { year }\end{array}$ & Date & Discharge & $\begin{array}{c}\text { Discharge } \\
\text { code }\end{array}$ & $\begin{array}{c}\text { Gage } \\
\text { height } \\
\text { (feet) }\end{array}$ & $\begin{array}{c}\text { Gage } \\
\text { height } \\
\text { code }\end{array}$ \\
\hline 1991 & Jan. 7 & 490 & & 5.22 & \\
\hline 1992 & Feb. 17 & 360 & & 4.76 & \\
\hline 1993 & Jan. 21 & 1,195 & & 6.99 & \\
\hline 1994 & June 25 & 450 & & 5.12 & \\
\hline 1995 & May 10 & 1,425 & & 7.44 & \\
\hline 1996 & Dec. 18 & 2,200 & & 8.59 & \\
\hline 1997 & July 19 & 910 & & 6.38 & \\
\hline 1998 & Sept. 28 & 1,250 & & 7.11 & \\
\hline 1999 & Mar. 14 & 840 & & 6.22 & \\
\hline 2000 & Mar. 29 & 320 & & 4.60 & \\
\hline 2001 & Mar. 3 & 318 & & 4.59 & \\
\hline 2002 & Sept. 26 & 720 & & 5.92 & \\
\hline 2003 & July 1 & 485 & & 5.21 & \\
\hline 2004 & Sept. 16 & 725 & & 5.96 & \\
\hline 2005 & July 6 & 1,200 & & 7.08 & \\
\hline 2006 & Dec. 15 & 130 & & 4.11 & \\
\hline 2007 & Nov. 15 & 1,300 & & 7.25 & \\
\hline 2008 & Apr. 5 & 940 & & 6.53 & \\
\hline 2009 & Mar. 28 & 900 & & 6.40 & \\
\hline 2010 & Jan. 21 & 620 & & 5.69 & \\
\hline 2011 & Sept. 5 & 331 & & 4.78 & \\
\hline 2012 & May 2 & 782 & & 6.13 & \\
\hline 2013 & Feb. 26 & 618 & & 5.69 & \\
\hline 2014 & Apr. 30 & 713 & & 5.95 & \\
\hline 2015 & May 17 & 685 & & 5.59 & \\
\hline
\end{tabular}


Appendix 2. Discharge and gage height data for streamgages in Alabama.-Continued

[--, date or data not available; $\mathrm{ft}$, foot; $\mathrm{ft}^{3} / \mathrm{s}$, cubic foot per second; mi, mile; $\mathrm{mi}^{2}$, square mile; qualification codes apply to the discharge data: 1 , discharge is a maximum daily average; 2 , discharge is an estimate; 4 , discharge is less than indicated value, which is the minimum recordable discharge at this site; 5 , discharge affected to unknown degree by regulation or diversion; 6 , discharge affected by regulation or diversion; 7, discharge is a historic peak; A, year of occurrence is unknown or not exact; $\mathrm{B}$, month or day of occurrence is unknown or not exact; E, only maximum peak available for this year. qualification codes apply to the gage height data: 1, gage height affected by backwater; 2, gage height not the maximum for the year; 3, gage height at different datum or at different site and datum; 5 , gage height is an estimate]

$\mathbf{0 2 3 6 0 5 0 0}$ East Fork Choctawhatchee River near Midland City L ocation-Lat $31^{\circ} 22^{\prime} 23^{\prime \prime}$, long $85^{\circ} 28^{\prime} 38^{\prime \prime}$, in NW 1/4 sec. 31, T. 5 N., R. 26 E., Dale County, Hydrologic Unit 03140201, 4 mi upstream from West Fork Choctawhatchee River and 4 mi north of Midland City.

Drainage area $-291 \mathrm{mi}^{2}$.

G age -Water-stage recorder 1953-63. Crest-stage gage 1964-70. Datum of gage is $179.10 \mathrm{ft}$ NGVD 29.

\begin{tabular}{|c|c|c|c|c|c|}
\hline $\begin{array}{c}\text { Water } \\
\text { year }\end{array}$ & Date & Discharge & $\begin{array}{c}\text { Discharge } \\
\text { code }\end{array}$ & $\begin{array}{c}\text { Gage } \\
\text { height } \\
\text { (feet) }\end{array}$ & $\begin{array}{c}\text { Gage } \\
\text { height } \\
\text { code }\end{array}$ \\
\hline 1953 & May 4 & 15,700 & & 23.82 & \\
\hline 1954 & Dec. 6 & 6,390 & & 18.20 & \\
\hline 1955 & Apr. 14 & 1,900 & & 10.52 & \\
\hline 1956 & Sept. 27 & 4,500 & & 16.94 & \\
\hline 1957 & Apr. 7 & 8,690 & & 19.90 & \\
\hline 1958 & Mar. 9 & 1,860 & & 10.40 & \\
\hline 1959 & Feb. 6 & 2,230 & & 12.40 & \\
\hline 1960 & Apr. 5 & 5,150 & & 17.54 & \\
\hline 1961 & Apr. 15 & 4,150 & & 16.55 & \\
\hline 1962 & Apr. 2 & 4,030 & & 16.26 & \\
\hline 1963 & Sept. 29 & 3,140 & & 13.95 & \\
\hline 1966 & Mar. 5 & 3,900 & & 16.00 & \\
\hline 1967 & Jan. 3 & 5,900 & & 18.15 & \\
\hline 1968 & Mar. 14 & 1,890 & & 9.80 & \\
\hline 1969 & May 15 & 6,840 & & 18.88 & \\
\hline 1970 & Apr. 1 & 9,840 & & 20.90 & \\
\hline 1990 & Mar. 17 & 27,500 & 7 & 28.18 & \\
\hline 1994 & July 6 & 31,000 & 2,7 & 29.30 & \\
\hline
\end{tabular}

02364000 Pea River at Elba

L ocation-Lat $31^{\circ} 24^{\prime} 48^{\prime \prime}$, long $86^{\circ} 03^{\prime} 47^{\prime \prime}$, in SE $1 / 4$ sec. 8 , T. 5 N., R. 20 E., Coffee County, Hydrologic Unit 03140202, at bridge on U.S. Highway 84 at Elba.

Drainage area-959 $\mathrm{mi}^{2}$.

G age - Water-stage recorder. Datum of gage is $159.24 \mathrm{ft}$ NGVD 29.

\begin{tabular}{|c|c|c|c|c|c|}
\hline $\begin{array}{c}\text { Water } \\
\text { year }\end{array}$ & Date & Discharge & $\begin{array}{l}\text { Discharge } \\
\text { code }\end{array}$ & $\begin{array}{c}\text { Gage } \\
\text { height } \\
\text { (feet) }\end{array}$ & $\begin{array}{c}\text { Gage } \\
\text { height } \\
\text { code }\end{array}$ \\
\hline 1929 & Mar & 68,000 & $2,7, \mathrm{~B}$ & 43.50 & \\
\hline 1936 & Jan. 21 & 24,300 & & 29.55 & \\
\hline 1937 & Apr. 6 & 25,000 & & 30.00 & \\
\hline 1938 & Mar. 17 & 34,500 & & 35.00 & \\
\hline 1939 & Feb. 28 & 12,900 & & 20.80 & \\
\hline 1940 & Feb. 18 & 7,900 & & 16.00 & \\
\hline 1941 & Mar. 7 & 3,520 & & 10.23 & \\
\hline 1942 & Apr. 10 & 7,350 & & 15.40 & \\
\hline 1943 & Jan. 19 & 20,400 & & 26.80 & \\
\hline 1944 & Mar. 24 & 19,000 & & 25.80 & \\
\hline 1945 & Apr. 29 & 5,270 & & 12.80 & \\
\hline 1946 & May 21 & 14,600 & & 22.30 & \\
\hline 1947 & Apr. 3 & 11,800 & & 19.80 & \\
\hline 1948 & Mar. 7 & 13,300 & & 21.20 & 5 \\
\hline 1949 & Nov. 30 & 13,200 & & 21.10 & 5 \\
\hline 1950 & Sept. 1 & 7,000 & & 15.00 & 5 \\
\hline 1951 & Mar. 29 & 5,720 & & 13.40 & 5 \\
\hline 1952 & Mar. 27 & 9,650 & & 17.80 & 5 \\
\hline 1953 & Dec. 4 & 17,500 & & 24.60 & 5 \\
\hline 1954 & Jan. 1 & 4,550 & & 11.79 & 5 \\
\hline 1955 & Apr. 14 & 16,200 & & 23.60 & 5 \\
\hline 1956 & Sept. 25 & 19,000 & & 26.00 & \\
\hline 1957 & Apr. 5 & 16,500 & & 24.10 & \\
\hline 1960 & Apr. 5 & 27,500 & & 31.45 & \\
\hline 1966 & Mar. 5 & 15,500 & & 23.20 & \\
\hline 1970 & June 4 & 17,000 & & 24.40 & \\
\hline 1971 & Mar. 27 & 18,500 & & 25.65 & \\
\hline 1972 & Mar. 3 & 11,700 & & 19.70 & 5 \\
\hline 1973 & Mar. 12 & 20,700 & & 27.00 & \\
\hline 1974 & Jan. 1 & 10,500 & & 18.60 & \\
\hline 1975 & Feb. 19 & 38,200 & & 37.26 & \\
\hline 1976 & May 15 & 11,900 & & 19.90 & \\
\hline 1977 & Nov. 29 & 8,750 & & 16.90 & 5 \\
\hline 1978 & Jan. 26 & 22,900 & & 28.60 & 5 \\
\hline 1979 & Mar. 4 & 12,400 & & 20.40 & 5 \\
\hline 1980 & Mar. 13 & 11,100 & & 19.15 & 5 \\
\hline
\end{tabular}


Appendix 2. Discharge and gage height data for streamgages in Alabama._- Continued

[--, date or data not available; ft, foot; $\mathrm{ft}^{3} / \mathrm{s}$, cubic foot per second; mi, mile; $\mathrm{mi}^{2}$, square mile; qualification codes apply to the discharge data: 1 , discharge is a maximum daily average; 2 , discharge is an estimate; 4 , discharge is less than indicated value, which is the minimum recordable discharge at this site; 5 , discharge affected to unknown degree by regulation or diversion; 6 , discharge affected by regulation or diversion; 7, discharge is a historic peak; A, year of occurrence is unknown or not exact; B, month or day of occurrence is unknown or not exact; E, only maximum peak available for this year. qualification codes apply to the gage height data: 1, gage height affected by backwater; 2, gage height not the maximum for the year; 3, gage height at different data or at different site and datum; 5 , gage height is an estimate]

\section{Pea River at Elba-Continued}

L ocation-Lat $31^{\circ} 24^{\prime} 48^{\prime \prime}$, long $86^{\circ} 03^{\prime} 47^{\prime \prime}$, in SE $1 / 4$ sec. 8 , T. 5 N.,

R. 20 E., Coffee County, Hydrologic Unit 03140202, at bridge on U.S. Highway 84 at Elba.

Drainage area $-959 \mathrm{mi}^{2}$.

Gage - Water-stage recorder. Datum of gage is $159.24 \mathrm{ft}$ NGVD 29.

\begin{tabular}{|c|c|c|c|c|c|}
\hline $\begin{array}{c}\text { Water } \\
\text { year }\end{array}$ & Date & Discharge & $\begin{array}{l}\text { Discharge } \\
\text { code }\end{array}$ & $\begin{array}{c}\text { Gage } \\
\text { height } \\
\text { (feet) }\end{array}$ & $\begin{array}{c}\text { Gage } \\
\text { height } \\
\text { code }\end{array}$ \\
\hline 1981 & Feb. 12 & 15,900 & & 23.40 & \\
\hline 1982 & Feb. 4 & 8,420 & & 16.55 & \\
\hline 1983 & May 20 & 13,500 & & 21.35 & \\
\hline 1984 & Mar. 25 & 8,900 & & 17.05 & \\
\hline 1985 & Feb. 6 & 10,900 & & 19.00 & \\
\hline 1986 & Mar. 15 & 15,600 & & 23.15 & \\
\hline 1987 & Feb. 28 & 6,630 & & 14.55 & \\
\hline 1988 & Mar. 4 & 10,700 & & 18.85 & \\
\hline 1989 & June 17 & 10,200 & & 18.30 & \\
\hline 1990 & Mar. 17 & 58,000 & & 43.28 & \\
\hline 1991 & Jan. 31 & 11,200 & & 19.30 & \\
\hline 1992 & Jan. 14 & 8,950 & & 17.10 & \\
\hline 1993 & Nov. 26 & 10,200 & & 18.33 & \\
\hline 1994 & July 7 & 42,500 & & 38.33 & \\
\hline 1995 & Feb. 12 & 13,600 & & 21.40 & \\
\hline 1996 & Oct. 5 & 16,200 & & 23.62 & \\
\hline 1997 & Feb. 15 & 8,760 & & 16.91 & \\
\hline 1998 & Mar. 6 & 45,000 & & 39.23 & \\
\hline 1999 & Mar. 14 & 12,600 & & 20.51 & \\
\hline 2000 & Mar. 20 & 3,430 & & 10.07 & \\
\hline 2001 & Mar. 4 & 15,100 & & 22.70 & \\
\hline 2002 & Mar. 13 & 3,460 & & 10.12 & \\
\hline 2003 & Apr. 8 & 10,000 & & 18.17 & \\
\hline 2004 & Sept. 17 & 15,100 & & 22.96 & \\
\hline 2005 & Apr. 1 & 16,800 & & 24.34 & \\
\hline 2006 & Mar. 10 & 6,100 & & 13.73 & \\
\hline 2007 & Apr. 15 & 8,800 & & 16.90 & \\
\hline 2008 & Feb. 1 & 10,800 & & 18.91 & \\
\hline 2009 & Apr. 1 & 16,700 & & 23.98 & \\
\hline 2010 & Dec. 15 & 31,900 & & 33.73 & \\
\hline 2011 & Mar. 10 & 5,500 & & 13.03 & \\
\hline 2012 & Sept. 4 & 7,660 & & 15.74 & \\
\hline 2013 & Feb. 26 & 11,500 & & 19.53 & \\
\hline 2014 & Apr. 8 & 18,700 & & 25.58 & \\
\hline 2015 & Apr. 19 & 7,730 & & 15.82 & \\
\hline
\end{tabular}

02479560 Escatawpa River near Agricola, Miss.

L ocation-Lat $30^{\circ} 48^{\prime} 12^{\prime \prime}$, long $88^{\circ} 27^{\prime} 31^{\prime \prime}$, in SW 1/4 SW 1/4 sec. 2, T. 3 S., R. 5 W., George County, Miss., Hydrologic Unit 03170008 , on county road 612, 2.5 mi west of AlabamaMississippi State line, $3.7 \mathrm{mi}$ east of Agricola, $6.7 \mathrm{mi}$ west of Wilmer, Ala., and 50.6 mi upstream from mouth.

Drainage area $-562 \mathrm{mi}^{2}$.

Gage -Water-stage recorder. Datum of gage is $50 \mathrm{ft} \mathrm{NGVD} 29$ (from topographic map).

\begin{tabular}{|c|c|c|c|c|c|}
\hline $\begin{array}{c}\text { Water } \\
\text { year }\end{array}$ & Date & Discharge & $\begin{array}{l}\text { Discharge } \\
\text { code }\end{array}$ & $\begin{array}{c}\text { Gage } \\
\text { height } \\
\text { (feet) }\end{array}$ & $\begin{array}{c}\text { Gage } \\
\text { height } \\
\text { code }\end{array}$ \\
\hline 1946 & Mar. 28 & 8,600 & 2 & -- & \\
\hline 1947 & Apr. 16 & 8,480 & 2 & -- & \\
\hline 1948 & Mar. 7 & 14,900 & 2 & -- & \\
\hline 1950 & Nov. 28 & 26,200 & 2 & -- & \\
\hline 1951 & Mar. 31 & 11,300 & 2 & -- & \\
\hline 1952 & May 20 & 5,280 & 2 & -- & \\
\hline 1953 & Feb. 25 & 6,000 & 2 & -- & \\
\hline 1955 & Dec. 8 & 5,920 & 2 & -- & \\
\hline 1956 & July 11 & 13,900 & 2 & -- & \\
\hline 1957 & Sept. 21 & 14,800 & 2 & -- & \\
\hline 1959 & Nov. 15 & 6,880 & 2 & -- & \\
\hline 1960 & May 7 & 14,600 & 2 & -- & \\
\hline 1961 & Feb. 25 & 17,400 & 2 & -- & \\
\hline 1963 & Dec. 13 & 21,100 & 2 & -- & \\
\hline 1964 & Apr. 28 & 7,930 & 2 & -- & \\
\hline 1965 & July 24 & 8,190 & 2 & -- & \\
\hline 1966 & Feb. 17 & 8,270 & 2 & -- & \\
\hline 1967 & Jan. 2 & 4,910 & 2 & -- & \\
\hline 1969 & Oct. 31 & 6,700 & 2 & -- & \\
\hline 1970 & May 5 & 5,330 & 2 & -- & \\
\hline 1972 & Dec. 17 & 6,690 & 2 & -- & \\
\hline 1973 & Sept. 14 & 7,890 & 2 & -- & \\
\hline 1974 & Apr. 17 & 11,700 & & 17.85 & \\
\hline 1975 & Apr. 12 & 12,500 & & 18.06 & \\
\hline 1976 & Oct. 3 & 6,285 & & 15.37 & \\
\hline 1977 & Apr. 1 & 8,500 & & 16.53 & \\
\hline 1978 & June 11 & 12,600 & & 18.10 & \\
\hline 1979 & Mar. 5 & 17,100 & & 19.67 & \\
\hline 1980 & Apr. 14 & 13,900 & & 18.56 & \\
\hline 1981 & Feb. 12 & 12,600 & & 18.20 & \\
\hline 1982 & Feb. 5 & 9,600 & & 16.96 & \\
\hline 1983 & Apr. 9 & 26,100 & & 22.39 & \\
\hline
\end{tabular}


Appendix 2. Discharge and gage height data for streamgages in Alabama.-Continued

[--, date or data not available; $\mathrm{ft}$, foot; $\mathrm{ft}^{3} / \mathrm{s}$, cubic foot per second; mi, mile; $\mathrm{mi}^{2}$, square mile; qualification codes apply to the discharge data: 1 , discharge is a maximum daily average; 2 , discharge is an estimate; 4 , discharge is less than indicated value, which is the minimum recordable discharge at this site; 5 , discharge affected to unknown degree by regulation or diversion; 6 , discharge affected by regulation or diversion; 7, discharge is a historic peak; A, year of occurrence is unknown or not exact; $\mathrm{B}$, month or day of occurrence is unknown or not exact; E, only maximum peak available for this year. qualification codes apply to the gage height data: 1, gage height affected by backwater; 2, gage height not the maximum for the year; 3, gage height at different datum or at different site and datum; 5 , gage height is an estimate]

\section{Escatawpa River near Agricola, Miss.-Continued} L ocation-Lat $30^{\circ} 48^{\prime} 12^{\prime \prime}$, long 88 $27^{\prime} 31^{\prime \prime}$, in SW 1/4 SW 1/4 sec. 2, T. 3 S., R. 5 W., George County, Miss., Hydrologic Unit 03170008 , on county road 612, $2.5 \mathrm{mi}$ west of AlabamaMississippi State line, $3.7 \mathrm{mi}$ east of Agricola, $6.7 \mathrm{mi}$ west of Wilmer, Ala., and 50.6 mi upstream from mouth.

Drainage area $-562 \mathrm{mi}^{2}$.

Gage - Water-stage recorder. Datum of gage is $50 \mathrm{ft}$ NGVD 29 (from topographic map).

\begin{tabular}{|c|c|c|c|c|c|c|c|c|c|c|c|}
\hline \multirow{2}{*}{\multicolumn{6}{|c|}{ (from topographic map). }} & \\
\hline & & & & & & \multirow[b]{2}{*}{$\begin{array}{c}\text { Water } \\
\text { year }\end{array}$} & \multirow[b]{2}{*}{ Date } & \multirow[b]{2}{*}{ Discharge } & \multirow[b]{2}{*}{$\begin{array}{l}\text { Discharge } \\
\text { code }\end{array}$} & \multirow{2}{*}{$\begin{array}{c}\text { Gage } \\
\text { height } \\
\text { (feet) }\end{array}$} & \multirow{2}{*}{$\begin{array}{c}\text { Gage } \\
\text { height } \\
\text { code }\end{array}$} \\
\hline \multirow{2}{*}{$\begin{array}{c}\text { Water } \\
\text { year }\end{array}$} & \multirow[t]{2}{*}{ Date } & \multirow[t]{2}{*}{ Discharge } & \multirow{2}{*}{$\begin{array}{l}\text { Discharge } \\
\text { code }\end{array}$} & \multirow{2}{*}{$\begin{array}{c}\text { Gage } \\
\text { height } \\
\text { (feet) }\end{array}$} & \multirow{2}{*}{$\begin{array}{c}\text { Gage } \\
\text { height } \\
\text { code }\end{array}$} & & & & & & \\
\hline & & & & & & 1954 & Dec. 6 & 9,200 & & 17.04 & \\
\hline 1984 & May 23 & 7,470 & & 16.24 & & 1955 & Dec. 6 & 443 & & 6.66 & \\
\hline 1985 & Sept. 25 & 13,100 & & 18.25 & & 1956 & Mar. 12 & 1,090 & & 9.71 & \\
\hline 1986 & Oct. 31 & 12,100 & & 17.84 & & 1950 & Apr 5 & $\begin{array}{l}1,090 \\
5080\end{array}$ & & 1470 & \\
\hline 1987 & Mar. 19 & 6,920 & & 15.42 & & 195/ & Apr. 5 & 5,080 & & 14.70 & \\
\hline 1988 & Mar. 5 & 7,000 & & 15.87 & & 1958 & Mar. 7 & 1,620 & & 10.93 & \\
\hline 1989 & May 21 & 5,600 & & 15.09 & & 1959 & Sept. 13 & 1,970 & & 11.50 & \\
\hline 1990 & Mar. 17 & 14,000 & 1,2 & -- & & 1960 & Apr. 3 & 1,520 & & 10.71 & \\
\hline 1991 & May 11 & 22,500 & & 19.91 & & 1961 & Apr. 12 & 4,570 & & 14.29 & \\
\hline 1992 & Feb. 19 & 8,080 & & 16.40 & & 1962 & Jan. 6 & 588 & & 8.01 & \\
\hline 1993 & Jan. 24 & 11,900 & & 17.47 & & 1963 & Jan. 21 & 690 & & 8.48 & \\
\hline 1994 & July 14 & 4,910 & & 14.51 & & 1964 & Apr. 27 & 5,470 & & 15.00 & \\
\hline 1995 & Feb. 13 & 7,990 & & 16.37 & & 1965 & July 11 & 559 & & 7.84 & \\
\hline 1996 & Dec. 20 & 10,400 & & 17.11 & & 1966 & Oct. 1 & 2,420 & & 12.13 & \\
\hline 1997 & June 22 & 4,880 & & 14.48 & & 1967 & Sept. 7 & 1,250 & & 10.18 & \\
\hline 1998 & Sept. 30 & 27,800 & & 22.81 & & 1968 & Dec. 11 & 330 & & 5.52 & \\
\hline 1999 & Feb. 2 & 8,350 & & 16.43 & & 1969 & July 24 & 4,220 & & 14.00 & \\
\hline 2000 & Oct. 11 & 3,900 & & 13.09 & & 1971 & Sept. 5 & 1,200 & & 10.04 & \\
\hline 2001 & Mar. 6 & $\begin{array}{r}13,800 \\
8,870\end{array}$ & & 18.60 & & 1987 & June 22 & 1,050 & & 10.37 & \\
\hline $\begin{array}{l}2002 \\
2003\end{array}$ & $\begin{array}{l}\text { Sept. } 28 \\
\text { July } 2\end{array}$ & $\begin{array}{r}8,870 \\
11,200\end{array}$ & & $\begin{array}{l}10.05 \\
17.49\end{array}$ & & 1988 & Sept. 17 & 1,060 & & 10.57 & \\
\hline 2004 & Apr. 30 & 6,590 & & 15.52 & & 1989 & June 9 & 9,470 & & 19.28 & \\
\hline 2005 & Apr. 2 & 9,400 & & 16.86 & & 1990 & Mar. 16 & 5,720 & & 16.77 & \\
\hline 2006 & Feb. 27 & 2,650 & & 10.72 & & 1991 & May 9 & 845 & & 10.00 & \\
\hline 2007 & Jan. 1 & 7,480 & & 16.00 & & 1992 & Jan. 13 & 4,570 & & 15.74 & \\
\hline 2008 & Sept. 4 & 7,150 & & 15.83 & & 1993 & Mar. 31 & 1,400 & & 11.32 & \\
\hline 2009 & Mar. 28 & 27,900 & & 22.82 & & 1994 & July 8 & 2,910 & & 13.89 & \\
\hline 2010 & Dec. 17 & 14,300 & & 18.79 & & 1995 & Mar. 8 & 1,610 & & 11.69 & \\
\hline 2011 & Mar. 13 & 6,400 & & 15.40 & & 1996 & Oct. 5 & 6,760 & & 17.56 & \\
\hline 2012 & Aug. 31 & 20,700 & & 20.79 & & 1997 & July 20 & 16,900 & & 22.78 & \\
\hline 2013 & May 3 & 11,200 & & 17.45 & & 1998 & Sept. 28 & 7,870 & & 18.31 & \\
\hline 2014 & Apr. 30 & 13,600 & & 18.30 & & 1999 & June 11 & 665 & & 9.05 & \\
\hline 2015 & May 17 & 6,750 & & 15.48 & & & & & & & \\
\hline
\end{tabular}

02378500 Fish River near Silver Hill

L ocation-Lat $30^{\circ} 32^{\prime} 43^{\prime \prime}$, long $87^{\circ} 47^{\prime} 55^{\prime \prime}$, NW 1/4 sec. 8, T. 6 S., R. 3 E., Baldwin County, Hydrologic Unit 03160205, on State Highway 104, 0.2 mi downstream from Caney Branch, $2.8 \mathrm{mi}$ west of Silver Hill, and 12 mi upstream from mouth.

Drainage area-55.3 $\mathrm{mi}^{2}$.

G age -Water-stage recorder. Datum of gage is $30 \mathrm{ft} \mathrm{NGVD} 29$ (from topographic map). 
Appendix 2. Discharge and gage height data for streamgages in Alabama.-Continued

[--, date or data not available; $\mathrm{ft}$, foot; $\mathrm{ft}^{3} / \mathrm{s}$, cubic foot per second; $\mathrm{mi}$, mile; $\mathrm{mi}^{2}$, square mile; qualification codes apply to the discharge data: 1 , discharge is a maximum daily average; 2 , discharge is an estimate; 4 , discharge is less than indicated value, which is the minimum recordable discharge at this site; 5 , discharge affected to unknown degree by regulation or diversion; 6 , discharge affected by regulation or diversion; 7, discharge is a historic peak; A, year of occurrence is unknown or not exact; B, month or day of occurrence is unknown or not exact; E, only maximum peak available for this year. qualification codes apply to the gage height data: 1, gage height affected by backwater; 2 , gage height not the maximum for the year; 3 , gage height at different data or at different site and datum;

5 , gage height is an estimate]

02378500 Fish River near Silver Hill-Continued

L ocation-Lat $30^{\circ} 32^{\prime} 43^{\prime \prime}$, long $87^{\circ} 47^{\prime} 55^{\prime \prime}$, NW 1/4 sec. 8, T. 6 S.,

R. 3 E., Baldwin County, Hydrologic Unit 03160205, on State

Highway 104, 0.2 mi downstream from Caney Branch, $2.8 \mathrm{mi}$

west of Silver Hill, and 12 mi upstream from mouth.

Drainage area $-55.3 \mathrm{mi}^{2}$.

Gage - Water-stage recorder. Datum of gage is $30 \mathrm{ft} \mathrm{NGVD} 29$ (from topographic map).

\begin{tabular}{|c|c|c|c|c|c|}
\hline $\begin{array}{l}\text { Water } \\
\text { year }\end{array}$ & Date & Discharge & $\begin{array}{l}\text { Discharge } \\
\text { code }\end{array}$ & $\begin{array}{c}\text { Gage } \\
\text { height } \\
\text { (feet) }\end{array}$ & $\begin{array}{c}\text { Gage } \\
\text { height } \\
\text { code }\end{array}$ \\
\hline 2000 & Mar. 30 & 600 & & 8.68 & \\
\hline 2001 & June 11 & 1,930 & & 12.39 & \\
\hline 2002 & Sept. 26 & 3,920 & & 15.08 & \\
\hline 2003 & July 1 & 3,050 & & 14.07 & \\
\hline 2004 & Sept. 16 & 4,970 & & 16.13 & \\
\hline 2005 & Apr. 1 & 11,400 & & 20.35 & \\
\hline 2006 & Apr. 22 & 344 & & 6.75 & \\
\hline 2007 & Oct. 18 & 541 & & 8.29 & \\
\hline 2008 & Sept. 1 & 3,130 & & 14.17 & \\
\hline 2009 & Mar. 28 & 7,300 & & 17.94 & \\
\hline 2010 & Jan. 21 & 1,830 & & 12.22 & \\
\hline 2011 & July 16 & 2,200 & & 12.87 & \\
\hline 2012 & June 10 & 1,020 & & 10.40 & \\
\hline 2013 & July 11 & 1,900 & & 11.84 & \\
\hline 2014 & Apr. 30 & 18,700 & & 23.06 & \\
\hline 2015 & Apr. 15 & 1,420 & & 10.48 & \\
\hline
\end{tabular}

02371200 Indian Creek near Troy

Location-Lat $31^{\circ} 48^{\prime} 50^{\prime \prime}$, long $86^{\circ} 07^{\prime} 15^{\prime \prime}$, in NE $1 / 4$ sec. 26 , T. 10

N., R. 19 E., Pike County, Hydrologic Unit 03140301, on U.S.

Highway 29, 3.5 mi upstream from mouth, and 9 mi west of Troy.

Drainage area $-8.87 \mathrm{mi}^{2}$.

G age -Water-stage recorder. Datum of gage is $354 \mathrm{ft}$ NGVD 29.

\begin{tabular}{lccccc}
\hline $\begin{array}{c}\text { Water } \\
\text { year }\end{array}$ & Date & Discharge & $\begin{array}{c}\text { Discharge } \\
\text { code }\end{array}$ & $\begin{array}{c}\text { Gage } \\
\text { height } \\
\text { (feet) }\end{array}$ & $\begin{array}{c}\text { Gage } \\
\text { height } \\
\text { code }\end{array}$ \\
\hline 1959 & Mar. 27 & 460 & & 5.42 & \\
1960 & Mar. 29 & 175 & & 4.48 & \\
1961 & Aug. 31 & 3,500 & & 7.60 &
\end{tabular}

02371200 Indian Creek near Troy-Continued

Location-Lat $31^{\circ} 48^{\prime} 50^{\prime \prime}$, long $86^{\circ} 07^{\prime} 15^{\prime \prime}$, in NE $1 / 4$ sec. 26 , T. 10 N., R. 19 E., Pike County, Hydrologic Unit 03140301, on U.S.

Highway 29, $3.5 \mathrm{mi}$ upstream from mouth, and $9 \mathrm{mi}$ west of Troy. Drainage area $-8.87 \mathrm{mi}^{2}$.

Gage-Water-stage recorder. Datum of gage is $354 \mathrm{ft} \mathrm{NGVD} 29$.

\begin{tabular}{|c|c|c|c|c|c|}
\hline $\begin{array}{l}\text { Water } \\
\text { year }\end{array}$ & Date & Discharge & $\begin{array}{l}\text { Discharge } \\
\text { code }\end{array}$ & $\begin{array}{c}\text { Gage } \\
\text { height } \\
\text { (feet) }\end{array}$ & $\begin{array}{c}\text { Gage } \\
\text { height } \\
\text { code }\end{array}$ \\
\hline 1962 & Mar. 31 & 535 & & 5.57 & \\
\hline 1963 & Jan. 20 & 177 & & 4.51 & \\
\hline 1964 & Apr. 27 & 323 & & 5.01 & \\
\hline 1965 & Sept. 30 & 285 & & 4.89 & \\
\hline 1966 & Mar. 4 & 342 & & 5.14 & \\
\hline 1967 & May 22 & 300 & & 5.00 & \\
\hline 1968 & Dec. 11 & 129 & & 4.26 & \\
\hline 1969 & Apr. 18 & 420 & & 5.29 & \\
\hline 1970 & June 3 & 1,740 & & 6.91 & \\
\hline 1971 & Mar. 25 & 1,340 & & 6.64 & \\
\hline 1972 & Mar. 1 & 684 & & 5.94 & \\
\hline 1973 & Dec. 31 & 752 & & 6.04 & \\
\hline 1974 & Apr. 4 & 629 & & 5.87 & \\
\hline 1975 & Feb. 17 & 3,950 & & 7.75 & \\
\hline 1976 & May 14 & 526 & & 5.71 & \\
\hline 1977 & Mar. 4 & 425 & & 5.52 & \\
\hline 1978 & Jan. 25 & 1,165 & & 6.45 & \\
\hline 1979 & Apr. 4 & 1,435 & & 6.65 & \\
\hline 1980 & Apr. 13 & 832 & & 6.14 & \\
\hline 1981 & Feb. 10 & 752 & & 6.04 & \\
\hline 1982 & Feb. 3 & 562 & & 5.77 & \\
\hline 1983 & Mar. 27 & 530 & & 5.74 & \\
\hline 1984 & Mar. 5 & 530 & & 5.74 & \\
\hline 1985 & Dec. 5 & 340 & & 5.37 & \\
\hline 1986 & Mar. 14 & 545 & & 5.77 & \\
\hline 1990 & Mar. 17 & 2,320 & 7 & 7.19 & \\
\hline
\end{tabular}


Appendix 2. Discharge and gage height data for streamgages in Alabama.-Continued

[--, date or data not available; $\mathrm{ft}$, foot; $\mathrm{ft}^{3} / \mathrm{s}$, cubic foot per second; mi, mile; $\mathrm{mi}^{2}$, square mile; qualification codes apply to the discharge data: 1 , discharge is a maximum daily average; 2 , discharge is an estimate; 4 , discharge is less than indicated value, which is the minimum recordable discharge at this site; 5 , discharge affected to unknown degree by regulation or diversion; 6 , discharge affected by regulation or diversion; 7, discharge is a historic peak; A, year of occurrence is unknown or not exact; $\mathrm{B}$, month or day of occurrence is unknown or not exact; E, only maximum peak available for this year. qualification codes apply to the gage height data: 1, gage height affected by backwater; 2, gage height not the maximum for the year; 3, gage height at different datum or at different site and datum;

5 , gage height is an estimate]

02360275 Judy Creek near Ozark

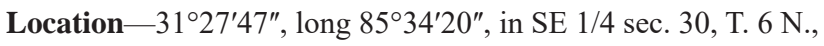
R. 25 E., Dale County, Hydrologic Unit 03140201, at bridge on county road, 1 mi upstream from mouth and 4.5 mi east of Ozark. Drainage area-102 $\mathrm{mi}^{2}$.

Gage Crest-stage gage. Datum not available.

\begin{tabular}{|c|c|c|c|c|c|}
\hline $\begin{array}{c}\text { Water } \\
\text { year }\end{array}$ & Date & Discharge & $\begin{array}{c}\text { Discharge } \\
\text { code }\end{array}$ & $\begin{array}{c}\text { Gage } \\
\text { height } \\
\text { (feet) }\end{array}$ & $\begin{array}{c}\text { Gage } \\
\text { height } \\
\text { code }\end{array}$ \\
\hline
\end{tabular}

\begin{tabular}{llrr}
\hline 1951 & Mar. 29 & 1,230 & 7.70 \\
1952 & Feb. 15 & 2,050 & 11.50 \\
1953 & May 4 & 13,500 & 19.00
\end{tabular}

1954 Dec. $6 \quad 6,200 \quad 16.50$

1955 Apr. $13 \quad 2,830 \quad 13.30$

1956 Sept. $25 \quad 12,000 \quad 18.70$

1957 Apr. $7 \quad 17,000 \quad 19.70$

1958 Apr. $10 \quad 1,600 \quad 9.00$

1959 Feb. $5 \quad 1,900 \quad 11.00$

1960 Apr. $5 \quad 5,600 \quad 16.10$

1961 Feb. $23 \quad 1,120 \quad 7.18$

1962 Apr. $1 \quad 2,280 \quad 12.20$

1963 Jan. $20 \quad 1,580$

1964 Mar. $3 \quad 3,860 \quad 14.70$

1965 Oct. $5 \quad 3,880 \quad 14.72$

1966 Mar. 5 2,340

12.33

1967 Jan. 3,000

15.75

9.44

12.24

12.30

9.77

11.78

16.14

6.76

1974 Apr. $4 \quad 1,020$

1975 Apr. $10 \quad 5,820$

1976 Oct. $17 \quad 2,560$

16.29

12.80

15.00

1977

Jan. 14

4,140

1990

Mar. 17

29,000

2,7

22.29

1994
13,000

\section{Lightwood Knot Creek at Babbie}

L ocation-Lat $31^{\circ} 16^{\prime} 14^{\prime \prime}$, long $86^{\circ} 18^{\prime} 49^{\prime \prime}$, in SE $1 / 4 \mathrm{sec}$. 35, T. 4 N., R. 17 E., Covington County, Hydrologic Unit 03140103, on U.S. Highway 84, 1 mi east of Babbie, 2 mi upstream from mouth, and 3.5 mi west of Opp.

Drainage area-114 $\mathrm{mi}^{2}$.

G age - Wire-weight gage prior to 1947. Water-stage recorder 1947-53. Crest-stage gage 1954-72. Datum of gage is $185 \mathrm{ft}$ NGVD 29 (from topographic map).

\begin{tabular}{|c|c|c|c|c|c|}
\hline $\begin{array}{c}\text { Water } \\
\text { year }\end{array}$ & Date & Discharge & $\begin{array}{l}\text { Discharge } \\
\text { code }\end{array}$ & $\begin{array}{c}\text { Gage } \\
\text { height } \\
\text { (feet) }\end{array}$ & $\begin{array}{c}\text { Gage } \\
\text { height } \\
\text { code }\end{array}$ \\
\hline 1944 & Sept. 11 & 12,500 & & 11.86 & \\
\hline 1945 & Apr. 29 & 1,900 & & 6.57 & \\
\hline 1946 & May 20 & 4,950 & & 8.60 & \\
\hline 1947 & Apr. 16 & 3,220 & & 7.52 & \\
\hline 1948 & Apr. 1 & 3,440 & & 7.58 & \\
\hline 1949 & Jan. 6 & 3,200 & & 7.48 & \\
\hline 1950 & Apr. 5 & 2,150 & & 6.80 & \\
\hline 1951 & Apr. 20 & 2,530 & & 7.10 & \\
\hline 1952 & Mar. 24 & 2,100 & & 7.42 & \\
\hline 1954 & Nov. 24 & 1,080 & & 6.40 & \\
\hline 1955 & Apr. 13 & 8,100 & & 10.10 & \\
\hline 1956 & Sept. 25 & 4,300 & & 8.20 & \\
\hline 1957 & Apr. 6 & 9,500 & & 10.80 & \\
\hline 1958 & Nov. 14 & 929 & & 6.20 & \\
\hline 1959 & June 3 & 2,100 & & 7.40 & \\
\hline 1960 & Apr. 4 & 3,200 & & 7.90 & \\
\hline 1961 & Apr. 2 & 4,700 & & 8.37 & \\
\hline 1962 & Apr. 1 & 6,060 & & 8.99 & \\
\hline 1963 & July 27 & 3,200 & & 7.47 & \\
\hline 1964 & Mar. 4 & 3,870 & & 7.79 & \\
\hline 1965 & Oct. 5 & 1,830 & & 6.61 & \\
\hline 1966 & Mar. 1 & 3,740 & & 7.64 & \\
\hline 1967 & Jan. 3 & 4,080 & & 7.77 & \\
\hline 1968 & Nov. 3 & 2,500 & & 6.98 & \\
\hline 1969 & May 19 & 1,570 & & 6.33 & \\
\hline 1970 & June 3 & 12,200 & & 11.31 & \\
\hline 1971 & Mar. 26 & 3,480 & & 7.37 & \\
\hline 1972 & May 9 & 2,150 & & 6.70 & \\
\hline 1975 & Apr. 13 & 21,600 & 7 & 14.70 & \\
\hline 1990 & Mar. 17 & 26,000 & 7 & 15.73 & \\
\hline 1998 & Mar. 8 & 37,900 & 7 & 19.54 & \\
\hline
\end{tabular}


Appendix 2. Discharge and gage height data for streamgages in Alabama._- Continued

[--, date or data not available; ft, foot; $\mathrm{ft}^{3} / \mathrm{s}$, cubic foot per second; mi, mile; $\mathrm{mi}^{2}$, square mile; qualification codes apply to the discharge data: 1 , discharge is a maximum daily average; 2 , discharge is an estimate; 4 , discharge is less than indicated value, which is the minimum recordable discharge at this site; 5 , discharge affected to unknown degree by regulation or diversion; 6 , discharge affected by regulation or diversion; 7, discharge is a historic peak; A, year of occurrence is unknown or not exact; $\mathrm{B}$, month or day of occurrence is unknown or not exact; E, only maximum peak available for this year. qualification codes apply to the gage height data: 1, gage height affected by backwater; 2, gage height not the maximum for the year; 3, gage height at different data or at different site and datum;

5 , gage height is an estimate]

02362240 Little Double Bridges Creek nr Enterprise, Ala.

L ocation-Lat $31^{\circ} 16^{\prime} 20^{\prime \prime}$, long $85^{\circ} 57^{\prime} 30^{\prime \prime}$ referenced to North American Datum of 1927, Coffee County, Ala., Hydrologic Unit 03140201, near right bank on downstream side of bridge on County Road 18, 8.4 mi southwest of Enterprise.

Drainage area-21 $\mathrm{mi}^{2}$.

Gage -Water-stage recorder. Elevation of gage is $195 \mathrm{ft}$ above NGVD of 1929, from topographic map.

\begin{tabular}{ccccc}
$\begin{array}{c}\text { Water } \\
\text { year }\end{array}$ Date Discharge & $\begin{array}{c}\text { Discharge } \\
\text { code }\end{array}$ & $\begin{array}{c}\text { Gage } \\
\text { height } \\
\text { (feet) }\end{array}$ & $\begin{array}{c}\text { Gage } \\
\text { height } \\
\text { code }\end{array}$ \\
\hline
\end{tabular}

$\begin{array}{llll}1986 & \text { Mar. 13 } & 827 & 8.21 \\ 1987 & \text { Mar. 30 } & 370 & 7.10\end{array}$

$1988 \quad$ Mar. $4 \quad 498$

1989 June $15 \quad 265 \quad 6.50$

1990 Mar. $17 \quad 7,950 \quad 13.90$

1991 Jan. $30 \quad 1,870 \quad 9.32$

1992 June $13 \quad 1,370$

8.80

$1993 \quad$ Mar. $31 \quad 2,750$

$1994 \quad$ July $6 \quad 14,200$

$1995 \quad$ Mar. $8 \quad 1,510$

1996 Oct. $4 \quad 3,100$

1997 Feb. $21 \quad 2,200$

1998 Mar. 8 13,900

1999 Jan. 23463

2000

2001

2002

Feb. 14

585

Mar. 3

1,280

May 30

358

2003

July 1

644

2004

2006

Sept. 16

1,490

Aug. 31

670

2007

Mar. 1

1,210

2008

2009

Feb. 1

1,370

Mar. 28

3,960

Dec. 14

3,630

2011

Dec. 1

316

2012

2013

Sept. 4

1,330

Aug. 20

690

Apr. 18

2,590

2015

Apr. 19

906

Dec. 30

1,490
02429000 Limestone Creek near Monroeville

L ocation-Lat $31^{\circ} 33^{\prime} 43^{\prime \prime}$, long $87^{\circ} 21^{\prime} 02^{\prime \prime}$, in NE 1/4 sec. 22 , T. 7 N., R. 7 E., Monroe County, Hydrologic Unit 03150204, on State Highway 41, 3 mi northwest of Monroeville, and 10 mi upstream from mouth.

Drainage area $-121 \mathrm{mi}^{2}$.

G age - Water-stage recorder. Datum of gage is $104.88 \mathrm{ft}$ NGVD 29.

\begin{tabular}{cccccc}
\hline $\begin{array}{c}\text { Water } \\
\text { year }\end{array}$ & Date & Discharge & $\begin{array}{c}\text { Discharge } \\
\text { code }\end{array}$ & $\begin{array}{c}\text { Gage } \\
\text { height } \\
\text { (feet) }\end{array}$ & $\begin{array}{c}\text { Gage } \\
\text { height } \\
\text { code }\end{array}$ \\
\hline 1929 & -- & -- & & 22.00 & \\
1952 & Mar. 23 & 3,200 & & 9.02 & \\
1953 & Dec. 10 & 1,575 & & 7.40 &
\end{tabular}

1954 Dec. $4 \quad 3,950 \quad 9.50$

1955 Apr. $14 \quad 8,600 \quad 11.50$

1956 July $8 \quad 5,100 \quad 10.13$

1957 Dec. $23 \quad 6,000 \quad 10.57$

$1958 \quad$ Nov. $14 \quad 2,700 \quad 8.62$

1959 June $10 \quad 2,180 \quad 8.29$

$1960 \quad$ Mar. $30 \quad 4,660 \quad 9.95$

$1961 \quad$ Feb. $25 \quad 30,600 \quad 16.28$

1962 Mar. $31 \quad 11,200 \quad 12.35$

1963 Mar. $6 \quad 1,600 \quad 7.47$

1964 Apr. $27 \quad 5,530 \quad 10.28$

1965 Sept. $30 \quad 10,200 \quad 12.05$

1966 Oct. $1 \quad 9,690 \quad 11.90$

$1967 \quad$ Jan. $1 \quad 2,710 \quad 8.64$

1968 Dec. $11 \quad 1,400 \quad 7.21$

1969 Mar. $24 \quad 2,210 \quad 8.20$

1970 June $3 \quad 3,190 \quad 8.98$

$1971 \quad$ Mar. $3 \quad 3,890 \quad 9.42$

1972 Mar. $3 \quad 1,370 \quad 7.03$

$1973 \quad$ Mar. $31 \quad 7,190 \quad 11.03$ 
Appendix 2. Discharge and gage height data for streamgages in Alabama.-Continued

[--, date or data not available; $\mathrm{ft}$, foot; $\mathrm{ft}^{3} / \mathrm{s}$, cubic foot per second; mi, mile; $\mathrm{mi}^{2}$, square mile; qualification codes apply to the discharge data: 1 , discharge is a maximum daily average; 2 , discharge is an estimate; 4 , discharge is less than indicated value, which is the minimum recordable discharge at this site; 5 , discharge affected to unknown degree by regulation or diversion; 6 , discharge affected by regulation or diversion; 7, discharge is a historic peak; A, year of occurrence is unknown or not exact; $\mathrm{B}$, month or day of occurrence is unknown or not exact; E, only maximum peak available for this year. qualification codes apply to the gage height data: 1, gage height affected by backwater; 2, gage height not the maximum for the year; 3, gage height at different datum or at different site and datum; 5 , gage height is an estimate]

\section{Murder Creek near Evergreen}

Location-Lat $31^{\circ} 25^{\prime} 06^{\prime \prime}$, long $86^{\circ} 59^{\prime} 12^{\prime \prime}$, in NW 1/4 sec. 8, T. 5 N., R. 11 E., Conecuh County, Hydrologic Unit 03140304, on U.S. Highway 31, 2.5 mi southwest of Evergreen, and at mile 35.6.

Drainage area-176 $\mathrm{mi}^{2}$.

Gage-Water-stage recorder. Datum of gage is $178.29 \mathrm{ft}$ NGVD 29.

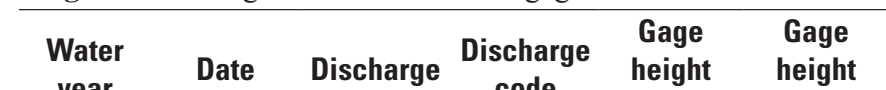

\begin{tabular}{llrlcc} 
year & Date & Discharge & $\begin{array}{c}\text { Discharge } \\
\text { code }\end{array}$ & $\begin{array}{c}\text { height } \\
\text { (feet) }\end{array}$ & $\begin{array}{c}\text { height } \\
\text { code }\end{array}$ \\
\hline 1929 & Mar & 90,000 & $2,7, B$ & 26.60 & 5 \\
1938 & Mar. 16 & 21,300 & & 16.65 & \\
1939 & Mar. 30 & 5,610 & & 12.27 & \\
1940 & Feb. 18 & 1,760 & & 10.08 &
\end{tabular}

1940

1941

1942

1943

Dec. 17

1,760

11.00

11.52

11.60

1944

Dec. 24 3,900

Mar. 22

4,090

12.77

1945

1946

Nov. 8

6,800

Apr. 29

2,090

10.32

12.57

11.00

1947

Apr. 2

3,180

Mar. 3

4,100

11.20

1949

1950

Nov. 27

10,000

Apr. 5

1,190

13.50

8.91

10.05

10.20

9.80

10.89

12.58

10.81

12.10

9.26

11.15

11.77

16.13

13.22

9.82

13.80

13.02

11.99

1966

Jan. 23

8,050

9.90
02374500 Murder Creek near Evergreen-Continued

L ocation-Lat $31^{\circ} 25^{\prime} 06^{\prime \prime}$, long $86^{\circ} 59^{\prime} 12^{\prime \prime}$, in NW 1/4 sec. 8, T. 5 N., R. 11 E., Conecuh County, Hydrologic Unit 03140304, on U.S. Highway 31, $2.5 \mathrm{mi}$ southwest of Evergreen, and at mile 35.6.

Drainage area-176 $\mathrm{mi}^{2}$.

G age - Water-stage recorder. Datum of gage is $178.29 \mathrm{ft}$ NGVD 29.

\begin{tabular}{llcccc}
\hline $\begin{array}{c}\text { Water } \\
\text { year }\end{array}$ & Date & Discharge & $\begin{array}{c}\text { Discharge } \\
\text { code }\end{array}$ & $\begin{array}{c}\text { Gage } \\
\text { height } \\
\text { (feet) }\end{array}$ & $\begin{array}{c}\text { Gage } \\
\text { height } \\
\text { code }\end{array}$ \\
\hline 1968 & Nov. 1 & 728 & & 7.90 & \\
1969 & May 19 & 1,400 & & 9.51 & \\
1970 & June 3 & 3,950 & & 11.43 & \\
1971 & Mar. 27 & 2,320 & & 10.44 &
\end{tabular}

$1971 \quad$ Mar. $27 \quad 2,320 \quad 10.44$

1973 Apr. $1 \quad 4,920 \quad 11.91$

$1974 \quad$ Sept. $9 \quad 3,890 \quad 11.40$

1975 Apr. $10 \quad 14,000 \quad 15.27$

1976 Dec. $31 \quad 5,930 \quad 12.46$

1977 Mar. $13 \quad 2,160 \quad 10.50$

$1978 \quad$ Jan. $25 \quad 6,110 \quad 12.54$

1979 Mar. $4 \quad 8,180 \quad 13.44$

1980 Apr. $13 \quad 8,510 \quad 13.58$

1981 Feb. $11 \quad 4,440 \quad 11.78$

1982 Feb. $4 \quad 3,000 \quad 11.00$

1983 Apr. $8 \quad 4,040 \quad 11.58$

1984 May $3 \quad 4,960 \quad 12.02$

1985 July $26 \quad 4,570 \quad 11.84$

1986 Mar. $14 \quad 2,390 \quad 10.54$

1987 Feb. $16 \quad 1,000 \quad$ E $\quad 8.67$

1988 Sept. $11 \quad 2,260 \quad$ E $~ 10.44$

1989 Mar. $30 \quad 2,400 \quad 10.56$

1990 Mar. $16 \quad 21,000 \quad 16.59$

1991 Mar. $2 \quad 2,300 \quad 10.45$

1992 Feb. $15 \quad 2,780 \quad 10.90$

$1993 \quad$ Nov. $25 \quad 3,900 \quad 11.50$

$1994 \quad$ July $8 \quad 1,750 \quad 9.91$

1995 Aug. $4 \quad 3,360 \quad 11.30$

1996 Oct. $5 \quad 5,750 \quad 12.52$

1997 Mar. $1 \quad 1,570 \quad 9.69$

1998 Mar. $8 \quad 7,300 \quad 13.20$ 
Appendix 2. Discharge and gage height data for streamgages in Alabama.-Continued

[--, date or data not available; $\mathrm{ft}$, foot; $\mathrm{ft}^{3} / \mathrm{s}$, cubic foot per second; $\mathrm{mi}$, mile; $\mathrm{mi}^{2}$, square mile; qualification codes apply to the discharge data: 1 , discharge is a maximum daily average; 2 , discharge is an estimate; 4 , discharge is less than indicated value, which is the minimum recordable discharge at this site; 5 , discharge affected to unknown degree by regulation or diversion; 6 , discharge affected by regulation or diversion; 7, discharge is a historic peak; A, year of occurrence is unknown or not exact; B, month or day of occurrence is unknown or not exact; E, only maximum peak available for this year. qualification codes apply to the gage height data: 1, gage height affected by backwater; 2 , gage height not the maximum for the year; 3 , gage height at different data or at different site and datum;

5 , gage height is an estimate]

\section{Murder Creek near Evergreen-Continued}

L ocation-Lat $31^{\circ} 25^{\prime} 06^{\prime \prime}$, long $86^{\circ} 59^{\prime} 12^{\prime \prime}$, in NW 1/4 sec. 8, T. 5 N., R. 11 E., Conecuh County, Hydrologic Unit 03140304, on U.S. Highway 31, 2.5 mi southwest of Evergreen, and at mile 35.6 .

Drainage area-176 $\mathrm{mi}^{2}$.

Gage -Water-stage recorder. Datum of gage is $178.29 \mathrm{ft}$ NGVD 29.

\begin{tabular}{|c|c|c|c|c|c|}
\hline $\begin{array}{c}\text { Water } \\
\text { year }\end{array}$ & Date & Discharge & $\begin{array}{c}\text { Discharge } \\
\text { code }\end{array}$ & $\begin{array}{c}\text { Gage } \\
\text { height } \\
\text { (feet) }\end{array}$ & $\begin{array}{c}\text { Gage } \\
\text { height } \\
\text { code }\end{array}$ \\
\hline 1999 & Mar. 15 & 2,650 & & 10.72 & \\
\hline 2000 & Oct. 11 & 440 & & 6.12 & \\
\hline 2001 & Mar. 4 & 12,500 & & 14.83 & \\
\hline 2002 & Oct. 14 & 1,650 & & 9.76 & \\
\hline 2003 & July 1 & 5,200 & & 12.31 & \\
\hline 2004 & Sept. 17 & 2,980 & & 10.97 & \\
\hline 2005 & July 12 & 3,620 & & 11.42 & \\
\hline 2006 & Feb. 27 & 1,200 & & 8.77 & \\
\hline 2007 & Nov. 17 & 3,040 & & 11.02 & \\
\hline 2008 & Dec. 31 & 1,920 & & 10.08 & \\
\hline 2009 & Mar. 28 & 2,560 & & 10.77 & \\
\hline 2010 & Dec. 15 & 7,160 & & 13.32 & \\
\hline 2011 & Feb. 6 & 1,750 & & 9.96 & \\
\hline 2012 & Feb. 19 & 1,180 & & 9.16 & \\
\hline 2013 & Feb. 11 & 4,300 & & 11.96 & \\
\hline 2014 & Apr. 8 & 4,050 & & 11.81 & \\
\hline 2015 & Apr. 18 & 1,610 & & 9.77 & \\
\hline
\end{tabular}

\section{Okatuppa Creek at Gilbertown}

L ocation-Lat $31^{\circ} 53^{\prime} 27^{\prime \prime}$, long $88^{\circ} 18^{\prime} 48^{\prime \prime}$, in SE $1 / 4$ sec. 30 , T. 11 N., R. 3 W., Choctaw County, Hydrologic Unit 03160201, on Highway 17, 0.8 mi northeast of Gilbertown, and 1.5 mi upstream from Bogueloosa Creek.

Drainage area-148 $\mathrm{mi}^{2}$.

Gage-Water-stage recorder. Datum of gage is $59.41 \mathrm{ft}$ NGVD 29.

\begin{tabular}{|c|c|c|c|c|c|}
\hline $\begin{array}{c}\text { Water } \\
\text { year }\end{array}$ & Date & Discharge & $\begin{array}{c}\text { Discharge } \\
\text { code }\end{array}$ & $\begin{array}{c}\text { Gage } \\
\text { height } \\
\text { (feet) }\end{array}$ & $\begin{array}{c}\text { Gage } \\
\text { height } \\
\text { code }\end{array}$ \\
\hline 1957 & Apr. 4 & 4,660 & & 12.00 & \\
\hline 1958 & Feb. 6 & 5,800 & & 13.40 & \\
\hline 1959 & Feb. 4 & 2,400 & & 8.60 & \\
\hline 1960 & Mar. 15 & 5,600 & & 13.30 & \\
\hline 1961 & Feb. 21 & 15,000 & & 17.40 & \\
\hline 1962 & Apr. 28 & 5,700 & 1 & 13.15 & \\
\hline 1963 & Jan. 20 & 2,850 & & 9.35 & \\
\hline 1964 & Apr. 7 & 7,100 & & 14.50 & \\
\hline 1965 & Jan. 23 & 6,350 & & 13.90 & \\
\hline 1966 & Feb. 12 & 5,830 & & 13.40 & \\
\hline 1967 & May 4 & 2,760 & & 9.26 & \\
\hline 1968 & Dec. 15 & 4,560 & & 11.89 & \\
\hline 1969 & Apr. 14 & 5,890 & & 13.47 & \\
\hline 1970 & Mar. 4 & 2,770 & & 9.28 & \\
\hline
\end{tabular}


Appendix 2. Discharge and gage height data for streamgages in Alabama.-Continued

[--, date or data not available; $\mathrm{ft}$, foot; $\mathrm{ft}^{3} / \mathrm{s}$, cubic foot per second; mi, mile; $\mathrm{mi}^{2}$, square mile; qualification codes apply to the discharge data: 1 , discharge is a maximum daily average; 2 , discharge is an estimate; 4 , discharge is less than indicated value, which is the minimum recordable discharge at this site; 5 , discharge affected to unknown degree by regulation or diversion; 6 , discharge affected by regulation or diversion; 7, discharge is a historic peak; A, year of occurrence is unknown or not exact; $\mathrm{B}$, month or day of occurrence is unknown or not exact; E, only maximum peak available for this year. qualification codes apply to the gage height data: 1, gage height affected by backwater; 2, gage height not the maximum for the year; 3, gage height at different datum or at different site and datum; 5 , gage height is an estimate]

\section{Patsaliga Creek at Luverne}

L ocation-Lat $31^{\circ} 43^{\prime} 27^{\prime \prime}$, long $86^{\circ} 16^{\prime} 42^{\prime \prime}$, in SW 1/4 sec. 29 , T. 9 N., R. 18 E., Crenshaw County, Hydrologic Unit 03140302, on U.S. Highway 331, 1 mi northwest of Luverne, and 3 downstream from Pond Creek.

Drainage area $-254 \mathrm{mi}^{2}$.

Gage -Water-stage recorder 1944-58. Crest-stage gage 1959-70. Datum of gage is $267.53 \mathrm{ft}$ NGVD 29.

\begin{tabular}{ccccc}
$\begin{array}{c}\text { Water } \\
\text { year }\end{array} \quad$ Date & Discharge & $\begin{array}{c}\text { Discharge } \\
\text { code }\end{array}$ & $\begin{array}{c}\text { Gage } \\
\text { height } \\
\text { (feet) }\end{array}$ & $\begin{array}{c}\text { Gage } \\
\text { height } \\
\text { code }\end{array}$ \\
\hline
\end{tabular}

\begin{tabular}{llrl}
\hline 1944 & Mar. 24 & 13,100 & 15.55 \\
1945 & Feb. 16 & 1,400 & 10.43 \\
1946 & Jan. 7 & 7,550 & 13.71
\end{tabular}

$\begin{array}{llll}1946 & \text { Jan. 7 } & 7,550 & 13.71 \\ 1947 & \text { Apr. 3 } & 5,560 & 12.90 \\ 1948 & \text { Mar. 7 } & 6,800 & 13.40\end{array}$

$\begin{array}{llrl}1949 & \text { Nov. } 28 & 16,700 & 16.80 \\ 1950 & \text { Apr. } 6 & 6,550 & 13.30 \\ 1951 & \text { Feb. } 4 & 1,690 & 10.60 \\ 1952 & \text { Mar. } 25 & 7,310 & 14.05\end{array}$

1953 Apr. $12 \quad 4,340$

1954 Dec. $6 \quad 7,280$

1955 Apr. $16 \quad 3,340 \quad 12.40$

1956 Mar. $14 \quad 2,240 \quad 11.40$

1957 Apr. $6 \quad 10,200 \quad 15.00$

1958 Mar. $9 \quad 5,160 \quad 13.40$

1959 Mar. $29 \quad 8,630 \quad 14.60$

1960 Apr. $4 \quad 15,500 \quad 16.40$

$1961 \quad$ Feb. $26 \quad 10,300 \quad 15.07$

1962 Apr. $1 \quad 6,110 \quad 13.79$

1963 Jan. $23 \quad 4,920 \quad 13.29$

$1964 \quad$ Apr. $28 \quad 14,500$

1965 Dec. $28 \quad 6,170 \quad 13.81$

1966 Mar. $4 \quad 7,830 \quad 14.37$

1967 Jan. $3 \quad 2,950 \quad 12.09$

1968 Mar. $15 \quad 2,060$

12.09

1969 Mar. $25 \quad 3,520 \quad 12.51$

1970 June $4 \quad 20,800 \quad 17.45$

$1990 \quad$ Mar. $17 \quad 37,000 \quad 7 \quad 20.29$

\section{Patsaliga Creek near Brantley}

L ocation-Lat $31^{\circ} 35^{\prime} 46^{\prime \prime}$, long $86^{\circ} 24^{\prime} 20^{\prime \prime}$, in NE $1 / 4$ sec. 12 , T. 7 N., R. 16 E., Crenshaw County, Hydrologic Unit 03140302, on State Highway 106, $3.0 \mathrm{mi}$ north of Leon, and 10.9 mi northwest of Brantley.

Drainage area- $442 \mathrm{mi}^{2}$

G age - Water-stage recorder. Datum of gage is $220 \mathrm{ft} \mathrm{NGVD} 29$ (from topographic map).

\begin{tabular}{|c|c|c|c|c|c|}
\hline $\begin{array}{c}\text { Water } \\
\text { year }\end{array}$ & Date & Discharge & $\begin{array}{l}\text { Discharge } \\
\text { code }\end{array}$ & $\begin{array}{c}\text { Gage } \\
\text { height } \\
\text { (feet) }\end{array}$ & $\begin{array}{c}\text { Gage } \\
\text { height } \\
\text { code }\end{array}$ \\
\hline 1975 & Feb. 19 & 15,200 & & 22.21 & \\
\hline 1976 & May 16 & 9,460 & & 20.73 & \\
\hline 1977 & Apr. 1 & 5,040 & & 19.29 & \\
\hline 1978 & Jan. 27 & 15,400 & & 22.05 & \\
\hline 1979 & Apr. 6 & 12,800 & & 21.46 & \\
\hline 1980 & Apr. 16 & 10,300 & & 20.90 & \\
\hline 1981 & Feb. 12 & 3,840 & & 17.93 & \\
\hline 1982 & Feb. 5 & 9,980 & & 20.82 & \\
\hline
\end{tabular}

1983 Mar. $8 \quad 6,080 \quad 19.79$

$1984 \quad$ Mar. $7 \quad 2,670 \quad 15.80$

1985 Mar. $2 \quad 3,520 \quad$ E $\quad 17.46$

1986 Mar. $16 \quad 9,690 \quad 20.76$

1987 Mar. $4 \quad 2,870 \quad$ E $\quad 16.25$

1988 Mar. $5 \quad 2,550 \quad$ E $\quad 15.51$

1989 June $19 \quad 9,110 \quad 20.79$

1990 Mar. $17 \quad 43,600 \quad 25.67$

1991 Mar. $5 \quad 6,700 \quad 20.00$

$1992 \quad$ Feb. $19 \quad 3,530 \quad 17.45$

$1993 \quad$ Nov. $27 \quad 14,200 \quad 21.83$

1994 July $10 \quad 4,870 \quad 19.13$

1995 Mar. $11 \quad 4,320 \quad 18.54$

1996 Oct. $8 \quad 7,620 \quad 20.36$

1997 May $1 \quad 5,970 \quad 19.73$

1998 Sept. $30 \quad 25,400 \quad 23.66$

$1999 \quad$ July $1 \quad 5,760 \quad 19.65$

$2000 \quad$ Mar. $22 \quad 1,090 \quad 10.71$

2001 Mar. $5 \quad 20,600 \quad 22.94$

2002 Mar. $22 \quad 2,320 \quad 15.07$

2003 Apr. $11 \quad 4,310 \quad 18.38$

2004 Sept. $19 \quad 11,700 \quad 21.36$ 
Appendix 2. Discharge and gage height data for streamgages in Alabama.-Continued

[--, date or data not available; ft, foot; $\mathrm{ft}^{3} / \mathrm{s}$, cubic foot per second; mi, mile; $\mathrm{mi}^{2}$, square mile; qualification codes apply to the discharge data: 1 , discharge is a maximum daily average; 2 , discharge is an estimate; 4 , discharge is less than indicated value, which is the minimum recordable discharge at this site; 5 , discharge affected to unknown degree by regulation or diversion; 6 , discharge affected by regulation or diversion; 7, discharge is a historic peak; A, year of occurrence is unknown or not exact; $\mathrm{B}$, month or day of occurrence is unknown or not exact; E, only maximum peak available for this year. qualification codes apply to the gage height data: 1 , gage height affected by backwater; 2, gage height not the maximum for the year; 3, gage height at different data or at different site and datum; 5 , gage height is an estimate]

02372250 Patsaliga Creek near Brantley-Continued

L ocation-Lat $31^{\circ} 35^{\prime} 46^{\prime \prime}$, long $86^{\circ} 24^{\prime} 20^{\prime \prime}$, in NE $1 / 4$ sec. 12 , T. 7

N., R. 16 E., Crenshaw County, Hydrologic Unit 03140302, on

State Highway 106, $3.0 \mathrm{mi}$ north of Leon, and 10.9 mi northwest of Brantley.

Drainage area $-442 \mathrm{mi}^{2}$.

Gage -Water-stage recorder. Datum of gage is $220 \mathrm{ft}$ NGVD 29 (from topographic map).

\begin{tabular}{llcccc}
\hline $\begin{array}{c}\text { Water } \\
\text { year }\end{array}$ & Date & Discharge & $\begin{array}{c}\text { Discharge } \\
\text { code }\end{array}$ & $\begin{array}{c}\text { Gage } \\
\text { height } \\
\text { (feet) }\end{array}$ & $\begin{array}{c}\text { Gage } \\
\text { height } \\
\text { code }\end{array}$ \\
\hline 2005 & Mar. 30 & 9,280 & & 20.83 & \\
2006 & May 14 & 4,740 & 18.96 & \\
2007 & Mar. 6 & 1,470 & 12.12 & \\
2008 & Aug. 29 & 2,620 & 16.48 & \\
2009 & Mar. 30 & 11,500 & 21.32 & \\
2010 & Dec. 16 & 12,700 & 21.69 & \\
2011 & Mar. 13 & 2,620 & 16.03 & \\
2012 & Apr. 5 & 1,750 & 13.16 & \\
2013 & Feb. 15 & 6,810 & 19.71 & \\
2014 & Apr. 9 & 11,800 & 21.65 & \\
2015 & Apr. 22 & 2,930 & 15.07 &
\end{tabular}

02363000 Pea River near Ariton

Location-Lat $31^{\circ} 35^{\prime} 41^{\prime \prime}$, long 85 $46^{\prime} 59^{\prime \prime}$, in SW 1/4 sec. 7, T.

7 N., R. 23 E., Dale County, Hydrologic Unit 03140202, on

Highway 231, $3.5 \mathrm{mi}$ west of Ariton and at mile 92.5.

Drainage area $-498 \mathrm{mi}^{2}$.

Gage-Water-stage recorder. Datum of gage is $246.72 \mathrm{ft}$ NGVD 29.

\begin{tabular}{llrrrr}
\hline $\begin{array}{c}\text { Water } \\
\text { year }\end{array}$ & Date & Discharge & $\begin{array}{c}\text { Discharge } \\
\text { code }\end{array}$ & $\begin{array}{c}\text { Gage } \\
\text { height } \\
\text { (feet) }\end{array}$ & $\begin{array}{c}\text { Gage } \\
\text { height } \\
\text { code }\end{array}$ \\
\hline 1929 & Mar & 50,000 & $2,7, B$ & 25.00 & \\
1939 & Mar. 1 & 13,000 & & 18.36 & \\
1940 & Feb. 20 & 7,290 & & 16.26 & \\
1941 & Mar. 12 & 1,190 & & 5.46 & \\
1942 & Feb. 20 & 4,541 & & 13.92 & \\
1943 & Mar. 22 & 19,100 & & 19.98 & \\
1944 & Mar. 24 & 18,600 & & 19.80 & \\
1945 & Apr. 29 & 1,730 & & 7.43 & \\
1946 & May 22 & 9,160 & & 17.57 & \\
1947 & Apr. 3 & 13,700 & & 18.80 & \\
1948 & Mar. 7 & 7,170 & & 16.10 &
\end{tabular}

02363000 Pea River near Ariton-Continued

L ocation-Lat $31^{\circ} 35^{\prime} 41^{\prime \prime}$, long $85^{\circ} 46^{\prime} 59^{\prime \prime}$, in SW 1/4 sec. 7, T. 7 N., R. 23 E., Dale County, Hydrologic Unit 03140202, on Highway 231, $3.5 \mathrm{mi}$ west of Ariton and at mile 92.5.

Drainage area $498 \mathrm{mi}^{2}$.

Gage-Water-stage recorder. Datum of gage is $246.72 \mathrm{ft}$ NGVD 29.

\begin{tabular}{|c|c|c|c|c|c|}
\hline $\begin{array}{l}\text { Water } \\
\text { year }\end{array}$ & Date & Discharge & $\begin{array}{l}\text { Discharge } \\
\text { code }\end{array}$ & $\begin{array}{c}\text { Gage } \\
\text { height } \\
\text { (feet) }\end{array}$ & $\begin{array}{c}\text { Gage } \\
\text { height } \\
\text { code }\end{array}$ \\
\hline 1949 & Nov. 29 & 13,700 & & 18.80 & \\
\hline 1950 & Apr. 6 & 2,500 & & 9.60 & \\
\hline 1951 & Apr. 21 & 1,900 & & 7.92 & \\
\hline 1952 & Mar. 26 & 11,600 & & 18.32 & \\
\hline 1953 & Sept. 28 & 9,680 & & 17.52 & \\
\hline 1954 & Dec. 7 & 9,030 & & 17.12 & \\
\hline 1955 & Apr. 16 & 4,360 & & 13.05 & \\
\hline 1956 & Sept. 27 & 8,240 & & 16.60 & \\
\hline 1957 & Dec. 25 & 9,190 & & 17.20 & \\
\hline 1958 & Mar. 11 & 4,090 & & 12.65 & \\
\hline 1959 & Mar. 29 & 7,190 & & 15.94 & \\
\hline 1960 & Apr. 4 & 22,000 & & 20.42 & \\
\hline 1961 & Apr. 2 & 7,840 & & 16.33 & \\
\hline 1962 & Apr. 3 & 5,000 & & 13.75 & \\
\hline 1963 & Jan. 22 & 4,480 & & 13.00 & \\
\hline 1964 & Mar. 5 & 7,110 & & 15.84 & \\
\hline 1965 & Oct. 6 & 10,600 & & 17.98 & \\
\hline 1966 & Mar. 5 & 10,900 & & 18.14 & \\
\hline 1967 & Jan. 3 & 5,900 & & 14.88 & \\
\hline 1968 & Mar. 14 & 4,330 & & 12.75 & \\
\hline 1969 & Apr. 21 & 3,540 & & 11.27 & \\
\hline 1970 & June 5 & 7,130 & & 15.86 & \\
\hline 1971 & Jan. 7 & 9,220 & & 17.22 & \\
\hline 1972 & Mar. 4 & 10,400 & & 17.91 & \\
\hline 1973 & Apr. 2 & 17,000 & & 19.57 & \\
\hline 1974 & Feb. 10 & 3,720 & & 11.64 & \\
\hline 1975 & Feb. 18 & 47,600 & & 24.38 & \\
\hline 1976 & Oct. 19 & 7,580 & & 16.16 & \\
\hline 1977 & Mar. 25 & 4,440 & & 12.74 & \\
\hline 1978 & Jan. 27 & 18,000 & & 19.88 & \\
\hline 1979 & Apr. 7 & 8,240 & & 16.60 & \\
\hline 1980 & Mar. 31 & 7,550 & & 16.14 & \\
\hline 1981 & Feb. 12 & 9,910 & & 17.62 & \\
\hline 1982 & Feb. 6 & 6,380 & & 15.26 & \\
\hline 1983 & Mar. 29 & 5,850 & $\mathrm{E}$ & 14.75 & \\
\hline
\end{tabular}


Appendix 2. Discharge and gage height data for streamgages in Alabama.-Continued

[--, date or data not available; $\mathrm{ft}$, foot; $\mathrm{ft}^{3} / \mathrm{s}$, cubic foot per second; mi, mile; $\mathrm{mi}^{2}$, square mile; qualification codes apply to the discharge data: 1 , discharge is a maximum daily average; 2 , discharge is an estimate; 4 , discharge is less than indicated value, which is the minimum recordable discharge at this site; 5 , discharge affected to unknown degree by regulation or diversion; 6 , discharge affected by regulation or diversion; 7, discharge is a historic peak; A, year of occurrence is unknown or not exact; B, month or day of occurrence is unknown or not exact; E, only maximum peak available for this year. qualification codes apply to the gage height data: 1, gage height affected by backwater; 2, gage height not the maximum for the year; 3, gage height at different datum or at different site and datum; 5 , gage height is an estimate]

\section{Pea River near Ariton-Continued}

Location-Lat $31^{\circ} 35^{\prime} 41^{\prime \prime}$, long $85^{\circ} 46^{\prime} 59^{\prime \prime}$, in SW 1/4 sec. 7, T.

7 N., R. 23 E., Dale County, Hydrologic Unit 03140202, on

Highway 231, $3.5 \mathrm{mi}$ west of Ariton and at mile 92.5.

Drainage area $498 \mathrm{mi}^{2}$.

Gage - Water-stage recorder. Datum of gage is $246.72 \mathrm{ft}$ NGVD 29.

\begin{tabular}{|c|c|c|c|c|c|}
\hline $\begin{array}{c}\text { Water } \\
\text { year }\end{array}$ & Date & Discharge & $\begin{array}{l}\text { Discharge } \\
\text { code }\end{array}$ & $\begin{array}{c}\text { Gage } \\
\text { height } \\
\text { (feet) }\end{array}$ & $\begin{array}{c}\text { Gage } \\
\text { height } \\
\text { code }\end{array}$ \\
\hline 1984 & Mar. 8 & 3,700 & $\mathrm{E}$ & 11.27 & \\
\hline 1985 & Feb. 7 & 2,070 & $\mathrm{E}$ & 7.51 & \\
\hline 1986 & Mar. 14 & 10,700 & $\mathrm{E}$ & 18.04 & \\
\hline 1987 & Mar. 31 & 3,620 & & 11.10 & \\
\hline 1988 & Mar. 5 & 3,050 & & 9.38 & \\
\hline 1989 & Mar. 26 & 2,780 & & 8.80 & \\
\hline 1990 & Mar. 17 & 47,700 & & 24.87 & \\
\hline 1991 & Mar. 5 & 6,530 & & 14.97 & \\
\hline 1992 & Jan. 16 & 7,430 & & 15.82 & \\
\hline 1993 & Nov. 28 & 7,110 & & 15.55 & \\
\hline 1994 & July 8 & 17,100 & & 19.53 & \\
\hline 1995 & Mar. 3 & 5,170 & & 13.11 & \\
\hline 1996 & Oct. 7 & 9,420 & & 17.05 & \\
\hline 1997 & Feb. 17 & 5,670 & & 13.89 & \\
\hline 1998 & Mar. 10 & 20,900 & & 20.43 & \\
\hline 1999 & Oct. 1 & 5,880 & & 14.67 & \\
\hline 2000 & Mar. 23 & 2,210 & & 7.04 & \\
\hline 2001 & Mar. 5 & 17,800 & & 19.70 & \\
\hline 2002 & Mar. 24 & 2,000 & & 6.57 & \\
\hline 2003 & July 4 & 5,230 & & 13.63 & \\
\hline 2004 & Jan. 28 & 3,970 & & 11.14 & \\
\hline 2005 & Mar. 29 & 12,500 & & 18.17 & \\
\hline 2006 & Mar. 10 & 3,260 & & 9.51 & \\
\hline 2007 & Apr. 18 & 3,080 & & 8.83 & \\
\hline 2008 & Aug. 26 & 9,510 & & 17.08 & \\
\hline 2009 & Mar. 17 & 10,500 & & 17.42 & \\
\hline 2010 & Dec. 15 & 16,200 & & 18.96 & \\
\hline 2011 & Mar. 13 & 3,040 & & 8.66 & \\
\hline 2012 & Feb. 23 & 1,520 & & 5.16 & \\
\hline 2013 & Feb. 14 & 7,620 & & 16.34 & \\
\hline 2014 & Apr. 9 & 10,800 & & 17.90 & \\
\hline 2015 & Apr. 21 & 2,900 & & 8.39 & \\
\hline
\end{tabular}

\section{Pigeon Creek near Thad}

L ocation-Lat $31^{\circ} 28^{\prime} 36^{\prime \prime}$, long 86 $36^{\prime} 30^{\prime \prime}$, in NE 1/4 sec. 21 , T. 6 N., R. 14 E., Covington County, Hydrologic Unit 03140303, downstream from State Highway 55, 2 mi southeast of Thad, $5.5 \mathrm{mi}$ southeast of McKenzie.

Drainage area-307 $\mathrm{mi}^{2}$.

G age -Water-stage recorder 1938-70. Datum of gage is $172.58 \mathrm{ft}$ NGVD 29.

\begin{tabular}{|c|c|c|c|c|c|}
\hline $\begin{array}{c}\text { Water } \\
\text { year }\end{array}$ & Date & Discharge & $\begin{array}{l}\text { Discharge } \\
\text { code }\end{array}$ & $\begin{array}{c}\text { Gage } \\
\text { height } \\
\text { (feet) }\end{array}$ & $\begin{array}{c}\text { Gage } \\
\text { height } \\
\text { code }\end{array}$ \\
\hline 1929 & Mar. & 24,000 & $2,7, \mathrm{~B}$ & 30.00 & \\
\hline 1938 & Mar. 18 & 14,400 & & 26.06 & \\
\hline 1939 & Aug. 18 & 12,600 & & 25.31 & \\
\hline 1940 & Feb. 18 & 3,740 & & 17.56 & \\
\hline 1941 & Dec. 20 & 2,180 & & 14.62 & \\
\hline 1942 & Dec. 27 & 5,730 & & 20.61 & \\
\hline 1943 & Mar. 23 & 13,400 & & 25.20 & \\
\hline 1944 & Apr. 28 & 12,800 & & 24.98 & \\
\hline 1945 & Apr. 28 & 1,570 & & 12.67 & \\
\hline 1946 & Jan. 8 & 12,600 & & 25.06 & \\
\hline 1947 & Apr. 5 & 4,220 & & 18.46 & \\
\hline 1948 & Mar. 7 & 7,540 & & 22.20 & \\
\hline 1949 & Nov. 29 & 17,100 & & 27.10 & \\
\hline 1950 & July 29 & 1,110 & & 10.13 & \\
\hline 1951 & Apr. 20 & 2,520 & & 14.90 & \\
\hline 1952 & Mar. 25 & 3,320 & & 16.60 & \\
\hline 1953 & May 4 & 2,430 & & 14.68 & \\
\hline 1954 & Dec. 7 & 5,860 & & 20.61 & \\
\hline 1955 & Apr. 14 & 3,500 & & 17.34 & \\
\hline 1956 & Mar. 17 & 1,830 & & 13.07 & \\
\hline 1957 & Apr. 7 & 5,930 & & 20.70 & \\
\hline 1958 & Mar. 19 & 1,700 & 1 & -- & \\
\hline 1959 & Mar. 30 & 3,670 & & 17.60 & \\
\hline 1960 & Apr. 1 & 9,680 & & 24.33 & \\
\hline 1961 & Feb. 27 & 17,700 & & 27.27 & \\
\hline 1962 & Apr. 3 & 3,970 & & 18.10 & \\
\hline 1963 & Jan. 23 & 3,720 & & 17.69 & \\
\hline 1964 & Apr. 29 & 19,000 & & 27.85 & \\
\hline 1965 & Jan. 25 & 8,500 & & 23.10 & \\
\hline 1966 & Mar. 4 & 7,120 & & 21.68 & \\
\hline 1967 & Sept. 13 & 8,300 & & 22.87 & \\
\hline 1968 & Mar. 16 & 1,740 & & 12.66 & \\
\hline 1969 & Sept. 22 & 2,190 & & 14.30 & \\
\hline 1970 & June 6 & 7,200 & & 22.19 & \\
\hline 1990 & Mar. 17 & 28,500 & 7 & 31.10 & \\
\hline
\end{tabular}


Appendix 2. Discharge and gage height data for streamgages in Alabama.-Continued

[--, date or data not available; ft, foot; $\mathrm{ft}^{3} / \mathrm{s}$, cubic foot per second; mi, mile; $\mathrm{mi}^{2}$, square mile; qualification codes apply to the discharge data: 1 , discharge is a maximum daily average; 2 , discharge is an estimate; 4 , discharge is less than indicated value, which is the minimum recordable discharge at this site; 5 , discharge affected to unknown degree by regulation or diversion; 6 , discharge affected by regulation or diversion; 7, discharge is a historic peak; A, year of occurrence is unknown or not exact; $\mathrm{B}$, month or day of occurrence is unknown or not exact; E, only maximum peak available for this year. qualification codes apply to the gage height data: 1, gage height affected by backwater; 2, gage height not the maximum for the year; 3, gage height at different data or at different site and datum;

5 , gage height is an estimate]

02479431 Pond Creek Near Deer Park, Ala.

L ocation-Lat $31^{\circ} 09^{\prime} 39^{\prime \prime}$, long 88 $21^{\circ} 43^{\prime \prime}$ referenced to North American Datum of 1927, Washington County, AL, Hydrologic Unit 03170008, near right bank on downstream side of bridge on County Road 9, 5 mi southwest of Deer Park, Alabama. (See attached map of site.)

Drainage area-20.4 $\mathrm{mi}^{2}$.

Gage -Water-stage recorder. Datum of gage is $128.00 \mathrm{ft}$ NGVD 29.

\begin{tabular}{|c|c|c|c|c|c|}
\hline $\begin{array}{l}\text { Water } \\
\text { year }\end{array}$ & Date & Discharge & $\begin{array}{l}\text { Discharge } \\
\text { code }\end{array}$ & $\begin{array}{c}\text { Gage } \\
\text { height } \\
\text { (feet) }\end{array}$ & $\begin{array}{c}\text { Gage } \\
\text { height } \\
\text { code }\end{array}$ \\
\hline 1977 & Mar. 4 & 1,400 & & 11.64 & \\
\hline 1978 & June 8 & 3,020 & & 18.09 & \\
\hline 1979 & Mar. 4 & 2,930 & & 17.74 & \\
\hline 1980 & May 18 & 2,990 & & 17.97 & \\
\hline 1981 & Feb. 10 & 2,530 & & 16.18 & \\
\hline 1982 & Feb. 3 & 1,750 & & 13.13 & \\
\hline 1983 & Apr. 7 & 3,780 & & 21.00 & \\
\hline 1984 & Feb. 27 & 833 & & 8.77 & \\
\hline 1985 & Sept. 23 & 1,720 & & 13.01 & \\
\hline 1986 & Oct. 28 & 960 & & 9.54 & \\
\hline 1987 & Nov. 24 & 643 & & 7.53 & \\
\hline 1988 & Mar. 4 & 1,100 & & 10.04 & \\
\hline 1989 & June 8 & 767 & & 8.21 & \\
\hline 1990 & May 13 & 1,480 & & 12.50 & \\
\hline 1991 & May 10 & 1,510 & & 12.61 & \\
\hline 1992 & Apr. 21 & 913 & & 9.40 & \\
\hline 1993 & Jan. 21 & 1,860 & & 14.09 & \\
\hline 1994 & Oct. 30 & 640 & & 7.39 & \\
\hline 1995 & Feb. 11 & 1,180 & & 11.15 & \\
\hline 1996 & Dec. 19 & 2,880 & & 17.68 & \\
\hline 1997 & Dec. 1 & 1,140 & & 10.93 & \\
\hline 1998 & Sept. 28 & 3,210 & & 18.72 & \\
\hline 1999 & Mar. 14 & 1,730 & & 13.54 & \\
\hline
\end{tabular}

02427300 Prairie Creek near Oak Hill

L ocation-Lat $31^{\circ} 55^{\prime} 37^{\prime \prime}$, long $87^{\circ} 06^{\prime} 21^{\prime \prime}$, in NW 1/4 sec. 18, T. 11 N., R. 10 E., Wilcox County, Hydrologic Unit 03150203, on State Highway 10, 1.4 mi west of Oak Hill and about 6 mi upstream from mouth.

Drainage area-10.3 $\mathrm{mi}^{2}$.

Gage -Water-stage recorder 1960-70. Crest-stage gage 1971-74. Datum of gage is $220 \mathrm{ft}$ NGVD 29.

\begin{tabular}{|c|c|c|c|c|c|}
\hline $\begin{array}{c}\text { Water } \\
\text { year }\end{array}$ & Date & Discharge & $\begin{array}{l}\text { Discharge } \\
\text { code }\end{array}$ & $\begin{array}{c}\text { Gage } \\
\text { height } \\
\text { (feet) }\end{array}$ & $\begin{array}{c}\text { Gage } \\
\text { height } \\
\text { code }\end{array}$ \\
\hline 1960 & May 7 & 1,320 & & 12.32 & \\
\hline 1961 & Feb. 24 & 1,690 & & 14.15 & \\
\hline 1962 & Dec. 12 & 748 & & 9.12 & \\
\hline 1963 & Jan. 20 & 652 & & 8.48 & \\
\hline 1964 & Apr. 27 & 854 & & 9.07 & \\
\hline 1965 & July 29 & 1,210 & & 11.43 & \\
\hline 1966 & Feb. 28 & 831 & & 8.82 & \\
\hline 1967 & Oct. 15 & 1,700 & & 14.19 & \\
\hline 1968 & Dec. 18 & 746 & & 8.30 & \\
\hline 1969 & Mar. 23 & 819 & & 8.82 & \\
\hline 1970 & Mar. 21 & 868 & & 9.16 & \\
\hline 1971 & Mar. 25 & 1,040 & & 10.30 & \\
\hline 1972 & Mar. 2 & 1,860 & & 15.00 & \\
\hline 1973 & Mar. 31 & 1,480 & & 13.08 & \\
\hline 1974 & Apr. 14 & 833 & & 8.92 & \\
\hline
\end{tabular}

\section{Pursley Creek near Camden}

L ocation-Lat $31^{\circ} 57^{\prime} 21^{\prime \prime}$, long $87^{\circ} 20^{\prime} 15^{\prime \prime}$, in SW 1/4 NE 1/4 sec. 2, T. 11 N., R. 7 E., Wilcox County, Hydrologic Unit 03150203, at bridge on State Highway 41, 1.2 mi northeast of Pebble Hill, $3.5 \mathrm{mi}$ southwest of Camden.

Drainage area $-64.3 \mathrm{mi}^{2}$.

G age - Water-stage recorder. Datum of gage is $58.78 \mathrm{ft}$ NGVD 29.

\begin{tabular}{|c|c|c|c|c|c|}
\hline $\begin{array}{l}\text { Water } \\
\text { year }\end{array}$ & Date & Discharge & $\begin{array}{l}\text { Discharge } \\
\text { code }\end{array}$ & $\begin{array}{c}\text { Gage } \\
\text { height } \\
\text { (feet) }\end{array}$ & $\begin{array}{c}\text { Gage } \\
\text { height } \\
\text { code }\end{array}$ \\
\hline 1951 & Mar. 29 & 3,200 & & 13.50 & \\
\hline 1953 & Apr. 6 & 2,600 & & 12.00 & \\
\hline 1954 & Dec. 4 & 630 & & 5.82 & \\
\hline 1955 & Apr. 11 & 7,000 & & 20.30 & \\
\hline 1956 & Mar. 16 & 3,050 & & 13.30 & \\
\hline 1957 & Apr. 5 & 7,000 & & 20.40 & \\
\hline
\end{tabular}


Appendix 2. Discharge and gage height data for streamgages in Alabama.-Continued

[--, date or data not available; $\mathrm{ft}$, foot; $\mathrm{ft}^{3} / \mathrm{s}$, cubic foot per second; mi, mile; $\mathrm{mi}^{2}$, square mile; qualification codes apply to the discharge data: 1 , discharge is a maximum daily average; 2 , discharge is an estimate; 4 , discharge is less than indicated value, which is the minimum recordable discharge at this site; 5 , discharge affected to unknown degree by regulation or diversion; 6 , discharge affected by regulation or diversion; 7, discharge is a historic peak; A, year of occurrence is unknown or not exact; $\mathrm{B}$, month or day of occurrence is unknown or not exact; E, only maximum peak available for this year. qualification codes apply to the gage height data: 1, gage height affected by backwater; 2, gage height not the maximum for the year; 3, gage height at different datum or at different site and datum; 5 , gage height is an estimate]

02427875 Pursley Creek near Camden-Continued

Location-Lat $31^{\circ} 57^{\prime} 21^{\prime \prime}$, long $87^{\circ} 20^{\prime} 15^{\prime \prime}$, in SW 1/4 NE 1/4 sec. 2 , T. 11 N., R. 7 E., Wilcox County, Hydrologic Unit 03150203, at bridge on State Highway 41, 1.2 mi northeast of Pebble Hill, 3.5 mi southwest of Camden.

Drainage area $-64.3 \mathrm{mi}^{2}$.

Gage-Water-stage recorder. Datum of gage is $58.78 \mathrm{ft}$ NGVD 29.

\begin{tabular}{|c|c|c|c|c|c|}
\hline $\begin{array}{c}\text { Water } \\
\text { year }\end{array}$ & Date & Discharge & $\begin{array}{l}\text { Discharge } \\
\text { code }\end{array}$ & $\begin{array}{c}\text { Gage } \\
\text { height } \\
\text { (feet) }\end{array}$ & $\begin{array}{c}\text { Gage } \\
\text { height } \\
\text { code }\end{array}$ \\
\hline 1958 & Mar. 7 & 5,400 & & 17.70 & \\
\hline 1959 & June 11 & 3,700 & & 14.80 & \\
\hline 1960 & Aug. 5 & 2,600 & & 11.90 & \\
\hline 1961 & Mar. 31 & 11,400 & & 25.90 & \\
\hline 1962 & Apr. 29 & 2,050 & & 10.95 & \\
\hline 1963 & Jan. 20 & 2,390 & & 11.80 & \\
\hline 1964 & Apr. 8 & 6,260 & & 19.03 & \\
\hline 1965 & Jan. 23 & 4,050 & & 15.56 & \\
\hline 1966 & Mar. 5 & 3,120 & & 13.63 & \\
\hline 1967 & Feb. 7 & 2,590 & & 12.30 & \\
\hline 1968 & Apr. 5 & 2,410 & & 11.86 & \\
\hline 1969 & May 19 & 2,980 & & 13.28 & \\
\hline 1970 & Mar. 22 & 3,070 & & 13.49 & \\
\hline
\end{tabular}

02364500 Pea River near Samson

L ocation-Lat $31^{\circ} 06^{\prime} 45^{\prime \prime}$, long $86^{\circ} 05^{\prime} 58^{\prime \prime}$, SW 1/4 sec. 25 , T. 2 N., R. 19 E., Geneva County, Hydrologic Unit 03140202, on State Highway 52, 3 mi west of Samson, $6.5 \mathrm{mi}$ upstream from Flat Creek, and at mile 29.8.

Drainage area-1,182 $\mathrm{mi}^{2}$.

Gage -Water-stage recorder. Datum of gage is $97.95 \mathrm{ft}$ NGVD 29. Prior to 1926, site at $1.5 \mathrm{mi}$ upstream at different datum.

\begin{tabular}{llrrcc}
\hline $\begin{array}{c}\text { Water } \\
\text { year }\end{array}$ & Date & Discharge & $\begin{array}{c}\text { Discharge } \\
\text { code }\end{array}$ & $\begin{array}{c}\text { Gage } \\
\text { height } \\
\text { (feet) }\end{array}$ & $\begin{array}{c}\text { Gage } \\
\text { height } \\
\text { code }\end{array}$ \\
\hline 1905 & Feb. 16 & 12,800 & 2 & -- & 3 \\
1906 & Dec. 25 & 9,500 & 2 & -- & 3 \\
1907 & Sept. 29 & 6,890 & 2 & -- & 3 \\
1908 & Mar. 28 & 14,100 & 2 & -- & 3 \\
1909 & Mar. 24 & 8,150 & 2 & -- & 3 \\
1910 & Mar. 3 & 8,550 & 2 & -- & 3 \\
1911 & Jan. 4 & 5,150 & 2 & -- & 3 \\
1912 & Apr. 24 & 16,000 & 2 & -- & 3 \\
1913 & Mar. 16 & 23,500 & 2 & -- & 3
\end{tabular}

02364500 Pea River near Samson-Continued

L ocation-Lat $31^{\circ} 06^{\prime} 45^{\prime \prime}$, long $86^{\circ} 05^{\prime} 58^{\prime \prime}$, SW 1/4 sec. 25 , T. 2 N., R. 19 E., Geneva County, Hydrologic Unit 03140202, on State Highway 52, 3 mi west of Samson, 6.5 mi upstream from Flat Creek, and at mile 29.8 .

Drainage area-1,182 $\mathrm{mi}^{2}$.

G age -Water-stage recorder. Datum of gage is $97.95 \mathrm{ft}$ NGVD 29. Prior to 1926, site at $1.5 \mathrm{mi}$ upstream at different datum.

\begin{tabular}{lccccc}
\hline $\begin{array}{c}\text { Water } \\
\text { year }\end{array}$ & Date & Discharge & $\begin{array}{c}\text { Discharge } \\
\text { code }\end{array}$ & $\begin{array}{c}\text { Gage } \\
\text { height } \\
\text { (feet) }\end{array}$ & $\begin{array}{c}\text { Gage } \\
\text { height } \\
\text { code }\end{array}$ \\
\hline 1923 & Mar. 20 & 12,200 & 2 & -- & 3 \\
1924 & Jan. 25 & 9,100 & 2 & -- & 3 \\
1925 & Jan. 20 & 30,000 & & 42.00 & 3 \\
1929 & Mar. 15 & 58,000 & 7 & 45.30 &
\end{tabular}

19

1936

1937 Apr. $7 \quad 23,400 \quad 35.8$

1938 Mar. $18 \quad 23,300 \quad 35.83$

1939 Mar. $2 \quad 14,200 \quad 28.80$

$1940 \quad$ Feb. $19 \quad 9,120 \quad 22.75$

1941 Dec. $28 \quad 4,170 \quad 13.55$

1942 Apr. $11 \quad 7,460 \quad 20.24$

$1943 \quad$ Jan. $21 \quad 19,200 \quad 33.22$

1944 Mar. $26 \quad 19,500 \quad 33.40$

1945 Apr. $30 \quad 5,920 \quad 17.40$

1946 May $22 \quad 16,000 \quad 30.50$

1947 Apr. $6 \quad 12,900 \quad 26.70$

1948 Mar. $8 \quad$ 15,300 29.60

1949 Dec. $1 \quad 15,700 \quad 29.90$

$1950 \quad$ Sept. $2 \quad 7,100 \quad 19.50$

1951 Mar. $30 \quad 7,100 \quad 19.50$

1952 Mar. $28 \quad 10,600 \quad 24.70$

$1953 \quad$ Sept. $28 \quad 14,700 \quad 29.10$

1954 Dec. $5 \quad 18,100 \quad 31.73$

1955 Apr. $15 \quad 14,700 \quad 29.10$

1956 Sept. $27 \quad 12,700 \quad 27.25$

1957 Apr. $7 \quad 18,500 \quad 32.00$

1958 Mar. $10 \quad 7,160 \quad 19.60$

$1959 \quad$ Feb. $5 \quad 9,090 \quad 22.70$

$1960 \quad$ Apr. $6 \quad 27,900 \quad 37.71$

1961 Apr. $15 \quad 12,400 \quad 27.35$

1962 Apr. $2 \quad 11,300 \quad 26.65$ 
Appendix 2. Discharge and gage height data for streamgages in Alabama.-Continued

[--, date or data not available; $\mathrm{ft}$, foot; $\mathrm{ft}^{3} / \mathrm{s}$, cubic foot per second; $\mathrm{mi}$, mile; $\mathrm{mi}^{2}$, square mile; qualification codes apply to the discharge data: 1 , discharge is a maximum daily average; 2 , discharge is an estimate; 4 , discharge is less than indicated value, which is the minimum recordable discharge at this site; 5 , discharge affected to unknown degree by regulation or diversion; 6 , discharge affected by regulation or diversion; 7, discharge is a historic peak; A, year of occurrence is unknown or not exact; B, month or day of occurrence is unknown or not exact; E, only maximum peak available for this year. qualification codes apply to the gage height data: 1, gage height affected by backwater; 2 , gage height not the maximum for the year; 3 , gage height at different data or at different site and datum; 5 , gage height is an estimate]

\section{Pea River near Samson-Continued}

L ocation-Lat $31^{\circ} 06^{\prime} 45^{\prime \prime}$, long $86^{\circ} 05^{\prime} 58^{\prime \prime}$, SW 1/4 sec. 25 , T. 2 N., R. 19 E., Geneva County, Hydrologic Unit 03140202, on State Highway 52, 3 mi west of Samson, $6.5 \mathrm{mi}$ upstream from Flat Creek, and at mile 29.8 .

Drainage area-1,182 $\mathrm{mi}^{2}$.

Gage -Water-stage recorder. Datum of gage is $97.95 \mathrm{ft}$ NGVD 29. Prior to 1926, site at $1.5 \mathrm{mi}$ upstream at different datum.

\begin{tabular}{|c|c|c|c|c|c|}
\hline $\begin{array}{c}\text { Water } \\
\text { year }\end{array}$ & Date & Discharge & $\begin{array}{l}\text { Discharge } \\
\text { code }\end{array}$ & $\begin{array}{c}\text { Gage } \\
\text { height } \\
\text { (feet) }\end{array}$ & $\begin{array}{c}\text { Gage } \\
\text { height } \\
\text { code }\end{array}$ \\
\hline 1963 & Jan. 22 & 9,170 & & 22.82 & \\
\hline 1964 & Mar. 5 & 10,700 & & 25.00 & \\
\hline 1965 & Dec. 30 & 9,750 & & 23.64 & \\
\hline 1966 & Mar. 6 & 14,500 & & 29.52 & \\
\hline 1967 & Jan. 3 & 10,900 & & 25.91 & \\
\hline 1968 & Mar. 13 & 6,860 & & 19.77 & \\
\hline 1969 & May 19 & 7,740 & & 20.57 & \\
\hline 1970 & June 5 & 15,700 & & 30.72 & \\
\hline 1975 & Feb. 21 & 30,800 & 7 & 38.06 & \\
\hline 2003 & Apr. 9 & 9,530 & & 23.90 & \\
\hline 2004 & Sept. 18 & 12,400 & & 27.32 & \\
\hline 2005 & Apr. 2 & 17,000 & & 31.51 & \\
\hline 2006 & Mar. 11 & 6,440 & & 19.12 & \\
\hline 2007 & Apr. 16 & 7,970 & & 21.56 & \\
\hline 2008 & Feb. 2 & 9,010 & & 22.59 & \\
\hline 2009 & Mar. 29 & 15,500 & & 30.52 & \\
\hline 2010 & Dec. 16 & 26,000 & & 37.83 & \\
\hline 2011 & Mar. 10 & 4,580 & & 15.96 & \\
\hline 2012 & Sept. 5 & 11,800 & & 26.09 & \\
\hline 2013 & Feb. 27 & 10,700 & & 24.88 & \\
\hline 2014 & Apr. 9 & 15,300 & & 29.48 & \\
\hline 2015 & Apr. 19 & 6,920 & & 19.96 & \\
\hline 2016 & Dec. 27 & 45,800 & & 42.02 & \\
\hline
\end{tabular}

02373000 Sepulga River near Mckenzie

L ocation-Lat $31^{\circ} 27^{\prime} 13^{\prime \prime}$, long $86^{\circ} 47^{\prime} 13^{\prime \prime}$, in SE $1 / 4$ sec. 30, T. 6 N., R. 13 E., Conecuh County, Hydrologic Unit 03140303, on U.S. Highway 31, 2.5 mi upstream from Piney Woods Creek, $5.5 \mathrm{mi}$ downstream from Persimmon Creek, and $7 \mathrm{mi}$ southwest of McKenzie.

Drainage area $470 \mathrm{mi}^{2}$.

Gage -Water-stage recorder. Datum of gage is $155.96 \mathrm{ft}$ NGVD 29.

\begin{tabular}{llcccc}
\hline $\begin{array}{c}\text { Water } \\
\text { year }\end{array}$ & Date & Discharge & $\begin{array}{c}\text { Discharge } \\
\text { code }\end{array}$ & $\begin{array}{c}\text { Gage } \\
\text { height } \\
\text { (feet) }\end{array}$ & $\begin{array}{c}\text { Gage } \\
\text { height } \\
\text { code }\end{array}$ \\
\hline 1929 & Mar & 60,000 & $2,7, B$ & 33.00 & 5 \\
1938 & Mar. 17 & 23,000 & & 24.50 & \\
1939 & Aug. 18 & 13,400 & & 19.48 &
\end{tabular}

$1940 \quad$ Feb. $20 \quad 9,000 \quad 16.00$

1941 Mar. $9 \quad 4,210 \quad 9.43$

1942 Dec. $26 \quad 7,100 \quad 13.46$

1943 Mar. $23 \quad 19,100 \quad 21.82$

1944 Mar. $31 \quad 22,100$

1945 May $1 \quad 3,040 \quad 8.11$

$1946 \quad$ Jan. $8 \quad 19,400 \quad 21.91$

1947 Apr. $4 \quad 9,470 \quad 16.54$

1948 Mar. $8 \quad 13,300 \quad 19.20$

$1949 \quad$ Nov. $28 \quad 21,200 \quad 23.60$

$1950 \quad$ July $30 \quad 2,950 \quad 7.70$

1951 Apr. $21 \quad 6,920 \quad 12.90$

1952 Mar. $25 \quad 5,880 \quad 11.60$

1953 May $5 \quad 3,370 \quad 8.29$

1954 Dec. $8 \quad 7,940 \quad 14.06$

1955 Apr. $16 \quad 9,550 \quad 15.86$

1956 Sept. $25 \quad 5,320 \quad 10.89$

1957 Apr. $7 \quad 14,000 \quad 19.80$

1958 Mar. $10 \quad 3,250 \quad 8.23$

$1959 \quad$ Feb. $7 \quad 3,760 \quad 8.77$

1960 Apr. $1 \quad 15,200 \quad 20.94$

1961 Feb. $26 \quad 23,300 \quad 24.70$

1962 Apr. $2 \quad 10,600 \quad 17.18$

$1963 \quad$ Jan. $23 \quad 5,750 \quad 11.60$

1964 Apr. $29 \quad 22,200 \quad 24.32$

1965 Jan. $25 \quad 16,100 \quad 21.45$

1966 Mar. $5 \quad 10,100 \quad 16.67$ 
Appendix 2. Discharge and gage height data for streamgages in Alabama.-Continued

[--, date or data not available; $\mathrm{ft}$, foot; $\mathrm{ft}^{3} / \mathrm{s}$, cubic foot per second; mi, mile; $\mathrm{mi}^{2}$, square mile; qualification codes apply to the discharge data: 1 , discharge is a maximum daily average; 2 , discharge is an estimate; 4 , discharge is less than indicated value, which is the minimum recordable discharge at this site; 5 , discharge affected to unknown degree by regulation or diversion; 6 , discharge affected by regulation or diversion; 7, discharge is a historic peak; A, year of occurrence is unknown or not exact; $\mathrm{B}$, month or day of occurrence is unknown or not exact; E, only maximum peak available for this year. qualification codes apply to the gage height data: 1, gage height affected by backwater; 2, gage height not the maximum for the year; 3, gage height at different datum or at different site and datum; 5 , gage height is an estimate]

\section{Sepulga River near Mckenzie-Continued}

L ocation-Lat $31^{\circ} 27^{\prime} 13^{\prime \prime}$, long $86^{\circ} 47^{\prime} 13^{\prime \prime}$, in SE $1 / 4$ sec. 30 , T. 6

N., R. 13 E., Conecuh County, Hydrologic Unit 03140303, on

U.S. Highway 31, 2.5 mi upstream from Piney Woods Creek,

$5.5 \mathrm{mi}$ downstream from Persimmon Creek, and $7 \mathrm{mi}$ southwest

of McKenzie.

Drainage area $-470 \mathrm{mi}^{2}$.

Gage - Water-stage recorder. Datum of gage is $155.96 \mathrm{ft}$ NGVD 29.

\begin{tabular}{|c|c|c|c|c|c|}
\hline $\begin{array}{c}\text { Water } \\
\text { year }\end{array}$ & Date & Discharge & $\begin{array}{c}\text { Discharge } \\
\text { code }\end{array}$ & $\begin{array}{c}\text { Gage } \\
\text { height } \\
\text { (feet) }\end{array}$ & $\begin{array}{c}\text { Gage } \\
\text { height } \\
\text { code }\end{array}$ \\
\hline 1967 & Sept. 14 & 8,700 & & 15.22 & \\
\hline 1968 & Nov. 2 & 2,800 & & 7.55 & \\
\hline 1969 & Mar. 26 & 4,660 & & 10.05 & \\
\hline 1970 & June 5 & 14,500 & & 20.40 & \\
\hline 1975 & Feb. 19 & 18,500 & & 22.80 & \\
\hline 1976 & Jan. 2 & 11,600 & & 17.96 & \\
\hline 1977 & Mar. 15 & 6,741 & & 12.53 & \\
\hline 1978 & Jan. 27 & 16,300 & & 21.62 & \\
\hline 1979 & Mar. 6 & 14,400 & & 20.34 & \\
\hline 1980 & Apr. 15 & 13,600 & & 18.68 & \\
\hline 1981 & Feb. 13 & 8,280 & & 13.14 & \\
\hline 1982 & Feb. 5 & 12,700 & & 17.77 & \\
\hline 1983 & Apr. 10 & 9,990 & & 16.33 & \\
\hline 1984 & May 3 & 5,940 & & 11.12 & \\
\hline 1985 & Feb. 7 & 5,020 & & 9.90 & \\
\hline 1986 & Mar. 16 & 5,260 & & 10.22 & \\
\hline 1987 & Mar. 2 & 3,860 & & 8.55 & \\
\hline 1988 & Mar. 6 & 4,780 & & 9.67 & \\
\hline 1989 & Apr. 12 & 6,930 & & 12.44 & \\
\hline 1990 & Mar. 18 & 29,100 & & 26.28 & \\
\hline 1991 & Mar. 5 & 7,010 & & 12.55 & \\
\hline 1992 & Feb. 19 & 5,900 & & 11.08 & \\
\hline 1993 & Dec. 19 & 11,500 & & 18.15 & \\
\hline
\end{tabular}

02373000 Sepulga River near Mckenzie-Continued

L ocation-Lat $31^{\circ} 27^{\prime} 13^{\prime \prime}$, long $86^{\circ} 47^{\prime} 13^{\prime \prime}$, in SE $1 / 4$ sec. 30 , T. 6 N., R. 13 E., Conecuh County, Hydrologic Unit 03140303, on U.S. Highway 31, 2.5 mi upstream from Piney Woods Creek, 5.5 mi downstream from Persimmon Creek, and 7 mi southwest of McKenzie.

Drainage area-470 $\mathrm{mi}^{2}$.

G age - Water-stage recorder. Datum of gage is $155.96 \mathrm{ft}$ NGVD 29.

\begin{tabular}{|c|c|c|c|c|c|}
\hline $\begin{array}{c}\text { Water } \\
\text { year }\end{array}$ & Date & Discharge & $\begin{array}{l}\text { Discharge } \\
\text { code }\end{array}$ & $\begin{array}{c}\text { Gage } \\
\text { height } \\
\text { (feet) }\end{array}$ & $\begin{array}{c}\text { Gage } \\
\text { height } \\
\text { code }\end{array}$ \\
\hline 1994 & July 10 & 12,000 & & 18.62 & \\
\hline 1995 & Mar. 10 & 6,580 & & 11.97 & \\
\hline 1996 & Mar. 30 & 8,500 & & 14.51 & \\
\hline 1997 & May 30 & 6,210 & & 11.48 & \\
\hline 1998 & Mar. 10 & 21,200 & & 24.00 & \\
\hline 1999 & Oct. 1 & 32,800 & & 27.19 & \\
\hline 2000 & Mar. 21 & 1,590 & & 6.11 & \\
\hline 2001 & Mar. 5 & 27,000 & & 25.73 & \\
\hline 2002 & Feb. 23 & 6,670 & & 12.09 & \\
\hline 2003 & July 3 & 9,440 & & 15.69 & \\
\hline 2004 & Sept. 19 & 6,560 & & 11.96 & \\
\hline 2005 & Apr. 3 & 11,900 & & 18.52 & \\
\hline 2006 & Feb. 28 & 3,520 & & 8.28 & \\
\hline 2007 & Mar. 3 & 2,890 & & 7.41 & \\
\hline 2008 & Aug. 27 & 3,100 & & 7.76 & \\
\hline 2009 & Mar. 30 & 11,700 & & 18.35 & \\
\hline 2010 & Dec. 16 & 21,400 & & 24.09 & \\
\hline 2011 & Mar. 12 & 4,160 & & 9.08 & \\
\hline 2012 & Sept. 7 & 4,620 & & 9.66 & \\
\hline 2013 & Feb. 14 & 9,280 & & 15.51 & \\
\hline 2014 & Apr. 9 & 15,600 & & 22.12 & \\
\hline 2015 & Apr. 19 & 5,870 & & 10.70 & \\
\hline
\end{tabular}


Appendix 2. Discharge and gage height data for streamgages in Alabama.-Continued

[--, date or data not available; $\mathrm{ft}$, foot; $\mathrm{ft}^{3} / \mathrm{s}$, cubic foot per second; $\mathrm{mi}$, mile; $\mathrm{mi}^{2}$, square mile; qualification codes apply to the discharge data: 1 , discharge is a maximum daily average; 2 , discharge is an estimate; 4 , discharge is less than indicated value, which is the minimum recordable discharge at this site; 5 , discharge affected to unknown degree by regulation or diversion; 6 , discharge affected by regulation or diversion; 7, discharge is a historic peak; A, year of occurrence is unknown or not exact; B, month or day of occurrence is unknown or not exact; E, only maximum peak available for this year. qualification codes apply to the gage height data: 1, gage height affected by backwater; 2 , gage height not the maximum for the year; 3 , gage height at different data or at different site and datum; 5 , gage height is an estimate]

\section{South Fork Cowikee Creek near Batesville}

L ocation-Lat $32^{\circ} 01^{\prime} 03^{\prime \prime}$, long $85^{\circ} 17^{\prime} 45^{\prime \prime}$, in SE $1 / 4$ sec. 14 , T. 12 N., R. 27 E., Barbour County, Hydrologic Unit 03130003, on county road, $1.2 \mathrm{mi}$ northeast of Batesville, $11.2 \mathrm{mi}$ northwest of Eufaula, and $13.0 \mathrm{mi}$ upstream from mouth.

Drainage area-112 $\mathrm{mi}^{2}$.

Gage - Water-stage recorder. Datum of gage is $200 \mathrm{ft} \mathrm{NGVD} 29$ (from topographic map).

\begin{tabular}{|c|c|c|c|c|c|}
\hline $\begin{array}{c}\text { Water } \\
\text { year }\end{array}$ & Date & Discharge & $\begin{array}{l}\text { Discharge } \\
\text { code }\end{array}$ & $\begin{array}{c}\text { Gage } \\
\text { height } \\
\text { (feet) }\end{array}$ & $\begin{array}{c}\text { Gage } \\
\text { height } \\
\text { code }\end{array}$ \\
\hline
\end{tabular}

\begin{tabular}{llll}
\hline 1964 & May 2 & 5,640 & 14.86 \\
1965 & Oct. 5 & 7,040 & 19.13 \\
1966 & Feb. 13 & 6,040 & 15.85
\end{tabular}

1967 Jan. $1 \quad 5,060$

1968 Mar. $12 \quad 6,810 \quad 18.37$

1969 Apr. $18 \quad 4,600 \quad 12.45$

1970 Mar. $30 \quad 5,220$

1971 Mar. $3 \quad 6,750$

1972 Mar. $2 \quad 5,270$

1973 Mar. $31 \quad$ 12,200

$1974 \quad$ Feb. 8 3,370

1975 Feb. $17 \quad$ 18,100

1976 Oct. $17 \quad 8,650$

1977 Mar. $22 \quad 3,510$

1978 Jan. $25 \quad 10,900$

1979 Apr. $4 \quad 4,730$

1980 Mar. $30 \quad 5,270$

1981 Feb. $10 \quad 5,800$

1982 Feb. $3 \quad 4,530$

1983 Mar. $6 \quad$ 4,680

1984 Mar. 5 2,000

1985 June $18 \quad 1,820$

1986 Mar. $13 \quad 7,550$

1987
Feb. $28 \quad 2,510$
13.79

18.18

13.92

27.40

10.12

37.08

24.58

14.19

28.03

17.26

18.34

19.40

16.82

17.15

10.01

E

9.39

22.79

11.17
02342933 South Fork Cowikee Creek near Batesville-Continued L ocation-Lat $32^{\circ} 01^{\prime} 03^{\prime \prime}$, long $85^{\circ} 17^{\prime} 45^{\prime \prime}$, in SE $1 / 4$ sec. 14 , T. 12 N., R. 27 E., Barbour County, Hydrologic Unit 03130003, on county road, $1.2 \mathrm{mi}$ northeast of Batesville, $11.2 \mathrm{mi}$ northwest of Eufaula, and 13.0 mi upstream from mouth.

Drainage area-112 $\mathrm{mi}^{2}$.

Gage - Water-stage recorder. Datum of gage is $200 \mathrm{ft} \mathrm{NGVD} 29$ (from topographic map).

\begin{tabular}{llcccc}
\hline $\begin{array}{c}\text { Water } \\
\text { year }\end{array}$ & Date & Discharge & $\begin{array}{c}\text { Discharge } \\
\text { code }\end{array}$ & $\begin{array}{c}\text { Gage } \\
\text { height } \\
\text { (feet) }\end{array}$ & $\begin{array}{c}\text { Gage } \\
\text { height } \\
\text { code }\end{array}$ \\
\hline 1988 & Mar. 4 & 2,340 & & 10.68 & \\
1989 & May 1 & 3,270 & & 13.33 & \\
1990 & Mar. 17 & 28,200 & & 43.40 & \\
1991 & & & & 16.15 &
\end{tabular}

1991 Mar. $2 \quad 3,820 \quad 16.15$

$1992 \quad$ Jan. $13 \quad 4,080 \quad 16.61$

$1993 \quad$ Dec. $17 \quad 5,490 \quad 19.40$

1994 July $4 \quad 13,100 \quad 31.17$

1995 Feb. $28 \quad 5,210 \quad 18.09$

1996 Feb. $2 \quad 5,820 \quad 19.32$

$1997 \quad$ Feb. $15 \quad 4,950 \quad 17.53$

1998 Mar. $8 \quad 9,190 \quad 25.48$

1999 Mar. $14 \quad 6,520 \quad 20.70$

$2000 \quad$ Mar. $20 \quad 3,210 \quad 13.66$

2001 Mar. $4 \quad 9,740 \quad 26.05$

$2002 \quad$ Nov. $25 \quad 4,880 \quad 17.37$

2003 July $1 \quad 5,200 \quad 18.02$

2004 Jan. $26 \quad 7,240 \quad 21.85$

2005 Mar. $27 \quad 9,160 \quad 25.11$

$2006 \quad$ Feb. $25 \quad 3,760 \quad 14.93$

2007 Apr. $15 \quad 5,110 \quad 17.83$

2008 Aug. $24 \quad 5,460 \quad 18.54$

2009 Mar. $28 \quad 8,520 \quad 24.05$

2010 Dec. $15 \quad 11,900 \quad 29.43$

2011 Mar. $9 \quad 4,400 \quad 16.37$ 
Appendix 2. Discharge and gage height data for streamgages in Alabama.-Continued

[--, date or data not available; $\mathrm{ft}$, foot; $\mathrm{ft}^{3} / \mathrm{s}$, cubic foot per second; mi, mile; $\mathrm{mi}^{2}$, square mile; qualification codes apply to the discharge data: 1 , discharge is a maximum daily average; 2 , discharge is an estimate; 4 , discharge is less than indicated value, which is the minimum recordable discharge at this site; 5 , discharge affected to unknown degree by regulation or diversion; 6 , discharge affected by regulation or diversion; 7, discharge is a historic peak; A, year of occurrence is unknown or not exact; $\mathrm{B}$, month or day of occurrence is unknown or not exact; E, only maximum peak available for this year. qualification codes apply to the gage height data: 1, gage height affected by backwater; 2, gage height not the maximum for the year; 3, gage height at different datum or at different site and datum; 5 , gage height is an estimate]

\section{Stevenson Creek near Headland}

L ocation-Lat $31^{\circ} 21^{\prime} 18^{\prime \prime}$, long $85^{\circ} 11^{\prime} 05^{\prime \prime}$, in SE $1 / 4$ sec. 36 , T. 5 N., R. 28 E., Henry County, Hydrologic Unit 03130004, on State Highway 134, 1 mi upstream from mouth, and 9.5 mi east of Headland.

Drainage area $-14.0 \mathrm{mi}^{2}$.

G age -Water-stage recorder 1960-65. Crest-stage gage 1966-74. Datum of gage is $150.39 \mathrm{ft}$ NGVD 29.

\begin{tabular}{|c|c|c|c|c|c|}
\hline $\begin{array}{l}\text { Water } \\
\text { year }\end{array}$ & Date & Discharge & $\begin{array}{l}\text { Discharge } \\
\text { code }\end{array}$ & $\begin{array}{c}\text { Gage } \\
\text { height } \\
\text { (feet) }\end{array}$ & $\begin{array}{c}\text { Gage } \\
\text { height } \\
\text { code }\end{array}$ \\
\hline 1960 & Apr. 4 & 728 & & 7.92 & \\
\hline 1961 & Apr. 15 & 1,070 & & 9.13 & \\
\hline 1962 & Jan. 6 & 3,120 & & 12.01 & \\
\hline 1963 & Feb. 11 & 978 & & 8.53 & \\
\hline 1964 & Mar. 2 & 2,200 & & 9.90 & \\
\hline 1965 & Apr. 19 & 2,530 & & 10.31 & \\
\hline 1966 & Feb. 28 & 872 & & 7.02 & \\
\hline 1967 & Dec. 31 & 396 & & 4.58 & \\
\hline 1968 & Dec. 10 & 784 & & 6.68 & \\
\hline 1969 & Mar. 18 & 633 & & 6.00 & \\
\hline 1970 & Dec. 25 & 458 & & 4.97 & \\
\hline 1971 & Mar. 26 & 1,500 & & 8.47 & \\
\hline 1972 & May 8 & 649 & & 6.08 & \\
\hline 1973 & June 7 & 1,420 & & 8.57 & \\
\hline 1974 & Jan. 2 & 2,180 & & 9.87 & \\
\hline
\end{tabular}

\section{Styx River near Elsanor, Ala.}

L ocation - Lat $30^{\circ} 36^{\prime} 20^{\prime \prime}$, long $87^{\circ} 32^{\prime} 50^{\prime \prime}$ referenced to North American Datum of 1927, Baldwin County, Ala., Hydrologic Unit 03140106, near left bank on downstream side of bridge on County Road 87, 0.2 mi downstream of Cowpen Creek, 5 mi northeast of Elsanor, and $11.4 \mathrm{mi}$ upstream of mouth.

Drainage area-192 $\mathrm{mi}^{2}$.

G age -Water-stage recorder. Elevation of gage is $20 \mathrm{ft}$ above NGVD of 1929, from topographic map.

\begin{tabular}{|c|c|c|c|c|c|}
\hline $\begin{array}{c}\text { Water } \\
\text { year }\end{array}$ & Date & Discharge & $\begin{array}{l}\text { Discharge } \\
\text { code }\end{array}$ & $\begin{array}{c}\text { Gage } \\
\text { height } \\
\text { (feet) }\end{array}$ & $\begin{array}{c}\text { Gage } \\
\text { height } \\
\text { code }\end{array}$ \\
\hline 1988 & Sept. 17 & 4,700 & & 13.18 & \\
\hline 1989 & June 9 & 17,500 & & 20.18 & \\
\hline 1990 & Mar. 17 & 17,200 & & 20.07 & \\
\hline 1991 & Jan. 31 & 4,060 & & 12.56 & \\
\hline 1992 & Jan. 14 & 9,670 & & 16.61 & \\
\hline 1993 & Mar. 31 & 5,750 & $\mathrm{E}$ & 14.05 & \\
\hline 1994 & July 8 & 7,690 & & 15.40 & \\
\hline 1995 & May 11 & 10,300 & & 16.97 & \\
\hline 1996 & Oct. 5 & 18,500 & & 20.50 & \\
\hline 1997 & July 21 & 15,900 & & 19.82 & \\
\hline 1998 & Sept. 29 & 48,000 & & 28.60 & \\
\hline 1999 & June 28 & 2,200 & 2 & 9.00 & 5 \\
\hline 2000 & Oct. 11 & 1,750 & & 7.73 & \\
\hline 2001 & Mar. 15 & 3,610 & & 12.01 & \\
\hline 2002 & Sept. 27 & 10,400 & & 17.38 & \\
\hline 2003 & July 2 & 12,000 & & 18.26 & \\
\hline 2004 & Feb. 27 & 3,650 & & 11.93 & \\
\hline 2005 & Apr. 1 & 14,900 & & 19.59 & \\
\hline 2006 & Sept. 13 & 1,170 & & 5.76 & \\
\hline 2007 & Nov. 17 & 1,590 & & 7.20 & \\
\hline 2008 & Nov. 17 & 1,590 & & 7.20 & \\
\hline 2009 & Mar. 29 & 13,900 & & 19.51 & \\
\hline 2010 & Jan. 22 & 10,100 & & 17.69 & \\
\hline 2011 & Sept. 5 & 3,430 & & 12.49 & \\
\hline 2012 & June 13 & 3,310 & & 12.79 & \\
\hline 2013 & July 12 & 3,580 & & 13.09 & \\
\hline 2014 & Apr. 30 & 17,300 & & 20.87 & \\
\hline 2015 & Apr. 16 & 3,670 & & 13.19 & \\
\hline
\end{tabular}


Appendix 2. Discharge and gage height data for streamgages in Alabama.-Continued

[--, date or data not available; ft, foot; $\mathrm{ft}^{3} / \mathrm{s}$, cubic foot per second; mi, mile; $\mathrm{mi}^{2}$, square mile; qualification codes apply to the discharge data: 1 , discharge is a maximum daily average; 2 , discharge is an estimate; 4 , discharge is less than indicated value, which is the minimum recordable discharge at this site; 5 , discharge affected to unknown degree by regulation or diversion; 6 , discharge affected by regulation or diversion; 7, discharge is a historic peak; A, year of occurrence is unknown or not exact; $\mathrm{B}$, month or day of occurrence is unknown or not exact; E, only maximum peak available for this year. qualification codes apply to the gage height data: 1, gage height affected by backwater; 2, gage height not the maximum for the year; 3, gage height at different data or at different site and datum;

5 , gage height is an estimate]

$\mathbf{0 2 3 7 7 5 0 0}$ Styx River near Loxley

L ocation-Lat $30^{\circ} 39^{\prime} 50^{\prime \prime}$, long $87^{\circ} 38^{\prime} 20^{\prime \prime}$, in SE $1 / 4$ sec. 26 , T. 4 S., R. 4 E., Baldwin County, Hydrologic Unit 03140106, on county road, 2 mi upstream from Hollinger Creek, and 7 mi northeast of Loxley.

Drainage area-92.2 $\mathrm{mi}^{2}$.

Gage -Water-stage recorder 1952-69, 1971. Crest-stage gage 1973-77. Datum of gage is $39 \mathrm{ft}$ NGVD 29.

\begin{tabular}{llllll}
\hline $\begin{array}{c}\text { Water } \\
\text { year }\end{array}$ & Date & Discharge & $\begin{array}{c}\text { Discharge } \\
\text { code }\end{array}$ & $\begin{array}{c}\text { Gage } \\
\text { height } \\
\text { (feet) }\end{array}$ & $\begin{array}{c}\text { Gage } \\
\text { height } \\
\text { code }\end{array}$ \\
\hline 1926 & Sept. & 27,500 & $2,7, B$ & 22.20 & \\
1952 & Sept. 19 & 1,730 & & 7.50 &
\end{tabular}

1952

1953

Sept. $19 \quad 1,730$

Apr. $12 \quad 1,790$

7.50

7.85

1954

Dec. $6 \quad 14,000$

1955

Apr. $14 \quad 2,520$

1956

Mar. 12

1,900

Apr. $5 \quad 7,430$

1957

Mar. 6

1,920

1959

Sept. 13

3,110

Apr. 4 3,780

1960

June 20

4,590

1961

Apr. 1

4,000

19.73

10.57

8.09

17.50

8.25

11.80

13.31

14.74

13.75

1963 Jan. $21 \quad 1,510$

6.48

17.91

1964

Apr. $27 \quad 7,820$

5.92

Aug. $9 \quad 1,430$

16.19

1966

Oct. 1

5,370

12.54

4.15

1968

Sept. 7

3,160

830

Aug. 19

4,320

Sept. 6

1,500

Mar. 31

3,220

1

14.82

1973

Feb. 8

3,620

1975

Aug. 1

19,000

Nov. $9 \quad 3,130$

1977

Mar. 31

1,920

1989

June $9 \quad$ 27,000

2,7

2,7

--

12.70

13.59

20.81

12.43

7.68

22.09

02342500 Uchee Creek near Fort Mitchell

L ocation-Lat $32^{\circ} 19^{\prime} 00^{\prime \prime}$, long $85^{\circ} 00^{\prime} 54^{\prime \prime}$, in SW 114 / sec. 3, T. 15 N., R. 30 E., Russell County, Hydrologic Unit 03130003, on State Highway 165, 2 mi south of Fort Mitchell, 4.8 mi downstream from Little Uchee Creek, and 5.3 mi upstream from mouth.

Drainage area- $322 \mathrm{mi}^{2}$.

Gage -Water-stage recorder. Datum of gage is $201.76 \mathrm{ft}$ NGVD 29. Prior to Sept. 1, 1953, at site 1,000 ft upstream at same datum, and Sept. 1, 1953 to Aug. 15, 1965, at present site at same datum, and Aug. 15, 1965 to Nov. 15, 1990, at site $120 \mathrm{ft}$ upstream at same datum.

\begin{tabular}{|c|c|c|c|c|c|}
\hline $\begin{array}{c}\text { Water } \\
\text { year }\end{array}$ & Date & Discharge & $\begin{array}{l}\text { Discharge } \\
\text { code }\end{array}$ & $\begin{array}{c}\text { Gage } \\
\text { height } \\
\text { (feet) }\end{array}$ & $\begin{array}{c}\text { Gage } \\
\text { height } \\
\text { code }\end{array}$ \\
\hline 1947 & Apr. 3 & 10,300 & & 14.73 & \\
\hline 1948 & July 11 & 13,000 & & 17.31 & \\
\hline 1949 & Nov. 27 & 25,000 & & 23.20 & \\
\hline 1950 & Mar. 7 & 4,270 & & 7.90 & \\
\hline 1951 & Apr. 23 & 1,860 & & 4.95 & \\
\hline 1952 & Mar. 25 & 11,900 & & 16.35 & \\
\hline 1953 & May 1 & 9,290 & & 13.65 & \\
\hline 1954 & Dec. 5 & 9,740 & & 12.40 & \\
\hline 1955 & July 12 & 5,310 & & 7.50 & \\
\hline 1956 & Mar. 17 & 4,680 & & 7.54 & \\
\hline 1957 & Apr. 6 & 11,600 & & 15.10 & \\
\hline 1958 & Mar. 8 & 21,100 & & 22.00 & \\
\hline 1959 & Mar. 6 & 3,200 & 1 & -- & \\
\hline 1960 & Apr. 3 & 9,400 & & 12.50 & \\
\hline 1961 & Feb. 25 & 14,800 & & 17.36 & \\
\hline 1962 & Apr. 13 & 7,430 & & 10.43 & \\
\hline 1963 & Jan. 21 & 4,800 & 1 & -- & \\
\hline 1964 & Apr. 9 & 55,100 & & 26.45 & \\
\hline 1965 & Oct. 6 & 10,200 & & 13.28 & \\
\hline 1966 & Mar. 4 & 16,500 & & 18.73 & \\
\hline 1967 & Jan. 2 & 4,720 & & 7.22 & \\
\hline 1968 & Mar. 13 & 8,910 & & 12.11 & \\
\hline 1969 & Apr. 19 & 11,200 & & 14.24 & \\
\hline 1970 & Mar. 21 & 5,660 & & 8.39 & \\
\hline 1971 & Mar. 4 & 13,500 & & 16.26 & \\
\hline 1972 & Jan. 13 & 3,480 & & 5.84 & \\
\hline 1973 & Dec. 22 & 11,200 & & 14.30 & \\
\hline
\end{tabular}


Appendix 2. Discharge and gage height data for streamgages in Alabama.-Continued

[--, date or data not available; $\mathrm{ft}$, foot; $\mathrm{ft}^{3} / \mathrm{s}$, cubic foot per second; mi, mile; $\mathrm{mi}^{2}$, square mile; qualification codes apply to the discharge data: 1 , discharge is a maximum daily average; 2 , discharge is an estimate; 4 , discharge is less than indicated value, which is the minimum recordable discharge at this site; 5 , discharge affected to unknown degree by regulation or diversion; 6 , discharge affected by regulation or diversion; 7, discharge is a historic peak; A, year of occurrence is unknown or not exact; $\mathrm{B}$, month or day of occurrence is unknown or not exact; E, only maximum peak available for this year. qualification codes apply to the gage height data: 1, gage height affected by backwater; 2, gage height not the maximum for the year; 3, gage height at different datum or at different site and datum; 5 , gage height is an estimate]

02342500 Uchee Creek near Fort Mitchell-Continued

Location-Lat $32^{\circ} 19^{\prime} 00^{\prime \prime}$, long $85^{\circ} 00^{\prime} 54^{\prime \prime}$, in SW 114/ sec. 3, T. 15 N., R. 30 E., Russell County, Hydrologic Unit 03130003, on State Highway 165, 2 mi south of Fort Mitchell, 4.8 mi downstream from Little Uchee Creek, and 5.3 mi upstream from mouth.

Drainage area $-322 \mathrm{mi}^{2}$.

G age - Water-stage recorder. Datum of gage is $201.76 \mathrm{ft}$ NGVD 29. Prior to Sept. 1, 1953, at site 1,000 ft upstream at same datum, and Sept. 1, 1953 to Aug. 15, 1965, at present site at same datum, and Aug. 15, 1965 to Nov. 15, 1990, at site $120 \mathrm{ft}$ upstream at same datum.

\begin{tabular}{|c|c|c|c|c|c|}
\hline $\begin{array}{l}\text { Water } \\
\text { year }\end{array}$ & Date & Discharge & $\begin{array}{c}\text { Discharge } \\
\text { code }\end{array}$ & $\begin{array}{c}\text { Gage } \\
\text { height } \\
\text { (feet) }\end{array}$ & $\begin{array}{c}\text { Gage } \\
\text { height } \\
\text { code }\end{array}$ \\
\hline 1974 & Apr. 5 & 4,430 & & 6.90 & \\
\hline 1975 & Apr. 15 & 10,100 & & 13.25 & \\
\hline 1976 & Apr. 1 & 9,380 & & 12.48 & \\
\hline 1977 & Oct. 8 & 8,630 & & 11.66 & \\
\hline 1978 & Jan. 26 & 15,700 & & 18.01 & \\
\hline 1979 & Apr. 5 & 12,800 & & 15.71 & \\
\hline 1980 & Apr. 14 & 7,350 & & 10.28 & \\
\hline 1981 & Apr. 2 & 18,500 & & 20.26 & \\
\hline 1982 & Feb. 4 & 8,910 & & 12.01 & \\
\hline 1983 & Mar. 6 & 9,850 & & 12.95 & \\
\hline 1984 & Mar. 26 & 5,530 & & 8.23 & \\
\hline 1985 & Feb. 7 & 4,510 & & 7.03 & \\
\hline 1986 & Mar. 19 & 6,200 & & 8.98 & \\
\hline 1987 & Feb. 28 & 8,430 & & 11.49 & \\
\hline 1988 & Feb. 3 & 4,580 & & 7.08 & \\
\hline 1989 & July 4 & 9,750 & & 12.86 & \\
\hline 1990 & Mar. 17 & 24,900 & & 23.18 & \\
\hline 1991 & Mar. 30 & 12,500 & & 15.40 & \\
\hline 1992 & Jan. 14 & 6,720 & & 9.88 & \\
\hline 1993 & Nov. 26 & 26,900 & & 23.64 & \\
\hline 1994 & July 8 & 25,600 & & 23.35 & \\
\hline
\end{tabular}

02342500 Uchee Creek near Fort Mitchell-Continued

L ocation-Lat $32^{\circ} 19^{\prime} 00^{\prime \prime}$, long $85^{\circ} 00^{\prime} 54^{\prime \prime}$, in SW 114/ sec. 3, T. 15 N., R. 30 E., Russell County, Hydrologic Unit 03130003, on State Highway 165, 2 mi south of Fort Mitchell, 4.8 mi downstream from Little Uchee Creek, and 5.3 mi upstream from mouth.

Drainage area-322 $\mathrm{mi}^{2}$.

G age -Water-stage recorder. Datum of gage is $201.76 \mathrm{ft}$ NGVD 29. Prior to Sept. 1, 1953, at site 1,000 ft upstream at same datum, and Sept. 1, 1953 to Aug. 15, 1965, at present site at same datum, and Aug. 15, 1965 to Nov. 15, 1990, at site $120 \mathrm{ft}$ upstream at same datum.

\begin{tabular}{|c|c|c|c|c|c|}
\hline $\begin{array}{c}\text { Water } \\
\text { year }\end{array}$ & Date & Discharge & $\begin{array}{l}\text { Discharge } \\
\text { code }\end{array}$ & $\begin{array}{c}\text { Gage } \\
\text { height } \\
\text { (feet) }\end{array}$ & $\begin{array}{c}\text { Gage } \\
\text { height } \\
\text { code }\end{array}$ \\
\hline 1995 & Feb. 18 & 6,180 & & 9.37 & \\
\hline 1996 & Oct. 5 & 9,640 & & 12.75 & \\
\hline 1997 & Apr. 28 & 9,970 & & 13.08 & \\
\hline 1998 & Mar. 9 & 18,200 & & 19.95 & \\
\hline 1999 & Feb. 1 & 3,620 & & 6.67 & \\
\hline 2000 & Mar. 20 & 4,730 & & 7.92 & \\
\hline 2001 & Mar. 4 & 15,200 & & 17.65 & \\
\hline 2002 & Feb. 7 & 1,600 & & 3.91 & \\
\hline 2003 & July 2 & 8,270 & & 11.32 & \\
\hline 2004 & Sept. 17 & 3,650 & & 6.71 & \\
\hline 2005 & Mar. 28 & 15,000 & & 17.53 & \\
\hline 2006 & Feb. 26 & 5,030 & & 8.24 & \\
\hline 2007 & Mar. 2 & 6,460 & & 9.64 & \\
\hline 2008 & Aug. 24 & 2,530 & & 5.77 & \\
\hline 2009 & Mar. 29 & 12,800 & & 16.36 & \\
\hline 2010 & Nov. 11 & 18,600 & & 19.95 & \\
\hline 2011 & Feb. 5 & 2,990 & & 6.44 & \\
\hline 2012 & Feb. 19 & 3,020 & & 6.48 & \\
\hline 2013 & Feb. 13 & 10,100 & & 14.23 & \\
\hline 2014 & Apr. 8 & 11,600 & & 17.34 & \\
\hline 2015 & Apr. 19 & 3,820 & & 8.60 & \\
\hline
\end{tabular}


Appendix 2. Discharge and gage height data for streamgages in Alabama.-Continued

[--, date or data not available; ft, foot; $\mathrm{ft}^{3} / \mathrm{s}$, cubic foot per second; mi, mile; $\mathrm{mi}^{2}$, square mile; qualification codes apply to the discharge data: 1 , discharge is a maximum daily average; 2 , discharge is an estimate; 4 , discharge is less than indicated value, which is the minimum recordable discharge at this site; 5 , discharge affected to unknown degree by regulation or diversion; 6 , discharge affected by regulation or diversion; 7, discharge is a historic peak; A, year of occurrence is unknown or not exact; $\mathrm{B}$, month or day of occurrence is unknown or not exact; E, only maximum peak available for this year. qualification codes apply to the gage height data: 1, gage height affected by backwater; 2, gage height not the maximum for the year; 3, gage height at different data or at different site and datum;

5 , gage height is an estimate]

02342150 Uchee Creek near Seale

L ocation-Lat $32^{\circ} 21^{\prime} 16^{\prime \prime}$, long $85^{\circ} 05^{\prime} 44^{\prime \prime}$, in NE $1 / 4$ sec. 26 , T. 16 N., R. 29 E., Russell County, Hydrologic Unit 03130003, at bridge on U.S. Highway 431, 6 mi northeast of Seale.

Drainage area-162 $\mathrm{mi}^{2}$.

Gage Crest-stage gage. Datum of gage is $275 \mathrm{ft}$ NGVD 29.

\begin{tabular}{|c|c|c|c|c|c|}
\hline $\begin{array}{c}\text { Water } \\
\text { year }\end{array}$ & Date & Discharge & $\begin{array}{l}\text { Discharge } \\
\text { code }\end{array}$ & $\begin{array}{c}\text { Gage } \\
\text { height } \\
\text { (feet) }\end{array}$ & $\begin{array}{c}\text { Gage } \\
\text { height } \\
\text { code }\end{array}$ \\
\hline 1951 & Mar. 20 & 761 & & 5.92 & \\
\hline 1952 & Mar. 25 & 5,590 & & 10.90 & \\
\hline 1953 & May 1 & 4,340 & & 10.40 & \\
\hline 1954 & Dec. 5 & 4,340 & & 10.40 & \\
\hline 1955 & July 12 & 3,450 & & 9.90 & \\
\hline 1956 & Mar. 17 & 2,000 & & 9.00 & \\
\hline 1957 & Apr. 5 & 6,140 & & 11.10 & \\
\hline 1958 & Mar. 8 & 14,100 & & 13.10 & \\
\hline 1959 & June 2 & 3,450 & & 9.90 & \\
\hline 1960 & Apr. 3 & 5,340 & & 10.80 & \\
\hline 1961 & Feb. 25 & 6,740 & & 11.29 & \\
\hline 1962 & Feb. 23 & 3,480 & & 9.93 & \\
\hline 1963 & Mar. 6 & 2,300 & & 9.20 & \\
\hline 1964 & Apr. 9 & 19,500 & & 14.06 & \\
\hline 1965 & Oct. 6 & 6,020 & & 11.06 & \\
\hline 1966 & Mar. 3 & 7,860 & & 11.65 & \\
\hline 1967 & Jan. 2 & 2,600 & & 9.39 & \\
\hline 1968 & Mar. 12 & 3,220 & & 9.85 & \\
\hline 1969 & Apr. 19 & 12,000 & & 12.69 & \\
\hline 1970 & Mar. 21 & 753 & & 5.88 & \\
\hline
\end{tabular}

02419000 Uphapee Creek near Tuskegee

L ocation-Lat $32^{\circ} 28^{\prime} 36^{\prime \prime}$, long 85 $41^{\prime} 42^{\prime \prime}$, in NE 1/4 sec. 12, T. 17 N., R. 23 E., Macon County, Hydrologic Unit 03150110, on State Highway 81, 1 mi upstream from Red Creek, and 4 mi north of Tuskegee.

Drainage area-333 $\mathrm{mi}^{2}$.

G age -Water-stage recorder. Datum of gage is $223.65 \mathrm{ft}$ NGVD 29.

\begin{tabular}{llrccc}
\hline $\begin{array}{c}\text { Water } \\
\text { year }\end{array}$ & Date & Discharge & $\begin{array}{c}\text { Discharge } \\
\text { code }\end{array}$ & $\begin{array}{c}\text { Gage } \\
\text { height } \\
\text { (feet) }\end{array}$ & $\begin{array}{c}\text { Gage } \\
\text { height } \\
\text { code }\end{array}$ \\
\hline 1929 & -- & 37,000 & $2,7, B$ & 30.00 & 5 \\
1940 & July 4 & 7,300 & & 15.30 & \\
1941 & Aug. 14 & 7,900 & & 15.80 &
\end{tabular}

1941 Aug. $14 \quad 7,900$

1942 Mar. $22 \quad$ 13,300 20.67

1943 Mar. $21 \quad 29,600 \quad 27.33$

1944 Apr. $27 \quad 26,500 \quad 26.20$

$1945 \quad$ Feb. $21 \quad 8,800 \quad 16.96$

1946 Jan. $7 \quad 15,200$

1947 Apr. $3 \quad 7,620 \quad 15.60$

$1948 \quad$ July $11 \quad 8,400 \quad 16.50$

$1949 \quad$ Nov. $27 \quad 29,600 \quad 27.30$

$1950 \quad$ Mar. $7 \quad 2,950 \quad 9.58$

$1951 \quad$ Mar. $20 \quad 1,430 \quad 6.47$

1952 Mar. $25 \quad 9,800 \quad 18.00$

1953 May $5 \quad 8,390 \quad 16.30$

1954 Dec. $5 \quad 12,400 \quad 20.40$

1955 Apr. $15 \quad 4,740 \quad 12.17$

1956 Mar. $17 \quad 5,890 \quad 13.64$

1957 Apr. $6 \quad 11,100 \quad 19.10$

1958 Mar. $8 \quad 20,400 \quad 23.70$

1959 Mar. $7 \quad 2,930 \quad 9.70$

1960 Apr. $4 \quad 7,250 \quad 15.06$

$1961 \quad$ Feb. $25 \quad 25,500 \quad 25.82$

1962 Apr. $1 \quad 6,700 \quad 14.53$

$1963 \quad$ Jan. $21 \quad 6,340 \quad 14.12$

1964 Apr. $9 \quad 32,200 \quad 28.18$

1965 Mar. $19 \quad 6,030 \quad 13.39$

1966 Mar. $4 \quad 12,500 \quad 20.14$

1967 Jan. $3 \quad 3,080 \quad 9.72$

1968 Mar. $13 \quad 6,620 \quad 14.42$

1969 Apr. $19 \quad 6,650 \quad 14.46$ 
Appendix 2. Discharge and gage height data for streamgages in Alabama.-Continued

[--, date or data not available; $\mathrm{ft}$, foot; $\mathrm{ft}^{3} / \mathrm{s}$, cubic foot per second; mi, mile; $\mathrm{mi}^{2}$, square mile; qualification codes apply to the discharge data: 1 , discharge is a maximum daily average; 2 , discharge is an estimate; 4 , discharge is less than indicated value, which is the minimum recordable discharge at this site; 5 , discharge affected to unknown degree by regulation or diversion; 6 , discharge affected by regulation or diversion; 7, discharge is a historic peak; A, year of occurrence is unknown or not exact; $\mathrm{B}$, month or day of occurrence is unknown or not exact; E, only maximum peak available for this year. qualification codes apply to the gage height data: 1, gage height affected by backwater; 2, gage height not the maximum for the year; 3, gage height at different datum or at different site and datum; 5 , gage height is an estimate]

02419000 Uphapee Creek near Tuskegee-Continued

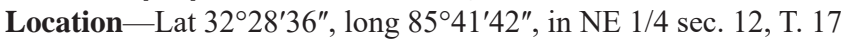
N., R. 23 E., Macon County, Hydrologic Unit 03150110, on State Highway 81, 1 mi upstream from Red Creek, and 4 mi north of

Tuskegee.

Drainage area $333 \mathrm{mi}^{2}$.

Gage -Water-stage recorder. Datum of gage is $223.65 \mathrm{ft}$ NGVD 29.

\begin{tabular}{|c|c|c|c|c|c|}
\hline $\begin{array}{c}\text { Water } \\
\text { year }\end{array}$ & Date & Discharge & $\begin{array}{l}\text { Discharge } \\
\text { code }\end{array}$ & $\begin{array}{c}\text { Gage } \\
\text { height } \\
\text { (feet) }\end{array}$ & $\begin{array}{c}\text { Gage } \\
\text { height } \\
\text { code }\end{array}$ \\
\hline 1970 & Mar. 21 & 11,500 & & 19.13 & \\
\hline 1971 & Mar. 3 & 13,400 & & 21.02 & \\
\hline 1972 & Mar. 3 & 3,650 & & 10.75 & \\
\hline 1973 & Apr. 1 & 11,000 & & 18.98 & \\
\hline 1974 & Jan. 31 & 3,200 & & 9.97 & \\
\hline 1975 & Apr. 15 & 10,600 & & 18.65 & \\
\hline 1976 & May 16 & 9,050 & & 17.00 & \\
\hline 1977 & Mar. 31 & 7,480 & & 15.35 & \\
\hline 1978 & Jan. 26 & 14,700 & & 21.63 & \\
\hline 1979 & Apr. 5 & 12,000 & & 20.01 & \\
\hline 1980 & Mar. 30 & 8,225 & & 16.26 & \\
\hline 1981 & Apr. 2 & 16,000 & & 22.23 & \\
\hline 1982 & Feb. 4 & 9,330 & & 17.29 & \\
\hline 1983 & Mar. 7 & 9,630 & & 17.61 & \\
\hline 1984 & Mar. 26 & 5,480 & & 13.13 & \\
\hline 1985 & July 29 & 3,230 & $\mathrm{E}$ & 10.06 & \\
\hline 1986 & Mar. 20 & 7,500 & & 15.39 & \\
\hline 1987 & Mar. 1 & 6,680 & & 14.49 & \\
\hline 1988 & Feb. 5 & 4,130 & & 11.37 & \\
\hline 1989 & June 16 & 8,870 & & 16.10 & \\
\hline 1990 & Mar. 17 & 28,400 & & 26.87 & \\
\hline 1991 & Mar. 30 & 11,700 & & 18.68 & \\
\hline 1992 & Jan. 14 & 6,760 & & 14.03 & \\
\hline
\end{tabular}

02419000 Uphapee Creek near Tuskegee-Continued

Location-Lat $32^{\circ} 28^{\prime} 36^{\prime \prime}$, long 85'41'42", in NE 1/4 sec. 12, T. 17 N., R. 23 E., Macon County, Hydrologic Unit 03150110, on State Highway 81, 1 mi upstream from Red Creek, and 4 mi north of Tuskegee.

Drainage area-333 $\mathrm{mi}^{2}$.

G age - Water-stage recorder. Datum of gage is $223.65 \mathrm{ft}$ NGVD 29.

\begin{tabular}{llllll}
\hline $\begin{array}{c}\text { Water } \\
\text { year }\end{array}$ & Date & Discharge & $\begin{array}{c}\text { Discharge } \\
\text { code }\end{array}$ & $\begin{array}{c}\text { Gage } \\
\text { height } \\
\text { (feet) }\end{array}$ & $\begin{array}{c}\text { Gage } \\
\text { height } \\
\text { code }\end{array}$ \\
\hline 1993 & Nov. 26 & 22,400 & & 24.50 & \\
1994 & July 8 & 23,200 & & 24.83 &
\end{tabular}

$1994 \quad$ July $8 \quad 23,200 \quad 24.83$

1995 Feb. $18 \quad 6,240 \quad 12.94$

$1996 \quad$ Mar. $7 \quad 10,600 \quad 17.60$

1997 Apr. $29 \quad 7,810 \quad 14.79$

1998 Mar. $9 \quad 21,500 \quad 24.07$

1999 Mar. $15 \quad 3,930 \quad 9.93$

2000 Mar. $21 \quad 2,580 \quad 7.83$

$2001 \quad$ Mar. $5 \quad$ 15,600 21.21

2002 Apr. $14 \quad 1,930 \quad 6.84$

$2003 \quad$ July $2 \quad 7,460 \quad 14.39$

2005 Mar. $28 \quad 17,600 \quad 22.23$

$2006 \quad$ Feb. $26 \quad 4,970 \quad 11.33$

2007 Mar. $3 \quad 5,270 \quad 11.71$

2008 Apr. $6 \quad 1,600 \quad 6.27$

2009 Mar. $29 \quad 8,100 \quad 15.11$

2010 Nov. $11 \quad 13,700 \quad 20.12$

2011 Mar. $11 \quad 1,710 \quad 6.47$

2012 Feb. $20 \quad 2,450 \quad 7.65$

2013 Feb. $13 \quad 9,050 \quad 16.10$

2014 Apr. $7 \quad 12,900 \quad 19.64$

2015 May $30 \quad 5,470 \quad 11.96$ 
Appendix 2. Discharge and gage height data for streamgages in Alabama.-Continued

[--, date or data not available; $\mathrm{ft}$, foot; $\mathrm{ft}^{3} / \mathrm{s}$, cubic foot per second; $\mathrm{mi}$, mile; $\mathrm{mi}^{2}$, square mile; qualification codes apply to the discharge data: 1 , discharge is a maximum daily average; 2 , discharge is an estimate; 4 , discharge is less than indicated value, which is the minimum recordable discharge at this site; 5 , discharge affected to unknown degree by regulation or diversion; 6 , discharge affected by regulation or diversion; 7, discharge is a historic peak; A, year of occurrence is unknown or not exact; B, month or day of occurrence is unknown or not exact; E, only maximum peak available for this year. qualification codes apply to the gage height data: 1, gage height affected by backwater; 2, gage height not the maximum for the year; 3, gage height at different data or at different site and datum; 5 , gage height is an estimate]

\section{West Fork Choctawhatchee River at Blue Springs}

L ocation-Lat $31^{\circ} 39^{\prime} 49^{\prime \prime}$ long $87^{\circ} 30^{\prime} 18^{\prime \prime}$, in SE $1 / 4$ sec. 14 , T. 8 N., R. 25 E., Barbour County, Hydrologic Unit 03140201, on State Highway 10 at Blue Springs, 4 mi downstream from Lindsey Creek.

Drainage area $-86.8 \mathrm{mi}^{2}$.

Gage-Water-stage recorder 1944-53. Crest-stage gage 1954-71. Datum of gage is $289.24 \mathrm{ft}$ NGVD 29.

\begin{tabular}{|c|c|c|c|c|c|}
\hline $\begin{array}{l}\text { Water } \\
\text { year }\end{array}$ & Date & Discharge & $\begin{array}{c}\text { Discharge } \\
\text { code }\end{array}$ & $\begin{array}{c}\text { Gage } \\
\text { height } \\
\text { (feet) }\end{array}$ & $\begin{array}{c}\text { Gage } \\
\text { height } \\
\text { code }\end{array}$ \\
\hline 1944 & Mar. 29 & 4,820 & & 9.10 & \\
\hline 1945 & Mar. 21 & 470 & & 4.68 & \\
\hline 1946 & Mar. 28 & 3,310 & & 7.92 & \\
\hline 1947 & June 23 & 2,280 & & 6.98 & \\
\hline 1948 & Mar. 7 & 1,660 & & 6.33 & \\
\hline 1949 & Nov. 27 & 2,550 & & 7.33 & \\
\hline 1950 & Apr. 5 & 586 & & 4.90 & \\
\hline 1951 & Mar. 30 & 970 & & 5.48 & \\
\hline 1952 & Mar. 24 & 1,900 & & 6.72 & \\
\hline 1953 & Apr. 10 & 3,730 & & 8.27 & \\
\hline 1954 & Dec. 4 & 2,850 & & 7.60 & \\
\hline 1955 & Feb. 7 & 2,510 & & 7.30 & \\
\hline 1956 & Sept. 26 & 9,200 & & 11.50 & \\
\hline 1957 & Apr. 6 & 7,100 & & 10.30 & \\
\hline 1958 & Mar. 2 & 630 & & 5.20 & \\
\hline
\end{tabular}

\section{West Fork Choctawhatchee River at Blue Springs-} Continued

L ocation-Lat $31^{\circ} 39^{\prime} 49^{\prime \prime}$ long $87^{\circ} 30^{\prime} 18^{\prime \prime}$, in SE 1/4 sec. 14, T. 8 N., R. 25 E., Barbour County, Hydrologic Unit 03140201, on State Highway 10 at Blue Springs, 4 mi downstream from Lindsey Creek.

Drainage area $-86.8 \mathrm{mi}^{2}$.

Gage -Water-stage recorder 1944-53. Crest-stage gage 1954-71. Datum of gage is $289.24 \mathrm{ft}$ NGVD 29.

\begin{tabular}{|c|c|c|c|c|c|}
\hline $\begin{array}{l}\text { Water } \\
\text { year }\end{array}$ & Date & Discharge & $\begin{array}{c}\text { Discharge } \\
\text { code }\end{array}$ & $\begin{array}{c}\text { Gage } \\
\text { height } \\
\text { (feet) }\end{array}$ & $\begin{array}{c}\text { Gage } \\
\text { height } \\
\text { code }\end{array}$ \\
\hline 1959 & Feb. 5 & 1,090 & & 5.80 & \\
\hline 1960 & Apr. 4 & 3,470 & & 8.10 & \\
\hline 1961 & Apr. 15 & 1,470 & & 6.24 & \\
\hline 1962 & Apr. 1 & 1,460 & & 6.23 & \\
\hline 1963 & Jan. 21 & 1,900 & & 6.70 & \\
\hline 1964 & Mar. 3 & 1,110 & & 5.82 & \\
\hline 1965 & Oct. 5 & 2,000 & & 6.80 & \\
\hline 1966 & Mar. 4 & 2,140 & & 6.94 & \\
\hline 1967 & Jan. 3 & 3,330 & & 7.99 & \\
\hline 1968 & Nov. 1 & 1,320 & & 6.08 & \\
\hline 1969 & Mar. 24 & 930 & & 5.60 & \\
\hline 1970 & Mar. 31 & 2,580 & & 7.36 & \\
\hline 1971 & Mar. 26 & 2,490 & & 7.28 & \\
\hline 1990 & Mar. 17 & 25,000 & 7 & 17.32 & \\
\hline 1994 & July 6 & 6,800 & 7 & 10.20 & \\
\hline
\end{tabular}

For more information about this publication, contact

Director, Lower Mississippi-Gulf Water Science Center

U.S. Geological Survey

640 Grassmere Park, Suite 100

Nashville, TN 37211

For additional information, visit

https://www.usgs.gov/centers/lmg-water/

Publishing support provided by

Lafayette Publishing Service Center 



\section{$\frac{12}{80}$}

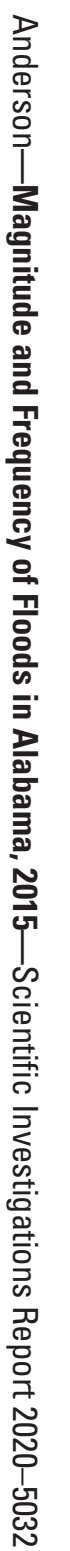

